\title{
Synthesis and Eualuation of Potential Ligands for Nuclear Waste Processing
}

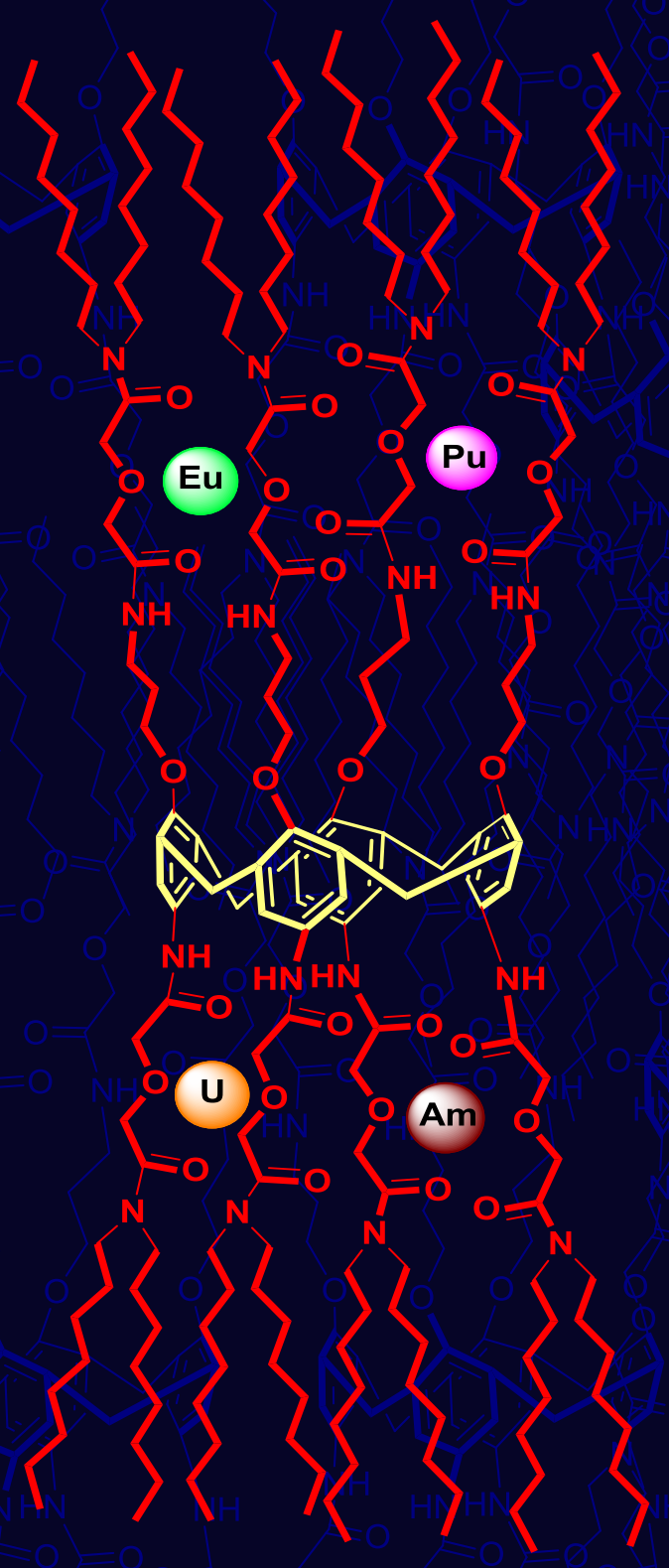

Mudassir labal 
Synthesis and Evaluation of Potential Ligands for Nuclear Waste Processing

Mudassir Iqbal 
Thesis Committee members:

Prof. dr. J. F. J. Engbersen University of Twente (Chairman)

Prof. dr. ir. J. Huskens $\quad$ University of Twente (Promotor)

Dr. W. Verboom University of Twente (Assistant-Promotor)

Prof. dr. J. J. L. M. Cornelissen University of Twente

Prof. dr. D. W. Grijpma University of Twente

Prof. dr. P. J. Dijkstra Soochow University, Suzhou, China

Prof. dr. ir. R. G. H. Lammertink University of Twente

Dr. G. Modolo RWTH Aachen University

The Dean of the faculty is prof. dr. G. van der Steenhoven.

The research work described in this thesis was carried out at the Molecular Nanofabrication group, University of Twente, The Netherlands. The research work was partially financed by the Higher Education Commission of Pakistan.

Publisher: Ipskamp Drukkers, Enschede, The Netherlands.

ISBN: 10.3990/1.9789036534291

doi: 10.3990/1.9789036534291

URL: http://dx.doi.org/10.3990/1.9789036534291

Copyright (C) Mudassir Iqbal, Enschede, 2012

All rights reserved. No part of this work may be reproduced by print, photocopy or any other means without prior permission in writing from the author. 


\title{
SYNTHESIS AND EVALUATION OF POTENTIAL LIGANDS FOR NUCLEAR WASTE PROCESSING
}

\author{
DISSERTATION \\ to obtain \\ the degree of doctor at the University of Twente, \\ on the authority of the rector magnificus, \\ prof. dr. H. Brinksma, \\ on account of the decision of the graduation committee, \\ to be publicly defended \\ on Thursday $18^{\text {th }}$ October, 2012 at $16.45 \mathrm{~h}$
}

by

\section{Mudassir Iqbal}

born on $1^{\text {st }}$ March, 1980

in Sargodha, Pakistan 
This dissertation has been approved by:

Promotor: Prof. dr. ir. Jurriaan Huskens

Assistant-promotor: Dr. Willem Verboom 
Q Dedicated to my Parents. for their CLave, Endless Support and Encouragement. 



\section{Table of Contents}

Chapter 1 General Introduction .................................................................................................................. 1

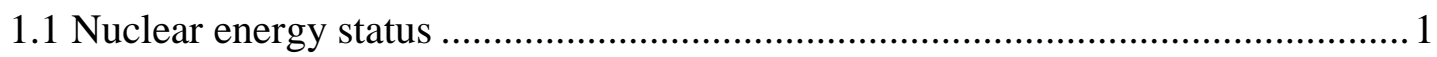

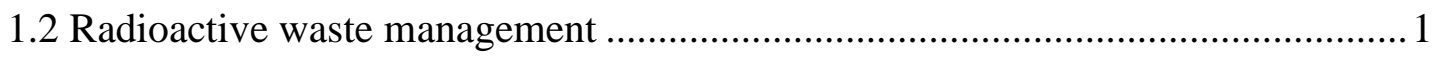

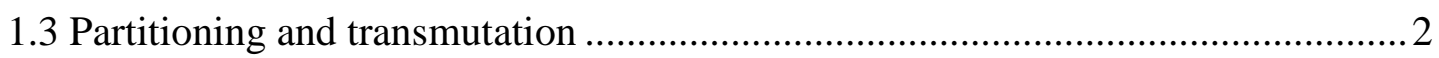

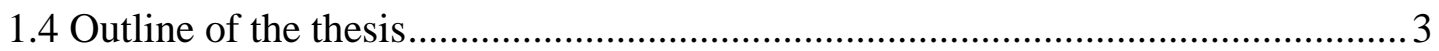

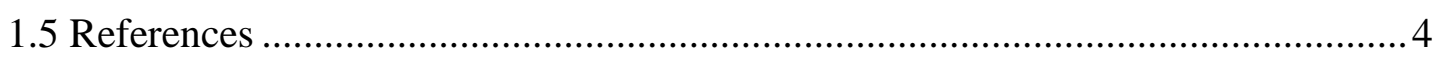

Chapter 2 Ligands for the Partitioning of Nuclear Waste..........................................................5

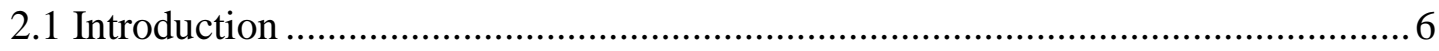

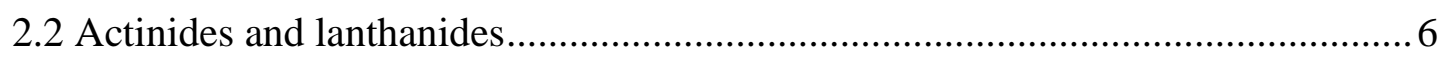

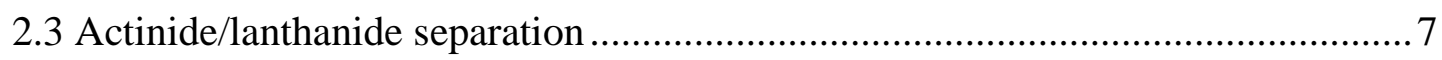

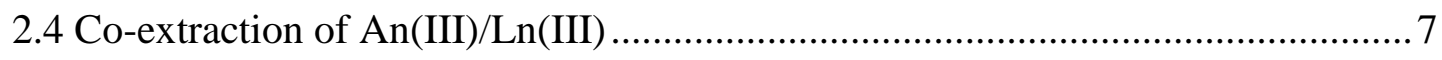

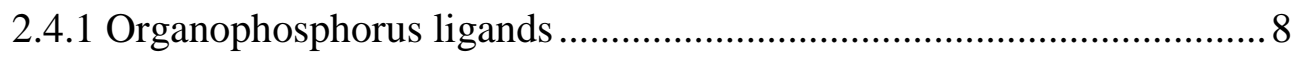

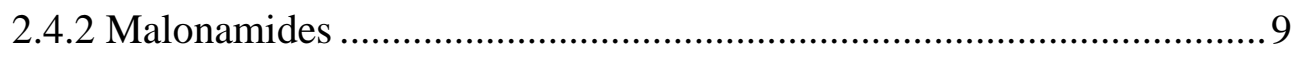

2.4.3 Glycolamides........................................................................... 10

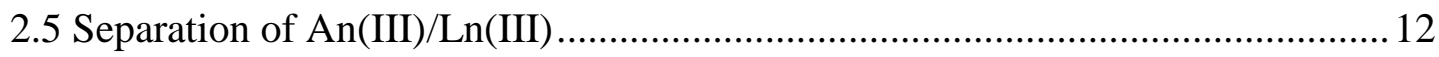

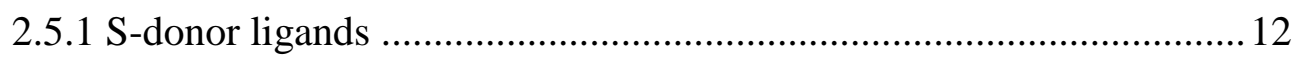

2.5.1.1 Extraction by thiophosphinic/phosphoric acids ................... 13

2.5.1.2 Radiation stability of thiophosphinic acids .......................... 19

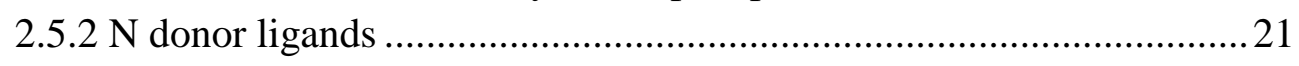

2.5.3 Extraction by synergistic mixtures ….......................................... 26

2.6 Water-soluble ligands/reagents for back-extraction ......................................... 31

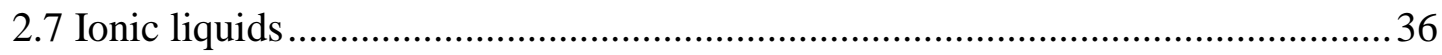

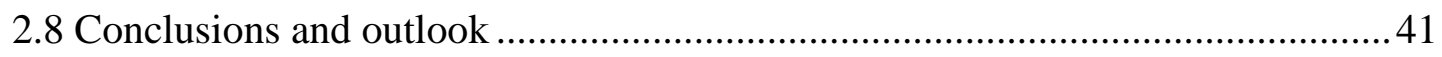

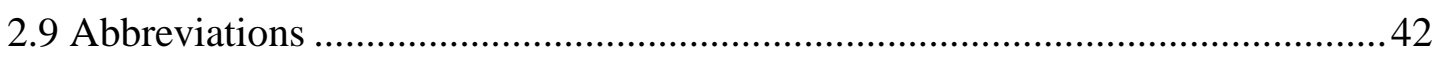

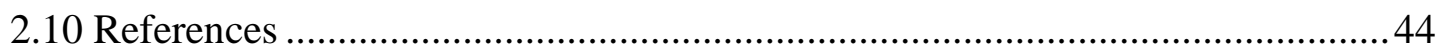

Chapter 3 Synthesis and Am/Eu extraction of Novel TODGA Derivatives................55

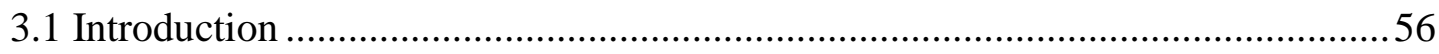

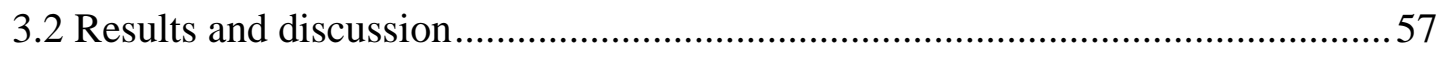

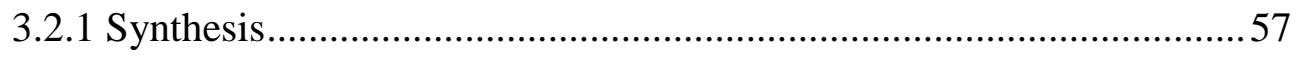

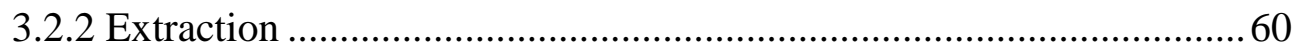

3.2.2.1 Extraction with central oxygen containing ligands ...............60 60

3.2.2.2 Extraction with central nitrogen containing ligands..............6 63

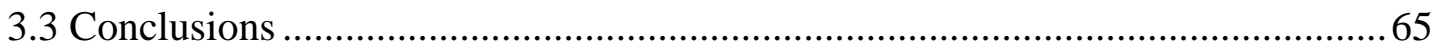

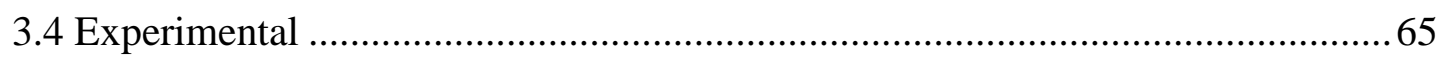

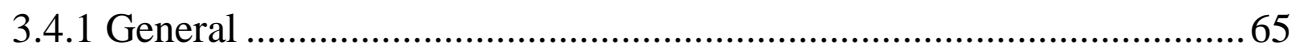

3.4.2 Extraction procedure .............................................................. 73

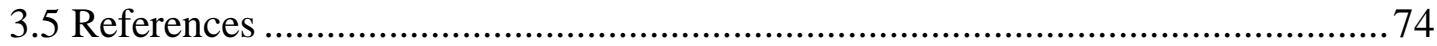




\section{Chapter 4 Synthesis and Evaluation of Novel Water-soluble Ligands for}

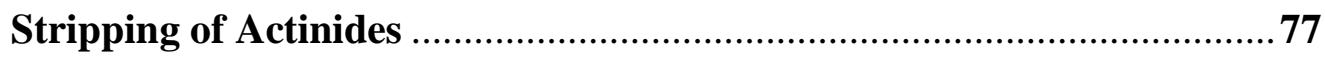

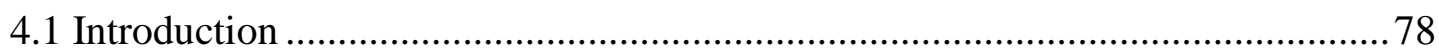

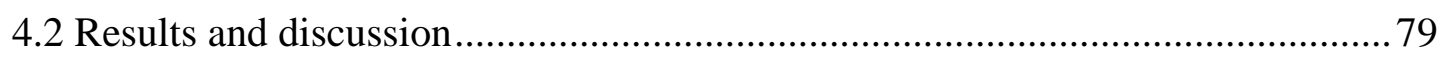

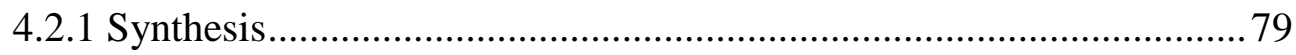

4.2.1.1 Water soluble TODGA-derived ligands................................ 79

4.2.1.2 Synthesis of S/O-containing ligands ...................................... 80

4.2.1.3 Synthesis of tripodal ligands ............................................. 82

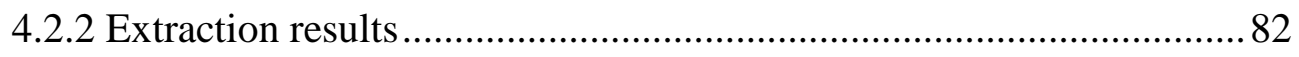

4.2.2.1 Water soluble TODGA-derived ligands............................... 83

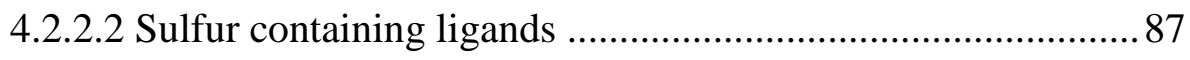

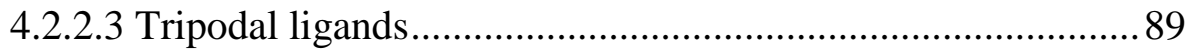

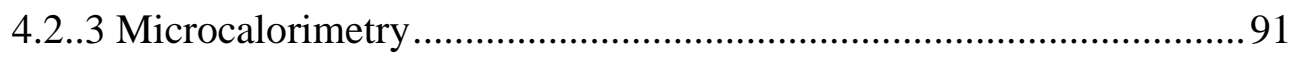

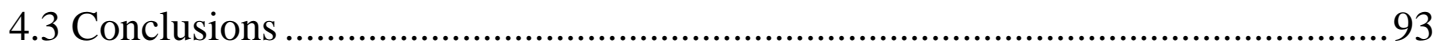

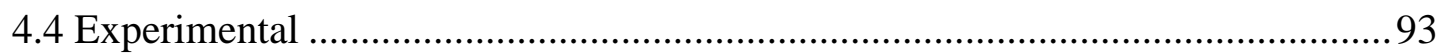

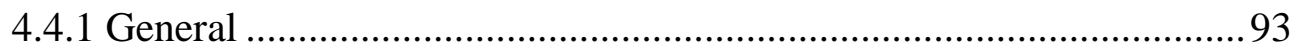

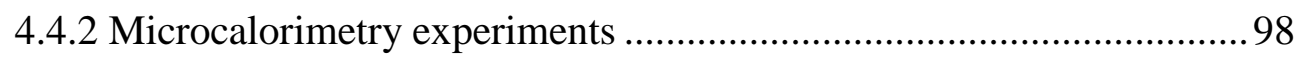

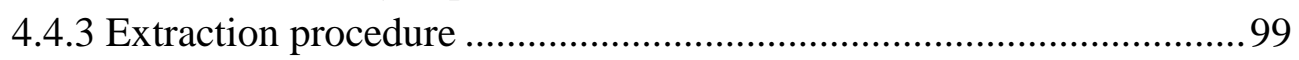

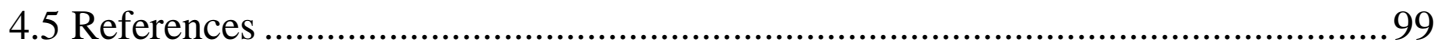

\section{Chapter 5 Preorganization of Diglycolamides on the Calix[4]arene}

Platform and its Effect on the Extraction of Am(III)/Eu(III) ................................103

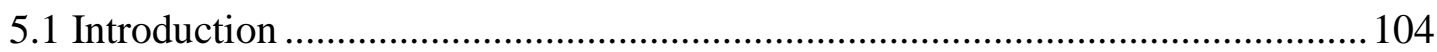

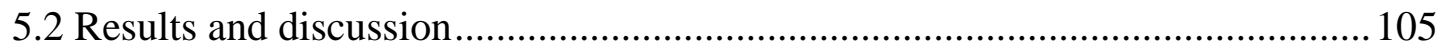

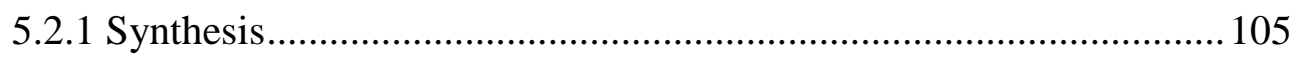

5.2.1.1 Narrow rim DGA-functionalized calix[4]arenes.................. 105

5.2.1.2 Narrow rim N-substituted DGA-functionalized

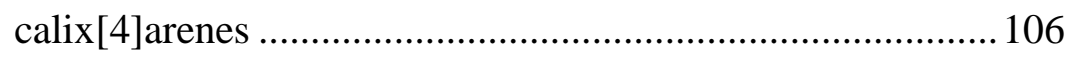

5.2.1.3 Wide rim DGA-functionalized calix[4]arenes .................... 108

5.2.1.4 Wide and narrow rim DGA-functionalized calix[4]arenes .. 108

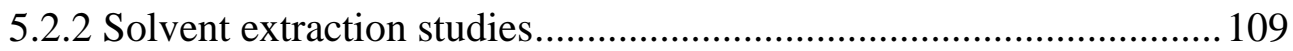

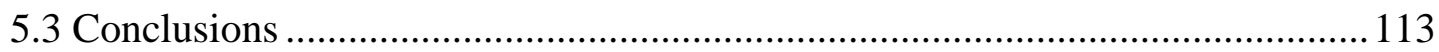

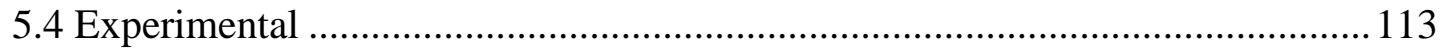

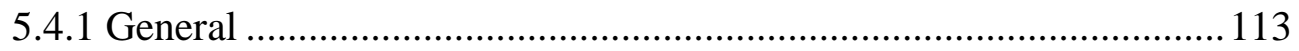

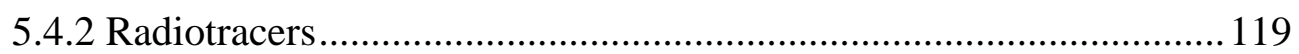

5.4.3 Solvent extraction studies.............................................................. 120

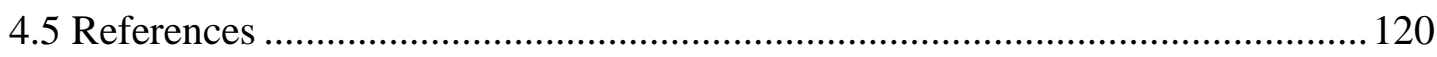


Chapter 6 Synthesis and Evaluation of Ligands with Mixed Amide and Phosphonate, Phosphinoxide, and Phosphonothioate Sites for An(III)/Ln(III) Extraction 123

6.1 Introduction 124

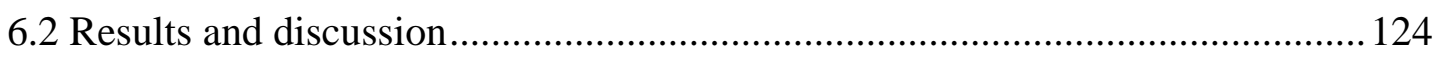

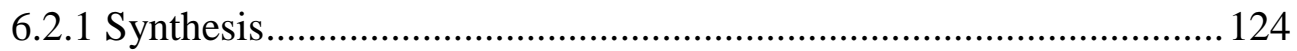

6.2.1.1 Mixed amides and $\mathrm{P}=\mathrm{O}$ ligands with central oxygen........... 124

6.2.1.2 Mixed amides and $\mathrm{P}=\mathrm{O}$ ligands with central nitrogen ......... 125

6.2.1.3 Tripodal mixed amides and phosphonates ......................... 126

6.2.1.4 $\mathrm{P}=\mathrm{S}$ containing ligands with central oxygen atom .............. 127

6.2.1.5 Methylene-bridged $\mathrm{P}=\mathrm{S}$ containing ligands ........................ 129

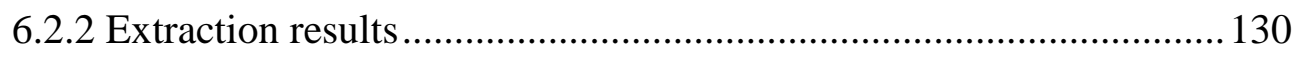

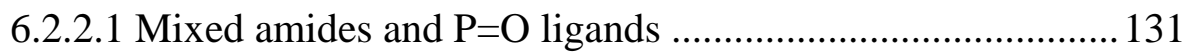

6.2.2.2 Tripodal mixed amides and phosphonates .......................... 133

6.2.2.3 $\mathrm{P}=\mathrm{S}$ containing ligands with central oxygen atom .............. 134

6.2.2.4 Methylene-bridged $\mathrm{P}=\mathrm{S}$ containing ligands ........................ 135

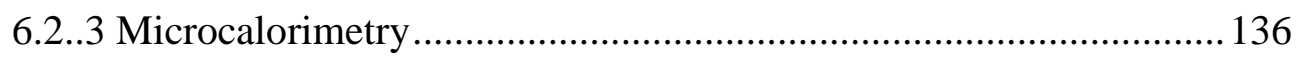

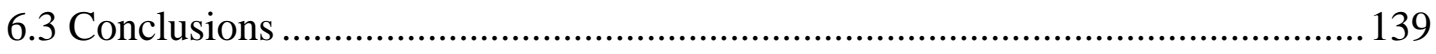

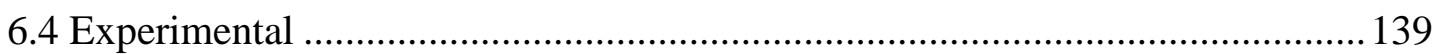

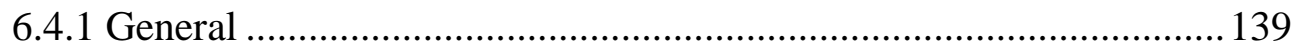

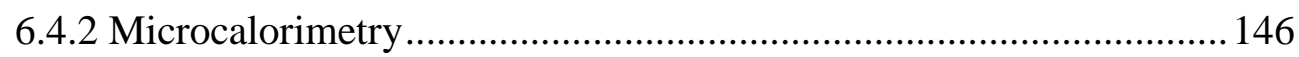

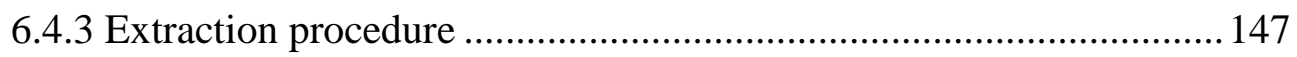

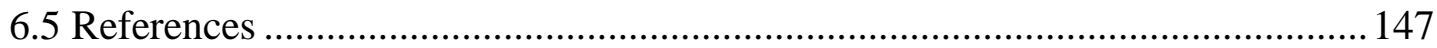

\section{Chapter 7 Extraction of Actinides by Tripodal and Calix[4]arene Diglycolamides} in Room Temperature Ionic Liquids ..........................................................................149

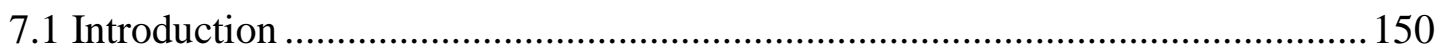

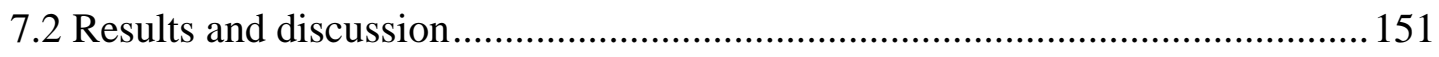

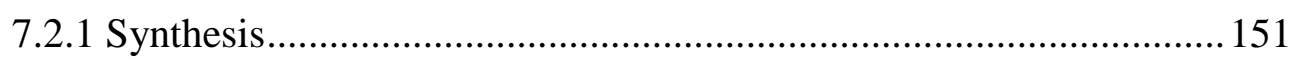

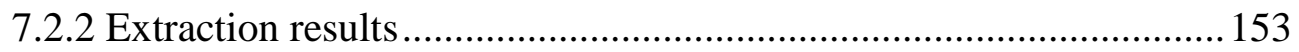

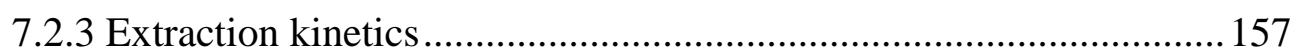

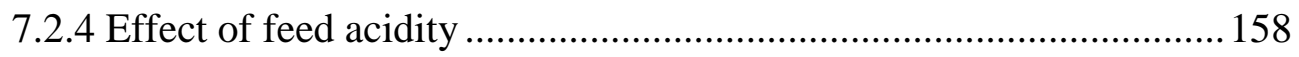

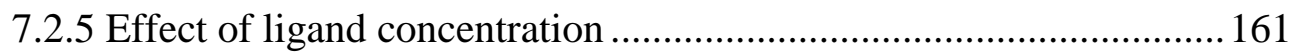

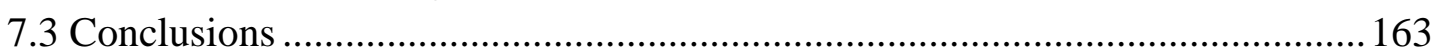

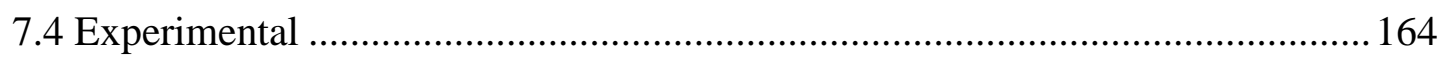

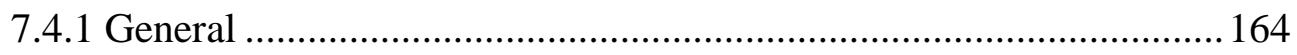

7.4.2 Synthesis of tripodal diglycolamide ………...................................... 164

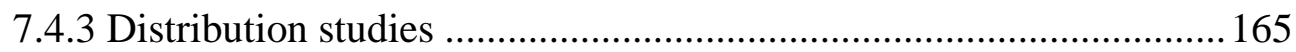

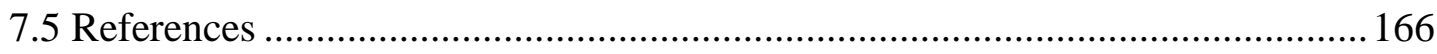


Chapter 8 DGA- and CMPO-based Task-Specific Ionic Liquids for Efficient Extraction of $\mathrm{An}(\mathrm{III}) / \mathrm{Ln}(\mathrm{III})$.......................................................................169

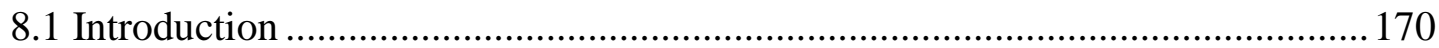

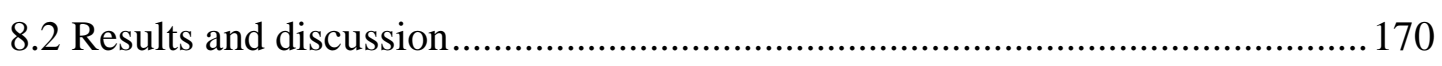

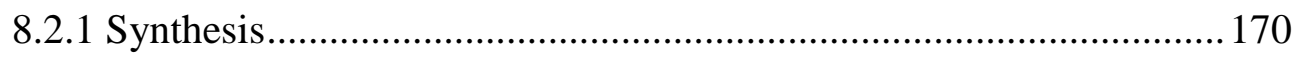

8.2.1.1 Diglycolamide-functionalized ionic liquids ....................... 170

8.2.1.2 CMPO-functionalized ionic liquids ................................. 171

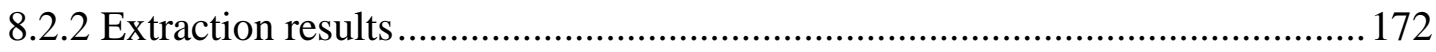

8.2.2.1 Extraction with DGA-FILs.............................................. 172

8.2.2.2 Extraction with CMPO-FILs ............................................... 177

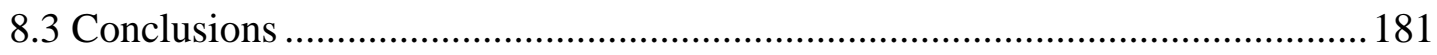

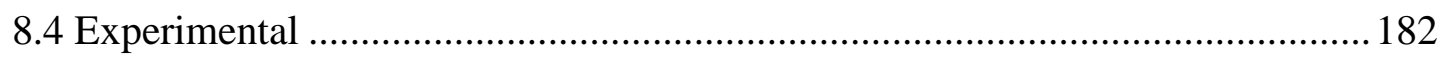

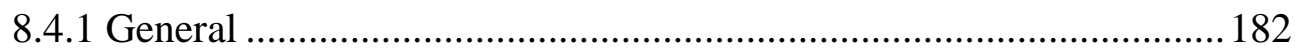

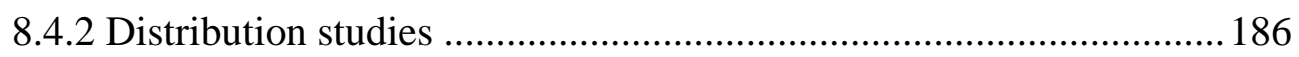

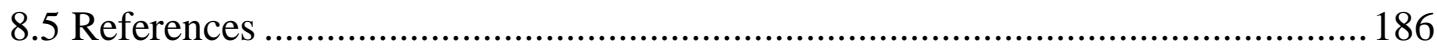

Summary and Outlook ..................................................................................................................................189

Samenvatting en Vooruitblik ......................................................................................................................193

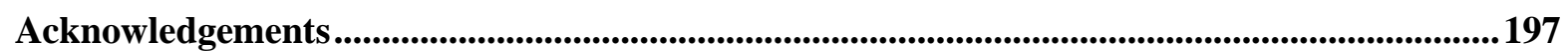

List of Publications................................................................................................................................................201

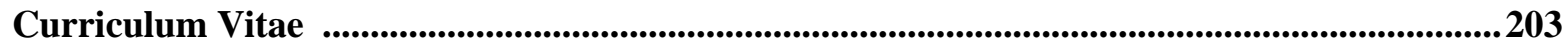




\section{Chapter}

\section{General Introduction}

\subsection{Nuclear energy status}

Nuclear energy is one of the alternative resources to meet the ever-increasing demands for energy, because of its non- $\mathrm{CO}_{2}$-emitting property in combination with the limited reserves of fossil fuels, their concomitantly increasing prices and their associated environmental and climatological risks. Currently, nuclear energy plants produce slightly less than $14 \%$ of the world's electricity and $5.7 \%$ of the total primary energy used worldwide. A total number of 441 nuclear reactors are working with a nuclear power capacity of $375 \mathrm{GW}(\mathrm{e})$. In addition, 67 reactors are under construction. ${ }^{1}$ Amongst these 67 , the construction of 16 new power reactors started in 2010. Revised projections of future nuclear power growth still indicate high expectations for nuclear power expansion. In the context of climate change concerns, as well as improved safety and performance records, some 65 countries are expressing interest in, considering, or actively planning for nuclear power. Nuclear power is projected to be consistently cheaper than other energy sources. ${ }^{2}$

\subsection{Radioactive waste management}

The high level waste (HLW) generated during the production of nuclear energy is of major concern. The annual accumulation of high level waste (HLW) with an average accumulation rate worldwide is approximately 850 cubic meters per year. ${ }^{2}$ This HLW, generated during spent nuclear fuel reprocessing, contains un-extracted $\mathrm{U}, \mathrm{Pu}$, minor actinides such as $\mathrm{Am}, \mathrm{Np}$, $\mathrm{Cm}$, lanthanides, fission product elements such as Tc, Pd, Zr, I, Cs, and Sr, transition metal elements including $\mathrm{Fe}, \mathrm{Ni}, \mathrm{Co}, \mathrm{Zr}$, and some salts of $\mathrm{Fe}, \mathrm{Al}$, and $\mathrm{Na}$. At present, the most accepted approach for the management of HLW is to vitrify the waste in a glass matrix followed by interim storage for $\sim 100$ years to allow the decay of heat-dissipating nuclides 


\section{Chapter 1}

such as ${ }^{137} \mathrm{Cs}$ and ${ }^{90} \mathrm{Sr}$ and its subsequent disposal in deep geological repositories. ${ }^{3}$ The halflives of a few minor actinides and some fission product elements range between a few hundreds to millions of years. Therefore, storing vitrified blocks for such a long period is not favorable from an economic, as well as an environmental safety point of view. There is also the possible risk of the migration of long-lived $\alpha$-emitting minor actinides from the repository to the aquatic environment, as in the past, more than three million liters of highly radioactive waste leaked into the surrounding soil of the Columbia Basin. ${ }^{4}$ As shown in Figure 1.1, if the actinides are not removed from the spent fuel, it will require millions of years to reduce its radiotoxicity to the level of natural uranium ore.
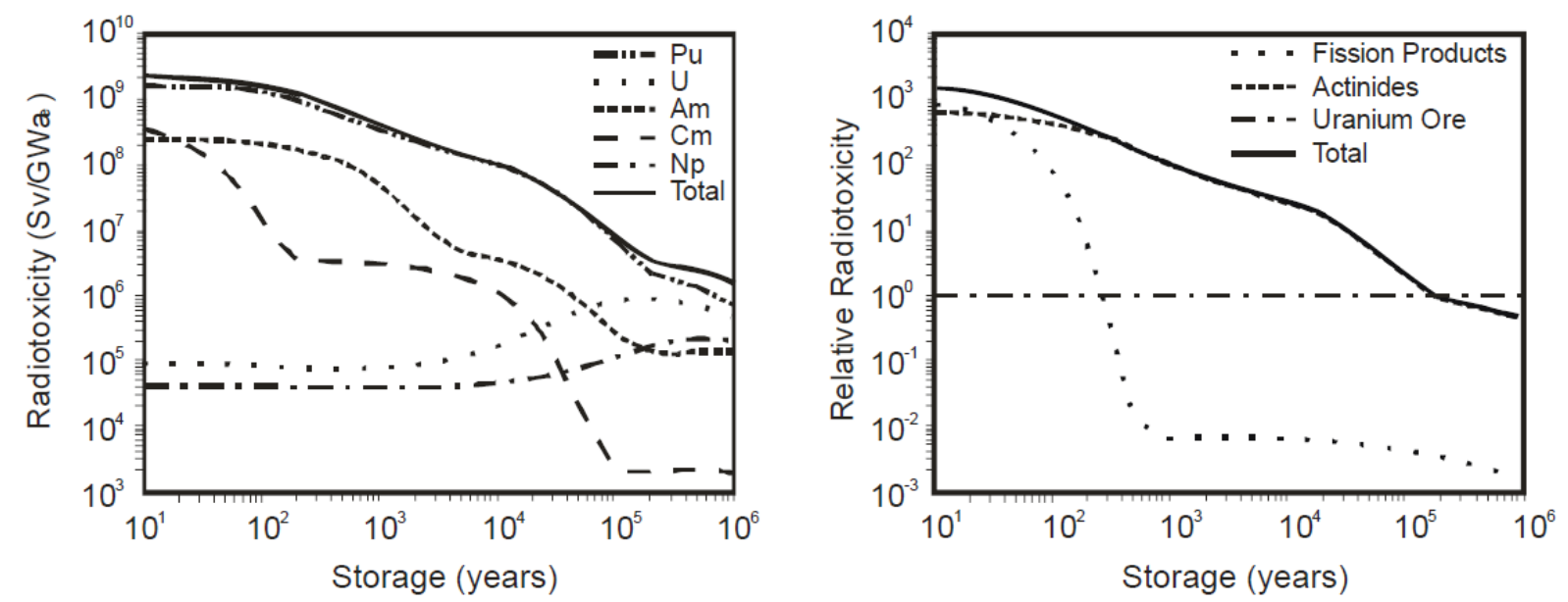

Figure 1.1. Left: The radiotoxicity per element of the actinides in spent $\mathrm{U}(\mathrm{VI}) \mathrm{O}_{2}$ fuel. $(\mathrm{GWae}$ is a measure of electricity produced and 1 GWae equals about two times the annual production of the Borssele nuclear power plant). Right: The radiotoxicity of the actinides and fission products in spent fuel relative to the uranium ore needed to manufacture the fuel. The storage time needed to reach a radiotoxicity level of uranium ore is about 200,000 years. ${ }^{5}$

\subsection{Partitioning and transmutation}

Several countries worldwide are currently exploring the strategy of P\&T (Partitioning and Transmutation), which aims to reduce the radiotoxicity of the waste by prior separation of uranium, plutonium, minor actinides, and other long-lived fission products. ${ }^{6}$ After partitioning, neutron bombardment (transmutation) of the minor actinides in dedicated reactors yields shorter-lived $\left(t_{1 / 2}=10^{1}\right.$ years $)$ or more stable elements. ${ }^{7}$ Due to the high neutron capture cross sections of the lanthanides and their bulk amount compared to actinides, partitioning is of great interest. There are several processes to separate metals, such as precipitation, ${ }^{8,9}$ electrolysis, ${ }^{10}$ ion exchange, ${ }^{11}$ and solvent extraction. In solvent extraction, the loss of extraction agent and the production of large amounts of organic waste are potential 
disadvantages. However, one distinct advantage in the processing of nuclear waste is that high selectivities can be obtained. For this reason solvent extraction is currently one of the most important techniques under investigation. A schematic view of the partitioning of waste by solvent extraction is presented in Figure 1.2, where $\mathrm{U}, \mathrm{Pu}$, and $\mathrm{Np}$ are partitioned in the PUREX (Plutonium URanium EXtraction) process. In the DIAMEX (DIAMide EXtraction) process actinides and lanthanides are co-extracted from the bulk containing corrosion and fission products. Finally, the actinides and lanthanides are mutually separated in the SANEX (Selective ActiNide EXtraction) process.

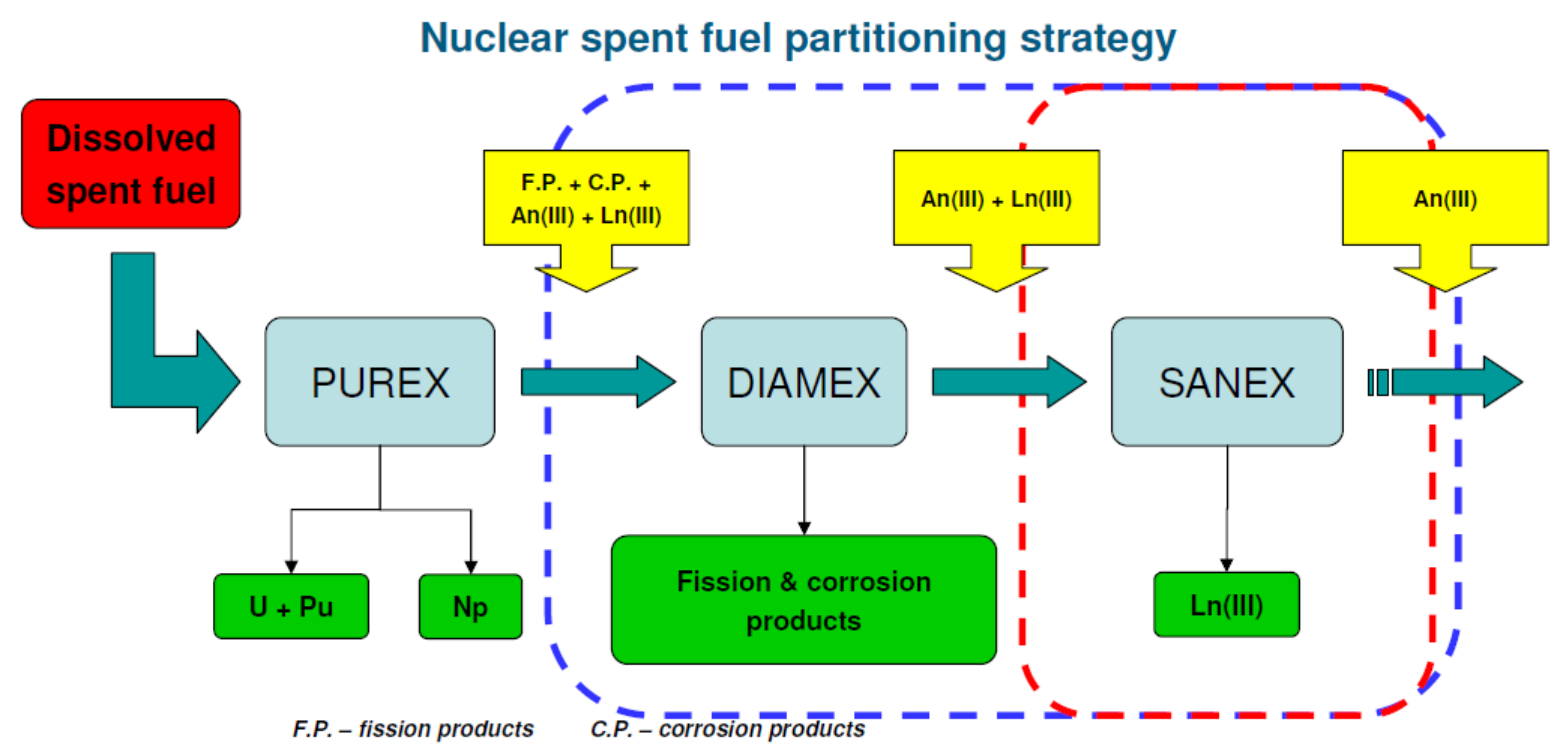

Figure 1.2. Schematic view of the partitioning process of actinides (courtesy of Dr. G. Modolo).

\subsection{Outline of the thesis}

The main objective of the research presented in this thesis is the development of new, potential ligands for actinide $(\mathrm{An}(\mathrm{III}))$ /lanthanide $(\mathrm{Ln}(\mathrm{III}))$ extraction from bulk nuclear waste and/or recovery of these $\mathrm{An}(\mathrm{III}) / \mathrm{Ln}$ (III) from the extracted organic phase by hydrophilic ligands for final processing. In Chapter 2 a brief description of the properties of An(III) and $\mathrm{Ln}(\mathrm{III})$ is given, followed by an overview of various $\mathrm{An}(\mathrm{III}) / \mathrm{Ln}(\mathrm{III})$ ligands, that have been developed in the recent years. Various ligands with $\mathrm{N}, \mathrm{S}$, and $\mathrm{O}$ donor atoms and their binding affinity for An(III) and $\mathrm{Ln}(\mathrm{III})$ are described.

Chapter 3 deals with the synthesis and extraction properties of new structural derivatives of the well-known TODGA (tetraoctyl diglycolamide) and some tripodal ligands. Chapter 4 describes the synthesis and stripping efficiency of new hydrophilic ligands bearing a 


\section{Chapter 1}

diglycolamide and tripodal backbone. In Chapter 5, the preorganization of diglycolamides on the calix[4] arene platform and its effect on the extraction of Eu(III) and Am(III) is described. Chapter 6 deals with hybrid types of ligands, synthesized by combining essential parts of various known ligands. The ligands contain either a diglycolamide or a malonamide backbone and have $\mathrm{P}=\mathrm{S}, \mathrm{P}=\mathrm{O}$, or amide donor sites. In Chapter 7, different methodologies for the synthesis of tripodal diglycolamide are described. The extraction ability of these tripodal diglycolamides and calix[4]arene-based diglycolamides in an imidazolium-based ionic liquid medium for various actinides and lanthanides is discussed. Diglycolamide- and CMPO-based task-specific ionic liquids and their extraction ability for various metal ions are the topic of Chapter 8.

\subsection{References}

1. The numbers are given until $31^{\text {st }}$ December 2010.

2. www.iaea.org Nuclear Technology Review, September 2011.

3. Baisden, P. A.; Choppin, G. R. Nuclear Waste Management and the Nuclear Fuel Cycle. In Radiochemistry and Nuclear Chemistry; Nagyl, S., Ed.; Encyclopedia of Life Support Systems (EOLSS); EOLSS Publishers: Oxford, U.K., 2007.

4. Johnson, J. Chem. Eng. News 1998, 76, 25.

5. Madic, C.; Ouvrier, N. Radiochim. Acta 2008, 96, 183.

6. (a) Actinide and Fission Product Partitioning and Transmutation Status and Assessment Report; OECD NEA: Paris, 1999. (b) Salvatores, M. Nucl. Eng. Des. 2005, 235, 805. (c) Salvatores, M.; Palmiotti, G. Prog. Part. Nucl. Phys. 2011, 66, 144. (d) Bourg, S.; Hill, C.; Caravaca, C.; Rhodes, C.; Ekberg, C.; Taylor, R.; Geist, A.; Modolo, G.; Cassayre, L.; Malmbeck, R.; Harrison, M.; Angelis, G.; Espartero, A.; Bouvet, S.; Ouvrier, N. Nucl. Eng. Des. 2011, 241, 3427.

7. Magill, J.; Berthou, V.; Haas, D.; Galy, J.; Schenkel, R.; Wiese, H.-W.; Heusener, G.; Tommasi, J.; Youinou, G. Nucl. Energy 2003, 42, 263.

8. Diamand, R. M. E. Environmental Chemistry, Plenum Press, New York, 1977.

9. Choppin, G. R.; Nash, K. L. Radiochim. Acta 1995, 70-71, 225.

10. Kuhn, A. T. Electrochemistry of Cleaner Environments, Plenum Press, New York, 1972.

11. Pérez de Ortiz, E. S. Ion Exchange, Science and Technology, Martinus Nijhoff Publishers, Dordrecht, 1986. 


\section{Chapter}

\section{2

\section{Ligands for the Partitioning of Nuclear Waste}

Solvent extraction is one of the most widely applied techniques for the processing of nuclear waste. In recent, years research in this area has led to a wide variety of ligands either for the co-extraction of actinides or for the separation of actinides and lanthanides. In this Chapter, an overview is given of the ligands that have been, and are being, developed, with a focus on the ligands involved in actinide/lanthanide separation. 


\section{Chapter 2}

\subsection{Introduction}

One of the major drawbacks of nuclear energy is the production of nuclear waste. Due to its radioactivity, treatment of this waste is an important issue and receives much attention. Many methods have been and are being developed with the goal to find a safe and cost effective treatment protocol. ${ }^{1,2}$ Most studies involve the partitioning (separation) of strongly radioactive elements. Nuclear waste predominately contains residual uranium ( 95 wt\%) and a small amount of the highly radiotoxic transuranium elements plutonium $(\sim 1 \mathrm{wt} \%)$, whereas the minor actinides (neptunium, americium, and curium, $0.1 \%$ ) are only a small part of the bulk. Partitioning generates groups of radionuclides with similar chemical properties, which can be managed in a safer way for long term storage or disposal. Currently, the most accepted approach for partitioning involves solvent extraction utilizing organic ligands. The schematic view of the partitioning process is presented in Chapter 1.

Recently, several reviews have been published dealing with extractions and processes based on CMPO, malonamide, and glycolamide ligands, ${ }^{3}$ more detailed properties of glycolamides, ${ }^{4}$ and preorganization of these ligands on molecular platforms. ${ }^{5}$ Two reviews describe the physicochemical and extraction properties of $\mathrm{N}$ donor ligands ${ }^{6}$ and more recently, a review covering the development of $\mathrm{N}$ and $\mathrm{S}$ donor ligands until the end of $2010 .^{7}$ In this Chapter, in addition to separations dealing with $\mathrm{N}, \mathrm{S}$ donor ligands, recent approaches for actinides $(\mathrm{An}) /$ lanthanides $(\mathrm{Ln})$ separations, viz. the use of synergistic mixtures, water-soluble ligands, and the application of ionic liquids in nuclear waste, are described. A summary of the $\mathrm{O}$ donor ligands that have been developed for the co-extraction of $\mathrm{An}(\mathrm{III}) / \mathrm{Ln}(\mathrm{III})$ is also given.

\subsection{Actinides and lanthanides}

The An and Ln are f-block elements, for which the $5 \mathrm{f}$ and $4 \mathrm{f}$ subshells, respectively, are being filled. Both actinides and lanthanides possess relatively similar chemical and physical properties ${ }^{8,9,10}$ e. g.

- Both are considered as hard acids in the Pearson classification (HSAB for Hard and Soft Acids and Bases). ${ }^{11}$ Their interactions with inorganic and organic ligands are therefore predominantly determined by electrostatic and steric factors.

- The trivalent cations of both An and Ln have the same electronic configuration within each group (apart from the filling of the f shell) and almost similar ionic radii.

- Both exist predominantly in their trivalent oxidation states in acidic aqueous solution.

- Both have a similar coordination sphere hydration number. 
Although both actinides and lanthanides are considered to be hard acids according to the HSAB theory, the higher spatial expansion of the $5 \mathrm{f}$ actinide orbitals with respect to the $4 \mathrm{f}$ lanthanide orbitals opens possibilities to discriminate them through their relative hardness. It has been postulated that an increased covalent nature in the interaction of $\mathrm{An}$ (III) with soft donor atoms and/or changes in coordination geometries account for these effects. ${ }^{12,13}$ Therefore, the introduction of soft donor atoms (e.g. S, N) into ligands may allow for the subtle discrimination between $\mathrm{An}(\mathrm{III})$ and $\mathrm{Ln}(\mathrm{III})$ via differences in the metal-ligand complex coordination, such as differences in binding stoichiometry, affinity and structure.

\subsection{Actinide/lanthanide separation}

Due to the large amount of lanthanides and fission products present in the spent fuel and the high neutron absorption cross section, the actinides must be separated from the lanthanides for proper transmutation in a dedicated reactor. Until now there is no process known that can selectively separate individual actinides from spent fuel directly. In most processes developed over the recent years first the lanthanides and actinides are co-extracted, followed by mutual group separation. The separation of An(III) can be achieved either by selective extraction into an organic phase or by selective complexation in the aqueous phase. Most of the processes described in literature extract the An(III) + Ln(III) containing solution issued from a first step process, which has separated the An(III) + Ln(III) fraction from a highly active PUREX (Plutonium URanium EXtraction) ${ }^{14}$ raffinate. The examples of such first step processes are TRUEX $^{15}{ }^{16}$ (TRans Uraniun EXtraction), TRPO ${ }^{17}$ (TRialkylPhosphine Oxide), DIAMEX ${ }^{18}$ (DIAMide EXtraction) and DIDPA ${ }^{19}$ (DiIsoDecyl Phosphoric Acid). The second step processes are SANEX (Selective ActiNide EXtraction), ALINA ${ }^{20}$ (Actinide-Lanthanide INtergroup separation from Acidic media), and some processes involving nitrogen polydentate ligands. The separation of actinides by complexing in the aqueous phase is achieved by processes like TALSPEAK ${ }^{21}$ (Trivalent Actinide Lanthanide Separations by Phosphorus-reagent Extraction from Aqueous Complexes) and Innovative SANEX. ${ }^{22}$

\subsection{Co-extraction of $\mathrm{An}(\mathrm{III}) / \mathrm{Ln}(\mathrm{III})$}

Oxygen donor ligands are generally considered as hard donors and they do not show any discrimination between $\mathrm{Ln}(\mathrm{III})$ and $\mathrm{An}(\mathrm{III})$. Some classical examples include the organophosphorus ligands, malonamides, and glycolamides. A schematic view of a process for the co-extraction of $\mathrm{An}(\mathrm{III}) / \mathrm{Ln}(\mathrm{III})$ is shown in Figure 2.1. 


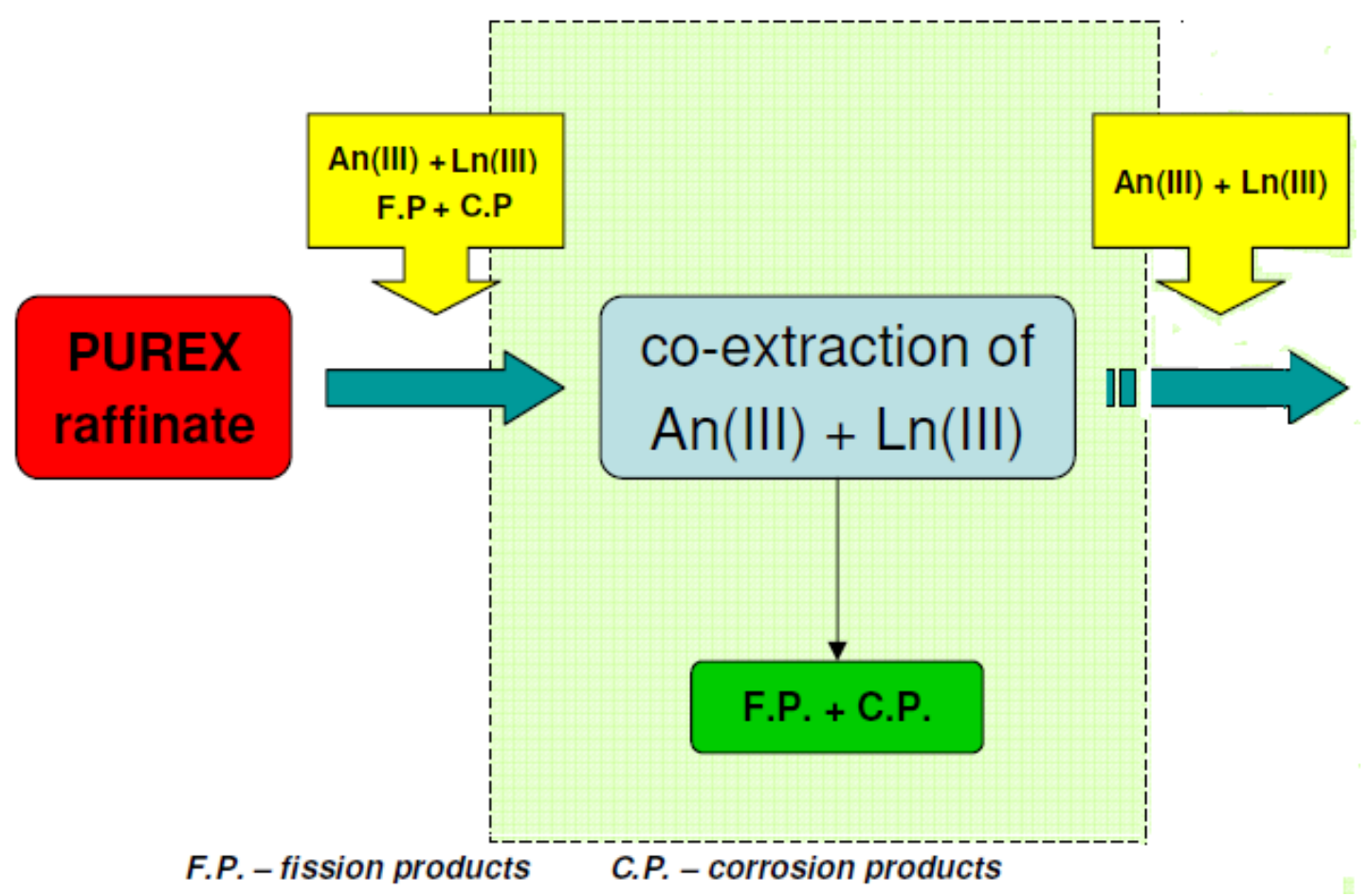

Figure 2.1. Co-extraction of $\mathrm{An}(\mathrm{III})$ and $\mathrm{Ln}(\mathrm{III})$ from the PUREX raffinate.

\subsubsection{Organophosphorus ligands}

Tri-n-butyl phosphate (TBP) (Chart 2.1) is being used on an industrial scale for the chemical separation of $\mathrm{U}(\mathrm{VI}) \mathrm{O}_{2}$ and $\mathrm{Pu}(\mathrm{IV})$ in a kerosene type aliphatic hydrocarbon solvent as the extractant. Almost all $\mathrm{U}$ and Pu can be separated from the depleted fuel by TBP. Diamylamyl phosphonate (Chart 2.1) has been evaluated as a substitute for TBP to extract $\mathrm{U}(\mathrm{VI}) \mathrm{O}_{2}$, $\mathrm{Th}(\mathrm{IV}), \mathrm{Pu}(\mathrm{IV})$, and Am(III) in $n$-dodecane. ${ }^{23}$ Trialkylphosphine oxides (Chart 2.1) have been used for the removal of transuranic elements (TRU) from highly active waste. Based on trialkylphosphine oxides, the TRPO process was developed in China. ${ }^{24}$

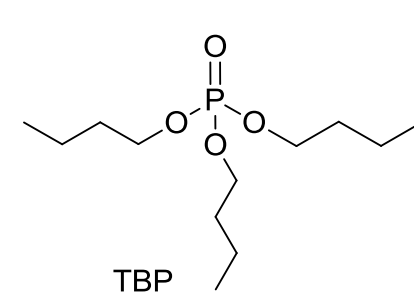

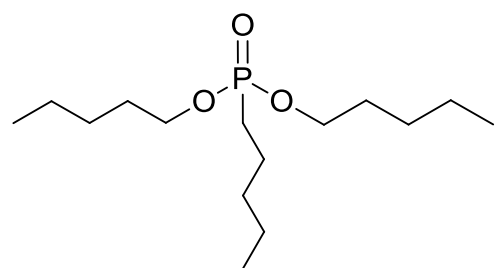

Diamylamyl phosphonate

Chart 2.1

The bidentate carbamoyl phosphonates were for the first time described for the extraction of actinides and lanthanides in the 1960s. ${ }^{25,26}$ Several derivatives of $\mathrm{CH}_{2}$-bridged organophosphorus ligands, e.g the bidentate neutral organophosphorus compounds 
(BNOPCs), ${ }^{27,28}$ diphenyl(diisobutylcarbamoylmethyl)phosphine oxide $\left(\mathrm{Ph}_{2} \mathrm{iBu}_{2}-\mathrm{CMPO}\right)^{29,30,31}$ and octyl(phenyl)- $N, N$-diisobutylcarbamoylmethyl phosphine oxide (CMPO) ${ }^{32,33,34,35}$ (Chart 2.2), have been extensively studied for the extraction of various actinides and lanthanides. The TRUEX process was developed in the 1980s, which uses a mixture of CMPO and $\mathrm{TBP}^{36}$ allowing all minor actinides and lanthanides to be extracted from a $\mathrm{HNO}_{3}$ medium.<smiles>[R][R](=O)P[R]([R])([R])[R]</smiles>
$R^{1}=B u, P h$
$R^{2}=B u, P h$
$n=1,2$<smiles>CC(C)CN(CC(C)C)C(=O)CP(=O)(c1ccccc1)c1ccccc1</smiles>

$\mathrm{Ph}_{2} \mathrm{Bu}_{2}-\mathrm{CMPO}$<smiles>CC(C)CN(CC(C)C)C(=O)CP(=O)([InH])c1ccccc1</smiles>

CMPO

\section{Chart 2.2}

\subsubsection{Malonamides}

The malonamides, bidentate oxygen donor ligands with the general formula $\mathrm{R}^{1} \mathrm{R}^{2}$ NCOCHR ${ }^{3} \mathrm{CONR}^{2} \mathrm{R}^{1}$, are used for the extraction of An(III) and $\mathrm{Ln}(\mathrm{III})$ from the PUREX raffinates since the 1980s. ${ }^{37}$ These extractants are completely incinerable and the amount of secondary waste produced can be significantly reduced compared to phosphorus-containing ligands.

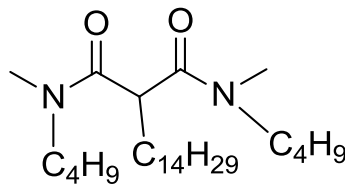

DMDBTDMA

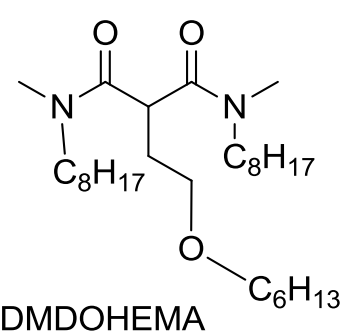

Chart 2.3

Extensive studies involving structural modifications ${ }^{38,39,40,41}$ led to the DIAMEX process. Initially, $N, N$ '-dimethyl- $N, N$ '-dibutyltetradecylmalonamide (DMDBTDMA; Chart 2.3) was considered the best malonamide for the DIAMEX process. This ligand extracts metal ions via the solvation mechanism and allows stripping in dilute $\mathrm{HNO}_{3}$ medium. Later, $N, N$ '-dimethyl$N, N$ '-dioctyl-2-(2-hexyloxyethyl)malonamide (DMDOHEMA; Chart 2.3) bearing an oxygen atom in its central chain, was found to be a better extractant than DMDBTDMA. The oxygen atom present reduces the amount of degradation and hydrolysis products. ${ }^{42}$ The relative 


\section{Chapter 2}

extractability of the actinides by malonamides follows the order $\mathrm{Pu}(\mathrm{IV})>\mathrm{U}(\mathrm{VI}) \mathrm{O}_{2}>\mathrm{Am}(\mathrm{III})$, while fission product ions such as $\mathrm{Eu}(\mathrm{III}), \mathrm{Zr}(\mathrm{IV})$, and $\mathrm{Fe}(\mathrm{III})$ are also significantly extracted in the acidity range of $3-4 \mathrm{~mol} / \mathrm{L} \mathrm{HNO}_{3}$.

Bicyclic malonamides (Chart 2.3) were described to be much stronger ligands than the linear derivatives. The extraction efficiency of these bicyclic malonamides increased from $D_{\mathrm{Eu}}(D$ is the distribution ratio, defined as the concentration of a metal in the organic phase relative to that in the aqueous phase e.g. $D=[M]_{\mathrm{org}} /[M]_{\mathrm{aq}}$ ) values of $5 \times 10^{-5}$ for acylic malonamide to 500 $\left(\sim 10^{7}\right.$ times higher) at $0.1 \mathrm{~mol} / \mathrm{L}$ ligand. ${ }^{43}$ In another study molecular mechanics calculations showed that the two amide oxygen atoms, occupying the lowest energy conformation, are ideally situated for binding a metal ion. ${ }^{44}$

\subsubsection{Glycolamides ${ }^{4}$}

Since malonamides have a weak binding efficiency towards actinide/lanthanide metal ions, relatively high extractant concentrations (0.5-1 mol/L) are required. During the late 1990s, Sasaki and Choppin showed that glycolamides have better extraction properties towards all actinides (An(III-IV)) from acidic waste solutions than malonamides. ${ }^{45,46}$ Extensive extraction studies have been performed with this very promising substance class. ${ }^{47}$ The change from a bidentate ligand (e.g. malonamide) to a tridentate diglycolamide (DGA) not only significantly increased the affinity for trivalent actinides, but also for the lanthanides. DGA derivatives with varying chain lengths on the amidic nitrogen have been synthesized and $N, N, N^{\prime}, N^{\prime}$-tetraoctyl diglycolamide (TODGA; Chart 2.4) was found to have the best properties in terms of extraction, solubility in aliphatic solvents, and stability. The nature of the $N$-substituents of DGA compounds plays an important role in the metal extraction. For instance, the $D_{\mathrm{U}}$ values decrease as the chain length increases along the series $\mathrm{C}_{3} \mathrm{H}_{7}>\mathrm{C}_{4} \mathrm{H}_{9}>$ $\mathrm{C}_{6} \mathrm{H}_{13}>\mathrm{C}_{8} \mathrm{H}_{17}{ }^{48}$ Although, the glycolamides with a short alkyl chain length are soluble in water, the actinide extractability decreases in the following order An(IV) > An(III) > An(VI) $>\mathrm{An}(\mathrm{V})$, whereas that of the fission products is relatively small, except for $\mathrm{Zr}(\mathrm{II})$, and $\mathrm{Sr}(\mathrm{II})$, and $\operatorname{Ln}(\mathrm{III})$. Contrary to the malonamides, the extraction of lanthanides increases with the atomic number in case of TODGA. 


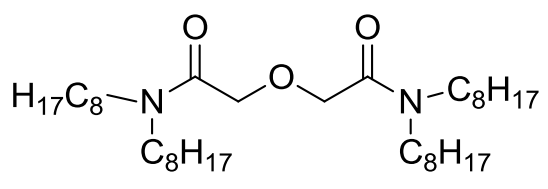

TODGA

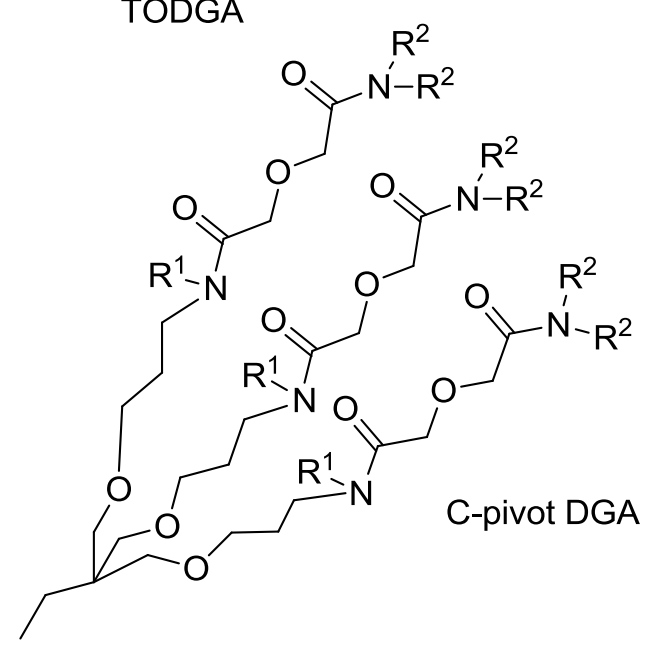<smiles>[R]CN([R])C(=O)COCC(=O)N([R])Cc1cc([R])cc(CN([R])C(=O)COCC(=O)N([R])[R])c1</smiles>

Bisdiglycolamides<smiles>CCC(C)(C)c1cc(C)c(OCCNC(=O)COCC(=O)N(C(C)C)C(C)C)c(C(C)(C)CC)c1</smiles>

Chart 2.4

The polarity of the organic diluents plays an important role in the extraction using DGAs. The $D_{\mathrm{M}(\mathrm{III})}$ values decrease in the order $n$-octanol $\sim n$-dodecane $>$ dichloromethane $>$ toluene $>$ chloroform, persumably because the oxygen donor atoms of the DGAs interact with aromatic and halogenated diluents. ${ }^{47,51 \mathrm{~g}}$ In polar diluents such as $n$-octanol, $\mathrm{M}(\mathrm{TODGA})_{2}\left(\mathrm{NO}_{3}\right)_{3}$ complexes are extracted, whereas metal complexes require three or more TODGA molecules to remain stable in nonpolar organic solvents such as toluene or $n$-dodecane, where $\mathrm{HNO}_{3}$ molecules are assumed to take part in the extraction. However, TODGA has a tendency to form a third phase in aliphatic solvents such as $n$-dodecane, particularly at high metal and $\mathrm{HNO}_{3}$ concentrations. ${ }^{49,50}$ In general, the DGAs have drawn attention as very effective ionophores for the complexation of f-elements. ${ }^{51}$

TODGA has been preorganized on the triphenoxymethane (also referred to as trityl) platform. ${ }^{52,53}$ DGA on the trityl platform (trityl DGA; Chart 2.4) has a better $S F$ ( $S F$ is the separation factor defined as the ratio of the distribution ratio of one metal to the other e.g. $S F$ $\left.=D_{\mathrm{M} 1} / D_{\mathrm{M} 2}\right)$ for $\mathrm{Eu}(\mathrm{III})$ over $\mathrm{Am}(\mathrm{III})\left(D_{\mathrm{Am}}>100\right.$, average $S F_{\mathrm{Eu} / \mathrm{Am}}=6.16$, at $10^{-3} \mathrm{~mol} / \mathrm{L}$ ligand concentration in $\mathrm{CH}_{2} \mathrm{Cl}_{2}$ from $3 \mathrm{~mol} / \mathrm{L}$ aqueous $\mathrm{HNO}_{3}$ ) which is slightly higher than for simple DGA chelators (i.e. $S F_{\mathrm{Eu} / \mathrm{Am}}=3.43$ in DCE (1,2-dichloroethane) and 0.81 in $\mathrm{CHCl}_{3}$ ). The diglycolamide functional groups preorganized at the C-pivot tripodal platform (C-pivot 


\section{Chapter 2}

DGA; Chart 2.4), showed to be very efficient extractants for Am(III) and Eu(III) with up to five times higher relative extraction ability for $\mathrm{Eu}(\mathrm{III})$. The distribution coefficients are up to 1000 times increased upon alkylation or arylation of the N-position of the diglycolamide moieties. The tripodal diglycolamides show a $1: 1$ metal-to-ligand stoichiometry. ${ }^{54}$ Bisdiglycolamides (Chart 2.4) bearing various substituents on the nitrogen atoms of diglycolamides showed a decrease in the $D$ value compared to the simple diglycolamides. ${ }^{55}$ However, the $S F_{\text {Eu/Am }}$ values were generally higher for bisdiglycolamides compared to the simple diglycolamides.

\subsection{Separation of An(III)/Ln(III)}

The separation of $\mathrm{An}$ (III) from the $\mathrm{Ln}(\mathrm{III})$ is one of the most challenging issues (see section 2.2). However, ligands with soft donor atoms such as $\mathrm{S}$ and $\mathrm{N}$ have the ability to separate them. In this section N, S donor ligands for the selective extraction of An(III) will be discussed. In addition, the use of synergistic mixtures for the separation of An(III) and Ln(III) will be considered. A schematic view of the SANEX process utilizing N, S donor ligands is shown in Figure 2.2.

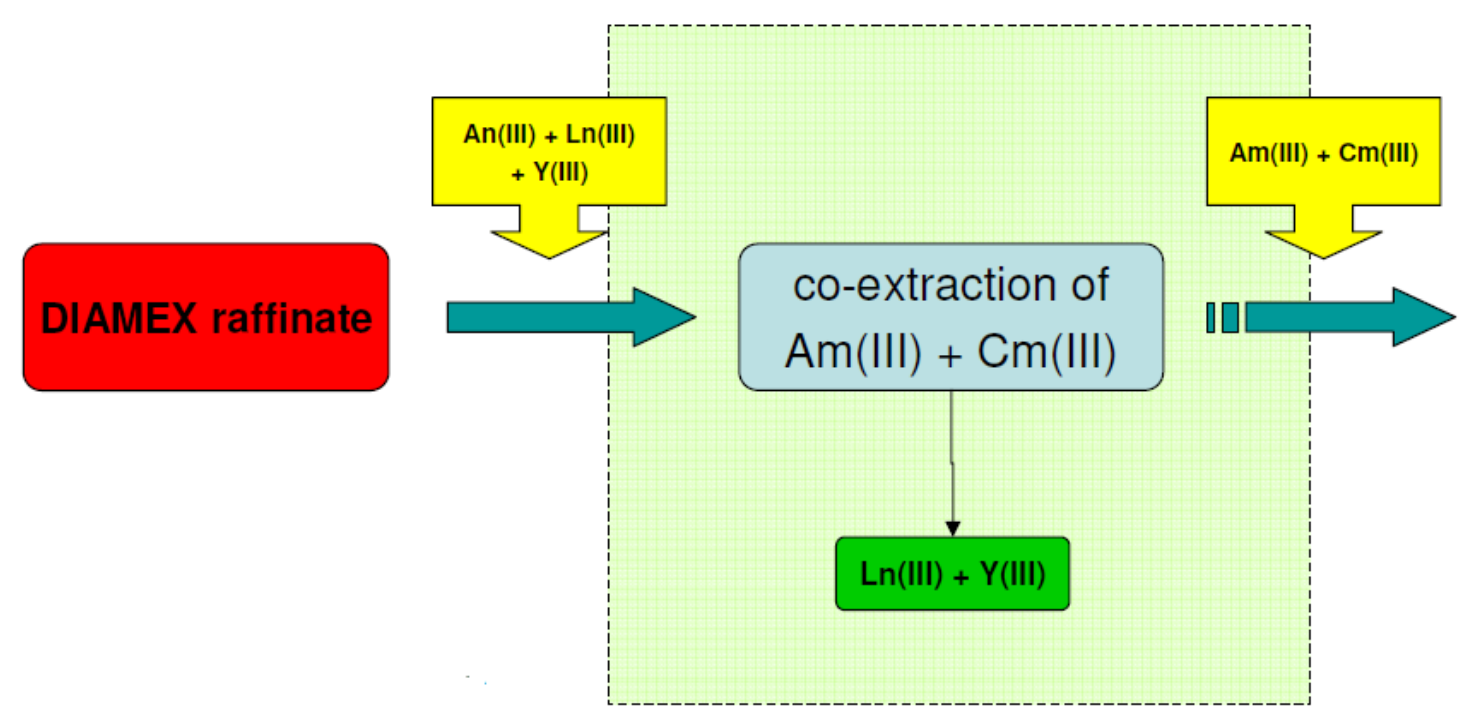

Figure 2.2. Schematic view of the SANEX process.

\subsubsection{S-donor ligands}

In general, sulfur-based soft-donor extractants show selectivity towards An(III). Among the S donor ligands, the most potent class represents the thiophosphinic/phosphoric acids. 


\subsubsection{Extraction by thiophosphinic/phosphoric acids}

Bis(2-ethylhexyl)dithiophosphoric acid (HDEHDTP; Chart 2.5) was the first example of this class showing selectivity for Am(III) over Eu(III) with a $S F$ value of 50, using a mixture of HDEHDTP and TBP as extractant. Further studies were carried out on a series of three phosphinic acids, namely bis(2,4,4-trimethylpentyl)phosphinic acid (CYANEX 272), bis(2,4,4-trimethylpentyl)monothiophosphinic acid (CYANEX 302), and bis(2,4,4-trimethylpentyl)dithiophosphinic acid (CYANEX 301) (Chart 2.5). Among these three ligands, the oxygen donor ligand CYANEX 272 showed the highest extraction efficiency, however, with no selectivity for Am(III). On the other hand, the dithio ligand CYANEX 301 exhibited a good selectivity for Am(III), while that of the monothio analog CYANEX 302 was less than that of CYANEX 301. This demonstrates the importance of the soft $\mathrm{S}$ atom in the extractants. ${ }^{56}$

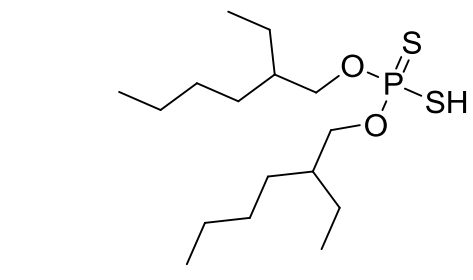

Bis(2-ethylhexyl)dithiophosphoric acid

HDEHDTP

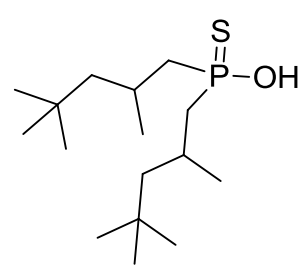

$\operatorname{Bis}(2,4,4$-trimethylpentyl)monothiophosphinic acid

CYANEX 302

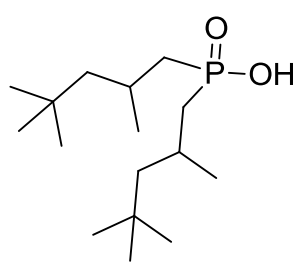

Bis(2,4,4-trimethylpentyl)phosphinic acid CYANEX 272

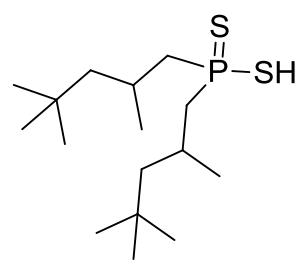

Bis(2,4,4-trimethylpentyl)dithiophosphinic acid CYANEX 301

\section{Chart 2.5}

Zhu et al. ${ }^{57}$ examined the synergistic effect of bis(2-ethylhexyl)dithiophosphoric acid and TBP on the separation of Am(III) from $\operatorname{Ln}(\mathrm{III})$, however, the separation factor obtained was less than 50. Commercial CYANEX 301 contains approximately $80 \%$ of bis $(2,4,4-$ trimethylpentyl)dithiophosphinic acid, however, after purification it as the ammonium salt by recrystallization from benzene, a high separation factor of $\mathrm{Am} / \mathrm{Eu}$ (> 5000) was achieved. ${ }^{58,59}$ In contrast, CYANEX 302 only showed a minor selectivity. 


\section{Chapter 2}

Jarvinen et al. ${ }^{60}$ examined the synergistic effect of TBP for three different dithiophosphinic acids viz. CYANEX 301, diphenyldithiophosphinic acid, and dicyclohexyldithiophosphinic acid (Chart 2.6). They found contradictory results as no separations were achieved with

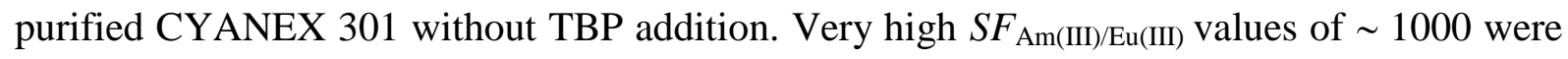
achieved in a single extraction step by dicyclohexyldithiophosphinic acid.

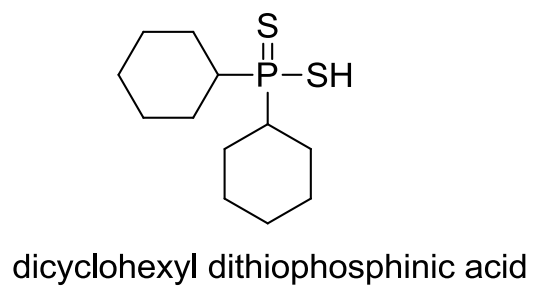

Chart 2.6

These contradictory results were further studied to examine the effect of purity on the selectivity with both purified and unpurified CYANEX 301. ${ }^{61}$ Commercial CYANEX 301 shows very interesting features for $\mathrm{Am}(\mathrm{III}) / \mathrm{Eu}(\mathrm{III})$ separations depending on the purity of its main constituent bis(2,2,4-trimethylpentyl)dithiophosphinic acid. Efficient separations were observed with non-purified CYANEX 301 for high Ln(III) concentrations, but not for low Ln(III) concentrations. Purified CYANEX 301, on the other hand, shows a very high selectivity for $\mathrm{Am}(\mathrm{III})$ (higher than $10^{3}$ ), both with tracer amounts and also with macro amounts of $\operatorname{Ln}(\mathrm{III})$ at $\mathrm{pH} 3-4$. This confirms the results of Zhu et al. ${ }^{57}$ (vide supra). These results indicate that the purity level of the extractants used has a great influence on the extraction behavior of tracer amounts of $\mathrm{Am}(\mathrm{III})$ and $\mathrm{Eu}(\mathrm{III})$. The impurities contained in CYANEX 301 are complexed by macro amounts of Eu(III), especially at high pH values. Thus it was demonstrated that commercial CYANEX 301 only has a high selectivity for Am(III) at high $\operatorname{Ln}(\mathrm{III})$ concentrations. ${ }^{61}$ Repetition of the extraction experiments after two days and after 15 days showed a gradual decrease in $S F$ values, which corresponds to gradual decomposition of CYANEX 301. Similar results were obtained by Hill et al. ${ }^{62}$ when the extraction experiments were carried out using synergistic mixtures (CYANEX 301 and TBP). Effective separation of Am(III) from macro amounts of lanthanides $\left(S F_{\mathrm{Am} / \mathrm{Eu}}>200\right)$ was obtained with a saponified CYANEX 301-TBP-kerosene solution. Am(III) was successfully separated (>99.99\%) from macro amounts of lanthanides with only <0.04\% lanthanides co-extraction using a countercurrent multistage process with 7 extraction, 3 scrubbing and 2 stripping stages at $\mathrm{pH} 2.7-2.8 .^{63}$ 
Although CYANEX 301 shows a high Am/Eu selectivity, its acidity is too low and it only becomes an effective extractant in the higher $\mathrm{pH}$ regions of aqueous solutions (i.e. $\mathrm{pH}>3$ ). Consequently, the $\mathrm{pH}$ value must be controlled or stabilized by a buffer during separation, especially if real waste solutions and Am(III) concentrations $>10^{-3} \mathrm{~mol} / \mathrm{L}$ are involved. Based on the positive results of $A n(I I I)$ and $\operatorname{Ln}($ III) separation by CYANEX 301, the new ligands diphenyldithiophosphinic acid, bis(chlorophenyl)- and bis(fluorophenyl)dithiophosphinic acids were developed (Chart 2.7). Compared to CYANEX 301, the alkyl chains were replaced by more electron-withdrawing substituents, such as stable aromatic rings, ${ }^{64,65}$ in order to increase the acidity of the ligands for extraction in the lower $\mathrm{pH}$ regions. Although, these ligands did not show any extraction in the absence of a synergist, it was possible to carry out the $\mathrm{An}(\mathrm{III}) / \mathrm{Ln}(\mathrm{III})$ separation with high selectivity even in strongly acidic medium (up to 1-mol/L $\mathrm{HNO}_{3}$ ) in the presence of TBP, trioctylphosphine oxide (TOPO), or tributylphosphine oxide (TBPO) as a synergist. The extraction ratios of Am(III) and Eu(III) strongly increase in the order $\left(\mathrm{C}_{6} \mathrm{H}_{5}\right)_{2} \mathrm{P}(\mathrm{S}) \mathrm{SH}<\left(\mathrm{F}-\mathrm{C}_{6} \mathrm{H}_{4}\right)_{2} \mathrm{P}(\mathrm{S}) \mathrm{SH}<\left(\mathrm{Cl}_{-} \mathrm{C}_{6} \mathrm{H}_{4}\right)_{2} \mathrm{P}(\mathrm{S}) \mathrm{SH}$. However, the selectivity in the investigated acidity range $(0.01-0.5 \mathrm{~mol} / \mathrm{L})$ decreases in the same order with Am/Eu separation factors of 230-280, 41-57, and 28-31, respectively. These ligands also showed better radiation stabilities than the aliphatic analogs. Since these aromatic dithiophosphinic acids are not soluble in $n$-dodecane, the extraction experiments were performed in toluene.

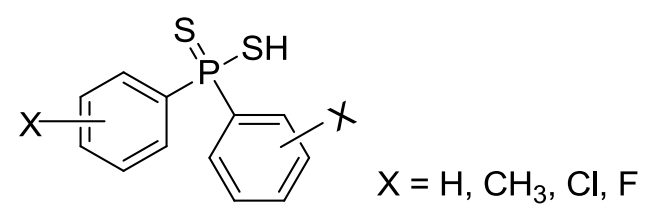

Chart 2.7

It was found that a synergistic mixture composed of bis(chlorophenyl)dithiophosphinic acid $\left[\left(\mathrm{Cl}-\mathrm{C}_{6} \mathrm{H}_{4}\right)_{2} \mathrm{P}(\mathrm{S}) \mathrm{SH}\right]$ and tris(2-ethylhexyl) phosphate (TEHP) showed an even higher affinity for trivalent actinides over lanthanides; ${ }^{66}$ a $S F_{\mathrm{Am} / \mathrm{Eu}}$ value of over 2000 and also a high $S F_{\mathrm{Am} / \mathrm{Cm}}$ value of 8 was obtained. A thermodynamic study of the extraction of Am(III), $\mathrm{Cm}(\mathrm{III})$, and nearly the whole lanthanide(III) series from $\mathrm{HNO}_{3}$ was carried out. ${ }^{67}$ It turned out that the interpretation of the extraction results is strongly influenced by the dimerization of the ligand $\left(\mathrm{Cl}-\mathrm{C}_{6} \mathrm{H}_{4}\right)_{2} \mathrm{P}(\mathrm{S}) \mathrm{SH}$. 


\section{Chapter 2}

Bis(o-trifluoromethylphenyl)dithiophosphinic acid (Chart 2.8) selectively extracts Am(III) with a $S F_{\text {Am/Eu }}$ value of $\sim 100,000$ at $\mathrm{pH} 2$. However, bis(3,5bis(trifluoromethyl)phenyl)dithiophosphinic acid shows a much lower selectivity for Am(III)

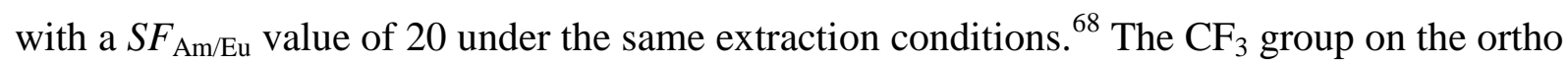
position has a strong electron-withdrawing inductive effect, making the sulfur atom softer and thus improves the SF. These thiophosphinic acids are more stable than CYANEX 301, showing very little degradation when contacted with water and $\mathrm{HNO}_{3}$ for prolonged periods.

To investigate the effect of an oxygen instead of a sulfur donor atom, the corresponding bis(o-trifluoromethylphenyl)phosphinic acid (Chart 2.8) was studied. ${ }^{69}$ This ligand extracts Am(III) by a similar mechanism to its S-containing analog, namely via an acidic cation-exchange mechanism and shows a selectivity for $\mathrm{Eu}(\mathrm{III})$ over $\mathrm{Am}$ (III) with a $S F$ value of 10. Studies of the acid dependence of the extraction of $\mathrm{Am}$ (III) and $\mathrm{Eu}$ (III) from $\mathrm{HNO}_{3}$ solutions by dithiophosphinic acid in phenyl trifluoromethyl sulfone (FS-13) solvent were carried out. ${ }^{70}$ The slopes of the $D_{\mathrm{Am}}$ values as a function of ligand concentration $(\mathrm{m}=3)$ indicated that three ligand molecules are involved in the extraction of metal ion. The authors concluded an acidic, cation-exchange extraction mechanism in which three protons are exchanged into the aqueous phase for each metal cation complex formed in the organic phase. The different slopes observed for the Eu(III) acid dependencies $(\mathrm{m}=1)$ indicate that the Eu-dithiophosphinic acid complex has a different stoichiometry, or $\mathrm{Eu}(\mathrm{III})$ has a fundamentally different interaction mode with these extractants. Phosphinic acid is a stronger extractant as $80 \%$ of the available Am(III) was extracted by this ligand, while only $20 \%$ of the available Am(III) was extracted by dithiophosphinic acid. 
<smiles>FC(F)(F)c1ccccc1P(=S)(S)c1ccccc1C(F)(F)F</smiles>

Bis(o-trifluoromethylphenyl) dithiophosphinic acid<smiles>FC(F)(F)c1cccc(P(=S)(S)c2ccccc2C(F)(F)F)c1</smiles>

(o-Trifluoromethylphenyl)(m-trifluoromethylphenyl) dithiophosphinic acid<smiles>FC(F)(F)c1cccc(P(=S)(S)c2cccc(C(F)(F)F)c2)c1</smiles>

Bis ( $m$-trifluoromethylphenyl) dithiophosphinic acid<smiles>FC(F)(F)c1cc(C(F)(F)F)cc(P(=S)(S)c2cc(C(F)(F)F)cc(C(F)(F)F)c2)c1</smiles>

Bis(3,5-bis(trifluoromethyl)phenyl) dithiophosphinic acid<smiles>O=P(O)(c1ccccc1C(F)(F)F)c1ccccc1C(F)(F)F</smiles>

Bis(o-trifluoromethylphenyl) phosphinic acid

Chart 2.8

In the case of a series of other symmetric and asymmetric aromatic dithiophosphinic acids, containing trifluoromethyl groups on the ortho-ortho, ortho-meta, and meta-meta positions, ${ }^{71}$ (Chart 2.8) the extraction data showed that a cation exchange mechanism is operating in these complexes (where $\mathrm{H}^{+}$is a leaving cation) and no nitrate ions accompanied the formed complexes into the organic phase. Two striking features of these extractants can be noted: First, the $S F_{\mathrm{Am} / \mathrm{Eu}}$ values are $10^{4}$ for bis(o-trifluoromethylphenyl)dithiophosphinic acid and (otrifluoromethylphenyl)-( $m$-trifluoromethylphenyl)dithiophosphinic acid, which is much higher than those of any previously known dithiophosphinic acids. Second, a very minor structural change, moving a $-\mathrm{CF}_{3}$ group only one carbon away from the metal coordination site, lowers the $S F$ value by an order of magnitude. The extraction efficiencies by these three dithiophosphinic acids are in the order having $\mathrm{CF}_{3}$ groups on the ortho-meta $\sim$ ortho-ortho > meta-meta positions.

Various dialkyldithiophosphinic acids with octyl, 1-methylheptyl, 2-ethylhexyl, and 2,4,4-trimethylpentane chains (Chart 2.9) were prepared and their extraction effeciencies were measured in toluene as the organic phase and $1 \mathrm{~mol} / \mathrm{L} \mathrm{NaNO}_{3}$ as the aqueous phase. ${ }^{72}$ The extractants with $n$-octyl (DODTPI), 1-methylheptyl (DMHDTPI), and 2-ethylhexyl (DEHDTPI) chains (Chart 2.9) have a better extraction efficiency than CYANEX 301, however, all extractants showed a $S F_{\mathrm{Am} / \mathrm{Eu}}$ value in the range of $1 \times 10^{4}$. 


\section{Chapter 2}

Extraction of $\mathrm{Am}(\mathrm{III})$ and $\mathrm{Eu}(\mathrm{III})$ from a $\mathrm{NaClO}_{4}$ medium with various phosphinic and phosphoric acids in xylene was investigated and the order of extraction selectivity for Am(III) was bis(2-ethylhexyl)dithiophosphinic acid (DEHDTPI) > HDEHDTP > bis(2ethylhexyl)monothiophosphinic acid (DEHMTPI) > bis(2-ethylhexyl)monothiophosphoric acid (DEHMTP), diheptyl- (DHPDTPI) > dihexyl- (DHXDTPI) > dinonyl- (DNDTPI), bis(1methylheptyl)- (DMHDTPI) > bis(2-ethylhexyl)- (DEHDTPI) > dioctyldithiophosphinic acid (DODTPI) (Chart 2.9). ${ }^{73}$ In general, the extraction selectivity for Am(III) is better with dithiophosphinic (or phosphoric) acids than with monothiophosphinic (or phosphoric) acids, whereas the extraction selectivity for Am(III) is better with phosphinic acids than with phosphoric acids. The branched alkyl chain-containing acids have higher extraction selectivity than straight alkyl chain-containing acids. An $S F_{\mathrm{Am} / \mathrm{Eu}}=2500$ was obtained by solvent extraction with $0.5 \mathrm{~mol} / \mathrm{L}$ DEHDTPI in toluene from a $1 \mathrm{~mol} / \mathrm{L} \mathrm{NaNO}_{3}$ solution.

$$
\begin{array}{ll}
\text { Ar-I: } \mathrm{R}^{1}=\mathrm{R}^{2}=\text { phenyl } \\
\text { Ar-II: } \mathrm{R}^{1}=\mathrm{R}^{2}=4 \text {-methyl } \\
\text { Ar-III: } \mathrm{R}^{1}=\mathrm{R}^{2}=2 \text {,3-dimethyl } \\
\text { Ar-IV: } \mathrm{R}^{1}=\mathrm{R}^{2}=4 \text {-chloro } \\
\text { Ar-V: } \mathrm{R}^{1}=\mathrm{R}^{2}=3,5 \text {-dichloro-4-methyl } \\
\text { Ar-VI: } \mathrm{R}^{1}=n \text {-octyl } \mathrm{R}^{2}=\text { phenyl } \\
\text { DHXDTPI: } \mathrm{R}^{1}=\mathrm{R}^{2}=n \text {-pentyl } \\
\text { DHPDTPI: } \mathrm{R}^{1}=\mathrm{R}^{2}=n \text {-heptyl } \\
\text { DODTPI: } \mathrm{R}^{1}=\mathrm{R}^{2}=n \text {-octyl } \\
\text { DNDTPI: } \mathrm{R}^{1}=\mathrm{R}^{2}=n \text {-nonyl } \\
\text { DMHDTPI: } \mathrm{R}^{1}=\mathrm{R}^{2}=1 \text {-methylheptyl } \\
\text { DEHDTPI: } \mathrm{R}^{1}=\mathrm{R}^{2}=2 \text {-ethylhexyl }
\end{array}
$$

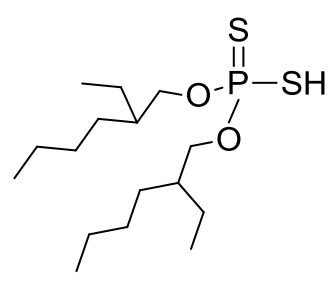

HDEHDTP

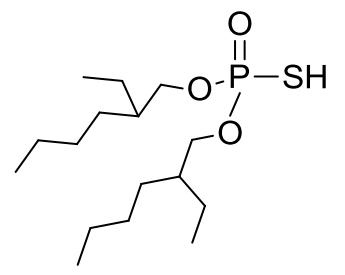

DEHMTP

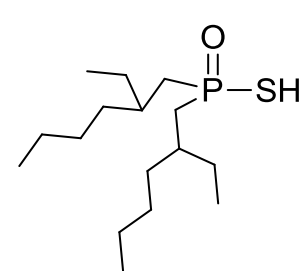

DEHMTPI

Chart 2.9

A systematic study on various symmetrical and asymmetrical mono-/dithiophosphoric acids and mono-/dithiophosphinic acids having dialkyl, diaryl groups or a combination of alkyl and aryl groups, summarized in Chart 2.9, showed that the selectivity for Am(III) over Eu(III) decreases in the order dithiophosphinic (or phosphoric) acids > monothiophosphinic acids > monothiophosphoric acids. The dithiophosphinic acids having branched alkyl chains showed higher selectivities for $\mathrm{Am}(\mathrm{III})$ over $\mathrm{Eu}(\mathrm{III})$ than those containing straight alkyl chains. ${ }^{74}$ 
Diaryldithiophosphinic acids have a better extraction performance than dialkyldithiophosphinic acids. Diaryldithiophosphinic acids bearing electron-withdrawing groups have a better separation capability, than those containing electron-donating groups. However, the latter ones have a stronger extraction power. The separation factor increases with decreasing extractant concentration, or increasing concentration of $\mathrm{Eu}\left(\mathrm{ClO}_{4}\right)_{3}$. An $S F_{\mathrm{Am} / \mathrm{Eu}}=2500$ was obtained by solvent extraction with $0.5 \mathrm{~mol} / \mathrm{L}$ DEHDTPI in toluene from $1 \mathrm{~mol} / \mathrm{L} \mathrm{NaNO}_{3}$ solution.

In conclusion, over the years, structural modifications of the thiophosphinic acids have led to improved extraction properties. The branched alkyl chain-containing thiophosphinic acids are more selective towards Am(III) over Eu(III) compared to straight alkyl chain-containing ones. The introduction of aromatic rings to thiophosphinic acids increases the efficiency of the ligands to extract at relativity higher acidities, however, it gives rise to decreasing $S F$ values. The introduction of electron-withdrawing groups on the aromatic rings, however, has a highly positve influence on the $S F$ values, but negatively influences the extraction efficiency.

\subsubsection{Radiation stability of thiophosphinic acids}

The thiophosphinic acids, which are active agents for the actinide/lanthanide separation undergo oxidation upon storage, even in the absence of hydrolysis and radiolysis. The oxidation products are the corresponding phosphinic acids, resulting from the replacement of $\mathrm{S}$ with $\mathrm{O}$ atoms. ${ }^{75} \mathrm{Bis}\left(2,4,4\right.$-trimethylpentyl)dithiophosphinic acid shows a high $S F_{\mathrm{Am} / \mathrm{Eu}}$ value of 5000 after purification of the commercial CYANEX $301 .^{76}$ However, the ligand is susceptible to hydrolytic degradation in the presence of $\mathrm{HNO}_{3}$. Sole et al. ${ }^{77}$ reported the degradation of $0.5 \mathrm{~mol} / \mathrm{L} \mathrm{CYANEX} 301$ in xylene when in contact with $5 \mathrm{~mol} / \mathrm{L} \mathrm{HNO}_{3}$ at 25 ${ }^{\circ} \mathrm{C}$. Infrared spectroscopy was used to demonstrate breaking of the $\mathrm{S}-\mathrm{H}$ and $\mathrm{P}-\mathrm{S}-\mathrm{H}$ linkages and the formation of S-O linkages. The alkyl chains were also cleaved upon acid exposure.

Similar products were formed upon radiolysis. Chen et al. ${ }^{76}$ reported that the acid content of samples of $0.5 \mathrm{~mol} / \mathrm{L}$ CYANEX 301 and $0.5 \mathrm{~mol} / \mathrm{L}$ pure $\mathrm{R}_{2} \mathrm{P}(\mathrm{S}) \mathrm{SH}$ in $n$-heptane, irradiated in the absence of an aqueous phase, decreased with absorbed $\gamma$ dose, up to $1000 \mathrm{kGy}$, possibly due to conversion of the more acidic thiophosphinic acid into phosphinic acids. Above $1000 \mathrm{kGy}$ the acidity began to increase, which was attributed to the formation of sulfuric acid.

${ }^{31} \mathrm{P}$ NMR techniques were used to identify $\mathrm{R}_{2} \mathrm{P}(\mathrm{S}) \mathrm{OH}$ and $\mathrm{R}_{2} \mathrm{P}(\mathrm{O}) \mathrm{OH}$ in irradiated solutions and oxidation of the starting compound occurred even in the absence of the aqueous phase. Decomposition of $\mathrm{R}_{2} \mathrm{P}(\mathrm{S}) \mathrm{SH}$ was more severe in the commercial CYANEX 301 solution, 


\section{Chapter 2}

namely $94 \%$ compared to $54 \%$ in the purified solution at $100 \mathrm{kGy}$. The distribution ratios for both americium and europium increased with the absorbed dose, but a clear interpretation of the results was confounded by the change in acidity of the irradiated solutions, which also affects the extraction efficiency.

The radiolysis of CYANEX 301 was investigated by irradiating both neat, purified $\mathrm{R}_{2} \mathrm{P}(\mathrm{S}) \mathrm{SH}$ and $0.5 \mathrm{~mol} / \mathrm{L} \mathrm{R}_{2} \mathrm{P}(\mathrm{S}) \mathrm{SH}$ in $n$-dodecane. Using ${ }^{1} \mathrm{H}$ NMR spectroscopy it was found that $80 \%$ of $\mathrm{R}_{2} \mathrm{P}(\mathrm{S}) \mathrm{SH}$ was decomposed at $1000 \mathrm{kGy}$, with the formation of $6 \% \mathrm{R}_{2} \mathrm{P}(\mathrm{S}) \mathrm{OH}$ and $5 \%$ $\mathrm{R}_{2} \mathrm{P}(\mathrm{O}) \mathrm{OH}$, in addition to numerous other unidentified products. For $0.5 \mathrm{~mol} / \mathrm{L} \mathrm{R}_{2} \mathrm{P}(\mathrm{S}) \mathrm{SH}$ in $n$-dodecane, irradiating over the range 0-700 kGy a steady increase in $D_{\mathrm{Eu}}$ was observed, due to the formation of monothiophosphinic and phosphinic acids. At an absorbed dose of $700 \mathrm{kGy}$, the $D_{\mathrm{Eu}}$ was nearly as high as $D_{\mathrm{Am}}$. The $S F_{\mathrm{Am} / \mathrm{Eu}}$ value dropped from 1000 to 10 at $\mathrm{pH} 3.3 .^{61}$

Saponified CYANEX 301 was irradiated using ${ }^{60} \mathrm{Co} \gamma$-rays as a $1 \mathrm{~mol} / \mathrm{L}$ solution in kerosene at varying dose rates. There was essentially no change in either $D_{\mathrm{Eu}}$ or $D_{\mathrm{Am}}$, to an absorbed dose of $42 \mathrm{kGy}$. The lack of an apparent effect on the solvent-extraction efficiency is probably due to the lower absorbed doses used in this study. ${ }^{78}$

Substitution of alkyl chains for aromatic groups in dithiophosphinic acids apparently increases the radiation stability, but this requires aromatic diluents and neutral organophosphorus compounds as synergists. Diphenyldithiophosphinic acid $\left(\mathrm{Ph}_{2} \mathrm{P}(\mathrm{S}) \mathrm{SH}\right)$ and bis(chlorophenyl)dithiophosphinic acid $\left((\mathrm{ClPh})_{2} \mathrm{P}(\mathrm{S}) \mathrm{SH}\right)$ were investigated for radiation stability as $0.5 \mathrm{~mol} / \mathrm{L}$ solutions in toluene with and without $0.25 \mathrm{~mol} / \mathrm{L}$ TBP as phase modifier. No aqueous phase was present during these irradiations. Over the range 100$700 \mathrm{kGy}$, the $D_{\text {Am }}$ slightly decreased, while $D_{\text {Eu }}$ greatly increased, resulting in a decreased separation factor. This interesting result was attributed to the radiolysis products originating from the added TBP. When TBP was added to irradiated TBP-free dithiophosphinic acid solutions, a subtle decrease in $S F_{\mathrm{Eu} / \mathrm{Am}}$ was observed. The difference in the effect of irradiation on the extraction of the two metals suggests that the actinides and lanthanides form different complexes in this mixed solvent system, which was confirmed by slope analysis. ${ }^{65}$

Bis(chlorophenyl)dithiophosphinic acid $\left((\mathrm{ClPh})_{2} \mathrm{P}(\mathrm{S}) \mathrm{SH}\right)$ was investigated for both radiolytic and hydrolytic stability. Upon addition of $2 \mathrm{~mol} / \mathrm{L} \mathrm{HNO}_{3},(\mathrm{ClPh})_{2} \mathrm{P}(\mathrm{S}) \mathrm{SH}$ in toluene solution was oxidized to give $(\mathrm{ClPh})_{2} \mathrm{P}(\mathrm{S}) \mathrm{OCH}_{3},(\mathrm{ClPh})_{2} \mathrm{P}(\mathrm{O}) \mathrm{SCH}_{3}$, and $(\mathrm{ClPh})_{2} \mathrm{P}(\mathrm{O}) \mathrm{OCH}_{3}$, after 1 day of exposure. Hydrolytic degradation did not occur upon exposure to $\mathrm{HCl}$ and was 
insignificant upon exposure to $\mathrm{H}_{2} \mathrm{SO}_{4}$. Furthermore, addition of nitrous acid scavengers such as hydrazine and urea mitigated the oxidation of $(\mathrm{ClPh})_{2} \mathrm{P}(\mathrm{S}) \mathrm{SH}$ exposed to $2 \mathrm{~mol} / \mathrm{L} \mathrm{HNO}_{3}{ }^{79}$ When $(\mathrm{ClPh})_{2} \mathrm{P}(\mathrm{S}) \mathrm{SH}$ was irradiated as a $0.5 \mathrm{~mol} / \mathrm{L}$ solution in toluene, about $40 \%$ was destroyed upon exposure to the high, absorbed dose of $2000 \mathrm{kGy}$, with only slightly more decomposition occurring in the presence of $0.5 \mathrm{~mol} / \mathrm{L} \mathrm{HNO}_{3} .{ }^{79}$ It was also demonstrated that the neat compound was more stable upon irradiation than in solution. When $0.5 \mathrm{~mol} / \mathrm{L}$ $(\mathrm{ClPh})_{2} \mathrm{P}(\mathrm{S}) \mathrm{SH}$ in toluene was irradiated (containing $0.15 \mathrm{~mol} / \mathrm{L}$ TOPO as phase modifier) in the presence of $0.5 \mathrm{~mol} / \mathrm{L} \mathrm{HNO}_{3}$, the $D_{\mathrm{Eu}}$ values remained nearly constant, while $D_{\mathrm{Am}}$ decreased due to loss of the dithiophosphinic acid. This resulted in a rapidly decreasing separation factor. However, the absorbed doses studied were high, and the $S F_{\mathrm{Am} / \mathrm{Eu}}$ was still $>10$ at an absorbed dose of $600 \mathrm{kGy}{ }^{76}$

Recently, bis(o-trifluoromethylphenyl)dithiophosphinic acid was studied for its hydrolytic stability, having a very high $S F_{\mathrm{Am} / \mathrm{Eu}}$ value of 100 000. It showed $28 \%$ degradation after 140 days contact of a $0.1 \mathrm{~mol} / \mathrm{L}$ solution in trifluoromethylsulfone diluent with a $0.01 \mathrm{~mol} / \mathrm{L}$ $\mathrm{HNO}_{3}$ solution of $1 \mathrm{~mol} / \mathrm{L}$ nitrate ion. ${ }^{76}$

From the described results, it is clear that the improvement of the stability of thiophosphinic acids under highly acidic and radiation conditions still remains a challenge. However, the stability has been improved by replacing alkyl chains by aromatic rings and in particular by electron-withdrawing group-containing aromatic rings, although this negatively influences the extraction efficiency.

\subsubsection{N donor ligands}

In general, due to the presence of the soft $\mathrm{N}$ atom, $\mathrm{N}$ donor ligands, show selectivity for An(III) over Ln(III). One of the most important classes are the 2,6-bis(5,6-dialkyl-1,2,4triazin-3-yl)pyridines, known as BTPs (Chart 2.10). ${ }^{80}$ These ligands show high $S F_{\text {Am/Eu }}$ values of $>100 .{ }^{81}$ These ligands extract An(III) by a solvation mechanism leading to the formation of $\mathrm{M}: \mathrm{L}_{3}$ complexes in which three bidentate ligands bind the trivalent metal cation. It is a completely anhydrated complex as shown by X-ray crystallography. ${ }^{82} 2,6$-Bis(5,6-dipropyl1,2,4-triazin-3-yl)pyridine (BTP) exhibited almost quantitative (99.85\%) extraction of Am(III) and $\mathrm{Cm}(\mathrm{III})$. The Am(III) extraction rate was found to linearly increase with the extractant concentration independent of the nitrate concentration. The $\mathrm{HNO}_{3}$ concentration indirectly influenced the extraction rate by lowering the concentration of BTP available for americium complexation. ${ }^{83}$ 
<smiles>[R]c1nnc(-c2cccc(-c3nnc([R])c([R])n3)n2)nc1[R]</smiles>

BTP<smiles>CC1(C)CCC(C)(C)c2nc(-c3cccc(-c4nnc5c(n4)C(C)(C)CCC5(C)C)n3)nnc21</smiles>

$\mathrm{CyMe}_{4}-\mathrm{BTP}$

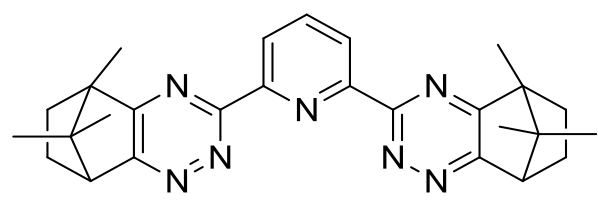

CA-BTP<smiles>[R]c1nnc(-c2cccc(-c3cccc(-c4nnc([R])c([R])n4)n3)n2)nn1</smiles>

BTBP<smiles>CC1(C)c2ccccc2C(C)(C)c2nc(-c3cccc(-c4nnc5c(n4)C(C)(C)c4ccccc4C5(C)C)n3)nnc21</smiles>

$\mathrm{BzMe}_{4}-\mathrm{BTP}$

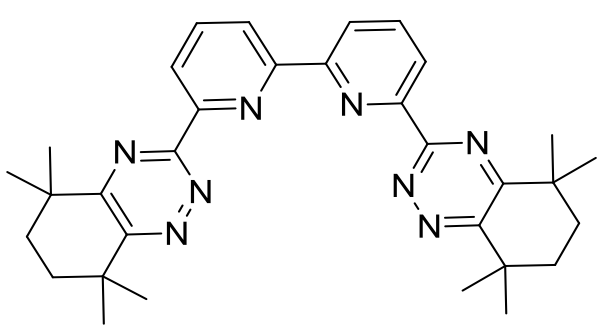

$\mathrm{CyMe}_{4}-\mathrm{BTBP}$

Chart 2.10

Further investigations showed that with $n \operatorname{Pr}$-BTP, the $D_{\text {Am }}$ value decreased to $80 \%$ after two days and to $50 \%$ upon contact with $1 \mathrm{~mol} / \mathrm{L} \mathrm{HNO}_{3}$ for two hours. ${ }^{84}$ It was demonstrated that BTP ligands bearing branched alkyl groups at the $\alpha$-position of the triazine ring are more stable than those bearing $n$-alkyl groups.

In various attempts to improve the hydrolytic stability, new tridentate nitrogen heterocyclic reagents were developed in which all $\alpha$-hydrogens were replaced by methyl groups, viz. 2,6bis(5,5,8,8-tetramethyl-5,6,7,8-tetrahydrobenzo[1,2,4]triazin-3-yl) pyridine (CyMe $\mathrm{CH}_{4}$-BTP) and 2,6-bis(9,9,10,10-tetramethyl-9,10-dihydro-1,2,4-triaza-anthran-3-yl) pyridine (BzMe 4 -BTP) (Chart 2.10). $\mathrm{CyMe}_{4}$-BTP is resistant to hydrolysis in $3 \mathrm{~mol} / \mathrm{L} \mathrm{HNO}_{3}$, whereas $\mathrm{BzMe}_{4}-\mathrm{BTP}$ is resistant to both acid hydrolysis and radiolysis. These ligands give rise to significantly enhanced separations of $\mathrm{Am}$ (III) from an $\mathrm{HNO}_{3}$ solution, with typical values for $\mathrm{CyMe}_{4}-\mathrm{BTP}$ of $D_{\mathrm{Am}}=500$ and $S F_{\mathrm{Am} / \mathrm{Eu}}=5000$ compared with $D_{\mathrm{Am}}=30$ and $S F_{\mathrm{Am} / \mathrm{Eu}}=400$ for $n \mathrm{Pr}-\mathrm{BTP}$ in $2 \mathrm{~mol} / \mathrm{L} \mathrm{HNO}_{3}{ }^{85}$ The nature of the organic diluents is very important (e.g. aliphatic, aromatic, and nitroaromatic), influencing the extraction properties due to the different stabilities of the BTPs in the different solvents. ${ }^{86,87}$ Bis-2,6-(5,6,7,8-tetrahydro-5,9,9-trimethyl-5,8-methano1,2,4-benzotriazin-3-yl)pyridine (CA-BTP; Chart 2.10) has been described as a new, 
optimized extracting agent for the separation of $\mathrm{Am}$ (III) and $\mathrm{Cm}$ (III) from $\mathrm{Ln}$ (III). The extraction properties of CA-BTP have been studied giving $S F_{\mathrm{Am} / \mathrm{Eu}}$ value of around 100 under conditions relevant to the SANEX process. CA-BTP has some advantages over other $\mathrm{N}$ donors like higher solubility, high stability towards $\mathrm{HNO}_{3}$, and fast extraction kinetics. ${ }^{88}$ Another class of soft $\mathrm{N}$ donor ligands are the BTBPs (6,6'-(5,6-dialkyl-1,2,4-triazin-3-yl)2,2'-bipyridines; Chart 2.10). ${ }^{89}$ The stoichiometry of the extracted M(III) complexes differs from that of BTP ligands as M:L $L_{2}$ complexes have been identified instead of $M: L_{3}$ for BTPs, as characterized by X-ray crystallography, slope analysis, and TRLFS. ${ }^{90}$ The alkylated BTBPs undergo rapid hydrolytic degradation in contact with $\mathrm{HNO}_{3}$, induced by the abstraction of a hydrogen from the pseudo-benzylic position of the triazine rings similar to the BTPs. ${ }^{84}$ To enhance the stability towards hydrolysis, $\mathrm{CyMe}_{4}$-BTBP (Chart 2.10), which is the current reference molecule for $\mathrm{An}(\mathrm{III}) / \mathrm{Ln}$ (III) separation, has successfully been designed. ${ }^{91}$

$\mathrm{Am}(\mathrm{III}) / \mathrm{Eu}(\mathrm{III})$ separation factors of more than 100 have been obtained with $\mathrm{CyMe}_{4}-\mathrm{BTBP}$, and back-extraction is feasible due to significantly lower distribution ratios (e.g., for the extraction from $\mathrm{HNO}_{3}$ into $10 \mathrm{mmol} / \mathrm{L}$ CyMe 4 -BTBP in 1-octanol, $D_{\mathrm{Am}}=10$ for $1 \mathrm{~mol} / \mathrm{L}$ $\mathrm{HNO}_{3}, D_{\mathrm{Am}}=0.1$ for $0.1 \mathrm{~mol} / \mathrm{L} \mathrm{HNO}_{3}$ ). However, compared to other actinide extractants such as TBP, malonamides, or diglycolamides, the extraction kinetics is still slow, requiring the addition of a phase-transfer catalyst such as DMDOHEMA ${ }^{91}$ or TODGA. ${ }^{92}$ Alternatively, cyclohexanone as a diluent has a positive impact on the kinetics, ${ }^{87,93}$ but taking into account some mutual solubility of cyclohexanone and $\mathrm{HNO}_{3}$ it is not useful under the required conditions. The solubility of $\mathrm{CyMe}_{4}-\mathrm{BTBP}$ in 1 -octanol, is only about $10 \mathrm{mmol} / \mathrm{L},{ }^{94}$ which may be too low for the extraction of nominal concentrations of $\mathrm{Am}$ (III) and $\mathrm{Cm}$ (III) (i.e., several $\mathrm{mmol} / \mathrm{L})$.

Of the new BTBP ligands 6,6'-bis(5,5,7,7-tetramethyl-5,7-dihydrofuro[3,4-e]-1,2,4-triazin-3yl)-2,2'-bipyridine $\quad\left(\mathrm{Cy}_{5}-\mathrm{O}-\mathrm{Me}_{4}\right.$-BTBP $), \quad$ and $\quad 6,6^{\prime}$-bis(5,5,7,7-tetramethyl-5,7dihydrothieno[3,4-e]-1,2,4-triazin-3-yl)-2,2'-bipyridine (Cy5-S-Me 4 -BTBP) (Chart 2.11), the former showed a higher affinity for Am(III) and selectivity for Am(III) over Eu(III). However, the $D_{\mathrm{Am}}$ and $S F_{\mathrm{Am} / \mathrm{Eu}}$ values of $\mathrm{CyMe}_{4}$-BTBP are both significantly higher than those of $\mathrm{Cy}_{5}-\mathrm{O}-\mathrm{Me}_{4}-\mathrm{BTBP}$ and $\mathrm{Cy}_{5}-\mathrm{S}_{-} \mathrm{Me}_{4}-\mathrm{BTBP}$ in cyclohexanone. Changing the diluent from cyclohexanone to 2-methylcyclohexanone led to a decrease in $D_{\mathrm{Am}}$, but an increase in $\mathrm{SF}_{\mathrm{Am} / \mathrm{Eu}}$ for $\mathrm{Cy}_{5}-\mathrm{S}_{-} \mathrm{Me}_{4}-\mathrm{BTBP}{ }^{95}$ 


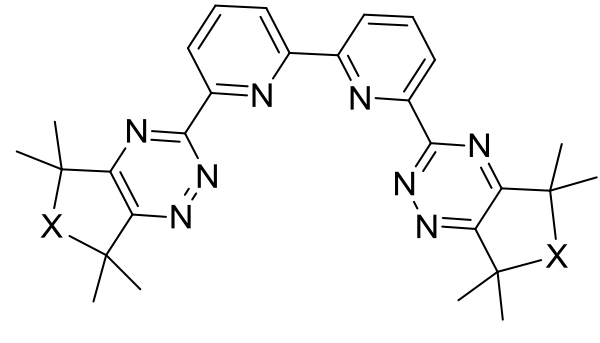

Cy5-O-Me 4 -BTBP: $\mathrm{X}=\mathrm{O}$ Cy5-S-Me ${ }_{4}$-BTBP: $X=S$

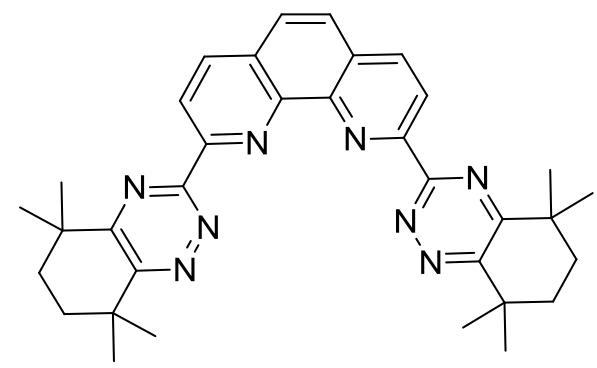

Cy ${ }_{4}$ BTPhen

Chart 2.11

In a recent study, preorganization of the donor atoms of bipyridine into a rigid cis-locked 1,10-phenanthroline system ( $\left.\mathrm{Cy}_{4} \mathrm{BTPhen}\right)$ (Chart 2.11) led to a rapid and highly efficient separation of actinides from lanthanides. The formation of a 1:2 bis-complex of a quadridentate bis-triazine ( $\mathrm{Cy}_{4} \mathrm{BTPhen}$ ) ligand with $\mathrm{Eu}(\mathrm{III})$ was shown by $\mathrm{X}$-ray crystallography. The ligand showed high $D_{\text {Am }}$ values up to 1000 at $4 \mathrm{~mol} / \mathrm{L} \mathrm{HNO}_{3}$ with an $S F_{\mathrm{Am} / \mathrm{Eu}}$ of 200-400 at various $\mathrm{HNO}_{3}$ concentrations. ${ }^{96}$ Theoretical calculations on BTBP and BTPhen clearly explain the fast extraction kinetics of BTPhen compared to BTBP, suggesting that the extraction of $\mathrm{Eu}(\mathrm{III})$ occurs at the interface via the protonated form of the ligand under acidic conditions. ${ }^{97}$

The terpyridine analog of BTBT, viz. 6,6'-bis(5,5,8,8-tetramethyl-5,6,7,8-tetrahydro-1,2,4benzotriazin-3-yl)-2,2':6',2''-terpyridine (CyMe ${ }_{4}$-BTTP) (Chart 2.12), containing aliphatic tetramethylcyclohexenyl rings to increase the solubility, was recently synthesized. ${ }^{98}{ }^{1} \mathrm{H}$ NMR and mass spectrometry studies indicated that the ligand forms 1:2 complexes with lanthanide(III) perchlorates, where the aliphatic rings are conformationally constrained, whereas 1:1 complexes are formed with lanthanide(III) nitrates in which the rings are conformationally flexible. In the absence of a phase-modifier, CyMe $\mathrm{C}_{4}$-BTTP in $n$-octanol showed a maximum $D_{\mathrm{Am}}$ value of 0.039 and a maximum $S F_{\mathrm{Am} / \mathrm{Eu}}$ value of 12.0 from $1 \mathrm{~mol} / \mathrm{L}$ $\mathrm{HNO}_{3}$. The metal(III) cations are extracted as $1: 1$ complexes from $\mathrm{HNO}_{3}$.<smiles>C[C@H](Br)[C@H](C)C(C)(C)C</smiles>

Chart 2.12 
Three new bis-triazine based ligands containing pyridine, furan, and thiophene rings viz. 5,5'bis-1,2,4-triazine-6-6'-pyridine (TAZP), 5,5'-bis-1,2,4-triazine-6-6'-furan (TAZF) and 5,5'bis-1,2,4-triazine-6-6'-thiophene (TAZT) (Chart 2.13) have been studied. Theoretical calculations predict that these ligands are selective for An(III), however, extraction results have not been reported yet. ${ }^{99}$

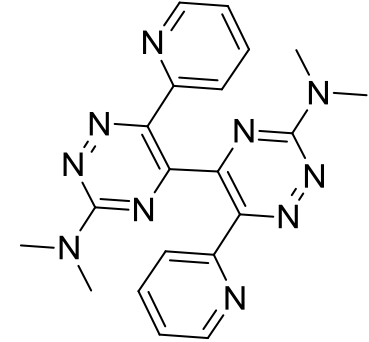

TAZP<smiles></smiles>

TAZF: $X=0$

TAZT: $X=S$

Chart 2.13

Recently, ligands have been developed based on a terpyridine core to which amide groups were attached. Solvent extraction studies with $N, N, N^{\prime}, N^{\prime}$-tetraalkyl-6,6' '-(2,2':6',2',terpyridine)diamides and $N, N^{\prime}$-diethyl- $N, N^{\prime}$-diphenyl-6,6' '-(2,2':6',2',-terpyridine)diamide (Chart 2.14) showed that these bitopic ligands extract actinides in different oxidation states (U(VI)O $\mathrm{O}_{2}, \mathrm{~Np}\left(\mathrm{~V}\right.$ and VI), $\mathrm{Pu}(\mathrm{IV}), \mathrm{Am}(\mathrm{III})$, and $\mathrm{Cm}(\mathrm{III})$ ) from $3 \mathrm{~mol} / \mathrm{L} \mathrm{HNO}_{3}$. These ligands showed moderate $D_{\mathrm{M}(\mathrm{III})}$ and $S F$ values for $\mathrm{Am}(\mathrm{III})$ and $\mathrm{Cm}(\mathrm{III})$ from $\mathrm{Ce}(\mathrm{III})$ and $\mathrm{Eu}(\mathrm{III})$. The introduction of phenyl rings on the amidic nitrogen increased the ligand efficiency. ${ }^{100}$<smiles>[R]N([R])C(=O)c1cccc(-c2cccc(-c3cccc(C(=O)N([R])[R])n3)n2)n1</smiles>

Chart 2.14

$$
\begin{aligned}
& \text { te-tpyda : } \mathrm{R}^{1}=\mathrm{R}^{2}=\mathrm{C}_{2} \mathrm{H}_{5} \\
& \text { tb-tpyda }: \mathrm{R}^{1}=\mathrm{R}^{2}=\mathrm{C}_{4} \mathrm{H}_{9} \\
& \text { to-tpyda }: \mathrm{R}^{1}=\mathrm{R}^{2}=\mathrm{C}_{8} \mathrm{H}_{17} \\
& \text { dedp-tpyda }: \mathrm{R}^{1}=\mathrm{C}_{2} \mathrm{H}_{5}, \mathrm{R}^{2}=\text { Phenyl }
\end{aligned}
$$

Various $\mathrm{N}$ donor ligands have been developed over the recent years that are soluble in a wide range of solvents. These $\mathrm{N}$ donor ligands are completely incinerable (only composed of $\mathrm{C}, \mathrm{H}$, $\mathrm{O}$, and $\mathrm{N}$ ). Several of them extract at $3 \mathrm{~mol} / \mathrm{L} \mathrm{HNO}_{3}$, comparable to nuclear waste conditions, with the high $D_{\mathrm{Am}}$ and $S F_{\mathrm{Am} / \mathrm{Eu}}$ values. 


\subsubsection{Extraction by synergistic mixtures}

Combining different ligands can increase the distribution ratio and/or selectivity for actinides/lanthanides. Two general approaches are applied for the separation of An(III) and $\mathrm{Ln}(\mathrm{III})$. In the first one, the separation of lanthanides from actinides is achieved by aqueousphase complexation of the actinides. In the second approach, actinides are selectively extracted into the organic phase. In such systems, the soft nitrogeN donor ligand selectivity forms complexes with actinides over the lanthanides and the other extractant helps to improve extraction.

DMDOHEMA displays only a very slight selectivity for Am(III) with an $S F_{\text {Am/Eu }}$ value of 1.7. ${ }^{101}$ However, by using DMDOHEMA and a mixture of HEDTA+citrate, an $S F_{\text {Eu/Am value }}$ of 12.5 was achieved. ${ }^{102}$ Gannaz et al. ${ }^{103}$ investigated a mixed solvent system containing DMDOHEMA and di- $n$-hexylphosphoric acid (HDHP) (Chart 2.15) resulting in a $S F_{\mathrm{Eu} / \mathrm{Am}}$ value of 10 .
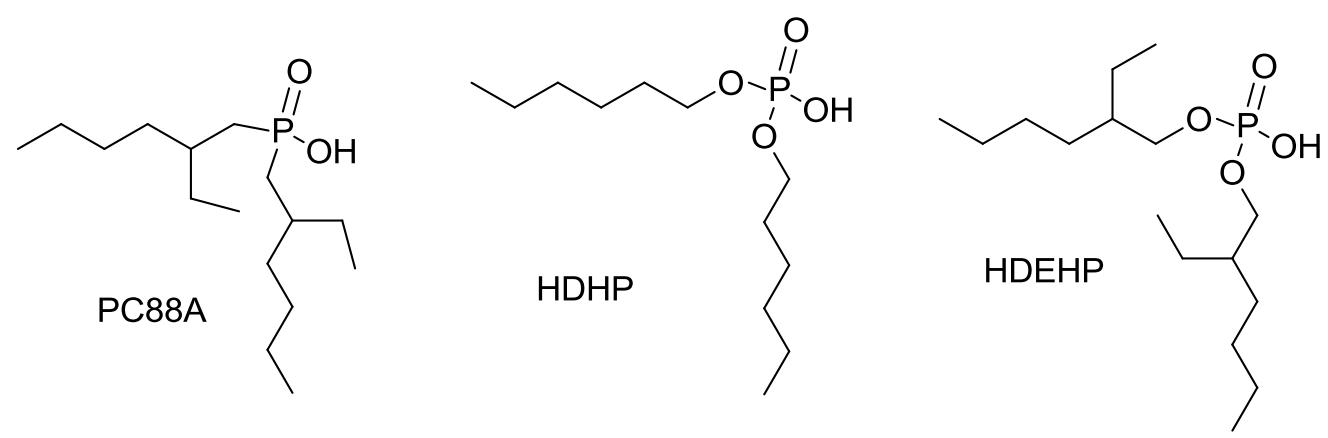

Chart 2.15

A mixed solvent system containing bis(2-ethylhexyl)phosphoric acid (HDEHP) (Chart 2.15) and CMPO dissolved in an n-paraffinic diluent was studied by Dhami et al. ${ }^{104}$ They reported that HDEHP suppressed the extraction of trivalent lanthanides and actinides from $\mathrm{HNO}_{3}$ solution compared to the TRUEX solvent in which the mixture of CMPO and TBP is used. It was assumed that this suppression is caused by interaction between HDEHP and CMPO, which lowers the effective CMPO concentration. Interestingly, bis(2-ethylhexyl)phosphinic acid (PC88A) (Chart 2.15) has been reported to synergize the extraction of $\mathrm{U}(\mathrm{VI}) \mathrm{O}_{2}$ by CMPO from phosphoric acid media. ${ }^{105}$ This might be due to the different type of interaction of CMPO to HDEHP or PC88A. Three diluents were considered to study their influence on the CMPO/HDEHP combined extractant system viz. m-nitrobenzotrifluoride (F-3), phenyl trifluoromethyl sulfone (FS-13), and Isopar (isoparrafinic solvent). ${ }^{106}$ An interesting 26 
observation was the reversal in selectivity in going from the aliphatic hydrocarbon diluent to fluorinated diluents. For a solvent system consisting of $0.2 \mathrm{~mol} / \mathrm{L} \mathrm{CMPO}$ and $0.6 \mathrm{~mol} / \mathrm{L}$ HDEHP, the $S F_{\mathrm{Eu} / \mathrm{Am}}$ was 1.55 with Isopar as a diluent, whereas they were 0.81 and 0.79 for the F-3 and FS-13 diluents, respectively.

Although $\mathrm{N}$ donor ligands mostly show selectivity for the actinide ions, their extracting power is often too low for practical applications. This limitation can be overcome to some extent by combining them with another extractant. An example of this approach is the combination of 2,4,6-tris(2-pyridyl)-1,3,5-triazine (TPTZ) (Chart 2.16) with 2-bromodecanoic acid. $S F_{\mathrm{Am} / \mathrm{Eu}}$ values of 8-10 were obtained for extractions into $0.1 \mathrm{~mol} / \mathrm{L}$ TPTZ/1 mol/L 2-bromodecanoic acid/1-decanol from $1 \mathrm{~mol} / \mathrm{L} \mathrm{KNO}_{3}$ adjusted to $\mathrm{pH} 1.8$ to 3 with $\mathrm{HNO}_{3}{ }^{107}$ TPTZ was also tested as a mixture with dinonylnaphthalene sulfonic acid (HDNNS) in tert-butylbenzene. The $S F_{\mathrm{Am} / \mathrm{Eu}}$ values were about the same as those for the TPTZ/2-bromodecanoic acid system, but HDNNS did allow for actinide extraction from slightly more acidic solutions $(0.1 \mathrm{~mol} / \mathrm{L}$ $\left.\mathrm{HNO}_{3}\right)$.<smiles>c1ccc(-c2nc(-c3ccccn3)nc(-c3ccccn3)n2)nc1</smiles>

TPTZ<smiles>c1ccc(-c2cccc(-c3ccccn3)n2)nc1</smiles>

tpy

Chart 2.16

Extraction of Am(III) and Eu(III) with TPTZ, alkylated TPTZ derivatives and 2,2',6',2', terpyridine (tpy) (Chart 2.16), and alkylated tpy derivatives mixed with 2-bromodecanoic acid were studied by Cordier et al. ${ }^{108}$ In the case of TPTZ, alkylation of the pyridyl rings resulted in increased extraction over the parent TPTZ, persumably due to increased lipophilicity of the ligand. Regardless of the alkylation of the pyridyl rings, no significant change in the $S F_{\mathrm{Am} / \mathrm{Eu}}(10)$ was observed. In contrast, alkylation of tpy resulted in a decrease of the extraction compared to the original tpy ligand, presumably because of an increase in the basicity of the ligand caused by the alkyl groups. Cordier et al. proposed the TPTZ/2bromodecanoic acid and tpy/2-bromodecanoic acid as synergistic mixtures. The synergistic nature of the tpy/2-bromodecanoic acid system was confirmed by Hagström et al. ${ }^{109}$ by continuous variation experiments in which the $\mathrm{Am}(\mathrm{III})$ and $\mathrm{Eu}(\mathrm{III})$ distribution ratios were 


\section{Chapter 2}

measured as a function of the tpy mole fraction. They observed that when Am(III) or Eu(III) (in $0.005-0.2 \mathrm{~mol} / \mathrm{L}^{H_{N O}}$ ) is extracted into $1 \mathrm{~mol} / \mathrm{L}$ 2-bromodecanoic acid in tertbutylbenzene, a significant increase in the distribution ratio occurs when $0.02 \mathrm{~mol} / \mathrm{L}$ tpy is added $\left(S F_{\mathrm{Am} / \mathrm{Eu}}=7-9\right)$. Since there is negligible extraction of $\mathrm{Am}(\mathrm{III})$ or $\mathrm{Eu}(\mathrm{III})$ into 0.02 $\mathrm{mol} / \mathrm{L}$ tpy under these conditions, it can be concluded that there is a synergistic interaction between 2-bromodecanoic acid and tpy.

Another tridentate nitrogen ligand 2-(3,5,5-trimethylhexanoyl-amino)-4,6-di(pyridine-2-yl)1,3,5-triazine (TMHADPTZ; Chart 2.17) showed an $S F_{\mathrm{Am} / \mathrm{Eu}}$ value of 10 in a synergistic system with octanoic acid. However, this system is very sensitive to $\mathrm{pH}$ and requires the feed to be buffered. ${ }^{110}$
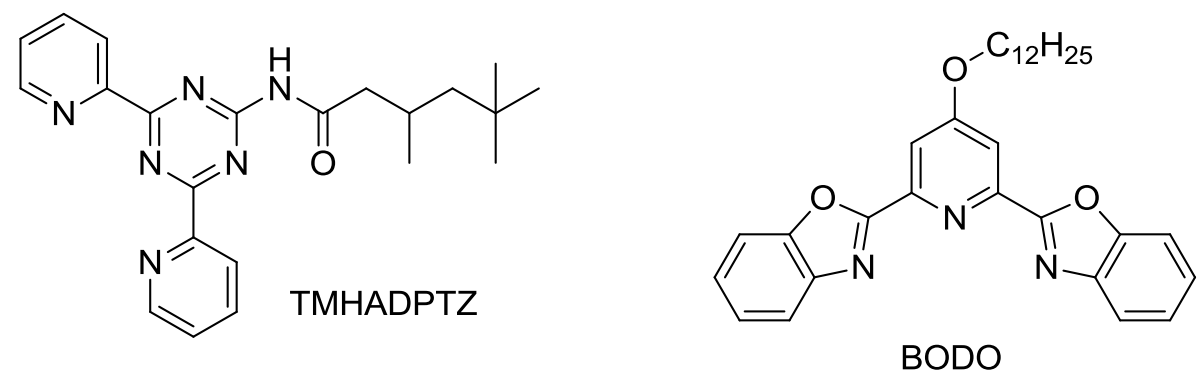

Chart 2.17

A synergistic mixture of 2,6-bis-(benzoxazolyl)-4-dodecyloxylpyridine (BODO; Chart 2.17) and 2-bromodecanoic acid was studied in nitrate and perchlorate medium by Andersson et $a l .{ }^{111} \mathrm{An} S F_{\mathrm{Am} / \mathrm{Eu}}$ value of 10-15 was achieved, depending on the nitrate ion concentration in the aqueous phase. Since BODO is less basic than tpy, extraction can be performed at relatively low $\mathrm{pH}$.

$N, N, N^{\prime}, N^{\prime}$-tetrakis(2-methylpyridyl) ethylenediamine (TPEN) (Chart 2.18) has been investigated in synergistic combination with HDEHP in 1-octanol. ${ }^{112}$ A maximum $S F_{\mathrm{Am} / \mathrm{Eu}}$ value of 4.1 was achieved at $\mathrm{pH} 4.8$ at $0.1 \mathrm{~mol} / \mathrm{L} \mathrm{NH}_{4} \mathrm{NO}_{3}$ into $0.01 \mathrm{~mol} / \mathrm{L}$ TPEN in 1-octanol without the addition of HDEHP. This separation factor is much lower than the expected value of 100 based on the complexation constants for the Am(III) and Eu(III) TPEN complexes in aqueous media. ${ }^{113}$ However, upon addition of $0.004 \mathrm{~mol} / \mathrm{L}$ HDEHP to $0.002 \mathrm{~mol} / \mathrm{L}$ TPEN in 1-octanol a significant increase in the extraction of $\mathrm{Am}(\mathrm{III})$ and $\mathrm{Eu}(\mathrm{III})$ from $0.1 \mathrm{~mol} / \mathrm{L}$ $\mathrm{NH}_{4} \mathrm{NO}_{3}$ occurred and the $S F_{\mathrm{Am} / \mathrm{Eu}}$ value was raised to 80 .

$\mathrm{N}$ donor ligands 2,6-bis(1-aryl-1H-tetrazol-5-yl)pyridines (ATPs; Chart 2.18) ${ }^{114}$ do not extract trivalent actinide and lanthanide ions from $\mathrm{HNO}_{3}$ media. However, synergistic extraction occurs when the acid form of chlorinated cobalt dicarbollide (CCD) is mixed with the ATP; 28 
the synergy between the two components is clearly demonstrated in continuous variation experiments. In this extractant system, the $\mathrm{Am}$ and $\mathrm{Eu}$ distribution ratios decrease with increasing $\mathrm{HNO}_{3}$ concentration. A solvent mixture consisting of $0.01 \mathrm{~mol} / \mathrm{L}$ ATP and 0.01 $\mathrm{mol} / \mathrm{L}$ CCD dissolved in F-3 showed good selectivity for Am(III) with an $S F_{\mathrm{Am} / \mathrm{Eu}}$ value of 70 75.<smiles>c1ccc(CN(CCN(Cc2ccccn2)Cc2ccccn2)Cc2ccccn2)nc1</smiles>

TPEN<smiles>[R]c1ccc(-n2nnnc2-c2cccc(-c3nnnn3-c3ccc([R])c([R])c3)n2)cc1[R]</smiles>

ATP

Chart 2.18

CYANEX $301(0.54 \mathrm{~mol} / \mathrm{L})$ yielded $S F_{\mathrm{Am} / \mathrm{Eu}}$ values of 5000, 16000, and 11000 with TBP, triphenyl phosphate (TPP), and diphenylsulphoxide (DPSO), respectively, as a synergist. ${ }^{115}$ CYANEX 301 was also studied in combination with the $\mathrm{N}$ donor ligands 2,2'-bipyridyl and 1,10-phenanthroline ${ }^{116}$ giving $S F_{\mathrm{Am} / \mathrm{Eu}}$ values of up to 40000 in the presence of $\mathrm{HNO}_{3}$. These $S F$ values are much higher than other cases in which TBP, TPP or DPSO are used as synergists due to the favorable soft-soft interaction of $\mathrm{N}$ donors with Am(III) compared to $\mathrm{Eu}(\mathrm{III})$. In the extracted species Am(III) is surrounded by three CYANEX and one auxillary ligand, whereas Eu(III) is centered around two CYANEX, one nitrate ion, and one auxillary ligand.

The extraction behavior towards Am(III) and Eu(III) of a mixture of CYANEX 301 and various neutral N, O, or S donor ligands was studied. ${ }^{117}$ CYANEX 301 in the presence of O donor auxiliary ligands, such as TBP and TOPO and corresponding ligands with S donor sites, viz. tri- $n$-butyl thiophosphate (TBTP) and tri-iso-butylphosphine sulphide (TiBPS) (Chart 2.19) gave rise to decreased $S F$ values compared to the parent CYANEX 301. 


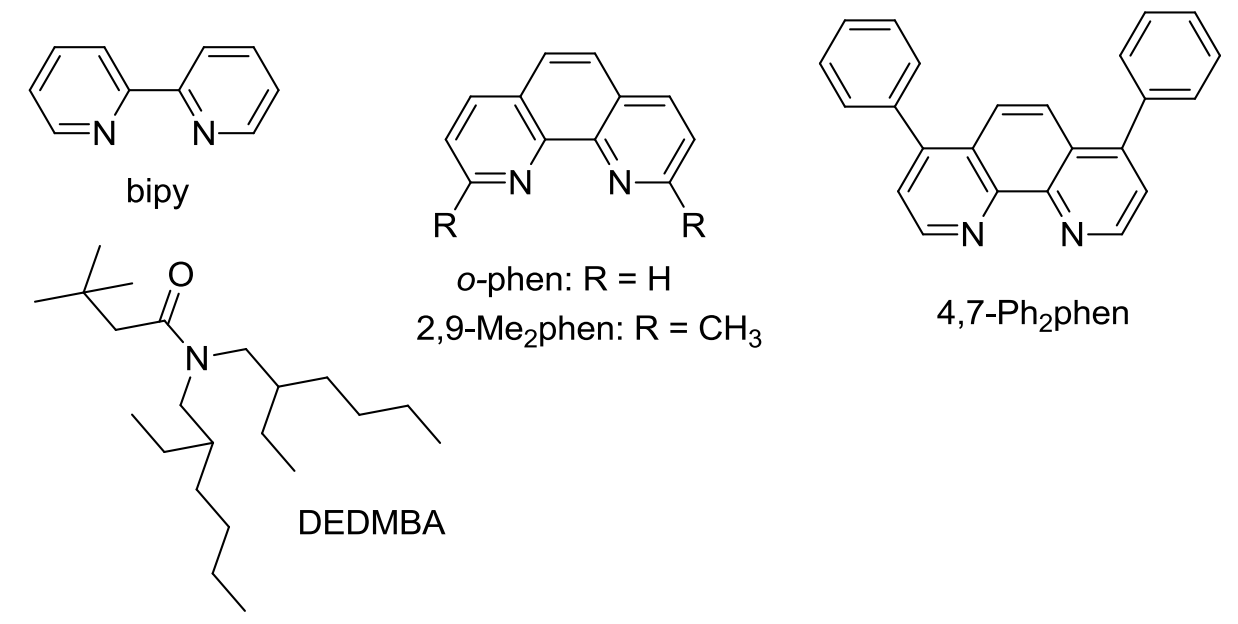

Chart 2.19

In another study, the extraction behavior of CYANEX 301 was investigated in the presence of various bi- and tridentate $\mathrm{N}$ donor ligands, viz. bipyridyl (bipy), $o$-phenanthroline (o-phen), 2,9-dimethylphenanthroline (2,9-Me 2 phen), 4,7-diphenylphenanthroline (4,7- $\mathrm{Ph}_{2}$ phen) and tripyridyl-triazine (TPTZ) (Chart 2.19). The synergistic system with $o$-phen gave rise to the highest $S F_{\mathrm{Am} / \mathrm{Eu}}$ values of 26500 with a high $D_{\mathrm{Am}}$ value of $204 .{ }^{118}$

CYANEX 301, TBP, tri-tert-butyl phosphate (TtBP), triphenyl phosphate (TPP), TOPO, CMPO, N,N-bis(ethyl-2-hexyl)dimethyl-2,2-butanamide (DEDMBA) and DMDBTDMA (Chart 2.3) were studied as synergistic mixtures for the separation of Am(III) and Eu(III). ${ }^{119}$ The addition of TBP, TtBP, TPP, and DOTA to CYANEX 301 increased the $S F_{\mathrm{Am} / \mathrm{Eu}}$ values, however, TOPO, CMPO, and DMDBTDMA decreased the $S F$ values with large increase in the extraction efficiency.

CYANEX $301(\mathrm{RH})$ dissolved in 1-octanol was investigated in the presence of various electroN donor additives such as trioctylamine (TOA), 1-octanol, TOPO, TBP, and TIBPS by means of IR spectroscopy. ${ }^{120}$ The formation of hydrogen bonded complexes (H-complexes) with proton transfer and the $\left[\mathrm{TOAH}^{+}\right]\left[\mathrm{R}^{-}\right]$ion pair were detected in the HR-TOA system. In mixtures of CYANEX 301 with TBP, TOPO, and TIBPS, during the formation of the Hcomplexes, hydrogen bonding without proton transfer was found to take place. The strength of the interaction between CYANEX 301 and ligand decreases in the series TOA > TOPO > TBP > TIBPS > 1-octanol, which corresponds to their relative basicity. Extraction of $\mathrm{Zn}$ (II) was performed and a decrease in extraction was observed in the series TOA > TOPO > TBP > 1-octanol, which corresponds with the order of the decreasing CYANEX 301 activity in the presence of the above additives. 
Recently, a mixture of BTBP and TBP was used to develop the GANEX (Group ActiNide EXtraction) process to separate the $\mathrm{An}(\mathrm{III})$ as a group from a mixture of $\mathrm{Ln}$ (III) and corrosion and fission products. $\mathrm{Am}(\mathrm{III}), \mathrm{Pu}(\mathrm{IV}), \mathrm{U}(\mathrm{VI}) \mathrm{O}_{2}$, and $\mathrm{Np}(\mathrm{IV})$ were extracted as a group by $0.01 \mathrm{~mol} / \mathrm{L} \mathrm{CyMe} \mathrm{CHTP}_{4}-30 \% \mathrm{TBP}$ in cyclohexanone, with negligible amount of lanthanides. However, some corrosion and fission products were co-extracted. ${ }^{121}$

The GANEX solution containing $\mathrm{CyMe}_{4}$-BTBP together with TBP in cyclohexanone was investigated for its stability towards aging as well as towards hydrolysis and $\gamma$-radiolysis. A large decrease in the concentration of $\mathrm{CyMe}_{4}$-BTBP was observed upon addition of $4 \mathrm{~mol} / \mathrm{L}$ $\mathrm{HNO}_{3}$. The radiolytic stability was confirmed for both low- as well as high-dose rates up to a total dose of $200 \mathrm{kGy}$. It was also observed that the radiolytic stability increased with the presence of $\mathrm{HNO}_{3}$ during the irradiation of the solvent. ${ }^{122}$ Direct selective extraction of trivalent actinides from a simulated PUREX raffinate was studied using a mixture of $\mathrm{CyMe}_{4}-$ BTBP and TODGA (1-cycle SANEX). The solvent showed a high selectivity for trivalent actinides with a high lanthanide separation factor. However, co-extraction of some fission product elements $(\mathrm{Cu}, \mathrm{Ni}, \mathrm{Zr}, \mathrm{Mo}, \mathrm{Pd}, \mathrm{Ag}$, and $\mathrm{Cd})$ from a simulated PUREX raffinate was also observed. The extraction of $\mathrm{Zr}$ and Mo could be suppressed using oxalic acid, but the use of the well-known $\mathrm{Pd}$ chelator $N$-(2-hydroxyethyl)-ethylenediamine- $N, N^{\prime}, N^{\prime}$-triacetic acid (HEDTA) was unsuccessful. However, the sulfur-bearing amino acid L-cysteine showed a good complexation of $\mathrm{Pd}$ and prevented its extraction into the organic phase without influencing the extraction of the trivalent actinides $\mathrm{Am}$ (III) and $\mathrm{Cm}(\mathrm{III}){ }^{123}$

The addition of a synergist is an interesting phenomenon to increase the efficiency of the ligands. By the careful choice of synergists, desired separation factors between lanthanides and actinides can be achieved. At present, $S F_{\mathrm{Am} / \mathrm{Eu}}$ values of up to 40,000 with some synergistic systems are achieved, whereas no system with a single extractant is known to achieve such a high $S F$ values.

\subsection{Water-soluble ligands/reagents for back-extraction}

Various reagents are being applied for the stripping of $\mathrm{An}(\mathrm{III}) / \mathrm{Ln}(\mathrm{III})$ from highly active nuclear waste. This concept has now led to the selective stripping of actinides by the use of selective ligands within the current European project ACSEPT $^{22}$ (Actinide reCycling by SEParation and Transmutation). In the so-called "innovative SANEX process" the trivalent actinides are recovered from the PUREX raffinate by means of one organic solvent, thus reducing the cycles and minimizing the waste. The process consists of an $\mathrm{An}(\mathrm{III}) / \mathrm{Ln}(\mathrm{III})$ 


\section{Chapter 2}

co-extraction step at high acidity and then the loaded solvent is subjected to several stripping steps (Figure 2.3). The first one concerns the selective stripping of trivalent actinides with a selective water-soluble agent, followed by the subsequent stripping of trivalent lanthanides.

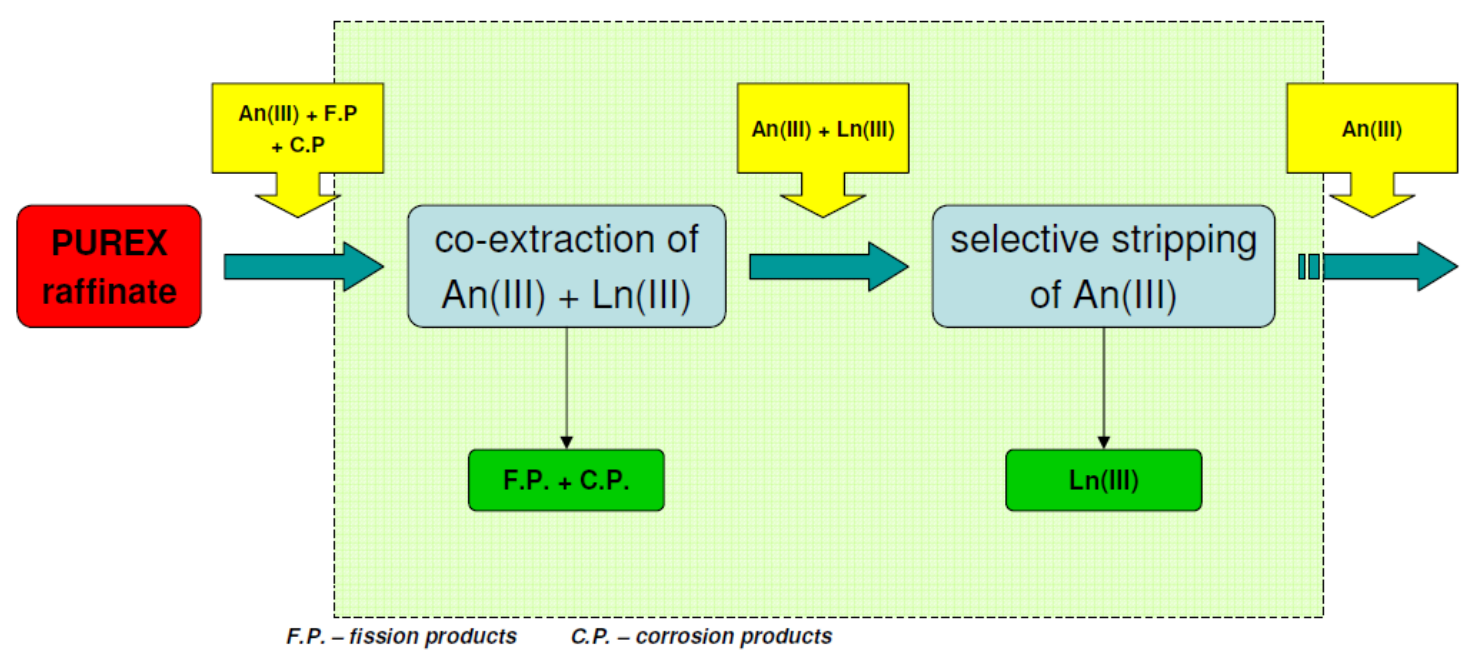

Figure 2.3. Schematic overview of the innovative SANEX process.

Carboxylic acids and aminopolycarboxylic acids have been studied as agents for the reverse extraction of An(III) in the TALSPEAK process. ${ }^{124}$ An organic phase containing a $0.2 \mathrm{~mol} / \mathrm{L}$ solution of HDEHP and an aqueous phase with $1 \mathrm{~mol} / \mathrm{L}$ carboxylic acids containing 0.05 mol/L DTPA at pH 3 were used to selectivity strip Am(III) from $\mathrm{La}(\mathrm{III}), \mathrm{Ce}(\mathrm{III})$, and Eu(III). The stripping of Am(III) and Pu(IV) from a solution of CMPO and TBP in $n$-dodecane (Truex solution, normally $0.2 \mathrm{~mol} / \mathrm{L} \mathrm{CMPO}+1.2 \mathrm{~mol} / \mathrm{L}$ TBP in $n$-dodecane) was performed following two schemes. In the first scheme, trivalent actinides and lanthanides are stripped by $0.04 \mathrm{~mol} / \mathrm{L} \mathrm{HNO}_{3}$, followed by $\sim 0.05 \mathrm{~mol} / \mathrm{L}$ oxalic acid for $\mathrm{Pu}(\mathrm{IV})$ and finally, $0.25 \mathrm{~mol} / \mathrm{L}$ sodium carbonate solution to remove $\mathrm{U}(\mathrm{VI}) \mathrm{O}_{2}$. In the second scheme, all the tri- and tetravalent metal ions are stripped together using a mixture of formic acid, hydrazine hydrate, and citric acid. ${ }^{125,126}$ The studies show that near quantitative recovery of $\mathrm{Am}(\mathrm{III})$ and $\mathrm{Pu}(\mathrm{IV})$ is feasible from an acid-bearing Truex solution. With appropriate concentrations of individual constituents, this stripping agent can be used for the recovery of actinides from a loaded Truex solution with any acid content. A slight modification of the stripping agent was made to selectively strip An(III). The stripping experiments by oxalic acid resulted in the recovery of all actinides as a group. Stripping experiments with $0.04 \mathrm{~mol} / \mathrm{L} \mathrm{HNO}_{3}$ removed most of $\mathrm{Am}(\mathrm{III})(+\mathrm{Cm}(\mathrm{III}))$ and a small portion of $\mathrm{Pu}(\mathrm{IV})$, while those with $0.05 \mathrm{~mol} / \mathrm{L} \mathrm{HNO}_{3}+0.05$ 
mol/L HF removed all the $\mathrm{Pu}(\mathrm{IV})$ and the remaining $\mathrm{Am}(\mathrm{III})$, and $0.25 \mathrm{~mol} / \mathrm{L} \mathrm{Na}_{2} \mathrm{CO}_{3}$ removed $\mathrm{U}(\mathrm{VI}) \mathrm{O}_{2}{ }^{127}$

It has been shown that a solution of vinylidene-1,1-diphosphonic acid or 1-hydroxyethane1,1-diphosphonic acid can strip the actinides from a simulated high level waste, which can be directly sent for vitrification. ${ }^{128}$ Neptunium and plutonium, extracted in the TBP phase, were stripped together using a mixture of $0.05 \mathrm{~mol} / \mathrm{L}$ ascorbic acid and $0.25 \mathrm{~mol} / \mathrm{L}$ hydrogen peroxide in $2.0 \mathrm{~mol} / \mathrm{L} \mathrm{HNO}_{3}$ solution. ${ }^{129}$ Stripping of $\mathrm{An}(\mathrm{III}) / \mathrm{Ln}(\mathrm{III})$ from an organic phase $(0.2 \mathrm{~mol} / \mathrm{L} \mathrm{CMPO}+1.2 \mathrm{~mol} / \mathrm{L}$ TBP in $n$-dodecane $)$ was also carried out with a $0.05 \mathrm{~mol} / \mathrm{L}$ potassium ferrocyanide solution. ${ }^{130}$ More than $98 \%$ of $\mathrm{Am}(\mathrm{III}), \mathrm{Pu}(\mathrm{IV}), \mathrm{Np}(\mathrm{III}), \mathrm{U}(\mathrm{VI}) \mathrm{O}_{2}$, and $\mathrm{Eu}(\mathrm{III})$ could be stripped in four contacts, whereas the stripping of $\mathrm{Zr}(\mathrm{IV})$ and $\mathrm{Ru}(\mathrm{III})$ was $94 \%$ and $92 \%$, respectively.

Various water soluble ligands $N, N, N^{\prime}, N^{\prime}$-tetramethyl- (TMDGA), $N, N, N^{\prime}, N^{\prime}$-tetraethyl(TEDGA), $N, N, N^{\prime}, N^{\prime}$-tetrapropyldiglycolamide (TPDGA), and $N, N$-dipropyldiglycolamic acid (DPDGAc) (Chart 2.20) were investigated for their ability to form complexes with, and to back-extract, different metal cations, ${ }^{131}$ not only An(III,IV) but also Ca(II), Sc(III), Y(III), $\mathrm{Zr}(\mathrm{IV}), \mathrm{La}(\mathrm{III}), \mathrm{Hf}(\mathrm{IV})$, and $\mathrm{Bi}(\mathrm{III})$. Their extraction efficiencies were compared with the corresponding carboxylic and aminopolycarboxylic acids diglycolic acid (DGAc), 3,6-dioxaoctanedioic acid (DODA), ethylenediamine- $N, N, N^{\prime}, N^{\prime}$-tetraacetic acid (EDTA), glycolic acid (GAc), 3-methyliminodiacetic acid (MIDAc), 2-phenylmalonic acid (PMAc), and 3-thiadiglycolic acid (TDGAc) (Chart 2.20).

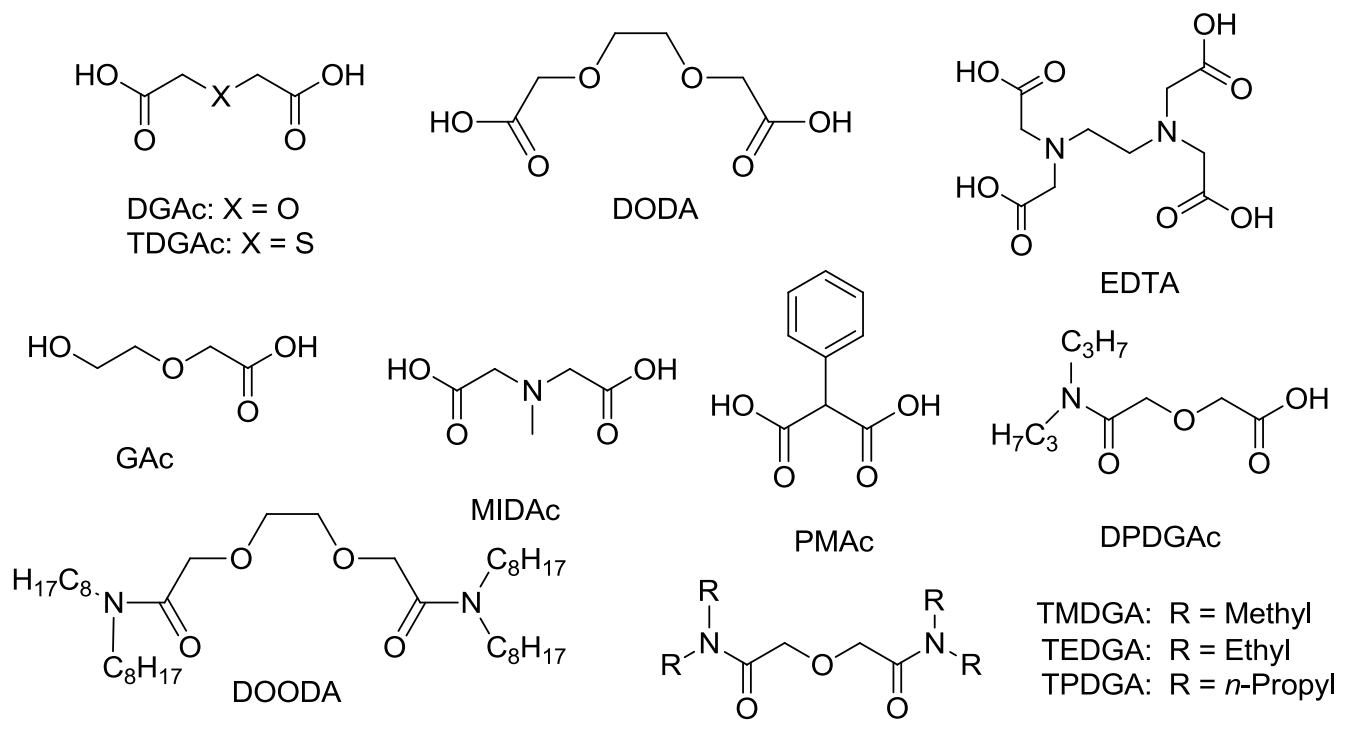

Chart 2.20 


\section{Chapter 2}

The diglycolamide-based ligands give more stable complexes with $\mathrm{Ca}(\mathrm{II}), \mathrm{Sc}(\mathrm{III}), \mathrm{Y}(\mathrm{III})$, $\mathrm{Zr}(\mathrm{IV}), \mathrm{La}(\mathrm{III}), \mathrm{Hf}(\mathrm{IV}), \mathrm{Bi}(\mathrm{III}), \mathrm{An}(\mathrm{III})$, and An(IV) than EDTA. They also have better water solubility independent of $\mathrm{pH}$. The ligand:metal complexation ratio was found to be $2: 1$ and 3:1 for $\mathrm{Am}(\mathrm{III})$ and $\mathrm{Pu}(\mathrm{IV})$, respectively, in the aqueous phase. ${ }^{132}$ Diglycolamides are neutral donors, so they can be used in acidic solution and are stronger An(III) stripping agents than multidentate carboxylic and aminopolycarboxylic acids. DPDGAc and other ligands from DGA series, having the same central frame $\left((\mathrm{O}) \mathrm{CCH}_{2} \mathrm{OCH}_{2} \mathrm{C}(\mathrm{O})\right)$, exhibited a high chelating ability for Am(III). Among the diglycolamide ligands, TEDGA is the best ligand for the back-extraction of $\mathrm{Am}(\mathrm{III})$ and other metals under acidic conditions. ${ }^{132}$

$N, N, N^{\prime}, N^{\prime}$-Tetraoctyl-3,6-dioxaoctanediamide (DOODA) extracts light lanthanides with high $D$ values into the organic phase, while TEDGA shows a strong complexing ability with middle and heavy lanthanides in the aqueous phase. Am(III) and $\mathrm{Cm}$ (III) behave as light lanthanides in this extraction system, therefore $\mathrm{Am}$ and $\mathrm{Cm}$ show a high separation from the middle lanthanides. Separation of actinides from the middle lanthanides was carried out by the combination of two neutral donors, DOODA (Chart 2.20) in the organic phase and TEDGA in the aqueous phase. ${ }^{133}$ Therefore, by using a $0.2 \mathrm{~mol} / \mathrm{L}$ solution of DOODA in the organic phase and $0.01 \mathrm{~mol} / \mathrm{L}$ TEDGA in the aqueous phase, $S F_{\mathrm{Am} / \mathrm{Ln}}$ values of $18.8,8.3$, and 18.8 were obtained for $\mathrm{Eu}, \mathrm{Sm}$, and $\mathrm{Gd}$, respectively, and using $0.3 \mathrm{~mol} / \mathrm{L}$ DOODA with the same concentration of TEDGA $S F_{\mathrm{Am} / \mathrm{Ln}}$ values of 40, 14.4, and 111 were obtained for Eu, Sm, and $\mathrm{Gd}$, respectively, in both cases at $5 \mathrm{~mol} / \mathrm{L} \mathrm{HNO}_{3}$.

Recently, some $\mathrm{N}$ donor tetrapodal hexadentate ligands, $N, N$-bis(2-pyridylmethyl)ethylenediamine- $N^{\prime}, N^{\prime}$-diacetic acid (Lpy) and $N, N$-bis(2-pyrazylmethyl)ethylenediamine$N^{\prime}, N^{\prime}$ - diacetic acid (Lpz) (Chart 2.21) have been designed to quantify the impact of softness on the coordination of f-block elements in aqueous solution, and in particular on the selectivity for Am(III) over Eu(III). ${ }^{134}$ The hard acetate groups in these ligands provide stability to the $\mathrm{An}(\mathrm{III})$ and $\mathrm{Ln}(\mathrm{III})$ complexes and two N-heterocyclic soft groups provide Am(III) versus Eu(III) selectivity. These ligands bearing pyridine or pyrazine $N$ donor moieties have the same complex structure in water as indicated by ${ }^{1} \mathrm{H}$ NMR spectroscopy and potentiometric analysis. Hydration numbers of 3 for the europium and terbium complexes, as assessed by luminescence lifetime measurements, indicated that the two ligands act as hexadentate donors in both systems. The selectivity for Am(III) over Eu(III) was increased by a factor of 8 when the pyridine-containing fragment $\mathrm{N}\left(\mathrm{CH}_{2} \mathrm{py}\right)_{2}$ was substituted by the softer 
pyrazine-containing fragment $\mathrm{N}\left(\mathrm{CH}_{2} \mathrm{pz}\right)_{2}$. However, the softer pyrazine-based ligand gave less stable complexes with the hard $\mathrm{Ln}(\mathrm{III})$ cations than the pyridine-based ligand. ${ }^{134}$<smiles>O=C(O)CN(CCN(Cc1ccccn1)Cc1ccccn1)CC(=O)O</smiles><smiles>O=C(O)CN(CCN(Cc1cnccn1)Cc1cnccn1)CC(=O)O</smiles><smiles>O=C(O)CN(CCN(Cc1ccccn1)Cc1ccccn1)Cc1ccccn1</smiles><smiles>O=C(O)CN(CCN(Cc1cnccn1)Cc1cnccn1)Cc1cnccn1</smiles>

Chart 2.21

Ligands with an enhanced soft character, bearing three pyridines (L3py) or three pyrazines (L3pz), (Chart 2.21) were synthesized ${ }^{135}$ and the related lanthanide complexes were studied in solution. Ligand L3py gave complexes with a coordination geometry similar to EDTA, i.e. a hexadentate coordination mode, as indicated by ${ }^{1} \mathrm{H}$ NMR spectroscopy and luminescence lifetimes. On the other hand, the softest ligand, L3pz, formed much less stable complexes.

The selective back-extraction of Am(III) from organic solutions containing $4 \mathrm{f}$ and $5 \mathrm{f}$ elements was tested with these water-soluble complexing agents. Ligand L3pz demonstrated poor stripping ability and selectivity. In contrast, the ligands Lpy, Lpz, and L3py gave interesting

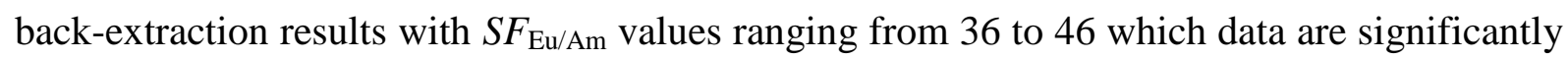
higher than for HEDTA.

More recently, pyrazine-based hydrophilic ligands (Chart 2.22) were described, which were synthesized by a Pd-based cross coupling reaction of chloropyrazine with phosphorus pronucleophiles. The ligands were tested for the selective stripping of $\mathrm{Am}(\mathrm{III})$ over $\mathrm{Eu}(\mathrm{III})$. Some of these ligands showed a good separation with a $S F_{\mathrm{Eu} / \mathrm{Am}}$ value of $40 .{ }^{136}$<smiles>O=[PH](O)c1cncc(P(=O)(O)O)n1</smiles><smiles>NOP(=O)(O)c1cncc(C(=O)N2CCOCC2)n1</smiles>

Chart 2.22 
Various $\mathrm{N}$ and $\mathrm{O}$ donor ligands have been developed in recent years for the selective stripping of An(III). These ligands find potential applications in the innovative SANEX process. In this process the trivalent actinides are recovered directly from the PUREX raffinate by means of water-soluble selective ligands, thus reducing the number of cycles in waste processing.

\subsection{Ionic liquids}

In comparison to traditional solvent-extraction processes, the metal-ion partitioning in ionic liquid (IL)-based extraction systems exhibits high complexity. The superior extraction efficiency and enhanced selectivity of some IL-based extraction systems, compared to those of traditional solvent systems, have received considerable attention.

Apart from a better efficiency, as solely measured via the $D$ values, two other aspects of IL systems are of high importance and might provide additional benefits. First, the $\mathrm{pH}$ dependence can be reversed and the selectivity from one cation to another can be alteed compared with traditional solvents. This is illustrated in the case of TODGA dissolved in either $\left.\left.\left[\mathrm{C}_{2} \mathrm{mim}\right]\right] \mathrm{Tf}_{2} \mathrm{~N}\right]$ or isooctane for the extraction of $\mathrm{Eu}(\mathrm{III}), \mathrm{La}(\mathrm{III})$, and $\mathrm{Lu}(\mathrm{III}) .{ }^{137} \mathrm{~A}$ reversal of the extraction efficiency as a function of $\left[\mathrm{HNO}_{3}\right]$ occurs in the IL system compared to isooctane as a solvent. In the IL system, extraction can be performed under much less acidic conditions. The selectivity of TODGA for the lanthanide series is in favor of the heavier $\mathrm{Ln}$ in isooctane and the lighter ones in $\left[\mathrm{C}_{\mathrm{n}} \mathrm{mim}\right]\left[\mathrm{Tf}_{2} \mathrm{~N}\right]$ (Chart 2.23). Similar results were found for transition metals. ${ }^{138}$

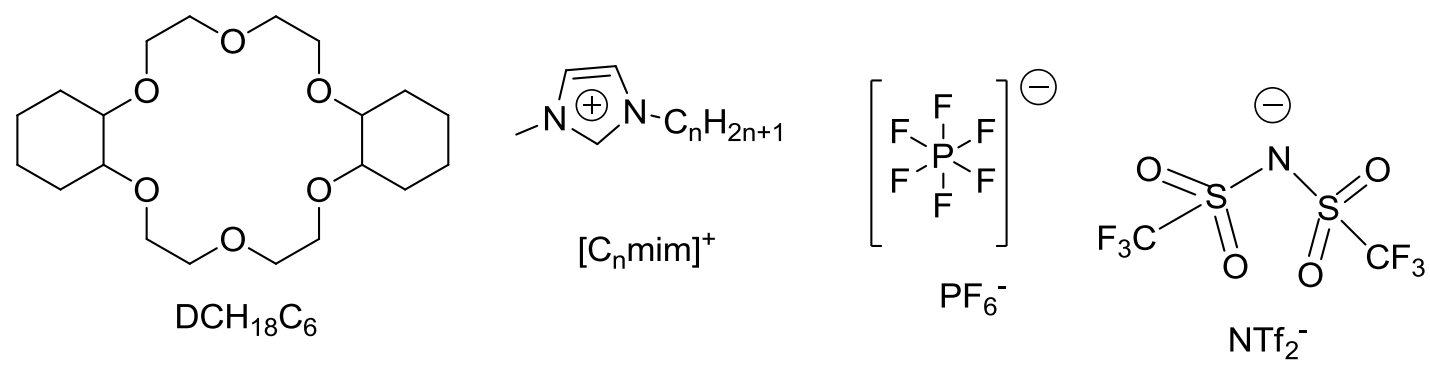

Chart 2.23

The second advantage concerns the synergistic extraction, which is an effective method for improving the extraction efficiency and selectivity in traditional solvent system extraction (see section 2.5.3). Stepinski et al. ${ }^{139}$ observed that the addition of TBP can markedly increase the extraction of $\mathrm{Sr}(\mathrm{II})$ from acidic nitrate media into $\left[\mathrm{C}_{\mathrm{n}} \mathrm{mim}\right]\left[\mathrm{NTf}_{2}\right]$ by $\mathrm{DCH}_{18} \mathrm{C}_{6}$ (Chart 
2.23). The alkyl chain length of the IL cation has important effects on the magnitude of the synergistic enhancement, which contributes to the design of IL-based synergistic systems.

Well-known ligands for An(III) such as TBP, CMPO, and TODGA have been investigated in combination with ionic liquids. TBP dissolved in $\left[\mathrm{C}_{4} \mathrm{mim}\right]\left[\mathrm{PF}_{6}\right] \mathrm{IL}$ medium was investigated for the extraction of $\mathrm{U}(\mathrm{VI}) \mathrm{O}_{2}$ and the results compared with those of a $\mathrm{TBP} / n$-dodecane system. The extraction of $\mathrm{U}(\mathrm{VI}) \mathrm{O}_{2}$ by a $1.1 \mathrm{~mol} / \mathrm{L} \mathrm{TBP} /\left[\mathrm{C}_{4} \mathrm{mim}\right]\left[\mathrm{PF}_{6}\right]$ system was similar to that by the traditional TBP/n-dodecane system, when the concentration of $\mathrm{HNO}_{3}$ was less than $4.0 \mathrm{~mol} / \mathrm{L}$. However, at high $\mathrm{HNO}_{3}$ (> $4 \mathrm{~mol} / \mathrm{L}$ ) concentrations, increased $\mathrm{U}(\mathrm{VI}) \mathrm{O}_{2}$ extraction was observed for the $\mathrm{TBP} /\left[\mathrm{C}_{4} \mathrm{mim}\right]\left[\mathrm{PF}_{6}\right]$ system. This may be attributed to ion exchange between $\left[\mathrm{PF}_{6}\right]^{-}$and anionic uranyl nitrate complexes $\left[\mathrm{UO}_{2}\left(\mathrm{NO}_{3}\right)_{3}\right]^{-140}$ The dissolution of $\mathrm{U}(\mathrm{VI}) \mathrm{O}_{2}$ in $[\mathrm{Bmim}]\left[\mathrm{Tf}_{2} \mathrm{~N}\right]$ with $\mathrm{TBP}$ resulted in a $\left(\mathrm{U}(\mathrm{VI}) \mathrm{O}_{2}\right): \mathrm{TBP}=1: 2$ complex. The maximum amount of $\mathrm{U}(\mathrm{VI}) \mathrm{O}_{2}$ dissolution is about $320 \mathrm{mg}$ in $3.6 \mathrm{~mL}$ of $[\mathrm{Bmim}]\left[\mathrm{Tf}_{2} \mathrm{~N}\right.$ ] containing $16.7 \%$ by volume of the $\operatorname{TBP}\left(\mathrm{HNO}_{3}\right)_{1.8}\left(\mathrm{H}_{2} \mathrm{O}\right)_{0.6}$ complex. ${ }^{141}$

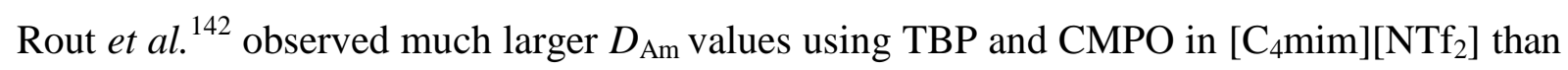
in $n$-dodecane. By using [ $\left.\mathrm{C}_{4} \operatorname{mim}\right]\left[\mathrm{NTf}_{2}\right]$ as diluents, the amount of CMPO needed for Am(III) extraction from HLLWs (3-4 mol/L in $\mathrm{HNO}_{3}$ ) can be minimized by a factor of 4. Similarly, using $0.2 \mathrm{~mol} / \mathrm{L} \mathrm{CMPO}-1.2 \mathrm{~mol} / \mathrm{L} \mathrm{TBP}, 40 \mathrm{mg} / \mathrm{mL}$ loading was achieved instead of $9 \mathrm{mg} / \mathrm{mL}$ for the $n$-dodecane system and no third phase formation was detected like in the $n$-dodecane system. The stoichiometry varied from $3: 1$ to $1: 1$ (L:M) depending on the metal concentration. $^{143}$

CMPO is a highly effective actinide extractant in traditional organic diluents. Visser et al. ${ }^{144}$ investigated CMPO in IL-based extraction of $\mathrm{Am}(\mathrm{III}), \mathrm{Pu}(\mathrm{IV}), \mathrm{Th}(\mathrm{IV})$, and [U(VI)O $\mathrm{O}_{2}$ ]. Distribution ratios of CMPO (CMPO and TBP) were at least an order of magnitude higher in $\left[\mathrm{C}_{4} \mathrm{mim}\right]\left[\mathrm{PF}_{6}\right]$ than in $n$-dodecane at $0.1 \mathrm{~mol} / \mathrm{L}$ ligand concentration. Nakashima et al. ${ }^{145,146}$ studied the extraction behavior of $\mathrm{Ce}(\mathrm{III}), \mathrm{Eu}(\mathrm{III})$, and $\mathrm{Y}(\mathrm{III})$ using $\mathrm{CMPO}$ in $\left[\mathrm{C}_{4} \mathrm{mim}\right]\left[\mathrm{PF}_{6}\right]$ and $n$-dodecane. The extraction efficiency of CMPO in $\left[\mathrm{C}_{4} \mathrm{mim}\right]\left[\mathrm{PF}_{6}\right]$ was exceedingly high compared to that in $n$-dodecane, presumably due to the high dielectric constant of the ILs. The extracted metal ions were effectively stripped using acetohydroxamic acid (AHA), ethylenediaminetetraacetic acid (EDTA), diethylenetriaminepentaacetic acid (DTPA), and citric acid, which form a water-soluble complex with metal ions. A highly concentrated aqueous solution of $\left[\mathrm{C}_{4} \mathrm{mim}\right][\mathrm{Cl}]$ can also sufficiently strip metal ions due to the cation exchange mechanism. Upon repeating the extraction and stripping processes four times, no appreciable decline in the extraction efficiency and no extraction hindrance by the 


\section{Chapter 2}

complexing agent were observed. In addition, the efficiency of CMPO for lanthanide ions in $\left[\mathrm{C}_{4} \mathrm{mim}\right]\left[\mathrm{PF}_{6}\right]$ was found to be better than in $n$-dodecane.

The effect of an added amount of an ionic liquid on the extraction of $\mathrm{Am}(\mathrm{III}), \mathrm{Pu}(\mathrm{IV})$, and $\mathrm{U}(\mathrm{VI}) \mathrm{O}_{2}$ has been recently investigated. ${ }^{147}$ The $D_{\text {Am }}$ value increased 126-fold upon addition of $50 \%\left[\mathrm{C}_{4} \mathrm{mim}\right]\left[\mathrm{PF}_{6}\right]$ to $0.1 \mathrm{~mol} / \mathrm{L}$ diphenyl(dibutyl)carbamoylmethyl phosphine oxide $\left(\mathrm{Ph}_{2} \mathrm{iBu}_{2}-\mathrm{CMPO}\right)$ in dichloroethane and 1040-fold upon addition of $50 \%\left[\mathrm{PH}_{4}\right]\left[\mathrm{PF}_{6}\right]$ (phosphonium hexafluorophosphate). The $D_{\mathrm{Pu}}$ and $D_{\mathrm{U}}$ values increased 135- and 272-fold, respectively, by addition of $50 \% \quad\left[\mathrm{C}_{4} \mathrm{mim}\right]\left[\mathrm{PF}_{6}\right]$ to $0.001 \mathrm{~mol} / \mathrm{L} \quad \mathrm{Ph}_{2} \mathrm{iBu}_{2}-\mathrm{CMPO}$ in dichloroethane.

The extraction of different lanthanides and Y nitrates by tetraphenylmethylenediphosphine dioxide, diphenyl(diethylcarbamoylmethyl)phosphine oxide and dibutyl(diethylcarbamoylmethyl)phosphine oxide was considerably increased in the presence of $\left[\mathrm{C}_{4} \mathrm{mim}\right]\left[\mathrm{PF}_{6}\right]$ compared to $n$-dodecane. A copolymer, based on macroporous styrene-divinylbenzene, seeded with a mixture of tetraphenylmethylenediphosphine oxide and $\left[\mathrm{C}_{4} \mathrm{mim}\right]\left[\mathrm{PF}_{6}\right]$ proved effective for the recovery of rare earth elements, $\mathrm{U}(\mathrm{VI}) \mathrm{O}_{2}$, and $\mathrm{Th}(\mathrm{IV})$ from $\mathrm{HNO}_{3}$ solutions. ${ }^{148}$

The extraction behavior of all lanthanides, except for Pm(III), was studied ${ }^{137}$ using TODGA in $\left[\mathrm{C}_{n} \operatorname{mim}\right]\left[\mathrm{NTf}_{2}\right](\mathrm{n}=2,4,6)$. Application of ILs as the extracting phase provided an unprecedented enhancement of the extraction performance of TODGA compared with that in isooctane. TODGA gave a higher selectivity for the middle lanthanides in the IL systems, but provided a higher selectivity for heavier lanthanides in isooctane. Shen et al. ${ }^{149}$ studied the extraction of $\left[\mathrm{U}(\mathrm{VI}) \mathrm{O}_{2}\right]$ in aqueous solution using $N, N, N^{\prime}, N$ '-tetrabutyl-3-oxapentanediamide (TBDA) and $N, N, N^{\prime}, N$ '-dimethyldibutyl-3-oxapentanediamide (MBDA) as an extractant in $\left[\mathrm{C}_{\mathrm{n}} \mathrm{mim}\right]\left[\mathrm{PF}_{6}\right](\mathrm{n}=4,6,8)$. The extraction capability of these diglycolamides was remarkably higher in ILs than in chloroform, particularly at low $\mathrm{HNO}_{3}$ concentration.<smiles>CN(C(=O)COCC(=O)N(C)c1ccccc1)c1ccccc1</smiles>

DMDPhOPDA

Chart 2.24

The extraction of various lanthanides and $\mathrm{Y}$ by $N, N^{\prime}$-dimethyl- $N, N^{\prime}$-diphenyl-1-3oxapentanediamide (DMDPhOPDA) (Chart 2.24) in 1,2-dichloroethane from aqueous media containing $\mathrm{ClO}_{4}^{-}, \mathrm{PF}_{6}^{-}$, and $\mathrm{Tf}_{2} \mathrm{~N}^{-}$anions or by DMDPhOPDA in 1,2-dichloroethane in the 38 
presence of $\left[\mathrm{C}_{4} \mathrm{mim}\right]\left[\mathrm{Tf}_{2} \mathrm{~N}\right]$ and $\left[\mathrm{C}_{4} \mathrm{mim}\right]\left[\mathrm{PF}_{6}\right]$ from $\mathrm{HNO}_{3}$ solutions was studied. The addition of $\mathrm{HPF}_{6}$ and $\mathrm{Tf}_{2} \mathrm{NH}$ or their salts to aqueous $\mathrm{HNO}_{3}$ solution led to an enhancement of Ln(III) extraction by DMDPhOPDA. The extraction efficiency towards Ln(III) for ILs with the $\mathrm{Tf}_{2} \mathrm{~N}^{-}$anion is higher than for ILs with the $\mathrm{PF}_{6}^{-}$anion. Apparently, there is a decrease in

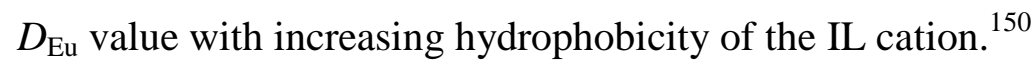

Zuo et al. ${ }^{151}$ studied the extraction and separation of Th(IV) using ILs, in which they found that pure $\left[\mathrm{C}_{8} \mathrm{mim}\right]\left[\mathrm{PF}_{6}\right]$ can be used for separating $\mathrm{Ce}(\mathrm{IV})$ from $\mathrm{Th}(\mathrm{IV})$ and $\mathrm{Ln}(\mathrm{III})$ in $\mathrm{HNO}_{3}$ medium. $\left[\mathrm{C}_{8} \mathrm{mim}\right]\left[\mathrm{PF}_{6}\right]$ showed a good distribution ratio for $\mathrm{Ce}(\mathrm{IV})$, whereas it was small for $\mathrm{Th}(\mathrm{IV})$ and negligible for $\mathrm{Ln}(\mathrm{III})$. The extraction behavior of $\mathrm{Ce}(\mathrm{IV})$ by $\left[\mathrm{C}_{8} \mathrm{mim}\right]\left[\mathrm{PF}_{6}\right]$ was attributed to the formation of $\left[\mathrm{Ce}\left(\mathrm{NO}_{3}\right)_{6}\right]^{2-}$, and the anion forms a new IL with the $\left[\mathrm{C}_{8} \mathrm{mim}\right]^{+}$ cation. The extraction of Th(IV) sulfate using primary amine $\mathrm{N} 1923\left[\left(\mathrm{C}_{\mathrm{n}} \mathrm{H}_{2 \mathrm{n}+1}\right)_{2} \mathrm{CHNH}_{2}(\mathrm{n}=\right.$ 9-11), Chart 2.25] in $\left[\mathrm{C}_{8} \mathrm{mim}\right]\left[\mathrm{PF}_{6}\right]$ was studied. ${ }^{152}$ The extraction behavior was quite different from that using $n$-heptane or dichloromethane as a diluent. A slope analysis revealed that the extraction mechanism was a reverse micellar solubilization, which was confirmed by polarized optical microscopy/transmission electron microscopy. Th(IV) was well-separated from $\mathrm{La}(\mathrm{III}), \mathrm{Ce}(\mathrm{III}), \mathrm{Nd}(\mathrm{III}), \mathrm{Gd}(\mathrm{III})$, and $\mathrm{Er}(\mathrm{III})$ by N1923/[C $\left.{ }_{8} \mathrm{mim}\right]\left[\mathrm{PF}_{6}\right]$ at low acidity, and a satisfactory stripping ratio was achieved using mixed strippants as citric acid, formic acid, and hydrazine hydrate.

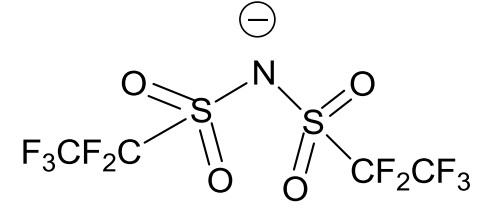

$\mathrm{BETI}^{-}$

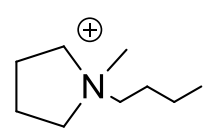

$\mathrm{C}_{4} \mathrm{mPy}$

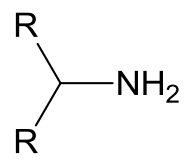

$\mathrm{R}=\mathrm{C} 9-\mathrm{C} 11$

N1923

Chart 2.25

Yoon et al. ${ }^{153}$ studied the extraction of Ce(III), Nd(III), Sm(III), Dy(III), and Yb(III) using HDEHP in $\left[\mathrm{C}_{\mathrm{n}} \mathrm{mim}\right]\left[\mathrm{PF}_{6}\right](\mathrm{n}=2,4)$ or $\left[\mathrm{C}_{4} \mathrm{mPy}\right]\left[\mathrm{PF}_{6}\right]($ Chart 2.25). They found that the IL system showed more than three times enhanced extractability of lanthanides than when $n$ hexane was used as a diluent. In general, the imidazolium-type IL system gave rise to a higher distribution ratio than the pyridinium-type IL system. The $\left[\mathrm{C}_{4} \mathrm{mPy}\right]\left[\mathrm{PF}_{6}\right]$ system gave a much higher selectivity to the heavy lanthanide $\mathrm{Yb}$ than the other systems. Rout et al. ${ }^{154}$ investigated the separation of $\mathrm{Am}(\mathrm{III})$ and $\mathrm{Eu}(\mathrm{III})$ in bis(2-ethylhexyl)diglycolic acid (HDEHDGA) and HDEHP with $\left[\mathrm{C}_{8} \mathrm{mim}\right]\left[\mathrm{NTF}_{2}\right] . S F_{\mathrm{Am} / \mathrm{Eu}}$ values of 35 and 150 were obtained 


\section{Chapter 2}

for HDEHP and HDEHDGA, respectively, using $0.05 \mathrm{~mol} / \mathrm{L}$ extractant in $\left[\mathrm{C}_{8} \mathrm{mim}\right]\left[\mathrm{NTF}_{2}\right]$. The use of HDEHP in $\left[\mathrm{C}_{\mathrm{n}} \mathrm{mim}\right]\left[\mathrm{Tf}_{2} \mathrm{~N}\right] /[\mathrm{BETI}]$ (bis(perfluoroethanesulfonyl)imide; Chart 2.25) has recently resulted in excellent extraction efficiencies and selectivities for some rare earth elements keeping the $\mathrm{An}(\mathrm{III})$ in the aqueous phase compared to that in diisopropylbenzene (DIPB), as in the conventional TALSPEAK process. ${ }^{155}$ Both cations and anions of the ILs exhibited obvious effects on the extraction processes, which are mainly considered to proceed via a cation exchange mechanism. The distribution ratios of applying ILs to the TALSPEAK process are quite attractive.

Recently, the focus has been shifted towards task-specific ILs or functionalized ILs (FILs). FILs contain an extracting moiety in their cationic and/or anionic part and behave simultaneously as the organic phase and the extracting agent. Imidazolium-based 2hydroxybenzylamine FILs (Chart 2.26) containing $\mathrm{Tf}_{2} \mathrm{~N}$ and $\mathrm{PF}_{6}$ anions, exhibited good extraction of Am(III), both in pure form as well as in solution. ${ }^{156}$ Some FILs containing quaternary ammonium cation bearing phosphoryl groups (Chart 2.26) achieved two orders of magnitude higher distribution ratios for $\mathrm{U}(\mathrm{VI}) \mathrm{O}_{2}$ than TBP in $\left[\mathrm{Me}_{3} \mathrm{BuN}\right]\left[\mathrm{NTf}_{2}\right]$ under the conditions of the PUREX process. ${ }^{157}$ A high distribution ratio $(D>1000)$ was obtained with tricaprylmethylammonium thiosalicylate [A336][TS] (Chart 2.26) for the extraction of ${ }^{238} \mathrm{U}$ from natural water, with a high selectivity compared to divalent magnesium and calcium ions, which showed no affinity to this IL. ${ }^{158}$

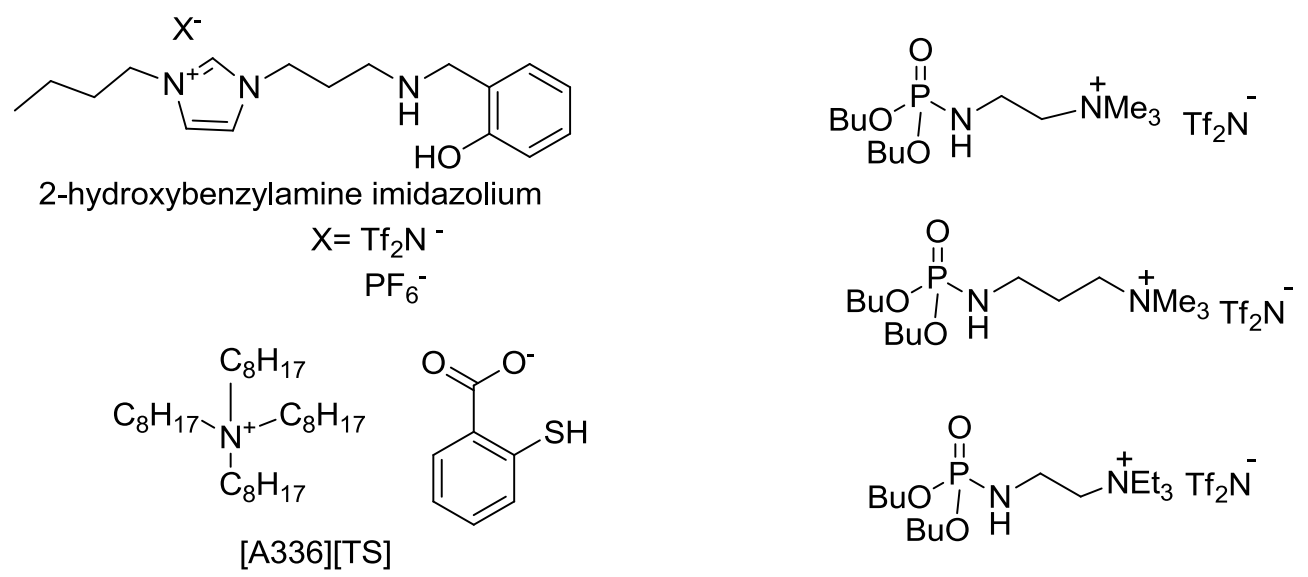

Chart 2.26

The extraction of $\mathrm{Pu}(\mathrm{IV}), \mathrm{U}(\mathrm{VI}) \mathrm{O}_{2}$, and $\mathrm{Am}(\mathrm{III})$ by a solution of [diethyl-2-(3methylimidazolium)ethylphosphonate $]\left[\mathrm{NTf}_{2}\right]\left([\mathrm{ImP}]\left[\mathrm{NTf}_{2}\right]\right)$ in $[\mathrm{Cnmim}]\left[\mathrm{NTf}_{2}\right](\mathrm{n}=4,8)$ was studied as a function of the $\mathrm{HNO}_{3}$ concentration. The distribution ratios of $\mathrm{Am}(\mathrm{III})$ and $\mathrm{U}(\mathrm{VI}) \mathrm{O}_{2}$ were significantly lower both in $[\mathrm{ImP}]\left[\mathrm{NTf}_{2}\right] /\left[\mathrm{C}_{\mathrm{n}} \operatorname{mim}\right]\left[\mathrm{NTf}_{2}\right]$ and $\left[\mathrm{C}_{\mathrm{n}} \operatorname{mim}\right]\left[\mathrm{NTf}_{2}\right]$ at 40 
all $\mathrm{HNO}_{3}$ concentrations, but unusually high separation factors for $\mathrm{Pu}(\mathrm{IV})$ from other actinides were obtained. ${ }^{159}$

Solid extractants, prepared by non-covalent immobilization of phosphonium ionic liquids on polyacrylonitrile (PAN) fibers, containing diphenyldiisobutyl-CMPO $\left(\mathrm{Ph}_{2} \mathrm{iBu}{ }_{2} \mathrm{CMPO}\right)$, were investigated for the extraction of $\mathrm{U}(\mathrm{VI}) \mathrm{O}_{2}, \mathrm{Pu}(\mathrm{IV})$, and $\mathrm{Eu}(\mathrm{III})$ in $0.5-5 \mathrm{~mol} / \mathrm{L} \mathrm{HNO}_{3}$ showing high extractions up to $99 \% .{ }^{160}$ CMPO-modified FILs were used as active agents of solid phase sorbents based on carbon nanotubes to give high separations of $\mathrm{Pu}(\mathrm{IV}), \mathrm{Am}(\mathrm{III}), \mathrm{Eu}(\mathrm{III})$, and $\mathrm{U}(\mathrm{VI}) \mathrm{O}_{2} \cdot{ }^{161}$ FIL-MA, a malonamide-based ionic liquid with imidazolium cation and $\mathrm{Tf}_{2} \mathrm{~N}$ anion (Chart 2.27), was more efficient and showed 12 times improved extraction of uranyl than the classical $N, N^{\prime}$-dimethyl- $N, N^{\prime}$-dibutylmalonamide (DMDBMA). ${ }^{162}$

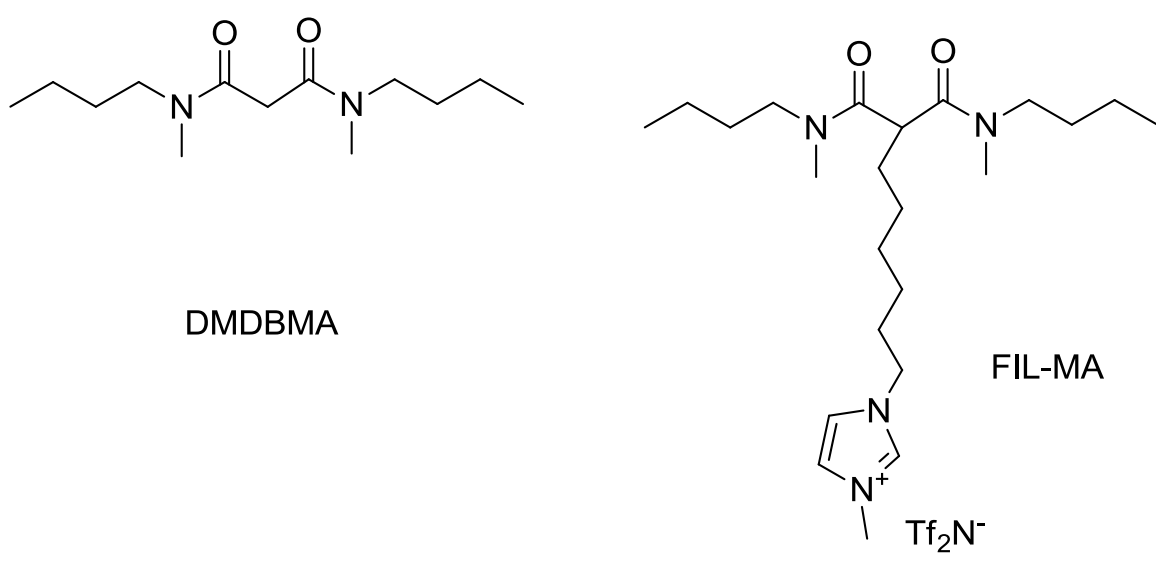

Chart 2.27

\subsection{Conclusions and outlook}

The separation of $\mathrm{An}(\mathrm{III})$ from $\mathrm{Ln}(\mathrm{III})$ remains a challenging problem. Based on present concepts, An(III) and $\operatorname{Ln}(\mathrm{III})$ are simultaneously extracted from acidic high-level liquid radioactive waste by an O-donor (e.g., CMPO, malonamides, and diglycolamides). Following the stripping of $\mathrm{An}(\mathrm{III})$ and $\mathrm{Ln}(\mathrm{III})$ into $0.01-0.5 \mathrm{~mol} / \mathrm{L} \mathrm{HNO}_{3}, \mathrm{An}(\mathrm{III})$ will be extracted selectively from the strip by an $\mathrm{N}$ donor and subsequent stripping of $\mathrm{An}(\mathrm{III})$. Although the diglycolamide-based ligands show superior extraction properties over CMPOs and malonamides, nevertheless, they also have some drawbacks. Various S- and N-based ligands have been developed for the separation of $\mathrm{An}(\mathrm{III})$ and $\mathrm{Ln}(\mathrm{III})$. The $\mathrm{N}$ donors are preferred over S donors, because they are completely incinerable. The $\mathrm{N}$ donor ligands, in particular the BTPs and BTBPs, have been thoroughly investigated and their radiolytic stability has also been improved by structural modifications. Some water-soluble ligands for selective stripping of $\mathrm{An}(\mathrm{III})$ have been developed in the recent years, which find potential applications in the 


\section{Chapter 2}

innovative SANEX process. The IL-based extraction method is a developing area in the nuclear waste field, which seems interesting as a green alternative of the traditional solvent systems. However, much research still needs to be done in this area.

The choice of the optimum extractant is not unambiguous. None of the studied extractants possesses all of the desired properties, such as high separation and extraction efficiency, high chemical and radiation stability, fast extraction kinetics, good solubility in solvents suitable for a nuclear process, and easy accessibility. Thus, a compromise must be found, corresponding to the desirability of each individual criterion. The considerable advances of recent years, both in process demonstrations as well as in better understanding of actinide extraction mechanisms, have set the stage for the development of even more efficient and robust ligands for use in more demanding future actinide/lanthanide separation processes (e.g., the GANEX process).

\subsection{Abbreviations}

$18 \mathrm{C} 6$

A336

An

BETI

BTP

BTBP

$\mathrm{C}_{\mathrm{n}} \operatorname{mim}$

$\mathrm{C}_{4} \mathrm{mPy}$

CMPO

CYANEX 272

CYANEX 301

CYANEX 302

D

DGA

DIAMEX

DIDPA

DMDBTDMA

DMDOHEMA

DMDPhOPDA 18-crown-6

tricaprylmethylammonium thiosalicylate

actinides

bis(perfluoroethanesulfonyl)imide

2,6-bis(5,6-dialkyl-1,2,4-triazin-3-yl)pyridine

6,6'-(5,6-dialkyl-1,2,4-triazin-3-yl)-2,2'-bipyridine

1-alkyl-3-methylimidazolium

$N$-alkyl- $N$-methylpyrrolidium

octyl(phenyl)- $N, N$-diisobutylcarbamoylmethyl phosphine oxide

bis(2,4,4-trimethylpentyl)phosphinic acid

bis(2,4,4-trimethylpentyl)dithiophosphinic acid

bis(2,4,4-trimethylpentyl)monothiophosphinic acid

distribution ratio

diglycolamide

diamide extraction

diisodecyl phosphoric acid

$N, N$ '-dimethyl- $N, N$ '-dibutyltetradecylmalonamide

$N, N$ '-dimethyl- $N, N$ '-dioctyl-2-(2-hexyloxyethyl)malonamide

$N, N$ '-dimethyl- $N, N$ '-dipheny-1-3-oxapentanediamide 
DOODA

DOTA

DPSO

DTPA

EDTA

F-3

FS-13

FIL

HDEHDTP

HDEHP

HDHP

HDNNS

IL

Imp

Ln

MA

$\mathrm{Me}_{3} \mathrm{BuN}$

$\mathrm{NTf}_{2}$

PC88A

$\mathrm{PF}_{6}$

PUREX

SANEX

SF

TALSPEAK

TBP

TBPO

TEHP

TiBPS

TOA

TMDGA

TEDGA

TPDGA
$N, N, N^{\prime}, N^{\prime}$-tetraoctyl-3,6-dioxaoctanediamide

$N, N$-bis(2-ethylhexyl)dimethyl-2,2-butanamide

diphenylsulfoxide

diethylenetriaminepentaacetic acid

ethylenediaminetetraacetic acid

$m$-nitrobenzotrifluoride

phenyl trifluoromethane sulfone

functionalized ionic liquids

bis(2-ethylhexyl)dithiophosphoric acid

bis(2-ethylhexyl)phosphoric acid

di-n-hexylphosphoric acid

dinonylnaphthalene sulfonic acid

ionic liquid

diethyl-2-(3-methylimidazolium)ethyl phosphonate

lanthanides

minor actinide

trimethylbutyl ammonium

bis(trifluoromethylsulfonyl)amide

bis(2-ethylhexyl)phosphinic acid

hexafluoro phosphate

plutonium uranium extraction

selective actinide extraction

separation factor

trivalent actinide lanthanide separations by phosphorus-reagent

extraction from aqueous complexes

tributyl phosphate

tributylphosphine oxide

tris(2-ethylhexyl) phosphate

tri-iso-butylphosphine sulphide

trioctylamine

tetramethyl diglycolamide

tetraethyl diglycolamide

tetrapropyl diglycolamide 


$\begin{array}{ll}\text { TODGA } & \text { tetraoctyl diglycolamide } \\ \text { TOPO } & \text { trioctylphosphine oxide } \\ \text { TPP } & \text { triphenyl phosphate } \\ \text { Tpy } & \text { terpyridine } \\ \text { TRPO } & \text { trialkylphosphine oxide } \\ \text { TRUEX } & \text { trans uraniun extraction } \\ \text { TS } & \text { thiosalicylate } \\ \text { TtBP } & \text { tri-tert-butyl phosphate }\end{array}$

\subsection{References}

1. Paiva, A. P.; Malik, P. J. Radioanal. Nucl. Chem. 2004, 261, 485.

2. Salvatores, M. Nucl. Eng. Des. 2005, 235, 805.

3. Ansari, S. A.; Pathak, P. N.; Mohapatra, P. K.; Manchanda, V. K. Sep. Purif. Rev. 2011, $40,43$.

4. Ansari, S. A.; Pathak, P.; Mohapatra, P. K.; Manchanda, V. K. Chem. Rev. 2012, 112, 1751.

5. Dam, H. H.; Verboom, W.; Reinhoudt, D. N. Chem. Soc. Rev. 2007, 36, 367

6. (a) Ekberg, C.; Fermvik, A.; Retegan, T.; Skarnemark, G.; Foreman, M. R. S.; Hudson, M. J.; Englund, S.; Nilsson, M. Radiochim. Acta 2008, 96, 225; (b) Kolarik, Z. Chem. Rev. 2008, 108, 4208.

7. Lewis, F. W.; Hudson, M. J.; Harwood, L. M. Synlett, 2011, 2609.

8. Nash, K. Solv. Extr. Ion Exch. 1993, 11, 729.

9. Nash, K. Sep. Sci. Technol. 1999, 34, 911.

10. Marcus, Y. Ion Properties, Marcel, Dekker, New York, 1997.

11. Pearson, R. G. J. Am. Chem. Soc. 1963, 85, 3533.

12. Diamond, R. M.; Street, K.; Seaborg, G. T. J. Am. Chem. Soc. 1954, 76, 1461. b) Jensen, M. P.; Bond, A. H. J. Am Chem Soc. 2002, 124, 9870.

13. a) Weaver, B.; Kappelmann, F. A.; J. Inorg. Nucl. Chem. 1968, 30, 263. b) Muscatello, A. C.; Horwitz, E. P.; Kalina, D. G.; Kaplan, L. Sep. Sci. Technol. 1982, 17, 859. c) Musikas, C.; Cuillerdier, C.; Livet, J.; Forchioni, A.; Chachaty, C. Inorg. Chem. 1983, 22, 2513.

14. McKibben, J. M.; Radiochim. Acta 1984, 36, 3. 
15. Horwiz, E. P.; Schulz, W. W. The TRUEX Process: A Vital Toll for Disposal of US Defense Nuclear Waste, Elsevier, London, 1991, 21.

16. Horwitz, E. P.; Kalina, D. G.; Diamond, V.; Vandegrift, D. G.; Schultz, W. W. Solv. Extr. Ion Exch. 1985, 3, 75.

17. Zhu, Y.; Jiao, R. Nucl. Technol. 1994, 108, 361.

18. Cuillerdier, C.; Musikas, C. Sep. Sci. Technol. 1991, 26, 1229.

19. Morita, Y.; Glatz, J. P.; Kubota, M.; Koch, L.; Pagliosa, G.; Roemer, K.; Nicholl, A. Solv. Extr. Ion Exch. 1996, 14, 385.

20. Modolo, G.; Seekamp, S.; Vijgen, H.; Scharf, K.; Baron, P. Recent developments in the ALINA process for An(III)/Ln(III) group separation during the partitioning of minor actinides, Proceedings of the 8th International Conference on Radioactive Waste Management and Enviromental Remediation. ICEM 01, Bruges, Belgium, Sept. 30-Oct. 4, 2001, ASME, 2001.

21. Liljenzin, J. O.; Persson, G.; Svantsesson, I.; Wingefors, S. Radiochim. Acta 1984, 35, 155.

22. See: www.acsept.org.

23. Brahmmananda, R. C. V. S.; Srinivasan, T . G.; Vasudeva Rao, P. R. Solv. Extr. Ion Exch. 2007, 25, 771.

24. Zhu,Y.; Jiao, R. Nucl. Technol. 1994, 108, 361.

25. Siddall, T. H. J. Inorg. Nucl. Chem. 1963, 25, 883.

26. Siddall, T. H. J. Inorg. Nucl. Chem. 1964, 26, 1991.

27. Rozen, A. M.; Volk, V. I.; Vakhrushin, A. Yu.; Zakharkin, B. S.; Kartasheva, N. A.; Krupnov, B. V.; Nikolotova, Z. I. Radiochemistry 1999, 41, 215.

28. Sasaki, Y.; Umetani, S. J. Nucl. Sci. Tech. 2006, 43, 794.

29. Kulyako, Y. M.; Malikov, D. A.; Chmutova, M. K.; Litvina, M. N.; Myasoedov, B. F. J. Alloys Compd. 1998, 271-273, 760.

30. Kulyako, Y.; Malikov, D.; Trofimov, T.; Chmutova, M.; Myasoedov B.; J. Nucl. Sci. Technol. 2002, Sup 3; 302.

31. Malofeeva, G. I.; Chmutova, M. K.; Rozhkova, L. S.; Petrukhin, O. M.; Spivakov, B. Ya.; Myasoedov, B. F. Radiochemistry 1998, 40, 241.

32. Kolarik, Z.; Horwitz, E. P. Solv. Extr. Ion Exch. 1988, 6, 247.

33. Mincher, B. J. Solv. Extr. Ion Exch. 1989, 7, 645. 
34. Nagasaki, S.; Kinoshita, K.; Wisnubroto, D. S.; Enokida, Y.; Suzuki, A. J. Nucl. Sci. Technol. 1992, 29, 671.

35. Mathur, J. N.; Murali, M. S.; Natarajan, P. R.; Badheka, L. P.; Banerji, A. Talanta 1992, $39,493$.

36. Horwitz, E. P.; Kalina, D. G.; Kaplan, L.; Mason, G. W.; Diamond, H. Sep. Sci. Technol. 1982, 17, 1261.

37. Musikas, C. Sep. Sci. Technol., 1988, 23, 1211.

38. Chan, G. Y. S.; Drew, M. G. B.; Hudson, M. J.; Iveson, P. B.; Liljenzin, J.; Skålberg, M.; Spjuth, L.; Madic, C. Dalton Trans. 1997, 649.

39. Mowafy, E. A.; Aly. H. F. Solv. Extr. Ion Exch. 2006, 24, 677.

40. Narita, H.; Yaita, T.; Tamura, K.; Tachimori, S. J. Radioanal. Nucl. Chem. 1999, 239, 381.

41. Spjuth, L.; Liljenzin, J. O.; Hudson, M. J.; Drew, M. G. B.; Iveson, P. B.; Madic, C. Solv. Extr. Ion Exch. 2000, 18, 1.

42. a) Madic, C.; Ouvrier, N. Radiochim. Acta 2008, 96, 183. b) Kumbhare, L. B.; Prabhu, D. R.; Mahajan, G. R.; Sriram S.; Manchanda, V. K. Nucl. Technol. 2002, 139, 253. c) Mahajan, G. R.; Prabhu, D. R.; Manchanda, V. K.; Badheka, L. P. Waste Manage. 1998, 18, 125. d) Purroy, D. S.; Baron, P.; Christiansen, B.; Glatz, J. P.; Madic, C.; Malmbeck, R.; Modolo, G. Sep. Purif. Technol. 2005, 45,157. e) Courson, O.; Lebrun, M.; Malmbeck, R.; Pagliosa, G.; Romer, K.; Satmark, B.; Glatz, J. P. Radiochim. Acta 2000, 88, 857. f) Malmbeck, R.; Courson, O.; Pagliosa, G.; Römer, K.; Sätmark, B.; Glatz, J. P.; Baron, P. R. Radiochim. Acta 2000, 88, 865. g) Serrano-Purroy, D.; Baron, P.; Christiansen, B.; Malmbeck, R.; Sorel, C.; Glatz, J. P. Radiochim. Acta 2005, 93, 351. h) Serrano-Purroy, D.; Christiansen, B.; Glatz, J. P.; Malmbeck, R.; Modolo, G. Radiochim. Acta 2005, 93, 357. i) Modolo, G.; Vijgen, H.; Serrano-Purroy, D.; Christiansen, B.; Malmbeck, R.; Sorel, C.; Baron, P. Sep. Sci. Technol. 2007, 42, 439.

43. Lumetta, G. J.; Rapko, B. M.; Garza, P. A.; Hay, B. P. J. Am. Chem. Soc. 2002, 124, 5644.

44. Lumetta, G. J.; Rapko, B. M.; Hay, B. P.; Garza, P. A.; Hutchison, J. E.; Gilbertson, R. D. Solv. Extr. Ion Exch. 2003, 21, 29.

45. Sasaki, Y.; Choppin, G. R. Anal. Sci. 1996, 12, 225.

46. Sasaki, Y.; Choppin, G. R. Radiochim. Acta 1998, 80, 85. 
47. (a) Tachimori, S.; Sasaki, Y.; Suzuki, S. Solv. Extr. Ion Exch. 2002, 20, 687. (b) Yaita, T.; Herlinger, A. W.; Thiyagarajan, P.; Jensen, M. P. Solv. Extr. Ion Exch. 2004, 22, 553. (c) Narita, H.; Yaita, T.; Tachimori, S. Solv. Extr. Ion Exch. 2004, 22, 135. (d) Suzuki, H.; Sasaki, Y.; Sugo, Y.; Apichaibuol, A.; Kimura, T. Radiochim. Acta 2004, 92, 463. (e) Zhu, Z. X.; Sasaki, Y.; Suzuki, H.; Suzuki, S.; Kimura, T. Anal. Chim. Acta 2004, 527, 163. (f) Sasaki, Y.; Zhu, Z. X.; Sugo, Y.; Suzuki, H.; Kimura, T. Anal. Sci. 2005, 21, 1171. (g) Sasaki, Y.; Rapold, P.; Arisaka, M.; Hirata, M.; Kimura, T.; Hill, C.; Cote, G. Solv. Extr. Ion Exch. 2007, 25, 187.

48. Mowafy, E.A.; Aly, H. F. Solv. Extr. Ion Exch. 2007, 25, 205.

49. Sasaki, Y.; Sugo, Y.; Suzuki, S.; Tachimori, S. Solv. Extr. Ion Exch. 2001, 19, 91.

50. Sasaki, Y.; Tachimori, S. Solv. Extr. Ion Exch. 2002, 20, 21.

51. (a) Pretsch, E.; Ammann, D.; Oswald, H. F.; Gugi, M.; Simon, W. Helv. Chim. Acta 1980, 63, 191. (b) Sasaki, Y.; Adachi, T.; Choppin, G. R. J. Alloys Compd. 1998, 271, 799. (d) Tachimori, S.; Suzuki, S.; Sasaki, Y.; Apichaibukol, A. Solv. Extr. Ion Exch. 2003, 21, 707. (e) Ansari, S. A.; Pathak, P. N.; Manchanda, V. K.; Husain, M.; Prasad, A. K.; Parmar, V. S. Solv. Extr. Ion Exch. 2005, 23, 463. (f) Sasaki, Y.; Sugo, Y.; Kitatsuji, Y.; Kirishima, A.; Kimura, T.; Choppin, G. R. Anal. Sci. 2007, 23, 727. (g) Sasaki, Y.; Zhu, Z. X.; Sugo, Y.; Kimura, T. J. Nucl. Sci. Technol. 2007, 44, 405.

52. Matloka, K.; Gelis, A.; Regalbuto, M.; Vandegrift, G.; Scott, M. J. Dalton Trans. 2005, 23,3719 .

53. Matloka, K.; Gelis, A.; Regalbuto, M.; Vandegrift, G.; Scott, M. J. Sep. Sci. Technol. 2006, 41, 2129.

54. Jańczewski, D.; Reinhoudt, D. N.; Verboom, W.; Hill, C.; Allignol, C.; Duchesne, M. New J. Chem. 2008, 32, 490.

55. Murillo, M. T.; Espartero, A. G.; Sánchez-Quesada, J.; de Mendoza, J.; Prados, P. Solv. Extr. Ion Exch. 2009, 27, 107.

56. Musikas, C.: Actinide-lanthanide group separation using sulfur and nitrogen donor extractants. Actinide/lanthanide separations. Proceedings of an International Symposium. World Scientific Publishing, Singapore, 1985, p. 19.

57. Zhu, Y. J.; Jiao, R. Z. Radiochim. Acta 1995, 69, 191.

58. Zhu, Y. J.; Song, C.; Jiao, R. GLOBAL 95 International Conference on Evaluation of Emerging Nuclear Fuel Cycle System, Versailles, France, 11-14 Sept, 1995, pp. 571-76. 
59. Zhu, Y. J.; Chen, J.; Jiao, R. Z. Solv. Extr. Ion Exch. 1996, 14, 61.

60. Jarvinen, G. D.; Barrans, Jr, R. E.; Schroeder, N. C.; Wade, K. L.; Jones, M. M.; Smith, B. F.; Mills, J. L.; Howard, G.; Freiser, H.; Muralidharan, S. Separation of f Elements, Nash, K. L.; Choppin, G. R. (Eds), Plenum Press, New York, 1995, p. 43.

61. Modolo, G.; Odoj, R. J. Radioanal. Nucl. Chem., 1998, 228, 83.

62. Hill, C.; Madic, C.; Baron, P.; Ozawa, M.; Tanaka. Y. J. Alloys Compd. 1998, 271-273, 1591.

63. Wang, X.; Zhu, Y.; Jiao. R. J. Radioanal. Nucl. Chem. 2002, 251, 487.

64. Modolo, G.; Odoj. R. J. Alloys Compd. 1998, 271-273, 248.

65. Modolo, G.; Odoj, R. Solv. Extr. Ion Exch. 1999, 17, 33.

66. Madic, C.; Testard, F.; Hudson, M. J.; Liljenzin, J. O.; Christiansen, B.; Ferrando, M.; Facchini, A.; Geist, A.; Modolo, G.; Gonzales-Espartero, A.; De Mendoza, J. PARTNEW - New Solvent Extraction Processes for Minor Actinides. Final Report. CEA-report 6066, 2004.

67. Modolo, G.; Nabet, S. Solv. Extr. Ion Exch. 2005, 23, 359.

68. Klaehn, J. R.; Peterman, D. R.; Harrup, M. K.; Tillotson, R. D.; Luther, T. A.; Law, J. D.; Daniels, L. M. Inorg. Chim. Acta 2008, 361, 2522.

69. Harrup, M. K.; Peterman, D. R.; Greenhalgh, M. R.; Luther, T. A.; Klaehn, J. R. Mater. Res. Soc. Symp. Proc. 2008, Vol. 1104..

70. Peterman, D. R.; Martin, L. R.; Klaehn, J. R.; Harrup, M. K.; Greenhalgh, M. R.; Luther, T. A. J. Radioanal. Nucl. Chem. 2009, 282, 527.

71. Peterman, D. R.; Greenhalgh, R. D.; Tillotson, R. D.; Klaehn, J. R.; Harrup, M. K.; Luther, T. A.; Law, J. D. Sep. Sci. Technol. 2010, 45, 1711.

72. Guoxin, T.; Yongjun, Z.; Jingming, X. Solv. Extr. Ion Exch. 2001, 19, 993.

73. Qichu, X.; Jianfeng, W.; Lixing, Z.; Yusheng, Y. J. Radioanal. Nucl. Chem. 2007, 273, 235.

74. Qichu, X.; Jianfeng, W.; Yinzhong, C.; Lixing, Z.; Yusheng, Y. Radiochim. Acta 2008, 96,771 .

75. Higgins, W. A. ; Vogel, P. W.; Craig, E.G. J. Am. Chem. Soc. 1955, 77, 1864.

76. Chen, J.; Jiao, R.; Zhu, Y. Solv. Extr. Ion Exch. 1996, 14, 555.

77. Sole, K. C.; Hiskey, J. B.; Ferguson, T. L. Solv. Extr. Ion Exch. 1993, 11, 783.

78. Zhu, Y. Radiochim. Acta 1995, 68, 95. 
79. Modolo, G.; Seekamp, S. Solv. Extr. Ion Exch. 2002, 20, 195.

80. Lewis, F. W.; Hudson, M. J.; Harwood, L. M. Synlett 2011, 2609.

81. Kolarik, Z.; Mullich, U.; Gassner, F. Solv. Extr. Ion Exch. 1999, 17, 23.

82. Berthet, J. C.; Miquel, Y.; Iveson, P. B.; Nierlich, M.; Thuery, P.; Madic, C.; Ephritikhine, M. Dalton Trans. 2002, 16, 3265.

83. Weigl, M; Geist, A.; Muellich, U.; Gompper, K. Solv. Extr. Ion Exch. 2006, 24, 845.

84. Hill, C.; Guillaneux, D.; Berthon, L.; Madic, D. J. Nucl. Sci. Tech. 2002, 39, 309.

85. Hudson, M. J.; Boucher, C. E.; Braekers, D.; Desreux, J. F.; Drew, M. G. B.; Foreman, M. R. S. J.; Harwood, L. M.; Hill, C.; Madic, C.; Marken, F. New J. Chem. 2006, 30, 1171.

86. Nilsson, M.; Andersson, S.; Ekberg, C.; Foreman, M . R. S.; Hudson, M. J.; Skarnemark, G. Radiochim. Acta 2006, 94, 103.

87. Retegan, T.; Ekberg, C.; Dubois, I.; Fermvik, A.; Skarnemark, G.; Wass, T. J. Solv. Extr. Ion Exch. 2007, 25, 417.

88. Trumm, S.; Geist, A.; Panak, P. J.; Fanghänel, T. Solv. Extr. Ion Exch. 2011, 29, 213.

89. Drew, M. G. B.; Foreman, M. R. S.; Hudson, M. J.; Madic, C. Inorg. Chem. Commun. 2005, 8, 239.

90. Trumm, S.; Lieser, G.; Foreman, M. R. S. J.; Panak, P. J.; Geist, A.; Fanghanel, T. Dalton Trans. 2010, 39, 923.

91. Geist, A.; Hill, C.; Modolo, G.; Foreman, M. R. S.; Weigl, M.; Gompper, K.; Hudson, M. J.; Madic, C. Solv. Extr. Ion Exch. 2006, 24, 463.

92. Modolo, G.; Sypula, M.; Geist, A.; Hill, C.; Sorel, C.; Malmbeck, R.; Magnusson, D.; Foreman, M. R. S. Development and Demonstration of a new SANEX Process for Actinide(III)/Lanthanide(III) Separation using a Mixture of CyMe4-BTBP and TODGA as a Selective Extractant. Actinide and Fission Product Partitioning and Transmutation, 10th Information Exchange Meeting, Mito, Japan, 6-10 October 2009. NEA No. 6420, OECDNEA, 2010, p. 235.

93. Retegan, T.; Berthon, L.; Ekberg, C.; Fermvik, A.; Skarnemark, G.; Zorz, N. Solv. Extr. Ion Exch. 2009, 27, 663.

94. Ekberg, C.; Aneheim, E.; Fermvik, A.; Foreman, M. R. S.; Löfström-Engdahl, E.; Retegan, T.; Spendlikova, I. J. Chem. Eng. Data 2010, 55, 5133. 
95. Harwood, L. M.; Lewis, F. W.; Hudson, M. J.; John, J.; Distler, P. Solv. Extr. Ion Exch. 2011, 29, 551.

96. Lewis, F. W.; Harwood, L. M.; Hudson, M. J.; Drew, M. G. B.; Desreux, J. F.; Vidick, G.; Bouslimani, N.; Modolo, G.; Wilden, A.; Sypula, M.; Vu, T. H.; Simonin, T. P. J. Am. Chem. Soc. 2011, 133, 13093.

97. Baney, G.; Schurhammer, R.; Wipff, G. Phys. Chem. Chem. Phys. 2011, 13, 2922.

98. Lewis, F. W.; Harwood, L. M.; Hudson, M. J.; Drew, M. G. B.; Modolo, G.; Sypula, M.; Desreux, J. F.; Bouslimani, N.; Vidick, G. Dalton Trans. 2010, 39, 5172.

99. Branowska, D.; Karczmarzyk, Z.; Rykowski, A.; Wysocki, W.; Olender, E.; UrbanczykLipkowska, Z.; Kalicki, P. J. Mol. Struct. 2010, 979, 186.

100. Marie, C.; Miguirditchian, M.; Guillaneux, D.; Bisson, J.; Pipelier, M.; Dubreuil, D. Solv. Extr. Ion Exch. 2011, 29, 292.

101. Mowafy, E. A.; Aly, H. F. Solv. Extr. Ion Exch. 2002, 20, 177.

102. Hérès, X.; Nicol, C.; Bisel, I.; Baron, P.; Ramain, L. PALADIN: A One Step Process for Actinides(III)/Fission Products Separation, GLOBAL '99: Nuclear Technology Bridging the Millennia, Jackson Hole, WY, August 29-September 3, 1999, American Nuclear Society, La Grange Park, Illinois; p. 585.

103. Gannaz, B.; Chiarizia, R.; Antonio, M. R.; Hill, C.; Cote, G. Solv. Extr. Ion Exch. 2007, 25,313 .

104. Dhami, P. S.; Chitnis, R. R.; Gopalakrishnan, V.; Wattal, P. K.; Ramanujam, A.; Bauri, A. K. Sep. Sci. Technol. 2001, 36, 325.

105. Singh, S. K.; Dhami, P. S.; Dakshinamoorthy, A.; Sundersanan, M. Sep. Sci. Technol. 2009, 44, 491.

106. Agafonova-Moroz, M. S.; Krasnikov, L. V.; Mishina, N. E.; Shadrin, A. Y.; Shmidt, O. V. Radiochemistry 2009, 51, 403.

107. Musikas, C. In Proc. Int. Symp. Actinide/Lanthanide Separations, Honolulu, Hawaii, USA, August 24-25, 1984, World Sci.: Singapore, 1985, 19.

108. Cordier, P. Y.; Hill, C.; Baron, P.; Madic, C.; Hudson, M. J., Liljenzin, J. O. J. Alloys Compd. 1998, 271-273, 738.

109. Hagström, I.; Spjuth, L.; Enarsson, A.; Liljenzin, J. O.; Skalberg, M.; Hudson, M. J.; Iveson, P. B.; Madic, C.; Cordier, P. Y.; Hill, C.; Francois, N. Solv. Extr. Ion Exch. 1999, 17,221 . 
110. Madic, C.; Lecomte, M.; Baron, P.; Boullis. B. C. R. Phys. 2002, 3, 797.

111. Andersson, S.; Ekberg, C.; Foreman, M. R. S.; Hudson, M. J.; Liljenzin, J. O.; Nilsson, M.; Skarnemark, G.; Spahiu, K. Solv. Extr. Ion Exch. 2003, 21, 621.

112. Watanabe, M.; Mirvaliev, R.; Tachimori, S.; Takeshita, K.; Nakano, Y.; Morikawa, K.; Chikazawa, T.; Mori, R. Solv. Extr. Ion Exch. 2004, 22, 377.

113. Jensen, M. P.; Morss, L. R.; Beitz, J. V.; Ensor, D. D. J. Alloys Compd. 2000, 303-304, 137.

114. Smirnov, I. V.; Chirkov, A. V.; Babain, V. A.; Pokrovskaya, E. Y.; Artamonova, T. A. Radiochim. Acta 2009, 97, 593.

115. Hill, C.; Madic, C.; Baron, P.; Ozawa, M.; Tanaka, Y. J. Alloys Compd. 1998, 159, 271.

116. Bhattacharyya, A.; Mohapatra, P. K.; Manchanda, V. K. Solv. Extr. Ion Exch. 2006, 24, 1.

117. Bhattacharyya, A.; Mohapatra, P. K.; Manchanda, V. K. J. Radioanal. Nucl. Chem. 2011, 288, 709.

118. Bhattacharyya, A.; Mohapatra, P. K.; Manchanda, V. K. Sep. Sci. Technol. 2011, 46, 376.

119. Ionova, G.; Ionov, S.; Rabbe, C.; Hill, C.; Madic, C.; Guillaumont, R.; Krupa, J. C. Solv. Extr. Ion Exch. 2001, 19, 391.

120. Grigorieva, N. A.; Pavlenko, N. I.; Pashkov, G. L.; Fleitlikh, I. Y.; Nikiforova, L. K. Solv. Extr. Ion Exch. 2010, 28, 510.

121. Aneheim, E.; Ekberg, C.; Fermvik, A.; Forema, M. R. St. J.; Retegan, T.; Skarnemark, G. Solv. Extr. Ion Exch. 2010, 28, 437.

122. Aneheim, E.; Ekberg, C.; Fermvik, A.; Forema, M. R. St. J.; Grüner, B.; Hájková, Z.; Kvicalová, M. Solv. Extr. Ion Exch. 2011, 29, 157.

123. Wilden, A.; Schreinemachers, C.; Sypula, M.; Modolo, G. Solv. Extr. Ion Exch. 2011, $29,190$.

124. Kosyakov, V. N.; Yerin, E. A. J. Radioanal. Chem. 1980, 56, 93.

125. Chitnis, R. R.; Wattal, P. K.; Ramanujam, A.; Dhami, P. S.; Gopalakrishnan, V.; Bauri, A. K.; Banerji, A. J. Radioanal. Nucl. Chem. 1999, 240, 727.

126. Chitnis, R. R.; Wattal, P. K.; Ramanujam, A.; Dhami, P. S.; Gopalakrishnan, V.; Bauri, A. K.; Banerji, A. J. Radioanal. Nucl. Chem. 1999, 240, 721. 
127. Mathur, J. N.; Murali, M. S.; Natarajan, P. R.; Badheka, L. P.; Banerji, A.; Ramanujam, A.; Dhami, P. S.; Gopalakrishnan, V.; Dhumwab, R. K.; Rao, M. K. Waste Manage. 1993, 13, 317.

128. Horwitz, E. P.; Schulz, W. W. The TRUEX Process: A vital tool for disposal of U.S. defence nuclear waste, Symp. on New Separation Chemistry for Radioactive Waste and Other Specific Applications, sponsored by the Commission of European Communities and the Italian Commission for Nuclear and Alternative Energy Sources, Rome, Italy, May 16-18, 1990.

129. Chitnis, R. R.; Wattal, P. K.; Ramanujam, A.; Dhami, P. S.; Gopalakrishnan, V.; Mathur, J. N.; Murali, M. S. Sep. Sci. Technol. 1998, 33, 1877.

130. Rizvi, G. H.; Mathur, J. N. J. Radioanal. Nucl. Chem. 2002, 253, 179.

131. Sasaki, Y.; Sugo, Y.; Kitatsuji, Y.; Kirishima, A.; Kimura, T.; Choppin, G. R. Anal. Sci. 2007, 23, 727 .

132. Sasaki, Y.; Suzuki, H.; Sugo, Y.; Kimura, T.; Choppin, G. R. Chem. Lett. 2006, 35, 256.

133. Sasaki, Y.; Morita, Y.; Kitatsuji, Y.; Kimura, T. Chem. Lett. 2010, 39, 898.

134. Heitzmann, M.; Bravard, F.; Gateau, C.; Boubals, N.; Berthon, C.; Pecaut, J.; Charbonnel, M. C.; Delangle, P. Inorg. Chem. 2009, 48, 246.

135. Heitzmann, M.; Gateau, C.; Chareyre, L.; Miguirditchian, M.; Charbonnel, M. C.; Delangle, P. New J. Chem. 2010, 34, 108.

136. Nikishkin, N. I.; Huskens, J.; Assenmacher, J.; Wilden, A.; Modolo, G.; Verboom, W. Org. Biomol. Chem. 2012, 10, 5443.

137. Shimojo, K.; Kurahashi, K.; Naganawa, H. Dalton Trans. 2008, 37, 5083.

138. Wei, T. G.; Yang, Z.; Chen, J. C. Anal. Chim. Acta 2003, 488, 183.

139. Stepinski, D. C.; Jensen, M. P.; Dzielawa, J. A.; Dietz, M. L. Green Chem. 2005, 7, 151.

140. Giridhar, P.; Venkatesan, K. A.; Srinivasan, T. G.; Rao, P. R. V. J. Radioanal. Nucl. Chem. 2005, 265, 31.

141. Wai, M. C.; Liao, Y.; Liao, W.; Tian, G.; Addleman, R. S.; Quacha, D.; Pasilisa, S. P. Dalton Trans. 2011, 40, 5039.

142. Rout, A.; Venkatesan, K. A.; Srinivasan, T. G.; Rao, P. R. V. Radiochim. Acta 2009, 97, 719.

143. Rout, A.; Venkatesan, K. A.; Srinivasan, T. G.; Rao, P. R. V. Sep. Purif. Technol. 2011, 76,238 . 
144. Visser, A. E.; Rogers, R. D. J. Solid State Chem. 2003, 171, 109.

145. Nakashima, K.; Kubota, F.; Maruyama, T.; Goto, M. Ind. Eng. Chem. Res. 2005, 44, 4368.

146. Nakashima, K.; Kubota, F.; Maruyama, T.; Goto, M. Anal. Sci. 2003, 19, 1097.

147. Pribylova, G. A. J. Radioanal. Nucl. Chem. 2011, $288,693$.

148. Turanov, A. N.; Karandashev, V.K.; Baulin, V. E. Radiochemistry 2008, 50, 229.

149. Shen, Y. L.; Tan, X. W.; Wang, L.; Wu, W. S. Sep. Purif. Technol. 2011, 78, 298.

150. Turanova, A. N.; Karandashev, V. K.; Baulin, V. E. Solv. Extr. Ion Exch. 2008, 26, 77.

151. Zuo, Y.; Liu, Y.; Chen, J.; Li, D. Q. Ind. Eng. Chem. Res. 2008, 47, 2349.

152. Zuo, Y.; Chen, J.; Li, D. Q. Sep. Purif. Technol. 2008, 63, 684.

153. Yoon, S. J.; Lee, J. G.; Tajima, H.; Yamasaki, A.; Kiyono, F.; Nakazato, T.; Tao, H. J. Ind. Eng. Chem. 2010, 16, 350.

154. Rout, A.; Karmakar, S.; Venkatesan, K. A.; Srinivasan, T. G.; Rao, P. R. V. Sep. Purif. Technol. 2011, 81, 109.

155. Sun, X. Q.; Bell, J. R.; Luo, H. M.; Dai, S. Dalton Trans. 2011, 40, 8019.

156. Ouadi, A.; Gadenne, B.; Hesemann, P.; Moreau, J. J. E.; Billard, I.; Gaillard, C.; Mekki, S.; Moutiers, G. Chem. Eur. J. 2006, 12, 3074.

157. Ouadi, A.; Klimchuk, O.; Gaillard, C.; Billard, I. Green Chem. 2007, 9, 1160.

158. Srncik, M.; Kogelnig, D.; Stojanovic, A.; Korner, W.; Krachler, R.; Wallner, G. Appl. Radiat. Isot. 2009, 67, 2146.

159. Rout, A.; Venkatesan, K. A.; Srinivasan, T. G.; Rao, P. R. V. Radiochim. Acta 2010, 98, 459.

160. Myasoedova, G. V.; Zakharchenko, E. A.; Molochnikova, N. P.; Myasoedov, B. F. Radiochemistry 2008, 50, 482.

161. Odinets, I. L.; Sharova, E. V.; Artyshin, O. I.; Lyssenko, K. A.; Nelyubina, Y. V.; Myasoedova, G. V.; Molochnikova, N. P.; Zakharchenro, E. A. Dalton Trans. 2010, 39, 4170.

162. Bonnaffé-Moity, M.; Ouadi, A.; Mazan, V.; Miroshnichenko, S.; Ternova, D.; Georg, S.; Sypula, M.; Gaillard, C.; Billard, I. Dalton Trans. 2012, 41, 7526. 



\section{Chapter}

\section{3}

\section{Synthesis and Am/Eu Extraction of Novel TODGA Derivatives*}

Various ligands with structural modifications of the TODGA skeleton were synthesized in good yields. These modifications include: i) the increase in chain length from one carbon to two carbons between the central ether oxygen atom and the amide moieties, ii) the addition of substituents on the carbon between the central oxygen atom and the amide moieties on one or both sides of the central oxygen, iii) the replacement of the central oxygen by a (substituted) nitrogen atom and, iv) synthesis of a rigidified glycolamide. The effect of these structural modifications on the extraction behavior toward Am(III) and Eu(III) at various nitric acid concentrations was studied. In most of the cases the extraction does not exceed that of TODGA in the entire acidity range of 0.001-4 mol/L $\mathrm{HNO}_{3}$. Monomethyl-TODGA derivative 10 a resembles that of TODGA at high nitric acid concentrations. However, at lower acidities its $\mathrm{D}$ values are much lower, which can be beneficial for back-extraction. The aza-tripodal ligands 18a,b show reverse extraction properties compared to TODGA concerning the $p H$ influence: with reasonable separation factor values 8 and 11, respectively at $p H 2$.

\footnotetext{
* This chapter is based on: Iqbal, M.; Huskens, J.; Sypula, M.; Modolo, G.; Verboom, W. Supramol. Chem. 2010, 22, 827.
} 


\section{Chapter 3}

\subsection{Introduction}

In the early 1990s, Stephan et al. ${ }^{1}$ reported the extraction of different metal ions using multidentate ligands such as diglycolamides (DGA). The DGA substance class with an ether group between both amide functions resembles the malonamides as outlined in Chapter 2 . During the late 1990s, Sasaki and Choppin recognized that these ligands are particularly suitable for extracting all actinides (An(III-VI)) from acidic waste solutions and are more efficient extractants than malonamides. ${ }^{2,3}$ Extensive extraction studies have been performed with this very promising substance class. ${ }^{4}$ The change from a bidentate ligand (e.g. malonamide) to a tridentate diglycolamide not only significantly increased the affinity for trivalent actinides but also for the lanthanides. Different DGAs have been synthesized and $N, N, N^{\prime}, N^{\prime}$-tetraoctyl-3-oxapentanediamide (TODGA, Chart 3.1) was found to have the best properties in terms of extraction, solubility in aliphatic solvents, and stability. However, TODGA has a tendency to form a third phase in aliphatic solvents such as $n$-dodecane, particularly at high metal and nitric acid concentrations. ${ }^{5,6}$ In general, the DGAs have drawn significant attention as highly effective ionophores for the complexation of f-elements. ${ }^{7}$ TODGA has been preorganized on various tripodal platforms ${ }^{8,9,10}$ and as bis(diglycolamides). ${ }^{11}$

To the best of our knowledge, with the exception of the replacement of the central oxygen atom with sulfur, ${ }^{50}$ the effect of structural modifications of TODGA on the extraction behavior has not yet been studied. In the case of malonamides small changes in the substitution pattern on the central carbon atom positively influenced the extraction ability. ${ }^{12}$ In this chapter, we describe the synthesis of different types of TODGA derivatives, among which the replacement of the central oxygen atom for NR groups, and their extraction properties for americium(III) and europium(III) are studied, simulating the extraction of actinides(III) and lanthanides(III) from nitric acid solutions.

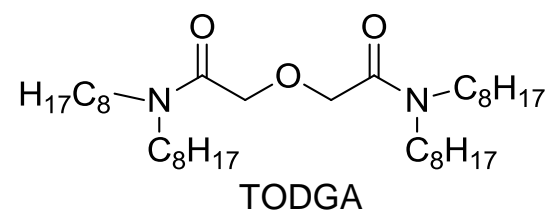

Chart 3.1 


\subsection{Results and discussion}

\subsubsection{Synthesis}

First 3,3'-oxybis( $N, N$-alkylpropanamides) 4a,b were praperad (Scheme 3.1) in which compared to TODGA the chain length between the central oxygen atom and amide moieties is enlarged. Commercially available 2-cyanoethyl ether (1) was converted to 3,3'oxydipropanoic acid (2) according to a slightly modified literature procedure; ${ }^{13}$ in our hands a longer reaction time ( $48 \mathrm{~h}$ instead of $24 \mathrm{~h}$ at $50{ }^{\circ} \mathrm{C}$ ) was needed to have full conversion. In literature ${ }^{14}$ dicarboxylic acid dichloride $\mathbf{3}$ has been prepared by reaction of dicarboxylic acid 2 with thionyl chloride. However, oxalyl chloride appeared to be a more suitable reagent due to its lower boiling point giving rise to an easier workup procedure. Subsequent reaction of diacid dichloride $\mathbf{3}$ with an appropriate dialkylamine afforded the target compounds $\mathbf{4 a}$ and $\mathbf{4 b}$ in $78 \%$ and $62 \%$ yield, respectively. The formation of $\mathbf{4 a , b}$ followed from its spectral data. The ${ }^{1} \mathrm{H}$ NMR spectra show a clear shift of the $\mathrm{CH}_{2} \mathrm{C}(\mathrm{O})$ triplet at $3.10 \mathrm{ppm}$ in $\mathbf{3}$ to about 2.58 ppm in $\mathbf{4 a , b .}$

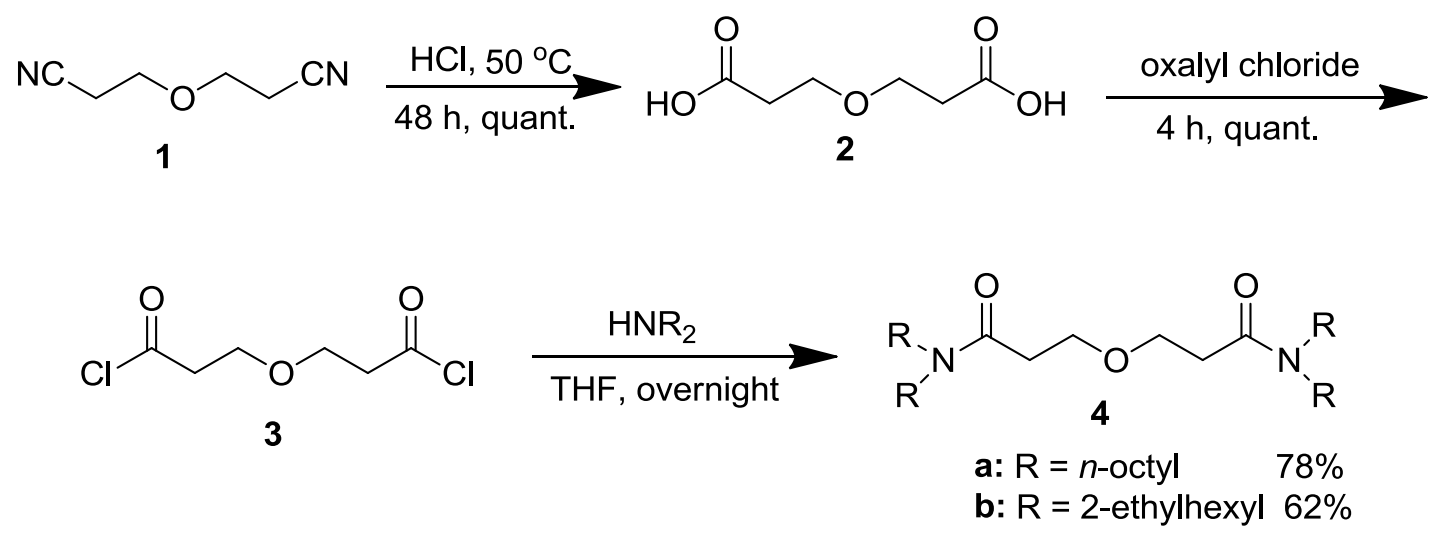

Scheme 3.1

The synthesis of a series of TODGA derivatives 10a-d having one or two substituents at the carbon atoms next to the central oxygen atom is summarized in Scheme 3.2. $\alpha$-Bromoesters 5a-c and $\alpha$-hydroxyesters $\mathbf{6 a}, \mathbf{b}$ were coupled in the presence of $\mathrm{NaH}$ in THF to give the diesters 7a-d in good yields. Saponification of the ester groups in 7a-d with $\mathrm{NaOH}$ in methanol afforded the dicarboxylic acids 8a-d. The TODGA derivatives 10a-d were prepared both by reaction of dicarboxylic acids 8a-d with dioctylamine in the presence of DCC and

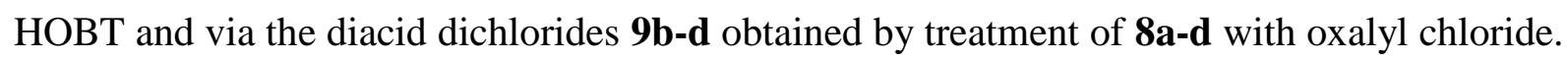
The DCC coupling method gave rise to higher yields (70-76\%) and less side products. The 
formation of the TODGA derivatives 10a-d was confirmed by the NMR analysis; the ESI mass spectra showed distinct $[\mathrm{M}+\mathrm{H}]$ peaks.

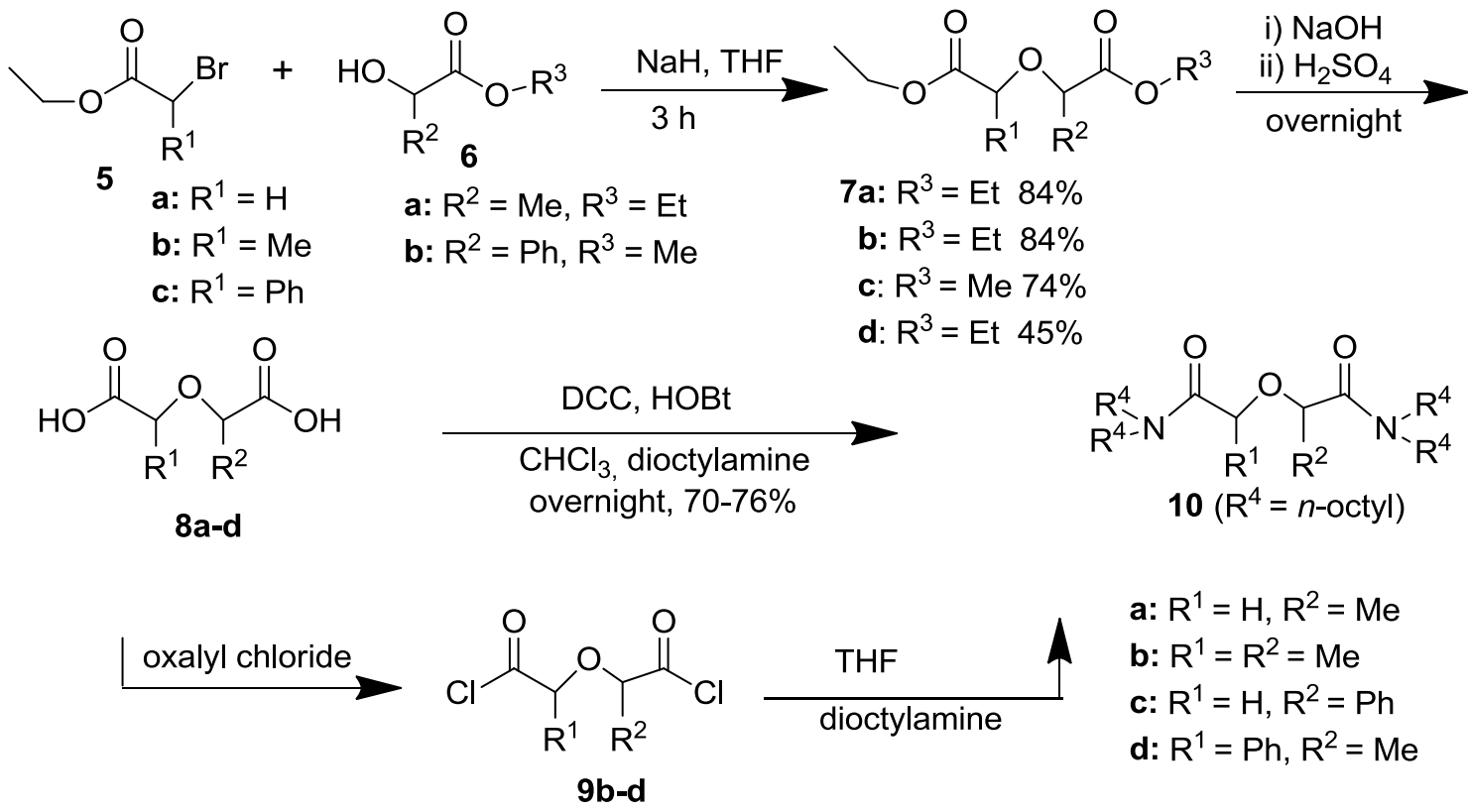

Scheme 3.2

The basicity of the central oxygen atom plays an important role in the extraction of metal ions with different nitric acid concentrations. ${ }^{15}$ The introduction of methyl and phenyl groups in TODGA derivatives will influence the basicity of the central oxygen atom. However, also steric interactions between these groups may play a role in the extraction. To study this effect the rigidified tetrahydrofuran TODGA derivative 14 was synthesized (Scheme 3.3). Starting from the commercially available furan-2,5-dicarboxylic acid (11) there are two ways for the synthesis of 14, viz. reduction of the furan ring as the first or the final step. Although the reduction of the ring in $\mathbf{1 1}$ has been described, ${ }^{16}$ we were not able to drive the reaction to completion and consequently we encountered separation problems and therefore, the second approach was followed. Furan 2,5-dicarboxylic acid (11) was converted into the corresponding dichloride 12 using oxalyl chloride in THF instead of thionyl chloride as described in literature. ${ }^{17}$ Subsequent reaction of 12 with dioctylamine gave $N, N, N^{\prime}, N^{\prime}$ tetraoctylfuran-2,5-dicarboxamide (13) in 50\% yield (over 2 steps), which was reduced at 10 bar $\mathrm{H}_{2}$ using $10 \% \mathrm{Pd} / \mathrm{C}$ as catalyst in methanol to give the target compound $\mathbf{1 4}$ in quantitative yield. The ${ }^{1} \mathrm{H}$ NMR spectrum of $\mathbf{1 3}$ exhibits a characteristic singlet for the furan ring at 6.92 ppm. However, upon reduction, the ${ }^{1} \mathrm{H}$ NMR spectrum of $\mathbf{1 4}$ shows the hydrogens of the 
tetrahydrofuran ring as multiplets at 1.96-2.11, 2.41-2.51, and 4.53-4.62 ppm (2H each) indicating the formation of diastereoisomers.<smiles>O=C(O)c1ccc(C(=O)O)o1</smiles>

11

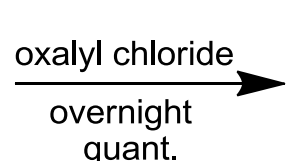

quant.

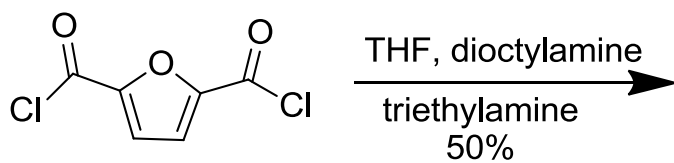

12<smiles>[R]NC(=O)c1ccc(C(=O)N([R])[R])o1</smiles>

13

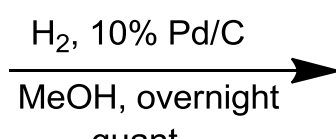

quant.

Scheme 3.3

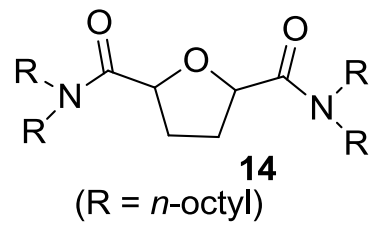

$(\mathrm{R}=n$-octyl)

In order to replace the central oxygen atom in TODGA by nitrogen, the approach depicted in Scheme 3.4 was employed. Since nitrogen is trivalent a third ligating group can be introduced as well giving tris-amides. $N, N$-dialkyl-2-chloroacetamides $(\mathbf{1 5 a}, \mathbf{b})$ were reacted with various amines in refluxing acetonitrile to obtain 16a-d containing different functional groups on nitrogen. The benzyl group of $\mathbf{1 6 a}, \mathbf{b}$ was removed by hydrogenolysis in the presence of $10 \%$ $\mathrm{Pd} / \mathrm{C}$ as a catalyst to yield $\mathbf{1 7 a} \mathbf{a}, \mathbf{b}$, which upon further treatment with acetyl chloride (in the case of 17a) gave 19, and with 15a,b afforded the tripodal ligands 18a,b. In the ${ }^{1} \mathrm{H}$ NMR spectra the characteristic singlet of $\mathbf{1 5 a}, \mathbf{b}$ at 4.03 ppm was shifted to $3.50-3.55$ ppm in $\mathbf{1 6 a}, \mathbf{b}$, and to $4.22 \mathrm{ppm}$ in the case of $\mathbf{1 6} \mathbf{d}$ along with the appearance of peaks in the aromatic region. The formation of $\mathbf{1 7} \mathbf{a}, \mathbf{b}$ from $\mathbf{1 6 a}, \mathbf{b}$ was indicated by the complete disappearance of the aromatic hydrogen atoms. The successful introduction of the third binding site in 18a,b was confirmed by the ESI mass spectrometry. In the case of $\mathbf{1 9}$, a characteristic singlet appeared in the ${ }^{1} \mathrm{H}$ NMR spectrum at $2.03 \mathrm{ppm}$ indicating the introduction of the acetyl moiety. 


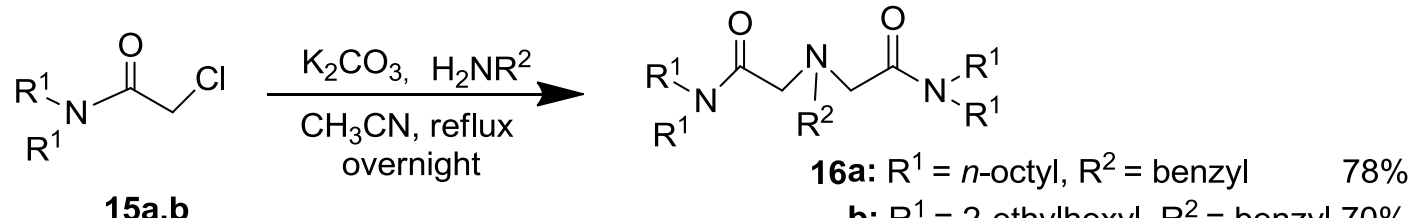

$$
\begin{aligned}
& \text { b: } R^{1}=2 \text {-ethylhexyl, } R^{2}=\text { benzyl } 70 \% \\
& \text { c: } \mathrm{R}^{1}=\mathrm{R}^{2}=n \text {-octyl } \quad 75 \% \\
& \text { d: } \mathrm{R}^{1}=n \text {-octyl, } \mathrm{R}^{2}=\text { phenyl } 65 \%
\end{aligned}
$$

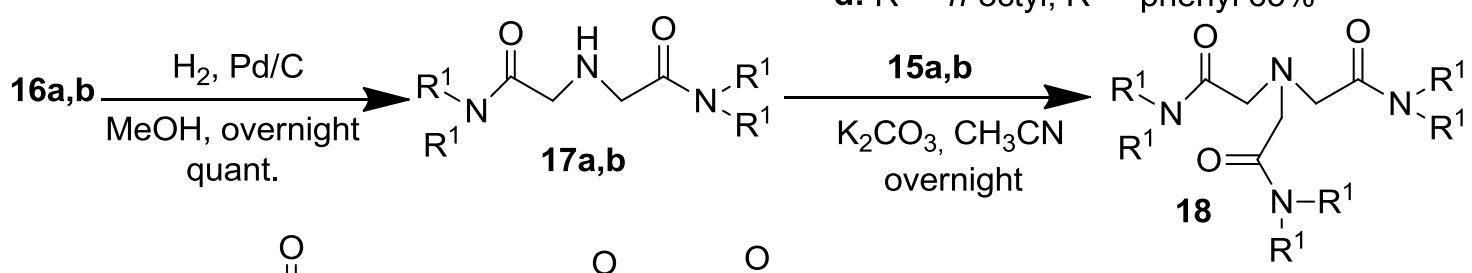

$17 a$

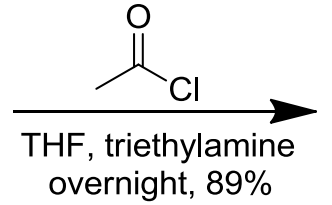<smiles>[R]NC(=O)CN(C(C)=O)C(=O)C(=O)N[R1]</smiles>

Scheme 3.4

$$
19 \mathrm{R}^{1}=n \text {-octyl }
$$

a: $\mathrm{R}^{1}=n$-octyl $\quad 75 \%$

b: $\mathrm{R}^{1}=2$-ethylhexyl $70 \%$

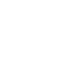


effective metal complexation is not possible due to the increased length of the aliphatic chain between the two amide groups (compared to TODGA), whereby the distance between the coordinating moieties too large.

In Figure 3.1 the extraction results of $\mathbf{1 0 a}$ and $\mathbf{1 0 b}$ are compared with those for TODGA. It is observed that with increasing nitric acid concentration of the aqueous phase, the extraction of $\mathrm{Am}$ and $\mathrm{Eu}$ increases. It has been reported that the TODGA molecules aggregate in the organic phase when the aqueous phase acidity is $\operatorname{low}^{7 \mathrm{e}}$. From the neutral character of the DGA and the strong $D_{\mathrm{M}}$ dependence on the $\mathrm{HNO}_{3}$ concentration, it is assumed that the new ligands act as solvating extractants. The metals are extracted as neutral species (e.g. $\mathrm{Am}\left(\mathrm{NO}_{3}\right)_{3} \mathrm{~L}_{\mathrm{n}}$, where $\mathrm{L}$ is the DGA and $\mathrm{n}$ is the number of ligands). Ligand 10a extracts Am and Eu more efficiently than 10b, although not as efficiently as TODGA. The order of extractability is TODGA > 10a > 10b. Only at high $\mathrm{HNO}_{3}$ concentrations of 3 and $4 \mathrm{~mol} / \mathrm{L}$ the distribution ratios for 10a are as high (over 100) as for TODGA. The differences in the extraction strength are attributed to the addition of one or two methyl groups, which decreases the flexibility of the molecule. In principle, one or two methyl groups will make the central oxygen atom more basic. Apparently, due to sterical constraints this does not result in better extraction properties compared to TODGA.

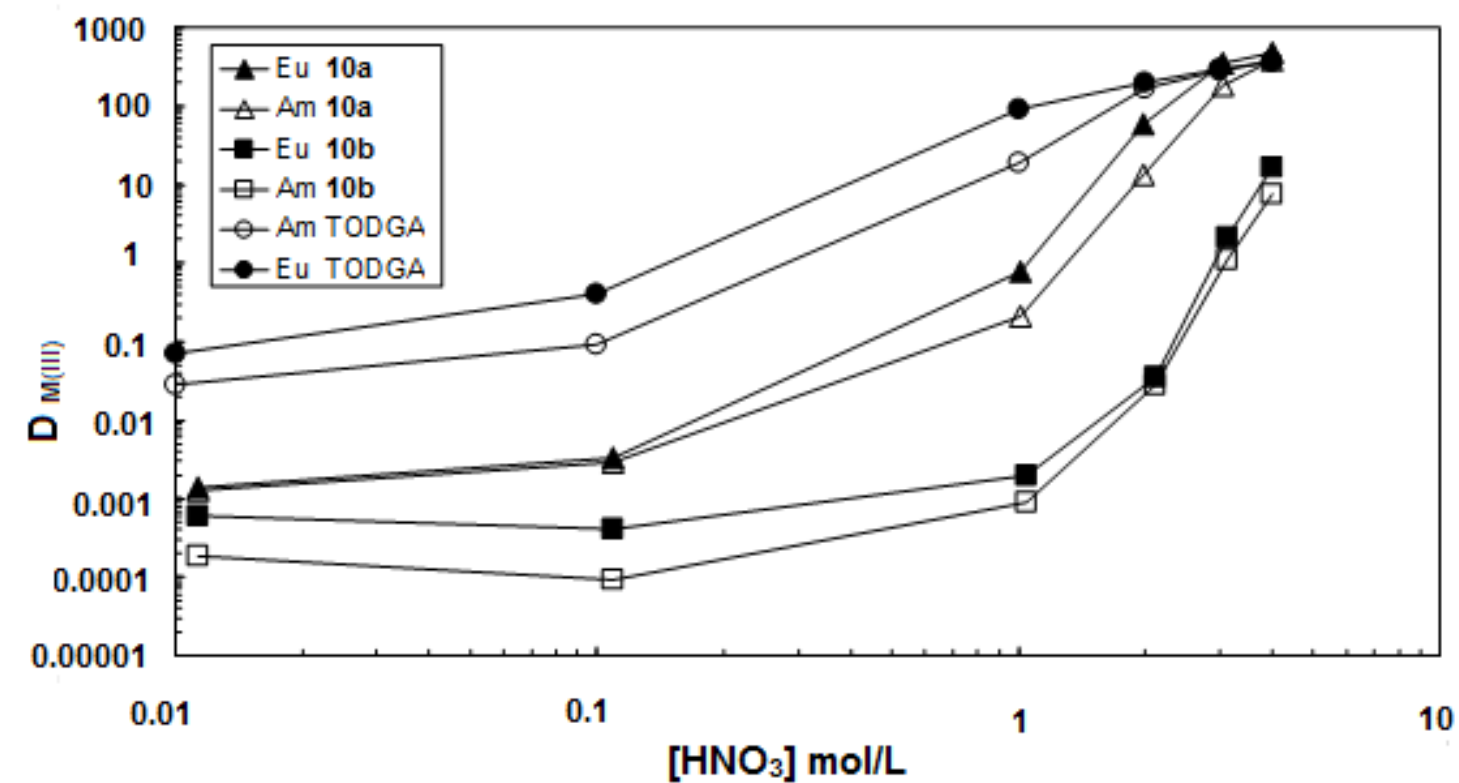

Figure 3.1. Nitric acid dependency for the extraction of ${ }^{241} \mathrm{Am}$ and ${ }^{152} \mathrm{Eu}$ by TODGA, 10a and 10b. Organic phase: $0.1 \mathrm{~mol} / \mathrm{L}$ of ligand in TPH. Aqueous phase: variable concentration of nitric acid, tracers: ${ }^{241} \mathrm{Am},{ }^{152} \mathrm{Eu}$, mixing time: $15 \mathrm{~min} ; \mathrm{T}=22{ }^{\circ} \mathrm{C} \pm 1{ }^{\circ} \mathrm{C}$. 
Ligands 10c and 10d do not extract Am and Eu as efficiently as TODGA. The addition of one phenyl group to the carbon atom between the central oxygen and the carbonyl of the carboxamide group decreases the extraction properties of the ligand (10c), although after adding a phenyl group on one side of the molecule and a methyl group on the other side (10d), the distribution ratios do not change significantly compared to 10c (Figure 3.2). The dominant influence of the phenyl group has clearly a strong negative (sterically and probably electronically) influence on the extraction behavior.

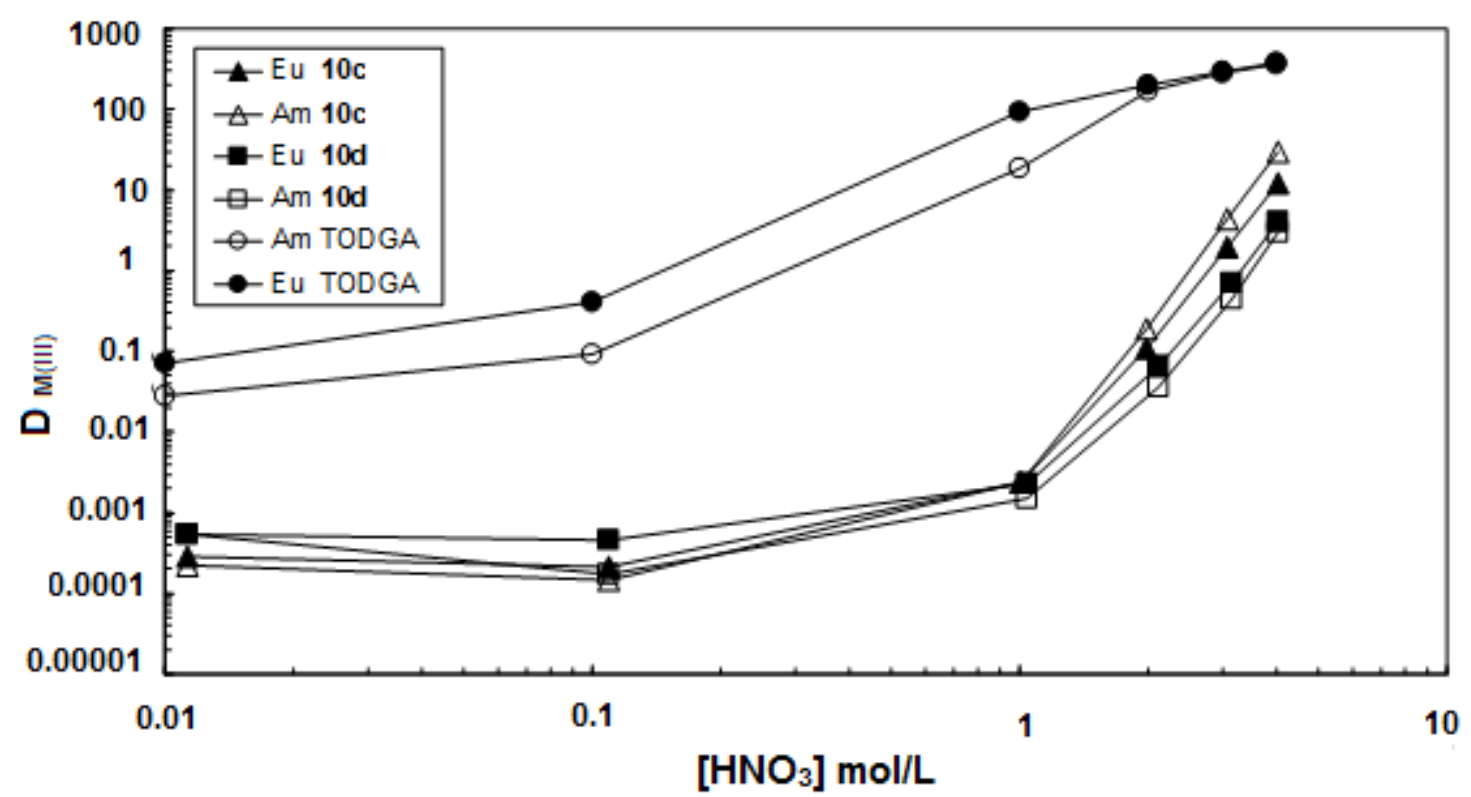

Figure 3.2. Nitric acid dependency for the extraction of ${ }^{241} \mathrm{Am}$ and ${ }^{152} \mathrm{Eu}$ by TODGA, 10c and 10d. Organic phase: $0.1 \mathrm{~mol} / \mathrm{L}$ of ligand in TPH. Aqueous phase: variable concentration of nitric acid, tracers: ${ }^{241} \mathrm{Am},{ }^{152} \mathrm{Eu}$, mixing time: $15 \mathrm{~min} ; \mathrm{T}=22{ }^{\circ} \mathrm{C} \pm 1{ }^{\circ} \mathrm{C}$.

Upon introduction of a tetrahydrofuran ring into the center of the molecule (14) the extraction of Am and Eu has been decreased compared to TODGA (Figure 3.3). In case of a furan ring (ligand 13) no extraction of Am nor Eu was detected. In the latter case the character of the oxygen atom in the aromatic furan ring is completely different compared to that in TODGA being less basic, ${ }^{18}$ explaining the lack of extraction. Surprisingly, the extraction behavior of tetrahydrofuran $\mathbf{1 4}$ is better than that of dimethyl-TODGA 10b, despite the lower concentration and the rigidification of the ligand.

From the different studied DGA-analogs, ligand 10a shows the best features with respect to further process optimization studies. As already discussed, 10a shows a high extraction capability towards actinides and lanthanides at high nitric acid concentration and the respective $D_{\text {Am,Eu }}$ values are comparable with those obtained for TODGA. Upon decreasing 
nitric acid concentration, however, the distribution ratios decrease much faster than for TODGA. At $0.1 \mathrm{~mol} / \mathrm{L}$ nitric acid the distribution ratios are approximately two orders of magnitude lower than for TODGA. This is favorable considering the conditions of the partitioning of high-level radioactive waste, since it facilitates the back-extraction step i.e. the strip solution can have a higher acidity or fewer back-extraction stages are necessary to achieve a complete reversible process. The results of a recently performed counter current test, fully described in refs 18,19 show that, during a TODGA-based actinide partitioning process, the complete stripping of actinides and lanthanides from a loaded organic phase was achieved with diluted nitric acid $(0.01 \mathrm{~mol} / \mathrm{L})$ using rather a large number of stripping stages (e.g. 12). Within this process, it was shown that TODGA also extracts nitric acid, which will be stripped back into the aqueous phase, thereby decreasing the stripping efficiency. So it can be supposed that this effect is not as important for 10a as extracting molecule, since stripping is not so sensitive on an acidity change.

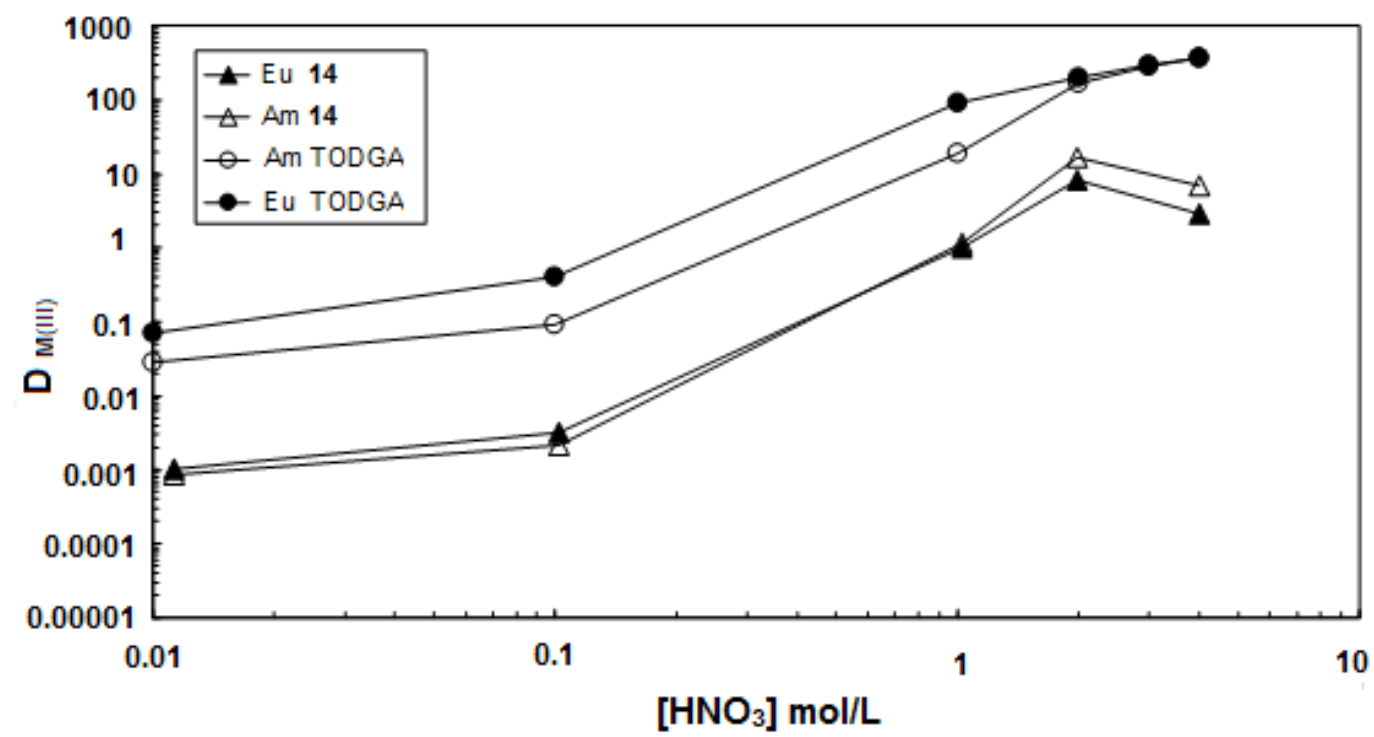

Figure 3.3. Nitric acid dependency for the extraction of ${ }^{241} \mathrm{Am}$ and ${ }^{152} \mathrm{Eu}$ by TODGA and $\mathbf{1 4}$. Organic phase: $0.1 \mathrm{~mol} / \mathrm{L}$ of TODGA or $0.08 \mathrm{~mol} / \mathrm{L}$ of $14 \mathrm{in} \mathrm{TPH.} \mathrm{Aqueous} \mathrm{phase:} \mathrm{variable}$ concentration of nitric acid, tracers: ${ }^{241} \mathrm{Am},{ }^{152} \mathrm{Eu}$, mixing time: 15 min TODGA (60 min ligand 14); $\mathrm{T}=22{ }^{\circ} \mathrm{C} \pm 1{ }^{\circ} \mathrm{C}$.

\subsubsection{Extraction of Am(III) and Eu(III) with ligands containing nitrogen as a central} atom

Several new ligands were synthesized composed of three donor atoms, namely two carbonyl oxygens and one nitrogen in the ether position of the central frame. Due to the softness of the nitrogen it is expected that, beside the extractability, probably also a discrimination between 


\section{Chapter 3}

the trivalent actinides and lanthanides can be achieved. The extraction studies, however, reveal that the ligands 16a,c,d, 17a, and 19 do not show any significant extraction of Am and $\mathrm{Eu}$ in the region between 0.01 and $4 \mathrm{~mol} / \mathrm{L}$ nitric acid. The distribution ratios for $\mathrm{Am}$ and $\mathrm{Eu}$ were below 0.1 for (16a,b and 17a) and even below 0.01 for 16d and 19. Similar ligands (Chart 3.2), where the nitrogen atom is bearing a methyl group, have already been studied by Sasaki et al. for the extraction of technetium(VII), rhenium(VII), palladium(II), and plutonium(IV). ${ }^{20}$

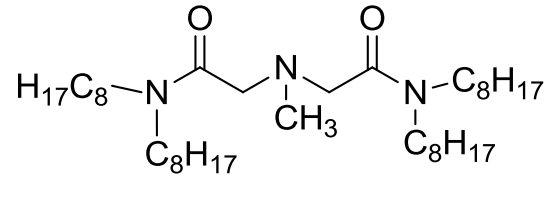

MIDOA

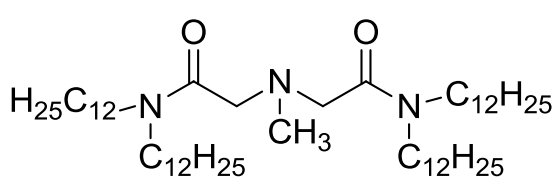

MIDDA

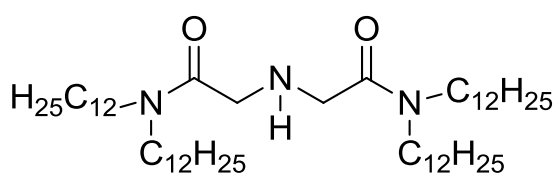

IDDA

Chart 3.2

In these cases, the extraction of oxometallates was observed and the results of slope analyses ( $\log D$ vs $\log$ [ligand]) indicate the species extracted to be 1:1 metal-ligand complexes. They found that the extraction of the oxometallates decreases with increasing nitric acid concentration. $\mathrm{Tc}(\mathrm{VII})$ and $\operatorname{Re}(\mathrm{VII})$ have remarkably high $D$ values in the range between 0.1 and $1 \mathrm{~mol} / \mathrm{L}$ nitric acid. The extraction of actinides was also studied using MIDOA (see Chart 3.2). For $\mathrm{Pu}(\mathrm{IV})$ the $D$ values increase up to a maximum at $3 \mathrm{~mol} / \mathrm{L}$ nitric acid with $D_{P u}$ of about 100 . The decrease at higher acidities was attributed to competition with nitric acid. The neptunium extraction as $\mathrm{Np}(\mathrm{V})$ was low $\left(D_{N p}<0.1\right)$ and the extraction of $\mathrm{Am}(\mathrm{III})$ was very low $\left(D_{\mathrm{Am}}<0.01\right)$ in the entire nitric acid concentration range of $0.1-6 \mathrm{~mol} / \mathrm{L}$. This is in agreement with our observations.

Ligands 18a and 18b differ from the above studied N-bearing ligands, because they contain a third amide function at the central $\mathrm{N}$ atom. They showed reverse extraction properties compared to TODGA: both ligands extract more efficiently Am and Eu at low nitric acid concentrations (Figure 3.4). Moreover, they show a higher affinity towards Am over Eu with separation factors $S F_{A m / E u}$ of 8 and 11 at $0.01 \mathrm{~mol} / \mathrm{L}$ nitric acid for $\mathbf{1 8 a}$ and $\mathbf{1 8 b}$, respectively. This type of ligands also act as tridentate chelating ligands, but not by coordination of the metal by the two carbonyl oxygens and the central $\mathrm{N}$ atom (vide supra), but instead by the 64 
additional oxygen of the third amide moiety. Since the $D$ values decrease with increasing acidity, it is assumed that the ligands (L) are protonated at the central $\mathrm{N}$ atom and the extraction of actinides $\left(\mathrm{M}^{3+}\right)$ can be explained by ion-pair extraction. This is only a hypothesis and needs to be studied very carefully by the well- known slope analysis.

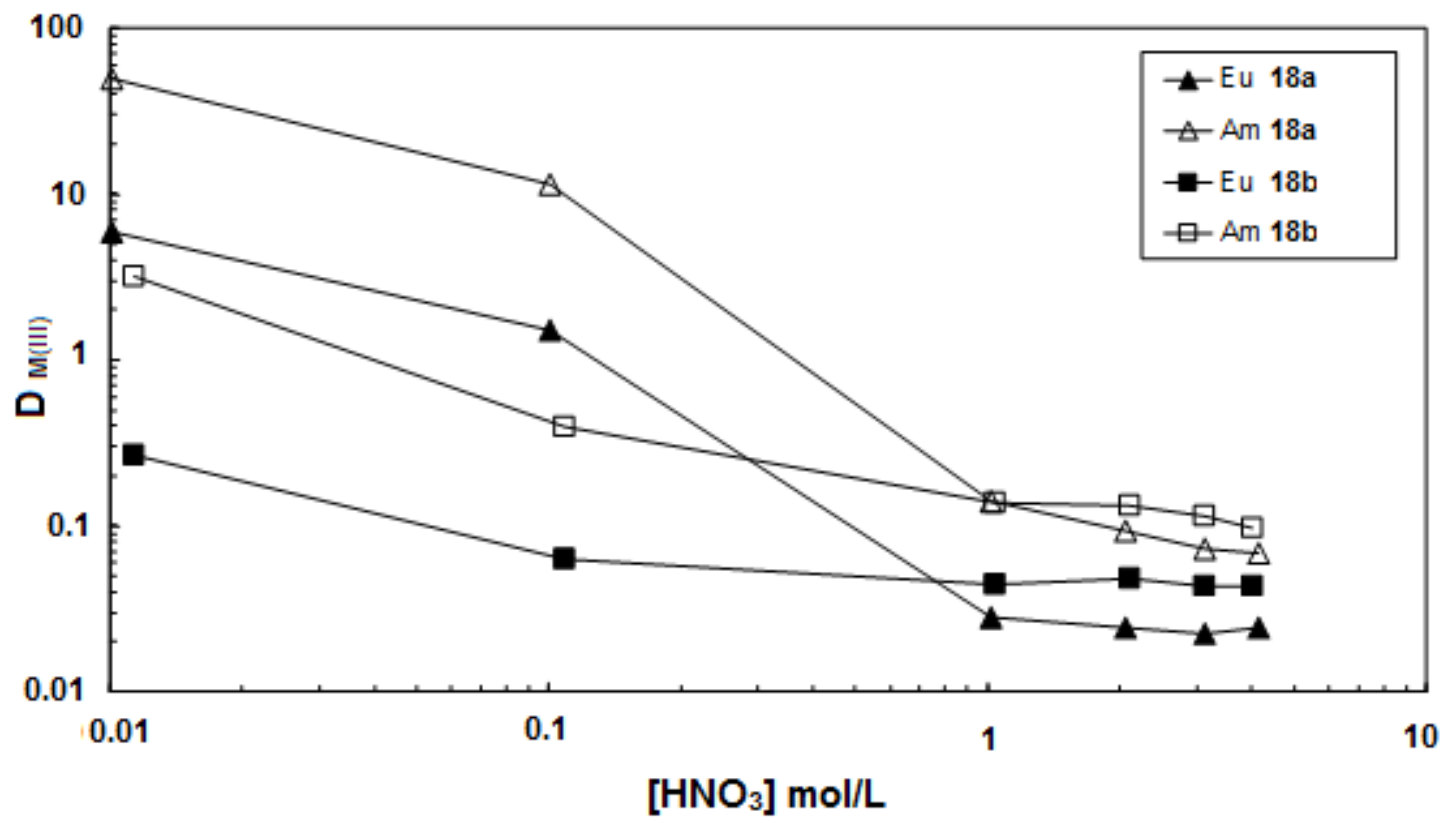

Figure 3.4. Nitric acid dependency for the extraction of ${ }^{241} \mathrm{Am}$ and ${ }^{152} \mathrm{Eu}$ by $\mathbf{1 8 a}$ and $\mathbf{1 8 b}$. Organic phase: $0.1 \mathrm{~mol} / \mathrm{L}$ of the ligand in TPH. Aqueous phase: variable concentration of nitric acid, tracers: ${ }^{241} \mathrm{Am},{ }^{152} \mathrm{Eu}$, mixing time: $15 \mathrm{~min}$; $\mathrm{T}=22{ }^{\circ} \mathrm{C} \pm 1{ }^{\circ} \mathrm{C}$.

\subsection{Conclusions}

Different types of structurally modified TODGA derivatives have been prepared. The extraction behavior of monomethyl-TODGA derivative 10a resembles that of the parent TODGA at high nitric acid concentrations. However, at $0.1 \mathrm{~mol} / \mathrm{L}$ nitric acid the $D$ values are about two orders of magnitude lower compared to TODGA, which is potentially useful for back-extraction processes. The aza-tripodal ligands $\mathbf{1 8 a}, \mathbf{b}$, in which probably three amide groups are involved in the complexation, exhibit a reverse complexation behavior compared to TODGA what the $\mathrm{pH}$ influence is concerned. At $0.01 \mathrm{~mol} / \mathrm{L}$ nitric acid interesting $D$ and $S F_{A m / E u}$ values are obtained. 


\subsection{Experimental}

\subsubsection{General}

All moisture-sensitive reactions were carried out under an argon atmosphere. The solvents and all reagents were obtained from commercial sources and used without further purification. Solvents were dried according to standard procedures and stored over molecular sieves. ${ }^{1} \mathrm{H}$ NMR and ${ }^{13} \mathrm{C}$ NMR spectra were recorded on a Varian Unity INOVA (300 MHz) spectrometer. ${ }^{1} \mathrm{H}$ NMR chemical shift values (300 MHz) are reported as $\delta$ using the residual solvent signal as an internal standard $\left(\mathrm{CDCl}_{3}, \delta 7.257\right) .{ }^{13} \mathrm{C}$ NMR chemical shift values (75 $\mathrm{MHz})$ are reported as $\delta$ using the residual solvent signal as an internal standard $\left(\mathrm{CDCl}_{3}, \delta\right.$ 77.0). All NMR spectra were recorded in $\mathrm{CDCl}_{3}$. Electrospray Ionization (positive mode) mass spectra were recorded on a WATERS LCT mass spectrometer. High resolution mass spectra were obtained with a JOEL T100CS AccuTOF mass spectrometer at the Radboud University at Nijmegen. Elemental analyses were performed using a Flash $200 \mathrm{CHN}$ analyzer of Thermo Scientific/Interscience. The extraction experiments were performed at Institute of Energy and Climate Research, Jülich, Germany. Analytical TLC was performed using Merck prepared plates (silica gel 60 F-254 on aluminum). Column chromatography was carried out on Merck silica gel 60 (230-400 mesh).

\section{General procedure for the preparation of 3,3'-oxybis(N,N-dialkylpropanamides)} $(4 a, b)$.

To a solution of a dialkylamine $(1 \mathrm{mmol})$ and triethylamine $(0.11 \mathrm{~g}, 1.1 \mathrm{mmol})$ in freshly distilled THF $(25 \mathrm{~mL})$ was added a solution of 3,3'-oxydipropanoyl chloride $3(0.1 \mathrm{~g}, 0.5$ $\mathrm{mmol})$ in THF $(5 \mathrm{~mL})$ at room temperature. The reaction mixture was stirred at room temperature overnight. When the reaction mixture was filtered, the solvent was evaporated under reduced pressure. The residue was dissolved in ethyl acetate $(50 \mathrm{~mL})$ and the resulting solution washed successively with $5 \% \mathrm{HCl}$ solution $(3 \times 50 \mathrm{~mL})$ and with water $(3 \times 50 \mathrm{~mL})$. The organic layer was dried over anhydrous $\mathrm{MgSO}_{4}$ and concentrated under reduced pressure to afford the pure products as oils.

\section{3,3'-Oxybis (N,N-dioctylpropanamide) (4a).}

Yield 78\%; ${ }^{1} \mathrm{H}$ NMR: $\delta$ 0.83-0.90 (12H, m, $\left.\mathrm{CH}_{3}\right), 1.18-1.36\left(40 \mathrm{H}, \mathrm{m}, \mathrm{CH}_{3}\left(\mathrm{CH}_{2}\right)_{5}\right), 1.48-1.54$ $\left(8 \mathrm{H}, \mathrm{m}, \mathrm{NCH}_{2} \mathrm{CH}_{2}\right), 2.58\left(4 \mathrm{H}, \mathrm{t}, J=6.9 \mathrm{~Hz}, \mathrm{OCH}_{2} \mathrm{CH}_{2}\right), 3.17-3.21\left(4 \mathrm{H}, \mathrm{m}, \mathrm{NCH}_{2}\right), 3.22(4 \mathrm{H}$, 
$\left.\mathrm{t}, J=6.9 \mathrm{~Hz}, \mathrm{NCH}_{2}\right), 3.75\left(4 \mathrm{H}, \mathrm{t}, J=6.9 \mathrm{~Hz}, \mathrm{OCH}_{2}\right) ;{ }^{13} \mathrm{C} \mathrm{NMR}: \delta 14.3,22.8,27.2,28.7,29.3$, 32.1, 45.4, 66.1, 169.8; ESI-MS m/z: $609.6[\mathrm{M}+\mathrm{H}]^{+}$; HRMS: m/z $609.5915[\mathrm{M}+\mathrm{H}]+$; calculated: 609.5934 for $\mathrm{C}_{38} \mathrm{H}_{77} \mathrm{~N}_{2} \mathrm{O}_{3}$.

\section{3,3'-Oxybis(N,N-bis(2-ethylhexyl)propanamide) (4b).}

Yield 62\%; ${ }^{1} \mathrm{H}$ NMR: $\delta$ 0.81-0.92 $\left(24 \mathrm{H}, \mathrm{m}, \mathrm{CH}_{3}\right), 1.09-1.40\left(32 \mathrm{H}, \mathrm{m}, \mathrm{CH}_{3} \mathrm{CH}_{2}, \mathrm{CH}_{3}\left(\mathrm{CH}_{2}\right)_{3}\right)$, 1.47-1.72 (4H, m, CH), $2.60\left(4 \mathrm{H}, \mathrm{t}, J=7.2 \mathrm{~Hz}, \mathrm{OCH}_{2} \mathrm{CH}_{2}\right), 3.76\left(4 \mathrm{H}, \mathrm{t}, J=7.2 \mathrm{~Hz}, \mathrm{OCH}_{2}\right)$, $3.12\left(4 \mathrm{H}, \mathrm{d}, J=7.5 \mathrm{~Hz}, \mathrm{NCH}_{2}\right), 3.24-3.27$ (4H, m, $\left.\mathrm{NCH}_{2}\right) ;{ }^{13} \mathrm{C} \mathrm{NMR}: \delta 11.4,14.2,23.1,23.9$, 29.2, 33.9, 37.1, 50.8, 67.1, 170.9; ESI-MS $m / z: 609.6[\mathrm{M}+\mathrm{H}]^{+}$and $627.6\left[\mathrm{M}+\mathrm{H}_{2} \mathrm{O}\right]^{+} ; \mathrm{HRMS}$ : $m / z 609.5914[\mathrm{M}+\mathrm{H}]^{+}$; calculated: 609.5934 for $\mathrm{C}_{38} \mathrm{H}_{77} \mathrm{~N}_{2} \mathrm{O}_{3}$.

\section{General procedure for the preparation of diesters $(7 a-d)$}

To a solution of the commercially available $\alpha$-bromopropanoates 5a-c $(1 \mathrm{mmol})$ and $\alpha$ hydroxyesters 6a,b $(1 \mathrm{mmol})$ in THF $(25 \mathrm{~mL})$ was added sodium hydride $(60 \%$ in oil, $0.04 \mathrm{~g}$, $1.1 \mathrm{mmol}$ ) portion-wise at $0{ }^{\circ} \mathrm{C}$. The reaction mixture was slowly brought to room temperature within a period of $1 \mathrm{~h}$, whereupon it was refluxed for $1 \mathrm{~h}$. The mixture was filtered and the solvent evaporated. The residue was dissolved in chloroform $(50 \mathrm{~mL})$, and the resulting solution was successively washed with $10 \% \mathrm{HCl}(3 \times 50 \mathrm{~mL})$, saturated $\mathrm{NaHCO}_{3}$ solution $(3 \times 50 \mathrm{~mL})$, and water $(50 \mathrm{~mL})$. The organic layer was dried over anhydrous $\mathrm{MgSO}_{4}$, concentrated under reduced pressure, and the residue was purified by chromatography $\left(\mathrm{SiO}_{2}, \mathrm{CH}_{2} \mathrm{Cl}_{2}\right)$.

\section{Ethyl 2-(2-ethoxy-2-oxoethoxy)propanoate (7a). ${ }^{21}$}

Yield 84\%; ${ }^{1} \mathrm{H}$ NMR: $\delta 1.25\left(6 \mathrm{H}, \mathrm{t}, J=6.0 \mathrm{~Hz}, \mathrm{CH}_{3}\right), 1.44\left(3 \mathrm{H}, \mathrm{d}, J=6.0 \mathrm{~Hz}, \mathrm{CHCH}_{3}\right), 4.00$ $4.27\left(7 \mathrm{H}, \mathrm{m}, \mathrm{OCH}, \mathrm{OCH}_{2}\right) ;{ }^{13} \mathrm{C} \mathrm{NMR}: \delta 14.3,18.5,61.2,66.9,75.2,170.1,172.8 ; \mathrm{MS}: \mathrm{m} / \mathrm{z}$ $205.1[\mathrm{M}+\mathrm{H}]^{+}$.

\section{Diethyl 2,2'-oxydipropanoate (7b).}

Yield 84\%; ${ }^{1} \mathrm{H}$ NMR: $\delta 1.28\left(6 \mathrm{H}, \mathrm{t}, J=6.0 \mathrm{~Hz}, \mathrm{CH}_{3}\right), 1.46\left(6 \mathrm{H}, \mathrm{d}, J=6.0 \mathrm{~Hz}, \mathrm{CHCH}_{3}\right), 4.09$ $(2 \mathrm{H}, \mathrm{q}, J=6.0 \mathrm{~Hz}, \mathrm{OCH}), 4.20\left(4 \mathrm{H}, \mathrm{q}, J=6.0 \mathrm{~Hz}, \mathrm{OCH}_{2}\right) ;{ }^{13} \mathrm{C} \mathrm{NMR}: \delta 14.3,18.5,61.3,80.5$, 173.0; MS: $m / z 219.1[\mathrm{M}+\mathrm{H}]^{+}$. 
Methyl 2-(2-ethoxy-2-oxoethoxy)-2-phenylacetate (7c).

Yield 74\%; ${ }^{1} \mathrm{H}$ NMR: $\delta 1.27\left(3 \mathrm{H}, \mathrm{t}, J=6.0 \mathrm{~Hz}, \mathrm{CH}_{3}\right), 3.72\left(3 \mathrm{H}, \mathrm{s}, \mathrm{OCH}_{3}\right), 4.14(2 \mathrm{H}, \mathrm{s}$, $\left.\mathrm{OCH}_{2}\right), 4.21\left(2 \mathrm{H}, \mathrm{q}, J=6.0 \mathrm{~Hz}, \mathrm{OCH}_{2} \mathrm{CH}_{3}\right), 5.18(1 \mathrm{H}, \mathrm{s}, \mathrm{OCH}), 7.32-7.47(5 \mathrm{H}, \mathrm{m}, \mathrm{ArH}) ;{ }^{13} \mathrm{C}$ NMR: $\delta 14.3,52.6,61.2,66.1,80.5,126.8,128.8,135.5,170.0,170.8 ;$ MS: $m / z 253.0$ $[\mathrm{M}+\mathrm{H}]^{+}$.

\section{Ethyl 2-(2-methoxy-2-oxo-1-phenylethoxy)propanoate (7d).}

Yield 45\%; ${ }^{1} \mathrm{H}$ NMR: $\delta 1.21\left(3 \mathrm{H}, \mathrm{t}, J=6.0 \mathrm{~Hz}, \mathrm{CH}_{3}\right), 1.25\left(3 \mathrm{H}, \mathrm{t}, J=6.0 \mathrm{~Hz}, \mathrm{CH}_{3}\right), 1.44$ $\left(1.5 \mathrm{H}, \mathrm{d}, J=6.0 \mathrm{~Hz}, \mathrm{CHCH}_{3}\right), 1.52\left(1.5 \mathrm{H}, \mathrm{d}, J=6.0 \mathrm{~Hz}, \mathrm{CHCH}_{3}\right), 3.95(0.5 \mathrm{H}, \mathrm{q}, J=6.0 \mathrm{~Hz}$, $\left.\mathrm{OCHCH}_{3}\right), 4.18\left(4.5 \mathrm{H}, \mathrm{m}, \mathrm{OCHCH}_{3}, \mathrm{OCH}_{2}\right), 5.06(1 \mathrm{H}, \mathrm{s}, \mathrm{ArCH}), 7.31-7.50(5 \mathrm{H}, \mathrm{m}, \mathrm{ArH})$; ${ }^{13} \mathrm{C}$ NMR: $\delta 14.3,18.6,61.4,67.3,80.5,126.7,128.9,135.5,170.6,170.8$; MS: $m / z 281.1$ $[\mathrm{M}+\mathrm{H}]^{+}$.

\section{General procedure for the preparation of dicarboxylic acids (8a-d)}

To a solution of diesters $7 \mathbf{a}-\mathbf{d}$ in methanol $(30 \mathrm{~mL})$ was slowly added $\mathrm{NaOH}(0.70 \mathrm{~g})$ at $0{ }^{\circ} \mathrm{C}$. The mixture was allowed to come to room temperature and was stirred overnight. The solvent was evaporated under reduced pressure. The white powder was dissolved in a minimum amount of water and was acidified with sulfuric acid. The solution was then extracted with diethyl ether $(3 \times 50 \mathrm{~mL})$. The extract was concentrated under reduced pressure to give the dicarboxylic acids 8a-d, which were used without further purification.

\section{General procedure for the preparation of substituted diglycolamides (10a-d)}

A mixture of dicarboxylic acids 8a-d (1 mmol), dioctylamine $(0.48 \mathrm{~g}, 2 \mathrm{mmol})$, triethylamine (0.21 g, $2 \mathrm{mmol})$, DCC (0.41 g, $2 \mathrm{mmol})$, and HOBT (0.27 g, $2 \mathrm{mmol})$ in chloroform (60 mL) was stirred overnight at room temperature. The solvent was evaporated and the resulting solid dissolved in $n$-hexane $(25 \mathrm{~mL})$. After filtration the solvent was evaporated under reduced pressure. The residue was purified by column chromatography $\left(\mathrm{SiO}_{2}, \mathrm{CH}_{2} \mathrm{Cl}_{2}: \mathrm{MeOH}=98: 2\right)$.

\section{2-(2-(Dioctylamino)-2-oxoethoxy)-N,N-dioctylpropanamide (10a).}

Yield 76\%; ${ }^{1} \mathrm{H}$ NMR: $\delta$ 0.83-0.92 $\left(12 \mathrm{H}, \mathrm{m}, \mathrm{CH}_{3}\right), 1.18-1.35\left(40 \mathrm{H}, \mathrm{m}, \mathrm{CH}_{3}\left(\mathrm{CH}_{2}\right)_{5}\right), 1.40(3 \mathrm{H}$, $\left.\mathrm{d}, J=6.0 \mathrm{~Hz}, \mathrm{CHCH}_{3}\right), 1.44-1.58\left(8 \mathrm{H}, \mathrm{m}, \mathrm{NCHCH}_{2}\right), 3.15-3.44\left(8 \mathrm{H}, \mathrm{m}, \mathrm{NCH}_{2}\right), 3.90(1 \mathrm{H}, \mathrm{d}$, $\left.J=12.0 \mathrm{~Hz}, \mathrm{OCH}_{2}\right), 4.28\left(1 \mathrm{H}, \mathrm{d}, J=12.0 \mathrm{~Hz}, \mathrm{OCH}_{2}\right), 4.55(1 \mathrm{H}, \mathrm{q}, J=6.0 \mathrm{~Hz}, \mathrm{OCH}) ;{ }^{13} \mathrm{C}$ NMR: $\delta 14.3,22.8,27.2,28.7,29.3,32.0,46.1,46.8,67.2,71.9,168.1,171.2 ;$ MS: $\mathrm{m} / z 595.4$ 68 
$[\mathrm{M}+\mathrm{H}]^{+}$and $613.5\left[\mathrm{M}+\mathrm{H}_{2} \mathrm{O}\right]^{+}$; HRMS: $m / z 595.5792[\mathrm{M}+\mathrm{H}]^{+}$; calculated: 595.5778 for $\mathrm{C}_{37} \mathrm{H}_{75} \mathrm{~N}_{2} \mathrm{O}_{3}$.

\section{2,2'-Oxybis(N,N-dioctylpropanamide) (10b).}

Yield 72\%; ${ }^{1} \mathrm{H}$ NMR: $\delta$ 0.83-0.92 $\left(12 \mathrm{H}, \mathrm{m}, \mathrm{CH}_{3}\right), 1.18-1.36\left(40 \mathrm{H}, \mathrm{m}, \mathrm{CH}_{3}\left(\mathrm{CH}_{2}\right)_{5}\right), 1.40(6 \mathrm{H}$, $\left.\mathrm{d}, J=6.6 \mathrm{~Hz}, \mathrm{CHCH}_{3}\right), 1.45-1.55\left(8 \mathrm{H}, \mathrm{m}, \mathrm{NCHCH}_{2}\right), 3.03-3.24\left(6 \mathrm{H}, \mathrm{m}, \mathrm{NCH}_{2}\right), 3.41-3.51$ $\left(2 \mathrm{H}, \mathrm{m}, \mathrm{NCH}_{2}\right), 4.22(2 \mathrm{H}, \mathrm{q}, J=6.6 \mathrm{~Hz}, \mathrm{OCH}) ;{ }^{13} \mathrm{C} \mathrm{NMR:} \delta 14.3,22.8,27.2,28.7,29.3,32.0$, 52.5, 74.5, 169.1; MS: $m / z 609.5[\mathrm{M}+\mathrm{H}]^{+}$; HHRMS: $\mathrm{m} / z 609.5930[\mathrm{M}+\mathrm{H}]^{+}$; calculated: 609.5934 for $\mathrm{C}_{38} \mathrm{H}_{77} \mathrm{~N}_{2} \mathrm{O}_{3}$.

\section{2-(2-(Dioctylamino)-2-oxoethoxy)-N,N-dioctyl-2-phenylacetamide (10c).}

Yield 70\%; ${ }^{1} \mathrm{H}$ NMR: $\delta$ 0.85-0.92 $\left(12 \mathrm{H}, \mathrm{m}, \mathrm{CH}_{3}\right), 1.18-1.36\left(40 \mathrm{H}, \mathrm{m}, \mathrm{CH}_{3}\left(\mathrm{CH}_{2}\right)_{5}\right), 1.44-1.58$ $\left(8 \mathrm{H}, \mathrm{m}, \mathrm{NCHCH}_{2}\right) 2.99-3.48\left(8 \mathrm{H}, \mathrm{m}, \mathrm{NCH}_{2}\right), 4.15\left(1 \mathrm{H}, \mathrm{d}, J=15.0 \mathrm{~Hz}, \mathrm{OCH}_{2}\right), 4.30(1 \mathrm{H}, \mathrm{d}, J$ $\left.=15.0 \mathrm{~Hz}, \mathrm{OCH}_{2}\right), 5.30(1 \mathrm{H}, \mathrm{s}, \mathrm{ArCH}), 7.30-7.37(3 \mathrm{H}, \mathrm{m}, \mathrm{ArH}), 7.47(2 \mathrm{H}, \mathrm{s}, \mathrm{ArH}) ;{ }^{13} \mathrm{C} \mathrm{NMR}$ : $\delta 14.3,22.8,27.2,28.7,29.3,32.0,46.2,48.2,67.5,80.0,128.5,129,136.5,168.5,169.5$, 171.5; MS: $m / z 657.8[\mathrm{M}+\mathrm{H}]^{+}$; HRMS: $m / z 657.5937[\mathrm{M}+\mathrm{H}]^{+}$; calculated: 657.5934 for $\mathrm{C}_{42} \mathrm{H}_{77} \mathrm{~N}_{2} \mathrm{O}_{3}$.

\section{2-(2-(Dioctylamino)-2-oxo-1-phenylethoxy)-N,N-dioctylpropanamide (10d).}

Yield 74\%; ${ }^{1} \mathrm{H}$ NMR: $\delta$ 0.85-0.92 (12H, m, $\left.\mathrm{CH}_{3}\right), 1.17-1.34\left(40 \mathrm{H}, \mathrm{m}, \mathrm{CH}_{3}\left(\mathrm{CH}_{2}\right)_{5}\right), 1.37(1.5 \mathrm{H}$, $\left.\mathrm{d}, J=6.6 \mathrm{~Hz}, \mathrm{CHCH}_{3}\right), 1.44\left(1.5 \mathrm{H}, \mathrm{d}, J=6.6 \mathrm{~Hz}, \mathrm{CHCH}_{3}\right), 1.44-1.58\left(8 \mathrm{H}, \mathrm{m}, \mathrm{NCHCH}_{2}\right)$, 2.90-3.50 (8H, m, $\left.\mathrm{NCH}_{2}\right), 4.27\left(0.5 \mathrm{H}, \mathrm{q}, J=6.6 \mathrm{~Hz}, \mathrm{CH}-\mathrm{CH}_{3}\right), 4.51(0.5 \mathrm{H}, \mathrm{q}, J=6.6 \mathrm{~Hz}, \mathrm{CH}-$ $\left.\mathrm{CH}_{3}\right), 5.11(0.5 \mathrm{H}, \mathrm{s}, \mathrm{ArCH}), 5.22(0.5 \mathrm{H}, \mathrm{s}, \mathrm{CH}-\mathrm{Ar}), 7.30-7.48(5 \mathrm{H}, \mathrm{m}, \mathrm{ArH}) ;{ }^{13} \mathrm{C} \mathrm{NMR}: \delta$ 14.3, 22.8, 27.2, 28.7, 29.3, 32.0, 47.5, 71.9, 79.2, 127.9, 129.1, 137.2, 169.5, 171.5; MS: $m / z$ $671.7[\mathrm{M}+\mathrm{H}]^{+}$; HRMS: $\mathrm{m} / z 671.6091[\mathrm{M}+\mathrm{H}]^{+}$; calculated: 671.6091 for $\mathrm{C}_{43} \mathrm{H}_{79} \mathrm{~N}_{2} \mathrm{O}_{3}$.

\section{N,N,N',N'-Tetraoctylfuran-2,5-dicarboxamide (13).}

To a solution of furan-2,5-dicarboxylic acid 11 ( $0.4 \mathrm{~g}, 2.56 \mathrm{mmol})$ in THF (25 mL) was added oxalyl chloride $(0.64 \mathrm{~g}, 5.1 \mathrm{mmol})$. The reaction mixture was refluxed overnight. The solvent and the excess of oxalyl chloride were evaporated under reduced pressure. The obtained furan-2,5-dicarbonyl dichloride 12 was directly reacted with dioctylamine $(1.54 \mathrm{~g}$, $6.4 \mathrm{mmol})$ in THF $(30 \mathrm{~mL})$ using triethylamine $(0.71 \mathrm{~g}, 7 \mathrm{mmol})$ as a base. The reaction mixture was stirred at room temperature overnight. The solvent was evaporated and the 
residue dissolved in dichloromethane $(50 \mathrm{~mL})$. The resulting solution was washed with $5 \%$ $\mathrm{HCl}$ solution $(3 \times 50 \mathrm{~mL})$ and with water $(3 \times 50 \mathrm{~mL})$. The organic layer was dried over anhydrous $\mathrm{MgSO}_{4}$ and concentrated under reduced pressure. The residue was purified by chromatography $\left(\mathrm{SiO}_{2}, \mathrm{CH}_{2} \mathrm{Cl}_{2}: \mathrm{MeOH}=97: 3\right)$ to afford 13 as an oil. Yield 50\%; ${ }^{1} \mathrm{H} \mathrm{NMR}: \delta$ 0.85-0.92 (12H, m, $\left.\mathrm{CH}_{3}\right), 1.18-1.35\left(40 \mathrm{H}, \mathrm{m}, \mathrm{CH}_{3}\left(\mathrm{CH}_{2}\right)_{5}\right), 1.54-1.58\left(8 \mathrm{H}, \mathrm{m}, \mathrm{NCH}_{2} \mathrm{CH}_{2}\right)$, 3.39-3.56 (8H, m, $\left.\mathrm{NCH}_{2}\right), 6.92(2 \mathrm{H}, \mathrm{s}, \mathrm{CH}) ;{ }^{13} \mathrm{C} \mathrm{NMR}: \delta 14.3,22.8,27.2,28.7,29.3,32.0$, 46.8, 140.2, 154.9, 169.9; MS: $m / z 603.6[\mathrm{M}+\mathrm{H}]^{+}$; HRMS: $m / z 603.5463[\mathrm{M}+\mathrm{H}]^{+}$; calculated: 603.5465 for $\mathrm{C}_{38} \mathrm{H}_{71} \mathrm{~N}_{2} \mathrm{O}_{3}$.

\section{$\mathrm{N}, \mathrm{N}, \mathrm{N}^{\prime}, \mathrm{N}^{\prime}$-Tetraoctyltetrahydrofuran-2,5-dicarboxamide (14).}

A solution of $13(0.69 \mathrm{~g}, 1.16 \mathrm{mmol})$ in methanol $(30 \mathrm{~mL})$ in the presence of $10 \% \mathrm{Pd} / \mathrm{C}(0.25$

g) was kept under 8 bar of $\mathrm{H}_{2}$ in an autoclave overnight. After removal of the catalyst by filtration the solvent was evaporated under reduced pressure, to afford $\mathbf{1 4}$ as an oil in quantitative yield. ${ }^{1} \mathrm{H}$ NMR: $\delta$ 0.85-0.94 $\left(12 \mathrm{H}, \mathrm{m}, \mathrm{CH}_{3}\right), 1.16-1.36\left(40 \mathrm{H}, \mathrm{m}, \mathrm{CH}_{3}\left(\mathrm{CH}_{2}\right)_{5}\right)$, 1.44-1.64 (8H, m, $\left.\mathrm{NCH}_{2} \mathrm{CH}_{2}\right), 1.96-2.11\left(2 \mathrm{H}, \mathrm{m}, \mathrm{CHCH}_{2}\right), 2.41-2.51\left(2 \mathrm{H}, \mathrm{m}, \mathrm{CHCH}_{2}\right), 3.15-$ $3.32\left(4 \mathrm{H}, \mathrm{m}, \mathrm{NCH}_{2}\right), 3.42-3.55\left(4 \mathrm{H}, \mathrm{m}, \mathrm{NCH}_{2}\right), 4.53-4.62(2 \mathrm{H}, \mathrm{m}, \mathrm{CH}) ;{ }^{13} \mathrm{C} \mathrm{NMR}: \delta 14.3$, 22.8, 27.2, 28.7, 29.3, 32.0, 46.4, 47.0, 78.1, 169.5; MS: $m / z: 607.6[\mathrm{M}+\mathrm{H}]^{+}$; HRMS: $m / z$ $607.5754[\mathrm{M}+\mathrm{H}]^{+}$; calculated: 607.5778 for $\mathrm{C}_{38} \mathrm{H}_{75} \mathrm{~N}_{2} \mathrm{O}_{3}$.

General procedure for the preparation of 2,2'-(alkylazanediyl)bis(N,Ndialkylacetamides) (16a-d)

A mixture of 2-chloro- $N, N$-dialkylacetamides $\mathbf{1 5 a}, \mathbf{b}^{22}$ (6.2 mmol), alkylamine/arylamine (3.1 $\mathrm{mmol}), \mathrm{K}_{2} \mathrm{CO}_{3}(1.2 \mathrm{~g}, 9 \mathrm{mmol})$, and $\mathrm{KI}(0.25 \mathrm{~g})$ in acetonitrile $(30 \mathrm{~mL})$ was refluxed overnight. The acetonitrile was evaporated and the residue was dissolved in ethyl acetate (50 $\mathrm{mL})$. The resulting solution was washed with dil. $\mathrm{HCl}(3 \times 50 \mathrm{~mL})$ and with water $(3 \times 50$ $\mathrm{mL}$ ). The organic layer was dried over anhydrous $\mathrm{MgSO}_{4}$ and concentrated under reduced pressure. The residue was purified by chromatography $\left(\mathrm{SiO}_{2}\right)$.

\section{2,2'-(Benzylazanediyl)bis(N,N-dioctylacetamide) (16a).}

Eluent: $\mathrm{CH}_{2} \mathrm{Cl}_{2}:$ EtOAc $=7: 3$; Oil; yield 78\%; ${ }^{1} \mathrm{H}$ NMR: $\delta$ 0.85-0.92 $\left(12 \mathrm{H}, \mathrm{m}, \mathrm{CH}_{3}\right), 1.17-1.34$ $\left(40 \mathrm{H}, \mathrm{m}, \mathrm{CH}_{3}\left(\mathrm{CH}_{2}\right)_{5}\right), 1.47-1.55\left(8 \mathrm{H}, \mathrm{m}, \mathrm{NCH}_{2} \mathrm{CH}_{2}\right), 3.10\left(4 \mathrm{H}, \mathrm{t}, J=7.5 \mathrm{~Hz}, \mathrm{NCH}_{2}\right), 3.22$ $\left(4 \mathrm{H}, \mathrm{t}, J=7.5 \mathrm{~Hz}, \mathrm{NCH}_{2}\right), 3.50\left(4 \mathrm{H}, \mathrm{s}, \mathrm{NCH}_{2} \mathrm{CO}\right), 3.84\left(2 \mathrm{H}, \mathrm{s}, \mathrm{NCH}_{2} \mathrm{Ph}\right), 7.20-7.42(5 \mathrm{H}, \mathrm{m}$, ArH); ${ }^{13} \mathrm{C}$ NMR: $\delta$ 14.3, 22.8, 27.2, 28.7, 29.3, 32.0, 46.3, 55.0, 59.4, 127.7, 128.8, 130.2, 70 
167.9; MS: $m / z$ 670.6 [M+H] $]^{+}$; HRMS: $m / z 670.6216[\mathrm{M}+\mathrm{H}]^{+}$; calculated: 670.6251 for $\mathrm{C}_{43} \mathrm{H}_{80} \mathrm{~N}_{3} \mathrm{O}_{2}$.

\section{2,2'-(Benzylazanediyl)bis(N,N-bis(2-ethylhexyl)acetamide) (16b).}

Eluent: $\mathrm{CH}_{2} \mathrm{Cl}_{2}:$ EtOAc $=7: 3$; Oil; yield 70\%; ${ }^{1} \mathrm{H}$ NMR: $\delta$ 0.81-0.92 $\left(24 \mathrm{H}, \mathrm{m}, \mathrm{CH}_{3}\right), 1.09-1.40$ $\left(32 \mathrm{H}, \mathrm{m}, \mathrm{CH}_{3} \mathrm{CH}_{2}, \mathrm{CH}_{3}\left(\mathrm{CH}_{2}\right)_{3}\right), 1.47-1.75(4 \mathrm{H}, \mathrm{m}, \mathrm{CH}), 3.10\left(4 \mathrm{H}, \mathrm{d}, J=7.5 \mathrm{~Hz}, \mathrm{NCH}_{2}\right), 3.18-$ 3.35 (4H, m, $\left.\mathrm{NCH}_{2}\right), 3.55$ (4H, s, $\left.\mathrm{NCH}_{2} \mathrm{CO}\right), 3.89$ (2H, s, $\left.\mathrm{NCH}_{2} \mathrm{Ph}\right), 7.20-7.42$ (5H, m, ArH); ${ }^{13}$ C NMR: $\delta$ 11.4, 14.2, 23.1, 23.9, 29.3, 34.0, 46.3, 55.0, 59.4, 127.7, 128.8, 130.2, 167.9; MS: $m / z 670.6[\mathrm{M}+\mathrm{H}]^{+}$; HRMS: $m / z 670.6226[\mathrm{M}+\mathrm{H}]^{+}$; calculated: 670.6251 for $\mathrm{C}_{43} \mathrm{H}_{80} \mathrm{~N}_{3} \mathrm{O}_{2}$.

\section{2,2'-(Octylazanediyl)bis(N,N-dioctylacetamide) (16c).}

Eluent: $\mathrm{CH}_{2} \mathrm{Cl}_{2}$ :EtOAc $=7: 3$; Oil; yield 75\%; ${ }^{1} \mathrm{H}$ NMR: $\delta$ 0.85-0.92 $\left(15 \mathrm{H}, \mathrm{m}, \mathrm{CH}_{3}\right), 1.17-1.34$ $\left(50 \mathrm{H}, \mathrm{m}, \mathrm{CH}_{3}\left(\mathrm{CH}_{2}\right)_{5}\right), 1.47-1.55\left(10 \mathrm{H}, \mathrm{m}, \mathrm{NCH}_{2} \mathrm{CH}_{2}\right), 2.67-2.73\left(2 \mathrm{H}, \mathrm{m}, \mathrm{NCH}_{2}\right), 3.22(8 \mathrm{H}, \mathrm{t}$, $\left.J=6.9 \mathrm{~Hz}, \mathrm{NCH}_{2}\right), 3.48-3.56\left(4 \mathrm{H}, \mathrm{m}, \mathrm{NCH}_{2} \mathrm{CO}\right) ;{ }^{13} \mathrm{C} \mathrm{NMR}: \delta$ 14.3, 22.8, 27.2, 28.7, 29.3, 32.0, 46.5, 49.1, 56.2; MS: $m / z 692.6[\mathrm{M}+\mathrm{H}]^{+}$; HRMS: $m / z 692.7000[\mathrm{M}+\mathrm{H}]^{+}$; calculated: 692.7033 for $\mathrm{C}_{44} \mathrm{H}_{90} \mathrm{~N}_{3} \mathrm{O}_{2}$.

\section{2,2'-(Phenylazanediyl)bis(N,N-dioctylacetamide) (16d).}

Eluent: $\mathrm{CH}_{2} \mathrm{Cl}_{2}$; Yield 65\%; mp 46-48 ${ }^{\circ} \mathrm{C}$; ${ }^{1} \mathrm{H}$ NMR: $\delta$ 0.85-0.92 $\left(12 \mathrm{H}, \mathrm{m}, \mathrm{CH}_{3}\right), 1.17-1.34$ $\left(40 \mathrm{H}, \mathrm{m}, \mathrm{CH}_{3}\left(\mathrm{CH}_{2}\right)_{5}\right), 1.47-1.54\left(8 \mathrm{H}, \mathrm{m}, \mathrm{NCH}_{2} \mathrm{CH}_{2}\right), 3.22\left(4 \mathrm{H}, \mathrm{t}, J=7.5 \mathrm{~Hz}, \mathrm{NCH}_{2}\right), 3.36$ $\left(4 \mathrm{H}, \mathrm{t}, J=7.5 \mathrm{~Hz}, \mathrm{NCH}_{2}\right), 4.22\left(4 \mathrm{H}, \mathrm{s}, \mathrm{OCH}_{2}\right), 6.42(2 \mathrm{H}, \mathrm{d}, J=7.8 \mathrm{~Hz}, \mathrm{ArH}), 6.64(1 \mathrm{H}, \mathrm{t}, J=$ $7.2 \mathrm{~Hz}, \mathrm{ArH}), 7.14(2 \mathrm{H}, \mathrm{t}, J=8.0 \mathrm{~Hz}, \mathrm{ArH}) ;{ }^{13} \mathrm{C}$ NMR: $\delta$ 14.3, 22.8, 27.2, 28.6, 29.4, 32.0, 46.5, 47.6, 53.9, 117.8, 112.5, 129.3, 149.0, 169.6; ESI-MS m/z: $656.8[\mathrm{M}+\mathrm{H}]^{+}$. Elem. Anal. Calculated for $\mathrm{C}_{42} \mathrm{H}_{77} \mathrm{~N}_{3} \mathrm{O}_{2}$ : C, 76.89; H, 11.83; N, 6.40. Found: C, 76.88; H, 11.86; N, 6.37; HRMS: $m / z 656.6069[\mathrm{M}+\mathrm{H}]^{+}$; calculated: 656.6094 for $\mathrm{C}_{42} \mathrm{H}_{78} \mathrm{~N}_{3} \mathrm{O}_{2}$.

\section{General procedure for the preparation of 2,2'-azanediylbis(N,N-dialkylacetamides)} $(17 a, b)$

A mixture of 16a,b $(1.5 \mathrm{mmol})$ and $10 \% \mathrm{Pd} / \mathrm{C}(0.25 \mathrm{~g})$ in ethanol $(40 \mathrm{~mL})$ was kept under $\mathrm{H}_{2}$ atmosphere overnight. After removal of the catalyst by filtration, the solvent was evaporated under reduced pressure to afford $\mathbf{1 7} \mathbf{a}, \mathbf{b}$ as oils in quantitative yield. 


\section{2,2'-Azanediylbis(N,N-dioctylacetamide) (17a).}

${ }^{1} \mathrm{H}$ NMR: $\delta$ 0.85-0.92 (12H, m, $\left.\mathrm{CH}_{3}\right), 1.17-1.34\left(40 \mathrm{H}, \mathrm{m}, \mathrm{CH}_{3}\left(\mathrm{CH}_{2}\right)_{5}\right), 1.47-1.54(8 \mathrm{H}, \mathrm{m}$, $\left.\mathrm{NCH}_{2} \mathrm{CH}_{2}\right), 2.62-2.82(1 \mathrm{H}$, br s, $\mathrm{NH}), 3.10\left(4 \mathrm{H}, \mathrm{t}, J=5.6 \mathrm{~Hz}, \mathrm{NCH}_{2}\right), 3.22(4 \mathrm{H}, \mathrm{t}, J=5.6 \mathrm{~Hz}$, $\mathrm{NCH}_{2}$ ), 3.50 (4H, s, $\left.\mathrm{NCH}_{2} \mathrm{CO}\right) ;{ }^{13} \mathrm{C}$ NMR: $\delta$ 14.3, 22.8, 27.2, 28.6, 29.5, 32.1, 42.6, 172.5; MS: $m / z, 580.5[\mathrm{M}+\mathrm{H}]^{+}$; HRMS: $m / z 580.5759[\mathrm{M}+\mathrm{H}]^{+}$; calculated: 580.5781 for $\mathrm{C}_{36} \mathrm{H}_{74} \mathrm{~N}_{3} \mathrm{O}_{2}$.

\section{2,2'-Azanediylbis(N,N-bis(2-ethylhexyl)acetamide) (17b).}

${ }^{1} \mathrm{H}$ NMR: $\delta$ 0.81-0.92 (24H, m, $\left.\mathrm{CH}_{3}\right), 1.09-1.40\left(32 \mathrm{H}, \mathrm{m}, \mathrm{CH}_{3} \mathrm{CH}_{2}, \mathrm{CH}_{3}\left(\mathrm{CH}_{2}\right)_{3}\right), 1.47-1.75$ $(4 \mathrm{H}, \mathrm{m}, \mathrm{CH}), 3.10\left(4 \mathrm{H}, \mathrm{d}, J=7.5 \mathrm{~Hz}, \mathrm{NCH}_{2}\right), 3.18-3.35\left(4 \mathrm{H}, \mathrm{m}, \mathrm{NCH}_{2}\right), 3.59(4 \mathrm{H}, \mathrm{s}$, $\left.\mathrm{NCH}_{2} \mathrm{CO}\right) ;{ }^{13} \mathrm{C}$ NMR: $\delta$ 11.4, 14.2, 23.1, 23.9, 29.3, 34.0, 42.6, 171.9; MS: $\mathrm{m} / \mathrm{z} 580.5$ $[\mathrm{M}+\mathrm{H}]^{+}$; HRMS: $m / z 580.5759[\mathrm{M}+\mathrm{H}]^{+}$; calculated: 580.5781 for $\mathrm{C}_{36} \mathrm{H}_{74} \mathrm{~N}_{3} \mathrm{O}_{2}$.

\section{General procedure for the preparation of nitrilotris( $\mathrm{N}, \mathrm{N}$-dialkylacetamides) $(18 a, b)$}

A mixture of 17a,b (1 mmol), 2-chloro- $N, N$-dialkylacetamides 15a,b (1 mmol), $\mathrm{K}_{2} \mathrm{CO}_{3}(0.41$ $\mathrm{g}, 3 \mathrm{mmol})$, and $\mathrm{KI}(0.25 \mathrm{~g})$ in acetonitrile $(30 \mathrm{~mL})$ was refluxed overnight. The acetonitrile was evaporated and the residue dissolved in ethyl acetate $(50 \mathrm{~mL})$. The resulting solution was washed with $5 \% \mathrm{HCl}(3 \times 50 \mathrm{~mL})$ and water $(3 \times 50 \mathrm{~mL})$, dried over anhydrous $\mathrm{MgSO}_{4}$, and evaporated under reduced pressure. The residue was purified by chromatography $\left(\mathrm{SiO}_{2}\right.$, $\mathrm{CH}_{2} \mathrm{Cl}_{2}: \mathrm{EtOAc}=7: 3$ ) to afford pure 18a,b as oils.

\section{$2,2^{\prime}, 2^{\prime \prime}$-Nitrilotris( $\mathbf{N}, \mathbf{N}-$ dioctylacetamide) $(18 a)$.}

Yield 75\%; ${ }^{1} \mathrm{H}$ NMR: $\delta$ 0.83-0.91 $\left(18 \mathrm{H}, \mathrm{m}, \mathrm{CH}_{3}\right), 1.11-1.36\left(60 \mathrm{H}, \mathrm{m}, \mathrm{CH}_{3}\left(\mathrm{CH}_{2}\right)_{5}\right), 1.36-1.57$ $\left(12 \mathrm{H}, \mathrm{m}, \mathrm{NCH}_{2} \mathrm{CH}_{2}\right), 3.15-3.31\left(12 \mathrm{H}, \mathrm{m}, \mathrm{NCH}_{2}\right), 3.62\left(6 \mathrm{H}, \mathrm{s}, \mathrm{NCH}_{2} \mathrm{CO}\right) ;{ }^{13} \mathrm{C} \mathrm{NMR}: \delta 14.3$, 22.8, 27.2, 28.7, 29.3, 32.0, 46.5, 52.5, 169.2; MS: $m / z 862.0[\mathrm{M}+\mathrm{H}]^{+}$and $884.0[\mathrm{M}+\mathrm{Na}]^{+}$; HRMS: $m / z, 861.8509[\mathrm{M}+\mathrm{H}]^{+}$; calculated: 861.8500 for $\mathrm{C}_{54} \mathrm{H}_{109} \mathrm{~N}_{4} \mathrm{O}_{3}$.

\section{2,2',2"'-Nitrilotris(N,N-bis(2-ethylhexyl)acetamide) (18b).}

Yield 70\%; ${ }^{1} \mathrm{H}$ NMR: $\delta$ 0.81-0.92 $\left(36 \mathrm{H}, \mathrm{m}, \mathrm{CH}_{3}\right), 1.09-1.40\left(48 \mathrm{H}, \mathrm{m}, \mathrm{CH}_{3} \mathrm{CH}_{2}, \mathrm{CH}_{3}\left(\mathrm{CH}_{2}\right)_{3}\right)$, 1.47-1.75 (6H, m, CH), 3.01-3.38 (12H, m, $\left.\mathrm{NCH}_{2}\right), 3.72\left(6 \mathrm{H}, \mathrm{s}, \mathrm{NCH}_{2} \mathrm{CO}\right) ;{ }^{13} \mathrm{C} \mathrm{NMR}: \delta 11.4$, 14.2, 23.1, 23.9, 29.3, 34.0, 37.4, 52.6, 169.3; MS: $m / z$ 862.0 [M+H] $]^{+}$; HRMS: $m / z$. 861.8527 $[\mathrm{M}+\mathrm{H}]^{+}$; calculated: 861.8500 for $\mathrm{C}_{54} \mathrm{H}_{109} \mathrm{~N}_{4} \mathrm{O}_{3}$. 


\section{2,2'-(Acetylazanediyl)bis(N,N-dioctylacetamide) (19).}

To a solution of $\mathbf{1 7 a}(1.15 \mathrm{~g}, 2 \mathrm{mmol})$ in freshly distilled THF (25 mL), containing triethylamine $(0.22 \mathrm{~g}, 2.1 \mathrm{mmol})$ as a base, was added dropwise a solution of acetyl chloride $(0.15 \mathrm{~g}, 2 \mathrm{mmol})$ in THF $(5 \mathrm{~mL})$. The reaction mixture was stirred at room temperature overnight. Subsequently, the reaction mixture was filtered and the solvent was evaporated under reduced pressure. The residue was dissolved in dichloromethane $(50 \mathrm{~mL})$. The resulting solution was washed with a saturated $\mathrm{NaHCO}_{3}$ solution $(3 \times 50 \mathrm{~mL})$ and with water $(3 \times 50$ $\mathrm{mL}$ ), dried over anhydrous $\mathrm{MgSO}_{4}$ and concentrated under reduced pressure. The residue was purified by column chromatography $\left(\mathrm{SiO}_{2}, \mathrm{CH}_{2} \mathrm{Cl}_{2}\right.$ :EtOAc $\left.=7: 3\right)$ to give pure 19 as an oil. Yield 89\%; ${ }^{1} \mathrm{H}$ NMR: $\delta$ 0.81-0.92 $\left(12 \mathrm{H}, \mathrm{m}, \mathrm{CH}_{3}\right), 1.09-1.39\left(40 \mathrm{H}, \mathrm{m}, \mathrm{CH}_{3}\left(\mathrm{CH}_{2}\right)_{5}\right), 1.36-1.56$ $\left(8 \mathrm{H}, \mathrm{m}, \mathrm{NCH}_{2} \mathrm{CH}_{2}\right), 2.03\left(3 \mathrm{H}, \mathrm{s}, \mathrm{COCH}_{3}\right), 3.10-3.32\left(8 \mathrm{H}, \mathrm{m}, \mathrm{NCH}_{2}\right), 4.26\left(4 \mathrm{H}, \mathrm{s}, \mathrm{NCH}_{2} \mathrm{CO}\right)$; ${ }^{13} \mathrm{C}$ NMR: $\delta 14.3,22.8,27.2,28.7,29.3,32.0,48.2,56.3,171.5,173.5$; MS: $m / z 622.7$ $[\mathrm{M}+\mathrm{H}]^{+}$; HRMS: $m / z 622.5907[\mathrm{M}+\mathrm{H}]^{+}$; calculated: 622.5887 for $\mathrm{C}_{38} \mathrm{H}_{76} \mathrm{~N}_{3} \mathrm{O}_{3}$.

\subsubsection{Extraction procedure}

All the ligands were dissolved in total petroleum hydrocarbon (TPH) to a preferable concentration of $0.1 \mathrm{~mol} / \mathrm{L}$. Only ligand 14 was tested at a concentration of $0.08 \mathrm{~mol} / \mathrm{L}$ due to a lack of the ligand. The obtained organic solvent was contacted with nitric acid of variable concentrations (0.01 to $4 \mathrm{~mol} / \mathrm{L})$ containing traces of $\mathrm{Am}(\mathrm{III})$ and $\mathrm{Eu}(\mathrm{III})$. Nitric acid solutions were prepared by diluting concentrated nitric acid (Merck KGa, Darmstadt, Germany) with Ultra-pure water. The acidity was checked by titration with $\mathrm{NaOH}$.

The batch extraction experiments were performed in $2 \mathrm{~mL}$ glass vials. $500 \mu \mathrm{L}$ of organic and aqueous phases were spiked with $10 \mu \mathrm{L}$ of radiotracer $\left({ }^{241} \mathrm{Am},{ }^{152} \mathrm{Eu}\right.$, approx. $\left.25 \mathrm{KBq} / \mathrm{mL}\right)$ and shaken by a vortex mixer for 15 min until equilibrium (in case of $\mathbf{1 4}$ the mixing time was $60 \mathrm{~min}$ ). Separation of the phases by centrifugation was followed by sampling of $200 \mu \mathrm{L}$ of each phase for analysis using high-purity germanium spectrometer system obtained from EG\&G Ortec, München, Germany and equipped with the gamma vision software. The $\gamma$-lines at $59.5 \mathrm{keV}$ and $121.8 \mathrm{keV}$ were examined for ${ }^{241} \mathrm{Am}$ and ${ }^{152} \mathrm{Eu}$, respectively. The distribution ratio was measured as the ratio between the radioactivity of an isotope in the organic and the aqueous phase. Distribution ratios between 0.1 and 100 exhibit a maximum error of $\pm 5 \%$. The error may be up to $\pm 20 \%$ for smaller and larger values. 


\subsection{Reference}

1. Stephan, H.; Gloe, K.; Beger, J.; Mühl, P. Solv. Extr. Ion Exch. 1991, 9, 459.

2. Sasaki, Y.; Choppin, G. R.; Anal. Sci. 1996, 12, 225.

3. Sasaki, Y.; Choppin, G. R. Radiochim. Acta 1998, 80, 85.

4. (a) Tachimori, S.; Sasaki, Y.; Suzuki, S. Solv. Extr. Ion Exch. 2002, 20, 687. (b) Yaita, T.; Herlinger, A. W.; Thiyagarajan, P.; Jensen, M. P. Solv. Extr. Ion Exch. 2004, 22, 553. (c) Narita, H.; Yaita, T.; Tachimori, S. Solv. Extr. Ion Exch. 2004, 22, 135. (d) Suzuki, H.; Sasaki, Y.; Sugo, Y.; Apichaibuol, A.; Kimura, T. Radiochim. Acta 2004, 92, 463. (e) Zhu, Z. X.; Sasaki, Y.; Suzuki, H.; Suzuki, S.; Kimura, T. Anal. Chim. Acta 2004, 527, 163. (f) Sasaki, Y.; Zhu, Z. X.; Sugo, Y.; Suzuki, H.; Kimura, T. Anal. Sci. 2005, 21, 1171. (g) Sasaki, Y.; Rapold, P.; Arisaka, M.; Hirata, M.; Kimura, T.; Hill, C.; Cote, G. Solv. Extr. Ion Exch. 2007, 25, 187.

5. Sasaki, Y.; Sugo, Y.; Suzuki, S.; Tachimori, S. Solv. Extr. Ion Exch. 2001, 19, 91.

6. Sasaki, Y.; Tachimori, S. Solv. Extr. Ion Exch. 2002, 20, 21.

7. (a) Pretsch, E.; Ammann, D.; Oswald, H. F.; Gugi, M.; Simon, W. HeIv. Chim. Acta 1980, 63, 191. (b) Sasaki, Y.; Adachi, T.; Choppin, G. R. J. Alloys Compd. 1998, 271, 799. (c) Sasaki, Y.; Sugo, Y.; Suzuki, S.; Tachimori, S. Solv. Extr. Ion Exch. 2001, 19, 91. (d) Tachimori, S.; Suzuki, S.; Sasaki, Y.; Apichaibukol, A. Solv. Extr. Ion Exch. 2003, 21, 707. (e) Ansari, S. A.; Pathak, P. N.; Manchanda, V. K.; Husain, M.; Prasad, A. K.; Parmar, V. S. Solv. Extr. Ion Exch. 2005, 23, 463. (f) Sasaki, Y.; Sugo, Y.; Kitatsuji, Y.; Kirishima, A.; Kimura, T.; Choppin, G. R. Anal. Sci. 2007, 23, 727. (g) Sasaki, Y.; Zhu, Z. X.; Sugo, Y.; Kimura, T. J. Nucl. Sci. Technol. 2007, 44, 405.

8. Matloka, K.; Gelis, A.; Regalbuto, M.; Vandegrift, G.; Scott, M. J. Dalton Trans. 2005, 23,3719 .

9. Matloka, K.; Gelis, A.; Regalbuto, M.; Vandegrift, G.; Scott, M. J. Sep. Sci. Technol. 2006, 41, 2129.

10. Jańczewski, D.; Reinhoudt, D. N.; Verboom, W.; Hill, C.; Allignol, C.; Duchesne, M. New J. Chem. 2008, 32, 490.

11. Murillo, M. T.; Espartero, A. G.; Sánchez-Quesada, J.; de Mendoza, J.; Prados, P. Solv. Extr. Ion Exch. 2009, 27, 107.

12. Mowafy, E. A.; Aly, H. F. Solv. Extr. Ion Exch. 2006, 24, 677.

13. White, D. E.; Jacobsen, E. N. Tetrahedron: Asymmetry 2003, 14, 3633. 
14. Pratt, J. A. E.; Sutherland, I. O.; Newton, R. F. J. Chem. Soc., Perkin Trans. 1988, 13.

15. Ansari, S. A.; Pathak, P. N.; Manchanda, V. K. Solv. Extr. Ion Exch. 2005, 23, 473.

16. Lew, B. W. US patent $322506619651221,1965$.

17. Nielek, S.; Lesiak, T. J. Prakt. Chem. (Leipzig) 1988, 330, 825.

18. Rauk, A.; Hunt, I. R.; Keay, B. A. J. Org. Chem. 1994, 59, 6808.

19. Magnusson, D.; Christiansen, B.; Glatz, J. P.; Malmbeck, R.; Modolo, G.; Serrano-Purroy, D.; Sorel, C. Solv. Extr. Ion Exch. 2009, 27, 26.

20. Sasaki, Y.; Ozawa, M.; Kimura, T.; Ohashi, K. Solv. Extr. Ion Exch. 2009, 27, 378.

21. Parsons, D. G. J. Chem. Soc., Perkin Trans. 1 1975, 245.

22. Kannan, S.; Gamare, J. S.; Chetty, K. V.; Drew, M. G. B. Polyhedron 2007, 26, 3810. 



\section{Chapter}

\section{4}

\section{Synthesis and Evaluation of Novel Water-Soluble Ligands for Stripping of Actinides"}

Different types of water-soluble ligands were synthesized and their capability was evaluated by solvent extraction studies to complex trivalent actinides. Hydrophilic diglycolamide (DGA) derivatives with a varying number of ethylene glycol groups, or containing sodium acetate moieties on the amidic nitrogen, show a decrease in back-extraction efficiency with increasing number of ethylene glycol units on the amidic nitrogen at various pH of the aqueous phase. Among the $P=S$ donating ligands, only the ligand with a malonamide backbone exhibits a high reverse extraction efficiency, although, with no selectivity for americium. Amongst the water-soluble tripodal ligands, i.e. the amide derivatives of nitrilotriacid with $\mathrm{N}, \mathrm{N}$-dimethyl (21) and N,N-bis(hydroxyethyl) (23) moieties, ligand 21 shows a pronounced selectivity for Am(III) over Eu(III), with a maximum separation factor of 11.1, while ligand 23 more efficiently complexes the radionuclides in the aqueous phase with a maximum separation factor of 5. Isothermal microcalorimetry experiments of the complexation of Eu(III) by ligands $2,7 a, 7 b, 21$, and 23 confirm the observed trend in the back-extraction properties being $2>7 a>7 b$ and $23>21$.

\footnotetext{
* This chapter is based on: Iqbal, M.; Huskens, J.; Sypula, M.; Modolo, G.; Verboom, W. New J. Chem. 2011, 35, 2591.
} 


\section{Chapter 4}

\subsection{Introduction}

Within the current European project ACSEPT $^{1}$ (Actinide reCycling by SEParation and Transmutation) the so-called "innovative SANEX" concept is studied; see Chapter 2. In this strategy, the trivalent actinides are recovered from the PUREX raffinate by means of one organic solvent, thus reducing the cycles and minimizing the waste. The process consists of an $\mathrm{An}(\mathrm{III}) / \mathrm{Ln}(\mathrm{III})$ co-extraction step at high acidity after which, the loaded solvent is subjected to several stripping steps. The first one concerns selective stripping of trivalent actinides with a selective water-soluble agent followed by the subsequent stripping of trivalent lanthanides.

$N, N, N^{\prime}, N$ '-tetraoctyl-3-oxapentanediamide (TODGA) is currently one of the innovative SANEX reference molecules due to its high distribution ratios for An and Ln from highly acidic media, ${ }^{2}$ high stability in aliphatic diluents, ${ }^{3}$ composition allowing destruction by combustion (C, H, O, N principle), and easy synthesis. ${ }^{4}$ Since it shows a slightly higher affinity towards trivalent lanthanides compared to trivalent actinides, the implementation of a selective water-soluble ligand forming stronger complexes with An(III) than Ln(III) would significantly increase the Ln/An separation factor. The most well-known hydrophilic ligand EDTA has been studied for the stripping of $\mathrm{Am}(\mathrm{III})$ and $\mathrm{Cm}$ (III) in the TALSPEAK (Trivalent Actinide Lanthanide Separations by Phosphorus- reagent Extraction from Aqueous Komplexes) process. ${ }^{5,6}$ A number of other carboxylic and polycarboxylic acids have been described by Sasaki et al. ${ }^{7}$ However, EDTA and other carboxylic acids have a limited solubility and the solubility also greatly depends on the $\mathrm{HNO}_{3}$ concentration of the aqueous solution. During the stripping process, there is always a competition between the organic and the aqueous phases to bind the metals, therefore strong ligands with a high solubility are required to selectively strip the actinide ions. Some water-soluble diglycolamides (tetraalkyl diglycolamide) have been studied by Sasaki et al., ${ }^{7,8}$ however, their water solubility is limited. Water-soluble $N$ donor ligands, containing pyridine and pyrazine rings described by Heitzmann et al. ${ }^{9,10}$ demonstrate a correlation of the selectivity for trivalent actinides (e.g. Am) over Ln with the $N$ donor groups coordinated to the cation.

This chapter deals with new water-soluble diglycolamides with varying lengths of ethylene glycol chains on the amidic nitrogens, and two novel water-soluble ligands with a backbone similar to diglycolamide ${ }^{4}$ and malonamide, ${ }^{11}$ but with the softer sulfur as coordinating atom instead of oxygen to study their back-extraction properties. 
As described in Chapter 3, lipophilic ligands ${ }^{12}$ 2,2',2' '-nitrilotris( $N, N$-dialkylacetamides) proved to be selective ligands for Am(III), however, only at lower nitric acid concentrations. Since back-extractions of An(III) and Ln(III) are performed at low nitric acid concentrations, we also synthesized water-soluble derivatives of these ligands to study their applicability for selective back-extraction.

\subsection{Results and discussion}

\subsubsection{Synthesis}

\subsubsection{Water-soluble TODGA-derived ligands}

The synthesis of water-soluble TODGA derivatives $\mathbf{2 , 7 a , b}$, and $\mathbf{1 0}$ is summarized in Schemes 4.1 and 4.2. First, diglycolyl dichloride (1) was reacted with diethanolamine in the presence of triethylamine in THF according to a literature procedure. ${ }^{13}$ However, a 1:1 mixture was obtained of the desired 2,2'-oxybis(N,N-bis(2-hydroxyethyl)acetamide) (2) and the $\mathrm{HCl}$ salt of diethanolamine as followed from ${ }^{1} \mathrm{H}$ NMR spectroscopy, which could not be separated. ${ }^{14}$ Ligand 2 could be synthesized in 67\% yield by reaction of diglycolyl dichloride (1) with diethanolamine in acetone using $\mathrm{K}_{2} \mathrm{CO}_{3}$ as a base (Scheme 4.1). In the ${ }^{1} \mathrm{H}$ NMR spectrum the peak for the methylene protons was shifted from $4.57 \mathrm{ppm}$ in $\mathbf{1}$ to $4.28 \mathrm{ppm}$ in $\mathbf{2}$.<smiles>O=C(Cl)COCC(=O)Cl</smiles>

1

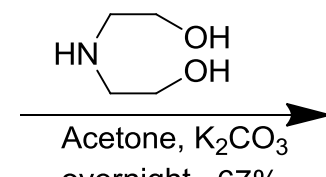

overnight $67 \%$

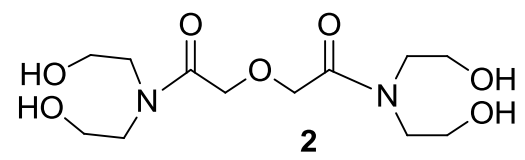

2

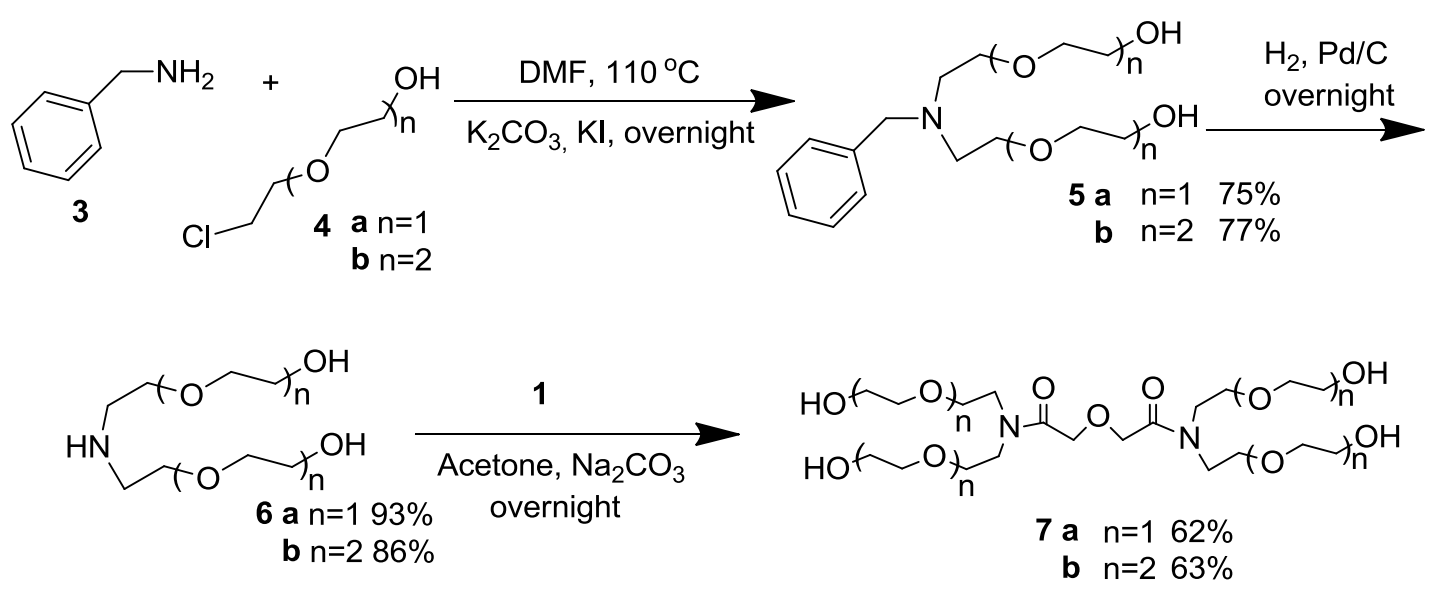

Scheme 4.1

To extend the glycol chain in ligand 2 the benzylamine (3) was reacted with 2-(2chloroethoxy)ethanol (4a) and 2-(2-(2-chloroethoxy)ethoxy)ethanol (4b) in the presence of 


\section{Chapter 4}

$\mathrm{K}_{2} \mathrm{CO}_{3}$ and $\mathrm{KI}$ in DMF at $110{ }^{\circ} \mathrm{C}$ to give 5a and $\mathbf{5 b}$, respectively. Removal of the benzyl group in $\mathbf{5 a}$ and $\mathbf{5 b}$ by hydrogenolysis with $10 \% \mathrm{Pd} / \mathrm{C}$ in methanol afforded $\mathbf{6 a}$ and $\mathbf{6 b}$, which subsequently were reacted with diglycolyl dichloride (1) in acetone to give the desired ligands 7a and 7b in 62 and 63\% yield, respectively (Scheme 4.1). In the ${ }^{1} \mathrm{H}$ NMR spectra the peak for the methylene protons was shifted from $4.57 \mathrm{ppm}$ in $\mathbf{1}$ to 4.31 and $4.29 \mathrm{ppm}$ in $\mathbf{7 a}$ and $\mathbf{7 b}$, respectively.

The next target molecule was ligand $\mathbf{1 0}$ containing four sodium acetate groups connected to the amidic nitrogen to induce water solubility. Diglycolyl dichloride (1) was reacted with dimethyl 2,2'-azanediyldiacetate (8) in the presence of triethylamine to obtain tetraester 9 in $80 \%$ yield. Subsequent hydrolysis of the ester groups in 9 using $\mathrm{NaOH}$ in methanol gave $N, N, N^{\prime}, N^{\prime}$-tetrasodium acetate diglycolamide (10) in quantitative yield (Scheme 4.2). In the ${ }^{1} \mathrm{H}$ NMR spectrum, the methyl ester peaks at 3.72 and 3.75 ppm of 9 completely disappeared upon hydrolysis.

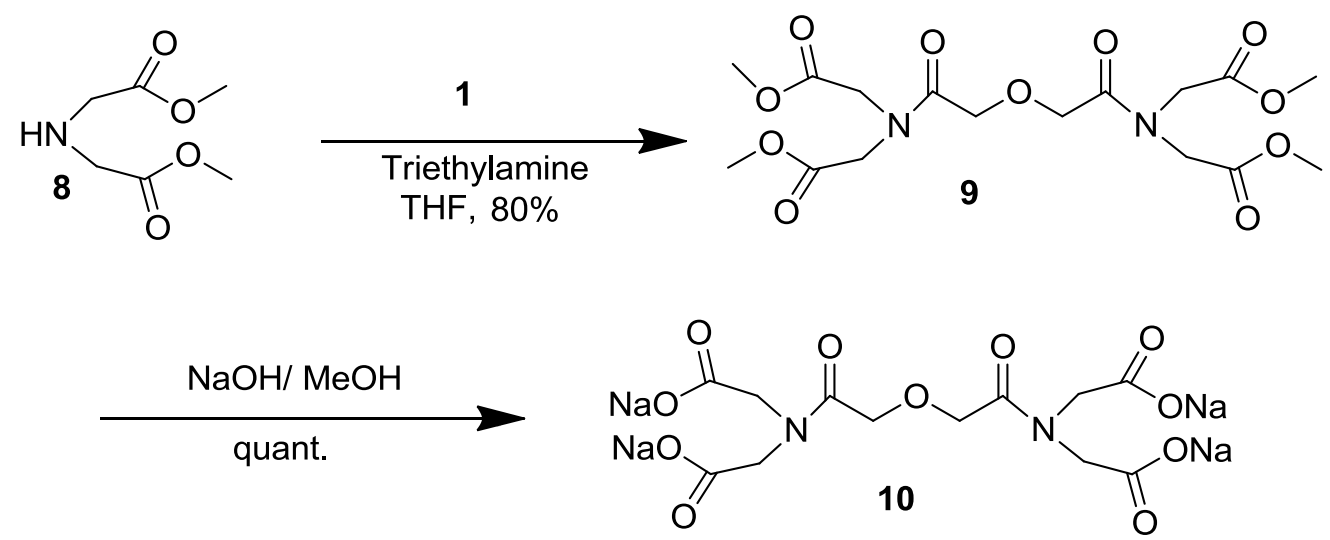

Scheme 4.2

\subsubsection{Synthesis of S/O-containing ligands}

\subsection{Water-soluble O-containing ligand with diglycolamide backbone}

(Diethoxyphosphoryl)methyl trifluoromethanesulfonate (11), prepared from diethyl (hydroxymethyl)phosphonate according to a literature procedure, ${ }^{15}$ was reacted with diethyl (hydroxymethyl)phosphonate in the presence of $\mathrm{NaH}$ as a base to give the desired tetraethyl (oxybis(methylene))bis(phosphonate) (12) in 74\% yield. In the ${ }^{1} \mathrm{H}$ NMR spectrum the doublet for the methylene hydrogens at $4.62 \mathrm{ppm}$ in $\mathbf{1 1}$ shifted to $3.85 \mathrm{ppm}$ in $\mathbf{1 2}$. The $\mathrm{P}=\mathrm{O}$ groups in 12 were converted into $\mathrm{P}=\mathrm{S}$ moieties using Lawesson's reagent to afford $\mathbf{1 3}$ in $80 \%$ yield. In this case in the ${ }^{1} \mathrm{H}$ NMR spectrum the methylene protons appeared at $4.10 \mathrm{ppm}$. Subsequent 80 
hydrolysis of $\mathbf{1 3}$ with conc. $\mathrm{HCl}$ gave the hydrophilic oxybis(methylene)diphosphonic acid (14) in 70\% yield and not the expected diphosphonothioic acid. Apparently, during the acid treatment the $\mathrm{P}=\mathrm{S}$ moieties were oxidized to $\mathrm{P}=\mathrm{O}$ groups. The ${ }^{1} \mathrm{H}$ NMR spectrum of $\mathbf{1 4}$ shows the methylene protons as a doublet at $3.62 \mathrm{ppm}$. All compounds showed characteristic $\mathrm{M}+1$ peaks in their mass spectra. The coupling reaction to give $\mathrm{P}=\mathrm{O}$ ligand $\mathbf{1 2}$ was also performed with a tosylate instead of a triflate group as leaving group. However, it gave rise to a much lower yield (34\%) of $\mathbf{1 2 .}$

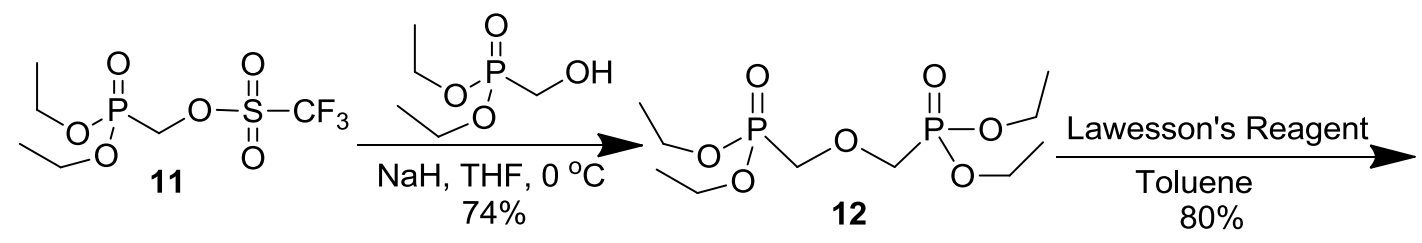<smiles>CCOP(=S)(COCP(=S)(OCC)OCC)OCC</smiles>

13

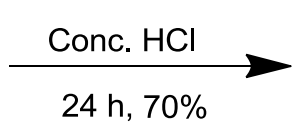

Scheme 4.3<smiles>O=P(O)(O)COCP(=O)(O)O</smiles>

14

\subsection{S-containing water-soluble ligand with malonamide backbone}

For the synthesis of ligand 17, with a malonamide backbone, first bis(dichlorophosphino)methane was quantitatively converted into bis(dichlorophosphorothioyl)methane (16) upon treatment with $\mathrm{PSCl}_{3}$. In contrast to a literature procedure, $\mathrm{AlCl}_{3}$ was not used as a Lewis acid catalyst. ${ }^{16}$

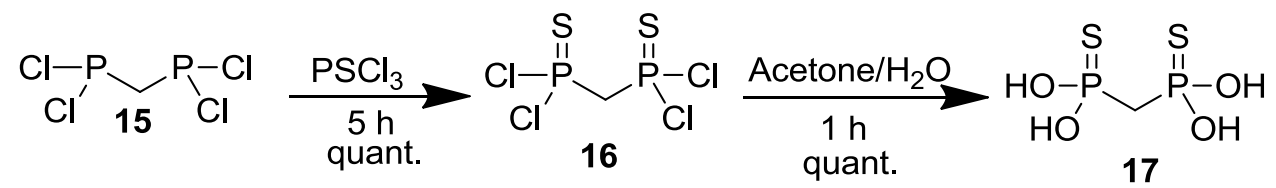

Scheme 4.4

Subsequently, 16 was reacted with aqueous acetone and complete hydrolysis occurred to give methylenediphosphonothioic $O, O$-acid (17). In the ${ }^{1} \mathrm{H}$ NMR spectrum the signal for the methylene hydrogens shifted from $4.48 \mathrm{ppm}$ in $\mathbf{1 6}$ to $2.99 \mathrm{ppm}$ in $\mathbf{1 7}$. The electrospray mass spectrum of $\mathbf{1 7}$ exhibited the correct $[\mathrm{M}+\mathrm{H}]^{+}$peak at $\mathrm{m} / \mathrm{z}, 208.9$. 


\section{Chapter 4}

\subsubsection{Synthesis of tripodal ligands}

Different methods were employed for the synthesis of the tripodal ligands 21 and 24 (Scheme 4.5). For the synthesis of ligand 212 -chloro- $N, N$-dimethylacetamide (18) was reacted with benzylamine in acetonitrile to get $\mathbf{1 9}$ in $81 \%$ yield. The methylene protons next to chlorine shifted from 4.06 in $\mathbf{1 8}$ to 3.74 ppm in $\mathbf{1 9}$ in the ${ }^{1} \mathrm{H}$ NMR spectrum. The benzyl group of $\mathbf{1 9}$ was cleaved by hydrogenation using $10 \% \mathrm{Pd} / \mathrm{C}$ to yield $\mathbf{2 0}$, which was subsequently reacted with 18 to afford ligand 21 in $75 \%$ yield. In the ${ }^{1} \mathrm{H}$ NMR spectrum the methylene protons appeared at $3.62 \mathrm{ppm}$ in $\mathbf{2 1}$. For the synthesis of tripodal ligand $\mathbf{2 3}$ the known triester $\mathbf{2 2}$ was reacted neat with diethanolamine to give the desired ligand 23 in $81 \%$ yield. In the ${ }^{1} \mathrm{H}$ NMR spectrum of 23 the peak for the methoxy groups in 22 at 3.67 ppm completely disappeared and characteristic triplets for the amidic $\mathrm{CH}_{2}$ protons were found at 3.31 and $3.39 \mathrm{ppm}$.

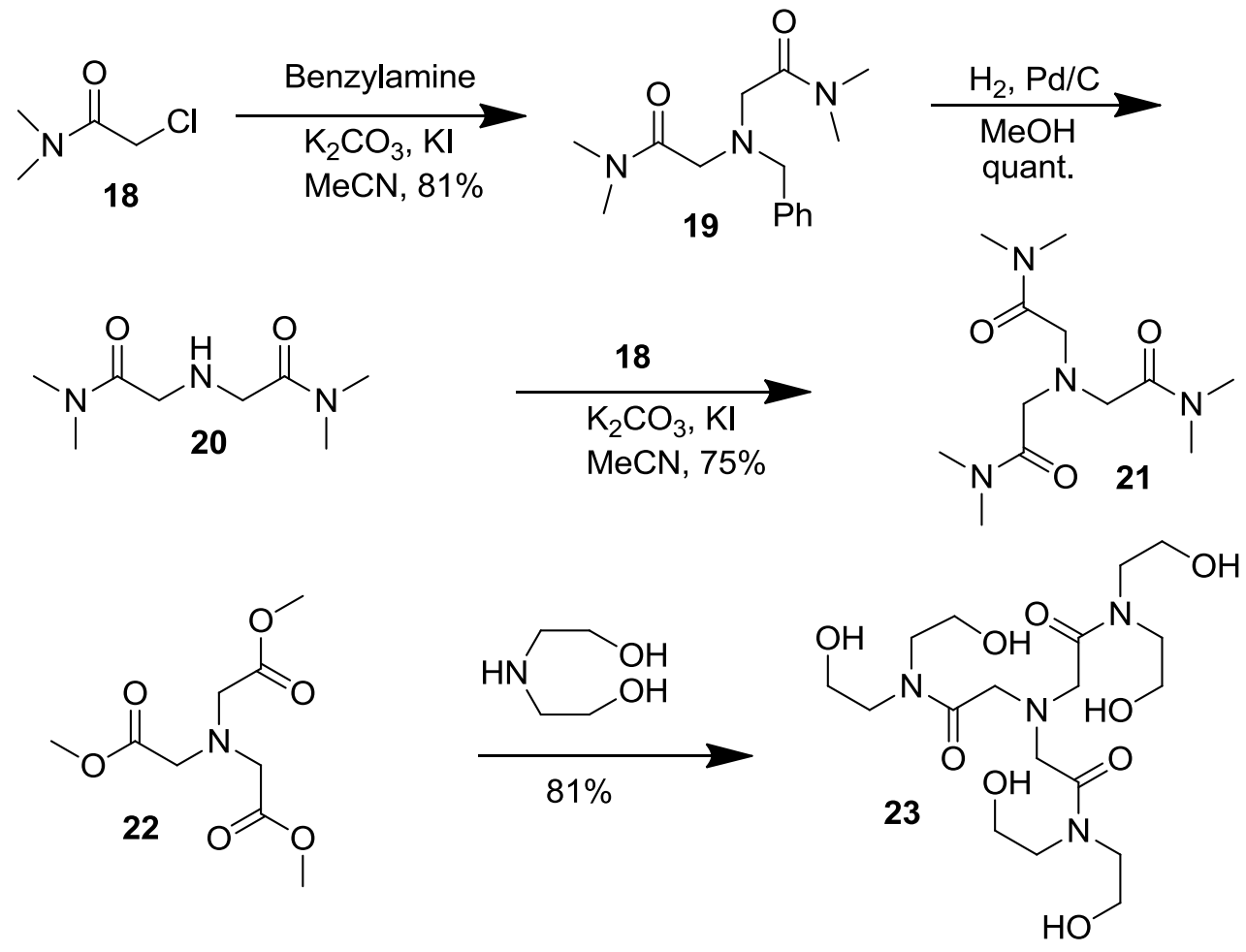

Scheme 4.5

\subsubsection{Extraction results}

Preliminary solvent extraction experiments were carried out to determine the back-extraction ability of the new water-soluble ligands. An organic solution containing TODGA + 5 vol\% 1-octanol in TPH (Total Petroleum Hydrocarbon/Hydrogenated tetrapropene) was used as solvent for the evaluation and comparison of the new ligands. 
A certain concentration of the ligand was dissolved in an aqueous $\mathrm{NH}_{4} \mathrm{NO}_{3}(0.5 \mathrm{~mol} / \mathrm{L})$ solution followed by $\mathrm{pH}$ adjustment and addition of traces of $\mathrm{Am}(\mathrm{III})+\mathrm{Eu}(\mathrm{III})$. The nitrate ion was used as salting-out agent to compensate the metal charge, since TODGA extracts metals only as neutral species (solvating extraction mechanism). ${ }^{17,18}$ The metal distribution ratio $D_{\mathrm{M}}$ was calculated according to equation 1 and the percentage of metal ions retained in the water phase after extraction using equation 2 . Therefore, the smaller $D_{\mathrm{M}}$ is, the more efficient the back-extraction is.

$$
\begin{aligned}
& D_{M}=[M]_{\text {org }} /[M]_{a q} \\
& \% M_{e q, a q}=\frac{1}{1+D_{M}} \times 100 \%
\end{aligned}
$$

The separation factor $(S F)$ between $\mathrm{Eu}(\mathrm{III})$ and $\mathrm{Am}(\mathrm{III})$ was calculated using equation 3.

$$
S F_{E u / A m}=D_{E u} / D_{A m}
$$

The higher the $S F_{\mathrm{Eu} / \mathrm{Am}}$ is, the better the selectivity of the water-soluble ligand for Am(III) versus $\mathrm{Eu}(\mathrm{III})$ is.

\subsubsection{Water-soluble TODGA derived ligands}

The four aliphatic groups of TODGA were substituted by four glycol chains of different length in order to increase its hydrophilicity to give the three new highly water-soluble diglycolamide ligands $\mathbf{2}, \mathbf{7 a}$, and $\mathbf{7 b}$. It can be anticipated that their utility for reverse-extraction experiments is in principle $\mathrm{pH}$ independent. ${ }^{7}$ In contrast, the solubility of (poly)carboxylic acids, such as EDTA, greatly depends on the $\mathrm{HNO}_{3}$ concentration of the aqueous solution.

Since TODGA shows only a slightly higher affinity for $\operatorname{Ln}(\mathrm{III})$ than An(III), no significant separation factor between these two element groups was expected from the hydrophilic derivatives studied here. The distribution ratios of ligand 2 for $\mathrm{Am}(\mathrm{III})$ and $\mathrm{Eu}(\mathrm{III})$ as a function of initial $\mathrm{pH}$ are shown in Figure 4.1. For comparison the reference extraction system (TODGA), in the absence of the hydrophilic ligand in the aqueous phase is expressed by the dotted lines. It can be seen that high distribution ratios for $\mathrm{Am}(\mathrm{III})$ and $\mathrm{Eu}(\mathrm{III})$ were obtained and they were not affected by the initial $\mathrm{pH}$ of the aqueous phase due to the salting-out effect of $\mathrm{NO}_{3}{ }^{-}$. The $D$ values for Am were between 34 and 76, whereas higher $D$ values (250-323) were obtained for Eu, resulting in separation factors $S F_{\mathrm{Eu} / \mathrm{Am}}$ between 4.5 and 7.2. 


\section{Chapter 4}

Ligand 2 with the shortest glycol chain at the $N$-amide of the DGA, showed a very strong complexation for ${ }^{241} \mathrm{Am}+{ }^{152} \mathrm{Eu}$ with increasing ligand concentration. At a fixed ligand concentration of $0.1 \mathrm{~mol} / \mathrm{L}$, the extraction of $\mathrm{Am}(\mathrm{III})$ and $\mathrm{Eu}(\mathrm{III})$ was progressively more suppressed with increasing $\mathrm{pH}$ of the aqueous solution (Figure 4.1). Even at $\mathrm{pH}_{\mathrm{ini}}=1$ the distribution ratios $D_{\mathrm{Am}}$ and $D_{\mathrm{Eu}}$ were 0.2 and 0.5 , respectively $\left(D_{\mathrm{M}}<1\right.$ expresses that $>50 \%$ of the metal is retained in the aqueous phase). As expected, no significant separation between $\mathrm{Eu}(\mathrm{III})$ and $\mathrm{Am}(\mathrm{III})$ was observed $\left(S F_{\mathrm{Eu} / \mathrm{Am}}<2\right)$. Moreover, the discrimination between both radionuclides decreased further with increasing $\mathrm{pH}_{\text {ini }}$ (Table 4.1). Decreasing the ligand 2 concentration below $0.1 \mathrm{~mol} / \mathrm{L}$ decreased the metals complexation in the aqueous phase and thus increased the extraction of $\mathrm{Am}(\mathrm{III})$ and $\mathrm{Eu}(\mathrm{III})$ into the organic phase (Figure 4.1). Only at $\mathrm{pH}=4$ and a low concentration of $0.01 \mathrm{~mol} / \mathrm{L}$ ligand 2 proved sufficient to keep most of $\mathrm{Am}(\mathrm{III})$ and $\mathrm{Eu}(\mathrm{III})$ in the aqueous phase. A lower initial $\mathrm{pH}$ resulted in $D$ values $>1(<50 \%$ metal kept in the aqueous phase).

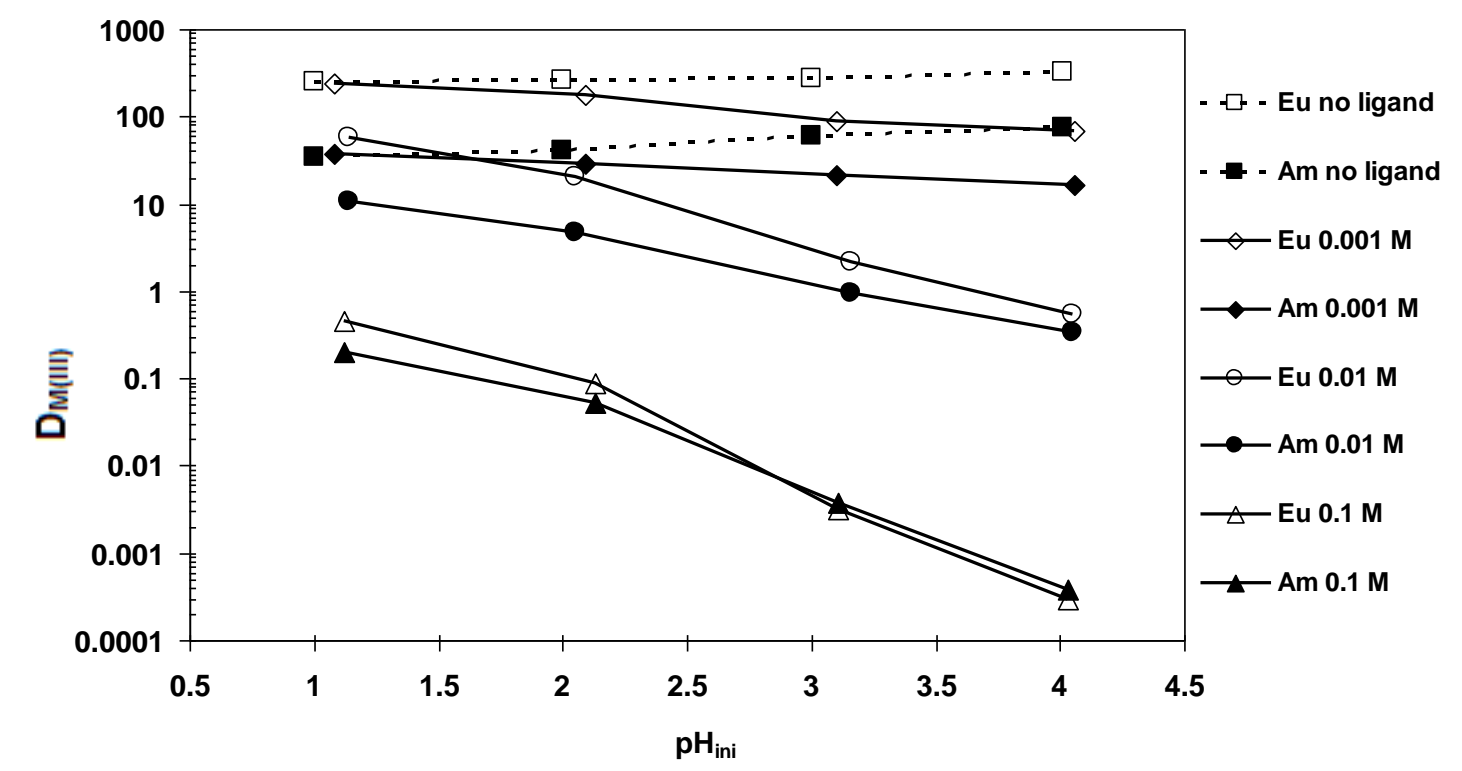

Figure 4.1. Initial $\mathrm{pH}_{\mathrm{aq}}$ and ligand (2) concentration dependency for the extraction of ${ }^{241} \mathrm{Am}$ and ${ }^{152} \mathrm{Eu}$. Organic phase: $0.2 \mathrm{~mol} / \mathrm{L}$ TODGA $+5 \mathrm{vol} \%$ 1-octanol in TPH. Aqueous phase: 0.5 $\mathrm{mol} / \mathrm{L} \mathrm{NH}_{4} \mathrm{NO}_{3}$, variable $\mathrm{pH}_{\text {ini }}$, variable concentrations of ligand 2, tracers: ${ }^{241} \mathrm{Am},{ }^{152} \mathrm{Eu}$, mixing time: $60 \mathrm{~min} ; T=22 \pm 1{ }^{\circ} \mathrm{C}$. 
Table 4.1. Percentages of retained ions in the aqueous phase and Eu/Am separation factors using ligand $2{ }^{a}$

\begin{tabular}{c|c|c|c|c}
\hline $\begin{array}{c}\text { Ligand } \\
\text { conc. } \\
{[\mathrm{mol} / \mathrm{L}]}\end{array}$ & $\mathrm{pH}_{\mathrm{ini}}$ & $\% \mathrm{Am}_{\mathrm{aq}, \mathrm{eq}}$ & $\% \mathrm{Eu}_{\mathrm{aq}, \mathrm{eq}}$ & $S F_{\mathrm{Eu} / \mathrm{Am}}$ \\
\hline & 1 & 2.83 & 0.40 & 7.2 \\
0 & 2 & 2.38 & 0.39 & 6.3 \\
& 3 & 1.64 & 0.37 & 4.5 \\
& 4 & 1.31 & 0.31 & 4.3 \\
\hline & 1 & 83.45 & 68.78 & 2.3 \\
0.1 & 2 & 95.01 & 91.78 & 1.7 \\
& 3 & 99.63 & 99.68 & 0.9 \\
\hline
\end{tabular}

${ }^{a}$ Calculated using eqs 2 and 3 on the distribution ratios from Figure 4.1.

The extraction kinetics of $\mathrm{Am}(\mathrm{III})$ and $\mathrm{Eu}(\mathrm{III})$ by $0.1 \mathrm{~mol} / \mathrm{L}$ ligand 2 at $\mathrm{pH} 3$ with formation of aqueous Am- and Eu-ligand 2 complexes is very fast. Only a mixing time of 5 min was required to suppress the $\mathrm{Am}+\mathrm{Eu}$ extraction by TODGA (Figure 4.2). The equilibrium values reached after $5 \mathrm{~min}$ did not further change up to $60 \mathrm{~min}$ mixing time, indicating the formation of strong and stable metal complexes.

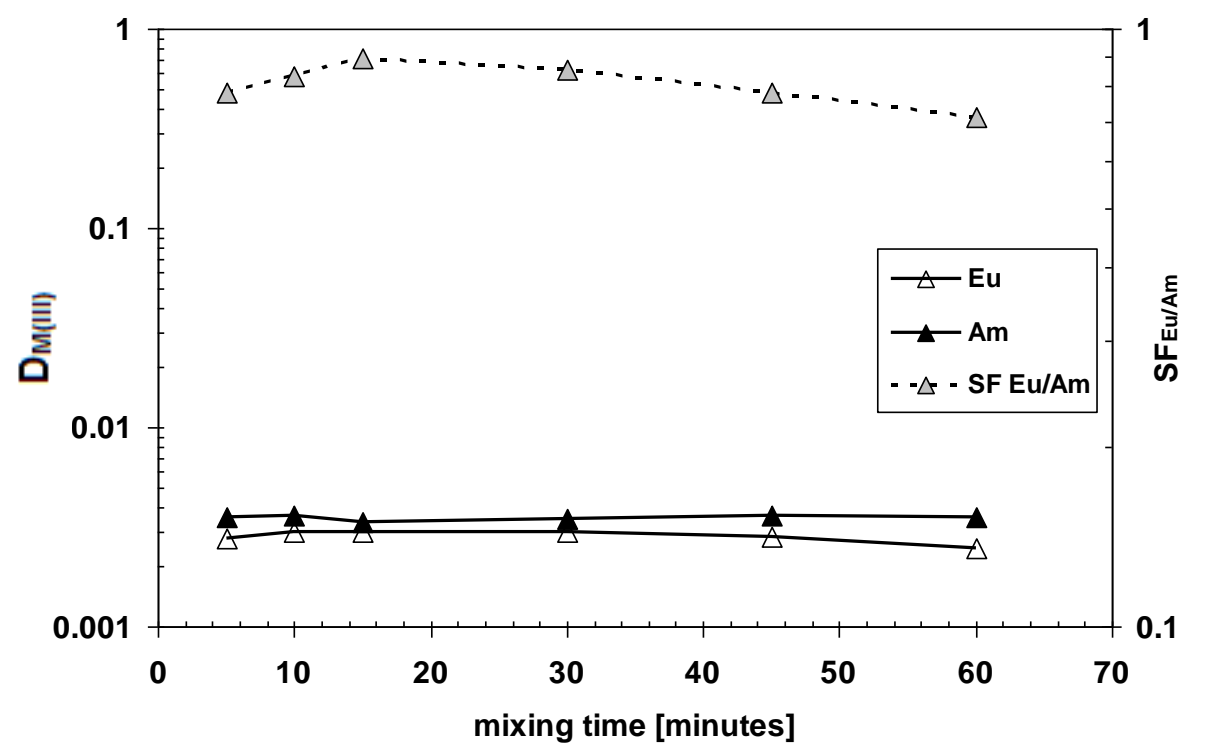

Figure 4.2. Mixing time dependency for the extraction of ${ }^{241} \mathrm{Am}$ and ${ }^{152} \mathrm{Eu}$. Organic phase: $0.2 \mathrm{~mol} / \mathrm{L}$ TODGA $+5 \mathrm{vol} \%$ 1-octanol in TPH. Aqueous phase: $0.5 \mathrm{~mol} / \mathrm{L} \mathrm{NH}_{4} \mathrm{NO}_{3}, 0.1$ $\mathrm{mol} / \mathrm{L}$ ligand (2), $\mathrm{pH}_{\mathrm{ini}}=3$, variable mixing time, tracers: ${ }^{241} \mathrm{Am},{ }^{152} \mathrm{Eu} ; T=22 \pm 1{ }^{\circ} \mathrm{C}$.

Compared to ligand $\mathbf{2}$, the chains of ligands $\mathbf{7 a}$ and $\mathbf{7 b}$ contain one and two more ethylene glycol units, increasing the hydrophilicity. Both new water-soluble DGA ligands $7 \mathbf{a}$ and $\mathbf{7 b}$ do 


\section{Chapter 4}

not complex $\mathrm{Am}(\mathrm{III})$ and $\mathrm{Eu}(\mathrm{III})$ as efficiently as ligand 2 (Figure 4.3). Only at $\mathrm{pH}_{\mathrm{ini}}=4$ ligand 7a kept most of the radionuclides in the aqueous phase $\left(D_{\mathrm{Am}}=0.5\right.$ and $\left.D_{\mathrm{Eu}}=0.92\right)$.

Surprisingly, higher distribution ratios were observed for ligand $\mathbf{7 b}$ in comparison with the TODGA reference extraction system (dotted lines) at $\mathrm{pH}<2$. At $\mathrm{pH} 3$, the values are

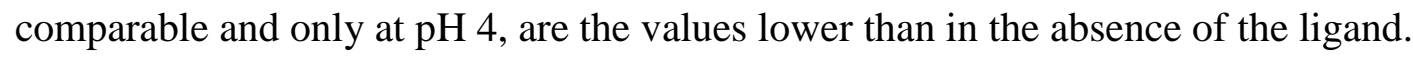

Finally, water-soluble diglycolamide derivative $\mathbf{1 0}$ was tested in which the aliphatic chains connected to the amidic nitrogen of the parent TODGA are substituted by four sodium acetate groups. This ligand was poorly soluble compared to the three ligands with glycol chains and a clear solution was obtained at a ligand concentration of $0.01 \mathrm{~mol} / \mathrm{L}$ in the aqueous phase. The distribution ratios $D_{\mathrm{Am}}$ and $D_{\mathrm{Eu}}$ decreased with increasing $\mathrm{pH}_{\mathrm{ini}}$. However, the complexes formed were not strong enough to prevent the metals extraction (Figure 4.3) by TODGA.

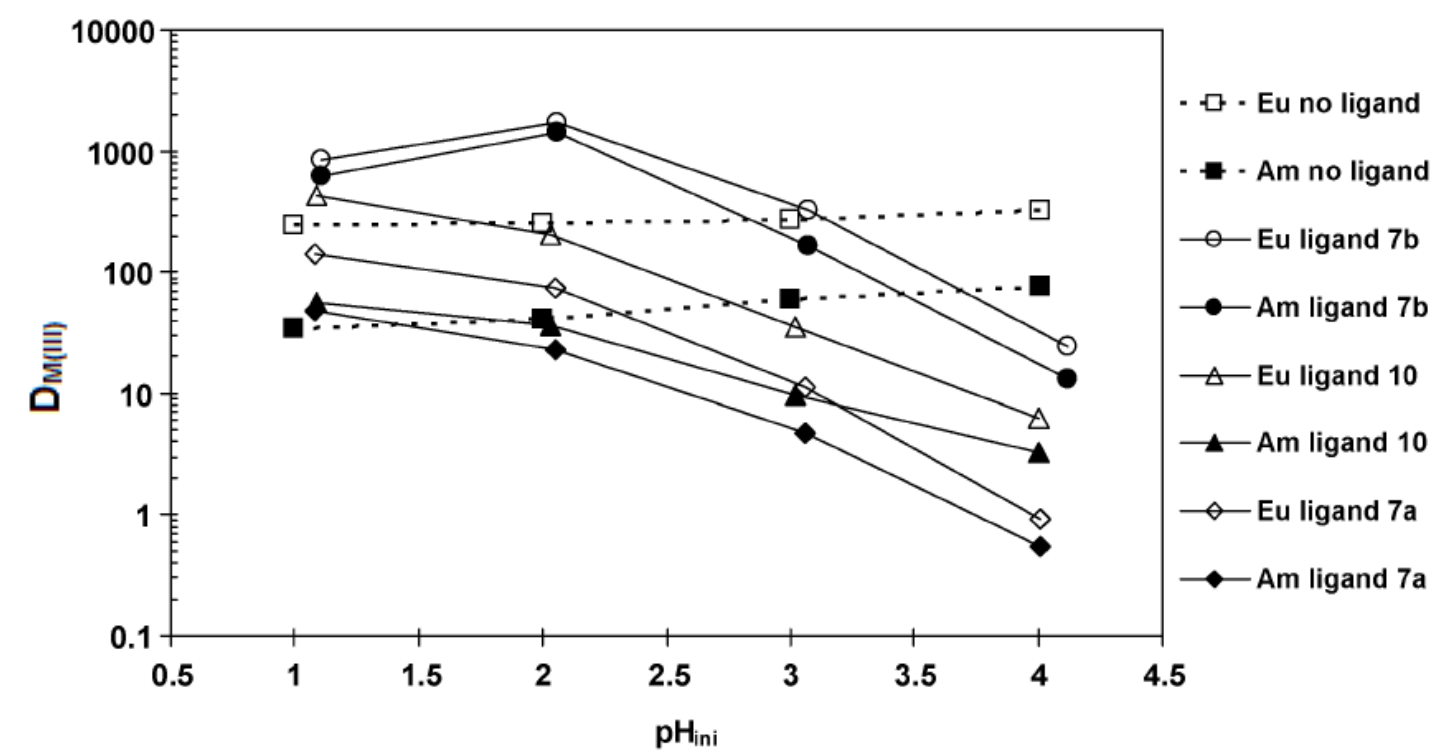

Figure 4.3. Initial $\mathrm{pH}_{\mathrm{aq}}$ dependency for the extraction of ${ }^{241} \mathrm{Am}$ and ${ }^{152} \mathrm{Eu}$. Organic phase: 0.2 $\mathrm{mol} / \mathrm{L}$ TODGA $+5 \mathrm{vol} \%$ 1-octanol in TPH. Aqueous phase: $0.5 \mathrm{~mol} / \mathrm{L} \mathrm{NH}_{4} \mathrm{NO}_{3}$, variable $\mathrm{pH}_{\mathrm{ini}}, 0.1 \mathrm{~mol} / \mathrm{L}$ ligand 7a, 7b $\left(0.01 \mathrm{~mol} / \mathrm{L}\right.$ ligand 10), tracers: ${ }^{241} \mathrm{Am},{ }^{152} \mathrm{Eu}$, mixing time: 60 $\min ; T=22 \pm 1{ }^{\circ} \mathrm{C}$.

Ligand 14, with a diglycolamide backbone, has a poor solubility in $0.5 \mathrm{~mol} / \mathrm{L} \mathrm{NH}_{4} \mathrm{NO}_{3}$ solution. After adjusting the ligand concentration to $0.03 \mathrm{~mol} / \mathrm{L}$, small un-dissolved residues were still visible in the solution. After centrifugation, the clear supernatant was used for the subsequent extraction experiment (see Figure 4.4). The distribution ratios of Am and Eu do not significantly decrease with increasing $\mathrm{pH}_{\text {ini. }}$. There is only a drop between $\mathrm{pH}_{\mathrm{ini}}=1$ and 2 whereas in the initial $\mathrm{pH}$ range of $2-4$, the $D_{\mathrm{Am}}$ and $D_{\mathrm{Eu}}$ values remain nearly constant around 10 and 40, respectively, indicating only poor complexation properties for reverse extraction. 
In addition, no increased complexation for the trivalent Am(III) was observed in comparison to the extraction system without the ligand, since the curves for $\mathrm{Eu} / \mathrm{Am}$ are nearly overlapping.

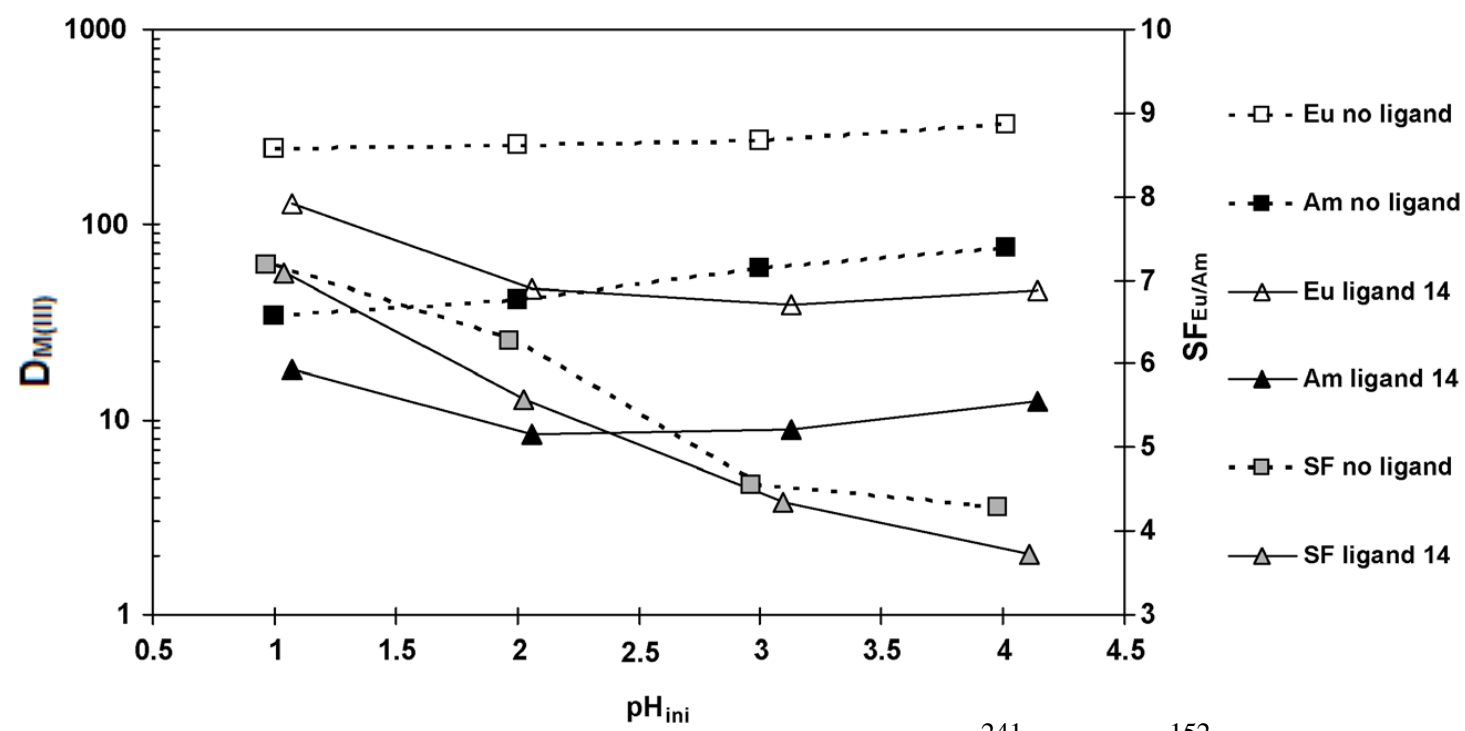

Figure 4.4. Initial $\mathrm{pH}_{\mathrm{aq}}$ dependency for the extraction of ${ }^{241} \mathrm{Am}$ and ${ }^{152} \mathrm{Eu}$. Organic phase: 0.2 $\mathrm{mol} / \mathrm{L}$ TODGA $+5 \mathrm{vol} \%$ 1-octanol in TPH. Aqueous phase: $0.5 \mathrm{~mol} / \mathrm{L} \mathrm{NH}_{4} \mathrm{NO}_{3}$, variable $\mathrm{pH}_{\text {ini }}$, ligand $14<0.03 \mathrm{~mol} / \mathrm{L}$, tracers: ${ }^{241} \mathrm{Am},{ }^{152} \mathrm{Eu}$, mixing time: $60 \mathrm{~min} ; T=22 \pm 1{ }^{\circ} \mathrm{C}$.

\subsubsection{Sulfur containing ligands}

In order to increase the selectivity for the trivalent actinides over the lanthanides two hydrophilic ligands containing sulphur-bearing groups were synthesised to study the impact of S-donors on their complexation properties. Sulfur containing ligand $\mathbf{1 7}$, bearing a malonamide backbone, showed a good solubility and allowed the preparation of a $0.1 \mathrm{~mol} / \mathrm{L}$ solution. At this high ligand concentration, both $\mathrm{Am}(\mathrm{III})$ and $\mathrm{Eu}(\mathrm{III})$ were strongly complexed in the aqueous phase in the entire $\mathrm{pH}_{\mathrm{ini}}$ range studied, thus their extraction by TODGA was significantly prevented (Figure 4.5). Over $90 \%$ of each metal remained in the aqueous phase at $\mathrm{pH}_{\mathrm{ini}}=1$, while over $99 \%$ at $\mathrm{pH}_{\mathrm{ini}}=2-4$ (Table 4.2).

Decreasing the ligand $\mathbf{1 7}$ concentration, the distribution ratios of $\mathrm{Am}(\mathrm{III})$ and $\mathrm{Eu}(\mathrm{III})$ increased, although in comparison with ligand 2 (c.f. Figure 4.1), the S-containing ligand appears to form more stable complexes, especially at lower $\mathrm{pH}_{\mathrm{ini}}$. The separation factor $S F_{\text {Eu/Am }}$ decreased from 2.0 to 0.8 with increasing $\mathrm{pH}_{\text {ini }}$ (Table 4.2). 


\section{Chapter 4}

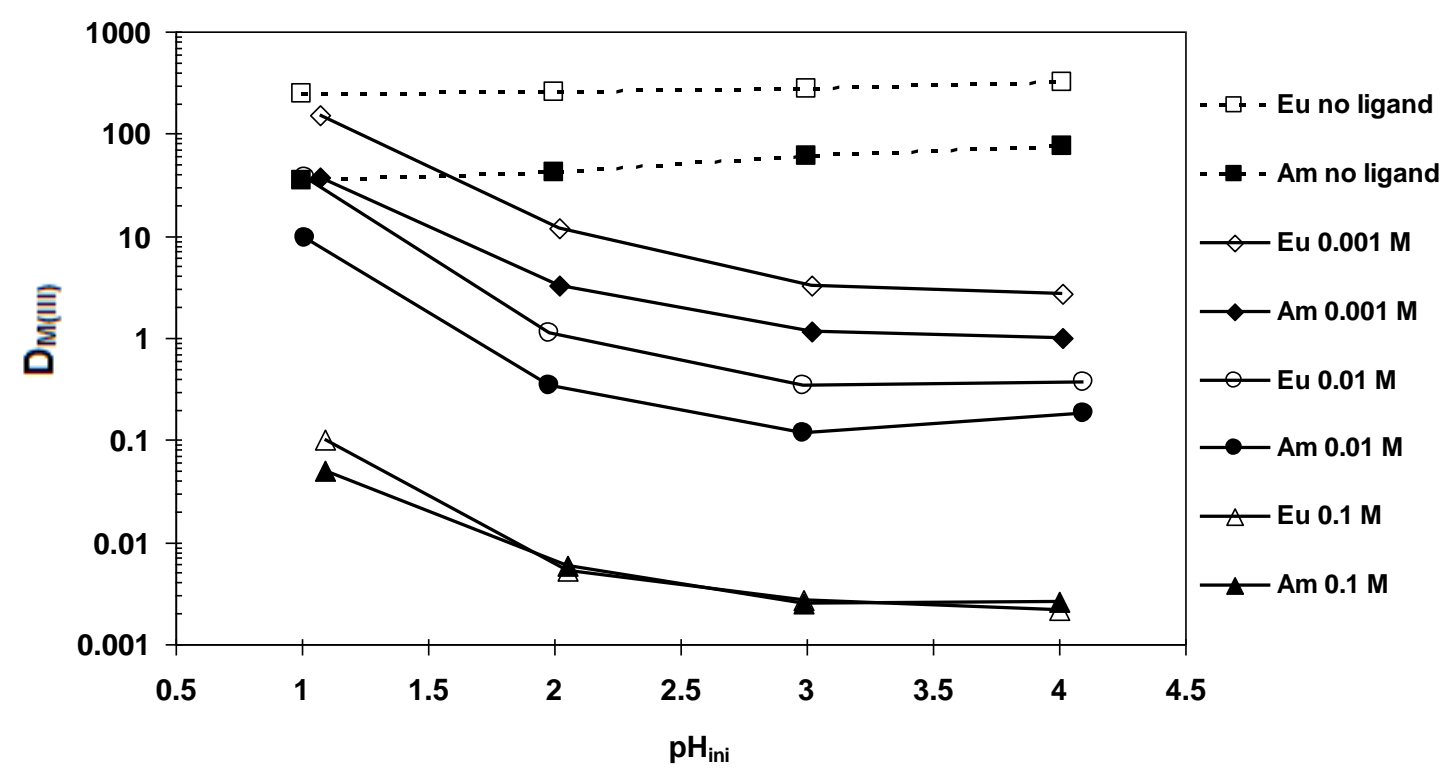

Figure 4.5. Initial $\mathrm{pH}_{\mathrm{aq}}$ and ligand 17 concentration dependency for the extraction of ${ }^{241} \mathrm{Am}$ and ${ }^{152} \mathrm{Eu}$. Organic phase: $0.2 \mathrm{~mol} / \mathrm{L}$ TODGA $+5 \mathrm{vol} \%$ 1-octanol in TPH. Aqueous phase: 0.5 $\mathrm{mol} / \mathrm{L} \mathrm{NH}_{4} \mathrm{NO}_{3}$, variable $\mathrm{pH}_{\text {ini }}$, variable concentration of ligand (17), tracers: ${ }^{241} \mathrm{Am},{ }^{152} \mathrm{Eu}$, mixing time: $60 \mathrm{~min} ; T=22 \pm 1{ }^{\circ} \mathrm{C}$.

Table 4.2. Percentages of retained ions in the aqueous phase and Eu/Am separation factors using ligand $17 .^{a}$

\begin{tabular}{l|l|l|l|l}
\hline $\begin{array}{l}\text { Ligand } \\
\text { conc. } \\
{[\mathrm{mol} / \mathrm{L}]}\end{array}$ & $\mathrm{pH}_{\mathrm{ini}}$ & $\% \mathrm{Am}_{\mathrm{aq}, \mathrm{eq}}$ & $\% \mathrm{Eu}_{\mathrm{aq}, \mathrm{eq}}$ & $\begin{array}{l}S F_{\mathrm{Eu} /} \\
\mathrm{Am}\end{array}$ \\
\hline & 1 & 2.83 & 0.40 & 7.2 \\
0 & 2 & 2.38 & 0.39 & 6.3 \\
& 3 & 1.64 & 0.37 & 4.5 \\
& 4 & 1.31 & 0.31 & 4.3 \\
\hline \multirow{3}{*}{0.1} & 1 & 95.16 & 90.70 & 2.0 \\
& 2 & 99.42 & 99.47 & 0.9 \\
& 3 & 99.75 & 99.73 & 1.0 \\
& 4 & 99.74 & 99.78 & 0.8 \\
\hline
\end{tabular}

${ }^{a}$ Calculated using eqs 2 and 3 on the distribution ratios from Figure 4.5.

Ligand 17 exhibits fast complexation kinetics and distribution ratios close to the equilibrium value are reached within 5 minutes of mixing time (Figure 4.6). Longer mixing times up to 60 minutes did not show any change, indicating the formation of strong and stable metal complexes. 


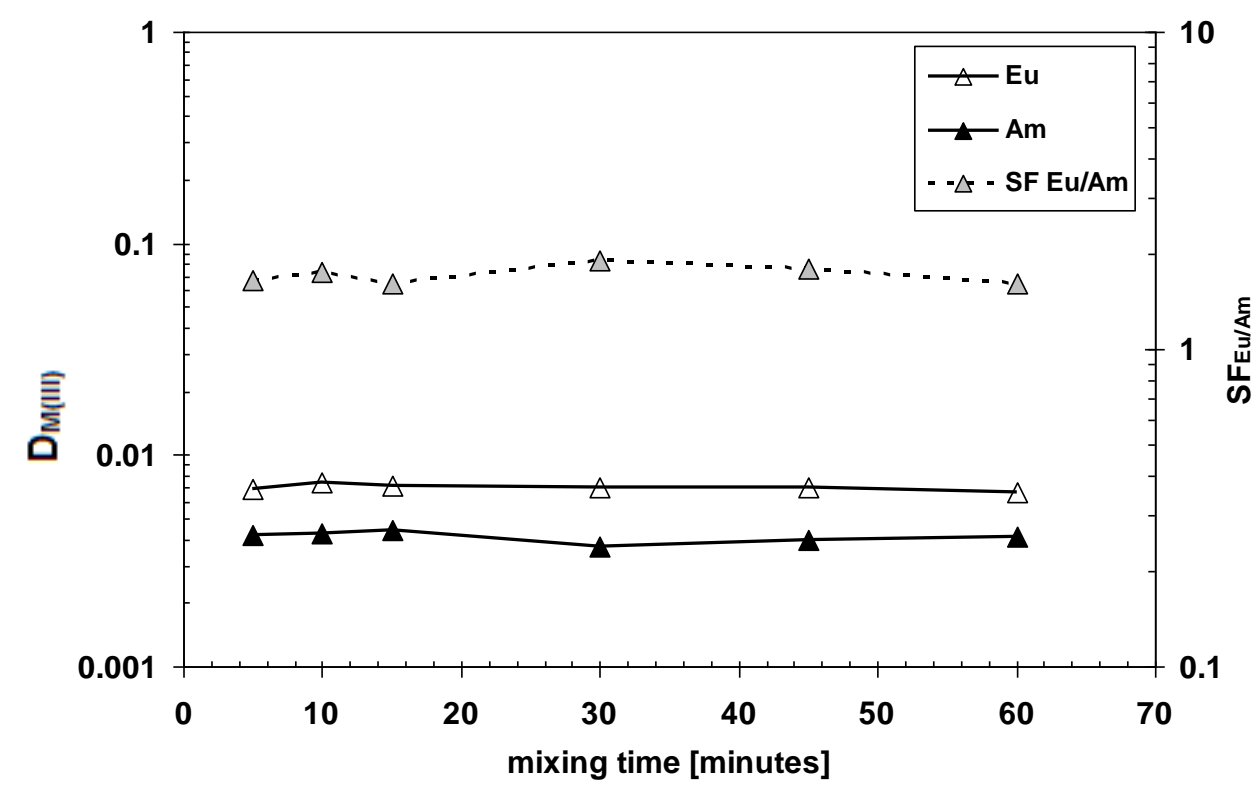

Figure 4.6. Mixing time dependency for the extraction of ${ }^{241} \mathrm{Am}$ and ${ }^{152} \mathrm{Eu}$. Organic phase: $0.2 \mathrm{~mol} / \mathrm{L}$ TODGA $+5 \mathrm{vol} \%$ 1-octanol in TPH. Aqueous phase: $0.5 \mathrm{~mol} / \mathrm{L} \mathrm{NH}_{4} \mathrm{NO}_{3}, 0.1$ $\mathrm{mol} / \mathrm{L}$ ligand 17, $\mathrm{pH}_{\mathrm{ini}}=4$, variable mixing time, tracers: ${ }^{241} \mathrm{Am},{ }^{152} \mathrm{Eu} ; T=22 \pm 1{ }^{\circ} \mathrm{C}$.

\subsubsection{Tripodal ligands}

The extraction results for the new tripodal ligands $\mathbf{2 1}$ and $\mathbf{2 3}$ are shown in Figures 4.7 and 4.8, respectively. The $S F_{\mathrm{Eu} / \mathrm{Am}}$ for ligand 21 decreases upon raising the $\mathrm{pH}_{\mathrm{ini}}$ of the aqueous phase. However, a higher selectivity for $\mathrm{Eu}(\mathrm{III})$ over $\mathrm{Am}(\mathrm{III})$ was obtained at a $\mathrm{pH}_{\text {ini }}$ range of 1-3 compared to the $S F_{\mathrm{Eu} / \mathrm{Am}}$ of the TODGA system without ligand (dotted line in Figure 4.7). The $S F_{\mathrm{Eu} / \mathrm{Am}}=11.1$ (ligand 21 at $\mathrm{pH}_{\mathrm{ini}}=2$ ) is comparable with the $S F_{\mathrm{Am} / \mathrm{Eu}}=11.8$ obtained for lipophilic ligand 2,2',2"'-nitrilotris( $N, N$-bis(2-ethylhexyl)acetamide) (24; see Chart 4.1)) at $\left[\mathrm{HNO}_{3}\right]=0.01 \mathrm{~mol} / \mathrm{L} .12$ At these conditions $72 \%$ of ${ }^{241} \mathrm{Am}$ and only $19 \%$ of ${ }^{152} \mathrm{Eu}$ remained in the aqueous phase. Over 98 and 92 percent of radionuclides were not extracted by TODGA at the highest tested $\mathrm{pH}_{\mathrm{ini}}=4\left({ }^{241} \mathrm{Am}\right.$ and ${ }^{152} \mathrm{Eu}$, respectively $)$. 


\section{Chapter 4}

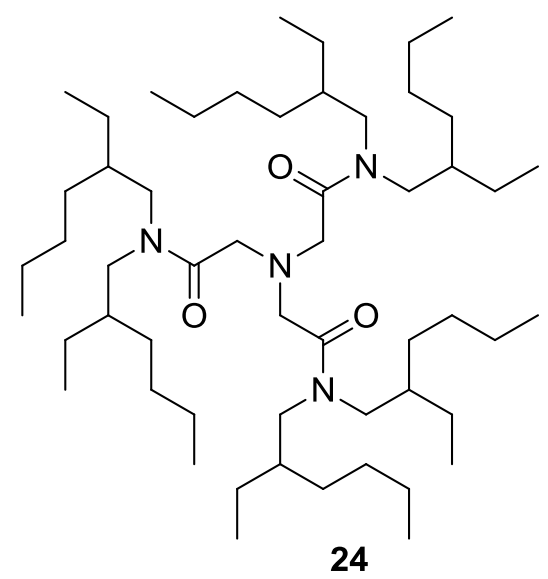

Chart 4.1. Structure of 2,2',2"'-nitrilotris( $N, N$-bis(2-ethylhexyl)acetamide)

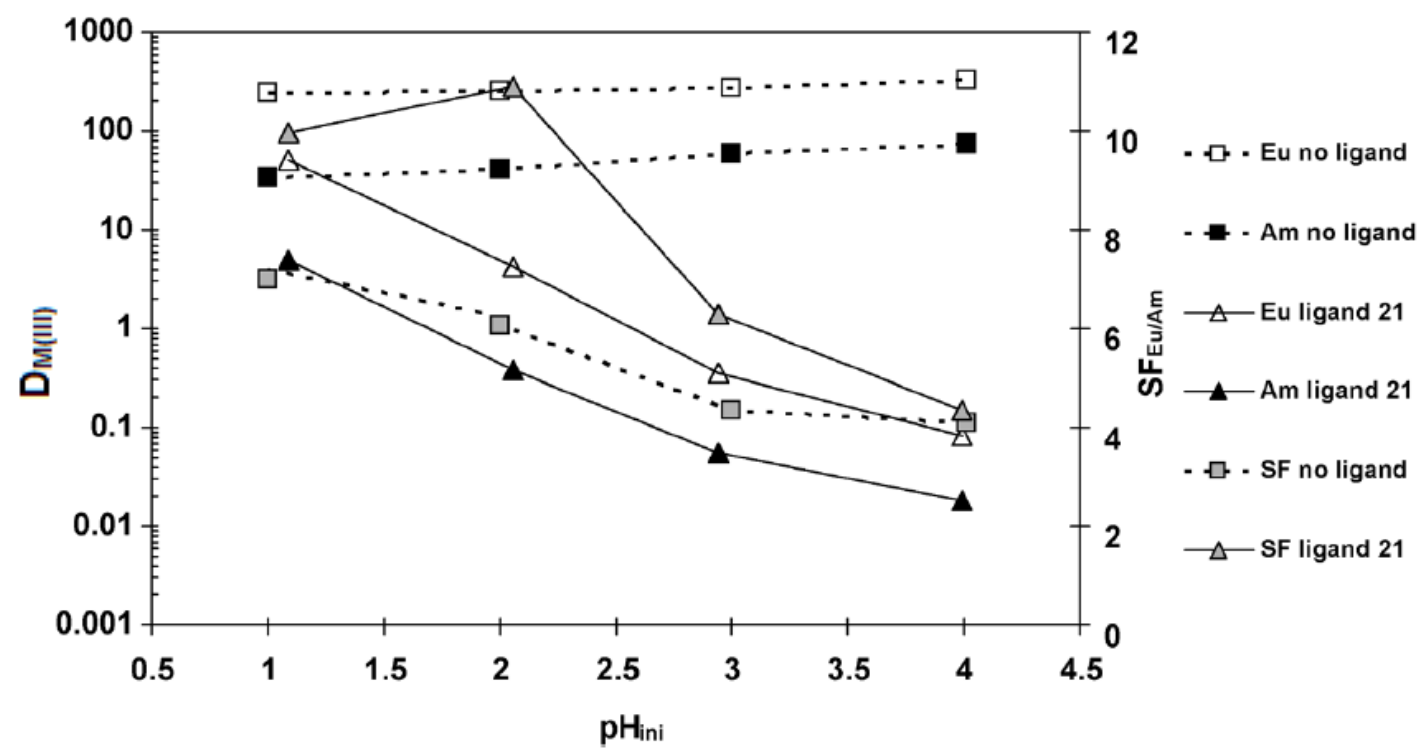

Figure 4.7. Initial $\mathrm{pH}_{\mathrm{aq}}$ dependency for the extraction of ${ }^{241} \mathrm{Am}$ and ${ }^{152} \mathrm{Eu}$. Organic phase: 0.2 $\mathrm{mol} / \mathrm{L}$ TODGA + 5 vol\% 1 -octanol in TPH. Aqueous phase: $0.5 \mathrm{~mol} / \mathrm{L} \mathrm{NH}_{4} \mathrm{NO}_{3}$, variable $\mathrm{pH}_{\text {ini }}, 0.07 \mathrm{~mol} / \mathrm{L}$ ligand 21, tracers: ${ }^{241} \mathrm{Am},{ }^{152} \mathrm{Eu}$, mixing time: $60 \mathrm{~min} ; T=22 \pm 1{ }^{\circ} \mathrm{C}$.

The results of the tripodal ligand $\mathbf{2 3}$ reveal a similar complexing behavior for ligand $\mathbf{2 1}$. Increase of the initial $\mathrm{pH}$ of the aqueous phase increased the metals complexation (Figure 4.8), however, low distribution ratios indicate that more metals are retained in the aqueous phase compared to ligand $\mathbf{2 1}$ for $\mathrm{pH}_{\mathrm{ini}}=3$ and 4 . This can be partly explained by the different concentrations of the two ligands, resulting from the poorer solubility of ligand 21.

The Eu/Am separation factors were almost the same as those of TODGA in the absence of the ligand: the $S F_{\mathrm{Eu} / \mathrm{Am}}$ at $\mathrm{pH}_{\mathrm{ini}}=1$ and 2 is actually even lower than in the reference experiment (dotted line). In conclusion, ligand 21 shows a good discrimination for Am(III), while ligand 23 is more efficient in stripping of $\mathrm{Am}(\mathrm{III})$ and Eu(III) simultaneously. 


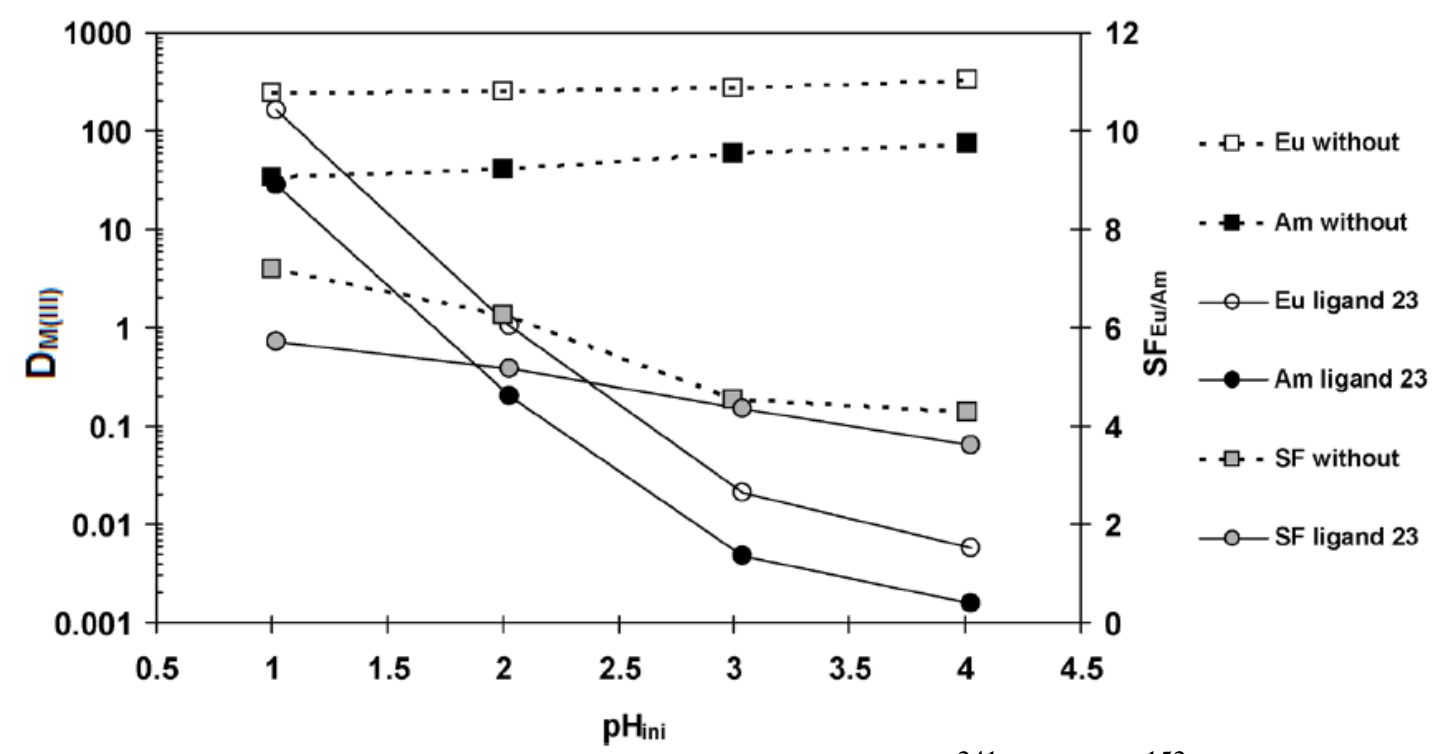

Figure 4.8. Initial $\mathrm{pH}_{\mathrm{aq}}$ dependency for the extraction of ${ }^{241} \mathrm{Am}$ and ${ }^{152} \mathrm{Eu}$. Organic phase: 0.2 mol/L TODGA + 5 vol\% 1-octanol in TPH. Aqueous phase: $0.5 \mathrm{~mol} / \mathrm{L} \mathrm{NH}_{4} \mathrm{NO}_{3}$, variable $\mathrm{pH}_{\text {ini }}, 0.1 \mathrm{~mol} / \mathrm{L}$ ligand 23, tracers: ${ }^{241} \mathrm{Am},{ }^{152} \mathrm{Eu}$, mixing time: $60 \mathrm{~min} ; T=22 \pm 1{ }^{\circ} \mathrm{C}$.

\subsubsection{Microcalorimetry}

Isothermal microcalorimetry (ITC) experiments were performed to study the difference in the binding strengths of ligands $\mathbf{2}, \mathbf{7 a}, \mathbf{b}, \mathbf{2 1}$, and 23 towards $\mathrm{Eu}(\mathrm{III})$ in water at $\mathrm{pH}$. The binding constants and the thermodynamic data are summarized in Table 4.3. Dilution experiments with ligands $\mathbf{2}, \mathbf{7 a}, \mathbf{b}, \mathbf{2 1}$ showed negligible heat effects indicating that no aggregation of the ligands takes place under the conditions used. For ligand $\mathbf{2 3}$ some dilution effect was observed, however after correcting the $\Delta \mathrm{H}$ complexation values for dilution, no significant change was observed in the $\Delta \mathrm{H}$ versus molar ratio plot. 
Table 4.3. Binding constants and thermodynamic parameters of the binding of ligands $\mathbf{2}, \mathbf{7 a}$, $\mathbf{7 b}, \mathbf{2 1}$, and 23 with $\mathrm{Eu}(\mathrm{III})$ determined with microcalorimetry in water at $25^{\circ} \mathrm{C}$.

\begin{tabular}{l|c|c|c|c}
\hline Ligand & \multicolumn{1}{c}{$K, \mathrm{~L} \mathrm{~mol}^{-1}$} & $\Delta G^{o}, \mathrm{kcal} \mathrm{mol}^{-1}$ & $\Delta H^{o}, \mathrm{kcal} \mathrm{mol}^{-1}$ & $T \Delta S^{o}, \mathrm{kcal} \mathrm{mol}^{-1}$ \\
\hline $\mathbf{2}^{b}$ & $6.4 \times 10^{5}$ & $-7.3 \pm 0.7$ & $-1.0 \pm 0.1$ & $6.3 \pm 0.8$ \\
& $7.3 \times 10^{3}$ & $-4.9 \pm 0.4$ & $-4.0 \pm 0.1$ & $0.9 \pm 0.5$ \\
\hline $\mathbf{7 a}$ & $2.2 \times 10^{3}$ & $-4.3 \pm 0.3$ & $-3.6 \pm 0.4$ & $0.7 \pm 0.7$ \\
\hline $\mathbf{7 b}$ & $7.1 \times 10^{2}$ & $-3.9 \pm 0.1$ & $-4.1 \pm 0.2$ & $-0.2 \pm 0.4$ \\
\hline $\mathbf{2 1}$ & $1.4 \times 10^{3}$ & $-4.2 \pm 0.1$ & $4.2 \pm 0.1$ & $8.4 \pm 0.3$ \\
& $3.5 \times 10^{2}$ & $-3.4 \pm 0.1$ & $3.9 \pm 0.1$ & $7.3 \pm 0.2$ \\
\hline $\mathbf{2 3}$ & $1.5 \times 10^{4}$ & $-5.5 \pm 0.2$ & $3.6 \pm 0.3$ & $9.1 \pm 0.5$ \\
\hline
\end{tabular}

${ }^{a}$ Ligand conc. $=10 \mathrm{mmol} / \mathrm{L}, \mathrm{Eu}(\mathrm{III})$ conc. $=0.166 \mathrm{mmol} / \mathrm{L} .{ }^{b}$ Ligands 2 and 23 show the best fit for 2:1 (L:M) complexation, so the thermodynamic parameters are given for both the 1:1 (first row) and 2:1 (second row) complexes.

From Table 4.3 it is clear that ligands $\mathbf{2}, \mathbf{7 a}$, and $\mathbf{7 b}$ show a complexation affinity towards $\mathrm{Eu}(\mathrm{III})$ in the order $\mathbf{2}>\mathbf{7 a}>\mathbf{7 b}$. The stripping efficiency in the back-extraction experiments described above can be explained by the binding efficiency of the different ligands towards $\mathrm{Eu}(\mathrm{III})$. Ligand $\mathbf{2}$ is the most efficient ligand as follows from both the extraction results as well as the ITC experiments. Ligand 2 shows an already sufficiently good fit for 2:1 (ligand:metal) complexation, however, ligands $\mathbf{7 a}$ and $\mathbf{7 b}$ show an already sufficiently good fit for a 1:1 stoichiometry, probably due to the lower affinity observed for these ligands. More importantly and most noticeably, the 1:1 complex of ligand $\mathbf{2}$ shows a relatively low binding enthalpy compared to ligands $\mathbf{7 a}$ and $\mathbf{7 b}$, which is fully compensated by a strongly positive entropy of binding for ligand $\mathbf{2}$. This is partially attributed to co-coordination of the hydroxyl groups of ligand $\mathbf{2}$ to the Eu center (associated with a negative enthalpy and a positive entropy of complexation owing to release of first coordination sphere water molecules) and partially to more negative entropies of binding for ligands $\mathbf{7 a}$ and $\mathbf{7 b}$ due to restricted degrees of conformational freedom of the ethylene glycol chains upon complexation. Ligand $\mathbf{7 b}$ has a lower $K$-value than ligand 7a, which apparently is not high enough for back-extraction (vide supra). The longer glycol chains on the amidic nitrogens in $\mathbf{7 b}$ compared to $\mathbf{7 a}$ weaken the binding capacity of the ligand. A similar effect was observed by Rao et al. ${ }^{19}$ for malonamide ligands by changing the substituents on the amidic nitrogen in acetonitrile. The ITC 92 
experiments performed here indicate that the poor performance of ligand $\mathbf{7 b}$ is due to a more negative entropy effect of binding for ligand $\mathbf{7 b}$ compared to $\mathbf{7 a}$.

The tripodal ligands $\mathbf{2 1}$ and $\mathbf{2 3}$ show the best fit for 2:1 and 1:1 (ligand:metal) complexation, respectively, in an endothermic process. ${ }^{20}$ Ligand $\mathbf{2 3}$ has a stronger binding affinity for Eu(III) than ligand 21 as clearly corresponds with the back extraction data (vide supra). The ITC data indicate that ligand $\mathbf{2 1}$ binds $\mathrm{Eu}$ in a 2:1 fashion, which is logical because of its tetradentate nature. The fact that ligand $\mathbf{2 3}$ binds more strongly but only shows a 1:1 stoichiometry indicates that, in this case, several of the ethylene glycol moieties are involved in binding. This is also evidenced by the more endothermic enthalpy of binding and more positive entropy of binding, both of which are in agreement with a higher loss of water from the first coordination sphere.

\subsection{Conclusions}

Different types of water-soluble ligands, partly based on the well-known TODGA and malonamide skeletons, have successfully been prepared. In most cases they show very good back-extraction properties of $\mathrm{Am}$ (III) and $\mathrm{Eu}$ (III) from a TODGA-containing organic phase, (dependent on the $\mathrm{pH}$ ), however, without selectivity. Only water-soluble tripodal ligands 21 and 23 exhibit a promising $S F_{\mathrm{Eu} / \mathrm{Am}}$ of 11.1 and 5, respectively. It is clearly demonstrated that microcalorimetry is an efficient method to determine the (trend in the) extraction efficiency of different ligands.

\subsection{Experimental}

\subsubsection{General}

All moisture-sensitive reactions were carried out under an argon atmosphere. The solvents and all reagents were obtained from commercial sources and used without further purification. All known compounds viz. $\mathbf{1}^{21}, \mathbf{1 1}^{15}, \mathbf{1 8}^{22}$, and $\mathbf{2 2}^{23}$ were prepared according to literature procedures. Solvents were dried according to standard procedures and stored over molecular sieves. ${ }^{1} \mathrm{H}$ NMR and ${ }^{13} \mathrm{C}$ NMR spectra were recorded on a Varian Unity INOVA (300 MHz) spectrometer in $\mathrm{CDCl}_{3}$ unless stated otherwise. ${ }^{1} \mathrm{H}$ NMR $(300 \mathrm{MHz})$ and ${ }^{13} \mathrm{C}$ NMR $(75 \mathrm{MHz})$ chemical shift values are reported as $\delta$ using the residual solvent signal as an internal standard. The IR spectrum was recorded on a Perkin Elmer Spectrum BX2 spectrometer. Electrospray Ionization (positive mode) mass spectra and high resolution mass spectra were recorded on a WATERS LCT mass spectrometer. Elemental analyses were performed using a 


\section{Chapter 4}

Flash $200 \mathrm{CHN}$ analyzer of Thermo Scientific/Interscience. The extraction experiments were performed at the Institute of Energy and Climate Research, Jülich, Germany. Analytical TLC was performed using Merck prepared plates (silica gel 60 F-254 on aluminum). Column chromatography was carried out on Merck silica gel 60 (230-400 mesh).

\section{2,2'-Oxybis(N,N-bis(2-hydroxyethyl)acetamide) (2).}

To a solution of diethanolamine $(0.86 \mathrm{~g}, 8.2 \mathrm{mmol})$ in acetone $(50 \mathrm{~mL})$ containing $\mathrm{K}_{2} \mathrm{CO}_{3}$ (2.26 g, $16.4 \mathrm{mmol})$ was added diglycolyl dichloride (1) $(0.68 \mathrm{~g}, 3.9 \mathrm{mmol})$ dropwise at $0{ }^{\circ} \mathrm{C}$ and the resulting mixture was stirred overnight at room temperature, filtered and the solvent was evaporated. The residue was dissolved several times in $\mathrm{CHCl}_{3}(25 \mathrm{~mL})$ and decanted to remove the excess of diethanolamine to afford $2(0.85 \mathrm{~g}, 67 \%)$ as an oil. ${ }^{1} \mathrm{H} \operatorname{NMR}\left(\mathrm{D}_{2} \mathrm{O}\right): \delta$ 3.28 and $3.36\left(4 \mathrm{H}, \mathrm{t}, J=6.0 \mathrm{~Hz}, \mathrm{NCH}_{2}\right), 3.56\left(8 \mathrm{H}, \mathrm{m}, \mathrm{CH}_{2} \mathrm{OH}\right), 4.29\left(4 \mathrm{H}, \mathrm{s}, \mathrm{OCH}_{2}\right) ;{ }^{13} \mathrm{C}$ $\mathrm{NMR}\left(\mathrm{D}_{2} \mathrm{O}\right): \delta 48.9,58.6,68.5,171.7 ; \mathrm{m} / z \quad 309.2[\mathrm{M}+\mathrm{H}]^{+}$. HRMS: $331.1418[\mathrm{M}+\mathrm{Na}]^{+}$; calculated: 331.1481 for $\mathrm{C}_{12} \mathrm{H}_{24} \mathrm{NaN}_{2} \mathrm{O}_{7}$.

\section{2,2'-(((Benzylazanediyl)bis(ethane-2,1-diyl))bis(oxy))diethanol (5a). ${ }^{24}$}

A mixture of 2-(2-chloroethoxy)ethanol (4a) (2.00 g, $16 \mathrm{mmol})$, benzylamine (0.86 g, 8.0 $\mathrm{mmol}), \mathrm{K}_{2} \mathrm{CO}_{3}(4 \mathrm{~g}, 29 \mathrm{mmol})$, and $\mathrm{KI}(2.5 \mathrm{~g})$ in DMF $(30 \mathrm{~mL})$ was heated at $110{ }^{\circ} \mathrm{C}$ overnight. The solvent was evaporated and the residue was dissolved in ethyl acetate $(50 \mathrm{~mL})$ and filtered. The filtrate was washed with $5 \% \mathrm{HCl}(2 \times 30 \mathrm{~mL})$ and water $(2 \times 30 \mathrm{~mL})$, dried over anhydrous $\mathrm{MgSO}_{4}$, and concentrated under reduced pressure to give 5a $(2.0 \mathrm{~g}, 77 \%)$ as an oil. ${ }^{1} \mathrm{H}$ NMR: $\delta$ 2.76-2.86 (4H, m, N( $\left.\left.\mathrm{CH}_{2}\right)_{2}\right), 3.56$ and $3.64\left(4 \mathrm{H}, \mathrm{t}, J=6.0 \mathrm{~Hz}, \mathrm{OCH}_{2}\right), 3.70$ $\left(4 \mathrm{H}, \mathrm{t}, J=6.0 \mathrm{~Hz}, \mathrm{CH}_{2} \mathrm{OH}\right), 3.83\left(2 \mathrm{H}, \mathrm{s}, \mathrm{NCH}_{2} \mathrm{Ph}\right), 7.27-7.45(5 \mathrm{H}, \mathrm{m}, \mathrm{ArH}) ; \mathrm{m} / z \quad 283.9$ $[\mathrm{M}+\mathrm{H}]^{+}$.

\section{9-Benzyl-3,6,12,15-tetraoxa-9-azaheptadecane-1,17-diol (5b). ${ }^{25}$}

It was synthesized by a similar procedure as described for 5a. 2-(2-(2Chloroethoxy)ethoxy)ethanol (4b) $(2.50 \mathrm{~g}, 14.4 \mathrm{mmol})$, benzylamine (0.82 g, $7.7 \mathrm{mmol})$, $\mathrm{K}_{2} \mathrm{CO}_{3}(4 \mathrm{~g}, 29 \mathrm{mmol})$ and $\mathrm{KI}(2.5 \mathrm{~g})$ afforded $\mathbf{5 b}(2.14 \mathrm{~g}, 75 \%)$ as an oil. ${ }^{1} \mathrm{H}$ NMR: $\delta 2.90$ $\left(4 \mathrm{H}, \mathrm{t}, J=6.0 \mathrm{~Hz}, \mathrm{~N}\left(\mathrm{CH}_{2}\right)_{2}\right), 3.50-3.84\left(22 \mathrm{H}, \mathrm{m}, \mathrm{OCH}_{2}\right.$ and $\left.\mathrm{NCH}_{2} \mathrm{Ph}\right), 7.20-7.36(5 \mathrm{H}, \mathrm{m}$, ArH). ${ }^{13} \mathrm{C}$ NMR: $\delta$ 50.7, 56.1, 57.9, 66.2, 67.0, 69.5, 122.2, 125.1, 126.0, 135.7; $\mathrm{m} / \mathrm{z} 372.2$ $[\mathrm{M}+\mathrm{H}]^{+}$. HRMS: $m / z 372.2046[\mathrm{M}+\mathrm{H}]^{+}$; calculated: 372.2386 for $\mathrm{C}_{19} \mathrm{H}_{34} \mathrm{NO}_{6}$. 


\section{2,2'-((Azanediylbis(ethane-2,1-diyl))bis(oxy))diethanol (6a).}

A solution of $5 \mathbf{a}(1.70 \mathrm{~g}, 6.0 \mathrm{mmol})$ in methanol $(30 \mathrm{~mL})$ in the presence of $10 \% \mathrm{Pd} / \mathrm{C}(0.5 \mathrm{~g})$ was kept under $\mathrm{H}_{2}$ atmosphere overnight. After removal of the catalyst by filtration the solvent was evaporated under reduced pressure to afford $6 \mathbf{a}(1.03 \mathrm{~g}, 93 \%)$ as an oil. ${ }^{1} \mathrm{H}$ NMR: $\delta 2.83\left(4 \mathrm{H}, \mathrm{t}, J=6.0 \mathrm{~Hz}, \mathrm{~N}\left(\mathrm{CH}_{2}\right)_{2}\right), 3.56-3.63\left(8 \mathrm{H}, \mathrm{m}, \mathrm{OCH}_{2}\right), 3.71(4 \mathrm{H}, \mathrm{t}, J=6.0 \mathrm{~Hz}$, $\left.\mathrm{CH}_{2} \mathrm{OH}\right), 4.04(2 \mathrm{H}$, br s, OH$) ; m / z 194.1[\mathrm{M}+\mathrm{H}]^{+}$.

\section{3,6,12,15-Tetraoxa-9-azaheptadecane-1,17-diol (6b). ${ }^{26}$}

It was synthesized by a similar procedure as described for $\mathbf{6 a}$. Starting from $\mathbf{5 b}(1.80 \mathrm{~g}, 4.8$ $\mathrm{mmol})$ and $10 \% \mathrm{Pd} / \mathrm{C}(0.5 \mathrm{~g})$ gave $6 \mathbf{b}(1.18 \mathrm{~g}, 87 \%)$ as an oil. ${ }^{1} \mathrm{H}$ NMR: $\delta 2.89(4 \mathrm{H}, \mathrm{t}, J=6.0$ $\left.\mathrm{Hz}, \mathrm{N}\left(\mathrm{CH}_{2}\right)_{2}\right), 3.56-3.74\left(20 \mathrm{H}, \mathrm{m}, \mathrm{OCH}_{2}\right), 4.09(2 \mathrm{H}$, br s, OH$) ; m / z 282.1[\mathrm{M}+\mathrm{H}]^{+} ; \mathrm{HRMS}$ : 282.1928 [M+H] $]^{+}$; calculated: 282.1917 for $\mathrm{C}_{12} \mathrm{H}_{27} \mathrm{NO}_{6}$.

\section{2,2'-Oxybis(N,N-bis(2-(2-hydroxyethoxy)ethyl)acetamide) (7a).}

The same procedure was adopted as described for 2 . Starting from $\mathbf{6 a}(0.80 \mathrm{~g}, 4.1 \mathrm{mmol})$, diglycolyl dichloride (1) $(0.35 \mathrm{~g}, 2.1 \mathrm{mmol})$, and $\mathrm{K}_{2} \mathrm{CO}_{3}(1.1 \mathrm{~g}, 8 \mathrm{mmol})$ afforded $7 \mathbf{a}(0.61 \mathrm{~g}$, $62 \%) .{ }^{1} \mathrm{H} \operatorname{NMR}\left(\mathrm{D}_{2} \mathrm{O}\right): \delta 3.37\left(4 \mathrm{H}, \mathrm{t}, J=6.0 \mathrm{~Hz}, \mathrm{CONCH}_{2}\right), 3.42-3.47\left(12 \mathrm{H}, \mathrm{m}, \mathrm{NCH}_{2}\right.$ and $\left.\mathrm{OCH}_{2}\right), 3.49-3.58\left(16 \mathrm{H}, \mathrm{m}, \mathrm{CH}_{2} \mathrm{OH}\right), 4.31\left(4 \mathrm{H}, \mathrm{s}, \mathrm{OCH}_{2}\right) ;{ }^{13} \mathrm{C} \mathrm{NMR}\left(\mathrm{D}_{2} \mathrm{O}\right): \delta 60.6,67.8,68.1$, 68.5, 71.8, 72.2, 171.7; HRMS: $m / z$ 485.2742 $[\mathrm{M}+\mathrm{H}]^{+}$; calculated: 485.2710 for $\mathrm{C}_{20} \mathrm{H}_{41} \mathrm{~N}_{2} \mathrm{O}_{11}$.

\section{2,2'-Oxybis(N,N-bis(2-(2-(2-hydroxyethoxy)ethoxy)ethyl)acetamide) (7b).}

The same procedure was adopted as described for the synthesis of 2 . Starting from $7 \mathbf{a}(0.90 \mathrm{~g}$, $3.2 \mathrm{mmol})$, diglycolyl dichloride (1) $(0.27 \mathrm{~g}, 1.6 \mathrm{mmol})$, and $\mathrm{K}_{2} \mathrm{CO}_{3}(1.0 \mathrm{~g}, 7.2 \mathrm{mmol})$ gave 7b $(0.66 \mathrm{~g}, 63 \%) .{ }^{1} \mathrm{H} \operatorname{NMR}\left(\mathrm{D}_{2} \mathrm{O}\right): \delta 3.36\left(4 \mathrm{H}, \mathrm{t}, J=6.0 \mathrm{~Hz}, \mathrm{NCH}_{2}\right), 3.42-3.47(12 \mathrm{H}, \mathrm{m}$, $\mathrm{NCH}_{2}$ and $\left.\mathrm{CH}_{2} \mathrm{OH}\right), 3.49-3.59\left(32 \mathrm{H}, \mathrm{m}, \mathrm{OCH}_{2}\right), 4.29\left(4 \mathrm{H}, \mathrm{s}, \mathrm{OCH}_{2}\right) ;{ }^{13} \mathrm{C} \mathrm{NMR}\left(\mathrm{D}_{2} \mathrm{O}\right): \delta 60.5$, 67.8, 68.1, 69.7, 70.2, 71.8, 171.5; HRMS: $\mathrm{m} / z 661.3782[\mathrm{M}+\mathrm{H}]^{+}$; calculated: 661.3759 for $\mathrm{C}_{28} \mathrm{H}_{57} \mathrm{~N}_{2} \mathrm{O}_{15}$.

\section{Tetramethyl 2,2',2",2"'"-((2,2'-oxybis(acetyl))bis(azanetriyl))tetraacetate (9).}

To a solution of dimethyl 2,2'-azanediyldiacetate (1.50 g, $9.3 \mathrm{mmol})$ in THF $(50 \mathrm{~mL})$, containing triethylamine $(1.0 \mathrm{~g}, 9.9 \mathrm{mmol})$ as a base was added diglycolyl dichloride (1) (0.79 $\mathrm{g}, 4.6 \mathrm{mmol})$ in THF $(15 \mathrm{~mL})$ dropwise at $0{ }^{\circ} \mathrm{C}$. The mixture was stirred at room temperature 


\section{Chapter 4}

overnight, whereupon the solvent was evaporated. The residue was dissolved in dichloromethane $(50 \mathrm{~mL})$, washed with $10 \% \mathrm{HCl}(2 \times 40 \mathrm{~mL})$, sat. aq. $\mathrm{NaHCO}_{3}(2 \times 40 \mathrm{~mL})$, and water $(50 \mathrm{~mL})$ to afford $9(1.51 \mathrm{~g}, 80 \%)$ as an oil. ${ }^{1} \mathrm{H}$ NMR: $\delta 3.72$ and $3.75(6 \mathrm{H}, \mathrm{s}$, $\mathrm{C}(\mathrm{O}) \mathrm{OMe}), 4.19$ and $4.21\left(4 \mathrm{H}, \mathrm{s}, \mathrm{NCH}_{2}\right), 4.27\left(4 \mathrm{H}, \mathrm{s}, \mathrm{OCH}_{2}\right) ;{ }^{13} \mathrm{C} \mathrm{NMR}: \delta 48.2,49.5,52.4$, 52.5, 52.8, 69.5, 169.5; HRMS: $\mathrm{m} / z, 421.1495[\mathrm{M}+\mathrm{H}]^{+}$; calculated: 421.1458 for $\mathrm{C}_{16} \mathrm{H}_{25} \mathrm{~N}_{2} \mathrm{O}_{11}$.

\section{Sodium 2,2',2",,2'"-((2,2'-oxybis(acetyl))bis(azanetriyl))tetraacetate (10).}

To a solution of 9 (1.00 g, $2.4 \mathrm{mmol})$ in a mixture of $\mathrm{MeOH}$ :water (8:2) (50 mL) was slowly added $\mathrm{NaOH}(0.38 \mathrm{~g}, 9.5 \mathrm{mmol})$ and the mixture was stirred at room temperature for $2 \mathrm{~d}$. The mixture was concentrated to dryness under reduced pressure to afford pure $\mathbf{1 0}$ (1.07 g, quant.) as a white solid. M.p. $284-286{ }^{\circ} \mathrm{C} .{ }^{1} \mathrm{H} \operatorname{NMR}\left(\mathrm{D}_{2} \mathrm{O}\right): \delta 3.67$ and $3.74\left(4 \mathrm{H}, \mathrm{s}, \mathrm{NCH}_{2}\right), 4.18(4 \mathrm{H}$, $\left.\mathrm{s}, \mathrm{OCH}_{2}\right) ;{ }^{13} \mathrm{C} \mathrm{NMR}\left(\mathrm{D}_{2} \mathrm{O}\right): \delta$ 51.6, 68.4, 171.3, 175.7, 176.4; HRMS: $m / z 453.0160[\mathrm{M}+\mathrm{H}]^{+}$; calculated: 453.0110 for $\mathrm{C}_{12} \mathrm{H}_{13} \mathrm{~N}_{2} \mathrm{Na}_{4} \mathrm{O}_{11}$.

\section{0,0,0',0'-Tetraethyl (oxybis(methylene))diphosphonothioate (13).}

To a solution of diethyl (hydroxymethyl)phosphonate $(0.73 \mathrm{~g}, 4.3 \mathrm{mmol}$ ) in THF (40 mL) was slowly added $\mathrm{NaH}$ at $0{ }^{\circ} \mathrm{C}$ portion wise. After stirring at this temperature for $1 \mathrm{~h}$, a solution of (diethoxyphosphoryl)methyl trifluoromethanesulfonate (11) (1.30 g, $4.3 \mathrm{mmol})$ in THF (15 $\mathrm{mL}$ ) was added and stirring was continued overnight at room temperature. The solvent was evaporated under reduced pressure and the residue was dissolved in chloroform $(30 \mathrm{~mL})$. The resulting solution was washed with $10 \% \mathrm{HCl}(3 \times 50 \mathrm{~mL})$ and water $(3 \times 50 \mathrm{~mL})$. The organic layer was dried over anhydrous $\mathrm{MgSO}_{4}$ and concentrated under reduced pressure, followed by purification of the residue with chromatography $\left(\mathrm{SiO}_{2}\right.$, dichloromethane) to afford $12(1.02 \mathrm{~g}$, $74 \%)$ as an oil. ${ }^{1} \mathrm{H}$ NMR: $\delta 1.32\left(12 \mathrm{H}, \mathrm{t}, J=7.0 \mathrm{~Hz}, \mathrm{CH}_{3}\right), 3.85\left(4 \mathrm{H}, \mathrm{d}, J=6.0 \mathrm{~Hz}, \mathrm{OCH}_{2} \mathrm{P}\right)$, $4.16\left(8 \mathrm{H}\right.$, pentet, $\left.J=7.0 \mathrm{~Hz}, \mathrm{OCH}_{2}\right) .{ }^{27}$ A solution of $12(1.00 \mathrm{~g}, 3.1 \mathrm{mmol})$ and Lawesson's reagent $(2.54 \mathrm{~g}, 6.2 \mathrm{mmol})$ was refluxed in toluene for $2 \mathrm{~h}$. After removal of the solvent the residue was separated by chromatography ( $\mathrm{SiO}_{2}$, dichloromethane) to afford $\mathbf{1 3}(0.87 \mathrm{~g}, 80 \%)$ as an oil. ${ }^{1} \mathrm{H}$ NMR: $\delta 1.33\left(12 \mathrm{H}, \mathrm{t}, J=7.0 \mathrm{~Hz}, \mathrm{CH}_{3}\right), 4.10\left(4 \mathrm{H}, \mathrm{d}, J=3.0 \mathrm{~Hz}, \mathrm{OCH}_{2} \mathrm{P}\right), 4.12-$ $4.25\left(8 \mathrm{H}, \mathrm{m}, \mathrm{OCH}_{2}\right) ;{ }^{13} \mathrm{C}$ NMR: $\delta 16.5,63.3,72.5,74.2$; HRMS: $m / z$ 351.0631[M+H] $]^{+}$; calculated: 351.0619 for for $\mathrm{C}_{10} \mathrm{H}_{25} \mathrm{O}_{5} \mathrm{P}_{2} \mathrm{~S}_{2}$. 


\section{(Oxybis(methylene))diphosphonic acid (14).}

A suspension of $13(0.90 \mathrm{~g}, 2.5 \mathrm{mmol})$ in conc. $\mathrm{HCl}(20 \mathrm{~mL})$ was heated in a sealed tube at $100{ }^{\circ} \mathrm{C}$ overnight. The $\mathrm{HCl}$ was evaporated under reduced pressure. The residue was dissolved in water $(25 \mathrm{~mL})$, and the solution was extracted with chloroform $(2 \times 25 \mathrm{~mL})$ to remove nonwater soluble impurities. Removal of the solvent under reduced pressure gave 14 (0.46 g, $70 \%)$ as a dense oil. ${ }^{1} \mathrm{H} \operatorname{NMR}\left(\mathrm{D}_{2} \mathrm{O}\right): \delta 3.62\left(4 \mathrm{H}, \mathrm{d}, J=9.0 \mathrm{~Hz}, \mathrm{OCH}_{2}\right) ;{ }^{13} \mathrm{C} \mathrm{NMR}\left(\mathrm{D}_{2} \mathrm{O}\right): \delta$ 66.9, 69.1; m/z 207.0 [M+H] $]^{+}$; HRMS: m/z $206.9833[\mathrm{M}+\mathrm{H}]^{+}$; calculated: 206.9824 for $\mathrm{C}_{2} \mathrm{H}_{9} \mathrm{O}_{7} \mathrm{P}_{2}$.

\section{Methylenediphosphonothioic dichloride (16).}

A mixture of thiophosphoryl chloride $(15 \mathrm{~mL})$ and bis(dichlorophosphino)methane (1.00 g, $4.5 \mathrm{mmol}$ ) was refluxed for $5 \mathrm{~h}$ at $115^{\circ} \mathrm{C}$. The excess of $\mathrm{PSCl}_{3}$ was evaporated under reduced pressure to afford 16 (1.3 g, quant.). ${ }^{1} \mathrm{H}$ NMR: $\delta 4.48$ (4H, t, $\left.J=15.0 \mathrm{~Hz}, \mathrm{PSCH}_{2} \mathrm{PS}\right)$. In the $\mathrm{ESI}^{+}$mass spectrum a characteristic pattern was observed at $\mathrm{m} / \mathrm{z} 278.8,281.8,283.8,285.8$ for $[\mathrm{M}+\mathrm{H}]^{+}$that corresponds to the calculated isotopic abundance of four chlorides.

\section{Methylenediphosphonothioic 0,0-acid (17).}

A mixture of $16(1.00 \mathrm{~g}, 3.5 \mathrm{mmol})$ in acetone:water $(1: 1)(50 \mathrm{~mL})$ was stirred at room temperature for $1 \mathrm{~h}$. The solvent was evaporated to give pure $\mathbf{1 7}(0.70 \mathrm{~g}$, quant.) as a solid. $\mathrm{mp}$ 131-133 ${ }^{\circ} \mathrm{C}$; Elem. Anal. Calculated for $\mathrm{CH}_{6} \mathrm{O}_{4} \mathrm{P}_{2} \mathrm{~S}_{2}$ : C, 5.8; H, 2.9. found: C, 5.95; H, 2.65; ${ }^{1} \mathrm{H} \operatorname{NMR}\left(\mathrm{CD}_{3} \mathrm{C}(\mathrm{O}) \mathrm{CD}_{3}\right): \delta 2.99\left(2 \mathrm{H}, \mathrm{t}, J=16.5 \mathrm{~Hz}, \mathrm{PSCH}_{2} \mathrm{PS}\right), 7.02(4 \mathrm{H}, \mathrm{br} \mathrm{s}, \mathrm{OH}) ;{ }^{13} \mathrm{C}$ NMR(D $\left.\mathrm{D}_{2} \mathrm{O}\right): \delta$ 57.7; HRMS: $m / z 208.9297[\mathrm{M}+\mathrm{H}]^{+}$; calculated: 208.9261 for $\mathrm{CH}_{7} \mathrm{O}_{4} \mathrm{P}_{2} \mathrm{~S}_{2}$.

\section{2,2'-(Benzylazanediyl)bis(N,N-dimethylacetamide) (19).}

A mixture of 2-chloro- $N, N$-dimethylacetamide (18) $(2.30 \mathrm{~g}, 18.9 \mathrm{mmol})$, benzylamine (1.00 g, $9.5 \mathrm{mmol}), \mathrm{K}_{2} \mathrm{CO}_{3}(5.2 \mathrm{~g}, 38 \mathrm{mmol})$ and $\mathrm{KI}(3 \mathrm{~g})$ in acetonitrile $(50 \mathrm{~mL})$ was refluxed for $6 \mathrm{~h}$. The acetonitrile was evaporated and the residue was dissolved in ethyl acetate $(50 \mathrm{~mL})$. The resulting solution was washed with dil. $\mathrm{HCl}(3 \times 50 \mathrm{~mL})$ and water $(3 \times 50 \mathrm{~mL})$. The organic layer was dried over anhydrous $\mathrm{MgSO}_{4}$ and concentrated under reduced pressure. The residue was purified by chromatography $\left(\mathrm{SiO}_{2}\right.$, EtOAc) to afford $\mathbf{1 9}(4.20 \mathrm{~g}, 81 \%)$ as an oil. ${ }^{1} \mathrm{H}$ NMR: $\delta 2.91$ and $2.95\left(6 \mathrm{H}, \mathrm{s}, \mathrm{NMe}_{2}\right), 3.74\left(4 \mathrm{H}, \mathrm{s}, \mathrm{NCH}_{2}\right), 4.02\left(2 \mathrm{H}, \mathrm{s}, \mathrm{NCH}_{2} \mathrm{Ph}\right) ;{ }^{13} \mathrm{C} \mathrm{NMR}: \delta$ 35.6, 36.9, 55.6, 59.1, 127.5, 128.5, 129.4, 138.3, 170.3; HRMS: $m / z 278.1875[\mathrm{M}+\mathrm{H}]^{+}$; calculated: 278.1869 for $\mathrm{C}_{15} \mathrm{H}_{24} \mathrm{~N}_{3} \mathrm{O}_{2}$. 


\section{2,2'-Azanediylbis(N,N-dimethylacetamide) (20).}

A mixture of $19(1.20 \mathrm{~g}, 4.3 \mathrm{mmol})$ and $10 \% \mathrm{Pd} / \mathrm{C}(0.5 \mathrm{~g})$ in ethanol $(40 \mathrm{~mL})$ was kept under $\mathrm{H}_{2}$ atmosphere overnight. After removal of the catalyst by filtration, the solvent was evaporated under reduced pressure to afford 20 (0.80 g, quant.) as a solid. mp 58-60 ${ }^{\circ} \mathrm{C}$; Elem. Anal. Calculated for $\mathrm{C}_{8} \mathrm{H}_{17} \mathrm{~N}_{3} \mathrm{O}_{2}$ : C, 51.3; H, 9.15; N, 22.4. found: C, 51.3; H, 9.0; N, 22.6. ${ }^{1} \mathrm{H}$ NMR: $\delta 2.98$ and $3.02\left(6 \mathrm{H}, \mathrm{s}, \mathrm{NMe}_{2}\right), 4.22\left(4 \mathrm{H}, \mathrm{s}, \mathrm{NCH}_{2}\right) ;{ }^{13} \mathrm{C} \mathrm{NMR}: \delta 35.6,36,3,50.1$, 170.5; HRMS: $m / z$ 188.1411 [M+H] $]^{+}$; calculated: 188.1399 for $\mathrm{C}_{8} \mathrm{H}_{18} \mathrm{~N}_{3} \mathrm{O}_{2}$.

\section{$2,2^{\prime}, 2^{\prime \prime}-$ Nitrilotris(N,N-dimethylacetamide) (21).}

A mixture of 20 (0.70 g, $3.7 \mathrm{mmol}), 2$-chloro- $N, N$-dimethylacetamide (18) (0.45 g, 3.7 $\mathrm{mmol}), \mathrm{K}_{2} \mathrm{CO}_{3}(1 \mathrm{~g}, 7.4 \mathrm{mmol})$, and $\mathrm{KI}(0.5 \mathrm{~g})$ in acetonitrile $(30 \mathrm{~mL})$ was refluxed overnight. The acetonitrile was evaporated and the residue dissolved in ethyl acetate $(50 \mathrm{~mL})$. The resulting solution was filtered and the solvent evaporated. The residue was dissolved in water $(50 \mathrm{~mL})$ and the solution was extracted with dichloromethane $(3 \times 50 \mathrm{~mL})$. Removal of the water afforded pure $21(0.74 \mathrm{~g}, 75 \%)$ as an oil. ${ }^{1} \mathrm{H}$ NMR: $\delta 2.92$ and $3.04\left(18 \mathrm{H}, \mathrm{s}, \mathrm{NMe}_{2}\right)$, $3.62\left(6 \mathrm{H}, \mathrm{s}, \mathrm{NCH}_{2}\right) ;{ }^{13} \mathrm{C}$ NMR: $\delta$ 35.6, 37.2, 55.9, 60.1, 169.8; HRMS: $\mathrm{m} / \mathrm{z} 295.1741$ [M+Na] $]^{+}$calculated: 295.1746 for $\mathrm{C}_{12} \mathrm{H}_{24} \mathrm{NaN}_{4} \mathrm{O}_{3}$.

\section{2,2',2"-Nitrilotris(N,N-bis(2-hydroxyethyl)acetamide) (23).}

A mixture of triester $22(2.00 \mathrm{~g}, 8.5 \mathrm{mmol})$ and diethanolamine $(2.70 \mathrm{~g}, 25.7 \mathrm{mmol})$ was heated as neat at $120{ }^{\circ} \mathrm{C}$ for $5 \mathrm{~h}$ to give $23(3.14 \mathrm{~g}, 81 \%)$ as an oil. ${ }^{1} \mathrm{H} \operatorname{NMR}\left(\mathrm{D}_{2} \mathrm{O}\right): \delta 3.31$ and $3.39\left(6 \mathrm{H}, \mathrm{t}, J=6.0 \mathrm{~Hz}, \mathrm{CONCH}_{2}\right), 3.51-4.01\left(18 \mathrm{H}, \mathrm{m}, \mathrm{NCH}_{2}\right.$ and $\left.\mathrm{CH}_{2} \mathrm{OH}\right) ;{ }^{13} \mathrm{C} \mathrm{NMR}\left(\mathrm{D}_{2} \mathrm{O}\right)$ : $\delta$ 48.1, 50.1, 55.6, 58.9, 173.0; HRMS: $m / z$ 453.2618 [M+H] ${ }^{+}$; calculated: 453.2561 for $\mathrm{C}_{18} \mathrm{H}_{37} \mathrm{~N}_{4} \mathrm{O}_{7}$.

\subsubsection{Microcalorimetry experiments}

Calorimetric measurements were carried out in water at $\mathrm{pH} 3$ and $25^{\circ} \mathrm{C}$ using a Microcal VPITC microcalorimeter with a cell volume of $1.4115 \mathrm{~mL}$. The $\mathrm{pH}$ of the solution was adjusted by $65 \% \mathrm{HNO}_{3}$. For studying the complexation of $\mathrm{Eu}(\mathrm{III})$ to the ligands $\mathbf{2}, \mathbf{7 a}, \mathbf{7 b}, \mathbf{2 1}$, and $\mathbf{2 3}$, aliquots of a $10.0 \mathrm{mM}$ aqueous solution of the ligands in the buret were added to a $0.166 \mathrm{mM}$ solution of $\mathrm{Eu}\left(\mathrm{NO}_{3}\right)_{3}$ in the calorimetric cell, monitoring the heat change after each addition. Dilution experiments showed that at the experimental concentrations none of the species described above showed significant aggregation behavior. 


\subsubsection{Extraction procedure}

All the aqueous solutions were prepared by dissolution of weighted amounts of the ligand in ultrapure water (resistivity, $18 \mathrm{M} \Omega \cdot \mathrm{cm}^{-1}$ ) containing $0.5 \mathrm{~mol} / \mathrm{L} \mathrm{NH}_{4} \mathrm{NO}_{3}$ (salting-out agent). The initial $\mathrm{pH}_{\text {ini }}$ of the aqueous phase was adjusted using ammonia or diluted nitric acid. The organic solvent consisted of $0.2 \mathrm{~mol} / \mathrm{L}$ TODGA (extractant) and 5 vol\% 1-octanol dissolved in TPH.

The organic phase was not loaded with Am and Eu followed by stripping as TODGA extracts significant amounts of $\mathrm{HNO}_{3}$ which would prevent obtaining reasonable results at $\mathrm{pH}>1$ without using a buffer. Instead each of the aqueous phases $(500 \mu \mathrm{L})$ were spiked with $10 \mu \mathrm{L}$ of radiotracer $\left({ }^{241} \mathrm{Am},{ }^{152} \mathrm{Eu}\right.$, approx. $\left.25 \mathrm{KBq} / \mathrm{mL}\right)$ and contacted with the organic solvent (500 $\mu \mathrm{L}$ ) by shaking for $60 \mathrm{~min}$ at $22^{\circ} \mathrm{C} \pm 1^{\circ}$ using an IKA Vibrax Orbital Shaker Model VXR $(2,200 \mathrm{rpm})$. After phase separation by centrifugation, $200 \mu \mathrm{L}$ aliquots of each phase were withdrawn for radio analysis. The kinetics extraction experiments were performed similarly as described above, except the phases contact time (mixing time). Activity measurements of the gamma-ray emitters were performed with a HPGe spectrometer, EG-G Ortec using the $\gamma$-lines at $59.5 \mathrm{keV}$, and $121.8 \mathrm{keV}$ for ${ }^{241} \mathrm{Am}$ and ${ }^{152} \mathrm{Eu}$, respectively. The distribution ratio $\mathrm{D}_{\mathrm{M}}$ was measured as the ratio between the radioactivity in the organic and the aqueous phase (Eq. 1). Distribution ratios between 0.01 and 100 exhibit a maximum error of $\pm 5 \%$. The error may be up to $\pm 20 \%$ for smaller and larger values. The acidities of the initial and final aqueous solutions were determined using a $691 \mathrm{Metrohm} \mathrm{pH}$ meter $(3 \mathrm{~mol} / \mathrm{L} \mathrm{KCl})$.

\subsection{References and Notes}

1. See: www.acsept.org.

2. Sasaki, Y.; Tachimori, S. Solv. Extr. Ion Exch. 2002, 20, 21.

3. Sasaki, Y.; Sugo, Y.; Suzuki, S.; Tachimori, S. Solv. Extr. Ion Exch. 2001, 19, 91.

4. Sasaki, Y.; Choppin, G. R. Anal. Sci. 1996, 12, 225.

5. Facchini, A.; Amato, L.; Nannicini, R. Sep. Sci. Technol. 1996, 31, 2245.

6. Danesi, P. R.; Cianetti, C.; Horwitz, E. P. Sep. Sci. Technol. 1982, 17, 507.

7. Sasaki, Y.; Sugo, Y.; Kitatsuji, Y.; Kirishima, A.; Kimura, T.; Choppin, G. R. Anal. Sci. 2007, 23, 727.

8. Sasaki, Y.; Suzuki, H.; Sugo, Y.; Kimura, T.; Choppin, G. R. Chem. Lett. 2006, 35, 256. 
9. Heitzmann, M.; Bravard, F.; Gateau, C.; Boubals, N.; Berthon, C.; Pécaut, J.; Charbonnel, M. C.; Delangle, P. Inorg. Chem. 2009, 48, 246.

10. Heitzmann, M.; Gateau, C.; Chareyre, L.; Miguirditchian, M.; Charbonnel, M. C.; Delangle, P. New J. Chem. 2010, 34, 108.

11. Spjuth, L.; Liljenzin, J. O.; Hudson, M. J.; Drew, M. G. B.; Iveson, P. B.; Madic, C. Solv. Extr. Ion Exch. 2000, 18, 1.

12. Iqbal, M.; Huskens, J.; Verboom, W.; Sypula, M.; Modolo, G. Supramol. Chem. 2010, 22, 827. (Chapter 3).

13. Bradshaw, J. S.; An, H.; Krakowiak, K. E.; Wang, T.; Zhu, C.; Izatt, R. M. J. Org. Chem. 1992, 57, 6112.

14. In a second attempt diglycolic acid was reacted with diethanolamine in THF using DCC as coupling reagent, however, no reaction occurred.

15. Xu, Y.; Flavin, M. T.; Xu, Z. Q. J. Org. Chem. 1996, 61, 7697.

16. Fild, M.; Handke, W. Z. Anorg. Allg. Chem. 1987, 555, 109.

17. Nave, S.; Modolo, G.; Madic, C.; Testard, F. Solv. Extr. Ion Exch. 2004, 22, 527.

18. Yaita, T.; Herlinger, A. W.; Thiyagarajan, P.; Jensen, M. P. Solv. Extr. Ion Exch. 2004, $22,527$.

19. Rao, L.; Zanonato, P. L.; Bernardo, P. D.; Bismondo, A. J. Chem. Soc., Dalton Trans. 2001, 1939.

20. Endothermic lanthanide complexes are well known in literature: a) Rao, L.; Zanonato, P. L.; Bernardo, P. D.; Bismondo, A. Inorg. Chim. Acta 2000, 306, 49. b) de Carvalho, R. G.; Choppin, G. R. J. Inorg. Nucl. Chem. 1967, 29, 737.

21. Louis, C.; Christine, D. M.; Claude, P.; Pierre, T. Tetrahedron Lett. 1989, 30, 1369.

22. Green, J. R.; Majewski, M.; Snieckus, V. Can. J. Chem. 2006, 84, 1397.

23. Wei, L. H.; He, Y. B.; Wu, J. L.; Qin, H. J.; Xu, K. X.; Meng, L. Z. Chin. J. Chem. 2005, 23,609 .

24. For another synthetic method see: Luk'yanenko, N. G.; Lobach, A. V.; Nazarova, N. Y.; Karpenko, L. P.; Lyamtseva, L. N. Khim. Geter. Soed. 1988, 687.

25. For another synthetic method see: Bordunov, A. V.; Hellier, P. C.; Bradshaw, J. S.; Dalley, N. K.; Kou, X.; Zhang, X. X.; Izatt, R. M. J. Org. Chem. 1995, 60, 6097.

26. For another synthetic method see: Maeda, H.; Furuyoshi, S.; Nakatsuji, Y.; Okahara, M. Tetrahedron 1982, 38, 3359. 
27. For a related synthesis and more spectral data see: Valentijn, A. R. P. M.; van den Berg, O.; van der Marel, G. A.; L. H. Cohen.; van Boom, J. H. Tetrahedron 1995, 51, 2099. 



\section{Chapter}

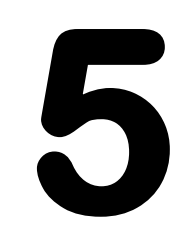

\section{Preorganization of Diglycolamides on the Calix[4]arene Platform and its Effect on the Extraction of Am(III)/Eu(III)*}

Different diglycolamide (DGA)-substituted calix[4]arene-based extractants were synthesized and evaluated for the extraction of Am(III) and Eu(III), representative trivalent actinide and lanthanide ions, respectively. On the narrow rim of the calix[4]arene platform, the DGA moiety was either 1,3-di- or tetrasubstituted with a varying spacer length between the oxygen and amide nitrogen atom. In addition, DGA groups were appended both to the wide rim and to both rims of calix[4]arenes and their efficiencies were compared for Am(III) and Eu(III) extraction at three different feed acidities. The extraction and separation efficiencies strongly depended on the N-alkyl substituent as well as the spacer length. 1,3-Di-DGA-substituted calix[4] arenes are inferior extractants to the corresponding tetrasubstitued ones. Narrow rim DGA-functionalized derivatives resulted in high extraction efficiencies, while the wide rim DGA-functionalized calix[4] arenes showed almost no extraction.

\footnotetext{
* This chapter is based on: Iqbal, M.; Mohapatra, P. K.; Ansari, S. A.; Huskens, J.; Verboom, W. Tetrahedron 2012, 68, 7840.
} 


\section{Chapter 5}

\subsection{Introduction}

The diglycolamides (DGA) are known for the extraction of metal ions since early $1990 \mathrm{~s}^{1}$ and extensive studies have been performed with this very promising class of ligands for the extraction of actinides $^{2}$ as described in Chapter 2. Similar to the malonamides, they form a third phase when contacted with moderate concentrations of nitric acid and hence, often require a phase modifier. A detailed study about DGAs has been presented in a recent review. $^{3}$

The extraction mechanism with $N, N, N$ ' $N^{\prime}$-tetra- $n$-octyl diglycolamide (TODGA) involves reverse micelle formation with four TODGA molecules, ${ }^{4}$ which has been supported by small angle neutron scattering ${ }^{4,5}$ and dynamic light scattering ${ }^{6}$ data. The proposed mechanism involves extraction of the metal ion into the core of the reverse micelle, which contains four units of the diglycolamide at $3 \mathrm{~mol} / \mathrm{L} \mathrm{HNO}_{3}$. The number of TODGA molecules in the reverse micelle is dependent on the feed acidity. As diluents have a very important role in the formation of reverse micelles, the extraction trends and also the species extracted vary significantly with the nature of the diluent. To exclude diluent effects, the ligating sites may be grouped together on a molecular platform.

It is known that calix[4] arenes appended with actinide specific ligating sites such as CMPO ${ }^{1 \mathrm{a}, 7}$ or picolinamides ${ }^{8}$ give rise to more efficient metal ion binding and extraction based on a co-operative complexation mechanism. In general, the positive effect of preorganization of ligands on a platform on the extraction efficiency has been demonstrated. ${ }^{9}$ We found that DGA groups preorganized at the C-pivot and trialkylphenyl platforms resulted in good extraction efficiencies for $\mathrm{Eu}(\mathrm{III})$ and $\mathrm{Am}(\mathrm{III}) .{ }^{10}$ In addition, we evaluated the performance of a tripodal diglycolamide in solvent extraction and supported liquid membranes for actinide extraction. $^{11}$

This Chapter deals with a systematic study of the effect of preorganization of DGA units on the calix[4]arene platform towards Am(III) and $\mathrm{Eu}(\mathrm{III})$ extraction. In addition to the number of DGA ligating sites, the influence of the spacer length and $\mathrm{N}$-alkylation of the DGA are also studied. 


\subsection{Results and discussion}

\subsubsection{Synthesis}

For the preparation of the different DGA-functionalized calix[4]arenes, we used an approach involving the reaction of appropriately amino-functionalized calix[4]arenes with the $p$-nitrophenol-activated diglycolamide 2 . Reaction of the amino-functionalized calix[4]arenes with the non-activated DGA 2 under peptide coupling conditions gave rise to much lower yields. The same holds for the reaction sequence: reaction of the amino-functionalized calix[4]arenes with diglycolic anhydride followed by reaction with di- $n$-octylamine.

\subsubsection{Narrow rim DGA-functionalized calix[4]arenes}

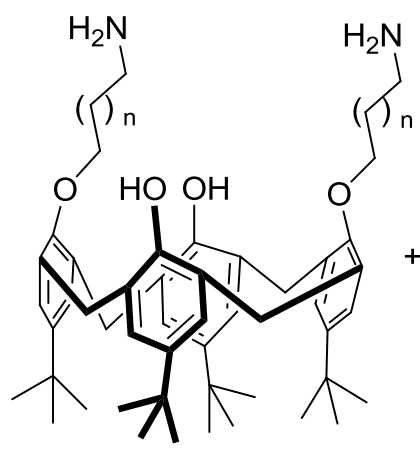

$1 a, b$<smiles>O=C(COCC(=O)N(OCl)OCl)Oc1ccc([N+](=O)[O-])cc1</smiles>

$$
\mathrm{a} n=1
$$$$
b n=2
$$
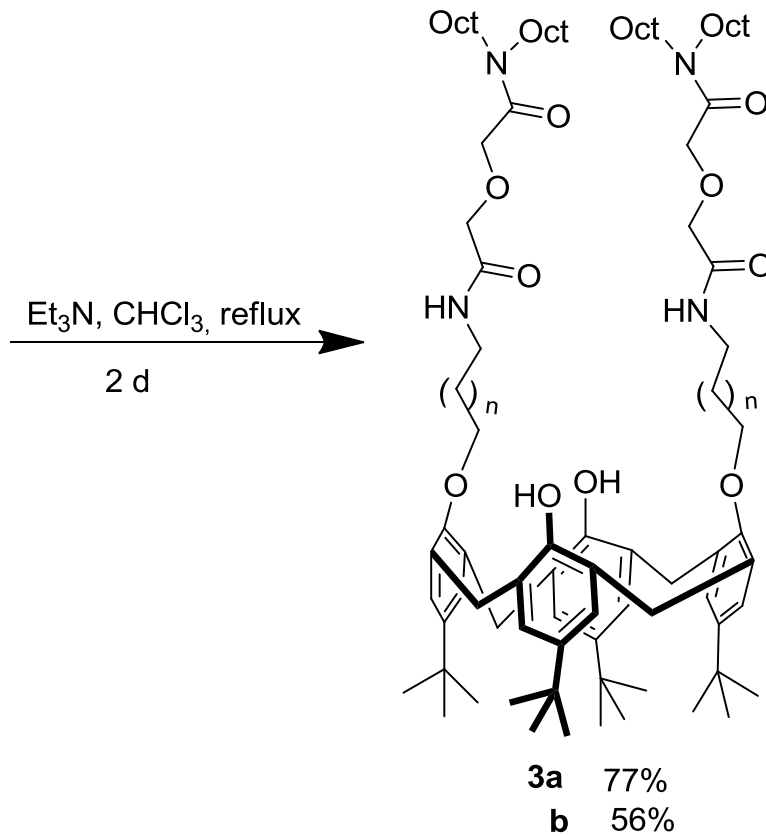

Scheme 5.1

The known ${ }^{12}$ 1,3-bis(aminoalkoxy)calix[4]arenes 1a,b were reacted with $p$-nitrophenyl activated DGA 2 in the presence of triethylamine as a base to give the di-DGA-calix[4]arenes 3a,b (Scheme 5.1). The formation of $\mathbf{3 a}, \mathbf{b}$ could be followed from the shift in the ${ }^{1} \mathrm{H}$ NMR spectra of the $\mathrm{CH}_{2} \mathrm{NH}$ methylene protons from 3.54 (1a) to 3.74 ppm (3a) and from 2.96 (1b) to $3.48 \mathrm{ppm}(\mathbf{3 b})$.

Two tetra-DGA-calixarenes (5a,b), with a C2 and C3 spacer between the calixarene platform and the DGA, were prepared in a similar way by reaction of cone tetrakis(aminoalkoxy)calix[4]arenes $\mathbf{4 a}, \mathbf{b}^{13}$ with $p$-nitrophenol-activated DGA 2 (Scheme 5.2). Also in these cases, its formation could be clearly followed from the shifts of the $\mathrm{CH}_{2} \mathrm{NH}$ 


\section{Chapter 5}

methylene protons from 3.17 (4a) to $3.89 \mathrm{ppm}(\mathbf{5 a})$ and from 2.91 (4b) to $3.46 \mathrm{ppm}(\mathbf{5 b})$ in the ${ }^{1} \mathrm{H}$ NMR spectra. In addition, the electrospray mass spectra exhibited the correct $\mathrm{M}+\mathrm{H}$ peaks.
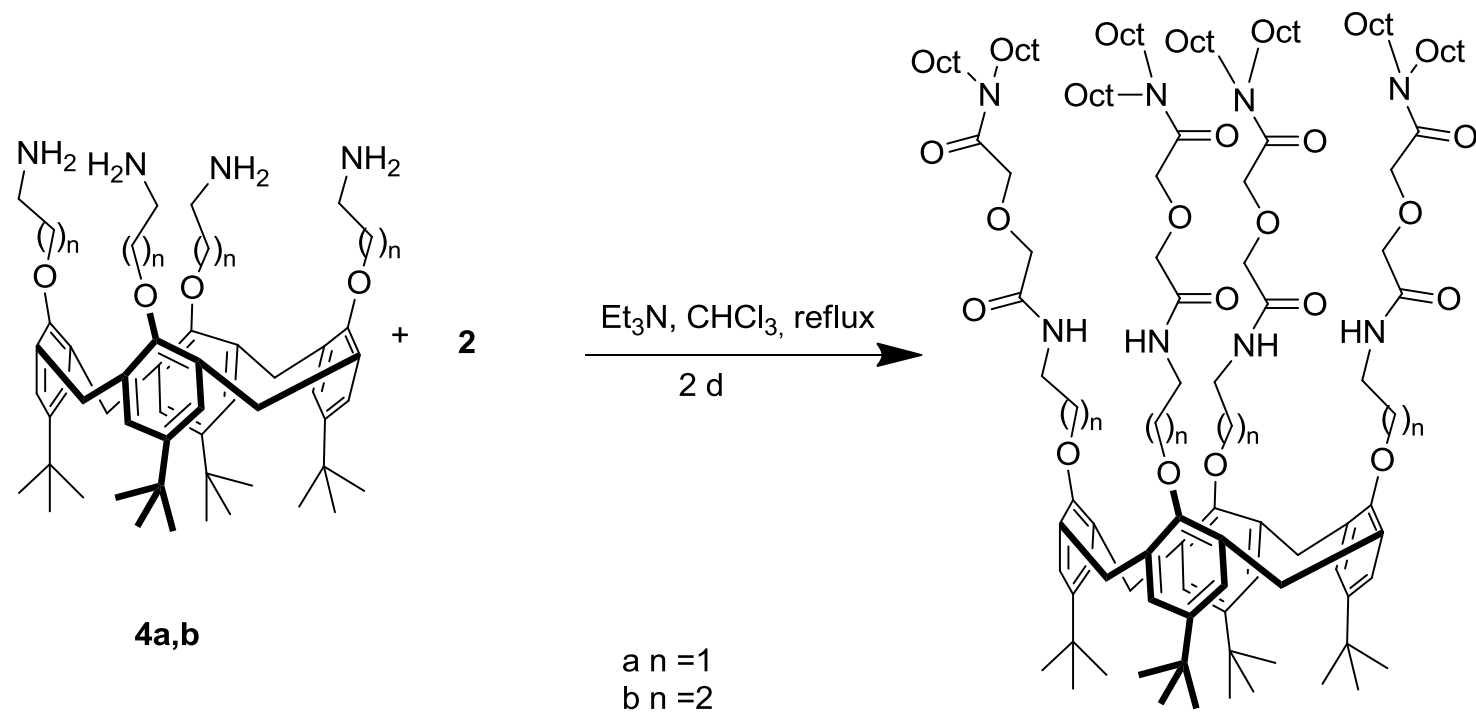

$$
\begin{aligned}
& a n=1 \\
& b n=2
\end{aligned}
$$

$$
\begin{array}{r}
\text { 5a } 52 \% \\
\text { b } 71 \%
\end{array}
$$

Scheme 5.2

\subsubsection{Narrow rim $N$-substituted DGA-functionalized calix[4]arenes}

Among the ligands described above, extraction experiments (vide infra) showed the tetraDGA-calix[4]arene 5b, with a spacer length of three, to be the most efficient one. Therefore, further improvement of this ligand was made by introduction of an alkyl group at the amide nitrogen atom close to the calixarene platform. It was anticipated that the introduction of this alkyl group would force the amide oxygens to point inwards to avoid steric crowding, thereby, preorganizing the molecule and consequently influencing its extraction efficiency. Therefore, three different tetra-DGA-calix[4]arenes 8a-c were prepared with $n$-propyl, $n$ octyl, and 3-pentyl groups, respectively, introduced at the amide nitrogen atom (Scheme 5.3). 


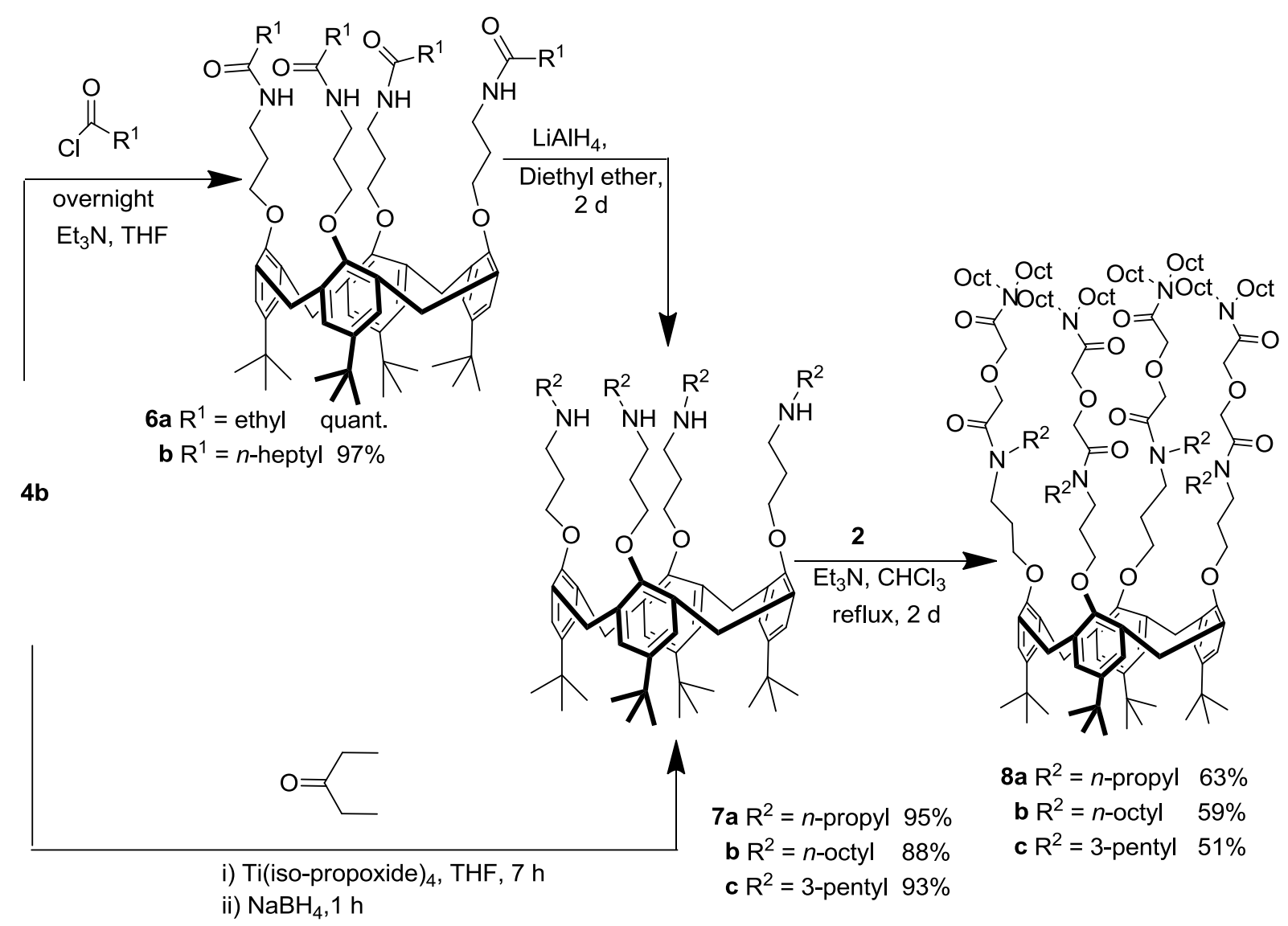

Scheme 5.3

The starting tetrakis(alkylaminoalkoxy)calix[4]arenes $\mathbf{7 a , b}$ were prepared by the reaction of 4b with propionyl and octanoyl chloride, respectively, to give $\mathbf{6 a}, \mathbf{b}$, followed by reduction of the amide carbonyl groups with $\mathrm{LiAlH}_{4}$. The corresponding 3-pentyl-substituted derivative 7c was synthesized by reductive amination. Tetrakis(aminopropoxy)calixarene (4b) was reacted with 3-pentanone in THF using Ti(iso-propoxide) $)_{4}$ as a catalyst, followed by in situ reduction of the formed imine by $\mathrm{NaBH}_{4}$ to afford 7c. In the cases of $\mathbf{6 a}, \mathbf{b}$ the reductive amination approach was not successful. The reduction of amides $\mathbf{6 a}, \mathbf{b}$ to amines $\mathbf{7 a}, \mathbf{b}$ was confirmed in their ${ }^{1} \mathrm{H}$ NMR spectra by the appearance of new triplets of the $\mathrm{NCH}_{2}$ groups at 2.60 and 2.61 ppm for $\mathbf{7 a}$ and $\mathbf{7 b}$ and the shift of the multiplets of the $\mathrm{NCH}_{2}$ groups from 3.34-3.48 and 3.34-3.47 ppm in 6a,b to distinct triplets at 2.73 and 2.74 ppm for $\mathbf{7 a}$ and $\mathbf{7 b}$, respectively. The formation of 7c was followed by the appearance in the ${ }^{1} \mathrm{H}$ NMR spectrum of the NCH proton at $2.36 \mathrm{ppm}$ and the shift of the $\mathrm{NCH}_{2}$ protons from $2.91 \mathrm{ppm}$ in $\mathbf{4 b}$ to $2.68 \mathrm{ppm}$ in $\mathbf{7 c}$. In all cases, the electrospray mass spectra showed the correct $\mathrm{M}+\mathrm{H}$ peaks.

In a similar way as described above, $\mathbf{7 a , b , c}$ were further reacted with $p$-nitrophenol-activated DGA 2 to convert them into the tetra-DGA-calix[4]arenes 8a-c. In the case of 8c, after 


\section{Chapter 5}

refluxing for 2 days in chloroform, the reaction was not yet complete as detected by ${ }^{1} \mathrm{H}$ NMR spectroscopy. This may be due to the sterically hindering isopentyl groups. However, by refluxing the mixture in toluene the reaction was completed after 1 day. The ${ }^{1} \mathrm{H}$ NMR spectra exhibited shifts of the signals of the $\mathrm{NCH}_{2}$ groups from $2.73 \mathrm{ppm}$ in 7a to 3.01-3.41 ppm in $\mathbf{8 a}$, from $2.74 \mathrm{ppm}$ in $\mathbf{7 b}$ to $3.02-3.44 \mathrm{ppm}$ in $\mathbf{8 b}$ and from $2.68 \mathrm{ppm}$ in $\mathbf{7 c}$ to $3.02-3.26 \mathrm{ppm}$ in 8c. Ligands 8a-c exhibited the correct $\mathrm{M}+\mathrm{H}$ peaks in the high resolution electrospray mass spectra.

\subsubsection{Wide rim DGA-functionalized calix[4]arene}

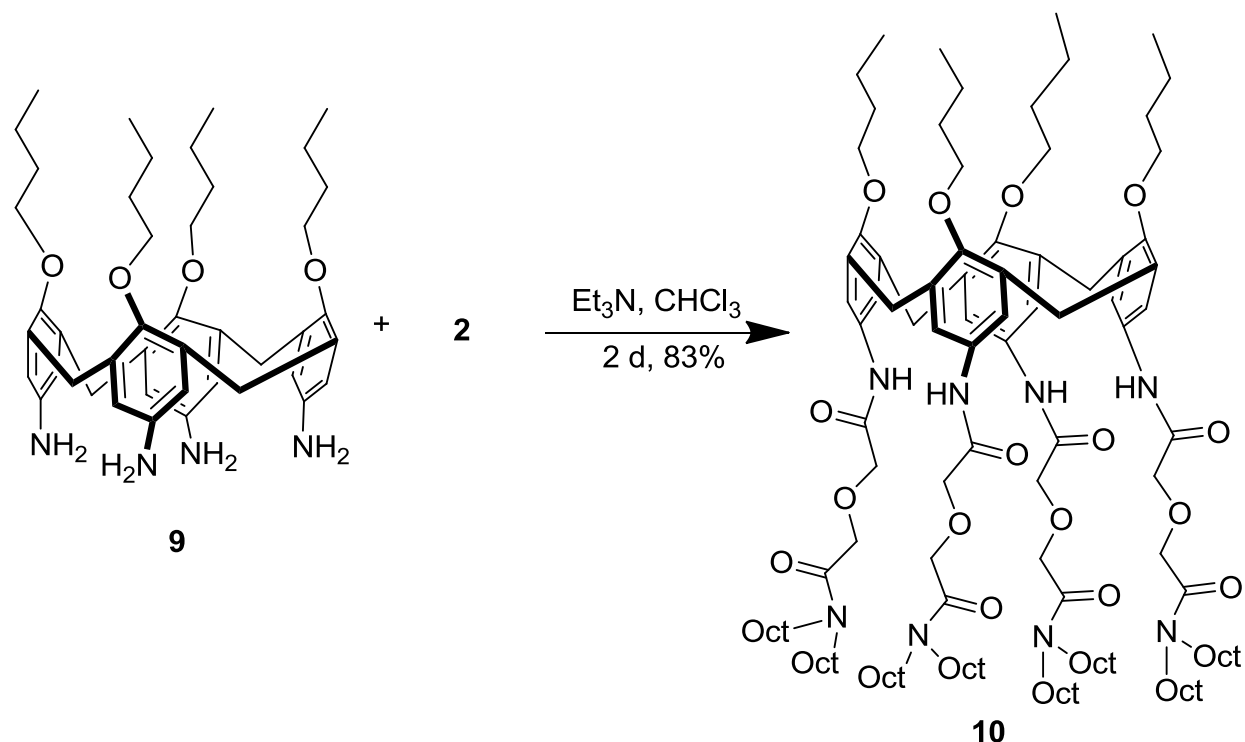

Scheme 5.4

The extraction results (vide infra) of the ligands described in the previous sections show that small changes greatly influence the extraction efficiency. Therefore, it was of interest to synthesize the wide rim DGA-functionalized calix[4]arene 10. It was prepared by the above described approach by reaction of the known ${ }^{14}$ tetrabutoxycalix[4]arene tetraamine 9 with $p$-nitrophenol-activated DGA 2 (Scheme 5.4). ${ }^{15}$ In addition to the electrospray mass spectrum, its formation was confirmed by the downfield shift of the aromatic protons in the ${ }^{1} \mathrm{H}$ NMR spectrum from $6.12 \mathrm{ppm}$ in $\mathbf{9}$ to $6.83 \mathrm{ppm}$ in $\mathbf{1 0}$.

\subsubsection{Wide and narrow rim DGA-functionalized calix[4]arene}

As a logical extension, ligand $\mathbf{1 3}$ was prepared bearing DGA moieties on both rims of the calix[4]arene skeleton. The required octaaminocalix[4]arene $\mathbf{1 2}$ was synthesized from tetrakis(phthalimido-1-propoxy)tetranitrocalix[4]arene $\mathbf{1 1}$ in a slightly different way to that 
described in literature. ${ }^{16}$ In the literature, 11 was reduced by hydrazine using $\mathrm{Pd} / \mathrm{C}$ as a catalyst to give $\mathbf{1 2}$ in $57 \%$ yield in a single step. However, using a two-step procedure involving first cleavage of the phthalimide groups by hydrazine followed by reduction of the nitro moieties, using Raney $\mathrm{Ni}$, as a catalyst afforded the desired product $\mathbf{1 2}$ in quantitative yield. This was further reacted with $p$-nitrophenol-activated DGA 2 to yield the desired ligand 13 (Scheme 5.5). The formation of $\mathbf{1 3}$ was clearly followed from the $\mathrm{M}+\mathrm{H}$ peak at $\mathrm{m} / \mathrm{z}$ 3427.6606 in the high resolution electrospray mass spectrum. In the ${ }^{1} \mathrm{H}$ NMR spectrum downfield shifts were observed for the $\mathrm{NCH}_{2}$ protons from 2.87 in $\mathbf{1 2}$ to $3.48 \mathrm{ppm}$ in $\mathbf{1 3}$, and of the aromatic hydrogens from 6.07 in $\mathbf{1 2}$ to $6.97 \mathrm{ppm}$ in $\mathbf{1 3}$.

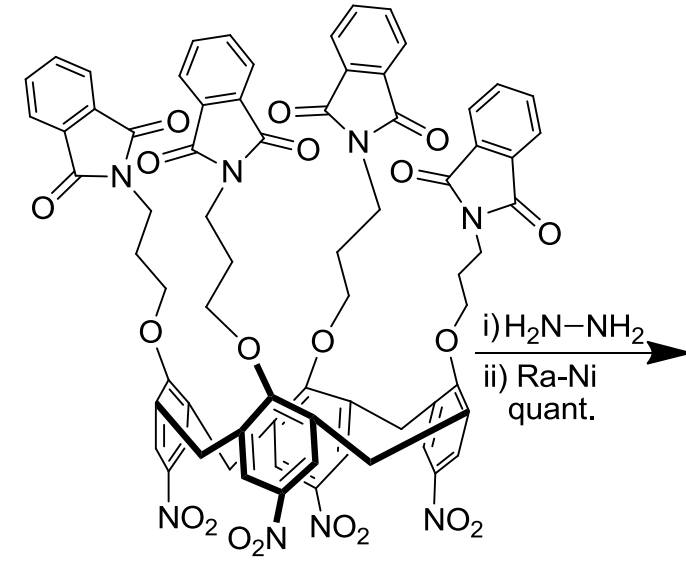

11

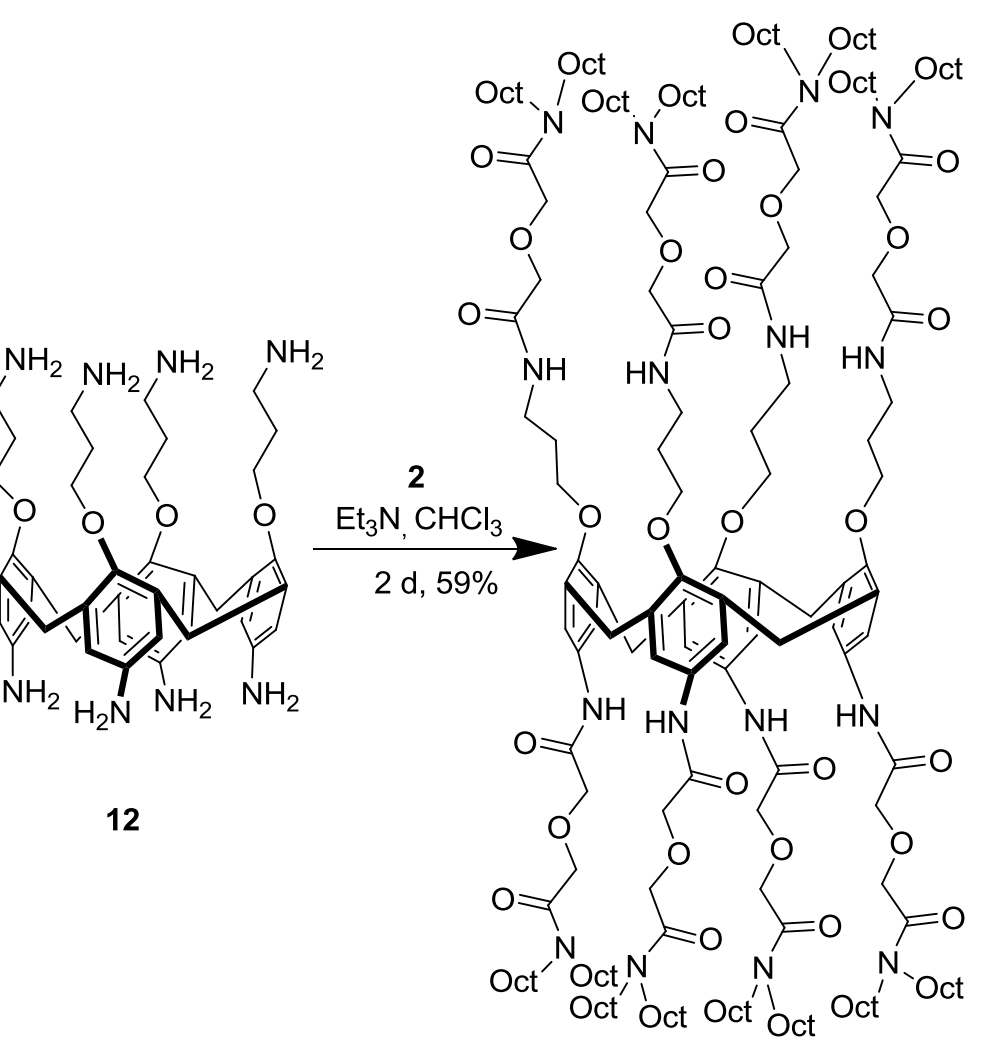

13

Scheme 5.5

\subsubsection{Solvent extraction studies}

The solvent extraction data of Am(III) and Eu(III) from three different feed acidities (0.01, 1.0 and $3.0 \mathrm{~mol} / \mathrm{L} \mathrm{HNO}_{3}$ ) using $1.0 \times 10^{-3} \mathrm{~mol} / \mathrm{L}$ solutions of the DGA-calix[4]arene ligands 3a,b, 5a,b, 8a-c, 10 and 12 in $5 \%$ iso-decanol + $95 \% n$-dodecane are summarized in Table 5.1. Iso-decanol was used as the phase modifier and turbid solutions were obtained when solutions without the phase modifier were contacted with $>3 \mathrm{~mol} / \mathrm{L}$ nitric acid. The extraction 


\section{Chapter 5}

of both $\mathrm{Am}$ (III) and $\mathrm{Eu}(\mathrm{III})$ was negligibly low when the calix[4] arene ligand contained two DGA moieties as in 3a,b. Of the narrow rim functionalized DGA-calix[4]arene ligands with a spacer length of three $(\mathbf{5 b}, \mathbf{8 a}, \mathbf{8 b}$, and $\mathbf{8 c}), \mathbf{5 b}$ was the least efficient, although, as expected for a TODGA ${ }^{3}$ derivative, the Eu/Am selectivity, was very good [separation factor (SF) 10 for $3 \mathrm{~mol} / \mathrm{L} \mathrm{HNO}_{3}$ as the feed]. Ligand $8 \mathbf{a}$ showed a significantly higher extraction efficiency than 5b. Although the $D_{\mathrm{Am}}$ and $D_{\mathrm{Eu}}$ (distribution ratio of Am and Eu, respectively) values increased by more than an order of magnitude compared to those obtained with $\mathbf{5} \mathbf{b}$, the selectivity decreased significantly (by more than 3 times at $3 \mathrm{~mol} / \mathrm{L} \mathrm{HNO}_{3}$ as the feed). The higher extraction ability of $\mathbf{8 a}$ is attributed to the enhanced organophilicity of the extractant due to the introduction of the $n$-propyl group at the amide $\mathrm{N}$ atom. However, the reason for the loss in selectivity is not clearly understood. With a further increase in the length of the alkyl chain from $n$-propyl to $n$-pentyl and $n$-pentyl to $n$-octyl groups, a further increase in the extraction of Am(III) takes place (by about 5 and 4 times for $\mathbf{8 b}$ and $\mathbf{8 c}$, respectively, as compared to 8a), while the increase in $\mathrm{Eu}(\mathrm{III})$ extraction was to a much lower extent (by about 1.5 times for both $\mathbf{8 b}$ and $\mathbf{8 c}$ as compared to $\mathbf{8 a}$ ). This means that the SF values are close to 1 at $3 \mathrm{~mol} / \mathrm{L} \mathrm{HNO}_{3}$, which increased to 6.25 and 4.87 for $\mathbf{8 b}$ and $\mathbf{8 c}$, respectively at 1 $\mathrm{mol} / \mathrm{L} \mathrm{HNO}_{3}$ as the feed. These results indicate that the role of the substituent at the amidic nitrogen close to the spacer is extremely important, influencing both the extraction as well as the separation efficiency. The higher extraction efficiency of the NR-DGA's 8a-c compared to the NH-DGA $\mathbf{5 b}$ may also be caused by the fact that the NR amide groups are more basic. In addition, the most favorable conformation for steric reasons is when the $\mathrm{N}$-alkyl groups are pointing outwards and consequently the amide oxygens are directing inwards. This will result in a more preorganized structure for metal complexation giving rise to higher extraction efficiencies for the NR-DGA's 8a-c compared to the NH-DGA 5b. Furthermore, alkyl group substitution enhances the lipophilicity of the extracted complex. Reducing the spacer length also plays an important role in the metal ion extraction. A spacer length of two, as in 5a, led to a significantly lower extraction of both $\mathrm{Am}(\mathrm{III})$ and $\mathrm{Eu}(\mathrm{III})$ at the three acidities as compared to the analogous extractant with a spacer length of three $(\mathbf{5 b})$. This is attributed to a better flexibility of the DGA arms in $\mathbf{5 b}$ leading to less strain during complexation.

The wide-rim-functionalized ligand $\mathbf{1 0}$ was also evaluated under the same conditions (Table 5.1). Surprisingly, the extraction of both $\mathrm{Am}(\mathrm{III})$ and $\mathrm{Eu}(\mathrm{III})$ was negligible at all the three acidities when a $1.0 \times 10^{-3} \mathrm{~mol} / \mathrm{L}$ ligand concentration was used. Increasing the ligand concentration 10 times, enhanced the extraction of metal ions (data presented between 110 
parentheses in Table 5.1). However, the $D$ values were significantly lower than those of the narrow rim functionalized DGA-calix[4]arenes at 10 times lower ligand concentration. Probably, the connection of the DGA to the calix[4]arene via an amide bond considerably reduces the flexibility of the ligand, which is reflected in a much lower extraction ability than that of the narrow rim DGA-calix[4]arenes described above. On the other hand, ligand 13, DGA-functionalized at both sides, showed a similar extraction trend as $\mathbf{8 a}$, with the difference that the $\mathrm{Eu}(\mathrm{III})$ extraction was lower at $1.0 \mathrm{~mol} / \mathrm{L} \mathrm{HNO}_{3}$ which showed a higher increase at 3 $\mathrm{mol} / \mathrm{L}_{\mathrm{HNO}_{3}}$, which was reflected in a higher SF value at higher acidity. One would expect for $\mathbf{1 3}$ an extraction behavior as that of $\mathbf{5 b}$. However, the higher extraction of the metal ions may be attributed to the presence of the four additional DGA moieties at the wide rim leading to a significantly higher lipophilicity of the extracted complex.

From Table 5.1 it is very clear that bringing together four DGA units on the narrow rim of a calix[4]arene gives rise to a huge enhancement of the extraction abilities towards Am(III) and $\mathrm{Eu}(\mathrm{III})$ compared to TODGA using the same conditions. Even at only $1 \mathrm{mmol} / \mathrm{L}$ ligand concentration, $D$ values of up to about 400 are obtained. This demonstrates the advantage of preorganization of ligating sites, while in this particular case, it will also have a positive effect in suppressing micelle formation (vide supra). On the other hand, in several cases, the $D$ values are rather low and consequently there will be a relatively large error in the corresponding $S F$ values, which makes it hard to compare the efficiencies. 


\section{Chapter 5}

Table 5.1 Extraction data of Am(III) and Eu(III) by DGA-calix[4]arenes 3a,b, 5a,b, 8a-c, 10, and 13; Ligand $=1 \mathrm{mmol} / \mathrm{L}$ in $5 \%$ iso-decanol $/ n$-dodecane; Temperature: $25^{\circ} \mathrm{C}$

\begin{tabular}{|c|c|c|c|c|}
\hline Ligand & {$\left[\mathrm{HNO}_{3}\right], \mathrm{M}$} & $D_{\mathrm{Am}}$ & $D_{\mathrm{Eu}}$ & $S F=D_{\mathrm{Eu}} / D_{\mathrm{Am}}$ \\
\hline \multirow[t]{3}{*}{ TODGA } & 0.01 & $<0.001$ & $<0.001$ & - \\
\hline & 1.0 & 0.001 & 0.01 & 10 \\
\hline & 3.0 & 0.02 & 0.97 & 48.5 \\
\hline \multirow[t]{3}{*}{$3 \mathbf{a}$} & 0.01 & $<0.001$ & 0.007 & $>7$ \\
\hline & 1.0 & $<0.001$ & 0.003 & $>3$ \\
\hline & 3.0 & 0.02 & 0.10 & 5 \\
\hline \multirow[t]{3}{*}{$3 b$} & 0.01 & $<0.001$ & $<0.001$ & 1 \\
\hline & 1.0 & 0.002 & 0.02 & 10 \\
\hline & 3.0 & 0.002 & 0.09 & 4.5 \\
\hline \multirow[t]{3}{*}{$5 \mathbf{a}$} & 0.01 & 0.001 & 0.05 & 50 \\
\hline & 1.0 & 0.06 & 0.21 & 3.5 \\
\hline & 3.0 & 0.60 & 4.03 & 7 \\
\hline \multirow[t]{3}{*}{$5 b$} & 0.01 & 0.01 & 0.09 & 9 \\
\hline & 1.0 & 0.10 & 0.71 & 7 \\
\hline & 3.0 & 1.80 & 17.8 & 10 \\
\hline \multirow[t]{3}{*}{$8 \mathbf{a}$} & 0.01 & 0.07 & 0.43 & 6 \\
\hline & 1.0 & 13.3 & 103 & 7.7 \\
\hline & 3.0 & 79.5 & 225 & 2.8 \\
\hline \multirow[t]{3}{*}{$8 b$} & 0.01 & 0.007 & 0.05 & 7 \\
\hline & 1.0 & 35.2 & 220 & 6.25 \\
\hline & 3.0 & 402 & 356 & 0.9 \\
\hline \multirow[t]{3}{*}{$8 c$} & 0.01 & 0.04 & 0.12 & 3 \\
\hline & 1.0 & 38.0 & 185 & 4.9 \\
\hline & 3.0 & 325 & 370 & 1.1 \\
\hline \multirow[t]{3}{*}{$10^{a}$} & 0.01 & $0.003(0.011)$ & $0.001(0.011)$ & $0.3(1)$ \\
\hline & 1.0 & $0.16(3.08)$ & $0.10(2.42)$ & $0.6(0.8)$ \\
\hline & 3.0 & $0.58(15.3)$ & $0.43(18.8)$ & $0.7(1.2)$ \\
\hline \multirow[t]{3}{*}{13} & 0.01 & 0.02 & 0.05 & 2.5 \\
\hline & 1.0 & 12.1 & 56.8 & 4.7 \\
\hline & 3.0 & 54.2 & 375 & 6.9 \\
\hline
\end{tabular}

${ }^{\mathrm{a}}$ The data between parentheses were obtained with a ten-fold higher ligand concentration, viz.10 $\mathrm{mmol} / \mathrm{L}$. 


\subsection{Conclusions}

This study clearly shows the advantages of the preorganization of ligating sites on a molecular platform, in this case a calix[4]arene, giving rise to much better extraction properties than in the case of the single ligand. The number of DGA units is essential. The di-DGA calix[4]arenes hardly show any extraction, while the tetra-DGA calix[4]arenes exhibit an excellent extraction ability. Comparison of the extraction data of tetra-DGA calix[4]arenes with different spacer lengths shows that a certain degree of flexibility is required. In addition, it is demonstrated that a subtle modification of the structure of the ligand, in this case $\mathrm{N}$ alkylation, significantly influences the extraction abilities.

\subsection{Experimental}

\subsubsection{General}

All moisture-sensitive reactions were carried out under an argon atmosphere. The solvents and all reagents were obtained from commercial sources and used without further purification. All known compounds viz. 1a, $, \mathbf{b}^{12} \mathbf{2},{ }^{17} \mathbf{4 a}, \mathbf{b},{ }^{13}$ and $\mathbf{9}^{14}$ were prepared according to literature procedures. Solvents were dried according to standard procedures and stored over molecular sieves. ${ }^{1} \mathrm{H}$ NMR and ${ }^{13} \mathrm{C}$ NMR spectra were recorded on a Varian Unity INOVA (300 MHz) spectrometer. ${ }^{1} \mathrm{H}$ NMR (300 MHz) and ${ }^{13} \mathrm{C}$ NMR (75 MHz) chemical shift values are reported as $\delta$ using the residual solvent signal as an internal standard. All NMR measurements are recorded in $\mathrm{CDCl}_{3}$ as a solvent unless mentioned otherwise. Infrared spectra were taken on a Thermo Scientific spectrometer. Electrospray Ionization (positive mode) mass spectra and high resolution mass spectra were recorded on a WATERS LCT mass spectrometer. Extraction studies were carried out at Bhabha Atomic Research Centre, India. Analytical TLC was performed using Merck prepared plates (silica gel 60 F-254 on aluminium). Column chromatography was carried out with Merck silica gel 60 (230-400 mesh).

\section{Di-DGA-calix[4]arene (3a).}

A mixture of 1a (1.60 g, $2.1 \mathrm{mmol})$, p-nitrophenol-activated DGA (2) (2.11 g, $4.4 \mathrm{mmol})$ and triethylamine $(0.46 \mathrm{~g}, 4.5 \mathrm{mmol})$ in chloroform $(50 \mathrm{~mL})$ was refluxed overnight. The crude reaction mixture was successively washed with $2 \mathrm{M} \mathrm{NaOH}(3 \times 50 \mathrm{~mL}), 1 \mathrm{M} \mathrm{HCl}(3 \times 50$ $\mathrm{mL})$, and water $(2 \times 50 \mathrm{~mL})$. The organic layer was concentrated under reduced pressure and the crude product was purified by column chromatography $\left(\mathrm{CH}_{2} \mathrm{Cl}_{2}: \mathrm{MeOH}, 96: 4\right)$ to give $\mathbf{3 a}$ 


\section{Chapter 5}

in 77\% yield $(2.20 \mathrm{~g})$ as a dense oil. ${ }^{1} \mathrm{H}$ NMR: $\delta$ 0.83-0.93 $\left(12 \mathrm{H}, \mathrm{m}, \mathrm{CH}_{3}\right), 0.99(18 \mathrm{H}, \mathrm{s}, t-\mathrm{Bu})$, 1.18-1.38 (58H, m, $t$-Bu and $\left.\mathrm{CH}_{3}\left(\mathrm{CH}_{2}\right)_{5}\right), 1.42-1.57\left(8 \mathrm{H}, \mathrm{m}, \mathrm{NCH}_{2} \mathrm{CH}_{2}\right), 2.26(4 \mathrm{H}$, pentet, $J=$ $\left.6.0 \mathrm{~Hz}, \mathrm{OCH}_{2} \mathrm{CH}_{2}\right), 3.03\left(4 \mathrm{H}, \mathrm{t}, J=7.5 \mathrm{~Hz}, \mathrm{NCH}_{2}\right), 3.21-3.33\left(8 \mathrm{H}, \mathrm{m}, \mathrm{ArCH}_{2} \mathrm{Ar}\right.$ and $\left.\mathrm{NCH}_{2}\right)$, $3.74\left(4 \mathrm{H}, \mathrm{t}, J=6.0 \mathrm{~Hz}, \mathrm{NHCH}_{2}\right), 4.05\left(4 \mathrm{H}, \mathrm{t}, J=6.0 \mathrm{~Hz}, \mathrm{ArOCH}_{2}\right), 4.11\left(4 \mathrm{H}, \mathrm{s}, \mathrm{OCH}_{2} \mathrm{CO}\right)$, $4.16\left(4 \mathrm{H}, \mathrm{s}, \mathrm{OCH}_{2} \mathrm{CO}\right), 4.25\left(4 \mathrm{H}, \mathrm{d}, J=12.0 \mathrm{~Hz}, \mathrm{ArCH}_{2} \mathrm{Ar}\right), 6.83(4 \mathrm{H}, \mathrm{s}, \mathrm{ArH}), 7.02(4 \mathrm{H}, \mathrm{s}$, $\mathrm{ArH}), 7.68$ (2H, s, ArOH), 8.09-8.18 (2H, m, NH); ${ }^{13} \mathrm{C}$ NMR: $\delta$ 14.3, 22.9, 27.3, 29.5, 31.3, 32.0, 34.1, 34.2, 69.7, 71.7, 125.7, 127.9, 132.9, 141.8, 147.2, 149.7, 150.7, 168.6, 170.4; IR $\left(v_{\max } / \mathrm{cm}^{-1}\right): 2954,2935,2855,1648,1484,1465,1361,1206,1124$. HRMS: $\mathrm{m} / z$. 1441.0741 [M+H] $]^{+}$; calculated: 1441.0882 for $\mathrm{C}_{90} \mathrm{H}_{144} \mathrm{~N}_{4} \mathrm{O}_{10}$.

\section{Di-DGA-calix[4]arene (3b).}

3b was prepared in an analogous way to $\mathbf{3 a}$, starting from $\mathbf{1 b}(1.16 \mathrm{~g}, 1.47 \mathrm{mmol})$ and $p$ nitrophenol-activated DGA (2) $(1.91 \mathrm{~g}, 4.0 \mathrm{mmol})$ in the presence of triethylamine $(0.42 \mathrm{~g}$, $4.1 \mathrm{mmol})$. After purification by column chromatography $\left(\mathrm{CHCl}_{3}: \mathrm{MeOH}, 98: 2\right) \mathbf{3 b}$ was obtained in 56\% yield $(1.20 \mathrm{~g})$ as a dense oil. ${ }^{1} \mathrm{H}$ NMR: $\delta 0.83-0.92\left(12 \mathrm{H}, \mathrm{m}, \mathrm{CH}_{3}\right), 0.95$ $(18 \mathrm{H}, \mathrm{s}, t-\mathrm{Bu}), 1.28\left(58 \mathrm{H}, \mathrm{m}, t-\mathrm{Bu}\right.$ and $\left.\mathrm{CH}_{3}\left(\mathrm{CH}_{2}\right)_{5}\right), 1.47-1.58\left(8 \mathrm{H}, \mathrm{m}, \mathrm{NCH}_{2} \mathrm{CH}_{2}\right), 1.90-2.07$ $\left(8 \mathrm{H}, \mathrm{m}, \mathrm{OCH}_{2} \mathrm{CH}_{2}, \mathrm{~N} \mathrm{CH}_{2} \mathrm{CH}_{2}\right), 3.05\left(4 \mathrm{H}, \mathrm{t}, J=6.0 \mathrm{~Hz}, \mathrm{NCH}_{2}\right), 3.29\left(12 \mathrm{H}, \mathrm{m}, \mathrm{ArCH}_{2} \mathrm{Ar}\right.$, $\left.\mathrm{NCH}_{2}\right), 3.48\left(4 \mathrm{H}, \mathrm{t}, J=6.0 \mathrm{~Hz}, \mathrm{NCH}_{2}\right), 3.99\left(4 \mathrm{H}, \mathrm{t}, J=6.0 \mathrm{~Hz}, \mathrm{OCH}_{2}\right), 4.08(4 \mathrm{H}, \mathrm{s}$, $\left.\mathrm{OCH}_{2} \mathrm{CO}\right), 4.20\left(4 \mathrm{H}, \mathrm{s}, \mathrm{COCH}_{2} \mathrm{O}\right), 4.26\left(4 \mathrm{H}, \mathrm{d}, J=12.0 \mathrm{~Hz}, \mathrm{ArCH}_{2} \mathrm{Ar}\right), 6.78(4 \mathrm{H}, \mathrm{s}, \mathrm{ArH})$, $7.03(4 \mathrm{H}, \mathrm{s}, \mathrm{ArH}), 7.42(2 \mathrm{H}, \mathrm{s}, \mathrm{OH}), 7.76(2 \mathrm{H}, \mathrm{t}, J=6.0 \mathrm{~Hz}, \mathrm{NH}) .{ }^{13} \mathrm{C}$ NMR $\delta: 14.3,22.9$, 27.3, 29.5, 31.3, 32.0, 34.8, 46.3, 47.0, 69.7, 71.8, 125.6, 128.0, 132.8, 141.6, 147.0, 150.1, 150.9, 168.3, 169.9. IR $\left(v_{\max } / \mathrm{cm}^{-1}\right): 2952,2923,2854,1647,1484,1465,1361,1200,1123$. HRMS: $m / z$ 1470.1365 [M+H] $]^{+}$; calculated: 1470.1274 for $\mathrm{C}_{92} \mathrm{H}_{149} \mathrm{~N}_{4} \mathrm{O}_{10}$.

\section{Tetra-DGA-calix[4]arene (5a).}

5a was prepared in an analogous way to $\mathbf{3 a}$, starting from $4 \mathbf{a}(0.76 \mathrm{~g}, 0.9 \mathrm{mmol})$ and $p$ nitrophenol-activated DGA (2) $(2.10 \mathrm{~g}, 4.5 \mathrm{mmol})$ in the presence of triethylamine $(0.5 \mathrm{~g}, 4.9$ mmol). After purification by column chromatography $\left(\mathrm{CHCl}_{3}: \mathrm{MeOH}, 97: 3\right)$, 5a was obtained in 52\% yield (1.04 g) as a dense oil. ${ }^{1} \mathrm{H}$ NMR: $\delta 0.83-0.92\left(24 \mathrm{H}, \mathrm{m}, \mathrm{CH}_{3}\right), 1.07(36 \mathrm{H}, \mathrm{s}, t-\mathrm{Bu})$, 1.21-1.35 (80H, m, $\left.\mathrm{CH}_{3}\left(\mathrm{CH}_{2}\right)_{5}\right), 1.43-1.51\left(16 \mathrm{H}, \mathrm{m}, \mathrm{NCH}_{2} \mathrm{CH}_{2}\right), 3.08-3.17(12 \mathrm{H}, \mathrm{m}$, $\left.\mathrm{ArCH}_{2} \mathrm{Ar}, \mathrm{CH}_{2} \mathrm{~N}\right), 3.26\left(8 \mathrm{H}, \mathrm{t}, J=6.0 \mathrm{~Hz}, \mathrm{NCH}_{2}\right), 3.89\left(8 \mathrm{H}, \mathrm{t}, J=6.0 \mathrm{~Hz}, \mathrm{NCH}_{2}\right), 4.06(8 \mathrm{H}, \mathrm{t}$, $\left.J=6.0 \mathrm{~Hz}, \mathrm{OCH}_{2}\right), 4.09\left(8 \mathrm{H}, \mathrm{s}, \mathrm{COCH}_{2} \mathrm{O}\right), 4.28\left(8 \mathrm{H}, \mathrm{s}, \mathrm{OCH}_{2} \mathrm{CON}\right), 4.34(4 \mathrm{H}, \mathrm{d}, J=12.0$ $\left.\mathrm{Hz}, \mathrm{ArCH}_{2} \mathrm{Ar}\right), 6.76(8 \mathrm{H}, \mathrm{s}, \mathrm{ArH}), 7.98(4 \mathrm{H}, \mathrm{t}, J=6.0 \mathrm{~Hz}, \mathrm{NH}) .{ }^{13} \mathrm{C} \mathrm{NMR}: \delta 14.3,22.9,27.1$, 114 
29.3, 31.6, 32.0, 34.1, 81.8, 103.1, 125.3, 133.7, 145.0, 148.6, 168.5, 170.4. IR $\left(v_{\max } / \mathrm{cm}^{-1}\right)$ : 2953, 2924, 2855, 1647, 1464, 1377, 1197, 1122, 1022. HRMS: m/z $2179.6992[\mathrm{M}+\mathrm{H}]^{+}$; calculated: 2179.7072 for $\mathrm{C}_{132} \mathrm{H}_{225} \mathrm{~N}_{8} \mathrm{O}_{16}$.

\section{Tetra-DGA-calix[4]arene (5b).}

5b was prepared in an analogous way to $\mathbf{3 a}$, starting from $4 \mathbf{b}(0.45 \mathrm{~g}, 0.5 \mathrm{mmol})$, pnitrophenol-activated DGA (2) (1.20 g, $2.5 \mathrm{mmol})$ and triethylamine (0.25 g, $2.5 \mathrm{mmol})$. The crude product was purified by column chromatography $\left(\mathrm{CH}_{2} \mathrm{Cl}_{2}: \mathrm{MeOH}, 96: 4\right)$ to afford calix[4]arene 4-DGA (5b) $(1.10 \mathrm{~g}, 71 \%)$ as a dense oil. ${ }^{1} \mathrm{H}$ NMR: $\delta 0.81-0.93\left(24 \mathrm{H}, \mathrm{m}, \mathrm{CH}_{3}\right)$, $1.07(36 \mathrm{H}, \mathrm{s}, t-\mathrm{Bu}), 1.17-1.35\left(80 \mathrm{H}, \mathrm{m}, \mathrm{CH}_{3}\left(\mathrm{CH}_{2}\right)_{5}\right), 1.43-1.60\left(16 \mathrm{H}, \mathrm{m}, \mathrm{NCH}_{2} \mathrm{CH}_{2}\right), 2.25$ $\left(8 \mathrm{H}\right.$, pentet, $\left.J=6.0 \mathrm{~Hz}, \mathrm{OCH}_{2} \mathrm{CH}_{2}\right), 3.04-3.16\left(12 \mathrm{H}, \mathrm{m}, \mathrm{ArCH}_{2} \mathrm{Ar}\right.$ and $\left.\mathrm{NCH}_{2}\right), 3.27(8 \mathrm{H}, \mathrm{t}, J=$ $\left.7.5 \mathrm{~Hz}, \mathrm{NCH}_{2}\right), 3.46\left(8 \mathrm{H}, \mathrm{t}, J=6.0 \mathrm{~Hz}, \mathrm{NHCH}_{2}\right), 3.89\left(8 \mathrm{H}, \mathrm{t}, J=6.0 \mathrm{~Hz}, \mathrm{ArOCH}_{2}\right), 4.09(8 \mathrm{H}$, s, $\left.\mathrm{OCH}_{2} \mathrm{CO}\right), 4.27\left(8 \mathrm{H}, \mathrm{s}, \mathrm{OCH}_{2} \mathrm{CO}\right), 4.34\left(4 \mathrm{H}, \mathrm{d}, J=12.0 \mathrm{~Hz}, \mathrm{ArCH}_{2} \mathrm{Ar}\right), 6.75(8 \mathrm{H}, \mathrm{s}, \mathrm{ArH})$, 7.97-8.07 (4H, m, NH); ${ }^{13} \mathrm{C}$ NMR: $\delta$ 14.3, 22.9, 27.1, 29.5, 31.6, 32.0, 34.0, 36.7, 46.3, 47.0, $60.1,133.9,144.8,153.5,168.4,170.0 ; \quad$ HRMS: $m / z 2234.7507[\mathrm{M}+\mathrm{H}]^{+}$, calculated: 2234.7665 for $\mathrm{C}_{136} \mathrm{H}_{233} \mathrm{~N}_{8} \mathrm{O}_{16}$.

\section{Calix[4]arene tetrakis(propionamide) (6a).}

To a solution of tetraamine $(\mathbf{4 b})(1.00 \mathrm{~g}, 1.1 \mathrm{mmol})$ in THF $(50 \mathrm{~mL})$ containing triethylamine $(0.50 \mathrm{~g}, 4.9 \mathrm{mmol})$ as a base was added propionyl chloride $(0.42 \mathrm{~g}, 4.4 \mathrm{mmol})$ in THF (15 $\mathrm{mL}$ ) dropwise at $0{ }^{\circ} \mathrm{C}$. The reaction mixture was stirred at room temperature overnight. The solvent was evaporated and the residue was dissolved in ethyl acetate $(100 \mathrm{~mL})$, washed with $5 \% \mathrm{HCl}(3 \times 50 \mathrm{~mL})$, and water $(3 \times 50 \mathrm{~mL})$. The organic layer was concentrated under reduced pressure to afford $6 \mathbf{a}\left(1.25 \mathrm{~g}\right.$, quantitative) as a dense oil. ${ }^{1} \mathrm{H}$ NMR: $\delta 1.07(36 \mathrm{H}, \mathrm{s}, t$ $\mathrm{Bu}), 1.19\left(12 \mathrm{H}, \mathrm{t}, J=7.5 \mathrm{~Hz}, \mathrm{CH}_{3}\right), 2.18-2.34\left(16 \mathrm{H}, \mathrm{m}, \mathrm{CH}_{3} \mathrm{CH}_{2}\right.$ and $\left.\mathrm{NCH}_{2} \mathrm{CH}_{2}\right), 3.12(4 \mathrm{H}, \mathrm{d}$, $\left.J=12.0 \mathrm{~Hz}, \mathrm{ArCH}_{2} \mathrm{Ar}\right), 3.34-3.48\left(8 \mathrm{H}, \mathrm{m}, \mathrm{CONCH}_{2}\right), 3.84\left(8 \mathrm{H}, \mathrm{t}, J=6.0 \mathrm{~Hz}, \mathrm{OCH}_{2}\right), 4.29$ $\left(4 \mathrm{H}, \mathrm{d}, J=12.0 \mathrm{~Hz}, \mathrm{ArCH}_{2} \mathrm{Ar}\right), 6.77(8 \mathrm{H}, \mathrm{s}, \mathrm{ArH}), 6.98(4 \mathrm{H}, \mathrm{brt}, J=6.0 \mathrm{~Hz}, \mathrm{NH}) .{ }^{13} \mathrm{C} \mathrm{NMR}$ : $\delta 11.0,14.3,29.2,32.1,33.0,40.8,60.1,133.9,144.8,153.5,168.4,173.9$. IR $\left(v_{\max } / \mathrm{cm}^{-1}\right)$ : 3291, 2952, 2922, 1644, 1548, 1462, 1360, 1192, 1122. HRMS: m/z $1101.7689[\mathrm{M}+\mathrm{H}]^{+}$; calculated: 1101.7619 for $\mathrm{C}_{68} \mathrm{H}_{101} \mathrm{~N}_{4} \mathrm{O}_{8}$. 


\section{Chapter 5}

\section{Calix[4]arene tetrakis(octanonamide) (6b).}

$\mathbf{6 b}$ was prepared in an analogous way to $\mathbf{6 a}$, starting from $\mathbf{4 b}(1.00 \mathrm{~g}, 1.1 \mathrm{mmol})$, triethylamine $(0.50 \mathrm{~g}, 4.9 \mathrm{mmol})$ and octanoyl chloride $(0.74 \mathrm{~g}, 4.4 \mathrm{mmol})$ to afford $\mathbf{6 b}$ as a dense oil (1.53 g, 97\%). ${ }^{1} \mathrm{H}$ NMR: $\delta$ 0.84-0.92 (12H, m, $\left.\mathrm{CH}_{3}\right), 1.07(36 \mathrm{H}, \mathrm{s}, t-\mathrm{Bu}), 1.21-1.39(40 \mathrm{H}, \mathrm{m}$, $\left.\mathrm{CH}_{3}\left(\mathrm{CH}_{2}\right)_{5}\right), 1.56-1.74\left(8 \mathrm{H}, \mathrm{m}, \mathrm{COCH}_{2} \mathrm{CH}_{2}\right), 2.23\left(16 \mathrm{H}, \mathrm{t}, J=7.5 \mathrm{~Hz}, \mathrm{COCH}_{2}\right.$ and $\left.\mathrm{NCH}_{2} \mathrm{CH}_{2}\right), 3.12\left(4 \mathrm{H}, \mathrm{d}, J=12.0 \mathrm{~Hz}, \mathrm{ArCH}_{2} \mathrm{Ar}\right), 3.34-3.47\left(8 \mathrm{H}, \mathrm{m}, \mathrm{CONCH}_{2}\right), 3.83(8 \mathrm{H}, \mathrm{t}, J$ $\left.=6.0 \mathrm{~Hz}, \mathrm{OCH}_{2}\right), 4.28\left(4 \mathrm{H}, \mathrm{d}, J=12.0 \mathrm{~Hz}, \mathrm{ArCH}_{2} \mathrm{Ar}\right), 6.76(8 \mathrm{H}, \mathrm{s}, \mathrm{ArH}), 7.03(4 \mathrm{H}, \mathrm{br} \mathrm{t}, J=$ $6.0 \mathrm{~Hz}, \mathrm{NH}) .{ }^{13} \mathrm{C}$ NMR: $\delta 14.3,22.8,27.1,27.4,29.3,31.6,32.0,32.6,60.1,133.9,144.8$, 153.5, 168.4, 173.2. IR $\left(v_{\max } / \mathrm{cm}^{-1}\right): 3307,2953,2923,2855,1640,1541,1466,1360,1197$, 1122. HRMS: $m / z$ 1382.0681 [M+H] $]^{+}$; calculated: 1382.0749 for $\mathrm{C}_{88} \mathrm{H}_{141} \mathrm{~N}_{4} \mathrm{O}_{8}$.

\section{Tetrakis(n-propylamino-n-propoxy)calix[4]arene (7a).}

A solution of $6 \mathbf{a}(1.00 \mathrm{~g}, 0.91 \mathrm{mmol})$ in diethyl ether $(80 \mathrm{~mL})$ was added dropwise to a suspension of $\mathrm{LiAlH}_{4}(0.27 \mathrm{~g}, 7.2 \mathrm{mmol})$ in diethyl ether $(75 \mathrm{~mL})$ at $0{ }^{\circ} \mathrm{C}$. The resulting mixture was stirred at room temperature for $2 \mathrm{~d}$. The reaction mixture was cooled to $0{ }^{\circ} \mathrm{C}$, whereupon $2 \mathrm{n} \mathrm{NaOH}(50 \mathrm{~mL})$ was added drop wise. Upon filtration, the product was extracted with diethyl ether $(2 \times 50 \mathrm{~mL})$. The solvent was evaporated to afford $7 \mathbf{a}$ as a dense oil (0.90 g, 95\%). ${ }^{1} \mathrm{H}$ NMR: $\delta 0.93\left(12 \mathrm{H}, \mathrm{t}, J=7.5 \mathrm{~Hz}, \mathrm{CH}_{3}\right), 1.07(36 \mathrm{H}, \mathrm{s}, t-\mathrm{Bu}), 1.52(8 \mathrm{H}$, q, $\left.J=7.5 \mathrm{~Hz}, \mathrm{CH}_{3} \mathrm{CH}_{2}\right), 2.19\left(8 \mathrm{H}, \mathrm{t}, J=7.5 \mathrm{~Hz}, \mathrm{OCH}_{2} \mathrm{CH}_{2}\right), 2.60$ and $2.73(8 \mathrm{H}, \mathrm{t}, J=7.5 \mathrm{~Hz}$, $\left.\mathrm{NCH}_{2}\right), 3.12\left(4 \mathrm{H}, \mathrm{d}, J=12.0 \mathrm{~Hz}, \mathrm{ArCH}_{2} \mathrm{Ar}\right), 3.93\left(8 \mathrm{H}, \mathrm{t}, J=7.5 \mathrm{~Hz}, \mathrm{OCH}_{2}\right), 4.38(4 \mathrm{H}, \mathrm{d}, J=$ $\left.12.0 \mathrm{~Hz}, \mathrm{ArCH}_{2} \mathrm{Ar}\right), 6.76$ (8H, s, ArH). ${ }^{13} \mathrm{C} \mathrm{NMR}: \delta$ 10.9, 23.1, 31.7, 34.0, 44.5, 49.3, 73.7, 125.1, 134.0, 144.5, 153.6. IR ( $\left.v_{\max } / \mathrm{cm}^{-1}\right): 2952,2924,2867,1461,1196,1122,1012,869$. HRMS: $m / z 1045.8501[\mathrm{M}+\mathrm{H}]^{+}$; calculated: 1045.8449 for $\mathrm{C}_{68} \mathrm{H}_{109} \mathrm{~N}_{4} \mathrm{O}_{4}$.

\section{Tetrakis(n-octylamino-n-propoxy)calix[4]arene (7b).}

7b was prepared in an analogous way to 7a starting from $\mathbf{6 b}(1.40 \mathrm{~g}, 1.0 \mathrm{mmol})$ and $\mathrm{LiAlH}_{4}$ $(0.31 \mathrm{~g}, 8.1 \mathrm{mmol})$ to afford $7 \mathbf{b}$ as a dense oil $(1.18 \mathrm{~g}, 88 \%) .{ }^{1} \mathrm{H}$ NMR: $\delta$ 0.83-0.92 $(12 \mathrm{H}, \mathrm{m}$, $\left.\mathrm{CH}_{3}\right), 1.07(36 \mathrm{H}, \mathrm{s}, t-\mathrm{Bu}), 1.22-1.37\left(40 \mathrm{H}, \mathrm{m}, \mathrm{CH}_{3}\left(\mathrm{CH}_{2}\right)_{5}\right), 1.48-1.67\left(8 \mathrm{H}, \mathrm{m}, \mathrm{NCH}_{2} \mathrm{CH}_{2^{-}}\right.$ octyl), $2.21\left(8 \mathrm{H}, \mathrm{t}, J=7.5 \mathrm{~Hz}, \mathrm{OCH}_{3} \mathrm{CH}_{2}\right), 2.61$ and $2.74\left(8 \mathrm{H}, \mathrm{t}, J=7.5 \mathrm{~Hz}, \mathrm{NCH}_{2}\right), 3.11(4 \mathrm{H}$, $\left.\mathrm{d}, J=12.0 \mathrm{~Hz}, \mathrm{ArCH}_{2} \mathrm{Ar}\right), 3.92\left(8 \mathrm{H}, \mathrm{t}, J=7.5 \mathrm{~Hz}, \mathrm{OCH}_{2}\right), 4.37(4 \mathrm{H}, \mathrm{d}, J=12.0 \mathrm{~Hz}$, $\left.\mathrm{ArCH}_{2} \mathrm{Ar}\right), 6.76$ (8H, s, ArH). ${ }^{13} \mathrm{C}$ NMR: $\delta 14.3,22.9,27.3,29.5,31.3,31.7,32.0,34.0,44.5$, 48.5, 73.7, 125.1, 134.0, 144.5, 153.6. IR $\left(v_{\max } / \mathrm{cm}^{-1}\right): 2942,2922,2853,1480,1199,1122$, 1015, 869. HRMS: $m / z$ 1326.1622 [M+H] ${ }^{+}$; calculated: 1326.1579 for $\mathrm{C}_{88} \mathrm{H}_{149} \mathrm{~N}_{4} \mathrm{O}_{4}$. 116 


\section{Tetrakis(3-pentylamino-n-propoxy)calix[4]arene (7c).}

To a solution of tetraamine $(\mathbf{4 b})(1.10 \mathrm{~g}, 1.2 \mathrm{mmol})$ in THF $(15 \mathrm{~mL})$ were added Ti(isopropoxide $)_{4}(5 \mathrm{~mL}, 17.0 \mathrm{mmol})$ and 3-pentanone $(0.45 \mathrm{~g}, 5.2 \mathrm{mmol})$. The resulting mixture was stirred at room temperature for $7 \mathrm{~h}$. Methanol $(15 \mathrm{~mL})$ was added to the reaction mixture, followed by portion-wise addition of $\mathrm{NaBH}_{4}(0.40 \mathrm{~g}, 10.5 \mathrm{mmol})$ and the reaction mixture was stirred for $1 \mathrm{~h}$. The reaction was quenched by $0.5 \mathrm{n} \mathrm{NaOH}(30 \mathrm{~mL})$. Upon filtration, the product was extracted with dichloromethane $(3 \times 50 \mathrm{~mL})$. The solvent was evaporated to yield 7c as a dense oil (1.35 g, 93\%). ${ }^{1} \mathrm{H}$ NMR: $\delta 0.88\left(24 \mathrm{H}, \mathrm{t}, J=7.5 \mathrm{~Hz}, \mathrm{CH}_{3}\right), 1.07(36 \mathrm{H}, \mathrm{s}, t$ $\mathrm{Bu}), 1.41\left(16 \mathrm{H}\right.$, pentet, $\left.J=7.5 \mathrm{~Hz}, \mathrm{CH}_{3} \mathrm{CH}_{2}\right), 2.17\left(8 \mathrm{H}, \mathrm{t}, J=7.5 \mathrm{~Hz}, \mathrm{OCH}_{3} \mathrm{CH}_{2}\right), 2.36(4 \mathrm{H}$, pentet, $J=6.0 \mathrm{~Hz}, \mathrm{NCH}), 2.68\left(8 \mathrm{H}, \mathrm{t}, J=6.0 \mathrm{~Hz}, \mathrm{NCH}_{2}\right), 3.12(4 \mathrm{H}, \mathrm{d}, J=15.0 \mathrm{~Hz}$, $\left.\mathrm{ArCH}_{2} \mathrm{Ar}\right), 3.96\left(8 \mathrm{H}, \mathrm{t}, J=7.5 \mathrm{~Hz}, \mathrm{OCH}_{2}\right), 4.40\left(4 \mathrm{H}, \mathrm{d}, J=15.0 \mathrm{~Hz}, \mathrm{ArCH}_{2} \mathrm{Ar}\right), 6.76(8 \mathrm{H}, \mathrm{s}$, ArH). ${ }^{13} \mathrm{C}$ NMR: $\delta 10.1,26.1,31.7,34.0,44.5,60.5,73.7,125.1,134.0,144.5$, 153.6. IR $\left(v_{\max } / \mathrm{cm}^{-1}\right): 2956,2924,2870,1479,1196,1122,1010,869$. HRMS: $m / z 1157.9633[\mathrm{M}+\mathrm{H}]^{+}$; calculated: 1157.9701 for $\mathrm{C}_{76} \mathrm{H}_{125} \mathrm{~N}_{4} \mathrm{O}_{4}$.

\section{Tetra-n-propylDGA-calix[4]arene (8a).}

8a was prepared in an analogous way as 3a starting from 7a $(1.10 \mathrm{~g}, 1.1 \mathrm{mmol})$ and $p$ nitrophenol-activated DGA (2) $(2.39 \mathrm{~g}, 5.0 \mathrm{mmol})$ in the presence of triethylamine $(0.51 \mathrm{~g}$, $5.0 \mathrm{mmol})$. After purification by column chromatography $\left(\mathrm{CH}_{2} \mathrm{Cl}_{2}: \mathrm{MeOH}, 95: 5 \rightarrow 8: 2\right) \mathbf{8 a}$ was obtained in $63 \%$ yield $(1.58 \mathrm{~g})$ as a dense oil. ${ }^{1} \mathrm{H}$ NMR: $\delta 0.82-0.94\left(\mathrm{~m}, 36 \mathrm{H}, \mathrm{CH}_{3}\right), 1.07$ (s, $36 \mathrm{H}, t-\mathrm{Bu}), 1.18-1.36\left(\mathrm{~m}, 120 \mathrm{H}, \mathrm{CH}_{3}\left(\mathrm{CH}_{2}\right)_{5}\right), 1.45-1.62\left(\mathrm{~m}, 16 \mathrm{H}, \mathrm{NCH}_{2} \mathrm{CH}_{2}\right), 2.10-2.25 \mathrm{~m}$, 8H, $\mathrm{OCH}_{2} \mathrm{CH}_{2}$ ), 3.01-3.41 (m, 36H, $\mathrm{ArCH}_{2} \mathrm{Ar}$ and $\mathrm{NCH}_{2}$ ), 3.91-4.04 (m, 8H, $\mathrm{ArOCH}_{2}$ ), 4.194.37 (m, 20H, $\left.\mathrm{OCH}_{2} \mathrm{CO}, \mathrm{ArCH}_{2} \mathrm{Ar}\right), 6.75$ (s, 8H, ArH). ${ }^{13} \mathrm{C} \mathrm{NMR}: \delta 11.4,14.3,22.8,27.1$, 27.8, 29.5, 81.6, 81.9, 34.0, 46.0, 47.1, 69.3, 125.3, 133.7, 144.7, 153.4, 168.6, 168.8, 169.6. IR $\left(v_{\max } / \mathrm{cm}^{-1}\right): 2956,2924,2870,1651,1479,1459,1360,1196,1122$. HRMS: $\mathrm{m} / \mathrm{z}$ 2402.9521 [M+H] $]^{+}$; calculated: 2402.9543 for $\mathrm{C}_{148} \mathrm{H}_{257} \mathrm{~N}_{8} \mathrm{O}_{16}$.

\section{Tetra-n-octylDGA-calix[4]arene (8b).}

$\mathbf{8 b}$ was prepared in an analogous way as 8a starting from $7 \mathbf{b}(1.00 \mathrm{~g}, 0.7 \mathrm{mmol})$ and $p$ nitrophenol-activated DGA (2) $(2.00 \mathrm{~g}, 4.2 \mathrm{mmol})$ in the presence of triethylamine $(0.51 \mathrm{~g}$, $5.0 \mathrm{mmol})$. Purification by column chromatography $\left(\mathrm{CH}_{2} \mathrm{Cl}_{2}: \mathrm{MeOH}, 95: 5 \rightarrow 8: 2\right)$ gave $\mathbf{8 b}$ in $59 \%$ yield $(1.19 \mathrm{~g})$ as a dense oil. ${ }^{1} \mathrm{H}$ NMR: $\delta 0.82-0.93\left(\mathrm{~m}, 36 \mathrm{H}, \mathrm{CH}_{3}\right), 1.07(\mathrm{~s}, 36 \mathrm{H}, t-\mathrm{Bu})$, 1.18-1.38 (m, $\left.120 \mathrm{H}, \mathrm{CH}_{3}\left(\mathrm{CH}_{2}\right)_{5}\right), 1.41-1.63\left(\mathrm{~m}, 24 \mathrm{H}, \mathrm{NCH}_{2} \mathrm{CH}_{2}\right), 2.10-2.24 \mathrm{~m}, 8 \mathrm{H}$, 


\section{Chapter 5}

$\mathrm{OCH}_{2} \mathrm{CH}_{2}$ ), 3.02-3.44 (m, 36H, $\mathrm{ArCH}_{2} \mathrm{Ar}$ and $\mathrm{NCH}_{2}$ ), 3.90-4.03 (m, 8H, $\left.\mathrm{ArOCH}_{2}\right), 4.19-4.31$ $\left(\mathrm{m}, 16 \mathrm{H}, \mathrm{OCH}_{2} \mathrm{CO}\right), 4.34\left(\mathrm{~d}, 4 \mathrm{H}, J=12.0 \mathrm{~Hz}, \mathrm{ArCH}_{2} \mathrm{Ar}\right), 6.75(\mathrm{~s}, 8 \mathrm{H}, \mathrm{ArH}) .{ }^{13} \mathrm{C} \mathrm{NMR}: \delta$ 14.3, 22.8, 27.1, 27.5, 29.4, 31.6, 32.0, 33.9, 47.2, 67.9, 69.2, 70.7, 125.2, 133.6, 144.8, 153.3, 168.6, 169.6. IR $\left(v_{\max } / \mathrm{cm}^{-1}\right): 2959,2923,2853,1637,1479,1464,1363,1201,1123$. HRMS: $m / z$ 2683.2700 [M+H] $]^{+}$; calculated: 2683.2673 for $\mathrm{C}_{168} \mathrm{H}_{297} \mathrm{~N}_{8} \mathrm{O}_{16}$.

\section{Tetra-3-pentylDGA-calix[4]arene (8c).}

8c was prepared in an analogous way to 8a, starting from 7c $(1.5 \mathrm{~g}, 1.3 \mathrm{mmol})$ and $p$ nitrophenol-activated DGA (2) $(2.77 \mathrm{~g}, 5.8 \mathrm{mmol})$ in the presence of triethylamine $(0.60 \mathrm{~g}$, $6.0 \mathrm{mmol}$ ), using toluene as a solvent. Purification by column chromatography $\left(\mathrm{CH}_{2} \mathrm{Cl}_{2}: \mathrm{MeOH}, 95: 5 \rightarrow 7: 3\right)$ gave $8 \mathrm{c}$ in $51 \%$ yield $(1.65 \mathrm{~g})$ as a dense oil. ${ }^{1} \mathrm{H}$ NMR (DMSO$\left.d_{6}\right): \delta$ 0.71-0.90 $\left(48 \mathrm{H}, \mathrm{m}, \mathrm{CH}_{3}\right.$ octyl and isopentyl), $1.01(36 \mathrm{H}, \mathrm{s}, t-\mathrm{Bu}), 1.13-1.30(80 \mathrm{H}, \mathrm{m}$, $\left.\mathrm{CH}_{3}\left(\mathrm{CH}_{2}\right)_{5}\right), 1.34-1.59\left(32 \mathrm{H}, \mathrm{m}, \mathrm{NCH}_{2} \mathrm{CH}_{2}\right.$-octyl and $\mathrm{CH}_{3} \mathrm{CH}_{2}$-isopentyl), 1.95-2.13 (8H, m, $\mathrm{OCH}_{2} \mathrm{CH}_{2}$ ), 3.02-3.26 (32H, m, NCH, $\mathrm{NCH}_{2}$ and $\left.\mathrm{ArCH}_{2} \mathrm{Ar}\right), 3.92-4.04\left(4 \mathrm{H}, \mathrm{m}, \mathrm{OCH}_{2}\right), 4.14$ and $4.16\left(8 \mathrm{H}, \mathrm{s}, \mathrm{OCH}_{2} \mathrm{CO}\right), 4.29\left(4 \mathrm{H}, \mathrm{d}, J=12.0 \mathrm{~Hz}, \mathrm{ArCH}_{2} \mathrm{Ar}\right), 6.71(8 \mathrm{H}, \mathrm{s}, \mathrm{ArH}) .{ }^{13} \mathrm{C} \mathrm{NMR}$ $\left(\mathrm{CDCl}_{3}\right): \delta 11.3,14.3,22.8,26.5,27.2,27.8,31.6,32.0,34.0,45.9,47.0,69.3,125.1,133.4$, 133.8, 144.5, 152.7, 168.6, 169.6. IR $\left(v_{\max } / \mathrm{cm}^{-1}\right): 2955,2923,2854,1642,1479,1460,1359$, 1200, 1121. HRMS: $m / z 2515.0811[\mathrm{M}+\mathrm{H}]^{+}$; calculated: 2515.0795 for $\mathrm{C}_{156} \mathrm{H}_{273} \mathrm{~N}_{8} \mathrm{O}_{16}$.

\section{Tetra-DGA-Calix[4]arene (wide rim) (10).}

10 was prepared in an analogous way as 3a starting from $9(0.50 \mathrm{~g}, 0.7 \mathrm{mmol})$ and $p$ nitrophenol-activated DGA (2) $(1.82 \mathrm{~g}, 3.8 \mathrm{mmol})$ in the presence of triethylamine $(0.40 \mathrm{~g}$, $4.0 \mathrm{mmol}$ ), using chloroform as a solvent. Purification by column chromatography $\left(\mathrm{CH}_{2} \mathrm{Cl}_{2}: \mathrm{MeOH}, 98: 2\right)$ afforded 10 in $83 \%$ yield (1.20 g) as a dense oil. ${ }^{1} \mathrm{H}$ NMR: $\delta 0.82-0.93$ (24H, m, $\mathrm{CH}_{3}$-octyl), 0.97 (12H, t, $J=7.5 \mathrm{~Hz}, \mathrm{CH}_{3}$-butyl), $1.18-1.36\left(80 \mathrm{H}, \mathrm{m}, \mathrm{CH}_{3}\left(\mathrm{CH}_{2}\right)_{5}\right)$, $1.42\left(8 \mathrm{H}\right.$, sextet, $J=7.5 \mathrm{~Hz}, \mathrm{CH}_{3} \mathrm{CH}_{2}$-butyl), 1.46-1.61 (16H, m, $\mathrm{NCH}_{2} \mathrm{CH}_{2}$-octyl), 1.85 (8H, pentet, $\left.J=7.5 \mathrm{~Hz}, \mathrm{OCH}_{2} \mathrm{CH}_{2}\right), 3.06-3.20\left(12 \mathrm{H}, \mathrm{m}, \mathrm{NCH}_{2}, \mathrm{ArCH}_{2} \mathrm{Ar}\right), 3.32(8 \mathrm{H}, \mathrm{t}, J=7.5 \mathrm{~Hz}$, $\left.\mathrm{NCH}_{2}\right), 3.84\left(8 \mathrm{H}, \mathrm{t}, J=7.5 \mathrm{~Hz}, \mathrm{OCH}_{2}\right), 4.17$ and $4.35\left(8 \mathrm{H}, \mathrm{s}, \mathrm{COCH}_{2} \mathrm{O}\right), 4.40(4 \mathrm{H}, \mathrm{d}, J=12.0$ $\left.\mathrm{Hz}, \mathrm{ArCH}_{2} \mathrm{Ar}\right), 6.83$ (8H, s, ArH), 9.28 (1H, br s, NH). ${ }^{13} \mathrm{C} \mathrm{NMR}: \delta$ 14.3, 19.6, 22.8, 27.1, 29.5, 32.1, 46.6, 47.3, 69.8, 71.9, 75.0, 121.7, 131.3, 135.2, 153.9, 167.7, 168.6. IR ( $v_{\max } / \mathrm{cm}^{-}$ $\left.{ }^{1}\right):$ 2954, 2922, 2853, 1646, 1541, 1464, 1420, 1211, 1126. HRMS: $m / z 2089.7678[\mathrm{M}+\mathrm{Na}]^{+}$; calculated: 2089.7715 for $\mathrm{C}_{124} \mathrm{H}_{208} \mathrm{~N}_{8} \mathrm{O}_{16} \mathrm{Na}$. 


\section{Calix[4]arene octaamine (12).}

Hydrazine hydrate $(15 \mathrm{~mL}, 300 \mathrm{mmol})$ was added to a suspension of $\mathbf{1 1}(2.00 \mathrm{~g}, 1.48 \mathrm{mmol})$ in ethanol $(60 \mathrm{~mL})$. The mixture was refluxed for $12 \mathrm{~h}$, cooled and then diluted with water $(100 \mathrm{ml})$. The precipitate formed was filtered and subsequently extracted with dichloromethane $(4 \times 50 \mathrm{~mL})$. The organic layer was then dried $\left(\mathrm{MgSO}_{4}\right)$ and the solvent evaporated to obtain the calix[4]arene tetraamine, which was used without further purification. It was directly dissolved into methanol $(50 \mathrm{~mL})$ and hydrazine hydrate $(5 \mathrm{~mL}$, $100 \mathrm{mmol})$ and a catalytic amount of Raney nickel $(0.5 \mathrm{~g})$ were added. The mixture was refluxed for $5 \mathrm{~h}$. After cooling to room temperature, the mixture was filtered. The filtrate was evaporated and the crude product was dissolved in dichloromethane $(50 \mathrm{~mL})$. The resulting solution was washed with water $(2 \times 50 \mathrm{~mL})$ to afford pure calix[4]arene octaamine 12 in quantitative yield.

\section{Octa-DGA-Calix[4]arene (13).}

13 was prepared in an analogous way as 3a starting from $12(0.45 \mathrm{~g}, 0.65 \mathrm{mmol})$ and $p$ nitrophenol-activated DGA (2) $(3.10 \mathrm{~g}, 6.5 \mathrm{mmol})$ in the presence of triethylamine $(0.65 \mathrm{~g}$, $6.5 \mathrm{mmol}$ ), using chloroform as a solvent. Purification by column chromatography $\left(\mathrm{CH}_{2} \mathrm{Cl}_{2}: \mathrm{MeOH}, 95: 5 \rightarrow 85: 15\right)$ gave 13 in $59 \%$ yield $(1.27 \mathrm{~g})$ as a dense oil. ${ }^{1} \mathrm{H}$ NMR $\left(\mathrm{CD}_{3} \mathrm{OD}\right): \delta$ 0.82-0.99 (48H, m, $\left.\mathrm{CH}_{3}\right), 1.20-1.44\left(160 \mathrm{H}, \mathrm{m}, \mathrm{CH}_{3}\left(\mathrm{CH}_{2}\right)_{5}\right), 1.46-1.68(32 \mathrm{H}, \mathrm{m}$, $\left.\mathrm{NCH}_{2} \mathrm{CH}_{2}\right), 2.23\left(8 \mathrm{H}\right.$, pentet, $\left.J=6.6 \mathrm{~Hz}, \mathrm{OCH}_{2} \mathrm{CH}_{2}\right), 3.10-3.27\left(20 \mathrm{H}, \mathrm{m}, \mathrm{ArCH}_{2} \mathrm{Ar}, \mathrm{NCH}_{2}-\right.$ octyl), 3.27-3.43 (16H, m, $\mathrm{NCH}_{2}$-octyl), $3.48\left(8 \mathrm{H}, \mathrm{t}, J=6.6 \mathrm{~Hz}, \mathrm{NCH}_{2}\right), 3.94-4.05(8 \mathrm{H}, \mathrm{m}$, $\left.\mathrm{OCH}_{2}\right), 4.07\left(16 \mathrm{H}, \mathrm{s}, \mathrm{OCH}_{2} \mathrm{CO}\right), 4.34,4.37\left(8 \mathrm{H}, \mathrm{s}, \mathrm{OCH}_{2} \mathrm{CO}\right), 4.48(4 \mathrm{H}, \mathrm{d}, J=12.0 \mathrm{~Hz}$, $\left.\mathrm{ArCH}_{2} \mathrm{Ar}\right), 6.97$ (8H, s, ArH). ${ }^{13} \mathrm{C} \mathrm{NMR}\left(\mathrm{CD}_{3} \mathrm{OD}\right): \delta$ 11.1, 12.7, 14.4, 16.0, 20.9, 22.6, 24.4, 27.0, 27.7, 29.3, 31.0, 31.9, 33.6, 69.0, 70.7, 73.1, 93.6, 120.2, 122.4, 131.9, 135.0, 153.4, 168.4, 169.2, 170.6. IR $\left(v_{\max } / \mathrm{cm}^{-1}\right): 2926,2890,2450,2068,1642,1469,1122,976,733$. HRMS: $m / z 3427.6606[\mathrm{M}+\mathrm{H}]^{+}$; calculated: 3427.6690 for $\mathrm{C}_{200} \mathrm{H}_{353} \mathrm{~N}_{16} \mathrm{O}_{28}$.

\subsubsection{Radiotracers}

The ${ }^{241} \mathrm{Am}$ tracer was purified by a procedure described elsewhere and its purity was checked by both gamma as well as alpha spectrometric analysis. ${ }^{18}{ }^{152,154} \mathrm{Eu}$ was purchased from BRIT (Board of Radiation Technology, India) and was used after checking its radiochemical purity. 


\section{Chapter 5}

Radiometric assay of ${ }^{241} \mathrm{Am}$ and ${ }^{152,154} \mathrm{Eu}$ was done by gamma counting using a $\mathrm{NaI}(\mathrm{Tl})$ scintillation detector (Para Electronics, India).

\subsubsection{Solvent extraction studies}

Solutions of the desired concentration $\left(1.0 \times 10^{-3} \mathrm{~mol} / \mathrm{L}\right)$ of the DGA-functionalized calixarenes were prepared in $n$-dodecane containing $5 \%$ iso-decanol as the phase modifier. The organic phases, containing the extractant, were agitated with an equal volume of the aqueous phase spiked with the requisite quantity of tracer (about $1.0 \times 10^{-8} \mathrm{Ci}^{241} \mathrm{Am}$ or ${ }^{152,154} \mathrm{Eu}$ ) in a rotary thermostated water bath for an hour at $25.0 \pm 0.1{ }^{\circ} \mathrm{C}$. The two phases were then centrifuged and assayed by taking suitable aliquots (typically $100 \mu \mathrm{L}$ ) from both the phases followed by counting using a $\mathrm{NaI}(\mathrm{Tl})$ scintillation counter interphased with a multichannel analyzer. The distribution ratio $(D)$ is defined as the ratio of the concentration of metal ion in the organic phase to that in the aqueous phase. Solvent extraction studies were carried out in triplicate and the standard deviation of the accepted data points was within $\pm 5 \%$ of the average value.

\subsection{References and notes}

1. Stephan, H.; Gloe, K.; Beger, J.; Mühl, P. Solv. Extr. Ion Exch. 1991, 9, 459.

2. (a) Tachimori, S.; Sasaki, Y.; Suzuki, S. Solv. Extr. Ion Exch. 2002, 20, 687; (b) Narita, H.; Yaita, T.; Tachimori, S. Solv. Extr. Ion Exch. 2004, 22, 135; (c) Sasaki, Y.; Rapold, P.; Arisaka, M.; Hirata, M.; Kimura, T.; Hill, C.; Cote, G. Solv. Extr. Ion Exch. 2007, 25, 187.

3. Ansari, S. A.; Pathak, P.; Mohapatra, P. K.; Manchanda, V. K. Chem. Rev. 2012, 112, 1751.

4. Jensen, M. P.; Yaita, T.; Chiarizia, R. Langmuir 2007, 23, 4765.

5. Yaita, T.; Herlinger, A. W.; Thiyagarajan, P.; Jensen, M. P. Solv. Extr. Ion Exch. 2004, $22,553$.

6. Pathak, P. N.; Ansari, S. A.; Kumar, S.; Tomar, B. S.; Manchanda, V. K. J. Colloid Interf. Sci. 2010, 342, 114.

7. (a) Mokhtari, B.; Porabdollah, K.; Dalali, N. J. Incl. Phenom. Macrocycl. Chem. 2011, 69, 1; (b) Schmidt, C.; Saadioui, M.; Böhmer, V.; Host, V.; Spirlet, M. R.; Desreux, J. F.; Brisach, F.; Arnaud-Neu, F.; Dozol, J.-F. Org. Biomol. Chem. 2003, 1, 4089. 
8. (a) Casnati, A.; Della Ca, N.; Fontanella, M.; Sansone, F.; Ugozzoli, F.; Ungaro, R.; Liger, K.; Dozol, J.-F. Eur. J. Org. Chem. 2005, 2338; (b) Macerata, E.; Sansone, F.; Baldini, L.; Ugozzoli, F.; Brisach, F.; Haddaoui, J.; Hubscher-Bruder, V.; Arnaud-Neu, F.; Mariani, M.; Ungaro, R.; Casnati, A. Eur. J. Org. Chem. 2010, 2675; (c) Galletta, M.; Baldini, L.; Sansone, F.; Ugozzolo, F.; Ungaro, R.; Casnati, A.; Mariani, M. Dalton Trans. 2010, 39, 2546.

9. Dam, H. H.; Reinhoudt, D. N.; Verboom, W. New J. Chem. 2007, 31, 1620.

10. Janczewski, D.; Reinhoudt, D. N.; Verboom, W.; Hill, C.; Allignol, C.; Duchesne, M.-T. New J. Chem. 2008, 32, 490.

11. Mohapatra, P. K.; Iqbal, M.; Raut, D. R.; Verboom, W.; Huskens, J.; Manchanda, V. K. J. Membr. Sci. 2011, 375, 141.

12. Metay, E.; Duclos, M. C.; Pellet-Rostaing, S.; Lemaire, M.; Schulz, J.; Kannappan, R.; Bucher, C.; Saint-Aman, E.; Chaix, C. Eur. J. Org. Chem. 2008, 25, 4304.

13. Barboso, S.; Carrera, A. G.; Matthews, S. E.; Arnaud-Neu, F.; Böhmer, V.; Dozol, J.; Rouquette, H.; Schwing-Weill, M. J. J. Chem. Soc., Perkin Trans. 2 1999, 719.

14. Zhengyi, L.; Jiejie, M.; Jiawen, C.; Yi, P.; Juli, J.; Leyong, W. Chin. J. Chem. 2009, 27, 2031.

15. A related compound has been prepared in a two-step procedure in a much lower overall yield: Alyapyshev, M. Yu.; Babain, V. A.; Boyko, V. I.; Eliseev, I. I.; Kirsanov, D. O.; Klimchuk, O. V.; Legin, A. V.; Mikhailina, E. S.; Rodik, R. V.; Smirnov, I. V. J. Incl. Phenom. Macrocycl. Chem. 2010, 67, 117. (b) Kirsanov, D.; Khaydukova, M.; Tkachenko, L.; Legin, A.; Babain, V. Electroanalysis 2012, 24, 121.

16. Danila, C.; Bolte, M.; Böhmer. V. Org. Biomol. Chem. 2005, 3, 172.

17. Mohapatra, P. K.; Iqbal, M.; Raut, D. R.; Verboom, W.; Huskens, J.; Godbole, S. V. Dalton Trans. 2012, 41, 360.

18. Mohapatra, P. K. Ph. D. Thesis, University of Bombay, 1993. 



\section{Chapter}

\section{6}

\section{Synthesis and Evaluation of Ligands with Mixed Amide and Phosphonate, Phosphinoxide, and Phosphonothioate Sites for An(III)/Ln(III) Extraction*}

Various organophosphorus ligands with a combination of different donor sites were synthesized and evaluated by solvent extraction studies for the complexation of Am(III)/Eu(III). Among the ligands with a glycolamide backbone, those bearing mixed amide and $P=O$ donor sites and a central oxygen or nitrogen atom, showed a reasonable extraction of $A m(I I I)$ and $E u(I I I)$. Ligands with a central oxygen atom exhibited selectivity towards $E u(I I I)$, and those with a central nitrogen atom towards Am(III). Ligands with $P=S$ donor sites and a glycolamide backbone did not show any reasonable extraction. Amongst the ligands with a malonamide backbone, a high extraction efficiency was observed for the ligand with electron-rich substituents on phosphorus, however, with almost no discrimination between Am(III) and Eu(III). The extraction efficiency of different ligands towards Eu(III) was confirmed by microcalorimetry.

\footnotetext{
* This chapter is based on: Iqbal, M.; Struijk, R. G.; Huskens, J.; Sypula, M.; Wilden, A.; Modolo, G.; Verboom, W. New J. Chem. 2012, 36, 2048.
} 


\section{Chapter 6}

\subsection{Introduction}

Multifunctional neutral organophosphorus compounds are already known as attractive extracting agents for a long time. ${ }^{1}$ A detailed description is given in Chapter 2. A number of ligands containing phosphoryl groups exhibits remarkable extraction and complexation properties towards actinides (An) and lanthanides $(\mathrm{Ln}){ }^{2}$ e.g. the carbamoylmethylphosphineoxides (CMPOs). It should be noted that malonamide ${ }^{3}$ and $\mathrm{CMPO}^{4}$ have a similar backbone structure, except that malonamide contains two amide groups and CMPO contains one amide and one phosphinoxide as donating groups. It was pointed out by Musikas that extractant molecules containing nitrogen or sulfur functionalities, which are softer than oxygen donors, offer a great potential to achieve the desired discrimination between $\mathrm{An}(\mathrm{III})$ and $\mathrm{Ln}(\mathrm{III}){ }^{5}$ A number of $\mathrm{P}=\mathrm{S}$ containing ligands e.g. Cyanex $301^{6}$ and aromatic dithiophosphinic acids ${ }^{7}$ are known to be selective for An(III) compared to $\operatorname{Ln}($ III).

Based on the above mentioned complexing properties of ligating sites in known extractants, the main objective of the work presented in this Chapter is to develop novel ligands, in the form of hybrids, in which these sites are combined in a different way on known ligand backbones. The synthesis and extraction properties will be described of both di- and tripodal ligands containing amide and/or phosphinoxide or (thio)phosphonate moieties. This Chapter focuses on the synthesis and characterization of these ligands. The complexation thermodynamics and complex stoichiometry of $\mathrm{Eu}(\mathrm{III})$ with several novel ligands is investigated using microcalorimetry. The performance of the novel ligands is furthermore tested in solvent extraction studies using trace amounts of Am(III) and Eu(III).

\subsection{Results and discussion}

\subsubsection{Synthesis}

\subsubsection{Mixed amides and phosphonates, phosphinoxides with central oxygen}

The CMPO type of ligands contain one amide and one phosphinoxide donor group and show a high affinity for hexa-, tetra-, and trivalent actinides including the lanthanides over most non-Ln fission products. The well-known TODGA ( $N, N, N^{\prime}, N^{\prime}$-tetra- $n$-octyl diglycolamide) exhibits a better extraction efficiency, ${ }^{8,9}$ however, showing pronounced selectivity for Eu(III) over Am(III) from $\mathrm{HNO}_{3}$ medium. Therefore, the TODGA structure was modified by replacing one of its amide group with $\mathrm{P}=\mathrm{O}$ donors to give 4a,b. A further modification 
involves the replacement of the central oxygen with a nitrogen atom, because of its softer donor character, to afford $\mathbf{7 a , b .}$

The synthesis of the mixed ligands phosphonate- $O$-acetamide (4a) and phosphinoxide- $O$ acetamide (4b) is summarized in Scheme 6.1. Reaction of di- $n$-butylphosphinoxide (1b) with paraformaldehyde using $\mathrm{K}_{2} \mathrm{CO}_{3}$ as a base in cyclohexane, gave the (hydroxymethyl)phosphinoxide $\mathbf{2 b}$ in quantitative yield, analogous to a procedure for $\mathbf{2 a} .^{10}$ Both $\mathbf{2 a}$ and $\mathbf{2 b}$ were reacted with 2-chloro- $N, N$-dioctylacetamide (3) in the presence of $\mathrm{NaH}$ to obtain the desired ligands $\mathbf{4 a}$ and $\mathbf{4 b}$ in $63 \%$ and $73 \%$ yield, respectively. The formation of 4a,b clearly followed from their ${ }^{1} \mathrm{H}$ NMR spectra by the shift of the peak at $4.05 \mathrm{ppm}$ for the $\mathrm{C}(\mathrm{O}) \mathrm{CH}_{2} \mathrm{Cl}$ group in 3 to $4.29 \mathrm{ppm}(\mathbf{4 a})$ and $4.17 \mathrm{ppm}(\mathbf{4 b})$ for the $\mathrm{C}(\mathrm{O}) \mathrm{CH}_{2} \mathrm{O}$ hydrogens. The mass spectra show peaks at $m / z$ of $506.4[\mathrm{M}+\mathrm{H}]^{+}$and $m / z 474.4[\mathrm{M}+\mathrm{H}]^{+}$for $\mathbf{4 a}$ and $\mathbf{4 b}$, respectively.
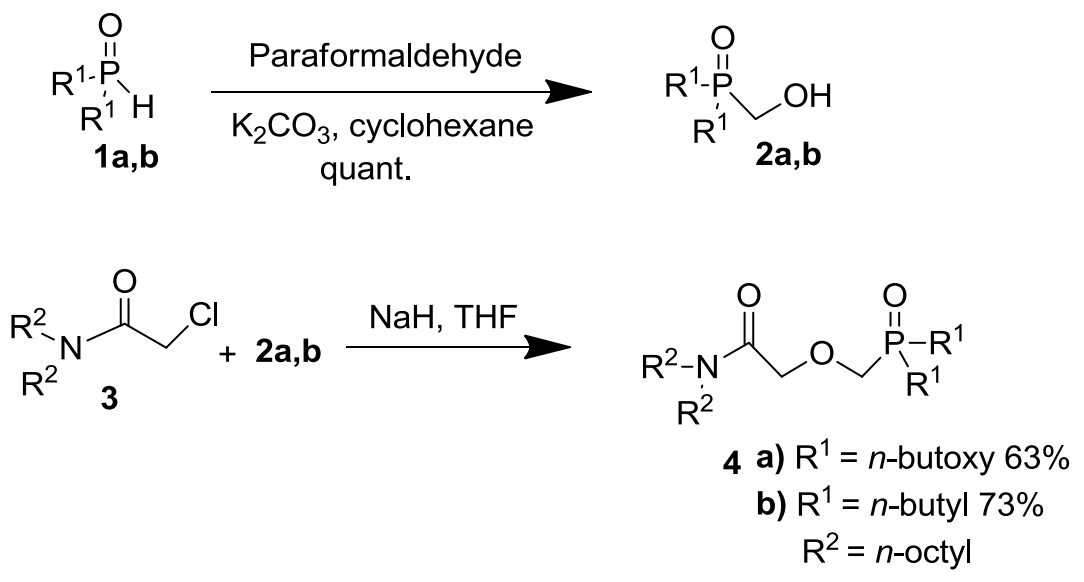

Scheme 6.1

\subsubsection{Mixed amides and phosphonates, phosphinoxides with central nitrogen}

The known compounds $\mathbf{5 a}, \mathbf{b}$ were reacted with 2-chloro- $N, N$-dioctylacetamide (3) to afford benzylacetamidephosphonate $\mathbf{6 a}$ and benzylacetamidephosphinoxide $\mathbf{6 b}$ in $62 \%$ and $73 \%$ yield, respectively. In the ${ }^{1} \mathrm{H}$ NMR spectra the peak for the $\mathrm{C}(\mathrm{O}) \mathrm{CH}_{2} \mathrm{Cl}$ group in $\mathbf{3}$ shifted from $4.05 \mathrm{ppm}$ to $3.81 \mathrm{ppm}$ and $3.94 \mathrm{ppm}$ in $\mathbf{6 a}$ and $\mathbf{6 b}$, respectively. The peak for the benzylic hydrogens is present at $3.53 \mathrm{ppm}$ in $\mathbf{6 a}$ and at $3.60 \mathrm{ppm}$ in $\mathbf{6} \mathbf{b}$. 

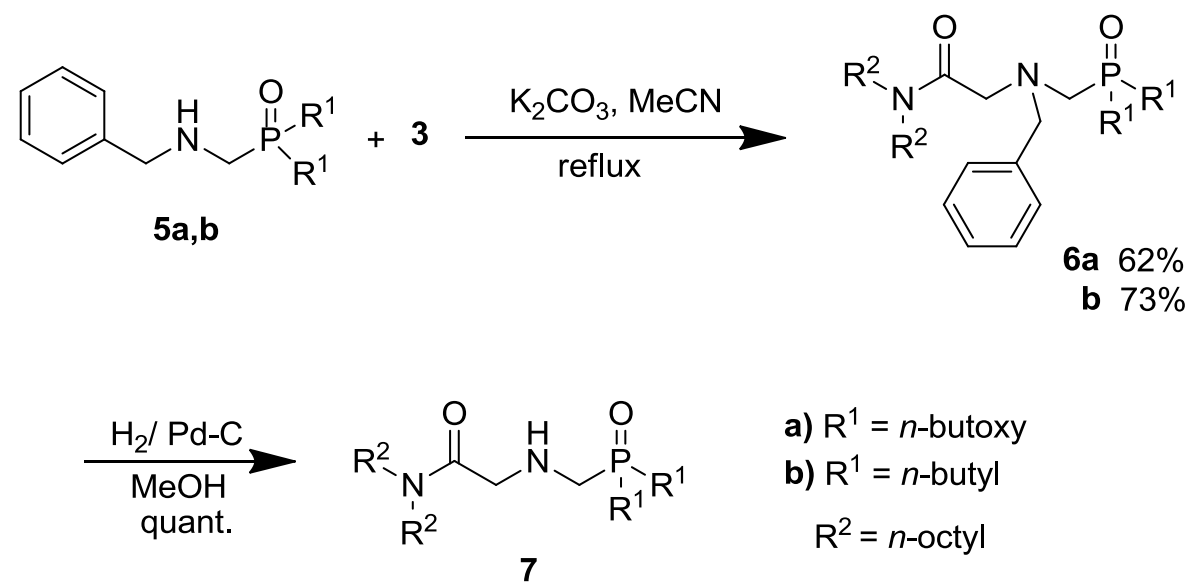

a) $\mathrm{R}^{1}=n$-butoxy

b) $\mathrm{R}^{1}=n$-butyl

$\mathrm{R}^{2}=n$-octyl

Scheme 6.2

In the next step, the benzyl group in $\mathbf{6 a}, \mathbf{b}$ was cleaved by $5 \% \mathrm{Pd} / \mathrm{C}$ in ethanol under hydrogen atmosphere to afford phosphonate- $N$-acetamide $\mathbf{7 a}$ and phosphinoxide- $N$-acetamide $\mathbf{7 b}$ in quantitative yields (Scheme 6.2). The cleavage of the benzyl group in $\mathbf{6 a , b}$ clearly followed from the disappearance of the peaks in the aromatic region in the ${ }^{1} \mathrm{H}$ NMR spectra. In addition, the doublet for the $\mathrm{PCH}_{2} \mathrm{~N}$ hydrogens shifted from $3.21 \mathrm{ppm}$ in $\mathbf{6 a}$ to $3.03 \mathrm{ppm}$ in $7 \mathbf{a}$ and from $3.06 \mathrm{ppm}$ in $\mathbf{6 b}$ to $3.00 \mathrm{ppm}$ in $\mathbf{7 b}$.

\subsubsection{Tripodal mixed amides and phosphonates}

Previously, we reported that a tripodal ligand with all amide donor groups showed interesting extraction properties with selectivity for Am(III) over Eu(III).$^{11}$ In this study, one and two amide groups were replaced by phosphonate groups to give tripodal ligands $\mathbf{9}$ and 13, respectively.

Tripodal diacetamidephosphonate 9, containing one phosphonate and two amide groups, was synthesized by reaction of azanediylbis( $N, N$-dialkylacetamide) (8) with di- $n$-butylphosphite (1a) in the presence of paraformaldehyde and $p$-toluenesulfonic acid in toluene in $60 \%$ yield by an analogous procedure as described for aminomethylphosphinoxides ${ }^{12}$ (Scheme 6.3). The ${ }^{1} \mathrm{H}$ NMR spectrum shows the characteristic $\mathrm{PCH}_{2} \mathrm{~N}$ doublet at $3.31 \mathrm{ppm}$. In the mass spectrum the parent peak is found at $m / z, 786.7[\mathrm{M}+\mathrm{H}]^{+}$. 


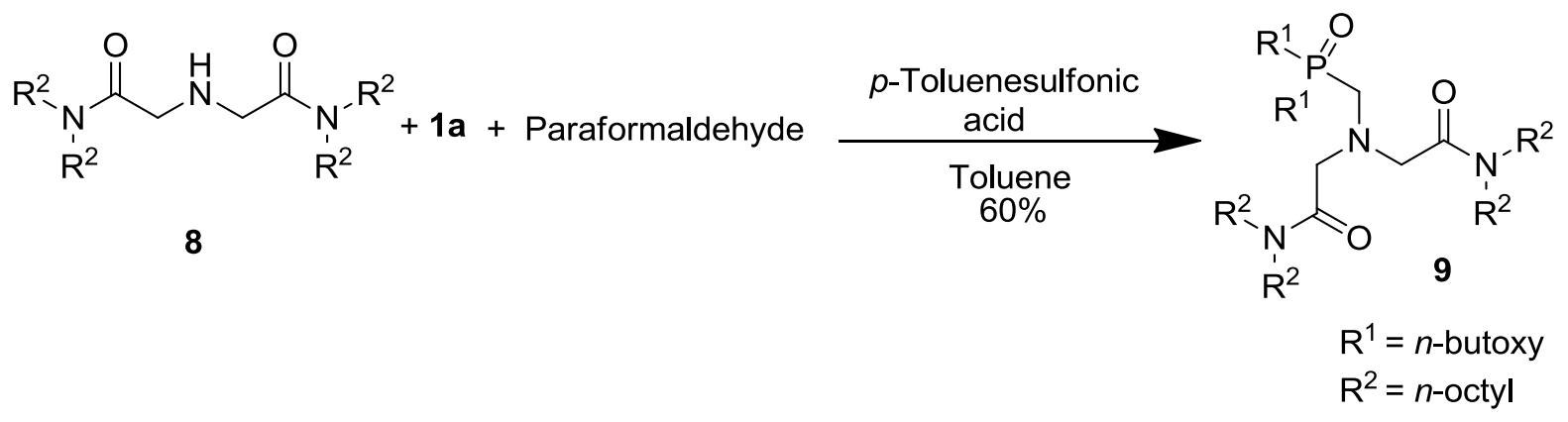

Scheme 6.3

For the synthesis of tripodal ligand 13, containing one amide and two phosphonate groups, benzylamine (10), paraformaldehyde, and di- $n$-butylphosphite (1a) were reacted in toluene by the same procedure ${ }^{12}$ to afford benzyldiphosphonate $\mathbf{1 1}$ in $72 \%$ yield. The benzyl group in $\mathbf{1 1}$ was hydrogenolyzed in ethanol using $10 \% \mathrm{Pd} / \mathrm{C}$ to give aminodiphosphate $\mathbf{1 2}$ in quantitative yield. The formation of $\mathbf{1 2}$ followed by the disappearance of the protons in the aromatic region in the ${ }^{1} \mathrm{H}$ NMR spectrum; the doublet for the $\mathrm{PCH}_{2} \mathrm{~N}$ group was shifted from $3.16 \mathrm{ppm}$ in 11 to $3.11 \mathrm{ppm}$ in 12. Finally, aminodiphosphate 12 was reacted with 2-chloro- $N, N$ dioctylacetamide (3) in acetonitrile to give tripodal acetamidediphosphate $\mathbf{1 3}$ in $78 \%$ yield (Scheme 6.4). In the ${ }^{1} \mathrm{H}$ NMR spectrum the $\mathrm{PCH}_{2} \mathrm{~N}$ peak shifted from $3.11 \mathrm{ppm}$ in $\mathbf{1 2}$ to 3.35 ppm in 13, and the $\mathrm{C}(\mathrm{O}) \mathrm{CH}_{2} \mathrm{Cl}$ peak of acetamide 3 shifted from $4.05 \mathrm{ppm}$ to $3.84 \mathrm{ppm}$ for the $\mathrm{C}(\mathrm{O}) \mathrm{CH}_{2} \mathrm{~N}$ group. In the mass spectrum the parent peak is found at $m / z 711.5[\mathrm{M}+\mathrm{H}]^{+}$.
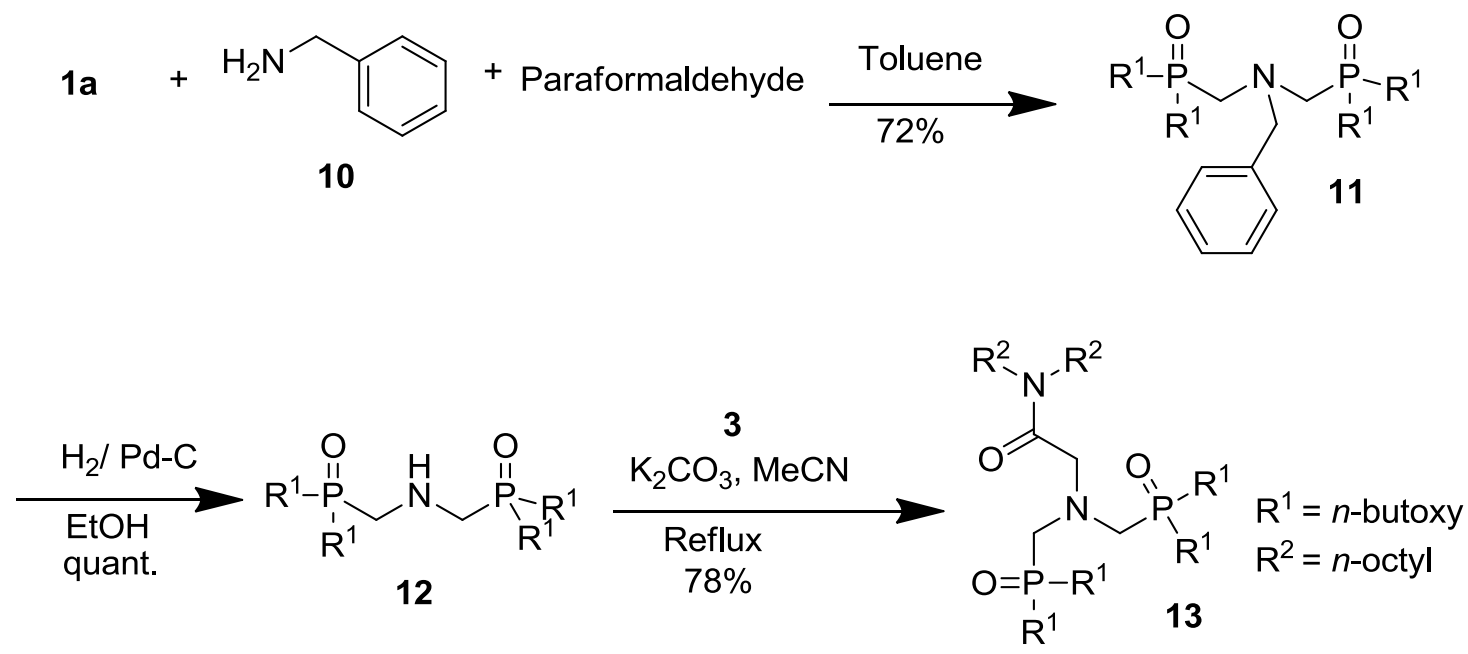

Scheme 6.4

\subsubsection{4 $P=S$ containing ligands with central oxygen atom}

As mentioned above, diglycolamides as TODGA have excellent extraction properties with a slight preference for $\mathrm{Eu}(\mathrm{III})$ over Am(III). Generally, S donors are selective for Am(III) over 


\section{Chapter 6}

$\mathrm{Eu}(\mathrm{III})$. Therefore, the amide groups in TODGA were replaced by $\mathrm{P}=\mathrm{S}$ containing sites to give ligands 16, 20, and 23.

The known (di-n-butoxyphosphoryl)methyl trifluoromethanesulfonate $\mathbf{1 4}$ was reacted with $\mathbf{2 a}$ using $\mathrm{NaH}$ as a base to give the desired diphosphonate 15. In the ${ }^{1} \mathrm{H}$ NMR spectrum, the doublet for the $\mathrm{PCH}_{2} \mathrm{O}$ hydrogens at $4.62 \mathrm{ppm}$ in $\mathbf{1 4}$ shifted to $3.84 \mathrm{ppm}$ in $\mathbf{1 5}$. The $\mathrm{P}=\mathrm{O}$ groups in $\mathbf{1 5}$ were converted into $\mathrm{P}=\mathrm{S}$ moieties using Lawesson's reagent to afford $\mathbf{1 6}$ in $80 \%$ yield (Scheme 6.5). In this case the $\mathrm{PCH}_{2} \mathrm{O}$ protons appeared at $4.07 \mathrm{ppm}$. Both compounds showed characteristic $[\mathrm{M}+\mathrm{H}]^{+}$peaks in their electrospray mass spectra.

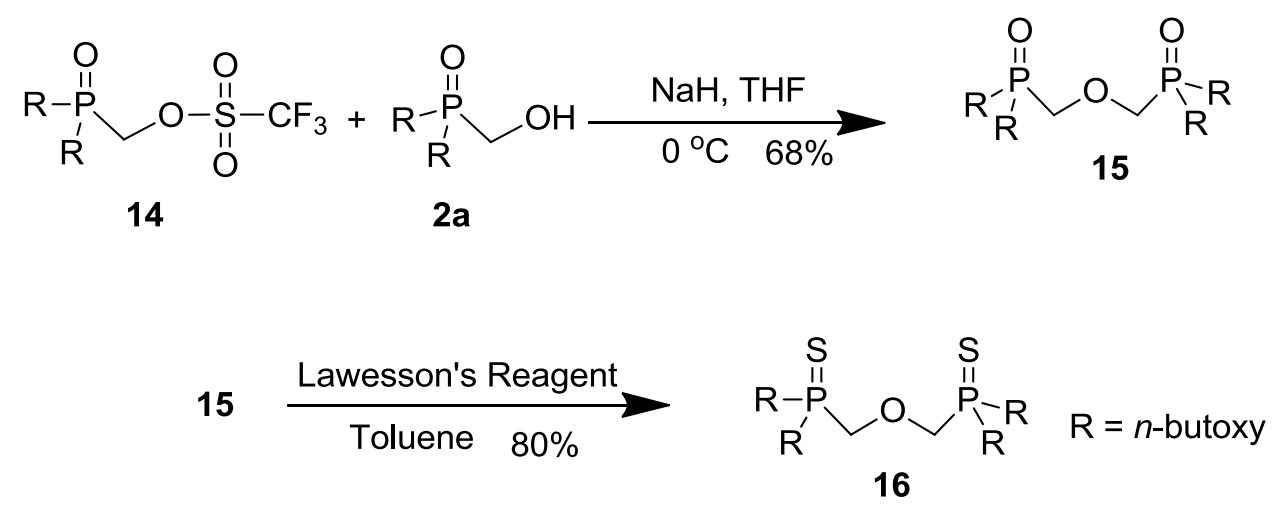

Scheme 6.5

Diphenylphosphine sulfide (17) was reacted with paraformaldehyde to get the hydroxymethylated compound $\mathbf{1 8}$ in quantitative yield. The introduction of the hydroxymethyl group clearly followed from the peak at $4.42 \mathrm{ppm}$ in its ${ }^{1} \mathrm{H}$ NMR spectrum. Upon conversion of the hydroxyl group in $\mathbf{1 8}$ into a tosylate, the resulting tosylate $\mathbf{1 9}$ was reacted with $\mathbf{1 8}$ in THF using $\mathrm{NaH}$ as a base to give the target (oxybis(methylene))bis(diphenylphosphine sulfide) (20) in $71 \%$ yield (Scheme 6.6). In the ${ }^{1} \mathrm{H}$ NMR spectrum, the peak for the methylene protons in $\mathbf{1 9}$ at $4.62 \mathrm{ppm}$ shifted to $4.42 \mathrm{ppm}$ in 20. The formation of $\mathbf{2 0}$ also followed from the correct $[\mathrm{M}+1]^{+}$peak at $\mathrm{m} / \mathrm{z} 479.1$ in the electrospray mass spectrum. 

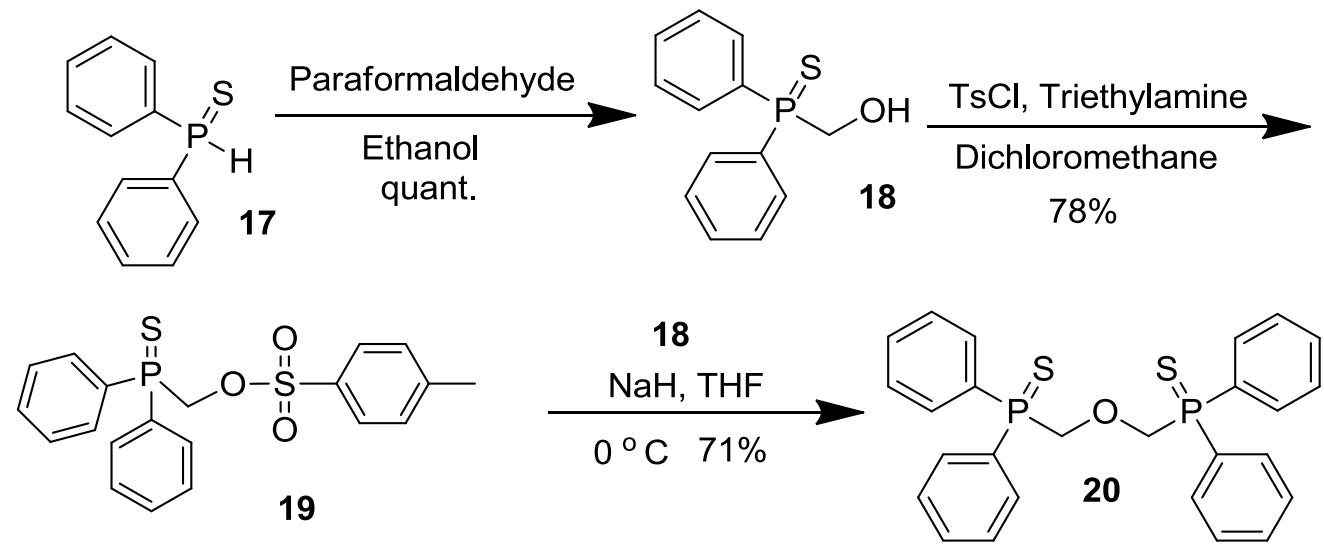

Scheme 6.6

Tosylate 21, prepared by tosylation of di-n-butyl(hydroxymethyl)phosphinoxide (2b), was reacted with compound $\mathbf{2 b}$ in the presence of $\mathrm{NaH}$ as a base in THF to give (oxybis(methylene))bis(di-n-butylphosphinoxide) (22) in 76\% yield (Scheme 6.7). Also, in this case, a characteristic shift of the $\mathrm{PCH}_{2} \mathrm{O}$ methylene resonances occurred in the ${ }^{1} \mathrm{H}$ NMR spectra, viz. from $4.15 \mathrm{ppm}$ in $\mathbf{2 1}$ to $3.88 \mathrm{ppm}$ in $\mathbf{2 2}$. The $\mathrm{P}=\mathrm{O}$ groups in $\mathbf{2 2}$ were converted into $\mathrm{P}=\mathrm{S}$ moieties with Lawesson's reagent to afford the target ligand $\mathbf{2 3}$ in $80 \%$ yield. In the ${ }^{1} \mathrm{H}$ NMR spectrum, the characteristic methylene protons are present at $3.91 \mathrm{ppm}$. The formation of these compounds was also confirmed by their $[\mathrm{M}+\mathrm{H}]^{+}$peaks in the electrospray mass spectra.

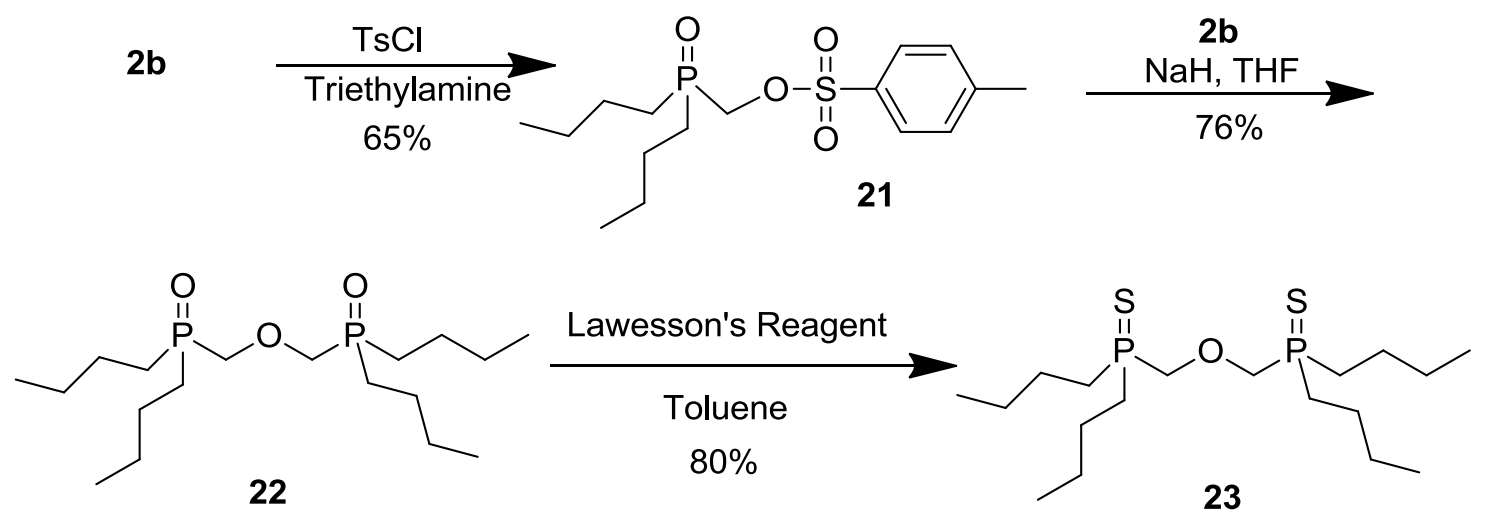

Scheme 6.7

\subsubsection{Methylene-bridged $P=S$ containing ligands}

The two lipophilic ligands $\mathbf{2 5}$ and $\mathbf{2 6}$ can in principle, be considered as malonamides in which the amides have been replaced by $\mathrm{P}=\mathrm{S}$ containing moieties, as described above for TODGA. The synthesis is summarized in Scheme 6.8. 


\section{Chapter 6}

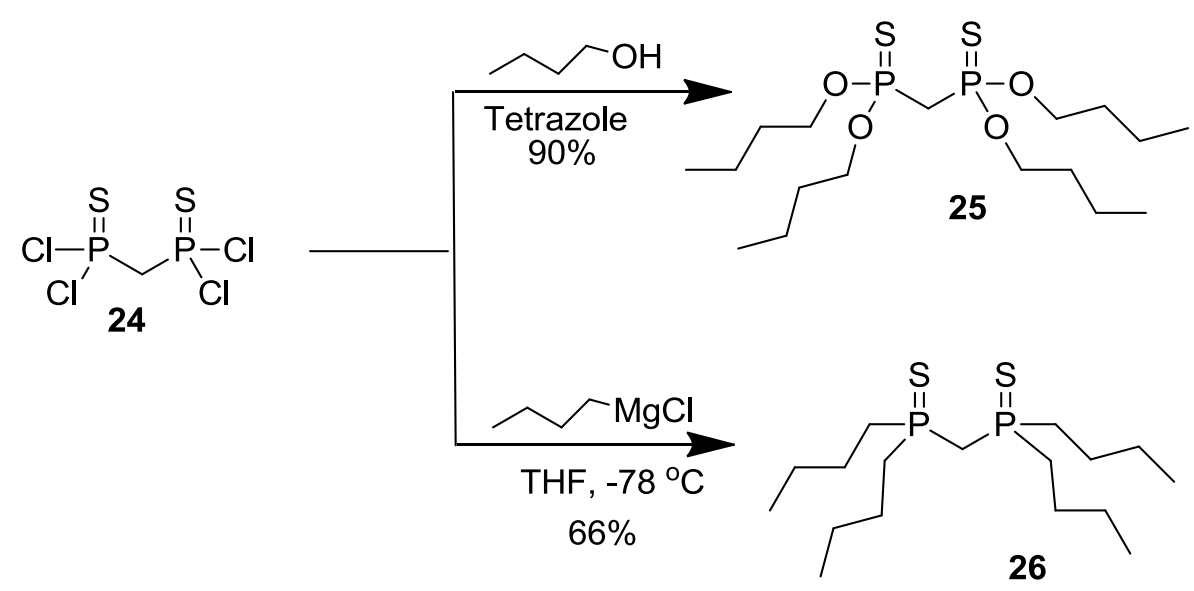

Scheme 6.8

Methylenediphosphonothioic dichloride (24) was reacted with different reagents to afford the desired potential ligands $\mathbf{2 5}$ and 26. Stirring of $\mathbf{2 4}$ with 1-butanol in the presence of tetrazole gave $O, O, O^{\prime}, O^{\prime}$-tetra- $n$-butyl methylenediphosphonothioate (25) in $90 \%$ yield in an analogous method as described for the corresponding $\mathrm{P}=\mathrm{O}$ compounds as a starting material. ${ }^{13}$ For the synthesis of methylenebis(di- $n$-butylphosphine sulfide) (26) compound 24 was reacted with $n$ butylmagnesium chloride at $-78{ }^{\circ} \mathrm{C}$; $n$-butyllithium gave unsatisfactory results. In the ${ }^{1} \mathrm{H}$ NMR spectra, the signal for the methylene hydrogens shifted from 4.48 ppm in 24 to 2.99 and 2.46 ppm in $\mathbf{2 5}$ and 26, respectively. These compounds were also characterized by their $[\mathrm{M}+1]^{+}$ peaks in the electrospray mass spectra.

\subsubsection{Extraction results}

All the synthesized ligands were tested using liquid-liquid extraction techniques. The extractabilities of $\mathrm{Am}(\mathrm{III})$ and $\mathrm{Eu}(\mathrm{III})$ by the lipophilic ligands are expressed by the distribution ratios, $D_{A m}$ and $D_{E u}$ (eqn 1). The distribution ratios of $\mathrm{Am}$ and $\mathrm{Eu}$ are plotted as a function of the initial nitric acid concentration.

$D_{\mathrm{M}}=[M]_{\mathrm{org}} /[M]_{\mathrm{aq}}$

The separation factor $S F_{\mathrm{A} / \mathrm{B}}$ is the ratio of the distribution ratios $D_{\mathrm{A}}$ and $D_{\mathrm{B}}$ (eqn 2), and describes the selectivity for A over B.

$S F_{\mathrm{A} / \mathrm{B}}=D_{\mathrm{A}} / D_{\mathrm{B}}$ 


\subsubsection{Mixed amides and phosphonates, phosphinoxides with central oxygen or nitrogen}

Similarly to CMPO, ligands $\mathbf{4 a , b}$ possess two different donating groups: one amide and one phosphonate (4a) or phosphinoxide (4b) moiety. The extraction of Am and Eu by ligand $4 \mathbf{a}$ increased with increasing nitric acid concentration in the aqueous phase (Figure 6.1). Above $1 \mathrm{~mol} / \mathrm{L} \mathrm{HNO}_{3}$, the $D$ values sharply increased reaching values of 4.6 and 0.9 at $4 \mathrm{~mol} / \mathrm{L}$ $\mathrm{HNO}_{3}$ for Eu and Am, respectively.

Phosphinoxide $\mathbf{4 b}$, which is more similar to CMPO, shows the same extraction behavior as CMPO at lower nitric acid concentrations. However, at $\left[\mathrm{HNO}_{3}\right]>0.1 \mathrm{~mol} / \mathrm{L}$ the $D$ values are lower. Apparently, the introduction of an oxygen atom between the phosphorus and the alkyl chain in ligand 4a has a negative influence on the extraction properties compared to CMPO. The extraction behavior of these two ligands are similar to that of CMPO, which is a neutral ligand extracting metals by the solvation mechanism. ${ }^{14}$ This may suggest the same extraction mechanism in the case of its derivatives $\mathbf{4 a , b}$. As for other diglycolamide-based ligands, ${ }^{8} \mathbf{4 a , b}$ exhibit a slight selectivity for Eu over Am. At $4 \mathrm{~mol} / \mathrm{L}$ nitric acid concentration, the Eu/Am separation factors are 2.5 (ligand $\mathbf{4 b}$ ) and 5.5 (ligand 4a). CMPO shows an opposite selectivity, favoring $\mathrm{Am}$ over $\mathrm{Eu}$ with $\mathrm{Am} / \mathrm{Eu}$ separation factors of approximately 2 at $4 \mathrm{~mol} / \mathrm{L} \mathrm{HNO}_{3}$.

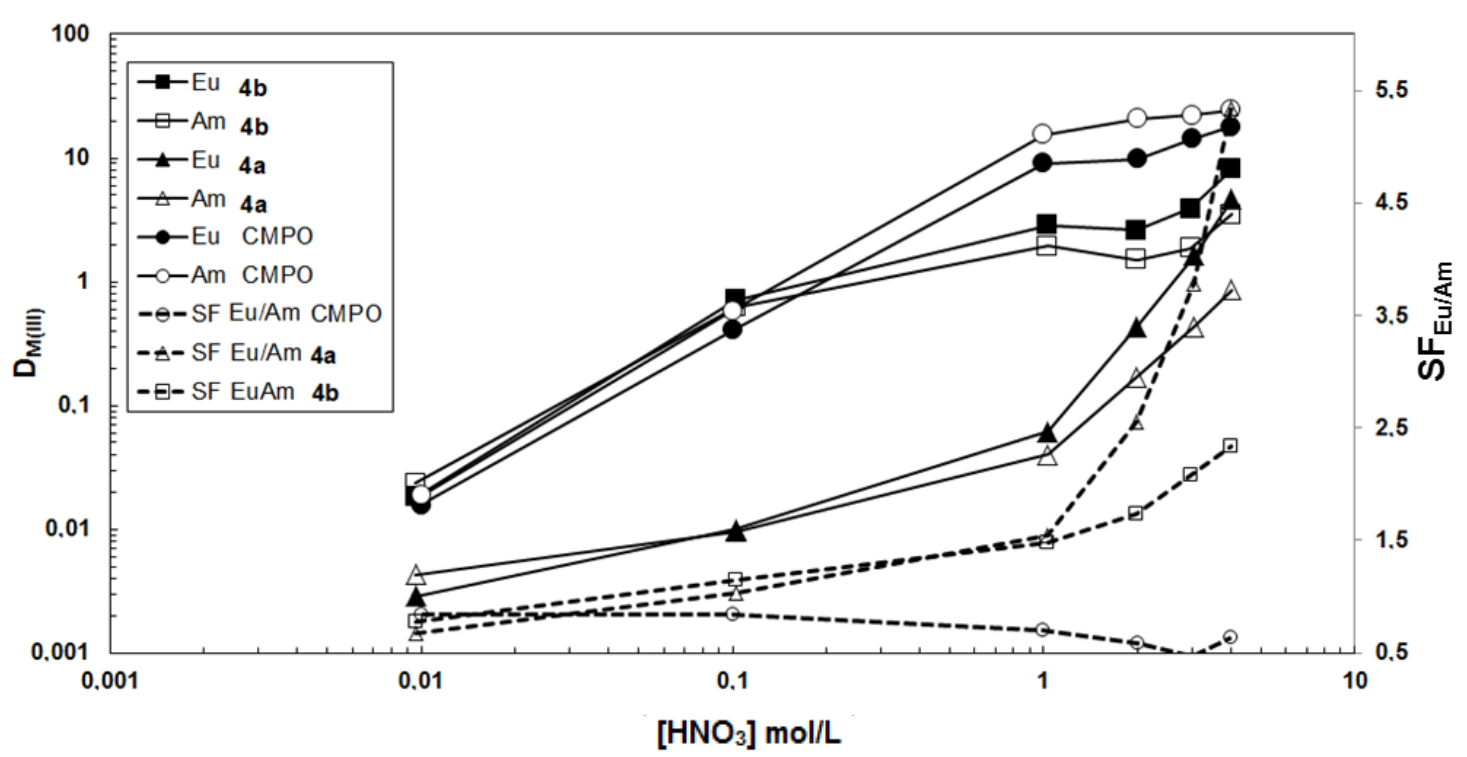

Figure 6.1. Nitric acid dependency for the extraction of ${ }^{241} \mathrm{Am}$ and ${ }^{152} \mathrm{Eu}$ by ligands $\mathbf{4 a , b}$ and comparison with CMPO/TBP.

Organic phase: $0.1 \mathrm{~mol} / \mathrm{L}$ of ligands $\mathbf{4 a , b}$ in TPH (hydrogenated tetrapropene) or $0.2 \mathrm{~mol} / \mathrm{L} \mathrm{CMPO} \mathrm{+}$ $1.4 \mathrm{~mol} / \mathrm{L}$ TBP in $n$-dodecane.

Aqueous phase: variable concentration of nitric acid, tracers: ${ }^{241} \mathrm{Am}$ and ${ }^{152} \mathrm{Eu}$, mixing time: $60 \mathrm{~min}$ (CMPO: $15 \mathrm{~min}$; enough to reach equilibrium); $T=22 \pm 1^{\circ} \mathrm{C}$. 


\section{Chapter 6}

The ligands $\mathbf{7 a , b}$ differ from $\mathbf{4 a , b}$ by the presence of a nitrogen instead of an oxygen as the central atom of the back-bone structure. This replacement resulted in a reversed metal extraction behavior (Figure 6.2), since the central nitrogen atom is protonated at higher nitric acid concentrations. In the case of both ligands $\mathbf{7 a , b}$, the metal extractability drastically decreased at nitric acid concentrations $>0.1 \mathrm{~mol} / \mathrm{L}$. In addition, precipitation occurred between the two equilibrated phases at $\left[\mathrm{HNO}_{3}\right] \geq 0.1 \mathrm{~mol} / \mathrm{L}$. The degree of precipitation increased upon increasing the acidity of the aqueous phase. It is assumed that the central nitrogen atom in both ligands is protonated, resulting in precipitation of the $\mathrm{HNO}_{3} \bullet$ ligand. At a nitric acid concentration of $0.01 \mathrm{~mol} / \mathrm{L}$, where the precipitation of the ligands did not take place, the $D$-values were higher for ligand $\mathbf{7 b}$ compared to $\mathbf{7 a}$.

A related lipophilic ligand, also with nitrogen in the center but containing two dioctylacetamide groups, instead of just one, has been studied recently. ${ }^{11}$ It did not show any significant extraction of $\mathrm{Eu}(\mathrm{III})$ or $\mathrm{Am}(\mathrm{III})\left(D_{M}<0.01\right)$. Therefore, we assume that the increase of the $D$ values at $0.01 \mathrm{~mol} / \mathrm{L} \mathrm{HNO}_{3}$ in the case of ligands $7 \mathbf{a}, \mathbf{b}$ is due to the phosphonate and phosphinoxide groups, respectively.

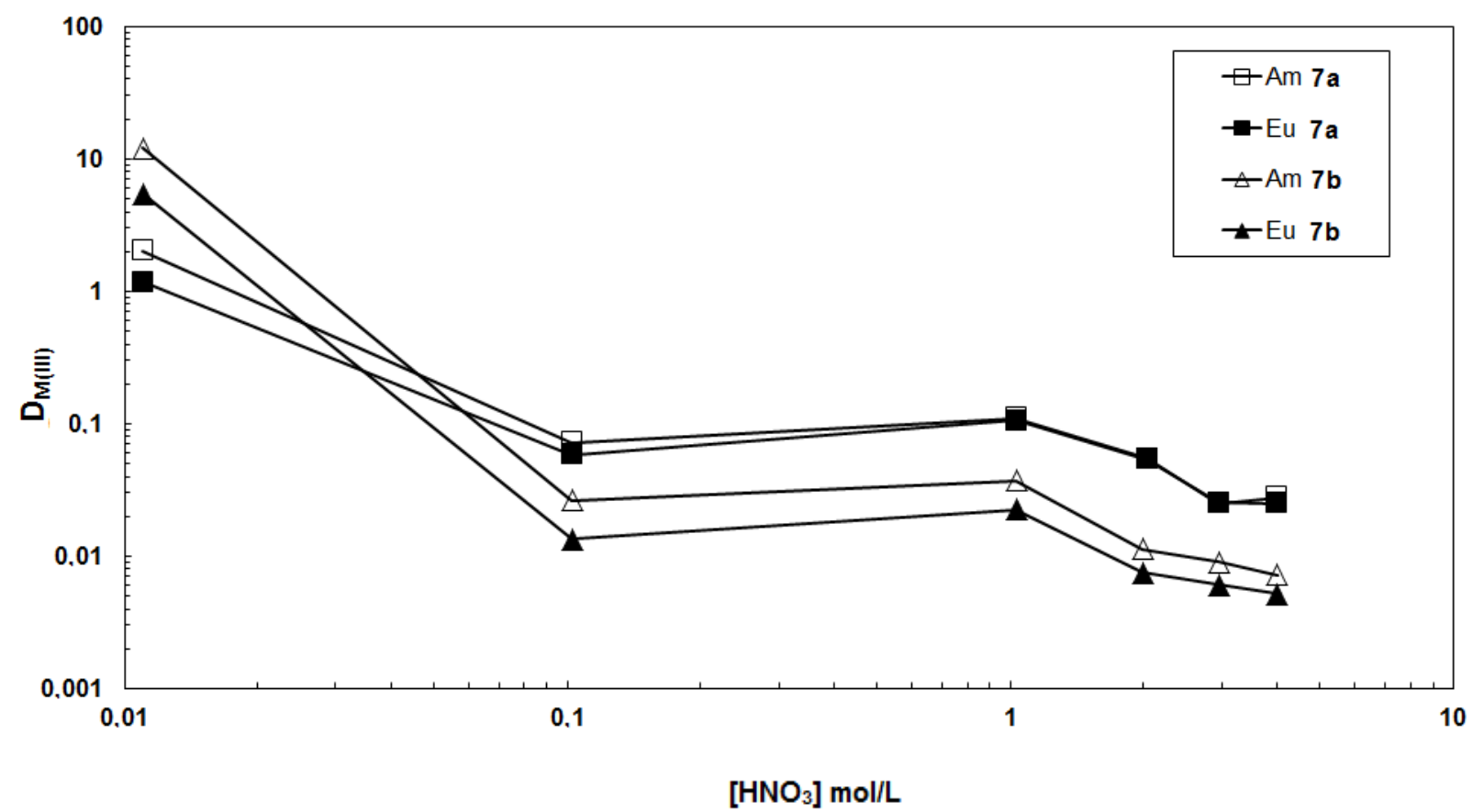

Figure 6.2. Nitric acid dependency for the extraction of ${ }^{241} \mathrm{Am}$ and ${ }^{152} \mathrm{Eu}$ by ligands $\mathbf{7 a}, \mathbf{b}$.

Organic phase: $0.1 \mathrm{~mol} / \mathrm{L}$ ligand in TPH.

Aqueous phase: variable concentration of nitric acid, tracers: ${ }^{241} \mathrm{Am}$ and ${ }^{152} \mathrm{Eu}$, mixing time: $60 \mathrm{~min} ; T$ $=22 \pm 1^{\circ} \mathrm{C}$. 


\subsubsection{Tripodal mixed amides and phosphonates}

The tripodal ligands $\mathbf{9}$ and $\mathbf{1 3}$ possess an additional third ligating group attached to the central nitrogen, compared to the ligands described above. Ligand $\mathbf{9}$, containing two acetamide and one phosphonate group, showed increasing metal extraction at nitric acid concentrations $>0.1 \mathrm{~mol} / \mathrm{L}$, although the distribution ratios did not exceed 0.25 at the highest acidity tested (Figure 6.3). When one of the acetamide functionalities of 9 was substituted by a phosphonate group (13), the metal distribution ratios somewhat increased, especially for Am. The extraction behavior as a function of $\mathrm{HNO}_{3}$ is more or less reversed compared to that of the related 2,2',2"-nitrilotris( $N, N$-dioctylacetamide) ligand, containing three acetamide groups, which showed higher $D$ values at lower acid concentration. ${ }^{11}$ It appears that in the cases of ligands 9 and 13, protonation of the central nitrogen atom is less pronounced than in the mentioned reference ligand. ${ }^{11}$ In contrast to the dipodal ligands $(\mathbf{7 a}, \mathbf{b})$, these results clearly show that replacement of an acetamide for a phosphonate group gives rise to much lower extraction ability at lower nitric acid concentrations. Ligands $\mathbf{9}$ and $\mathbf{1 3}$ show lower extraction at $0.1 \mathrm{~mol} / \mathrm{L} \mathrm{HNO}_{3}$ compared to 0.01 , persumably due to protonation of the nitrogen atom. The $D$ values are increased upon further increase in $\mathrm{HNO}_{3}$ concentration. It might be due to the fact that nitrogen is not involved in the extraction at higher $\mathrm{HNO}_{3}$ concentrations, and only three $\mathrm{P}=\mathrm{O}$ or $\mathrm{C}=\mathrm{O}$ groups are involved in extraction, where extraction is simply expected to increase upon increasing nitrate ion concentration, (metal is extracted in combination with nitrate ions). 


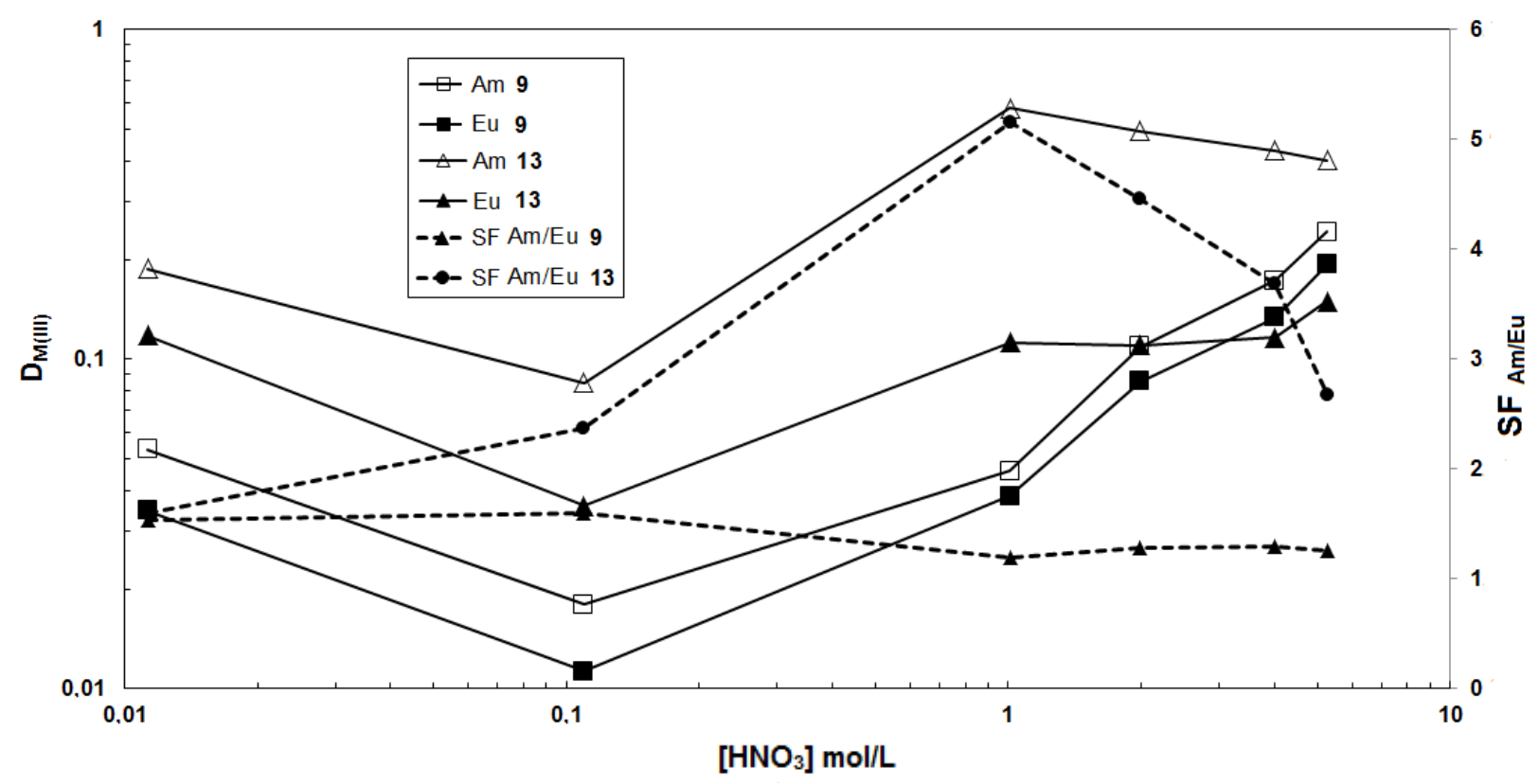

Figure 6.3. Nitric acid dependency for the extraction of ${ }^{241} \mathrm{Am}$ and ${ }^{152} \mathrm{Eu}$ by ligands $\mathbf{9}$ and $\mathbf{1 3}$. Organic phase: $0.1 \mathrm{~mol} / \mathrm{L}$ ligand in TPH.

Aqueous phase: variable concentration of nitric acid, tracers: ${ }^{241} \mathrm{Am}$ and ${ }^{152} \mathrm{Eu}$, mixing time: $60 \mathrm{~min} ; T$ $=22 \pm 1{ }^{\circ} \mathrm{C}$.

\subsubsection{3 $P=S$ containing ligands with central oxygen atom}

As mentioned above, DGA ligands with their three oxygen atoms in the backbone structure are very efficient for trivalent actinide and lanthanide complexation. One may expect selectivity for $\mathrm{Am}(\mathrm{III})$ over $\mathrm{Eu}(\mathrm{III})$ by replacing $\mathrm{C}=\mathrm{O}$ by $\mathrm{P}=\mathrm{S}$ groups in DGA ligands, however, in addition to low $D$ values the substitution of the carbonyl oxygens for the softer sulfurs in the lipophilic ligands 16, 20, and 23 gave no significant separation between Am and Eu. Ligands 20 and $\mathbf{2 3}$ even showed no extraction ( $D$ ratios below detection limit; see Figure 6.4) in the entire tested acidity range. It appears like that there is some influence on the extraction behavior upon the introduction of an oxygen between the butyl chain and the phosphorus into the structure of ligand $\mathbf{2 3}$ resulting in ligand 16, which showed some minor extraction of the metals at low acid concentration (Figure 6.4).

Ligand 20 showed a lower metal extractability than 16, which may result from its poor solubility in TPH. In the extraction experiments, the concentration of $\mathbf{2 0}$ was 10 times lower than that of $\mathbf{1 6 .}$ 


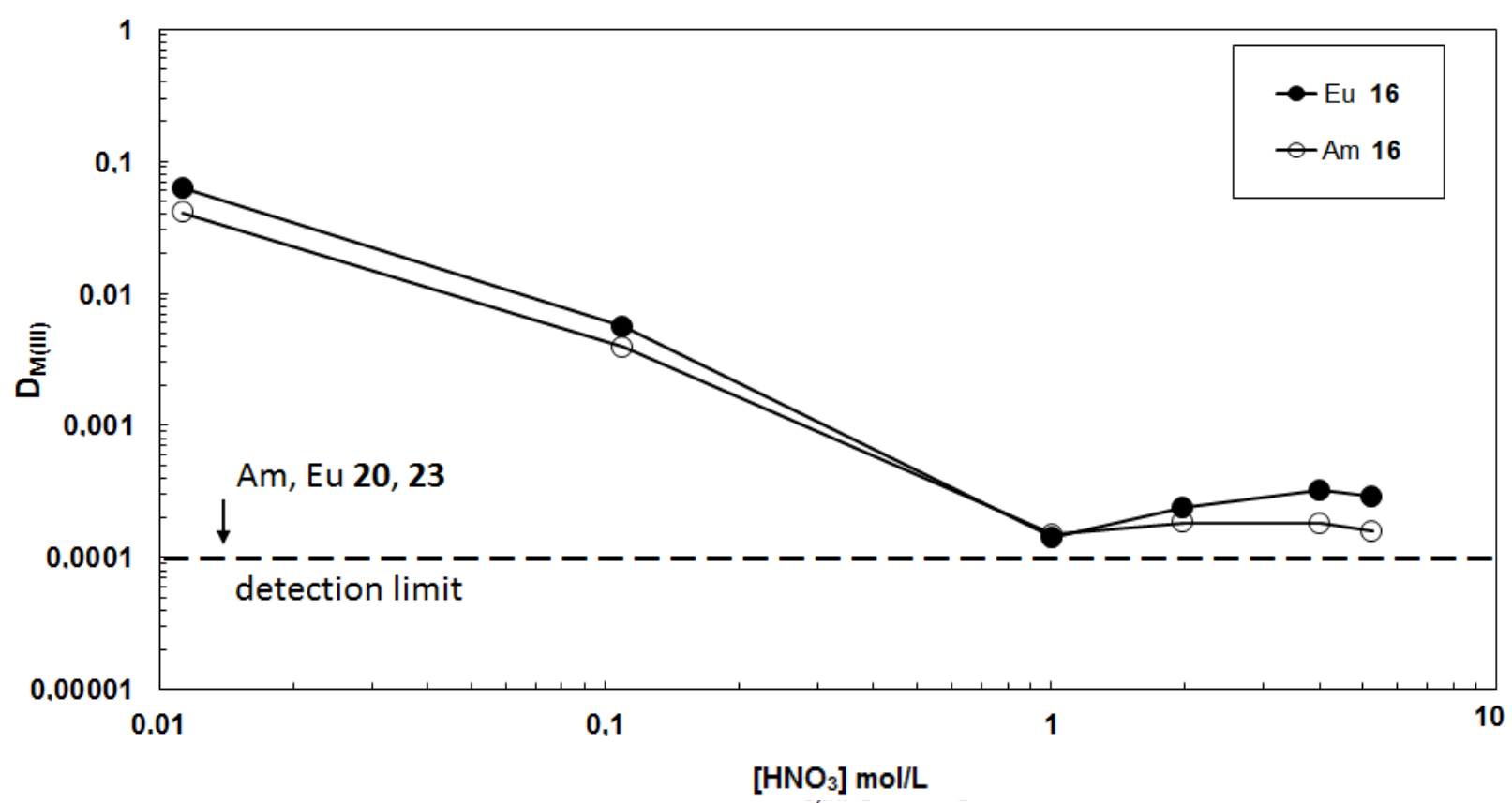

Figure 6.4. Nitric acid dependency for the extraction of ${ }^{241} \mathrm{Am}$ and ${ }^{152} \mathrm{Eu}$ by ligand $\mathbf{1 6 .}$ Organic phase: $0.1 \mathrm{~mol} / \mathrm{L} 16 \mathrm{in} \mathrm{TPH}$.

Aqueous phase: variable concentration of nitric acid, tracers: ${ }^{241} \mathrm{Am}$ and ${ }^{152} \mathrm{Eu}$, mixing time: $60 \mathrm{~min} ; T$ $=22 \pm 1^{\circ} \mathrm{C}$.

\subsubsection{Methylene-bridged $P=S$ containing ligands}

The two lipophilic ligands $\mathbf{2 5}$ and $\mathbf{2 6}$ represent good examples of the influence of the side chain of the phosphine sulfide on its electronic properties. Ligand $\mathbf{2 5}$ has four butoxy groups, thus increasing the basicity of the donor S-atoms, while ligand $\mathbf{2 6}$ has four butyl chains, less affecting the basicity. Ligand 26 gave Eu and Am distribution ratios of around 0.1 at low acidity (Figure 6.5). Above $0.1 \mathrm{~mol} / \mathrm{L} \mathrm{HNO}_{3}$ the $D$ values even decreased further to 0.001 at $4 \mathrm{~mol} / \mathrm{L} \mathrm{HNO}_{3}$. At an acid concentration of $5 \mathrm{~mol} / \mathrm{L}$, precipitation of the ligand occurred, probably due to oxidation of the sulfur.

The butoxy groups of ligand $\mathbf{2 5}$ make the sulfur donor atoms much more electron rich as compared to those in $\mathbf{2 6}$, resulting in an increased affinity to the trivalent metals. Distribution ratio values over 100 were obtained in the acidic range $0.01-1 \mathrm{~mol} / \mathrm{L}$, followed by a decrease at higher nitric acid concentration, which may be due to oxidation of the sulfur. Another explanation could be competition between the metal ions and nitric acid extraction. However, after phase contact at 4 and $5 \mathrm{~mol} / \mathrm{L} \mathrm{HNO}_{3}$, a white emulsion between the organic and the aqueous phase was visible in the samples, indicating a low hydrolytic stability. Nevertheless, no loss of Am or Eu was observed. 


\section{Chapter 6}

Cuillerdier et al. ${ }^{3}$ studied unsubstituted malonamides containing $n$-butyl or $n$-octyl groups to give maximal $D_{\mathrm{Am}}$ values of 0.5 and 1.2 , respectively, in the acid range of $2-4 \mathrm{~mol} / \mathrm{L}^{-\mathrm{HNO}_{3}}$. Compared to the results obtained with ligand $\mathbf{2 5}$, it clearly demonstrates that the replacement of a dialkyl amide for a butyl phosphonothioate moiety leads to higher distribution ratios of $\mathrm{Am}(\mathrm{III})$ and $\mathrm{Eu}(\mathrm{III})$.

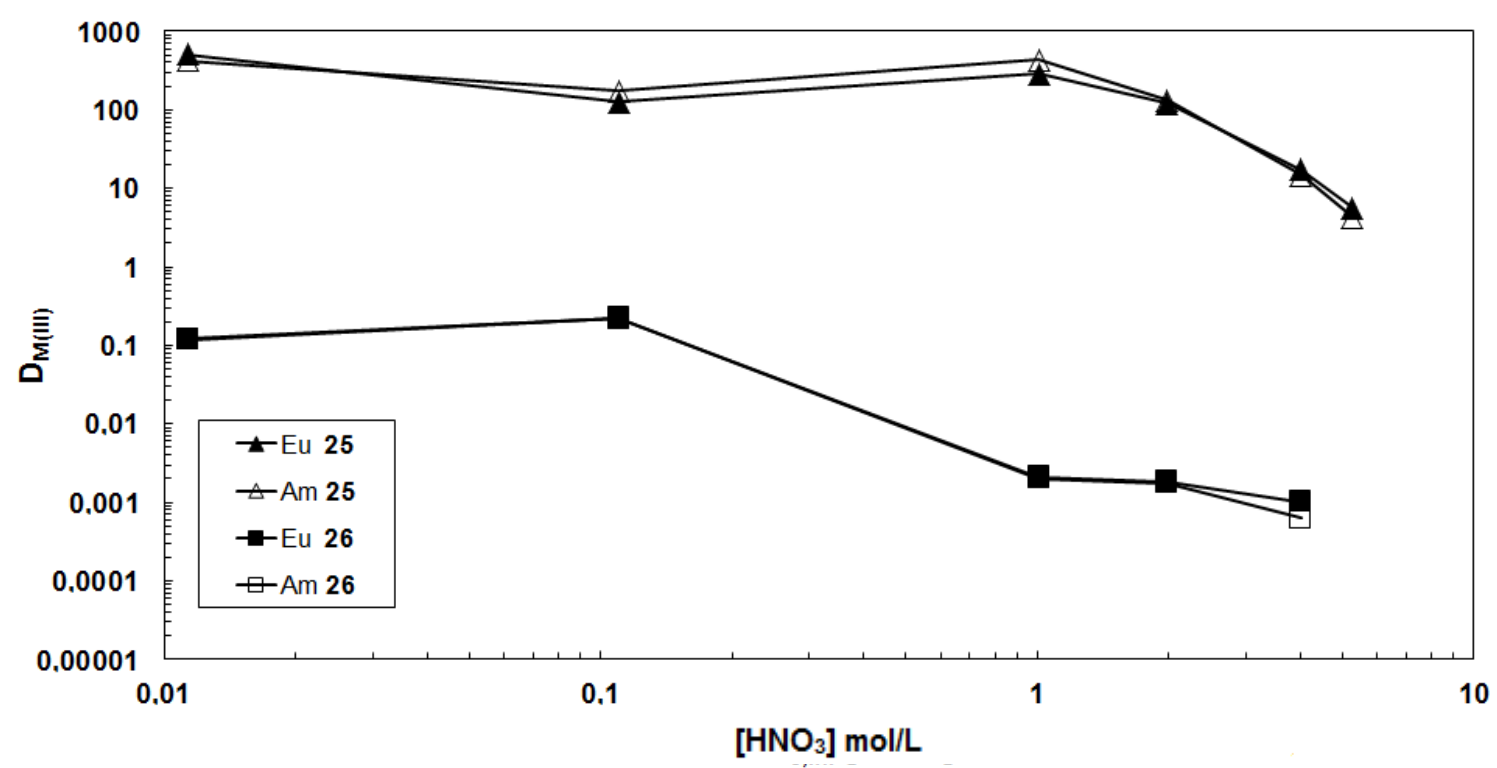

Figure 6.5. Nitric acid dependency for the extraction of ${ }^{241} \mathrm{Am}$ and ${ }^{152} \mathrm{Eu}$ by ligands $\mathbf{2 5}$ and $\mathbf{2 6}$.

Organic phase: $0.1 \mathrm{~mol} / \mathrm{L} 26$ in TPH or $0.09 \mathrm{~mol} / \mathrm{L} 25+0.003 \mathrm{~mol} / \mathrm{L} 1$-octanol in TPH (due to a lower solubility of 25 in pure TPH).

Aqueous phase: variable concentration of nitric acid, tracers: ${ }^{241} \mathrm{Am}$ and ${ }^{152} \mathrm{Eu}$, mixing time: $60 \mathrm{~min} ; T$ $=22 \pm 1{ }^{\circ} \mathrm{C}$.

\subsubsection{Microcalorimetry}

Microcalorimetry (ITC) experiments were performed to quantitatively study the difference in the binding capacities of ligands $4 \mathbf{a}, \mathbf{b}, \mathbf{7 a}, \mathbf{b}, \mathbf{9}, \mathbf{1 3}$, and 25 towards $\mathrm{Eu}(\mathrm{III})$, to determine the stoichiometry of the complexes, and to gain insight into the thermodynamics of the ligand:metal complexation. It should be kept in mind, however, that these cannot be directly compared, since the microcalorimetric experiments could not be carried out under similar conditions as the extractions were done due to solubility reasons. ITC experminents were not performed with the other ligands, since they did not exhibit any reasonable extraction. The experiments were performed in acetonitrile, since the ligands were not soluble in water; $\mathrm{Eu}(\mathrm{III})$ perchlorate was used because of its solubility in acetonitrile. Therefore, a $2 \mathrm{mmol} / \mathrm{L}$ ligand solution was titrated with a $0.066 \mathrm{mmol} / \mathrm{L} \mathrm{Eu}(\mathrm{III})$ perchlorate solution, except for 
ligands 4a,b where a $1 \mathrm{mmol} / \mathrm{L}$ ligand solution was titrated against $0.033 \mathrm{mmol} / \mathrm{L} \mathrm{Eu(III),} \mathrm{due}$ to the high binding constants at higher concentrations. The binding constants and the thermodynamic data are summarized in Table 1. Dilution experiments with ligands $\mathbf{4 a}, \mathbf{b}, \mathbf{7 b}$, 13, and 25 showed negligible heat effects, indicating that no aggregation of the ligands takes place under the conditions used. However, in the dilution experiments with ligands $7 \mathbf{a}$ and 9 endothermic heat effects were observed, indicating deaggregation of the ligand upon dilution. The binding constants $(K)$ and $\Delta H$ values for ligand:metal complexation were corrected by compensating $K$ and $\Delta H$ for dilution and deaggregation (when applicable) in the plots of $\Delta H$ versus molar ratio.

Table 6.1 Binding constants and thermodynamic parameters of the binding of ligands $\mathbf{4 a}, \mathbf{b}$, 7a,b, 9, 13, and 25 with $\mathrm{Eu}(\mathrm{III})$ determined with microcalorimetry in acetonitrile at $30{ }^{\circ} \mathrm{C} .{ }^{a}$

\begin{tabular}{|c|c|c|c|c|}
\hline Ligand & $K, \mathrm{~L} \mathrm{~mol}^{-1}$ & $\Delta G^{o}, \mathrm{kcal} \mathrm{mol}^{-1}$ & $\Delta H^{o}, \mathrm{kcal} \mathrm{mol}^{-1}$ & $T \Delta S^{o}, \mathrm{kcal} \mathrm{mol}^{-1}$ \\
\hline $4 a$ & $2.5 \times 10^{4}$ & -6.0 & -1.0 & 5.0 \\
\hline $4 b$ & $4.7 \times 10^{5}$ & -7.7 & -0.6 & 7.1 \\
\hline $7 \mathbf{a}^{b}$ & $\begin{array}{l}4.4 \times 10^{5} \\
1.8 \times 10^{4}\end{array}$ & $\begin{array}{l}-7.7 \\
-6.1\end{array}$ & $\begin{array}{l}-7.9 \\
-4.8\end{array}$ & $\begin{array}{c}-0.2 \\
1.3\end{array}$ \\
\hline $7 b^{b}$ & $\begin{array}{l}2.3 \times 10^{5} \\
5.7 \times 10^{4}\end{array}$ & $\begin{array}{l}-7.3 \\
-6.5\end{array}$ & $\begin{array}{l}-9.7 \\
-20.9\end{array}$ & $\begin{array}{l}-2.4 \\
-14.4\end{array}$ \\
\hline 9 & $2.0 \times 10^{4}$ & -5.9 & -1.5 & 4.4 \\
\hline 13 & $3.3 \times 10^{4}$ & -6.1 & -19.4 & -13.3 \\
\hline $25^{b}$ & $\begin{array}{l}7.6 \times 10^{4} \\
5.8 \times 10^{4}\end{array}$ & $\begin{array}{l}-6.7 \\
-6.5\end{array}$ & $\begin{array}{l}-4.8 \\
-0.7\end{array}$ & $\begin{array}{l}1.9 \\
5.8\end{array}$ \\
\hline
\end{tabular}

${ }^{a}$ Ligand conc. (burette) $=2 \mathrm{mmol} / \mathrm{L}, \mathrm{Eu}(\mathrm{III})$ conc. (cell) $=0.066 \mathrm{mmol} / \mathrm{L}$; in case of $\mathbf{4 a , b}$ the concentrations are halved. ${ }^{b}$ Data showed the best fit for 2:1 (L:M) complexation, so the thermodynamic parameters are given for both the 1:1 (first row) and 2:1 (second row) complexes.

From Table 6.1, it is clear that the central oxygen atom containing ligands $\mathbf{4 a}$ and $\mathbf{4 b}$ show a complexation affinity towards $\mathrm{Eu}(\mathrm{III})$ with $\mathbf{4 b}$ having a slightly higher stability constant than 4a and a sufficiently good fit for 1:1 (ligand:metal) complexation. The order is in line with the 


\section{Chapter 6}

extraction data obtained at low $\mathrm{HNO}_{3}$ concentration (Fig. 6.1). For ligand 4a, the binding enthalpy is less exothermic than that of $\mathbf{4 b}$, however, $\mathbf{4 b}$ has higher positive entropy compared to $4 \mathbf{a}$ showing the stronger release of solvent molecules from the coordination sphere.

The ligands $\mathbf{7 a}$ and $\mathbf{7 b}$ show a complexation affinity towards Eu(III) in a comparable order with a sufficiently good fit for 2:1 (ligand:metal) complexation. For ligand 7a the first binding enthalpy is exothermic as expected with a slightly negative entropy. However, the second binding enthalpy is less exothermic, which in turn is compensated by the positive entropy owing to the release of solvent molecules from the coordination sphere. Ligand $\mathbf{7 b}$ shows a comparable exothermic binding enthalpy with a slightly negative entropy. However, it exhibits a stronger exothermic binding enthalpy for the second ligand binding, which is compensated by a large negative entropy.

The tripodal ligand 9, with two dioctylamide moieties and one dibutyl phosphonate, also shows the best fit for 1:1 (ligand:metal) complexation, in an exothermic process. It has a binding affinity for $\mathrm{Eu}(\mathrm{III})$ with a negative binding enthalpy, however, the more positive entropy indicates the release of solvent molecules from the coordination sphere. Ligand 13, with two butylphosphonate, and one dioctylamide group, shows stronger binding efficiency than 9, also showing the best fit for 1:1 (ligand:metal) complexation, which clearly corresponds with the extraction data (Fig. 6.3). This can be partly attributed to the presence of two sterically hindering dioctyl groups and partly to the presence of two butyl phosphonate groups that act as stronger donors than the amide moieties. Ligand $\mathbf{1 3}$ shows a high exothermic enthalpy and a high negative entropy of binding. These ligands did also not show good extraction efficiencies due to the protonation of the central nitrogen atom under the highly acidic extraction conditions.

Ligand 25 shows a good fit for 2:1 (ligand:metal) complexation with a first negative binding enthalpy and positive entropy. In the second binding step the ligand exhibits a less negative enthalpy, which is compensated by a larger positive entropy indicating the release of solvent molecules from the coordination sphere. It shows the highest extraction efficiency compared to all other ligands (Figure 6.5) although this is not reflected in the $K$ values in Table 6.1. It should be kept in mind, however, that these cannot be directly compared, since the microcalorimetric experiments could not be carried out under similar conditions as the extractions were done, due to solubility reasons. 


\subsection{Conclusions}

Different types of hybrid-like ligands were successfully prepared in which essential parts of well-known ligands were combined. Interestingly, the mixed type ligand $\mathbf{4 a}$ shows a steeper acid dependency than CMPO, which makes the development of a partitioning process much easier, since the stripping of the actinides from a loaded phase is more facile. Surprisingly, the diphosphonothioate-based malonamide analogue 25 exhibited a much better extraction efficiency than the regular malonamides. For the ligands studied, the extraction trend was clearly demonstrated by microcalorimetry.

\subsection{Experimental}

\subsubsection{General}

All moisture-sensitive reactions were carried out under an argon atmosphere. The solvents and all reagents were obtained from commercial sources and used without further purification. All known compounds viz. $\mathbf{2} \mathbf{a}^{10}, \mathbf{3}^{11}, \mathbf{5 a}, \mathbf{b}^{15}, \mathbf{8}^{11}, \mathbf{1 4}^{16}$ and $\mathbf{2 4}{ }^{17}$ were prepared according to literature procedures. Solvents were dried according to standard procedures and stored over molecular sieves. ${ }^{1} \mathrm{H}$ NMR and ${ }^{13} \mathrm{C}$ NMR spectra were recorded on a Varian Unity INOVA (300 MHz) spectrometer. ${ }^{1} \mathrm{H}$ NMR (300 MHz) and ${ }^{13} \mathrm{C}$ NMR (75 MHz) chemical shift values are reported as $\delta$ using the residual solvent signal as an internal standard. Electrospray Ionization (positive mode) mass spectra and high resolution mass spectra were recorded on a WATERS LCT mass spectrometer. Elemental analyses were performed using a Flash 200 CHN analyzer of Thermo Scientific/Interscience. The extraction experiments were performed at the Institute of Energy and Climate Research, Jülich, Germany. Analytical TLC was performed using Merck prepared plates (silica gel 60 F-254 on aluminum). Column chromatography was carried out on Merck silica gel 60 (230-400 mesh).

\section{Di-n-butyl(hydroxymethyl)phosphinoxide (2b).}

A mixture of $\mathbf{1 b}(0.81 \mathrm{~g}, 5.0 \mathrm{mmol}), \mathrm{K}_{2} \mathrm{CO}_{3}(0.04 \mathrm{~g}, 0.3 \mathrm{mmol})$, and paraformaldehyde $(0.18$ $\mathrm{g}, 6 \mathrm{mmol})$ in cyclohexane $(15 \mathrm{~mL})$ was stirred at room temperature for $48 \mathrm{~h}$. The mixture was filtered and concentrated under reduced pressure to give $\mathbf{2 b}$ in quantitative yield. ${ }^{1} \mathrm{H}$ NMR: $\delta 0.92\left(6 \mathrm{H}, \mathrm{t}, J=7.4 \mathrm{~Hz}, \mathrm{CH}_{3}\right), 1.31-1.44\left(4 \mathrm{H}, \mathrm{m}, \mathrm{CH}_{2} \mathrm{CH}_{3}\right), 1.48-1.60(4 \mathrm{H}, \mathrm{m}$, $\left.\mathrm{PCH}_{2} \mathrm{CH}_{2}\right), 1.65-1.79\left(4 \mathrm{H}, \mathrm{m}, \mathrm{PCH}_{2}\right), 3.87\left(2 \mathrm{H}, \mathrm{d}, J=6.4 \mathrm{~Hz}, \mathrm{PCH}_{2} \mathrm{O}\right) . \mathrm{MS}: m / z 193.1$ $[\mathrm{M}+\mathrm{H}]^{+}$; calculated: 193.2 for $\mathrm{C}_{9} \mathrm{H}_{22} \mathrm{O}_{2} \mathrm{P}$. 


\section{Chapter 6}

\section{Di-n-butyl ((2-(di-n-octylamino)-2-oxoethoxy)methyl)phosphonate (4a).}

A suspension of $2 \mathbf{a}(0.69 \mathrm{~g}, 3.1 \mathrm{mmol}),(\mathrm{NaH} 60 \%)$ in oil $(0.15 \mathrm{~g}, 3.5 \mathrm{mmol})$, and $\mathrm{KI}(0.52 \mathrm{~g}$, $3.1 \mathrm{mmol})$ in dry THF $(25 \mathrm{~mL})$ was stirred for $1 \mathrm{~h}$ at $0{ }^{\circ} \mathrm{C}$. A solution of 2-chloro- $N, N$ dioctylacetamide (3) (1.00 g, $3.5 \mathrm{mmol})$ in THF (25 mL) was added dropwise to the mixture over $20 \mathrm{~min}$ at $0{ }^{\circ} \mathrm{C}$ followed by stirring at room temperature for $3 \mathrm{~h}$. The reaction mixture was quenched with saturated $\mathrm{NH}_{4} \mathrm{Cl}$ solution $(10 \mathrm{~mL})$. The solvent was evaporated and the residue was dissolved in ethyl acetate $(30 \mathrm{~mL})$. The resulting solution was washed with water $(3 \times 25 \mathrm{~mL})$. The organic layer was dried with anhydrous $\mathrm{MgSO}_{4}$ and concentrated under reduced pressure. The residue was separated by chromatography $\left(\mathrm{SiO}_{2}\right.$, ethyl acetate: methanol, 95:5) to afford $4 \mathbf{a}(0.98 \mathrm{~g}, 63 \%)$ as an oil. ${ }^{1} \mathrm{H}$ NMR: $\delta 0.80-1.01\left(12 \mathrm{H}, \mathrm{m}, \mathrm{CH}_{3}\right)$, 1.20-1.30 (20H, s, $\left.\left(\mathrm{CH}_{2}\right)_{5} \mathrm{CH}_{3}\right), 1.39\left(4 \mathrm{H}\right.$, sextet, $\left.J=7.0 \mathrm{~Hz}, \mathrm{CH}_{2} \mathrm{CH}_{3}\right), 1.52(4 \mathrm{H}, \mathrm{s}$, $\left.\mathrm{NCH}_{2} \mathrm{CH}_{2}\right), 1.66\left(4 \mathrm{H}\right.$, pentet, $\left.J=7.0 \mathrm{~Hz}, \mathrm{OCH}_{2} \mathrm{CH}_{2}\right), 3.07,3.20\left(2 \mathrm{H}, \mathrm{t}, J=6.4 \mathrm{~Hz}, \mathrm{CH}_{2} \mathrm{~N}\right)$, $3.95\left(2 \mathrm{H}, \mathrm{d}, J=7.7 \mathrm{~Hz}, \mathrm{PCH}_{2} \mathrm{O}\right), 4.03-4.17\left(4 \mathrm{H}, \mathrm{m}, \mathrm{POCH}_{2}\right), 4.29\left(2 \mathrm{H}, \mathrm{s}, \mathrm{C}(\mathrm{O}) \mathrm{CH}_{2} \mathrm{O}\right) .{ }^{13} \mathrm{C}$ NMR: $\delta 14.0,18.5,27.0,29.3,31.8,49.6,67.0,69.0,166.5$. HRMS: $m / z 506.3988[\mathrm{M}+\mathrm{H}]^{+}$; calculated: 506.3974 for $\mathrm{C}_{27} \mathrm{H}_{57} \mathrm{NO}_{5} \mathrm{P}$.

\section{2-((Di-n-butylphosphoryl)methoxy)-N,N-di-n-octylacetamide (4b).}

Based on the same procedure as described for $\mathbf{4 a}$, starting from $\mathbf{2 b}(0.60 \mathrm{~g}, 3.1 \mathrm{mmol}), \mathrm{NaH}$ (60\% in oil) (0.15 g, $3.5 \mathrm{mmol}), \mathrm{KI}(0.52 \mathrm{~g}, 3.1 \mathrm{mmol})$, and 3 (0.98 g, $3.1 \mathrm{mmol})$ afforded $4 \mathbf{b}$ $\left(1.07 \mathrm{~g}, 73 \%\right.$ yield) as an oil. ${ }^{1} \mathrm{H}$ NMR: $\delta$ 0.81-1.01 $\left(12 \mathrm{H}, \mathrm{m}, \mathrm{CH}_{3}\right), 1.18-1.26(20 \mathrm{H}, \mathrm{m}$, $\left.\left(\mathrm{CH}_{2}\right)_{5} \mathrm{CH}_{3}\right), 1.34-1.62\left(12 \mathrm{H}, \mathrm{m}, \mathrm{CH}_{2} \mathrm{CH}_{2} \mathrm{CH}_{3}, \mathrm{NCH}_{2} \mathrm{CH}_{2}\right), 1.64-1.80$ (4H, m, $\left.\mathrm{PCH}_{2} \mathrm{CH}_{2}\right)$, $3.05,3.22\left(2 \mathrm{H}, \mathrm{t}, J=6.4 \mathrm{~Hz}, \mathrm{CH}_{2} \mathrm{~N}\right), 3.83\left(2 \mathrm{H}, \mathrm{d}, J=6.4 \mathrm{~Hz}, \mathrm{PCH}_{2} \mathrm{O}\right), 4.17(2 \mathrm{H}, \mathrm{s}$, $\left.\mathrm{COCH}_{2} \mathrm{O}\right) .{ }^{13} \mathrm{C}$ NMR: $\delta$ 14.0, 22.7, 24.7, 27.0, 29.3, 31.8, 49.6, 69.0, 166.5. HRMS: $\mathrm{m} / \mathrm{z}$ 474.4099 $[\mathrm{M}+\mathrm{H}]^{+}$; calculated: 474.4076 for $\mathrm{C}_{27} \mathrm{H}_{57} \mathrm{NO}_{3} \mathrm{P}$.

\section{Di-n-butyl ((benzyl(2-(di-n-octylamino)-2-oxoethyl)amino)methyl)phosphonate (6a).}

A mixture of 5a $(1.00 \mathrm{~g}, 3.0 \mathrm{mmol}), 3(1.11 \mathrm{~g}, 3.3 \mathrm{mmol}), \mathrm{KI}(0.47 \mathrm{~g}, 3 \mathrm{mmol})$ and $\mathrm{K}_{2} \mathrm{CO}_{3}$ $(1.32 \mathrm{~g}, 9.5 \mathrm{mmol})$ in acetonitrile $(50 \mathrm{~mL})$ was refluxed for $48 \mathrm{~h}$. The solvent was evaporated and the residue was dissolved in dichloromethane $(200 \mathrm{~mL})$. The resulting solution was washed with water $(3 \times 50 \mathrm{ml})$. The organic layer was dried with anhydrous $\mathrm{MgSO}_{4}$ and concentrated under reduced pressure, followed by purification by chromatography $\left(\mathrm{SiO}_{2}\right.$, ethyl acetate) to give $\mathbf{6 a}\left(1.28 \mathrm{~g}, 62 \%\right.$ yield) as an oil. ${ }^{1} \mathrm{H}$ NMR: $\delta 0.82-0.95\left(12 \mathrm{H}, \mathrm{m}, \mathrm{CH}_{3}\right)$, 
1.20-1.30 (20H, m, $\left.\left(\mathrm{CH}_{2}\right)_{5} \mathrm{CH}_{3}\right), 1.39\left(4 \mathrm{H}\right.$, sextet, $\left.J=7.0 \mathrm{~Hz}, \mathrm{CH}_{2} \mathrm{CH}_{3}\right), 1.42-1.46(4 \mathrm{H}, \mathrm{m}$, $\left.\mathrm{NCH}_{2} \mathrm{CH}_{2}\right), 1.63\left(4 \mathrm{H}\right.$, pentet, $\left.J=7.0 \mathrm{~Hz}, \mathrm{OCH}_{2} \mathrm{CH}_{2}\right), 3.05,3.25(2 \mathrm{H}, \mathrm{t}, J=6.5 \mathrm{~Hz}$, $\left.\mathrm{CONCH}_{2}\right), 3.21\left(2 \mathrm{H}, \mathrm{d}, J=9.9 \mathrm{~Hz}, \mathrm{PCH}_{2} \mathrm{~N}\right), 3.53\left(2 \mathrm{H}, \mathrm{s}, \mathrm{NCH}_{2} \mathrm{Ar}\right), 3.92-4.07(4 \mathrm{H}, \mathrm{m}$, $\left.\mathrm{POCH}_{2}\right), 3.81\left(2 \mathrm{H}, \mathrm{s}, \mathrm{C}(\mathrm{O}) \mathrm{CH}_{2} \mathrm{~N}\right), 7.21-7.39(5 \mathrm{H}, \mathrm{m}, \mathrm{ArH}) . \mathrm{MS}: \mathrm{m} / z 595.7[\mathrm{M}+\mathrm{H}]^{+}$; calculated: 595.5 for $\mathrm{C}_{34} \mathrm{H}_{64} \mathrm{~N}_{2} \mathrm{O}_{4} \mathrm{P}$.

\section{2-(Benzyl((di-n-butylphosphoryl)methyl)amino)-N,N-di-n-octylacetamide (6b).}

Using the same procedure as described for $\mathbf{6 a}$, starting from $\mathbf{5 b}(0.89 \mathrm{~g}, 3.0 \mathrm{mmol}), \mathbf{3}(1.11 \mathrm{~g}$, $3.3 \mathrm{mmol}), \mathrm{KI}(0.47 \mathrm{~g}, 3 \mathrm{mmol})$, and $\mathrm{K}_{2} \mathrm{CO}_{3}(1.32 \mathrm{~g}, 9.5 \mathrm{mmol})$ gave $6 \mathbf{b}$ as an oil $(1.42 \mathrm{~g}$, $73 \%$ yield). ${ }^{1} \mathrm{H}$ NMR: $\delta$ 0.82-0.95 $\left(12 \mathrm{H}, \mathrm{m}, \mathrm{CH}_{3}\right), 1.15-1.23\left(20 \mathrm{H}, \mathrm{m},\left(\mathrm{CH}_{2}\right)_{5} \mathrm{CH}_{3}\right), 1.30-1.60$ $\left(12 \mathrm{H}, \mathrm{m}, \mathrm{NCH}_{2} \mathrm{CH}_{2}, \mathrm{PCH}_{2}\left(\mathrm{CH}_{2}\right)_{2}\right), 1.60-1.74\left(4 \mathrm{H}, \mathrm{m}, \mathrm{PCH}_{2}\right), 3.05,3.25(2 \mathrm{H}, \mathrm{t}, J=6.5 \mathrm{~Hz}$, $\left.\mathrm{CONCH}_{2}\right), 3.06\left(2 \mathrm{H}, \mathrm{d}, J=4.7 \mathrm{~Hz}, \mathrm{PCH}_{2} \mathrm{~N}\right), 3.60\left(2 \mathrm{H}, \mathrm{s}, \mathrm{NCH}_{2} \mathrm{Ar}\right), 3.94\left(2 \mathrm{H}, \mathrm{s}, \mathrm{C}(\mathrm{O}) \mathrm{CH}_{2} \mathrm{~N}\right)$, 7.25-7.51 (5H, m, ArH). MS: $m / z$ 563.4 $[\mathrm{M}+\mathrm{H}]^{+}$; calculated: 563.5 for $\mathrm{C}_{34} \mathrm{H}_{64} \mathrm{~N}_{2} \mathrm{O}_{2} \mathrm{P}$.

\section{Di-n-butyl (((2-(di-n-octylamino)-2-oxoethyl)amino)methyl)phosphonate (7a).}

A suspension of $6 \mathbf{a}(1.00 \mathrm{~g}, 1.7 \mathrm{mmol})$ and $5 \% \mathrm{Pd} / \mathrm{C}(300 \mathrm{mg})$ in ethanol $(50 \mathrm{~mL})$ was stirred under hydrogen atmosphere overnight. The catalyst was filtered off and the ethanol was removed under reduced pressure to give $7 \mathbf{a}$ as an oil in quantitative yield. ${ }^{1} \mathrm{H}$ NMR: $\delta 0.90$ $0.95\left(12 \mathrm{H}, \mathrm{m}, \mathrm{CH}_{3}\right), 1.24-1.30\left(20 \mathrm{H}, \mathrm{m},\left(\mathrm{CH}_{2}\right)_{5} \mathrm{CH}_{3}\right), 1.39\left(4 \mathrm{H}\right.$, sextet, $\left.J=7.0 \mathrm{~Hz}, \mathrm{CH}_{3} \mathrm{CH}_{2}\right)$, 1.50-1.54 (4H, m, $\left.\mathrm{NCH}_{2} \mathrm{CH}_{2}\right), 1.66\left(4 \mathrm{H}\right.$, pentet, $\left.J=7.0 \mathrm{~Hz}, \mathrm{POCH}_{2} \mathrm{CH}_{2}\right), 3.15,3.30(2 \mathrm{H}, \mathrm{t}, J$ $\left.=6.5 \mathrm{~Hz}, \mathrm{CONCH}_{2}\right), 3.03\left(2 \mathrm{H}, \mathrm{d}, J=11.9 \mathrm{~Hz}, \mathrm{PCH}_{2} \mathrm{~N}\right), 3.52\left(2 \mathrm{H}, \mathrm{s}, \mathrm{COCH}_{2} \mathrm{~N}\right), 4.06-4.15$ (4H, m, POCH 2$).{ }^{13} \mathrm{C}$ NMR: $\delta$ 14.0, 18.5, 22.7, 24.0, 24.4, 27.0, 29.3, 31.8, 50.0, 66.9, 164.0. HRMS: $m / z 505.4119[\mathrm{M}+\mathrm{H}]^{+}$; calculated: 505.4134 for $\mathrm{C}_{27} \mathrm{H}_{57} \mathrm{~N}_{2} \mathrm{O}_{4} \mathrm{P}$.

\section{2-(((Di-n-butylphosphoryl)methyl)amino)-N,N-di-n-octylacetamide (7b).}

$\mathbf{7 b}$ was synthesized by the same procedure as described for 7a, starting from $\mathbf{6 b}(1.20 \mathrm{~g}, 2.1$ $\mathrm{mmol})$ and $5 \% \mathrm{Pd} / \mathrm{C}(300 \mathrm{mg})$ in ethanol $(50 \mathrm{~mL})$ in quantitative yield. ${ }^{1} \mathrm{H}$ NMR: $\delta 0.85-0.95$ $\left(12 \mathrm{H}, \mathrm{m}, \mathrm{CH}_{3}\right), 1.20-1.28\left(20 \mathrm{H}, \mathrm{m},\left(\mathrm{CH}_{2}\right)_{5} \mathrm{CH}_{3}\right), 1.26-1.65\left(12 \mathrm{H}, \mathrm{m}, \mathrm{NCH}_{2} \mathrm{CH}_{2}\right.$, $\left(\mathrm{PCH}_{2}\left(\mathrm{CH}_{2}\right)_{2}\right), 1.65-1.78\left(4 \mathrm{H}, \mathrm{m}, \mathrm{PCH}_{2}\right), 3.00\left(2 \mathrm{H}, \mathrm{d}, J=7.9 \mathrm{~Hz}, \mathrm{PCH}_{2} \mathrm{~N}\right), 3.04,3.21(2 \mathrm{H}, \mathrm{t}, J$ $\left.=6.6 \mathrm{~Hz}, \mathrm{CONCH}_{2}\right), 3.54\left(2 \mathrm{H}, \mathrm{s}, \mathrm{COCH}_{2} \mathrm{~N}\right) .{ }^{13} \mathrm{C} \mathrm{NMR:} \delta$ 14.0, 22.7, 24.0, 24.4, 27.0, 29.3, 31.3, 49.0, 52.6, 164.0. HRMS: $m / z$ 473.4229 $[\mathrm{M}+\mathrm{H}]^{+}$; calculated: 473.4236 for $\mathrm{C}_{27} \mathrm{H}_{58} \mathrm{~N}_{2} \mathrm{O}_{2} \mathrm{P}$. 


\section{Chapter 6}

\section{Di-n-butyl ((bis(2-(di-n-octylamino)-2-oxoethyl)amino)methyl)phosphonate (9).}

A mixture of $8(0.80 \mathrm{~g}, 1.5 \mathrm{mmol})$, paraformaldehyde $(0.05 \mathrm{~g}, 1.6 \mathrm{mmol}), 1 \mathrm{a}(0.31 \mathrm{~g}, 1.6$ $\mathrm{mmol}), p$-toluenesulfonic acid $(0.10 \mathrm{~g})$, and molecular sieves in toluene $(35 \mathrm{~mL})$ was refluxed for $48 \mathrm{~h}$. Subsequently, $\mathrm{K}_{2} \mathrm{CO}_{3}(0.50 \mathrm{~g})$ was added and the mixture was refluxed for $10 \mathrm{~min}$. After cooling to room temperature, the mixture was washed with water $(1 \times 20 \mathrm{~mL})$, dried with anhydrous $\mathrm{MgSO}_{4}$, and concentrated under reduced pressure. The residue was purified by chromatography ( $\mathrm{SiO}_{2}$, ethyl acetate) to afford $9(0.65 \mathrm{~g}, 60 \%)$ as an oil. ${ }^{1} \mathrm{H}$ NMR: $\delta 0.82-$ $0.94\left(18 \mathrm{H}, \mathrm{m}, \mathrm{CH}_{3}\right), 1.23-1.30\left(40 \mathrm{H}, \mathrm{s},\left(\mathrm{CH}_{2}\right)_{5} \mathrm{CH}_{3}\right), 1.38\left(4 \mathrm{H}\right.$, sextet, $\left.J=7.0 \mathrm{~Hz}, \mathrm{CH}_{3} \mathrm{CH}_{2}\right)$, 1.43-1.47 (8H, m, $\left.\mathrm{NCH}_{2} \mathrm{CH}_{2}\right), 1.62\left(4 \mathrm{H}\right.$, pentet, $\left.J=7.0 \mathrm{~Hz}, \mathrm{OCH}_{2} \mathrm{CH}_{2}\right), 3.17,3.23(4 \mathrm{H}, \mathrm{t}, J=$ $\left.6.6 \mathrm{~Hz}, \mathrm{CONCH}_{2}\right), 3.31\left(2 \mathrm{H}, \mathrm{d}, J=9.3 \mathrm{~Hz}, \mathrm{PCH}_{2} \mathrm{~N}\right), 3.74\left(4 \mathrm{H}, \mathrm{s}, \mathrm{NCH}_{2} \mathrm{CO}\right), 4.07(4 \mathrm{H}, \mathrm{q}, J=$ $\left.7.0 \mathrm{~Hz}, \mathrm{CH}_{2} \mathrm{OP}\right),{ }^{13} \mathrm{C}$ NMR: $\delta$ 14.0, 18.5, 22.7, 27.0, 29.3, 31.8, 49.3, 60.0, 60.5, 66.9, 167.5 . HRMS: $m / z 786.6879[\mathrm{M}+\mathrm{H}]^{+}$; calculated: 786.6853 for $\mathrm{C}_{45} \mathrm{H}_{93} \mathrm{~N}_{3} \mathrm{O}_{5} \mathrm{P}$.

\section{Tetra-n-butyl ((benzylazanediyl)bis(methylene))bis(phosphonate) (11).}

A mixture of benzylamine (10) $(0.43 \mathrm{~g}, 4.0 \mathrm{mmol})$, paraformaldehyde $(0.24 \mathrm{~g}, 8.0 \mathrm{mmol}), \mathbf{1 a}$ (1.79 g, $8.0 \mathrm{mmol})$, $p$-toluenesulfonic acid $(0.11 \mathrm{~g})$, and molecular sieves in toluene $(35 \mathrm{~mL})$ was refluxed for $24 \mathrm{~h}$. Subsequently, $\mathrm{K}_{2} \mathrm{CO}_{3}(0.50 \mathrm{~g})$ was added and the mixture was refluxed for $10 \mathrm{~min}$. The mixture was washed with water $(1 \times 20 \mathrm{~mL})$, dried with anhydrous $\mathrm{MgSO}_{4}$, and concentrated under reduced pressure. The residue was purified by chromatography $\left(\mathrm{SiO}_{2}\right.$, ethyl acetate) to give $11(1.49 \mathrm{~g}, 72 \%)$ as an oil. ${ }^{1} \mathrm{H}$ NMR: $\delta$ 0.85-0.96 $\left(12 \mathrm{H}, \mathrm{m}, \mathrm{CH}_{3}\right), 1.37$ $\left(8 \mathrm{H}\right.$, sextet, $\left.J=7.0 \mathrm{~Hz}, \mathrm{CH}_{2} \mathrm{CH}_{3}\right), 1.62\left(8 \mathrm{H}\right.$, pentet, $\left.J=7.0 \mathrm{~Hz}, \mathrm{OCH}_{2} \mathrm{CH}_{2}\right), 3.16(4 \mathrm{H}, \mathrm{d}, J=$ 9.2 Hz, $\left.\mathrm{PCH}_{2} \mathrm{~N}\right), 3.94-4.09\left(8 \mathrm{H}, \mathrm{m}, \mathrm{POCH}_{2}\right), 4.02\left(2 \mathrm{H}, \mathrm{s}, \mathrm{ArCH}_{2} \mathrm{~N}\right), 7.22-7.40(5 \mathrm{H}, \mathrm{m}, \mathrm{ArH})$. MS: $m / z 520.3[\mathrm{M}+\mathrm{H}]^{+}$; calculated: 520.3 for $\mathrm{C}_{25} \mathrm{H}_{48} \mathrm{NO}_{6} \mathrm{P}_{2}$.

\section{Tetra-n-butyl (azanediylbis(methylene))bis(phosphonate) (12).}

A suspension of $11(1.30 \mathrm{~g}, 2.5 \mathrm{mmol})$ and $10 \% \mathrm{Pd} / \mathrm{C}(300 \mathrm{mg})$ in ethanol $(50 \mathrm{~mL})$ was stirred under a hydrogen atmosphere (10 bar) at room temperature for $72 \mathrm{~h}$. The catalyst was filtered off and the solvent was removed under reduced pressure, to give $\mathbf{1 2}$ as an oil in quantitative yield. ${ }^{1} \mathrm{H}$ NMR: $\delta$ 0.82-0.97 $\left(12 \mathrm{H}, \mathrm{m}, \mathrm{CH}_{3}\right), 1.37\left(8 \mathrm{H}\right.$, sextet, $\left.J=7.0 \mathrm{~Hz}, \mathrm{CH}_{2} \mathrm{CH}_{3}\right), 1.63(8 \mathrm{H}$, pentet, $\left.J=7.0 \mathrm{~Hz}, \mathrm{OCH}_{2} \mathrm{CH}_{2}\right), 3.11\left(4 \mathrm{H}, \mathrm{d}, J=10.6 \mathrm{~Hz}, \mathrm{PCH}_{2} \mathrm{~N}\right), 4.02-4.14\left(8 \mathrm{H}, \mathrm{m}, \mathrm{POCH}_{2}\right)$. MS: $m / z 430.3[\mathrm{M}+\mathrm{H}]^{+}$; calculated: 430.2 for $\mathrm{C}_{18} \mathrm{H}_{42} \mathrm{NO}_{6} \mathrm{P}_{2}$. 
Tetra-n-butyl (((2-(di-n-octylamino)-2-oxoethyl)azanediyl)bis(methylene))bis(phosphonate) (13).

A mixture of 3 (0.60 g, $1.9 \mathrm{mmol}), 12(0.77 \mathrm{~g}, 1.8 \mathrm{mmol}), \mathrm{K}_{2} \mathrm{CO}_{3}(0.74 \mathrm{~g}, 5.4 \mathrm{mmol})$, and $\mathrm{KI}$ $(0.27 \mathrm{~g}, 1.8 \mathrm{mmol})$ in acetonitrile $(50 \mathrm{~mL})$ was refluxed for $48 \mathrm{~h}$. The solvent was evaporated and the residue was dissolved in dichloromethane $(200 \mathrm{~mL})$. The resulting solution was washed with water $(3 \times 50 \mathrm{~mL})$. The organic layer was dried with anhydrous $\mathrm{MgSO}_{4}$ and concentrated under reduced pressure. The residue was purified by chromatography $\left(\mathrm{SiO}_{2}\right.$, dichloromethane: methanol, 97:3) to give $\mathbf{1 3}$ (1.04 g, 78\% yield) as an oil. ${ }^{1} \mathrm{H}$ NMR: $\delta$ 0.82$0.96\left(18 \mathrm{H}, \mathrm{m}, \mathrm{CH}_{3}\right), 1.23-1.29\left(20 \mathrm{H}, \mathrm{m},\left(\mathrm{CH}_{2}\right)_{5} \mathrm{CH}_{3}\right), 1.38\left(8 \mathrm{H}\right.$, sextet, $\left.J=7.0 \mathrm{~Hz}, \mathrm{CH}_{2} \mathrm{CH}_{3}\right)$, 1.49-1.53 (4H, m, $\left.\mathrm{NCH}_{2} \mathrm{CH}_{2}\right), 1.63\left(8 \mathrm{H}\right.$, pentet, $\left.J=7.0 \mathrm{~Hz}, \mathrm{OCH}_{2} \mathrm{CH}_{2}\right), 3.16,3.23(2 \mathrm{H}, \mathrm{t}, J=$ $\left.6.6 \mathrm{~Hz}, \mathrm{CONCH}_{2}\right), 3.35\left(4 \mathrm{H}, \mathrm{d}, J=9.3 \mathrm{~Hz}, \mathrm{PCH}_{2} \mathrm{~N}\right), 3.84\left(2 \mathrm{H}, \mathrm{s}, \mathrm{NCH}_{2} \mathrm{C}(\mathrm{O})\right), 4.06-4.15(8 \mathrm{H}$, m, $\left.\mathrm{CH}_{2} \mathrm{OP}\right) .{ }^{13} \mathrm{C}$ NMR: $\delta$ 14.0, 18.5, 22.7, 27.0, 29.3, 31.8, 49.3, 58.2, 66.9, 167.5. HRMS: $m / z 711.5194[\mathrm{M}+\mathrm{H}]^{+}$; calculated: 711.5206 for $\mathrm{C}_{36} \mathrm{H}_{77} \mathrm{~N}_{2} \mathrm{O}_{7} \mathrm{P}_{2}$.

\section{Tetra-n-butyl (oxybis(methylene))bis(phosphonate) (15).}

To a solution of $\mathbf{2 a}(1.00 \mathrm{~g}, 4.4 \mathrm{mmol})$ in THF (40 mL) was slowly added $\mathrm{NaH}$ (60\% in oil) $(0.2 \mathrm{~g}, 5 \mathrm{mmol})$ portionwise at $0{ }^{\circ} \mathrm{C}$. After stirring at this temperature for $1 \mathrm{~h}$, a solution of (din-butoxyphosphoryl)methyl trifluoromethanesulfonate (14) (1.58 g, $4.4 \mathrm{mmol})$ in THF (15 $\mathrm{mL}$ ) was added and stirring was continued overnight at room temperature. The solvent was evaporated and the residue was dissolved in chloroform $(30 \mathrm{~mL})$. The resulting solution was washed with $10 \% \mathrm{HCl}(3 \times 50 \mathrm{~mL})$ and water $(3 \times 50 \mathrm{~mL})$. The organic layer was dried over anhydrous $\mathrm{MgSO}_{4}$ and concentrated under reduced pressure, followed by purification of the residue with chromatography $\left(\mathrm{SiO}_{2}\right.$, dichloromethane) to afford $\mathbf{1 5}(1.29 \mathrm{~g}, 68 \%)$ as an oil. ${ }^{1} \mathrm{H}$ NMR: $\delta 0.95\left(12 \mathrm{H}, \mathrm{t}, J=7.0 \mathrm{~Hz}, \mathrm{CH}_{3}\right), 1.39\left(8 \mathrm{H}\right.$, sextet, $\left.J=7.0 \mathrm{~Hz}, \mathrm{CH}_{3} \mathrm{CH}_{2}\right), 1.63(8 \mathrm{H}$, pentet, $\left.J=7.0 \mathrm{~Hz}, \mathrm{OCH}_{2} \mathrm{CH}_{2}\right), 3.84\left(4 \mathrm{H}, \mathrm{d}, J=7.5 \mathrm{~Hz}, \mathrm{OCH}_{2} \mathrm{P}\right), 4.10(8 \mathrm{H}$, pentet, $J=7.0 \mathrm{~Hz}$, $\left.\mathrm{OCH}_{2}\right) .{ }^{13} \mathrm{C}$ NMR: $\delta 13.8,18.9,32.6,66.9,72.5,74.2$. HRMS $m / z 431.2339[\mathrm{M}+\mathrm{H}]^{+}$; calculated: 431.2328 for $\mathrm{C}_{18} \mathrm{H}_{41} \mathrm{O}_{7} \mathrm{P}_{2}$.

\section{0,0,0',0'-Tetra-n-butyl (oxybis(methylene))diphosphonothioate (16).}

A solution of 15 (1.00 g, $2.3 \mathrm{mmol})$ and Lawesson's reagent (1.88 g, $4.6 \mathrm{mmol})$ was refluxed in toluene for $2 \mathrm{~h}$. After removal of the solvent the residue was separated by chromatography $\left(\mathrm{SiO}_{2}\right.$, dichloromethane) to afford $\mathbf{1 6}(0.85 \mathrm{~g}, 80 \%)$ as an oil. ${ }^{1} \mathrm{H}$ NMR: $\delta 0.95(12 \mathrm{H}, \mathrm{t}, J=7.0$ 


\section{Chapter 6}

$\left.\mathrm{Hz}, \mathrm{CH}_{3}\right), 1.39\left(8 \mathrm{H}\right.$, sextet, $\left.J=7.0 \mathrm{~Hz}, \mathrm{CH}_{2} \mathrm{CH}_{3}\right), 1.63\left(8 \mathrm{H}\right.$, pentet, $\left.J=7.0 \mathrm{~Hz}, \mathrm{OCH}_{2} \mathrm{CH}_{2}\right)$, $4.18\left(4 \mathrm{H}, \mathrm{d}, J=7.5 \mathrm{~Hz}, \mathrm{OCH}_{2} \mathrm{P}\right), 4.07\left(8 \mathrm{H}\right.$, pentet, $\left.J=7.0 \mathrm{~Hz}, \mathrm{OCH}_{2}\right) .{ }^{13} \mathrm{C} \mathrm{NMR}: \delta 13.8$, 18.9, 32.6, 66.9, 72.3, 74.1. HRMS: $m / z$ 463.1893 $[\mathrm{M}+\mathrm{H}]^{+}$; calculated 463.1871 for $\mathrm{C}_{18} \mathrm{H}_{41} \mathrm{O}_{5} \mathrm{P}_{2} \mathrm{~S}_{2}$.

\section{(Hydroxymethyl)diphenylphosphine sulfide (18).}

A mixture of 17 (1.00 g, $4.6 \mathrm{mmol})$, paraformaldehyde (0.15 g, $5.0 \mathrm{mmol})$, and $\mathrm{K}_{2} \mathrm{CO}_{3}(0.07$ $\mathrm{g}, 0.5 \mathrm{mmol})$ in ethanol $(15 \mathrm{~mL})$ was refluxed for $4 \mathrm{~h}$. The mixture was cooled to room temperature, filtered and the solvent was evaporated under reduced pressure to afford $\mathbf{1 8}$ in quantitative yield. ${ }^{1} \mathrm{H}$ NMR: $\delta 3.03(1 \mathrm{H}, \mathrm{bs}, \mathrm{OH}), 4.42\left(2 \mathrm{H}, \mathrm{s}, \mathrm{CH}_{2}\right), 7.43-7.61(6 \mathrm{H}, \mathrm{m}, \mathrm{ArH})$, 7.70-7.83 (4H, m, ArH). MS: $m / z 248.2[\mathrm{M}+\mathrm{H}]^{+}$; calculated: 248.0 for $\mathrm{C}_{13} \mathrm{H}_{13} \mathrm{OPS}$.

\section{(Oxybis(methylene))bis(diphenylphosphine sulfide) (20).}

To a solution of $18(1.00 \mathrm{~g}, 4.0 \mathrm{mmol})$ in dichloromethane $(50 \mathrm{~mL})$, containing triethylamine $(0.50 \mathrm{~g}, 4.9 \mathrm{mmol})$ as a base, was added $p$-toluenesulfonyl chloride $(0.76 \mathrm{~g}, 4 \mathrm{mmol})$. The resulting mixture was stirred overnight at room temperature, washed with water $(3 \times 50 \mathrm{~mL})$, and the solvent was removed under reduced pressure to afford tosylate $19(1.25 \mathrm{~g}, 78 \%)$. ${ }^{1} \mathrm{H}$ NMR: $\delta 4.62\left(4 \mathrm{H}, \mathrm{d}, J=6.0 \mathrm{~Hz}, \mathrm{OCH}_{2} \mathrm{P}\right), 7.27(2 \mathrm{H}, \mathrm{d}, J=7.5 \mathrm{~Hz}, \mathrm{ArH}), 7.42-7.52(4 \mathrm{H}, \mathrm{m}$, $\mathrm{ArH})$, 7.53-7.62 (4H, m, ArH), 7.72-7.83 (4H, m, ArH). To a solution of 18 (1.00 g, 4.0 $\mathrm{mmol})$ in THF $(50 \mathrm{~mL})$ was added $\mathrm{NaH}(60 \%$ in oil $)(0.20 \mathrm{~g}, 5.0 \mathrm{mmol})$ at $0{ }^{\circ} \mathrm{C}$. After stirring at this temperature for $1 \mathrm{~h}$, a solution of $19(1.61 \mathrm{~g}, 4 \mathrm{mmol})$ in THF (10 mL) was added dropwise at $0{ }^{\circ} \mathrm{C}$. The resulting mixture was stirred at room temperature overnight. The solvent was evaporated and the residue was dissolved in dichloromethane $(50 \mathrm{~mL})$, washed with $10 \% \mathrm{HCl}(2 \times 50 \mathrm{~mL})$, and water $(2 \times 50 \mathrm{~mL})$. The organic layer was concentrated under reduced pressure and the residue was purified by chromatography $\left(\mathrm{SiO}_{2}\right.$, dichloromethane:methanol, 97:3) to afford $20(0.84 \mathrm{~g}, 71 \%)$ as a solid. (found $\mathrm{C}, 65.16 ; \mathrm{H}$, 5.11. $\mathrm{C}_{26} \mathrm{H}_{24} \mathrm{OP}_{2} \mathrm{~S}_{2}$ requires C, 65.26; H, 5.06\%). m.p. 104-106 ${ }^{\circ} \mathrm{C} .{ }^{1} \mathrm{H}$ NMR: $\delta 4.42(4 \mathrm{H}, \mathrm{d}, J$ $\left.=6.0 \mathrm{~Hz}, \mathrm{OCH}_{2} \mathrm{P}\right), 7.33-7.42(8 \mathrm{H}, \mathrm{m}, \mathrm{ArH}), 7.48-7.56(4 \mathrm{H}, \mathrm{m}, \mathrm{ArH}), 7.65-7.75(8 \mathrm{H}, \mathrm{m}, \mathrm{ArH})$. ${ }^{13} \mathrm{C}$ NMR: $\delta 75.5,76.3,128.7,129.9,131.0,132.2 . \mathrm{MS}: \mathrm{m} / \mathrm{z} 479.1[\mathrm{M}+\mathrm{H}]^{+}$; calculated: 479.1 for $\mathrm{C}_{26} \mathrm{H}_{25} \mathrm{OP}_{2} \mathrm{~S}_{2}$. 
(Oxybis(methylene))bis(di-n-butylphosphinoxide) (22).

To a solution of $\mathbf{2 b}(1.50 \mathrm{~g}, 7.8 \mathrm{mmol})$ in dichloromethane $(50 \mathrm{~mL})$, containing triethylamine as a base $(0.81 \mathrm{~g}, 8.0 \mathrm{mmol})$, was added $p$-toluenesulfonyl chloride $(1.48 \mathrm{~g}, 7.8 \mathrm{mmol})$. The resulting mixture was stirred overnight at room temperature, washed with water $(3 \times 50 \mathrm{~mL})$, and the solvent was removed under reduced pressure to afford (dibutylphosphoryl)methyl 4methylbenzenesulfonate 21 (1.85 g, 65\%). ${ }^{1} \mathrm{H}$ NMR: $\delta 0.89\left(6 \mathrm{H}, \mathrm{t}, J=7.5 \mathrm{~Hz}, \mathrm{CH}_{3}\right), 1.32$ 1.48 (4H, m, $\left.\mathrm{CH}_{3} \mathrm{CH}_{2}\right), 1.50-1.64\left(4 \mathrm{H}, \mathrm{m}, \mathrm{PCH}_{2} \mathrm{CH}_{2}\right), 1.66-1.83\left(4 \mathrm{H}, \mathrm{m}, \mathrm{PCH}_{2}\right)$, ), $4.15(2 \mathrm{H}$, $\left.\mathrm{d}, J=7.5 \mathrm{~Hz}, \mathrm{OCH}_{2} \mathrm{P}\right), 7.39(2 \mathrm{H}, \mathrm{d}, J=8.1 \mathrm{~Hz}, \mathrm{ArH}), 7.80(2 \mathrm{H}, \mathrm{d}, J=8.1 \mathrm{~Hz}, \mathrm{ArH})$. To a solution of $\mathbf{2 b}(1.00 \mathrm{~g}, 5.2 \mathrm{mmol})$ in THF $(50 \mathrm{~mL})$ was added $\mathrm{NaH}(60 \%$ in oil) $(0.24 \mathrm{~g}, 6.0$ $\mathrm{mmol})$ at $0{ }^{\circ} \mathrm{C}$. After stirring at this temperature for $1 \mathrm{~h}$, a solution of 21 (1.80 g, $\left.5.2 \mathrm{mmol}\right)$ in THF $(10 \mathrm{~mL})$ was added dropwise at $0{ }^{\circ} \mathrm{C}$. The resulting mixture was stirred at room temperature overnight. The solvent was evaporated and the residue was dissolved in dichloromethane $(50 \mathrm{~mL})$, washed with $10 \% \mathrm{HCl}(2 \times 50 \mathrm{~mL})$, and water $(2 \times 50 \mathrm{~mL})$. The organic layer was concentrated under reduced pressure and the residue was purified by chromatography $\left(\mathrm{SiO}_{2}\right.$, dichloromethane) to give $22(0.80 \mathrm{~g}, 76 \%)$ as an oil. ${ }^{1} \mathrm{H}$ NMR: $\delta 0.94\left(12 \mathrm{H}, \mathrm{t}, J=7.2 \mathrm{~Hz}, \mathrm{CH}_{3}\right), 1.42\left(8 \mathrm{H}\right.$, sextet, $\left.J=7.2 \mathrm{~Hz}, \mathrm{CH}_{3} \mathrm{CH}_{2}\right), 1.51-1.67$ $\left(8 \mathrm{H}, \mathrm{m}, \mathrm{PCH}_{2} \mathrm{CH}_{2}\right), 1.67-1.84\left(8 \mathrm{H}, \mathrm{m}, \mathrm{PCH}_{2}\right), 3.88\left(4 \mathrm{H}, \mathrm{d}, J=6.0 \mathrm{~Hz}, \mathrm{OCH}_{2} \mathrm{P}\right) .{ }^{13} \mathrm{C} \mathrm{NMR}: \delta$ 13.9, 24.1, 28.7, 29.3, 71.6, 72.9. HRMS: $m / z 367.2549[\mathrm{M}+\mathrm{H}]^{+}$; calculated: 367.2531 for $\mathrm{C}_{18} \mathrm{H}_{41} \mathrm{O}_{3} \mathrm{P}_{2}$.

\section{(Oxybis(methylene))bis(di-n-butylphosphine sulfide) (23).}

A solution of 22 ( $0.80 \mathrm{~g}, 2.2 \mathrm{mmol})$ and Lawesson's reagent (1.76 g, $4.4 \mathrm{mmol})$ was refluxed in toluene for $2 \mathrm{~h}$. After removal of the solvent the residue was separated by chromatography $\left(\mathrm{SiO}_{2}\right.$, dichloromethane) to afford $23(0.68 \mathrm{~g}, 80 \%)$ as a solid. (found $\mathrm{C}, 54.22 ; \mathrm{H}, 10.07$. $\mathrm{C}_{18} \mathrm{H}_{40} \mathrm{OP}_{2} \mathrm{~S}_{2}$ requires C, 54.24; H, 10.12\%). m.p. 81-83 ${ }^{\circ} \mathrm{C} .{ }^{1} \mathrm{H}$ NMR: $\delta 0.95(12 \mathrm{H}, \mathrm{t}, J=7.5$ $\left.\mathrm{Hz}, \mathrm{CH}_{3}\right), 1.44\left(8 \mathrm{H}\right.$, sextet, $\left.J=6.0 \mathrm{~Hz}, \mathrm{CH}_{3} \mathrm{CH}_{2}\right), 1.53-1.96\left(16 \mathrm{H}, \mathrm{m}, \mathrm{PCH}_{2} \mathrm{CH}_{2}\right), 3.91(4 \mathrm{H}, \mathrm{d}$, $\left.J=6.0 \mathrm{~Hz}, \mathrm{OCH}_{2} \mathrm{P}\right) .{ }^{13} \mathrm{C}$ NMR: $\delta$ 13.9, 24.1, 28.5, 29.1, 71.8, 72.6. MS: $m / z 399.3[\mathrm{M}+\mathrm{H}]^{+}$; calculated: 399.2 for $\mathrm{C}_{18} \mathrm{H}_{41} \mathrm{OP}_{2} \mathrm{~S}_{2}$.

\section{0,0,0',0'-Tetra-n-butyl methylenediphosphonothioate (25).}

To a solution of methylenediphosphonothioic dichloride $24(1.00 \mathrm{~g}, 3.5 \mathrm{mmol})$ and $1 \mathrm{H}$ tetrazole $(0.04 \mathrm{~g}, 0.6 \mathrm{mmol})$ in toluene $(50 \mathrm{~mL})$, was added a solution of 1-butanol $(1.05 \mathrm{~g}$, 


\section{Chapter 6}

$14.0 \mathrm{mmol})$ and diisopropylethylamine $(2.27 \mathrm{~g}, 17.6 \mathrm{mmol})$ in toluene $(20 \mathrm{~mL})$ dropwise over a $2 \mathrm{~h}$ period. After stirring overnight at room temperature, the diisopropylethylammonium and tetrazonium salts were removed by filtration. Upon evaporation of the solvent the product was purified by chromatography ( $\mathrm{SiO}_{2}$, dichloromethane) to afford tetraester $\mathbf{2 5}(1.35 \mathrm{~g}, 90 \%)$ as an oil. ${ }^{1} \mathrm{H}$ NMR: $\delta 0.93\left(12 \mathrm{H}, \mathrm{t}, J=7.5 \mathrm{~Hz}, \mathrm{CH}_{3}\right), 1.40\left(8 \mathrm{H}\right.$, sextet, $\left.J=7.5 \mathrm{~Hz}, \mathrm{CH}_{3} \mathrm{CH}_{2}\right), 1.64$ $\left(8 \mathrm{H}\right.$, pentet, $\left.J=7.5 \mathrm{~Hz}, \mathrm{OCH}_{2} \mathrm{CH}_{2}\right), 2.99\left(2 \mathrm{H}, \mathrm{t}, J=18.0 \mathrm{~Hz}, \mathrm{PCH}_{2} \mathrm{P}\right), 3.98-4.18(8 \mathrm{H}, \mathrm{m}$, $\left.\mathrm{OCH}_{2}\right) .{ }^{13} \mathrm{C}$ NMR: $\delta$ 13.9, 19.0, 32.4, 42.3, 67.1. HRMS: $\mathrm{m} / z$ 433.1778 [M+H] ${ }^{+}$; calculated: 433.1765 for $\mathrm{C}_{17} \mathrm{H}_{39} \mathrm{O}_{4} \mathrm{P}_{2} \mathrm{~S}_{2}$.

\section{Methylenebis(di-n-butylphosphine sulfide) (26).}

To a solution of $24(1.00 \mathrm{~g}, 3.5 \mathrm{mmol})$ in THF $(50 \mathrm{~mL})$, a solution of $n$-butylmagnesium chloride (2 M in THF) (7.5 mL, $15.0 \mathrm{mmol})$ was added dropwise over a period of half an hour at $-78{ }^{\circ} \mathrm{C}$. The resulting mixture was brought to room temperature in $2 \mathrm{~h}$, followed by overnight stirring at room temperature. The solvent was evaporated and the residue was dissolved in dichloromethane $(50 \mathrm{~mL})$. The resulting solution was washed with $10 \% \mathrm{HCl}(2 \times$ $30 \mathrm{~mL})$, water $(2 \times 30 \mathrm{~mL})$, and brine $(2 \times 30 \mathrm{~mL})$. The organic layer was concentrated under reduced pressure and the crude product was purified by chromatography $\left(\mathrm{SiO}_{2}\right.$, dichloromethane) to afford $26(0.85 \mathrm{~g}, 66 \%)$ as a colorless oil. ${ }^{1} \mathrm{H}$ NMR: $\delta 0.95(12 \mathrm{H}, \mathrm{t}, J=$ $\left.7.5 \mathrm{~Hz}, \mathrm{CH}_{3}\right), 1.46\left(8 \mathrm{H}\right.$, sextet, $\left.J=7.5 \mathrm{~Hz}, \mathrm{CH}_{3} \mathrm{CH}_{2}\right), 1.54-1.67\left(8 \mathrm{H}, \mathrm{m}, \mathrm{PCH}_{2} \mathrm{CH}_{2}\right), 2.07-2.21$ $\left(8 \mathrm{H}, \mathrm{m}, \mathrm{PCH}_{2}\right), 2.46\left(2 \mathrm{H}, \mathrm{t}, J=12.0 \mathrm{~Hz}, \mathrm{PCH}_{2} \mathrm{P}\right) .{ }^{13} \mathrm{C}$ NMR: $\delta 13.9,24.0,24.7,31.8,32.8$, 33.6. $\mathrm{ES}^{+} \mathrm{MS}: \mathrm{m} / z 369.1993[\mathrm{M}+\mathrm{H}]^{+}$; calculated: 369.1968 for $\mathrm{C}_{17} \mathrm{H}_{39} \mathrm{P}_{2} \mathrm{~S}_{2}$.

\subsubsection{Microcalorimetry}

Calorimetric measurements were carried out in acetonitrile using a Microcal VP-ITC microcalorimeter with a cell volume of $1.4115 \mathrm{~mL}$. For studying the complexation of Eu(III) to the ligands $\mathbf{7 a}, \mathbf{b}, \mathbf{9}, \mathbf{1 3}$, and $\mathbf{2 5}$, aliquots of a $2.0 \mathrm{mmol} / \mathrm{L}$ solution $(10 \mu \mathrm{L})$ of the ligands $(1.0 \mathrm{mmol} / \mathrm{L}$ for ligands $\mathbf{4 a , b})$ in the burette were added to a $0.066 \mathrm{mmol} / \mathrm{L}$ solution $(0.033$ $\mathrm{mmol} / \mathrm{L}$ for ligands $\mathbf{4 a}, \mathbf{b}$ ) of $\mathrm{Eu}(\mathrm{III})$ perchlorate in the calorimetric cell, monitoring the heat change after each addition. 


\subsubsection{Extraction procedure}

All the lipophilic ligands were dissolved in TPH (hydrogenated tetrapropene) to a preferable concentration of $0.1 \mathrm{~mol} / \mathrm{L}$. Complete dissolution of ligands 20 and 25 was only obtained by decreasing their concentration or addition of 1-octanol. The obtained organic solvent was contacted with nitric acid of variable concentrations $(0.01-5 \mathrm{~mol} / \mathrm{L})$ containing traces of $\mathrm{Am}(\mathrm{III})$ and $\mathrm{Eu}(\mathrm{III})$. Nitric acid solutions were prepared by diluting concentrated nitric acid (Merck KGa, Darmstadt, Germany) with ultrapure water (resistivity, $18 \mathrm{M} \Omega \mathrm{cm}$ ). Their acidity was checked by titration with $\mathrm{NaOH}$.

The batch extraction experiments were performed in $2 \mathrm{~mL}$ glass vials. Organic and aqueous phases $(500 \mu \mathrm{L})$ were spiked with $10 \mu \mathrm{L}$ of radiotracer $\left({ }^{241} \mathrm{Am},{ }^{152} \mathrm{Eu}\right.$, approx. $\left.25 \mathrm{kBq} / \mathrm{mL}\right)$ and shaken by a vortex mixer for $60 \mathrm{~min}$ at $22 \pm 1{ }^{\circ} \mathrm{C}$ using an IKA Vibrax Orbital Shaker Model VXR (2,200 rpm). Experiments using CMPO were conducted with 15 min. phase contact time, which is enough to reach equilibrium. Separation of the phases by centrifugation was followed by sampling of $200 \mu \mathrm{L}$ of each phase for analysis using a high-purity germanium spectrometer system purchased from EG\&G Ortec, München, Germany, equipped with the gamma vision software. The $\gamma$-lines at 59.5 and $121.8 \mathrm{keV}$ were examined for ${ }^{241} \mathrm{Am}$ and ${ }^{152} \mathrm{Eu}$, respectively. The distribution ratio $\mathrm{D}$ was measured as the ratio between the radioactivity of an isotope in the organic and the aqueous phase. Distribution ratios between 0.1 and 100 exhibit a maximum error of $\pm 5 \%$. The error may be up to $\pm 20 \%$ for smaller and larger values.

The kinetics extraction experiments were performed similarly as described above, except the phases contact time (mixing time).

\subsection{References}

1. Horwitz, E. P.; Kalina, D. G. Solv. Extr. Ion Exch. 1984, 2, 179.

2. a) Weaver, B. Ion Exchange and Solvent Extraction, edited by Marinsky, J. A.; Marcus, Y. (Marcel Dekker, New York, 1974), Vol. 6; b) Nash, K. L. Solv. Extr. Ion Exch. 1993, 11,729 .

3. Cuillerdier, C.; Musikas, C.; Hoel, P.; Nigond, L.; Vitart, X. Sep. Sci. Technol. 1991, 26, 1229.

4. Schulz, W. W.; Horwitz, E. P. The TRUEX process: Removal/recovery of TRU Elements from Acidic Waste Solutions; I Chem E Symposium Series, No. 103, 1987. 
5. Musikas, C. In Actinide-Lanthanide Group Separation Using Sulfur and Nitrogen Donor Extractants, Symposium on Americium and Curium Chemistry and Technology, International Chemical Congress of Pacific Basin Societies, Honolulu, 1985; Choppin, G. R., Navratil, J. D., Schulz, W. W., Eds.; World Scientific: Honolulu, HI, 1985; pp 19.

6. Zhu, Y. Radiochim. Acta 1995, 68, 95.

7. (a) Modolo, G.; Kluxen, P.; Geist, A. Radiochim. Acta 2010, 98, 193. (b) Modolo, G.; Odoj, R. Solv. Extr. Ion Exch. 1999, 17, 33. (c) Modolo, G.; Odoj, R. J. Alloys Compd. 1998, 271-273, 248.

8. For a recent review see: Ansari, S. A.; Pathak, P.; Mohapatra, P. K.; Manchanda, V. K. Chem. Rev. 2012, 112, 1751.

9. For recent examples see: a) Mohapatra, P. K.; Iqbal, M.; Raut, D. R.; Verboom, W.; Huskens, J.; Godbole, S. V. Dalton Trans. 2012, 41, 360 and b) Sengupta, A.; Mohapatra, P. K.; Iqbal, M.; Verboom, W.; Huskens, J. Dalton Trans. Accepted for publication, 2012, 41, 6970. (Chapter 5).

10. Jeanmaire, T.; Hervaud, Y.; Boutevin, B. Phosphorus Sulfur Silicon Relat. Elem. 2002, $177,1137$.

11. Iqbal, M.; Huskens, J.; Verboom, W.; Sypula, M.; Modolo, G. Supramol. Chem. 2010, 22, 827. (Chapter 3).

12. Cherkasov, R. A.; Garifzyanov, A. R.; Talan, A. S.; Davletshin, R. R.; Kurnosova, N. V. Russian J. Gen. Chem. 2009, 79, 1835.

13. Stepinski, D. C.; Nelson, D.W.; Zalupski, P.R.; Herlinger, A.W. Tetrahedron 2001, 57, 8637.

14. Schulz, W. W.; Horwitz, E. P. Sep. Sci. Technol. 1988, 23, 1191.

15. Rojas-Quijano, F. A.; Benyo, E. T.; Tircso, G.; Kalman, F. K.; Baranyai, Z.; Aime, S.; Sherry, A. D.; Kovacs, Z. Chem. Eur. J. 2009, 15, 13188.

16. Yibo, X.; Michael T.; Flavin.; Ze-Qi X. J. Org. Chem. 1996, 61, 7697.

17. Iqbal, M.; Huskens, J.; Sypula, M.; Modolo, G.; Verboom, W. New J. Chem. 2011, 35, 2591. (Chapter 4). 


\section{Chapter}

\section{Extraction of Actinides by Tripodal and Calix[4]arene Diglycolamides in Room Temperature Ionic Liquids*}

Extraction of Am(III) from acidic feed solutions was investigated using a tripodal diglycolamide (T-DGA) in three room temperature ionic liquids (RTILs), viz. C $\mathrm{Cmim}^{+}{ }^{+} \mathrm{NTf}_{2}{ }^{-}$, $C_{6}$ mim $^{+} . N T f_{2}^{-}$, and $C_{8}$ mim $^{+} . N T f_{2}^{-}$. The $\mathrm{D}_{\text {Am }}$ values decreased with increasing carbon chain length in the RTILs, which was related to the solubility of the RTIL in the aqueous phase. The distribution behavior of Am(III) was also studied with calix[4]arene diglycolamide extractants dissolved in $\mathrm{C}_{8} \mathrm{mim}^{+} \cdot \mathrm{PF}_{6}^{-}$. Apart from Am(III), extraction of $\mathrm{Pu}(\mathrm{IV}), \mathrm{U}(\mathrm{VI}) \mathrm{O}_{2}$, Eu(III), Sr(II), and Cs(I) by T-DGA as well as C4DGA was also investigated, since they have significant implications in radioactive waste processing. The stoichiometry of complexes was determined by slope analysis for both the T-DGA and C4DGA extractants. The use of RTILs gave rise to significantly improved extraction properties compared to the commonly used $\mathrm{n}$-dodecane and an unusual increase in separation factor values was observed, which can lead to selective separation of Am from wastes containing a mixture of $U(V I) O_{2}, P u(I V)$, and Am(III).

\footnotetext{
* This Chapter is based on: a) Sengupta, A.; Mohapatra, P. K.; Iqbal, M.; Huskens, J.; Verboom, W. Dalton Trans. 2012, 41, 6970. b) Sengupta, A.; Mohapatra, P. K.; Iqbal, M.; Huskens, J.; Verboom, W. RSC Adv. 2012, 2, 7492.
} 


\section{Chapter 7}

\subsection{Introduction}

Growing concern for the environment has led to an increasing interest in room temperature ionic liquids (RTILs) as an alternative to molecular diluents in myriad applications including synthesis, ${ }^{1}$ catalysis, ${ }^{2}$ separation, ${ }^{3}$ and electrochemistry. ${ }^{4}$ Of these, application of RTILs to separation science has increased significantly as can be seen from the rapid rise in the number of publications in this area in the last decade, due to their unique characteristics of high thermal stability and low volatility.

Actinide extraction using ionic liquids include the extraction of $\mathrm{U}(\mathrm{VI}) \mathrm{O}_{2}$ by tributylphosphate (TBP) in 1-butyl-3-methylimidazolium hexafluorophosphate $\left[\mathrm{C}_{4} \mathrm{mim}^{+} . \mathrm{PF}_{6}{ }^{-}\right]$, where an increased extraction of $\mathrm{U}$ was observed. $^{5}$ In another study, octyl(phenyl)- $N, N$ diisobutylcarbamoylmethyl phosphine oxide (CMPO) dissolved in RTILs has been used for the extraction of Am(III) from slightly acidic feed solutions, giving rise to much larger $D_{\mathrm{Am}}$ values compared to CMPO in $n$-dodecane. The amount of CMPO needed for Am(III) extraction from high level liquid waste (HLLW) (3-4 mol/L in $\mathrm{HNO}_{3}$ ) can be reduced by a factor of 4 (see Chapter 2). ${ }^{6}$

The diglycolamide extractants, such as $N, N, N^{\prime}, N^{\prime}$-tetra- $n$-octyl diglycolamide (TODGA), have been found significantly more effective for minor actinide partitioning as compared to CMPO. ${ }^{7}$ On the other hand, diglycolamides appended on tripodal and calix[4]arene platforms are more efficient than TODGA itself ${ }^{8,9,10}$ (Chapter 5).

In view of the improved extraction and separation behavior of extractants in RTILs, and the increased efficiency of diglycolamide-functionalized tripodal and calix[4]arene ligands for An(III), it was of interest to carry out actinide extractions with these ligands in RTILs. This Chapter deals with the extraction of Am(III) from acidic feed solutions using a tripodal diglycolamide (T-DGA) in three commercially available RTILs, viz. $\mathrm{C}_{\mathrm{n}} \operatorname{mim}^{+} \cdot \mathrm{NTf}_{2}{ }^{-},(\mathrm{n}=4,6$, 8) (1-alkyl-3-methylimidazolium bis(trifluoromethane)sulfonimide) (Chart 7.1). In addition, the extraction of Am(III) was also carried out using calix[4]arene-tetrakis-diglycolamide (C4DGA) and calix[4]arene-bis-diglycolamide compounds (C2DGA and C2DGA-Bu) (Chart 7.2) in $\mathrm{C}_{8} \mathrm{mim}^{+} \cdot \mathrm{PF}_{6}^{-}$. In addition to the effects of equilibration time and feed acidity, the stoichiometry of the complexes is studied for both extractants. 


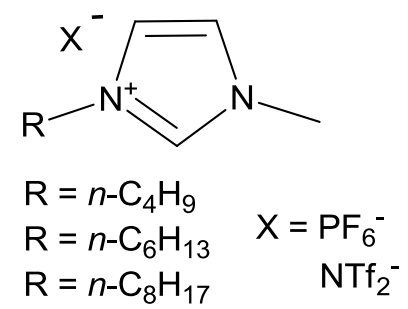

Chart 7.1. Structure of 1-alkyl-3-methylimidazolium RTILs

\subsection{Results and discussion}

\subsubsection{Synthesis}

The tripodal diglycolamide (T-DGA) was synthesized by three different approaches. In the first approach the known tripodal amine 1 was reacted with $N, N$ '-di- $n$-octyl diglycolamic acid (2) via peptide coupling in an analogous procedure reported earlier for a related derivative ${ }^{8}$ to give T-DGA in $25 \%$ yield. The low yield may be caused by the bulky isopentyl groups on the nitrogens of the tripodal amine. The formation of T-DGA clearly followed from the ${ }^{1} \mathrm{H}$ NMR spectrum as the $\mathrm{CH}_{2} \mathrm{~N}$ peak at $2.66 \mathrm{ppm}$ and the $\mathrm{NCH}$ signal at $2.37 \mathrm{ppm}$ in $\mathbf{1}$ shifted to a multiplet at 3.10-3.47 ppm. The MALDI mass spectrum showed the $[\mathrm{M}+\mathrm{H}]^{+}$and $[\mathrm{M}+\mathrm{Na}]^{+}$ peaks at $m / z 1535.0$ and 1557.0, respectively. To increase the yield, another approach was followed, in which tripodal amine $\mathbf{1}$ was first reacted with the more reactive diglycolic anhydride to give tripodal glycolamic acid (3). In the second step, $\mathbf{3}$ was further reacted with $n$-dioctylamine to afford the desired T-DGA; in this way the yield was raised to $50 \%$. In the third approach tripodal amine 1 was reacted with $p$-nitrophenol activated DGA (4) to afford T-DGA in 74\% yield. (Scheme 7.1).

The DGA-functionalized calix[4]arene derivatives (Chart 7.2) were synthesized as described in Chapter 5. 


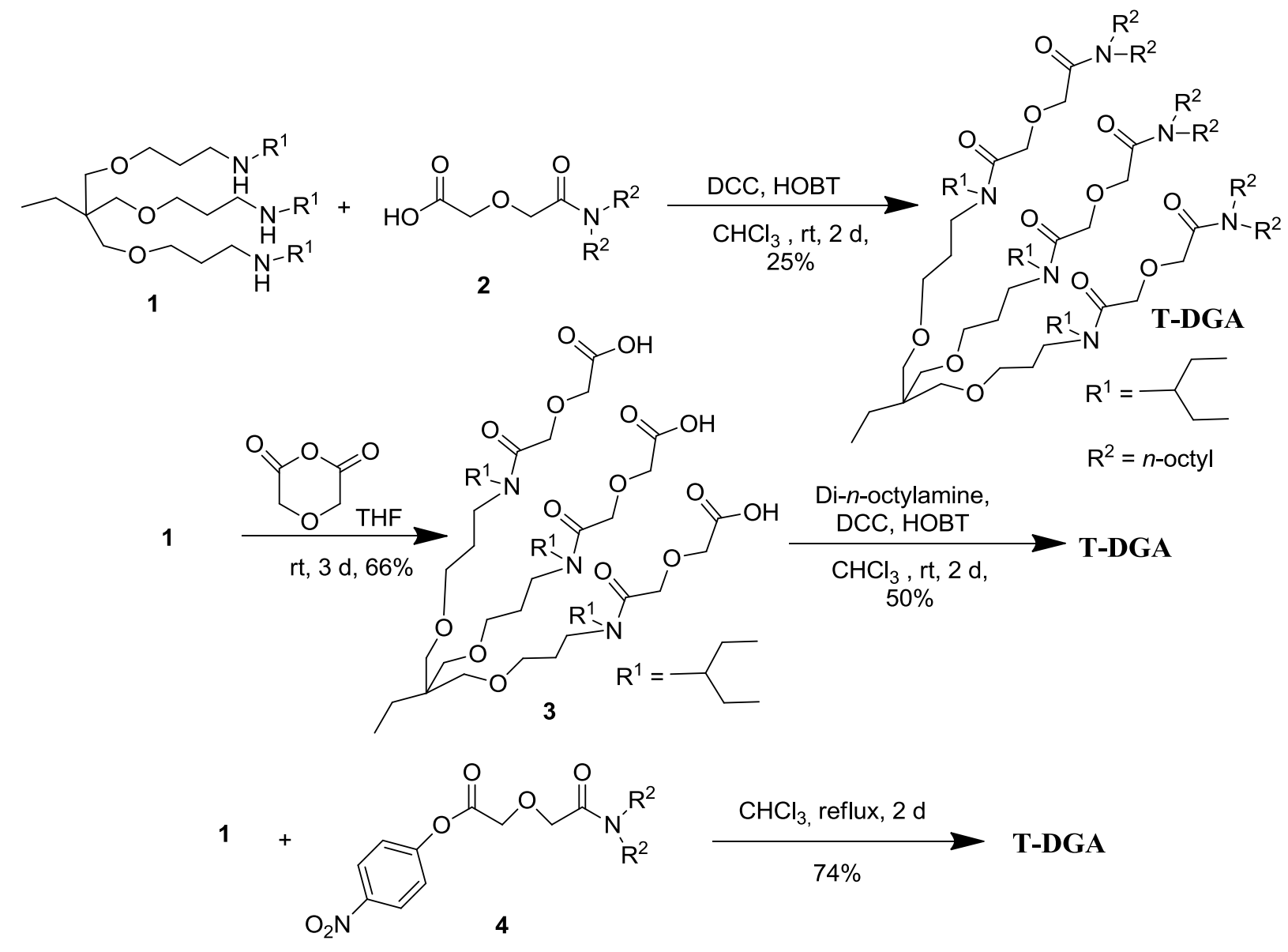

Scheme 7.1

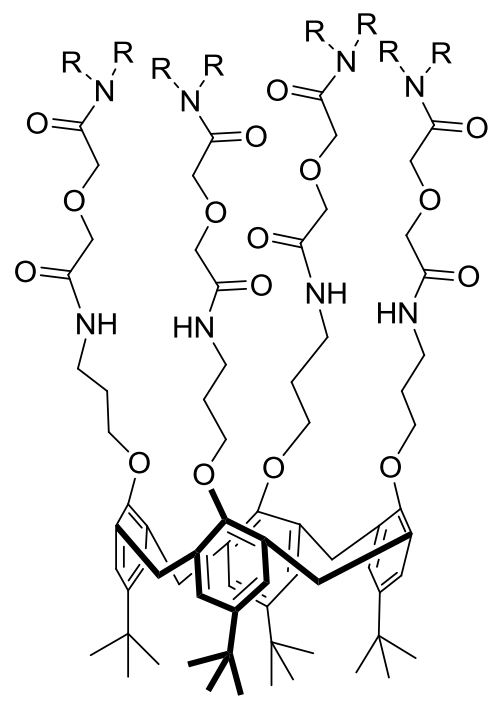

C4DGA

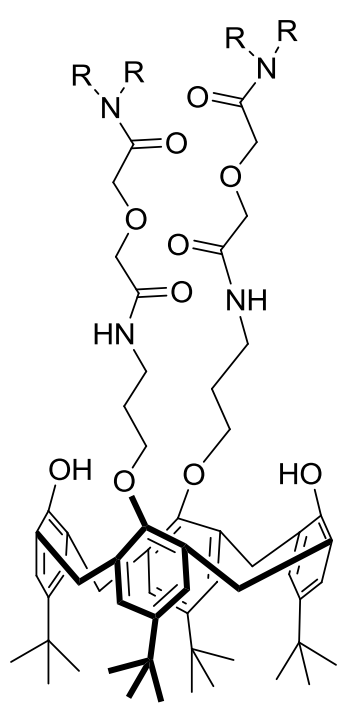

C2DGA

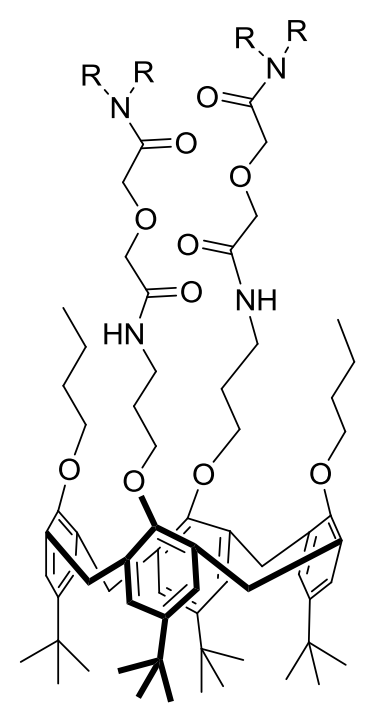

C2DGA-Bu

$\mathrm{R}=\mathrm{C}_{8} \mathrm{H}_{17}$

Chart 7.2. DGA-functionalized calix[4]arenes 


\subsubsection{Extraction results}

The extraction of Am(III) was performed with T-DGA and the DGA-functionalized calix[4]arene derivatives in different RTILs, and the results were compared to those of TODGA. No extraction was observed with the RTIL in the absence of the extractants, however, a sharp increase in the $D_{\mathrm{M}}$ values was observed when a small amount of the extractant was added to the ionic liquid phase.

The extraction data for $\mathrm{Am}(\mathrm{III})$ are listed in Table 7.1 with the three ionic liquids, viz. $\mathrm{C}_{4} \mathrm{mim}^{+} . \mathrm{NTf}_{2}{ }^{-}, \mathrm{C}_{6} \mathrm{mim}^{+} . \mathrm{NTf}_{2}{ }^{-}$and $\mathrm{C}_{8} \mathrm{mim}^{+} . \mathrm{NTf}_{2}{ }^{-}$and a $1.0 \mathrm{mmol} / \mathrm{L}$ concentration of both the extractants T-DGA and TODGA. The observed trend of Am(III) extraction is $\mathrm{C}_{4} \mathrm{mim}^{+} \cdot \mathrm{NTf}_{2}{ }^{-}>$ $\mathrm{C}_{6} \mathrm{mim}^{+} . \mathrm{NTf}_{2}^{-}>\mathrm{C}_{8} \mathrm{mim}^{+} . \mathrm{NTf}_{2}^{-}$, which is similar to that reported for several systems such as the extraction of $\mathrm{Am}(\mathrm{III})$ and $\mathrm{U}(\mathrm{VI}) \mathrm{O}_{2}$ using CMPO and TODGA as the extractants, respectively. ${ }^{11}$ Apparently, the relatively higher aqueous solubility of the butyl form of the ionic liquid was responsible for the higher metal ion extraction as will be discussed below. Comparison of the $D_{\mathrm{Am}}$ values of T-DGA in RTILs with those in $n$-dodecane, shows that the RTIL system has a much higher extraction efficiency.

Table 7.1. Distribution constants of Am(III), $D_{\text {Am }}$, vs. nitric acid feed concentration using 1.0 $\mathrm{mmol} / \mathrm{L}$ TODGA and T-DGA in RTILs and a reference solvent mixture.

\begin{tabular}{|c|c|c|c|c|c|c|c|}
\hline \multirow[t]{2}{*}{$\begin{array}{c}{\left[\mathrm{HNO}_{3}\right],} \\
\mathrm{mol} / \mathrm{L}\end{array}$} & \multicolumn{3}{|c|}{$\begin{array}{c}D_{\text {Am }} \text { in T-DGA in different } \\
\text { RTILs }\end{array}$} & \multirow{2}{*}{$\begin{array}{c}D_{\text {Am }} \text { in } \\
\text { T-DGA in } \\
n \text {-dodecane } \\
+ \text { iso-dodecanol }\end{array}$} & \multicolumn{3}{|c|}{$\begin{array}{c}D_{\mathrm{Am}} \text { in TODGA }{ }^{\mathrm{a}} \text { in different } \\
\text { RTILs }\end{array}$} \\
\hline & $\begin{array}{l}\mathrm{C}_{4} \mathrm{mim}^{+} . \\
\mathrm{NTf}_{2}^{-}\end{array}$ & $\begin{array}{l}\mathrm{C}_{6} \mathrm{mim}^{+} . \\
\mathrm{NTf}_{2}^{-}\end{array}$ & $\begin{array}{l}\mathrm{C}_{8} \mathrm{mim}^{+} . \\
\mathrm{NTf}_{2}^{-}\end{array}$ & & $\begin{array}{l}\mathrm{C}_{4} \mathrm{mim}^{+} . \\
\mathrm{NTf}_{2}^{-}\end{array}$ & $\begin{array}{l}\mathrm{C}_{6} \mathrm{mim}^{+} . \\
\mathrm{NTf}_{2}^{-}\end{array}$ & $\begin{array}{c}\mathrm{C}_{8} \mathrm{mim}^{+} \\
\cdot \\
\mathrm{NTf}_{2}^{-}\end{array}$ \\
\hline 0.01 & $1.5 \times 10^{3}$ & 161 & 105 & 0.02 & 208 & 28 & 26 \\
\hline 0.5 & 91 & 32 & 3 & 0.05 & 0.7 & 0.3 & 0.2 \\
\hline 3 & 0.6 & 0.2 & 0.2 & 30 & 0.3 & 0.1 & 0.1 \\
\hline
\end{tabular}

${ }^{\text {a }}$ The $D_{\mathrm{Am}}$ values for TODGA in $n$-dodecane are $<0.03$ at all $\mathrm{HNO}_{3}$ concentrations.

Apart from the $\mathrm{Am}(\mathrm{III})$ ion, the extraction of several actinide ions, viz. $\mathrm{U}(\mathrm{VI}) \mathrm{O}_{2}$ and $\mathrm{Pu}(\mathrm{IV})$, was also investigated using $0.5 \mathrm{~mol} / \mathrm{L} \mathrm{HNO}_{3}$ as the aqueous feed and $1.0 \mathrm{mmol} / \mathrm{L} \mathrm{T}$-DGA in the ionic liquids as the extractant; the results are listed in Table 7.2. Distribution ratio data for some important fission product elements were also determined under identical experimental conditions. The results are included for comparison purposes, the extraction trend being 


\section{Chapter 7}

$\mathrm{Eu}(\mathrm{III})>\mathrm{Am}(\mathrm{III})>\mathrm{Pu}(\mathrm{IV})>\mathrm{U}(\mathrm{VI}) \mathrm{O}_{2} \sim \mathrm{Sr}(\mathrm{II})>\mathrm{Cs}(\mathrm{I})$. The extractability trend for these elements is similar to that reported for the T-DGA $-n$-dodecane / iso-decanol system. ${ }^{10}$

Table 7.2. Distribution constants of actinides and fission product elements using $1.0 \mathrm{mmol} / \mathrm{L}$ T-DGA in different RTILs. Feed: $0.5 \mathrm{~mol} / \mathrm{L} \mathrm{HNO}_{3}$.

\begin{tabular}{cccc}
\hline & \multicolumn{3}{c}{$D$ values of T-DGA in RTILs } \\
\cline { 2 - 4 } Metal ions & $\mathrm{C}_{4} \mathrm{mim}^{+} . \mathrm{NTf}_{2}{ }^{-}$ & $\mathrm{C}_{6} \mathrm{mim}^{+} . \mathrm{NTf}_{2}{ }^{-}$ & $\mathrm{C}_{8} \mathrm{mim}^{+} . \mathrm{NTf}_{2}{ }^{-}$ \\
\hline $\mathrm{Am}(\mathrm{III})$ & 91 & 33 & 3.1 \\
$\mathrm{Pu}(\mathrm{IV})$ & 30 & 17 & 1.4 \\
$\mathrm{U}(\mathrm{VI}) \mathrm{O}_{2}$ & 0.2 & 0.2 & 0.06 \\
$\mathrm{Cs}(\mathrm{I})$ & 0.1 & 0.04 & 0.02 \\
$\mathrm{Sr}(\mathrm{II})$ & 0.3 & 0.1 & 0.02 \\
$\mathrm{Eu}(\mathrm{III})$ & 219 & 64 & 13 \\
\hline
\end{tabular}

The extraction of $\mathrm{Am}(\mathrm{III})$ by $\mathrm{C} 4 \mathrm{DGA}$ at $3 \mathrm{~mol} / \mathrm{L} \mathrm{HNO}_{3}$ was carried out using $\mathrm{C}_{8} \mathrm{mim}^{+} \cdot \mathrm{PF}_{6}^{-}$, which displayed a good solubility of the extractant and resulted in reasonably high distribution values. The extraction efficiency of Am(III) by C4DGA was compared to those of the calix-2DGA ligands (abbreviated as C2DGA and C2DGA-Bu (Chart 7.2)) and TODGA. The concentration of the three calix-DGA ligands and TODGA was taken as $5 \times 10^{-4} \mathrm{~mol} / \mathrm{L}$ and $1 \times 10^{-2} \mathrm{~mol} / \mathrm{L}$, respectively. The results of Am(III) extraction from $3 \mathrm{~mol} / \mathrm{L} \mathrm{HNO}_{3}$ feed solutions (with an equilibration time of $3 \mathrm{~h}$ ) are listed in Table 7.3. The results showed a significantly lower extraction of Am(III) with the C2DGA compounds, clearly indicating that the C4DGA is far more effective for metal ion extraction. This is in line with our previous observations on the extraction behavior of the three calix[4]arene DGA compounds in $n$-dodecane as the organic diluent as described in Chapter $5 .{ }^{12}$ Because of the high $D$ values of the $\mathrm{C} 4 \mathrm{DGA}$ ligand at $3 \mathrm{~mol} / \mathrm{L} \mathrm{HNO}_{3}$ (comparable to nuclear waste), further studies were only performed with this ligand. T-DGA, on the other hand, exhibited very low $D_{\text {Am }}$ values at 3 $\mathrm{mol} / \mathrm{L}_{\mathrm{HNO}}$. Therefore, further studies with T-DGA were carried out at $0.5 \mathrm{~mol} / \mathrm{L}^{\mathrm{HNO}_{3}}$. Because of the different conditions used, a real comparison of C4DGA and T-DGA ligands is not possible at this stage. 
Table 7.3. Distribution constants of $A m(I I I), D_{\mathrm{Am}}$, for the diglycolamide-functionalized calix[4]arenes $\left(5 \times 10^{-4} \mathrm{~mol} / \mathrm{L}\right)$ and TODGA $\left(1 \times 10^{-2} \mathrm{~mol} / \mathrm{L}\right)$ in $\mathrm{C}_{8} \mathrm{mim}^{+} . \mathrm{PF}_{6}^{-}$from $3 \mathrm{~mol} / \mathrm{L}$ $\mathrm{HNO}_{3}$.

\begin{tabular}{cc}
\hline Extractant & $D_{\text {Am }}$ \\
\hline C4DGA & 194 \\
C2DGA & 0.7 \\
C2DGA-Bu & 0.2 \\
TODGA & 75 \\
\hline
\end{tabular}

Solvent extraction studies of several other actinide ions such as $\mathrm{U}(\mathrm{VI}) \mathrm{O}_{2}, \mathrm{~Np}(\mathrm{IV}), \mathrm{Pu}(\mathrm{IV})$, and $\mathrm{Pu}(\mathrm{VI}) \mathrm{O}_{2}$ were carried out and the results are listed in Table 7.4. As most radioactive wastes consist of long lived fission product nuclides such as ${ }^{90} \mathrm{Sr}\left(\mathrm{t}_{1 / 2}=28.5 \mathrm{y}\right)$ and ${ }^{137} \mathrm{Cs}\left(\mathrm{t}_{1 / 2}=\right.$ $30.1 \mathrm{y}$ ), distribution data of $\mathrm{Sr}(\mathrm{II})$ and $\mathrm{Cs}(\mathrm{I})$ are also included using C4DGA as well as TODGA in $\mathrm{C}_{8} \mathrm{mim}^{+} \cdot \mathrm{PF}_{6}^{-}$at concentrations of $5 \times 10^{-5}$ and $1 \times 10^{-2} \mathrm{~mol} / \mathrm{L}$, respectively, from 3 $\mathrm{mol} / \mathrm{L} \mathrm{HNO}_{3}$ feed solutions (Table 7.4). It is interesting to note that the $D$ values for Am(III), $\mathrm{Np}(\mathrm{IV})$, and $\mathrm{Pu}(\mathrm{IV})$ are significantly higher compared to those obtained with other metal ions, viz. $\mathrm{Pu}(\mathrm{VI}) \mathrm{O}_{2}, \mathrm{U}(\mathrm{VI}) \mathrm{O}_{2}, \mathrm{Cs}(\mathrm{I})$, and $\mathrm{Sr}(\mathrm{II})$, indicating the effectiveness of TODGA in RTIL for minor actinide partitioning. The $D_{\mathrm{Am}}$ values with C4DGA are much higher than those obtained with TODGA, while the $D$ values for $\mathrm{U}(\mathrm{VI}) \mathrm{O}_{2}$ and $\mathrm{Pu}(\mathrm{VI}) \mathrm{O}_{2}$ are significantly lower with C4DGA than with TODGA. The low extraction of $U$ and $\mathrm{Pu}$ in the hexavalent oxidation state is rather surprising and may be based on the unusual coordination geometry of the actinyl ion. 


\section{Chapter 7}

Table 7.4. Distribution constants of actinides and fission products from $3 \mathrm{~mol} / \mathrm{L} \mathrm{HNO}_{3}$ feed solutions using TODGA $\left(1 \times 10^{-2} \mathrm{~mol} / \mathrm{L}\right)$ and C4DGA $\left(5 \times 10^{-5} \mathrm{~mol} / \mathrm{L}\right)$ as the extractant in $\mathrm{C}_{8} \mathrm{mim}^{+} . \mathrm{PF}_{6}^{-}$.

\begin{tabular}{ccc}
\hline & \multicolumn{2}{c}{$D$ values of DGA extractants } \\
\cline { 2 - 3 } Metal ion & TODGA & C4DGA \\
\hline $\mathrm{Am}(\mathrm{III})$ & 75 & 21 \\
$\mathrm{Pu}(\mathrm{IV})$ & 31 & 0.6 \\
$\mathrm{Pu}(\mathrm{VI}) \mathrm{O}_{2}$ & 1.2 & 0.2 \\
$\mathrm{U}(\mathrm{VI}) \mathrm{O}_{2}$ & 0.5 & 7 \\
$\mathrm{~Np}(\mathrm{IV})$ & 4 & 0.02 \\
$\mathrm{Sr}(\mathrm{II})$ & 0.02 & 0.2 \\
$\mathrm{Cs}(\mathrm{I})$ & 0.1 &
\end{tabular}

The separation factors $(S F$, defined as the ratio of the distribution constants for a pair of metal ions) were determined for the metal ion pairs $\mathrm{Am}-\mathrm{U}$ and $\mathrm{Am}-\mathrm{Pu}$ for both extractants, viz. TODGA and C4DGA, in $\mathrm{C}_{8} \mathrm{mim}{ }^{+} \cdot \mathrm{PF}_{6}{ }^{-}$as well as in $n$-dodecane, and the results are listed in Table 7.5. It is interesting to note that $S F$ values for Am are enhanced in the ionic liquid medium as compared to $n$-dodecane. Moreover, C4DGA was found to be a superior extractant from the actinide separation point of view. Concludingly, C4DGA in $\mathrm{C}_{8} \mathrm{mim}^{+} \cdot \mathrm{PF}_{6}{ }^{-}$results in a highly efficient separation system for Am(III) extraction.

Table 7.5. Separation factors, $S F$, for $\mathrm{Am}(\mathrm{III})$ relative to $\mathrm{U}(\mathrm{VI}) \mathrm{O}_{2}$ and $\mathrm{Pu}(\mathrm{IV})$ with TODGA $\left(1.0 \times 10^{-2} \mathrm{~mol} / \mathrm{L}\right)$ and C4DGA $\left(5.0 \times 10^{-4} \mathrm{~mol} / \mathrm{L}\right)$ in ionic liquid and $n$-dodecane

\begin{tabular}{ccc}
\hline Solvent system & $S F_{\mathrm{Am} / \mathrm{U}}$ & $S F_{\mathrm{Am} / \mathrm{Pu}}$ \\
\hline TODGA in $n$-dodecane & 132 & 25 \\
TODGA in $\mathrm{C}_{8} \mathrm{mim}^{+} . \mathrm{PF}_{6}^{-}$ & 144 & 63 \\
C4DGA in $n$-dodecane & 45 & 173 \\
$\mathrm{C}^{-}$ & 1021 & 303 \\
\hline
\end{tabular}




\subsubsection{Extraction kinetics}

Although extraction kinetics is an important parameter in all liquid-liquid extraction studies, it has great significance in studies involving ionic liquids, as these diluents invariably display higher viscosities than molecular diluents. The extraction kinetics of Am(III) was studied from a feed solution containing $1.0 \times 10^{-3} \mathrm{~mol} / \mathrm{L}$ T-DGA in $\mathrm{C}_{\mathrm{n}} \mathrm{mim}^{+} \mathrm{NTf}_{2}^{-}$. Figure 7.1 represents the extraction kinetics data up to a period of $4 \mathrm{~h}$, in which complete equilibration was seen for all three RTILs. The rate of equilibration follows the trend: $\mathrm{C}_{4} \mathrm{mim}^{+} . \mathrm{NTf}_{2}{ }^{-}>$ $\mathrm{C}_{6} \mathrm{mim}^{+} . \mathrm{NTf}_{2}^{-}>\mathrm{C}_{8} \mathrm{mim}^{+} . \mathrm{NTf}_{2}^{-}$, suggesting that while $1 \mathrm{~h}$ is sufficient for the $n$-butyl derivative, $2 \mathrm{~h}$ are required for both the $n$-hexyl and $n$-octyl derivatives of the ionic liquids. Although the $D_{\mathrm{Am}}$ values with $\mathrm{C}_{6} \mathrm{mim}^{+} . \mathrm{NTf}_{2}{ }^{-}$as the diluent are between those observed with $\mathrm{C}_{4} \mathrm{mim}^{+} . \mathrm{NTf}_{2}^{-}$and $\mathrm{C}_{8} \mathrm{mim}^{+} . \mathrm{NTf}_{2}^{-}$, the equilibration time for $\mathrm{C}_{6} \mathrm{mim}^{+} . \mathrm{NTf}_{2}^{-}$is similar to that of $\mathrm{C}_{8} \mathrm{mim}^{+} \cdot \mathrm{NTf}_{2}^{-}$.

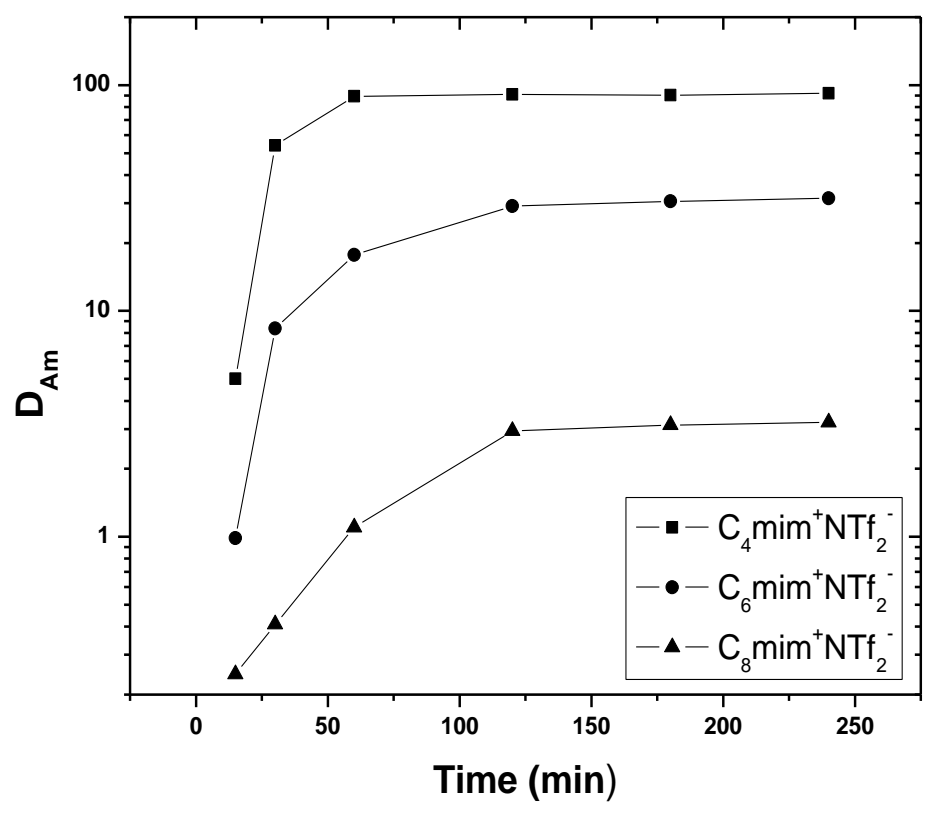

Figure 7.1. Distribution constants of $\mathrm{Am}(\mathrm{III}), D_{\mathrm{Am}}$, vs. time using $1.0 \mathrm{mmol} / \mathrm{L}$ T-DGA in $\mathrm{C}_{\mathrm{n}} \mathrm{mim}^{+} \cdot \mathrm{NTf}_{2}^{-}(\mathrm{n}=4,6,8)$ and a $0.5 \mathrm{~mol} / \mathrm{L} \mathrm{HNO}_{3}$ feed.

The extraction kinetics was also studied using $1.0 \mathrm{mmol} / \mathrm{L} \mathrm{C} 4 \mathrm{DGA}$ in $\mathrm{C}_{8} \mathrm{mim}^{+} \cdot \mathrm{PF}_{6}{ }^{-}$(Figure 7.2) from a $3 \mathrm{~mol} / \mathrm{L} \mathrm{HNO}_{3}$ feed solution and compared with TODGA under similar conditions. The distribution ratio of Am(III) increased up to a plateau with $D_{\mathrm{Am}} \sim 700$ within $180 \mathrm{~min}$. In case of TODGA, the $D_{\mathrm{Am}}$ value for $\mathrm{C}_{8} \mathrm{mim}^{+} \cdot \mathrm{PF}_{6}{ }^{-}$increased to a plateau with a $D_{\text {Am }}$ value of $\sim 70$, also within $180 \mathrm{~min}$. The time required to attain the maximum $D_{\text {Am }}$ values 


\section{Chapter 7}

is similar for both TODGA and C4DGA. The extraction kinetics for T-DGA and C4DGA in RTILs is much slower compared to the $15 \mathrm{~min}$ of equilibration time in cases when the molecular solvents are used as diluents.

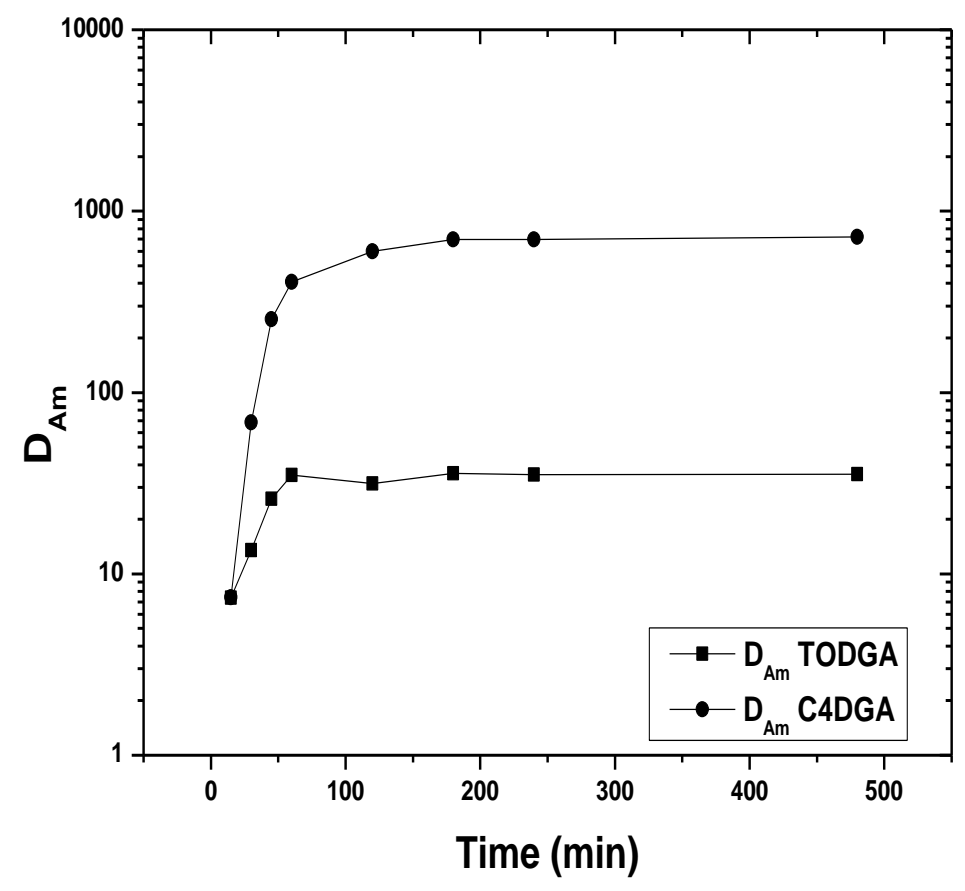

Figure 7.2. Distribution constants of $\mathrm{Am}(\mathrm{III}), D_{\mathrm{Am}}$, vs. time using $1.0 \mathrm{mmol} / \mathrm{L}$ C4DGA and $10 \mathrm{mmol} / \mathrm{L}$ TODGA in $\mathrm{C}_{8} \mathrm{mim}^{+} . \mathrm{PF}_{6}{ }^{-}$and $3 \mathrm{~mol} / \mathrm{L} \mathrm{HNO}_{3}$ feed.

\subsubsection{Effect of the feed acidity}

Usually, for solvating type extractants, such as TBP, CMPO, and TODGA, the extraction of the metal ion increases with increasing feed acidity, which is based on an increase in the counter anion concentration which helps in the formation of neutral extractable species as per equation 1:

$$
\mathrm{M}^{\mathrm{n}+}{ }_{\mathrm{aq}}+\mathrm{nNO}_{3}{ }_{\mathrm{aq}}^{-}+\mathrm{mL}_{\mathrm{org}} \leftrightarrow\left[\mathrm{M}\left(\mathrm{NO}_{3}\right)_{\mathrm{n}} \cdot \mathrm{L}_{\mathrm{m}}\right]_{\mathrm{org}}
$$

where, species with the subscript 'org' indicate those present in the organic phase, while those with subscript 'aq' indicate species in the aqueous phase. Although this trend has invariably been observed in molecular diluents such as $n$-dodecane, ${ }^{13}$ an entirely different trend, i.e., decrease in the metal ion extraction with increasing aqueous feed acidity, has been reported in 
ionic liquids as the diluent due to an entirely different extraction mechanism ${ }^{14}$ as indicated in equation 2.

$$
\begin{gathered}
\operatorname{Am}(\mathrm{III})_{\mathrm{aq}}+\mathrm{qH}^{+}{ }_{\mathrm{IL}}+\mathrm{mL}_{\mathrm{IL}}+(3-\mathrm{q}) \mathrm{C}_{\mathrm{n}} \mathrm{mim}^{+}{ }_{\mathrm{IL}}+3 \mathrm{X}_{\mathrm{IL}}^{-} \leftrightarrow \\
{\left[\mathrm{Am}(\mathrm{L})_{\mathrm{m}}{ }^{3+} \cdot 3 \mathrm{X}^{-}\right]_{\mathrm{IL}}+\mathrm{qH}^{+}{ }_{\mathrm{aq}}+(3-\mathrm{q}) \mathrm{C}_{\mathrm{n}} \mathrm{mim}^{+}{ }_{\mathrm{aq}}}
\end{gathered}
$$

Eqn. 2 describes that, in case of RTILs as the organic phase, a cation exchange mechanism is operating, where metal ions are extracted into the IL phase while $\mathrm{C}_{\mathrm{n}} \mathrm{mim}^{+}$cations move into the aqueous phase. It is well known that ILs easily dissolve $\mathrm{HNO}_{3},{ }^{15}$ and large amounts of acids are readily transferred to the IL phase during the phase equilibration stage of extraction experiments. ${ }^{16}$ Therefore, adding larger amounts of acid to the aqueous phase (lower $\mathrm{pH}$ ) drives the equilibrium of eqn 2 to the left which explains the decrease of $D_{\text {Am }}$ values upon increase of $\left[\mathrm{H}^{+}\right]$in the aqueous phase. Upon increasing the alkyl chain length in $\mathrm{C}_{\mathrm{n}} \mathrm{mim}^{+}$, the $D_{\text {Am }}$ value is expected to decrease due to a decrease in solubility of the $\mathrm{C}_{\mathrm{n}} \mathrm{mim}^{+}$cation in the aqueous phase. The slower extraction kinetics upon increase of the alkyl chain length in the $\mathrm{C}_{\mathrm{n}} \mathrm{mim}^{+}$cation (Figures 7.1 and 7.2) can potentially also be explained by the cation exchange mechanism, where the rate of solubilization of the $\mathrm{C}_{\mathrm{n}} \mathrm{mim}^{+}$cations in the aqueous phase is expected to decrease upon increase in chain length. The other possible reason for the observed slower extraction kinetics upon increase of the alkyl chain length in the $\mathrm{C}_{\mathrm{n}} \mathrm{mim}^{+}$cation might be the increase in viscosity of the IL for longer chain lengths.

Figure 7.3 shows the dependence of the distribution ratio of Am(III) with the change in the feed nitric acid concentration for the ionic liquid solvents containing $1.0 \mathrm{mmol} / \mathrm{L}$ T-DGA. It is clear that an increase in the feed nitric acid concentration led to a decrease in the $D_{\mathrm{M}}$ values in the acidity range of $0.01-3 \mathrm{~mol} / \mathrm{L}$, beyond which a slight increase was noticed. The decrease in $D_{\mathrm{Am}}$ values was less significant for $\mathrm{C}_{6} \mathrm{mim}^{+} . \mathrm{NTf}_{2}{ }^{-}$and $\mathrm{C}_{8} \mathrm{mim}^{+} . \mathrm{NTf}_{2}{ }^{-}$as compared to when $\mathrm{C}_{4} \mathrm{mim}^{+}$. $\mathrm{NTf}_{2}^{-}$was used as the ionic liquid (Figure 7.3). The extraction profiles obtained in the present study indicate that, apparently, a cation-exchange mechanism is responsible for the extraction of Am(III) into the ionic liquid phase. As shown in Figure 7.3, there is a marginal increase in the $D_{\mathrm{Am}}$ values for all three ionic liquids beyond $3 \mathrm{~mol} / \mathrm{L} \mathrm{HNO}_{3}$, which can be attributed to a change of mechanism with nitrate ion participation, as described previously for a crown ether extraction system. ${ }^{17}$ 


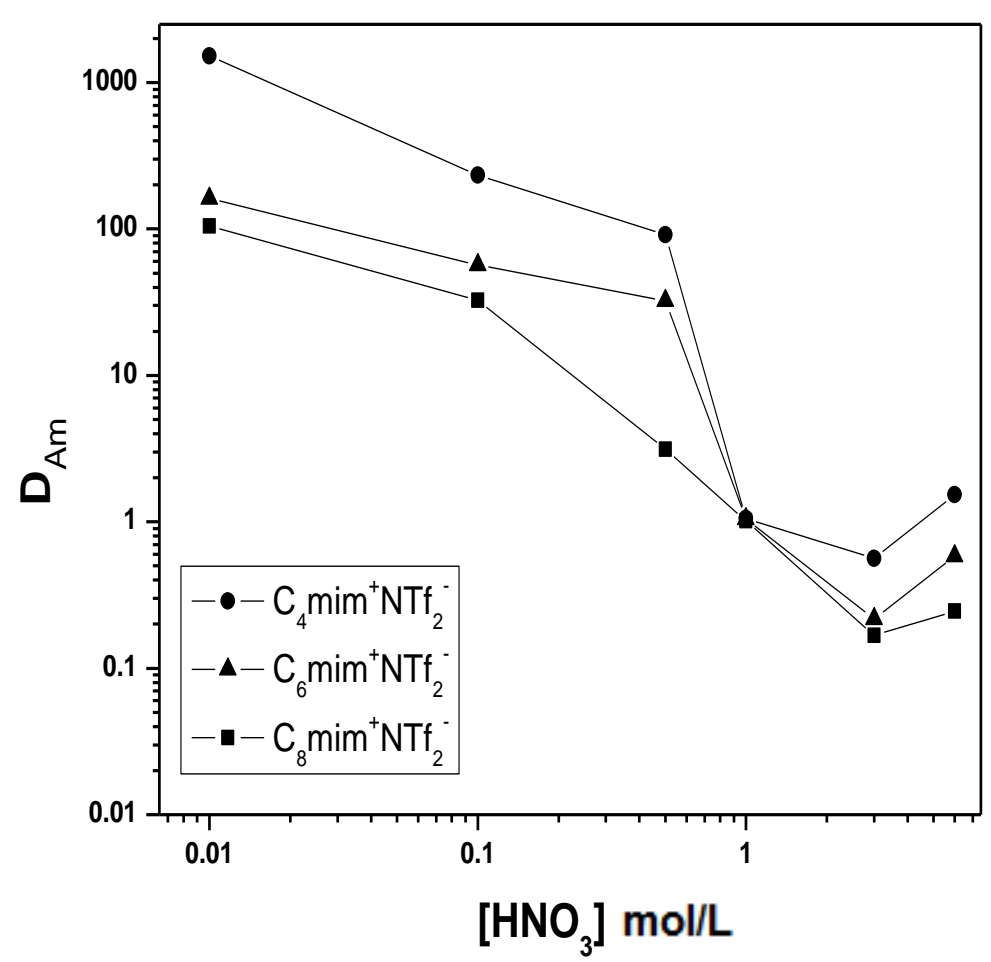

Figure 7.3. Distribution constants of $\mathrm{Am}(\mathrm{III}), D_{\mathrm{Am}}$, vs. the aqueous nitric acid concentration upon extraction into $1.0 \mathrm{mmol} / \mathrm{L}$ T-DGA in $\mathrm{C}_{\mathrm{n}} \mathrm{mim}^{+} . \mathrm{NTf}_{2}^{-}(\mathrm{n}=4,6,8)$ after 3 h equilibration.

The variation of the $D_{\mathrm{Am}}$ value with the feed acidity was studied using $7 \times 10^{-4} \mathrm{~mol} / \mathrm{L} \mathrm{C} 4 \mathrm{DGA}$ in $\mathrm{C}_{8} \mathrm{mim}^{+} \cdot \mathrm{PF}_{6}{ }^{-}$as the organic phase with a phase ratio of $1: 1(\mathrm{v} / \mathrm{v})$. The equilibration time was kept at $3 \mathrm{~h}$ to assure complete equilibration. The $D_{\mathrm{Am}}$ value decreased with increasing aqueous feed acidity (Figure 7.4). The $D_{\text {Am }}$ values decreased more drastically at 0.01 to 1 $\mathrm{mol} / \mathrm{L} \mathrm{HNO}_{3}$ with a much lower subsequent decrease upon further increase in the aqueous phase acid concentration. Also in this case, a cation-exchange mechanism is responsible for the extraction of americium from nitric acid feed to the ionic liquid phase. 


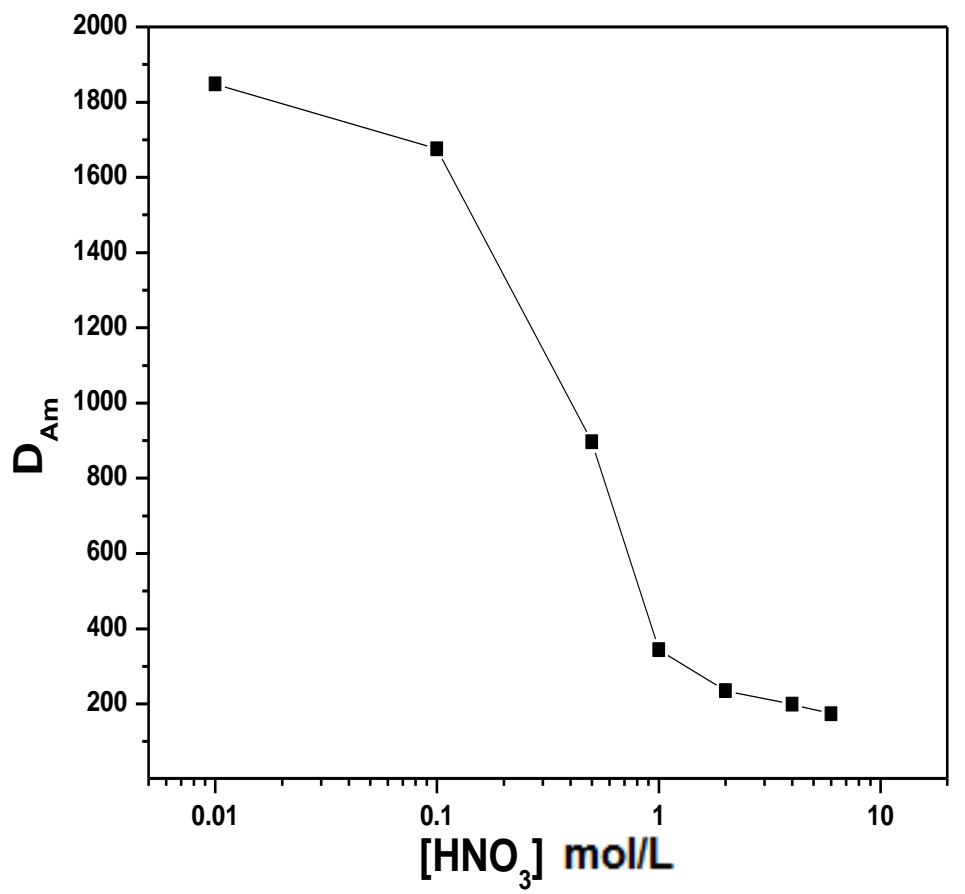

Figure 7.4. Distribution constants of $\mathrm{Am}(\mathrm{III}), D_{\mathrm{Am}}$, vs. the aqueous nitric acid concentration upon extraction into $7 \times 10^{-4} \mathrm{~mol} / \mathrm{L}$ C4DGA in $\mathrm{C}_{8} \mathrm{mim}^{+} . \mathrm{PF}_{6}^{-}$.

\subsubsection{Effect of ligand concentration}

The stoichiometry of complexation can be derived by varying the ligand concentration. The equilibrium extraction constant, $K_{\mathrm{ex}}$, using eqn. 2 , can be written as:

$$
\begin{aligned}
& K_{\mathrm{ex}}=\left[\mathrm{Am}(\mathrm{L})_{\mathrm{m}}{ }^{3+} \cdot 3 \mathrm{X}_{\mathrm{IL}}^{-}\right]\left[\mathrm{H}^{+}{ }_{\mathrm{aq}}\right]^{\mathrm{q}}\left[\mathrm{C}_{\mathrm{n}} \mathrm{mim}^{+}{ }_{\mathrm{aq}}\right]^{3-\mathrm{q}} / \\
& {\left[\mathrm{Am}(\mathrm{III})_{\mathrm{aq}}\right]\left[\mathrm{H}_{\mathrm{IL}}^{+}\right]^{\mathrm{q}}\left[\mathrm{L}_{\mathrm{IL}}\right]^{\mathrm{m}}\left[\mathrm{C}_{\mathrm{n}} \mathrm{mim}^{+}{ }_{\mathrm{IL}}\right]^{3-\mathrm{q}}\left[\mathrm{X}_{\mathrm{IL}}^{-}\right]^{3}}
\end{aligned}
$$

The experimentally determined distribution ratio $D_{\mathrm{Am}}$ is the ratio of the total concentration of Am(III) in the IL phase relative to the total concentration of Am(III) in the aqueous phase, given as:

$$
D_{\mathrm{Am}}=\left[\mathrm{Am}(\mathrm{L})_{\mathrm{m}}{ }^{3+} \cdot 3 \mathrm{X}_{\mathrm{IL}}^{-}\right] /\left[\mathrm{Am}(\mathrm{III})_{\mathrm{aq}}\right]
$$

Putting the value of $D_{\mathrm{Am}}$ in eqn. (3)

$$
K_{\mathrm{ex}}=D_{\mathrm{Am}}\left[\mathrm{H}_{\mathrm{aq}}^{+}\right]^{\mathrm{q}}\left[\mathrm{C}_{\mathrm{n}} \operatorname{mim}^{+}{ }_{\mathrm{aq}}\right]^{3-\mathrm{q}} /\left[\mathrm{H}^{+}{ }_{\mathrm{IL}}\right]^{\mathrm{q}}\left[\mathrm{L}_{\mathrm{IL}}\right]^{\mathrm{m}}\left[\mathrm{C}_{\mathrm{n}} \operatorname{mim}_{\mathrm{IL}}^{+}\right]^{3-\mathrm{q}}\left[\mathrm{X}_{\mathrm{IL}}^{-}\right]^{3}
$$

Taking the logarithm of the rearranged eqn. at varying ligand concentration, while keeping the concentrations of nitric acid and the IL constant, yields: 


\section{Chapter 7}

$$
\log D_{\mathrm{Am}}=\log K_{\mathrm{ex}}+\operatorname{mlog}\left[\mathrm{L}_{\mathrm{IL}}\right]+\text { constant }
$$

The extraction of Am(III) with varying T-DGA concentrations was investigated using all the three RTILs from aqueous feed solutions of $0.1 \mathrm{~mol} / \mathrm{L} \mathrm{HNO}_{3}$ and the time of equilibration was kept at $3 \mathrm{~h}$. The results, presented in Figure 7.5, show an increase in Am(III) extraction with increasing T-DGA concentration for all the ionic liquids. The slope values of the plots of $\log D_{\text {Am }}$ vs. $\log$ [T-DGA], which indicate the ligand to metal stoichiometry m (eqn. 6), are close to $1\left(1.17 \pm 0.05\right.$ for $\mathrm{C}_{4} \mathrm{mim}^{+} . \mathrm{NTf}_{2}{ }^{-} ; 1.13 \pm 0.02$ for $\mathrm{C}_{6} \mathrm{mim}^{+} . \mathrm{NTf}_{2}{ }_{2}$; and $1.05 \pm 0.04$ for $\mathrm{C}_{8} \mathrm{mim}^{+}$.NTf ${ }_{2}{ }^{-}$) suggesting that one T-DGA unit is present in the extracted species, which is in sharp contrast to the extracted species in the molecular diluents, where two T-DGA units were present. $^{10}$

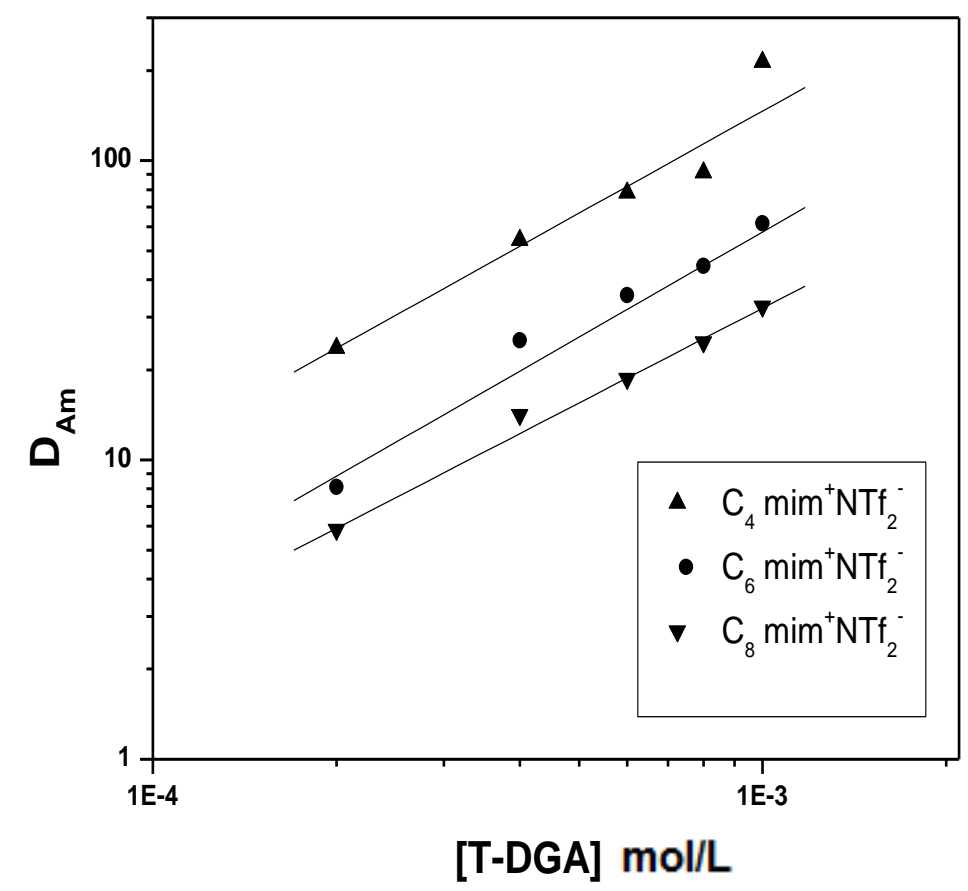

Figure 7.5. Distribution ratio of $\mathrm{Am}(\mathrm{III}), D_{\mathrm{Am}}$, vs. the T-DGA concentration upon extraction from $0.5 \mathrm{~mol} / \mathrm{L} \mathrm{HNO}_{3}$ feed into $\mathrm{C}_{\mathrm{n}} \operatorname{mim}^{+} . \mathrm{NTf}_{2}{ }^{-}(\mathrm{n}=4,6,8)$.

A similar study was carried out to determine the stoichiometry of the complex formed by americium with C4DGA in $\mathrm{C}_{8} \mathrm{mim}^{+} \cdot \mathrm{PF}_{6}{ }^{-}$. The $D_{\text {Am }}$ vs. [C4DGA] plot (Figure 7.6) yielded a slope value of 1.8, indicating the extraction of both $\mathrm{ML}$ and $\mathrm{ML}_{2}$ species from $3 \mathrm{~mol} / \mathrm{L} \mathrm{HNO}_{3}$ with the latter being present predominantly in the ionic liquid phase. 


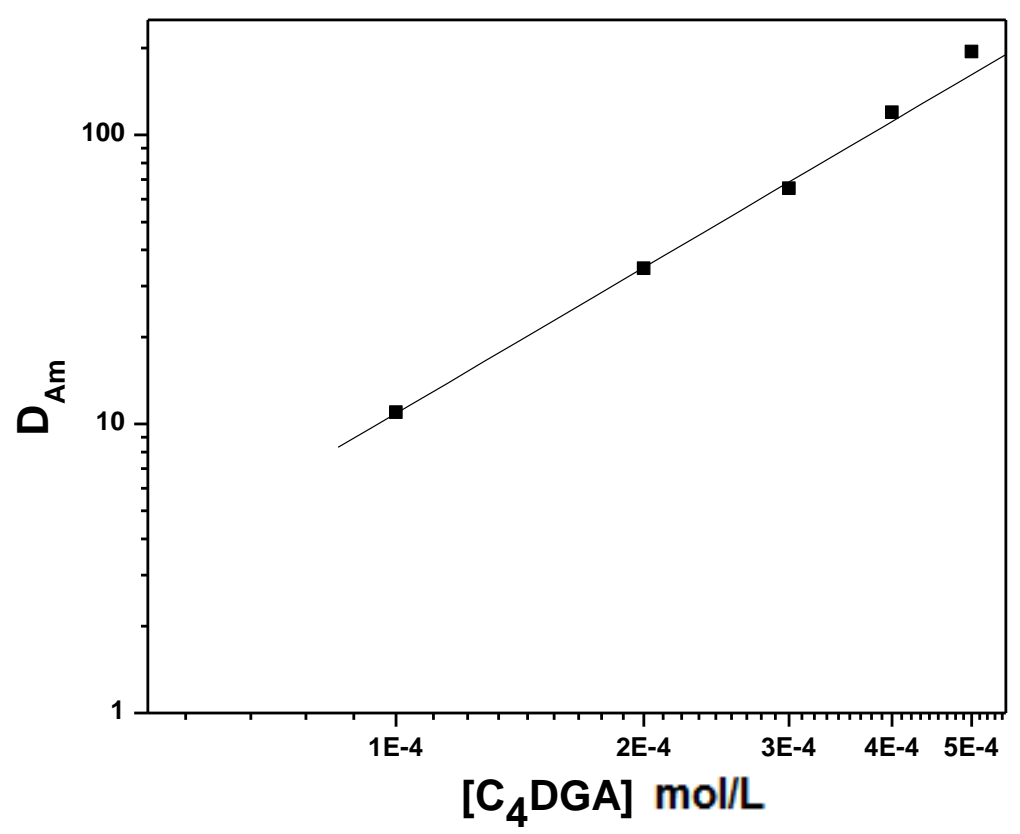

Figure 7.6. Distribution ratio of $\mathrm{Am}(\mathrm{III}), D_{\mathrm{Am}}$, vs. the C4DGA concentration upon extraction from $3 \mathrm{~mol} / \mathrm{L} \mathrm{HNO}_{3}$ feed into $\mathrm{C}_{8} \mathrm{mim}^{+} \cdot \mathrm{PF}_{6}{ }^{-}$.

\subsection{Conclusions}

Solvent extraction studies of Am(III) using a tripodal diglycolamide (T-DGA) and calix[4]arene diglycolamide extractants performed in room temperature ionic liquids instead of the conventional $n$-dodecane as the diluent showed improved extraction efficiency and separation factors. T-DGA and C4DGA are far superior extractants than TODGA. The unusually high extraction of Am(III) by T-DGA or C4DGA can be attributed to the unique ion-exchange extraction mechanism in the RTIL medium. Generally, slower extraction kinetics were observed in RTILs compared to that in molecular solvents, possibly because of a higher viscosity of the ILs and possibly due to a different extraction mechanism taking place in the RTILs. The trivalent ions were highly extracted as compared to tetra- and hexavalent ions, which is very useful for the selective separation of Am(III) from mixture of various actinides. The use of the proposed solvent system is a more efficient and viable 'green' alternative to the TODGA-based solvent systems with molecular diluents. 


\subsection{Experimental}

\subsubsection{General}

${ }^{1} \mathrm{H}$ NMR and ${ }^{13} \mathrm{C}$ NMR spectra were recorded on a Varian Unity INOVA (300 MHz) spectrometer. ${ }^{1} \mathrm{H}$ NMR (300 MHz) and ${ }^{13} \mathrm{C}$ NMR (75 MHz) chemical shift values are reported as $\delta$ using the residual solvent signal as an internal standard. All NMR measurements were recorded in $\mathrm{CDCl}_{3}$ as a solvent. Electrospray Ionization (positive mode) mass spectra and high resolution mass spectra were recorded on a WATERS LCT mass spectrometer. Analytical TLC was performed using Merck prepared plates (silica gel 60 F-254 on aluminium). Column chromatography was carried out with Merck silica gel 60 (230-400 mesh). C4DGA and C2DGA compounds were prepared as described in Chapter 5. Extraction studies were carried out at Bhabha Atomic Research Centre, India.

\subsubsection{Synthesis of tripodal diglycolamide T-DGA.}

(First approach). A mixture of glycolamic acid 2 (7.90 g, $22.2 \mathrm{mmol}), \quad$ N,N'dicyclohexylcarbodiimide (DCC) (4.58 g, $22.2 \mathrm{mmol}$ ), 1-hydroxybenzotriazole (HOBT) (3.00 g, $22.2 \mathrm{mmol})$ and tripodal amine $1(2.95 \mathrm{~g}, 5.71 \mathrm{mmol})$ in dry chloroform (100 mL) was stirred at room temperature for $48 \mathrm{~h}$. Filtration of the precipitate and evaporation of the solvent gave an oil that was subsequently purified by column chromatography $\left(\mathrm{SiO}_{2}\right.$, $\mathrm{CH}_{2} \mathrm{Cl}_{2}: \mathrm{MeOH}=95: 5 \rightarrow 7: 3$ ) to afford impure T-DGA as an oil. The obtained product was dissolved in $\mathrm{CHCl}_{3}(50 \mathrm{~mL})$. The resulting solution was washed with $1 \mathrm{M} \mathrm{HCl}(20 \mathrm{~mL})$ and $\mathrm{H}_{2} \mathrm{O}(3 \times 20 \mathrm{~mL})$ to give, after solvent evaporation, T-DGA as an oil $(2.19 \mathrm{~g} .25 \%)$.

(Second approach).A solution of tripodal amine 1 (1.70 g, $3.29 \mathrm{mmol})$ and diglycolic anhydride $(1.14 \mathrm{~g}, 9.89 \mathrm{mmol})$ in THF $(60 \mathrm{~mL})$ was stirred at room temperature for 3 days. The solvent was evaporated and the residue was dissolved in chloroform $(50 \mathrm{~mL})$ and the resulting solution washed with $1 \mathrm{M} \mathrm{HCl}$ solution $(2 \times 30 \mathrm{~mL})$ and water $(3 \times 30 \mathrm{~mL})$. Evaporation of the solvent afforded tripodal tricarboxylic acid 3 as an oil $(1.87 \mathrm{~g}, 66 \%) .{ }^{1} \mathrm{H}$ NMR: $\delta 0.80-0.95\left(21 \mathrm{H}, \mathrm{t}, J=7.5 \mathrm{~Hz}, \mathrm{CH}_{3}\right), 1.42-1.65\left(14 \mathrm{H}, \mathrm{m}, \mathrm{CH}_{3} \mathrm{CH}_{2}\right), 1.80-1.91(6 \mathrm{H}$, $\left.\mathrm{m}, \mathrm{OCH}_{2} \mathrm{CH}_{2}\right), 2.99-3.19(3 \mathrm{H}, \mathrm{m}, \mathrm{NCH}), 3.20-3.35\left(6 \mathrm{H}, \mathrm{m}, \mathrm{NCH}_{2}\right), 3.36-3.55(12 \mathrm{H}, \mathrm{m}$, $\left.\mathrm{OCH}_{2}\right), 4.21\left(6 \mathrm{H}, \mathrm{s}, \mathrm{OCH}_{2} \mathrm{CO}\right), 4.39\left(6 \mathrm{H}, \mathrm{s}, \mathrm{OCH}_{2} \mathrm{CO}\right)$; MS (MALDI) $\mathrm{m} / \mathrm{z} 864.7[\mathrm{M}+\mathrm{H}]^{+}$, $886.6[\mathrm{M}+\mathrm{Na}]^{+}, 902.6[\mathrm{M}+\mathrm{K}]^{+}$.

A solution of 3 (1.70 g, $1.96 \mathrm{mmol})$, dioctylamine (1.42 g, $5.90 \mathrm{mmol})$, DCC (1.24 g, 5.96 $\mathrm{mmol})$, HOBT $(0.80 \mathrm{~g}, 5.96 \mathrm{mmol})$, and triethylamine $(0.64 \mathrm{~g}, 6.33 \mathrm{mmol})$ in $\mathrm{CHCl}_{3}(60 \mathrm{~mL})$ 
was stirred at room temperature for 2 days. The solvent was evaporated and the residue was dissolved in $n$-hexane $(100 \mathrm{~mL})$ and filtered. The solvent was evaporated and the residue purified with column chromatography $\left(\mathrm{SiO}_{2}, \mathrm{CH}_{2} \mathrm{Cl}_{2}: \mathrm{MeOH}=95: 5 \rightarrow 7: 3\right)$ to yield T-DGA as an oil $(1.50 \mathrm{~g}, 50 \%)$.

(Third approach). A mixture of 1 (1.10 g, $2.1 \mathrm{mmol}), p$-nitrophenol activated DGA ${ }^{12}$ (4) $(3.20 \mathrm{~g}, 6.6 \mathrm{mmol})$ and triethylamine $(0.65 \mathrm{~g}, 6.5 \mathrm{mmol})$ in chloroform $(70 \mathrm{~mL})$ was refluxed for $2 \mathrm{~d}$. The crude reaction mixture was successively washed with $2 \mathrm{M} \mathrm{NaOH}$ solution $(3 \times 50$ $\mathrm{mL}), 1 \mathrm{M} \mathrm{HCl}(3 \times 50 \mathrm{~mL})$, and water $(2 \times 50 \mathrm{~mL})$. The organic layer was concentrated under reduced pressure and the crude product was purified by column chromatography $\left(\mathrm{SiO}_{2}\right.$, $\left.\mathrm{CH}_{2} \mathrm{Cl}_{2}: \mathrm{MeOH}=95: 5 \rightarrow 7: 3\right)$ to afford T-DGA as an oil $(2.42 \mathrm{~g}, 74 \%) .{ }^{1} \mathrm{H}$ NMR: $\delta 0.80-0.91$ $\left(39 \mathrm{H}, \mathrm{m}, \mathrm{CH}_{3}\right), 1.20-1.35$ (74H, m, $\mathrm{CH}_{3}\left(\mathrm{CH}_{2}\right)_{5}$-octyl, $\mathrm{CHCH}_{2}$-isopentyl, $\left.\mathrm{CH}_{3} \mathrm{CH}_{2}\right), 1.44-1.61$ $\left(12 \mathrm{H}, \mathrm{m}, \mathrm{NCH}_{2} \mathrm{CH}_{2}\right), 1.63-1.92\left(6 \mathrm{H}, \mathrm{m}, \mathrm{OCH}_{2} \mathrm{CH}_{2}\right), 3.10-3.47\left(33 \mathrm{H}, \mathrm{m}, \mathrm{NCH}, \mathrm{NCH}_{2}\right.$, $\left.\mathrm{OCH}_{2}\right), 4.25-4.37\left(12 \mathrm{H}, \mathrm{m}, \mathrm{OCH}_{2} \mathrm{CO}\right) ;{ }^{13} \mathrm{C} \mathrm{NMR}: 8.1,11.3,11.4,14.3,20.2,22.8,23.6,25.8$, 26.5, 29.2, 29.5, 30.6, 31.4, 31.9, 33.4, 39.0, 40.7, 43.4, 47.8, 58.8, 60.2, 68.9, 69.3, 69.4, 69.8, 71.7, 168.8, 169.0, 169.8, 169.9, 170.2, 170.3; MS (MALDI) m/z $1535.0[\mathrm{M}+\mathrm{H}]^{+}$, 1557.0 $[\mathrm{M}+\mathrm{Na}]^{+}$. HRMS: $m / z$ 1534.3447 $[\mathrm{M}+\mathrm{H}]^{+}$; calculated: 1534.3425 for $\mathrm{C}_{90} \mathrm{H}_{177} \mathrm{~N}_{6} \mathrm{O}_{12}$.

\subsubsection{Distribution studies}

The distribution studies were carried out by equilibrating the organic phase, containing the required concentration of the extractants in room temperature ionic liquids, as well as the aqueous phase, with required feed acidity, spiked with ${ }^{241} \mathrm{Am}$ tracer, with a phase ratio of $1: 1$ in a thermostated water bath at $25 \pm 0.1{ }^{\circ} \mathrm{C}$. The tubes were centrifuged for $5 \mathrm{~min}$ to enable phase separation. After centrifugation, both phases were separated and assayed radiometrically. The distribution ratio, $D_{\mathrm{M}}$, was defined as the ratio of the activity per unit volume in the organic phase to that in the aqueous phase. Typically, the concentration of americium was in the range of $10^{-6}-10^{-7} \mathrm{~mol} / \mathrm{L}$. The thermodynamic parameters were calculated from temperature variation studies carried out in the temperature range of 20-40 ${ }^{\circ} \mathrm{C}$. The experiments were carried out in duplicate with a precision of $\pm 5 \%$. 


\subsection{References}

1. (a) Adam, C. J.; Earle, M. J.; Sneddon, K. R. Green Chem. 2000, 2, 21. (b) Toma, G.; Gotov, B.; Solkaniova, E. Green Chem. 2000, 2, 149. (c) Song, C. E.; Roh, E. J. Chem. Commun. 2000, 837. (d) Dyson, P. J.; Ellis, D. J.; Parker, D. C.; Welton, T. Chem. Commun. 1999, 25.

2. Welton, T. Chem. Rev. 1999, 99, 2071.

3. (a) Huddleston, J. G.; Willauer, H. D.; Swatloski, R. P.; Visser, A. N.; Rogers, R. D. Chem. Commun. 1998, 1765. (b) Blanchard, L. A.; Hancut, D.; Beckman, E. J.; Brennecke, J. F. Nature 1999, 399, 28. (c) Fadeev, A. G.; Meagher, M. M. Chem. Commun. 2001, 295. (d) Cocalia, V. A.; Jensen, M. P.; Holbrey, J. D.; Spear, S. K.; Stepinski, D. C.; Rogers, R. D. Dalton Trans. 2005, 1966. (e) Visser, A. E.; Rogers, R. D. J. Solid State Chem. 2003, 171, 109.

4. (a) Sun, I. W.; Hussey, C. L. Inorg. Chem. 1989, 28, 2731. (b) Hussey, C. L.; in: Mamantov, G.; Popov, A. I. (Eds.), Chemistry of Nonaqueous Solvents: Current Progress, VCH, New York, 1994.

5. Giridhar, P.; Venkatesan, K. A.; Srinivasan, T. G.; Rao, P. R. V. J. Radioanal. Nucl. Chem. 2005, 265, 31 .

6. Rout, A.; Venkatesan, K. A.; Srinivasan, T. G.; Rao, P. R. V. Radiochim. Acta 2009, 97, 719.

7. (a) Gujar, R. B.; Ansari, S. A.; Prabhu, D. R.; Mohapatra, P. K.; Pathak, P. N.; Sengupta, A.; Thulasidas, S. K.; Manchanda, V. K. Solv. Extr. Ion Exch. 2012, 30, 156. (b) Modolo, G.; Hanna, A.; Schreinemachers, C.; Vijgen, H. Solv. Extr. Ion Exch. 2007, 25, 703.

8. Jańczewski, D.; Reinhoudt, D. N.; Verboom, W.; Hill, C.; Allignol, C.; Duchesne, M. T. New J. Chem. 2008, 32, 490.

9. Jańczewski, D.; Reinhoudt, D. N.; Verboom, W.; Malinowska, E.; Pietrzak, M.; Hill, C.; Allignole, C. New J. Chem. 2007, 31, 109.

10. Mohapatra, P. K.; Iqbal, M.; Raut, D. R.; Verboom, W.; Huskens, J.; Manchanda, V. K. J. Membr. Sci. 2011, 375, 141.

11. Cocalia, V. A.; Jensen, M. P.; Holbrey, J. D.; Spear, S. K.; Stepinski, D. C.; Rogers, R. D. Dalton Trans. 2005, 1966.

12 Mohapatra, P. K.; Iqbal, M.; Raut, D. R.; Verboom, W.; Huskens, J.; Godbole, S. V. Dalton Trans. 2012, 41, 360. 
13. Gujar, R. B.; Ansari, S. A.; Murali, M. S.; Mohapatra, P. K.; Manchanda, V. K. J. Radioanal. Nucl. Chem. 2010, 284, 377.

14. Billard, I.; Ouadi, A.; Gaillard, C. Anal. Bioanal. Chem. 2011, 400, 1555.

15. Billard, I.; Gaillard, C.; Hennig, C. Dalton Trans. 2007, 4214.

16. Billard, I.; Ouadi, A.; Jobin, E.; Champion, J.; Gaillard, C.; Georg. S. Solv. Extr. Ion Exch. 2011, 29, 577.

17. Dietz, M. L.; Stepinski, D. C. Green Chem. 2005, 7, 747. 



\section{Chapter}

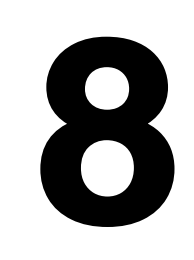

\section{DGA- and CMPO-based Task-Specific Ionic Liquids for Efficient Extraction of An(III)/Ln(III) ${ }^{*}$}

Two diglycolamide-functionalized ionic liquids (DGA-FILs) were prepared and evaluated for their extraction behavior towards actinide and fission product ions. They showed exceptionally high distribution ratio values with the actinide and lanthanide ions with relatively low extraction of $\mathrm{Cs}(\mathrm{I}), \mathrm{Sr}(\mathrm{II})$, and $\mathrm{U}(\mathrm{VI}) \mathrm{O}_{2}$. In addition, $\mathrm{CMPO}$-functionalized ionic liquids (CMPO-FILs) were synthesized and evaluated for various metal ions. The CMPO-FIL with the NTf ${ }_{2}^{-}$counter ion dissolved in room temperature ionic liquids (RTILs) exhibited high distribution ratios for trivalent, tetravalent and hexavalent actinide ions.

\footnotetext{
"Part of this chapter is based on: Sengupta, A.; Mohapatra, P. K.; Iqbal, M.; Huskens, J.; Verboom, W. Chem. Eur. J. Submitted for publication.
} 


\section{Chapter 8}

\subsection{Introduction}

Solvent extraction is the most commonly accepted technique in the partitioning and transmutation strategy (P\&T) for the separation of $\mathrm{An}(\mathrm{III}) / \mathrm{Ln}(\mathrm{III})$ in the reprocessing of high level nuclear waste (see Chapter 1). Growing concern for the environment has led to an increased interest in room temperature ionic liquids (RTILs) as an alternative to volatile organic solvents in many areas of chemistry including separations by liquid-liquid extractions. ${ }^{1,2,3}$ RTILs also have many advantages over the traditional solvent systems because of their unique chemical and physical properties e.g. negligible vapor pressure, nonflammability, tuneable viscosity, and a unique solvation mechanism. These properties make them good candidates for extraction systems (See also Chapter 2).

$\mathrm{CMPO}^{4,5}$ and diglycolamides ${ }^{6,7}$ dissolved in ionic liquids have been studied for the extraction of actinides and lanthanides. Functionalized ionic liquids (FILs) have been studied for the extraction of $\mathrm{U}(\mathrm{VI}) \mathrm{O}_{2},{ }^{8} \mathrm{Pu}(\mathrm{IV}),{ }^{9}$ and $\mathrm{Am}(\mathrm{III}) .{ }^{10,11}$ Since diglycolamides and CMPOs exhibit high extraction efficiency for An(III) ions in traditional solvents as well as in ionic liquid medium and in view of the higher extraction efficiency of the FILs, it was of great interest to study diglycolamide- and CMPO-functionalized ionic liquids. This Chapter deals with the synthesis of diglycolamide- and CMPO-functionalized ionic liquids containing bis(trifluoromethane)sulfonamide $\left(\mathrm{NTf}_{2}\right)$ and hexafluorophosphate $\left(\mathrm{PF}_{6}\right)$ as counter anions. Ionic liquids with a functional group covalently tethered to the cationic or anionic part are commonly termed task-specific ionic liquids (TSILs). The extraction efficiency of these FILs towards various actinide, lanthanide, and fission product cations has been evaluated.

\subsection{Results and discussion}

\subsubsection{Synthesis}

\subsubsection{Diglycolamide-functionalized ionic liquids}

The synthesis of diglycolamide-based ionic liquids 5 and $\mathbf{6}$ is summarized in Scheme 8.1. Commercially available 3-(1H-imidazol-1-yl)propan-1-amine (1) was treated with the known 2-(2-(dioctylamino)-2-oxoethoxy)acetic $\operatorname{acid}^{12}$ (2) in chloroform using $N, N^{\prime}$ dicyclohexylcarbodiimide (DCC) and 1-hydroxybenzotriazole (HOBT) as coupling agent to afford imidazole-diglycolamide 3 . The formation of $\mathbf{3}$ clearly followed from the ${ }^{1} \mathrm{H}$ NMR spectrum as the $\mathrm{CH}_{2}$ amine peak at $2.74 \mathrm{ppm}$ in $\mathbf{1}$ shifted to $3.34 \mathrm{ppm}$ in $\mathbf{3}$. The formation of $\mathbf{3}$ was also concluded from the $\mathrm{M}+\mathrm{H}$ peak at $\mathrm{m} / \mathrm{z} 465.4$ in the electrospray mass spectrum. 
Compound 3 was further reacted with $n$-butyl bromide in acetonitrile for 2.5 days to give ionic liquid 4 with bromide as the counter-anion. The NCHN imidazole peak at $7.57 \mathrm{ppm}$ in the ${ }^{1} \mathrm{H}$ NMR spectrum of $\mathbf{3}$ was shifted to $10.46 \mathrm{ppm}$ for $\mathbf{4}$. The bromide counter-anion of $\mathbf{4}$ was exchanged with either hexafluorophosphate or bis(trifluoromethane)sulfonamide by treating with the corresponding sodium and lithium salts, to give FILs $\mathbf{5}$ and $\mathbf{6}$, respectively. Their formation is evident from the shift of the peak at $10.46 \mathrm{ppm}$ in the ${ }^{1} \mathrm{H}$ NMR spectrum of 4 to 8.93 ppm for 5 and $9.01 \mathrm{ppm}$ for $\mathbf{6}$. The high resolution electrospray mass spectra of $\mathbf{5}$ and 6 clearly showed $[\mathrm{M}]^{+}$peaks as well as the $[\mathrm{M}+\mathrm{NaPF} 6]^{+}$and $\left[\mathrm{M}+\mathrm{NaNTf}_{2}\right]^{+}$peaks for $\mathbf{5}$ and 6 respectively.
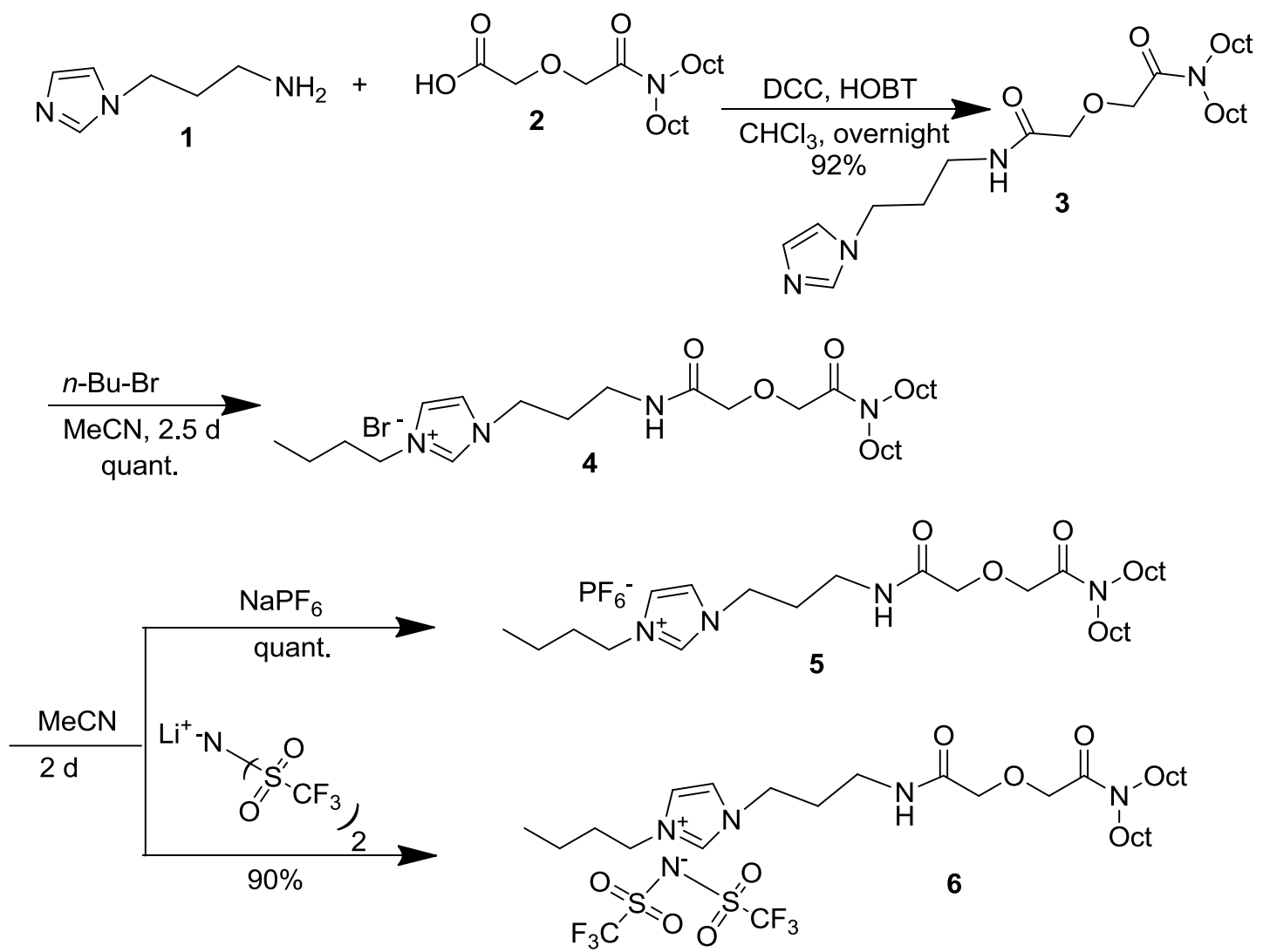

Scheme 8.1

\subsubsection{CMPO-functionalized ionic liquids}

The known CMPO-imidazole propanamide $7,{ }^{9}$ synthesized in two steps by reaction of diphenyl phosphite with ethyl bromoacetate and subsequent reaction of the formed product with 3-(1H-imidazol-1-yl)propan-1-amine (1), was reacted with $n$-dodecyl bromide in refluxing acetonitrile to afford ionic liquid 8. In the ${ }^{1} \mathrm{H}$ NMR spectra, the NCHN proton shifted from 7.32 in $\mathbf{7}$ to $10.00 \mathrm{ppm}$ in $\mathbf{8}$. The formation of $\mathbf{8}$ also followed from the $[\mathrm{M}]^{+}$peak at $m / z, 536.3400$ in the high resolution electrospray mass spectrum. The bromide counter- 


\section{Chapter 8}

anion in 8 was exchanged with either hexafluorophosphate or bis(trifluoromethane)sulfonamide by treating with the corresponding sodium and lithium salts, to give FILs 9 and 10, respectively (Scheme 8.2). From the ${ }^{1} \mathrm{H}$ NMR spectra, their formation followed from the shift of the peak at $10.00 \mathrm{ppm}$ in $\mathbf{8}$ to $8.83 \mathrm{ppm}$ in $\mathbf{9}$ and 8.97 ppm in 10. FILs 9 and $\mathbf{1 0}$ also showed the corresponding $\left[\mathrm{M}+\mathrm{PF}_{6}+\mathrm{H}\right]^{+}$and $\left[\mathrm{M}+\mathrm{NTf}_{2}+\mathrm{H}\right]^{+}$ peaks respectively, in the high resolution electrospray mass spectrum.
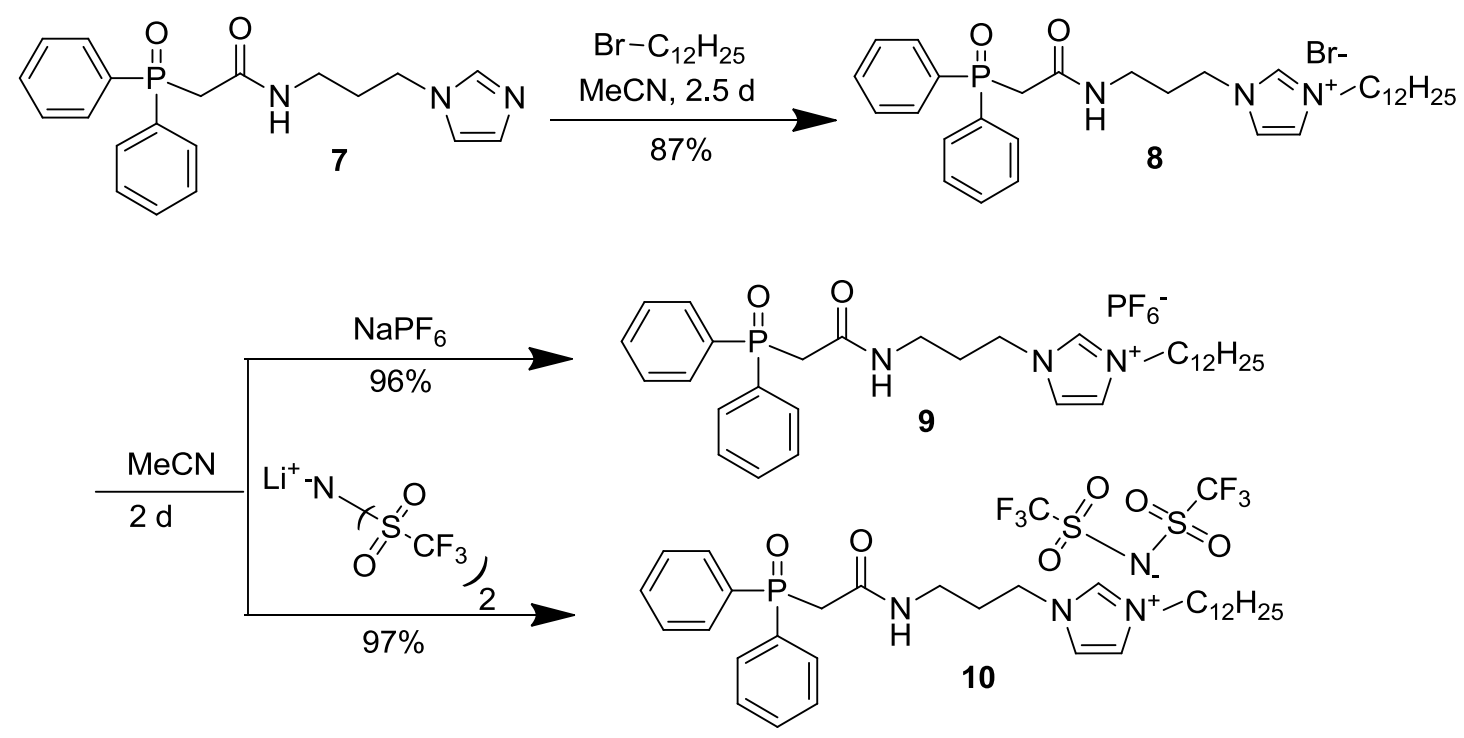

Scheme 8.2

\subsubsection{Extraction results}

\subsubsection{Extraction with DGA-FILs}

The solvent extraction data of actinide and fission product ions using DGA-FILs $\mathbf{5}$ and $\mathbf{6}$ are listed in Table 8.1. For comparison purpose, the distribution data with the analogous diglycolamide extractant, TODGA, in ILs such as Bmim.PF 6 (1-butyl-3-methylimidazolium hexafluorophosphate) and Bmim.NTf 2 (1-butyl-3-methylimidazolium bis(trifluoromethane)sulfonimide), as well as data with TODGA in $n$-dodecane are also included in the table. Three significant observations can be made. Firstly, the $D$ values of TODGA are significantly higher in the IL-based solvent systems than those in $n$-dodecane as the diluent. Moreover, the DGA-FILs exhibit exceptionally higher $D$ values than the TODGA-IL solvent system. Secondly, the time required to attain equilibrium $D$ values was much higher (about $6 \mathrm{~h}$ ) in case of the two DGA-functionalized ILs as compared to the TODGA-IL solvent systems (about $1 \mathrm{~h}$ ), which can be attributed to the high viscosity of the FILs (Figure 8.1). Thirdly, where the $D$ values obtained with the trivalent actinide and 
lanthanide ions are unusually high compared to analogous solvent systems, the hexavalent uranyl ion and the $\mathrm{Cs}(\mathrm{I})$ and $\mathrm{Sr}(\mathrm{II})$ ions are practically unextracted. Similar to TODGA in $n$-dodecane, the distribution ratio values follow the trend: $\mathrm{Eu}(\mathrm{III})>\mathrm{Am}(\mathrm{III})>>\mathrm{Pu}(\mathrm{IV})>$ $\mathrm{Np}(\mathrm{IV})>>\mathrm{U}(\mathrm{VI}) \mathrm{O}_{2} \gg \mathrm{Sr}(\mathrm{II}) \sim \mathrm{Cs}(\mathrm{I})$.

Table 8.1 Distribution data of some relevant major actinide and fission product element ions. ${ }^{a}$

\begin{tabular}{llllllll}
\hline \multirow{2}{*}{\multicolumn{1}{c}{ Ligand }} & \multicolumn{7}{c}{ Distribution coefficient $^{a}$} \\
\cline { 2 - 8 } & $\mathrm{Am}(\mathrm{III})$ & $\mathrm{Np}(\mathrm{IV})^{d}$ & $\mathrm{Pu}(\mathrm{IV})^{e}$ & $\mathrm{U}(\mathrm{VI}) \mathrm{O}_{2}$ & $\mathrm{Eu}(\mathrm{III})$ & $\mathrm{Cs}(\mathrm{I})$ & $\mathrm{Sr}(\mathrm{II})$ \\
\hline DGA-FIL-PF $_{6}(\mathbf{5})^{b}$ & $1.1 \times 10^{3}$ & 152 & 378 & 9 & $1.0 \times 10^{4}$ & 0.2 & 0.3 \\
DGA-FIL-NTf $_{2}(\mathbf{6})^{b}$ & $2.2 \times 10^{3}$ & 187 & 572 & 12 & $1.1 \times 10^{4}$ & 0.3 & 0.5 \\
TODGA in Bmim.PF $_{6}{ }^{c}$ & 8 & 2.1 & 5 & 0.3 & 14 & 0.03 & 0.06 \\
TODGA in Bmim.NTf $_{2}{ }^{c}$ & 12 & 4 & 7 & 1.2 & 42 & 0.07 & 0.08 \\
TODGA in $n$-dodecane $^{c}$ & 1.4 & 0.3 & 0.6 & 0.09 & 3 & $5.0 \times 10^{-3}$ & $4.0 \times 10^{-3}$ \\
\hline
\end{tabular}

${ }^{a}$ Feed: $3 \mathrm{~mol} / \mathrm{L} \mathrm{HNO}_{3} \cdot{ }^{b}$ Used as pure. ${ }^{c}$ Concentration of the extractant: $1.0 \times 10^{-2} \mathrm{M} .{ }^{d}$ Hydroxylamine and ferrous sulfamate were used as the reducing agents. ${ }^{e} \mathrm{NaNO}_{2}$ was used as the holding oxidant.

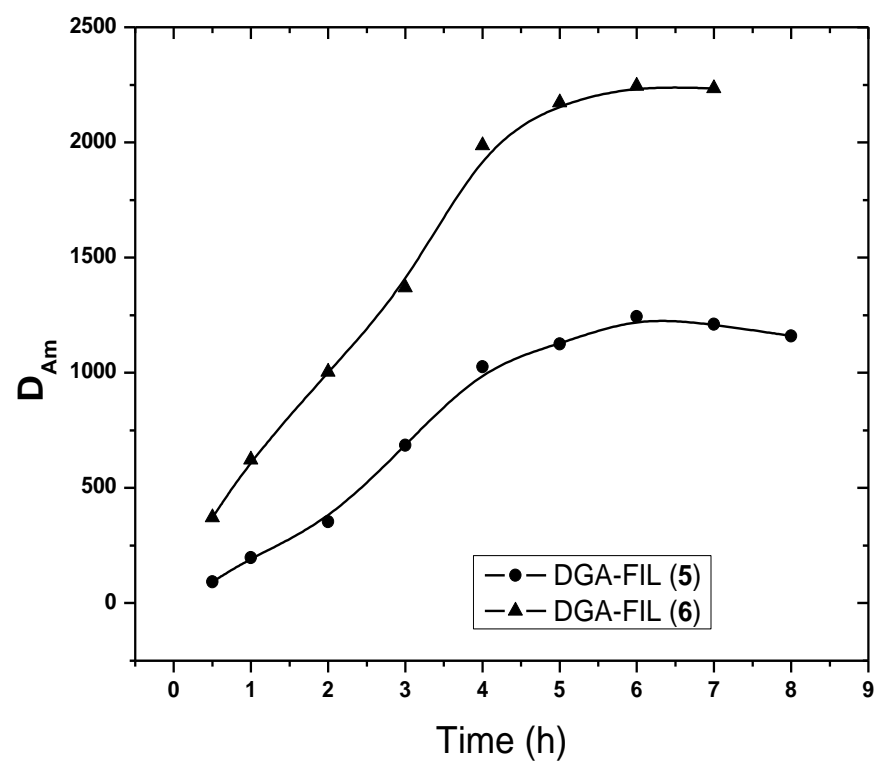

Figure 8.1. $D_{\mathrm{Am}}$ values as a function of time for DGA-FILs 5 and $\mathbf{6}$ at $3 \mathrm{~mol} / \mathrm{L} \mathrm{HNO}_{3}$.

The results of the $D_{\mathrm{Am}}$ values by pure DGA-FILs as a function of $\mathrm{HNO}_{3}$ concentration showed a decrease in $D_{\mathrm{Am}}$ values upon increasing $\mathrm{HNO}_{3}$ concentration (Figure 8.2a), which appearss to be consistent with the typical extraction behavior obtained with other IL extraction systems. ${ }^{13,14}$ Shimojo et al ${ }^{13}$ carried out the extraction of metal ions by TODGA dissolved in ILs and found that the extraction decreases as a function of $\left[\mathrm{H}^{+}\right]$on use of either 


\section{Chapter 8}

$\mathrm{HNO}_{3}$ or $\mathrm{H}_{2} \mathrm{SO}_{4}$. This showed at the same time that no anion is involved in the extraction mechanism. The following mechanism was proposed for TODGA dissolved in IL.

$$
\mathrm{Am}(\mathrm{III})_{\mathrm{aq}}+\mathrm{nTODGA}_{\mathrm{IL}}+3 \mathrm{Bmim}^{+} \mathrm{X}_{\mathrm{IL}}^{-}==\operatorname{Am}\left(\mathrm{TODGA}_{\mathrm{n}}{ }^{3+} \cdot 3 \mathrm{X}_{\mathrm{IL}}^{-}+3 \mathrm{Bmim}^{+}{ }_{\mathrm{aq}}\right.
$$

Where $\mathrm{Bmim}^{+}$is the cation (1-butyl-3-methylimidazolium), $\mathrm{X}^{-}$is the counter anion $\left(\mathrm{PF}_{6}^{-}\right.$ or $\mathrm{NTf}_{2}^{-}$). Eqn (1) suggests that with increasing extraction of Am(III), more and more $\mathrm{Bmim}^{+}$gets into the aqueous phase. This equation duly accounts for the $\mathrm{Bmim}^{+}$ dependence of the extraction efficiency but does not reproduce the effect of $\mathrm{H}^{+}$obtained with $\mathrm{HNO}_{3}$ and $\mathrm{H}_{2} \mathrm{SO}_{4}$. It was found by Billard et al. ${ }^{15}$ that $\mathrm{HNO}_{3}$ dissolves easily in BmimTf $_{2} \mathrm{~N}$ and large amounts of acid are readily transferred to the IL phase during the phase equilibration stage of extraction experiments. For example, for an initial $\mathrm{HNO}_{3}$ concentration of $7.4 \mathrm{~mol} / \mathrm{L}$, the remaining $\mathrm{HNO}_{3}$ concentration in water after equilibration with pure $\mathrm{C}_{4} \operatorname{mimTf}_{2} \mathrm{~N}$ is only $5.8 \mathrm{~mol} / \mathrm{L} .{ }^{16}$ Therefore, considering that the extraction is driven by a cation-exchange process involving both $\mathrm{H}^{+}$and $\mathrm{Bmim}^{+}$, the following type of mechanism was proposed by Billard et al.: ${ }^{17}$

$$
\begin{aligned}
& \operatorname{Am}(\mathrm{III})_{\mathrm{aq}}+\mathrm{nH}^{+}{ }_{\mathrm{IL}}+\mathrm{mTODGA}_{\mathrm{IL}}+3 \mathrm{Bmim}^{+} \mathrm{X}_{\mathrm{IL}}^{-}== \\
& \operatorname{Am}(\mathrm{TODGA})_{\mathrm{m}}{ }^{3+} \cdot 3 \mathrm{X}_{\mathrm{IL}}^{-}+\mathrm{nH}^{+}{ }_{\mathrm{aq}}+(3-\mathrm{n}) \mathrm{Bmim}^{+}{ }_{\mathrm{aq}}
\end{aligned}
$$

On the other hand, with a pure DGA-FIL, as $\mathrm{Bmim}^{+}$is a part of the complexing entity, there is almost no chance of getting it partitioned into the aqueous phase, because of much increased lipophilicity of its octyl groups. Hence, the possible extraction mechanism can be given as:

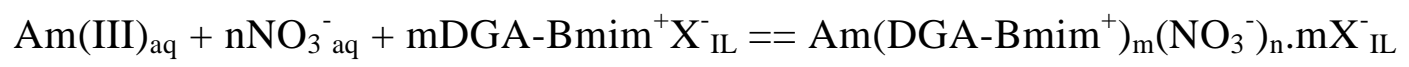

In this case, the $D_{\text {Am }}$ should increase with increasing nitrate ion concentration. This was confirmed by carrying out extraction experiments as a function of the nitrate ion concentration in the aqueous phase at a constant $\mathrm{HNO}_{3}$ concentration of $0.01 \mathrm{~mol} / \mathrm{L}$ (Fig $8.2 \mathrm{~b}$ ), where a slope value of 1 for the $\log D_{\mathrm{Am}} \mathrm{vs} a\left[\mathrm{NO}_{3}\right]$ plots suggested the presence of one $\mathrm{NO}_{3}{ }^{-}$in the extracted species. However, upon increasing $\mathrm{HNO}_{3}$ concentration, although there is an increase in nitrate ion concentration, $D_{\mathrm{Am}}$ was found to decrease. This suggests that this process involves an $\mathrm{H}^{+}$driven cation exchange mechanism, where upon increase in $\left[\mathrm{H}^{+}\right]$in the aqueous phase, $D_{\mathrm{Am}}$ decreases: 


$$
\begin{aligned}
& \mathrm{Am}(\mathrm{III})_{\mathrm{aq}}+\mathrm{nH}^{+}{ }_{\mathrm{IL}}+\mathrm{mNO}_{3 \mathrm{aq}}^{-}+\mathrm{pDGA}^{-} \mathrm{Bmim}^{+} \mathrm{X}_{\mathrm{IL}}^{-}== \\
& \operatorname{Am}\left(\mathrm{DGA}_{-\mathrm{Bmim}}^{+}\right)_{\mathrm{p}}\left(\mathrm{NO}_{3}{ }^{-}\right)_{\mathrm{m}} \cdot \mathrm{pX}_{\mathrm{IL}}^{-}+\mathrm{nH}^{+}{ }_{\mathrm{aq}}
\end{aligned}
$$

Another possibility of decreasing $D_{\text {Am }}$ with increase in $\mathrm{HNO}_{3}$ concentration might be due to the formation of species like HTODGA ${ }^{+} . \mathrm{X}_{\mathrm{IL}}{ }^{-}$, however, this can be refuted as the $D_{\mathrm{Am}}$ value of TODGA in molecular diluents is well known to increase with increasing $\mathrm{HNO}_{3}$ concentration.
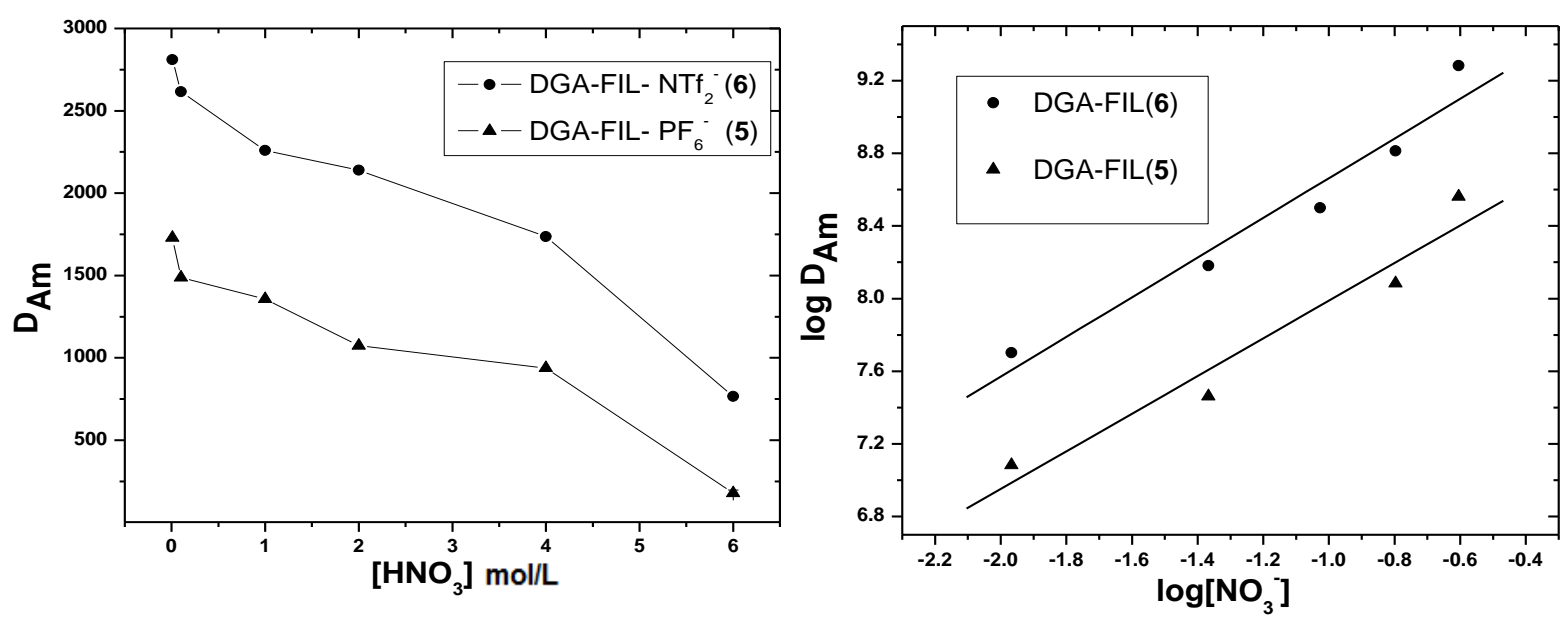

Figure 8.2 left: a) Relative extraction profiles of Am(III) in DGA-FILs 5 and $\mathbf{6}$ as a function of aqueous phase acid concentration. right: b) Dependence of Am(III) extraction on aqueous phase nitrate ion concentration; Aqueous phase: $0.01 \mathrm{~mol} / \mathrm{L} \mathrm{HNO}_{3}+$ varying amounts of $\mathrm{NaNO}_{3}$.

The stoichiometry of the metal:ligand complexation can be determined by slope analysis. According to eqn. 3 , the equilibrium constant $\left(K_{\mathrm{ex}}\right)$ can be written as.

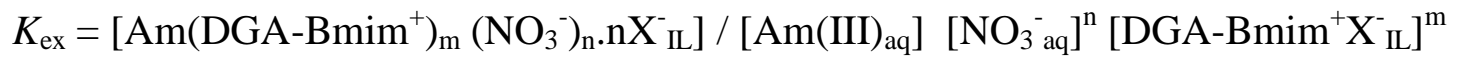

The experimentally determined distribution ratio $D_{\mathrm{Am}}$ is the ratio of the total concentration of Am(III) in the IL phase to the total concentration of Am(III) in the aqueous phase given as:

$$
D_{\mathrm{Am}}=\left[\mathrm{Am}\left(\mathrm{DGA}-\mathrm{Bmim}^{+}\right)_{\mathrm{m}}\left(\mathrm{NO}_{3}^{-}\right)_{\mathrm{n}} \cdot \mathrm{nX}_{\mathrm{IL}}^{-}\right] /\left[\mathrm{Am}(\mathrm{III})_{\mathrm{aq}}\right]
$$

Putting the value of $D_{\mathrm{Am}}$ in eqn 5 gives:

$$
K_{\mathrm{ex}}=D_{\mathrm{Am}} /\left[\mathrm{NO}_{3}{ }_{\mathrm{aq}}^{-}\right]^{\mathrm{n}}\left[\mathrm{DGA}-\mathrm{Bmim}^{+} \mathrm{X}_{\mathrm{IL}}^{-}\right]^{\mathrm{m}}
$$




\section{Chapter 8}

Taking the logarithm of the rearranged eqn. 7 yields:

$$
\log D_{\mathrm{Am}}=\log K_{\mathrm{ex}}+\operatorname{nlog}\left[\mathrm{NO}_{3}{ }^{-}\right]_{\mathrm{aq}}+\operatorname{mlog}\left[\mathrm{DGA}-\mathrm{Bmim}^{+} \mathrm{X}_{\mathrm{IL}}^{-}\right]
$$

At constant $\left[\mathrm{NO}_{3}{ }^{-}\right]$, a plot of $\log D_{\mathrm{Am}}$ vs $\log$ (ligand concentration) according to eqn. (8) yields a straight line where $\mathrm{m}$ is the slope, which is equal to the number of ligand molecules involved in the extraction. Similarly, a plot of $\log D_{\mathrm{Am}} \mathrm{Vs} \log \left[\mathrm{NO}_{3}{ }^{-}\right]$gives $\mathrm{n}$ as the slope value, which is equal to the number of nitrate ions involved in the extraction at constant ligand concentration. The DGA-FIL concentration variation experiments (carried out using Bmim. $\mathrm{PF}_{6}$ or Bmim. $\mathrm{NTf}_{2}$ as the diluents) indicated a 1:2 metal:ligand stoichiometry (Figure 8.3). These results suggest that the extracted species is $\mathrm{Am}(\mathrm{DGA}-\mathrm{Bmim})_{2}$. $\left(\mathrm{NO}_{3}\right)(\mathrm{X})_{4}$, where $\mathrm{X}$ is either $\mathrm{PF}_{6}^{-}$or $\mathrm{NTf}_{2}^{-}$. The aggregate formation in the case of TODGA in molecular diluents also occurs in the DGA-FILs whereby very large aggregates were formed as confirmed by dynamic light scattering and small angle neutron scattering studies. The DGAFILs 5 and 6 also displayed a unique separation behavior. While the Am/Sr separation factor ${ }^{18}$ values were 350, 140, and 150 for TODGA in $n$-dodecane, Bmim.PF 6 , and Bmim.NTf respectively, these increased substantially to $3.6 \times 10^{3}$ and $4.8 \times 10^{3}$ for $\mathbf{5}$ and $\mathbf{6}$, respectively. Similarly, the Am/U separation factor values (which are in the range of 10-26 for TODGA systems) increased by one order of magnitude to 128 and 180 for $\mathbf{5}$ and $\mathbf{6}$, respectively (Table 8.1). 


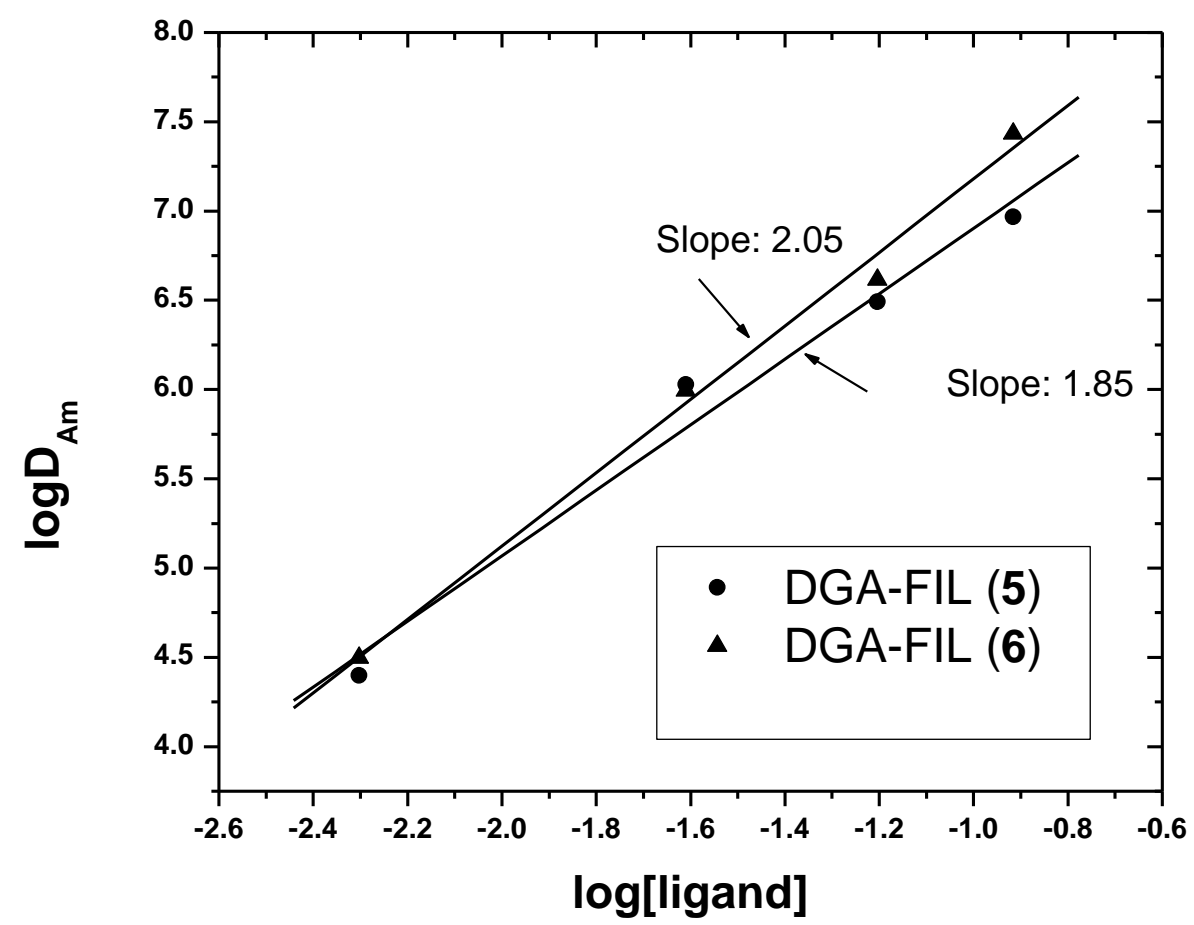

Figure 8.3. Effect of variation of DGA-FILs 5 and $\mathbf{6}$ concentration on the distribution of Am(III).

\subsubsection{Extraction with CMPO-FILs}

The CMPO-functionalized ionic liquids (CMPO-FILs) 9 and $\mathbf{1 0}$ were tested for various actinides and fission products in RTILs. CMPO-FIL 9, having the $\mathrm{PF}_{6}$ counter ion, exhibited no extraction efficiency for actinides. The solvent extraction data of actinide and fission product ions using CMPO-FIL 10, with $\mathrm{NTf}_{2}$ as the counter ion, dissolved in three different RTILs $\left[\mathrm{C}_{\mathrm{n}} \operatorname{mim}(\mathrm{n}=4,6,8)\right]\left[\mathrm{NTf}_{2}\right]$ (1-alkyl-3-methylimidazolium.NTf $\left.\mathrm{NT}_{2}\right)$ is given in Table 8.2. For comparison purpose, the distribution data of CMPO, dissolved in the same ILs, as well as data of CMPO in $n$-dodecane are also included in the table. The $D$ values for all metal ions are significantly higher in the IL-based solvent systems than those in $n$-dodecane as the diluent. Moreover, the $D$ values for all the actinides and lanthanides decrease with increase of the alkyl chain length of the imidazolium cation $\left(\mathrm{C}_{\mathrm{n}} \mathrm{mim}^{+}\right)$, when CMPO-FIL (10) is used as the extractant. A similar trend was observed for CMPO in an IL system. However, in case of tetra- and hexavalent actinides, the $D$ values seem to be independent of the alkyl chain length of the imidazolium cation. The $D$ values obtained for $\mathrm{Pu}(\mathrm{IV})$ are unusually high for both CMPO and CMPO-FIL $\mathbf{1 0}$ in the IL system as compared to the $n$-dodecane systems. The 


\section{Chapter 8}

$\mathrm{Cs}(\mathrm{I})$ and $\mathrm{Sr}(\mathrm{II})$ ions are practically unextracted in $n$-dodecane as well as in ILs. With CMPO in $n$-dodecane, the distribution ratio values follow the trend: $\mathrm{U}(\mathrm{VI}) \mathrm{O}_{2}>\mathrm{Pu}(\mathrm{IV})>\mathrm{Am}(\mathrm{III})>$ $\mathrm{Eu}(\mathrm{III})>\mathrm{Cs}(\mathrm{I}) \sim \mathrm{Sr}(\mathrm{II})$. However, while using CMPO and CMPO-FIL 10 in ILs a different trend is observed: $\mathrm{Pu}(\mathrm{IV})>\mathrm{U}(\mathrm{VI}) \mathrm{O}_{2}>\mathrm{Am}(\mathrm{III})>\mathrm{Eu}(\mathrm{III})>\mathrm{Cs}(\mathrm{I}) \sim \mathrm{Sr}(\mathrm{II})$.

Table 8.2 Distribution data of some important actinide and fission product element ions. ${ }^{a}$

\begin{tabular}{|c|c|c|c|c|c|c|}
\hline \multirow{2}{*}{ Solvent system ${ }^{\mathrm{b}}$} & \multicolumn{6}{|c|}{ Distribution coefficient } \\
\hline & Am(III) & $\mathrm{Pu}(\mathrm{IV})^{c}$ & $\mathrm{U}(\mathrm{VI}) \mathrm{O}_{2}$ & $\overline{\mathrm{Eu}(\mathrm{III})}$ & $\mathrm{Cs}(\mathrm{I})$ & $\operatorname{Sr}(\mathrm{II})$ \\
\hline CMPO-FIL (10) in $\mathrm{C}_{4} \mathrm{mim}^{+}$. $\mathrm{NTf}_{2}^{-}$ & 42 & $3.5 \times 10^{3}$ & 257 & 30 & 0.1 & $<0.01$ \\
\hline CMPO-FIL (10) in $\mathrm{C}_{6} \mathrm{mim}^{+} \cdot \mathrm{NTf}_{2}^{-}$ & 26 & $2.2 \times 10^{3}$ & 155 & 16 & 0.03 & $<0.01$ \\
\hline CMPO-FIL (10) in $\mathrm{C}_{8} \mathrm{mim}^{+} \cdot \mathrm{NTf}_{2}^{-}$ & 16 & 852 & 94 & 2.2 & 0.02 & $<0.01$ \\
\hline $\mathrm{CMPO}$ in $\mathrm{C}_{4} \mathrm{mim}^{+} . \mathrm{NTf}_{2}^{-}$ & 364 & $1.4 \times 10^{3}$ & 53 & 172 & 0.09 & $<0.01$ \\
\hline $\mathrm{CMPO}$ in $\mathrm{C}_{6} \mathrm{mim}^{+} . \mathrm{NTf}_{2}^{-}$ & 139 & $2.8 \times 10^{3}$ & 57 & 62 & 0.03 & $<0.01$ \\
\hline $\mathrm{CMPO}$ in $\mathrm{C}_{8} \mathrm{mim}^{+} . \mathrm{NTf}_{2}^{-}$ & 76 & $2.8 \times 10^{3}$ & 52 & 34 & 0.01 & $<0.01$ \\
\hline CMPO in $n$-dodecane & 1.3 & 7.55 & 20 & 1.1 & 0.01 & $<0.01$ \\
\hline
\end{tabular}

${ }^{a}$ Feed: 3 mol/L $\mathrm{HNO}_{2} .{ }^{b}$ Concentration of the extractant: $0.1 \mathrm{~mol} / \mathrm{L} .{ }^{c} \mathrm{NaNO}$, was used as the holding

The extraction by CMPO-FIL (10) in three different ILs for Am(III) at various $\mathrm{HNO}_{3}$ concentrations is presented in Figure 8.4. There are two possible mechanisms for the extraction of Am(III) viz. by the involvement of the nitrate ion (eqn. 9) and by a cation exchange mechanism (eqn. 10).

$$
\begin{aligned}
& \mathrm{Am}(\mathrm{III})_{\mathrm{aq}}+3 \mathrm{NO}_{3}{ }^{-}+\mathrm{nCMPO}-\mathrm{Dmim}^{+} \mathrm{X}_{\mathrm{IL}}^{-}=\mathrm{Am}\left(\mathrm{CMPO}-\mathrm{Dmim}^{+}\right)_{\mathrm{n}}{ }^{(3+\mathrm{n})+} \cdot\left(\mathrm{NO}_{3}{ }^{-}\right)_{3} \cdot \mathrm{nX}_{\mathrm{IL}}^{-}
\end{aligned}
$$

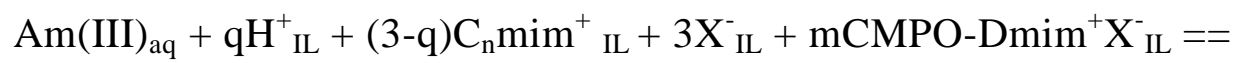

$$
\begin{aligned}
& \mathrm{Am}\left(\mathrm{CMPO}-\mathrm{Dmim}^{+}\right)_{\mathrm{m}}{ }^{(3+\mathrm{m})+} .(3+\mathrm{m}) \mathrm{X}_{\mathrm{IL}}^{-}+(3-\mathrm{q}) \mathrm{C}_{\mathrm{n}} \mathrm{mim}^{+}{ }_{\mathrm{aq}}+\mathrm{qH}^{+}{ }_{\mathrm{aq}}
\end{aligned}
$$

Where $\mathrm{C}_{\mathrm{n}}$ mim represents the ionic liquid with $(\mathrm{n}=2,4,6)$, and $\mathrm{Dmim}^{+}$is the imidazolium part of CMPO-FIL (10) having an $n$-dodecyl chain. If the extraction of Am(III) is driven by the involvement of the nitrate ion (eqn 9), then $D_{\mathrm{Am}}$ is expected to be independent of the alkyl chain length in the cationic part of the ionic liquid. However, the extraction efficiency by CMPO-FIL (10) in ILs is in the order $\mathrm{C}_{4} \mathrm{mim}^{+}>\mathrm{C}_{6} \mathrm{mim}^{+}>\mathrm{C}_{8} \mathrm{mim}^{+}$(Figure 8.4), which is due to a better solubility of $\mathrm{C}_{4} \mathrm{mim}^{+}$in the aqueous phase. This suggests that, in this ligand system, Am(III) is extracted by a cation exchange mechanism (eqn. 10). Since the ILs are known to dissolve nitric acid, ${ }^{15,16}$ there is a competition of $\mathrm{H}^{+}$and $\mathrm{C}_{\mathrm{n}} \mathrm{mim}^{+}$cations for migration into the 
aqueous phase. Therefore, $D_{\mathrm{Am}}$ decreases with increase in $\mathrm{HNO}_{3}$ concentration, due to increase in $\left[\mathrm{H}^{+}\right]$in the aqueous phase. (Figure 8.4).

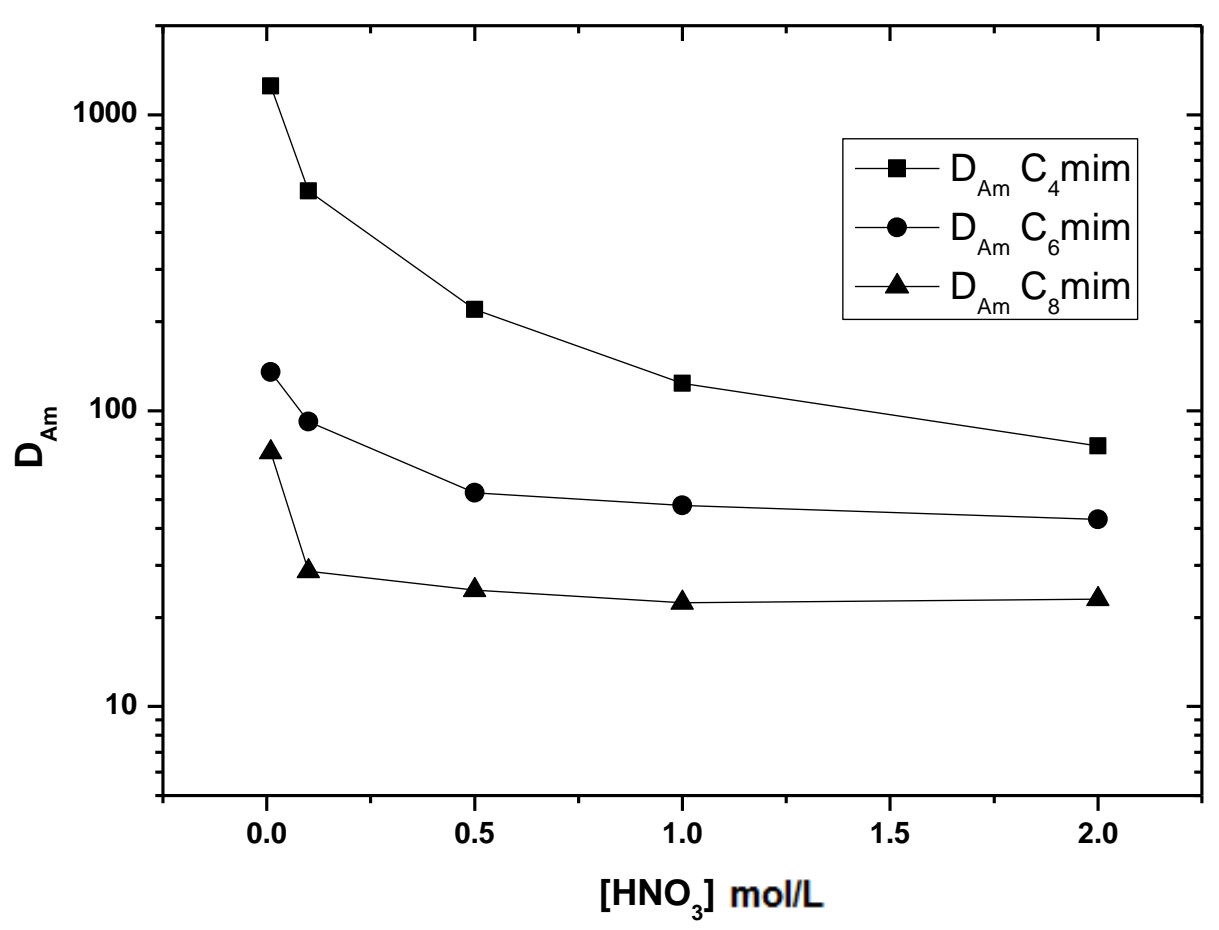

Figure 8.4. Relative extraction of Am(III) by CMPO-FIL (10) in various ILs as a function of aqueous phase acid concentration.

The effect of the concentration of CMPO-FIL (10) on the extraction of Am(III) in three different RTILs $\left[\mathrm{C}_{\mathrm{n}} \operatorname{mim}\right]\left[\mathrm{NTf}_{2}\right]$ was investigated and the stoichiometry of the metal:ligand complexes was determined. Equation 11 can be derived from eqn. 10 in the same way as for eqn. 8, keeping the concentrations of nitric acid and IL constant.

$$
\log D_{\mathrm{Am}}=\log K_{\mathrm{ex}}+\operatorname{mlog}\left[\mathrm{CMPO}-\mathrm{Dmim}^{+}\right]+\text {constant }
$$

A plot of $D_{\text {Am }}$ values as a function of CMPO-FIL concentration yielded slope values of 2.86, 2.79, and 2.76 obtained in ionic liquids with $\mathrm{n}=4,6$, and 8, respectively (Figure 8.5). This suggests that three molecules of CMPO-FIL (10) are complexed to Am(III) during its extraction. 


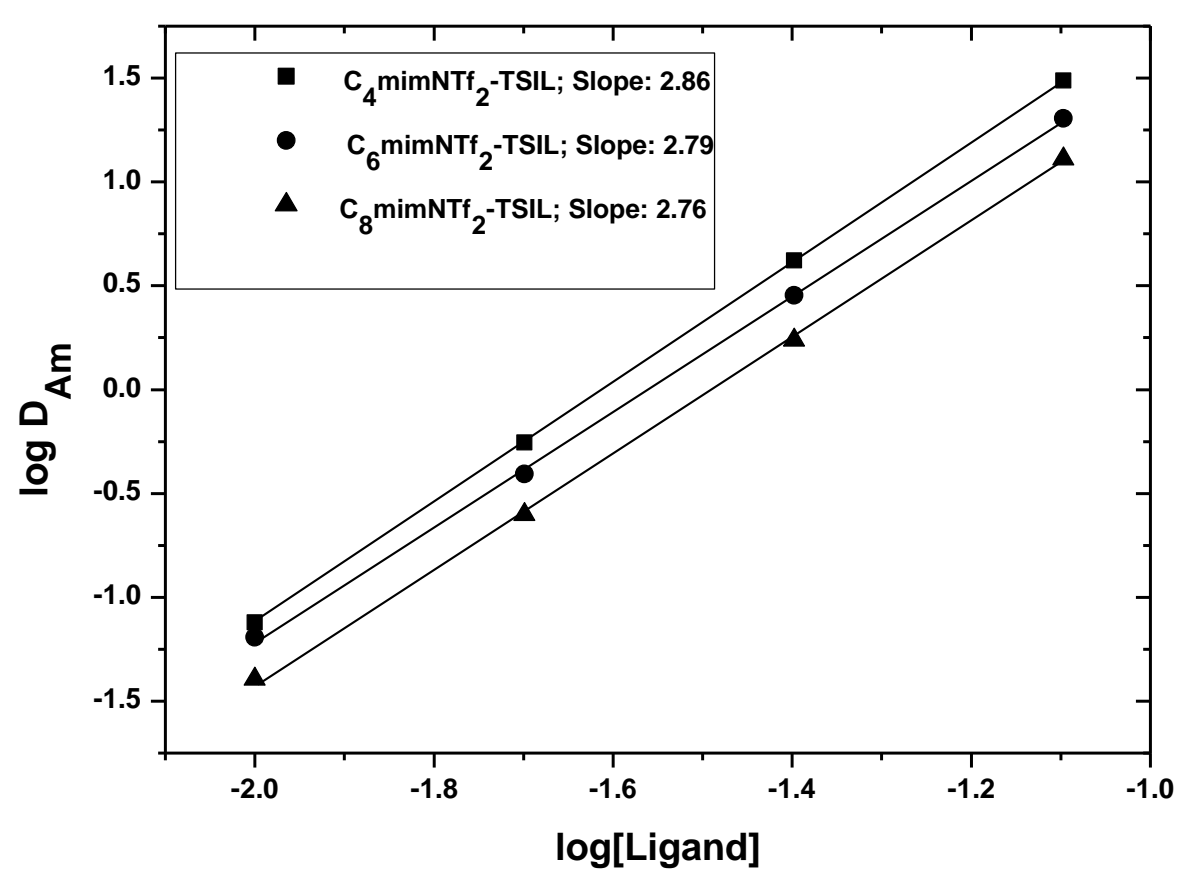

Figure 8.5. Effect of variation of CMPO-FIL (10) concentration versus Am(III) distribution. Aqueous phase: $3 \mathrm{~mol} / \mathrm{L} \mathrm{HNO}_{3}$. Organic phase: CMPO-FIL in $\mathrm{C}_{\mathrm{n}}$ min. $\mathrm{NTf}_{2}$.

The $D_{\text {Am }}$ values as a function of time were studied for CMPO-FIL (10) dissolved in three different RTILs $\left[\mathrm{C}_{\mathrm{n}} \mathrm{mim}\right]\left[\mathrm{NTf}_{2}\right](\mathrm{n}=2,4,6)$ at $3 \mathrm{~mol} / \mathrm{L}_{\mathrm{HNO}}$. The time required to attain equilibrium $D$ values was about $30 \mathrm{~min}$ in all three ionic liquids (Figure 8.6). In contrast, in case of the DGA-FILs, the equilibrium $D$ values were reached in about $6 \mathrm{~h}$ (Figure 8.1), suggesting that the FILs dissolved in ILs more readily achieve $D$ equilibrium values. This is attributed to the higher viscosity of the DGA-FILs compared to the solution of CMPO-FIL (10) in $\mathrm{C}_{\mathrm{n}} \operatorname{mim} . \mathrm{NTf}_{2}$. 


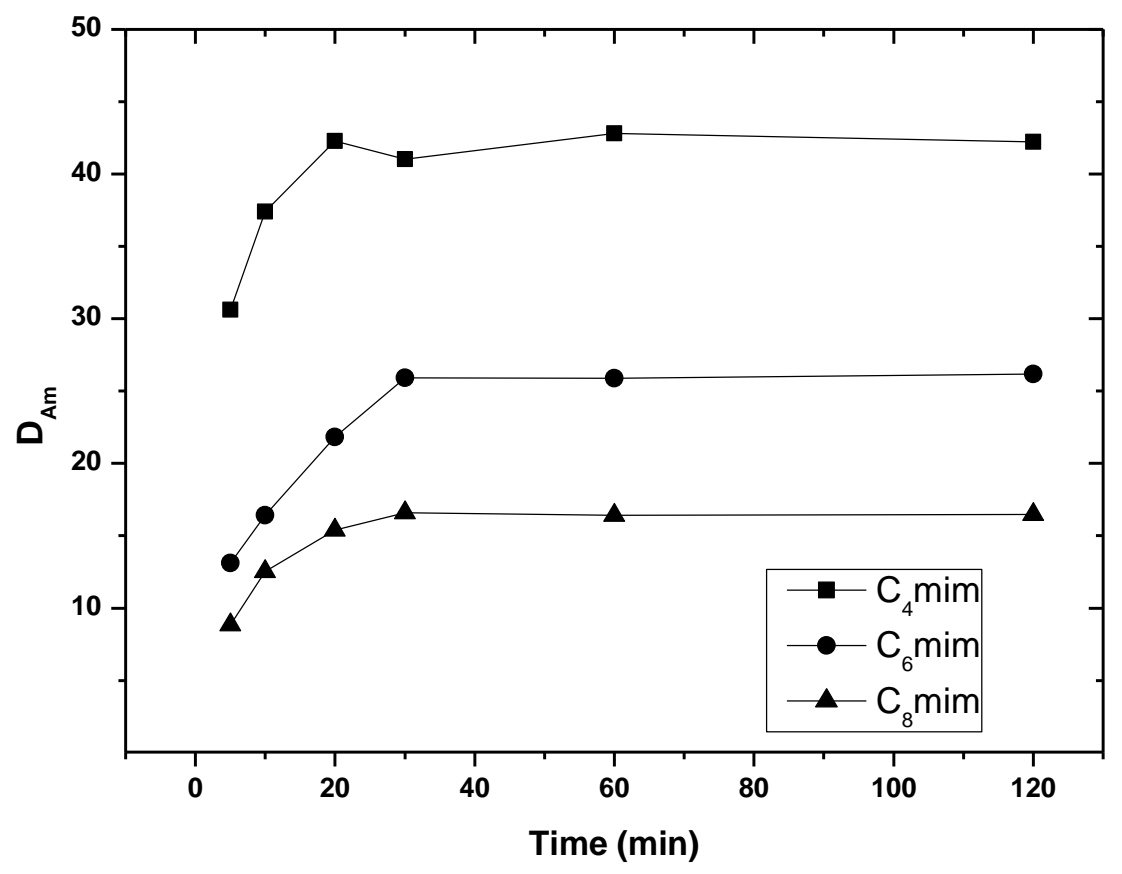

Figure 8.6. $D_{\mathrm{Am}}$ values as a function of time for CMPO-FIL (10) in $\left[\mathrm{C}_{\mathrm{n}} \mathrm{mim}\right]\left[\mathrm{NTf}_{2}\right]$ at 3 $\mathrm{mol} / \mathrm{L} \mathrm{HNO}_{3}$.

\subsection{Conclusions}

Diglycolamide-functionalized ionic liquids (DGA-FILs) and CMPO-functionalized ionic liquids (CMPO-FILs) were prepared and evaluated for their extraction behavior towards actinides/lanthanides and fission product ions. DGA-FILs showed exceptionally high distribution ratio values for the tested metal ions in the order $\mathrm{Eu}$ (III) $>\mathrm{Am}$ (III) $>>\mathrm{Pu}$ (IV) > $\mathrm{Np}(\mathrm{IV})$, with relatively insignificant extraction of $\mathrm{Cs}(\mathrm{I}), \mathrm{Sr}(\mathrm{II})$, and $\mathrm{U}(\mathrm{VI}) \mathrm{O}_{2}$. Among the CMPO-FILs, only that with the $\mathrm{NTf}_{2}{ }^{-}$counter ion, dissolved in room temperature ionic liquids (RTILs), exhibited high distribution ratios for all the tri-, tetra-, and hexavalent ions in the order $\mathrm{Pu}(\mathrm{IV})>\mathrm{U}(\mathrm{VI}) \mathrm{O}_{2}>\mathrm{Am}(\mathrm{III})>\mathrm{Eu}(\mathrm{III})$ with negligible extraction of $\mathrm{Cs}(\mathrm{I})$ and $\mathrm{Sr}(\mathrm{II})$. The excellent extraction properties of DGA-FILs and CMPO-FILs, makes them very suitable for future application in radioactive waste processing. 


\subsection{Experimental}

\subsubsection{General}

All reactions were carried out under an argon atmosphere. The solvents and all reagents were obtained from commercial sources and used without further purification. Compound $\mathbf{2}$ was prepared according to a literature procedure. ${ }^{19}$ Solvents were dried according to standard procedures and stored over molecular sieves. ${ }^{1} \mathrm{H}$ NMR and ${ }^{13} \mathrm{C}$ NMR spectra were recorded on a Varian Unity INOVA (300 MHz) spectrometer. ${ }^{1} \mathrm{H}$ NMR (300 MHz) and ${ }^{13} \mathrm{C}$ NMR (75 $\mathrm{MHz}$ ) chemical shift values were reported as $\delta$ using the residual solvent signal as an internal standard. All NMR measurements are recorded in $\mathrm{CDCl}_{3}$ as a solvent. Electrospray Ionization (positive mode) high resolution mass spectra were recorded on a WATERS LCT mass spectrometer. The extraction experiments were carried out at Bhabha Atomic Research Centre, India. Analytical TLC was performed using Merck prepared plates (silica gel 60 F254 on aluminum). Column chromatography was carried out with Merck silica gel 60 (230400 mesh).

\section{2-(2-((3-(1H-imidazol-1-yl)propyl)amino)-2-oxoethoxy)-N,N-di-n-octylacetamide} (3).

A solution of 3-(1H-imidazol-1-yl)propan-1-amine (1) $(44.0 \mathrm{~g}, 123.0 \mathrm{mmol}), \mathrm{N}, \mathrm{N}-$ dioctylglycolic acid (2) (18.0 g, $143.8 \mathrm{mmol})$, DCC (30.00 g, $145.4 \mathrm{mmol})$, and HOBT (19.6 $\mathrm{g}, 145.4 \mathrm{mmol})$ in chloroform $(600 \mathrm{~mL})$ was stirred overnight at room temperature. The solvent was evaporated and the residue was dissolved in diethyl ether $(500 \mathrm{~mL})$. The resulting solution was filtered and the filtrate was washed with $5 \% \mathrm{HCl}$ solution $(2 \times 250$ $\mathrm{mL}$ ). The organic layer was dried with anhydrous $\mathrm{MgSO}_{4}$ and concentrated under reduced pressure. The residue was purified by column chromatography $\left(\mathrm{CHCl}_{3}: \mathrm{MeOH}, 95: 5 \rightarrow\right.$ 90:10) to afford 3 (52.5 g, 92\%) as a colorless oil. ${ }^{1} \mathrm{H}$ NMR: $\delta$ 0.84-0.96 $\left(6 \mathrm{H}, \mathrm{m}, \mathrm{CH}_{3}\right), 1.17-1.40$ $\left(20 \mathrm{H}, \mathrm{m},\left(\mathrm{CH}_{2}\right)_{5}\right), 1.46-1.62\left(4 \mathrm{H}, \mathrm{m}, \mathrm{NCH}_{2} \mathrm{CH}_{2}\right), 2.05\left(4 \mathrm{H}\right.$, pentet, $\left.J=7.5 \mathrm{~Hz}, \mathrm{ImCH}_{2} \mathrm{CH}_{2}\right)$, $3.10\left(4 \mathrm{H}, \mathrm{t}, J=7.5 \mathrm{~Hz}, \mathrm{NCH}_{2}\right), 3.34\left(8 \mathrm{H}, \mathrm{t}, J=7.5 \mathrm{~Hz}, \mathrm{NCH}_{2}\right.$ and $\left.\mathrm{NHCH}_{2}\right), 4.08$ and 4.26 $\left(2 \mathrm{H}, \mathrm{s}, \mathrm{OCH}_{2}\right), 4.17\left(2 \mathrm{H}, \mathrm{t}, J=7.5 \mathrm{~Hz}, \mathrm{ImCH}_{2}\right), 7.00(1 \mathrm{H}, \mathrm{s}, \mathrm{ImH}), 7.06(1 \mathrm{H}, \mathrm{s}, \mathrm{ImH}), 7.57$ $(1 \mathrm{H}, \mathrm{s}, \mathrm{ImH}), 8.43(1 \mathrm{H}, \mathrm{br} \mathrm{s}, \mathrm{NH}) .{ }^{13} \mathrm{C} \mathrm{NMR}: \delta 14.3,22.8,27.2,29.4,32.0,35.8,45.5,46.7$, 47.1, 70.2, 72.8, 103.5, 120.0, 136.6, 168.9, 170.6. HRMS calculated for $\mathrm{C}_{26} \mathrm{H}_{49} \mathrm{~N}_{4} \mathrm{O}_{3}$ 465.3805 $[\mathrm{M}+\mathrm{H}]^{+}$, found 465.3806. 


\section{3-n-Butyl-1-(3-(2-(2-(di-n-octylamino)-2-oxoethoxy)acetamido)propyl)-1H-imidazol-}

\section{3-ium bromide (4).}

To a solution of DGA-imidazole-propanamide $3(5.00 \mathrm{~g}, 10.7 \mathrm{mmol})$ in anhydrous $\mathrm{CH}_{3} \mathrm{CN}$ (70 mL), $n$-butyl bromide $(1.76 \mathrm{~g}, 12.9 \mathrm{mmol})$ was added dropwise at $0{ }^{\circ} \mathrm{C}$. The mixture was stirred at room temperature for $12 \mathrm{~h}$, followed by reflux at $80-85^{\circ} \mathrm{C}$ for $2.5 \mathrm{~d}$. The solvent was evaporated under reduced pressure and the resulting residue was washed with $n$-hexane $(3 \times 50 \mathrm{~mL})$. Residual solvent was removed under reduced pressure and the residue was dried under vacuum for $5 \mathrm{~h}$ to afford 4 as a dense oil in quantitative yield. ${ }^{1} \mathrm{H}$ NMR: $\delta$ 0.84-0.93 $\left(6 \mathrm{H}, \mathrm{m}, \mathrm{CH}_{3}\right), 0.98\left(3 \mathrm{H}, \mathrm{t}, J=7.5 \mathrm{~Hz}, \mathrm{CH}_{3}(\mathrm{Bu})\right), 1.20-1.36\left(20 \mathrm{H}, \mathrm{m},\left(\mathrm{CH}_{2}\right)_{5}\right), 1.41(2 \mathrm{H}$, sextet, $\left.J=7.5 \mathrm{~Hz}, \mathrm{CH}_{3} \mathrm{CH}_{2}(\mathrm{Bu})\right), 1.47-1.62\left(4 \mathrm{H}, \mathrm{m}, \mathrm{NCH}_{2} \mathrm{CH}_{2}\right), 1.92(4 \mathrm{H}$, pentet, $J=7.5$ $\left.\mathrm{Hz}, \mathrm{ImCH}_{2} \mathrm{CH}_{2}(\mathrm{Bu})\right), 2.24\left(4 \mathrm{H}\right.$, pentet, $\left.J=7.5 \mathrm{~Hz}, \mathrm{ImCH}_{2} \mathrm{CH}_{2}\right), 3.12(4 \mathrm{H}, \mathrm{t}, J=7.5 \mathrm{~Hz}$, $\left.\mathrm{NCH}_{2}\right), 3.27-3.41\left(8 \mathrm{H}, \mathrm{m}, \mathrm{NCH}_{2}\right.$ and $\left.\mathrm{NHCH}_{2}\right), 4.19$ and $4.41\left(2 \mathrm{H}, \mathrm{s}, \mathrm{OCH}_{2}\right), 4.30(2 \mathrm{H}, \mathrm{t}, J=$ $\left.7.5 \mathrm{~Hz}, \mathrm{ImCH}_{2}\right), 4.48\left(2 \mathrm{H}, \mathrm{t}, J=7.5 \mathrm{~Hz}, \operatorname{ImCH}_{2}(\mathrm{Bu})\right), 7.23(1 \mathrm{H}, \mathrm{s}, \operatorname{ImH}), 7.65(1 \mathrm{H}, \mathrm{s}, \operatorname{ImH})$, $8.71\left(1 \mathrm{H}\right.$, br s, NH), 10.46 (1H, s, ImH). ${ }^{13} \mathrm{C}$ NMR: $\delta$ 13.7, 14.3, 19.7, 22.8, 27.2, 29.4, 31.9, 36.2, 46.4, 47.3, 50.0, 69.8, 71.6, 121.8, 123.2, 137.4, 168.8, 170.9. HRMS calculated for $\mathrm{C}_{30} \mathrm{H}_{57} \mathrm{BrN}_{4} \mathrm{NaO}_{3}$ 623.3512, 625.3491 [M+NaBr $]^{+}$, found 623.3532, 625.3483.

\section{General procedure for ion exchange}

To a solution of an ionic liquid, containing bromide as anion, (1 equiv) in anhydrous $\mathrm{CH}_{3} \mathrm{CN}$, a solution of $\mathrm{NaPF}_{6}$ or bis(trifluoromethane)sulfonimide lithium salt (2.5 equiv) in anhydrous $\mathrm{CH}_{3} \mathrm{CN}$ was added. The mixture was stirred at room temperature for $48 \mathrm{~h}$. The solvent was evaporated under reduced pressure and water $(50 \mathrm{~mL})$ was added to the solid residue. The product was extracted into $\mathrm{CH}_{2} \mathrm{Cl}_{2}(3 \times 50 \mathrm{~mL})$ and the organic phase was washed with water $(3 \times 50 \mathrm{~mL})$. The collected organic layers were dried over $\mathrm{MgSO}_{4}$, filtered and the solvent was evaporated. The obtained ionic liquid was dried under vacuum for $4 \mathrm{~h}$.

\section{3-n-Butyl-1-(3-(2-(2-(di-n-octylamino)-2-oxoethoxy)acetamido)propyl)-1H-imidazol-}

3-ium hexafluorophosphate (5) was prepared from DGA-imidazole-propanamide-butyl bromide (4) (20.0 g, $33.2 \mathrm{mmol})$ in $\mathrm{CH}_{3} \mathrm{CN}(70 \mathrm{~mL})$ and $\mathrm{NaPF}_{6}(14.0 \mathrm{~g}, 83.1 \mathrm{mmol})$ in $\mathrm{CH}_{3} \mathrm{CN}(35 \mathrm{~mL})$ as a dense oil in quantitative yield. ${ }^{1} \mathrm{H}$ NMR: $\delta 0.84-0.93\left(6 \mathrm{H}, \mathrm{m}, \mathrm{CH}_{3}\right), 0.97$ $\left(3 \mathrm{H}, \mathrm{t}, J=7.5 \mathrm{~Hz}, \mathrm{CH}_{3}(\mathrm{Bu})\right), 1.21-1.35\left(20 \mathrm{H}, \mathrm{m},\left(\mathrm{CH}_{2}\right)_{5}\right), 1.39(2 \mathrm{H}$, sextet, $J=7.5 \mathrm{~Hz}$, $\left.\mathrm{CH}_{3} \mathrm{CH}_{2}(\mathrm{Bu})\right), 1.47-1.62\left(4 \mathrm{H}, \mathrm{m}, \mathrm{NCH}_{2} \mathrm{CH}_{2}\right), 1.88\left(4 \mathrm{H}\right.$, pentet, $J=7.5 \mathrm{~Hz}, \mathrm{ImCH}_{2} \mathrm{CH}_{2}$ 
(Bu)), $2.14\left(4 \mathrm{H}\right.$, pentet, $\left.J=7.5 \mathrm{~Hz}, \mathrm{ImCH}_{2} \mathrm{CH}_{2}\right), 3.12\left(4 \mathrm{H}, \mathrm{t}, J=7.5 \mathrm{~Hz}, \mathrm{NCH}_{2}\right), 3.27-3.41$ $\left(8 \mathrm{H}, \mathrm{m}, \mathrm{NCH}_{2}\right.$ and $\left.\mathrm{NHCH}_{2}\right), 4.17$ and $4.37\left(2 \mathrm{H}, \mathrm{s}, \mathrm{OCH}_{2}\right), 4.17\left(2 \mathrm{H}, \mathrm{t}, J=7.5 \mathrm{~Hz}, \mathrm{ImCH}_{2}\right)$, $4.28\left(2 \mathrm{H}, \mathrm{t}, J=7.5 \mathrm{~Hz}, \mathrm{ImCH}_{2}(\mathrm{Bu})\right), 7.23(1 \mathrm{H}, \mathrm{s}, \mathrm{ImH}), 7.50(1 \mathrm{H}, \mathrm{s}, \mathrm{ImH}), 8.58(1 \mathrm{H}$, br s, $\mathrm{NH}), 8.93(1 \mathrm{H}, \mathrm{s}, \mathrm{ImH}) .{ }^{13} \mathrm{C}$ NMR: $\delta 13.5,14.3,19.6,22.8,27.0,29.5,32.0,46.7,47.2,50.1$, 70.1, 122.2, 122.8, 136.3, 169.4, 171.5. HRMS calculated for $\mathrm{C}_{30} \mathrm{H}_{57} \mathrm{~F}_{6} \mathrm{~N}_{4} \mathrm{NaO}_{3} \mathrm{P} 689.3970$ $\left[\mathrm{M}+\mathrm{PF}_{6}+\mathrm{Na}\right]^{+}$, found 689.3991 .

\section{3-n-Butyl-1-(3-(2-(2-(di-n-octylamino)-2-oxoethoxy)acetamido)propyl)-1H-imidazol-}

3-ium bis(trifluoromethane)sulfonimide (6) was prepared from 4 (24.0 g, 39.9 mmol) in $\mathrm{CH}_{3} \mathrm{CN}(70 \mathrm{~mL})$ and bis(trifluoromethane)sulfonimide lithium salt $(28.6 \mathrm{~g}, 99.7 \mathrm{mmol})$ in $\mathrm{CH}_{3} \mathrm{CN}(35 \mathrm{~mL})$ as an oil $(28.1 \mathrm{~g})$ in $90 \%$ yield. ${ }^{1} \mathrm{H}$ NMR: $\delta 0.83-0.93\left(6 \mathrm{H}, \mathrm{m}, \mathrm{CH}_{3}\right), 0.97$ $\left(3 \mathrm{H}, \mathrm{t}, J=7.5 \mathrm{~Hz}, \mathrm{CH}_{3}(\mathrm{Bu})\right), 1.21-1.36\left(20 \mathrm{H}, \mathrm{m},\left(\mathrm{CH}_{2}\right)_{5}\right), 1.39(2 \mathrm{H}$, sextet, $J=7.5 \mathrm{~Hz}$, $\left.\mathrm{CH}_{3} \mathrm{CH}_{2}(\mathrm{Bu})\right), 1.47-1.62\left(4 \mathrm{H}, \mathrm{m}, \mathrm{NCH}_{2} \mathrm{CH}_{2}\right), 1.88\left(4 \mathrm{H}\right.$, pentet, $J=7.5 \mathrm{~Hz}, \mathrm{ImCH}_{2} \mathrm{CH}_{2}$ (Bu)), $2.13\left(4 \mathrm{H}\right.$, pentet, $\left.J=7.5 \mathrm{~Hz}, \mathrm{ImCH}_{2} \mathrm{CH}_{2}\right), 3.10\left(4 \mathrm{H}, \mathrm{t}, J=7.5 \mathrm{~Hz}, \mathrm{NCH}_{2}\right), 3.26-3.38$ $\left(8 \mathrm{H}, \mathrm{m}, \mathrm{NCH}_{2}\right.$ and $\left.\mathrm{NHCH}_{2}\right), 4.12$ and $4.32\left(2 \mathrm{H}, \mathrm{s}, \mathrm{OCH}_{2}\right), 4.19\left(2 \mathrm{H}, \mathrm{t}, J=7.5 \mathrm{~Hz}, \mathrm{ImCH}_{2}\right)$, $4.29\left(2 \mathrm{H}, \mathrm{t}, J=7.5 \mathrm{~Hz}, \operatorname{ImCH}_{2}(\mathrm{Bu})\right), 7.24(1 \mathrm{H}, \mathrm{s}, \operatorname{ImH}), 7.56(1 \mathrm{H}, \mathrm{s}, \operatorname{ImH}), 8.34(1 \mathrm{H}$, br s, $\mathrm{NH}), 9.01(1 \mathrm{H}, \mathrm{s}, \mathrm{ImH}) .{ }^{13} \mathrm{C} \mathrm{NMR}: \delta 13.6,14.4,19.6,22.8,27.1,29.5,32.0,46.7,47.2,50.1$, 70.1, 117.9, 122.3, 122.7, 136.3, 169.6, 171.4. HRMS calculated for $\mathrm{C}_{32} \mathrm{H}_{57} \mathrm{~F}_{6} \mathrm{~N}_{5} \mathrm{NaO}_{7} \mathrm{~S}_{2}$ 824.3507, $\left[\mathrm{M}+\mathrm{NTf}_{2}+\mathrm{Na}\right]^{+}$found 824.3532.

\section{1-(3-(2-(Diphenylphosphoryl)acetamido)propyl)-3-dodecyl-1H-imidazol-3-ium bromide $(8)$.}

To a solution of CMPO-imidazole-propanamide $7^{9}$ (26.00 g, $\left.70.8 \mathrm{mmol}\right)$ in $\mathrm{CH}_{3} \mathrm{CN}(150 \mathrm{~mL})$, $n$-dodecyl bromide $(21.2 \mathrm{~g}, 85.0 \mathrm{mmol})$ was added dropwise at $0{ }^{\circ} \mathrm{C}$. The mixture was stirred at room temperature for $12 \mathrm{~h}$, followed by reflux at $80-85^{\circ} \mathrm{C}$ for $2.5 \mathrm{~d}$. Then the solvent was evaporated under reduced pressure and the resulting residue was washed with $n$-hexane $(3 \times$ $100 \mathrm{~mL}$ ). Residual solvent was removed under reduced pressure and the residue was dried under vacuum for $5 \mathrm{~h}$ to afford $8(37.9 \mathrm{~g}, 87 \%)$ as a dense oil. ${ }^{1} \mathrm{H}$ NMR: $\delta 0.85(3 \mathrm{H}, \mathrm{t}, J=7.5$ $\left.\mathrm{Hz}, \mathrm{CH}_{3}\right), 1.16-1.36$ (18H, m, $\left.\left(\mathrm{CH}_{2}\right)_{9}\right), 1.75-1.90\left(2 \mathrm{H}, \mathrm{m}, \mathrm{ImCH}_{2} \mathrm{CH}_{2}\right.$ (dodec.)), 1.97-2.10 $\left(2 \mathrm{H}, \mathrm{m}, \mathrm{ImCH}_{2} \mathrm{CH}_{2}\right), 3.02-3.14\left(2 \mathrm{H}, \mathrm{m}, \mathrm{NCH}_{2}\right), 3.68\left(2 \mathrm{H}, \mathrm{d}, J=12.0 \mathrm{~Hz}, \mathrm{PCH}_{2} \mathrm{CO}\right), 4.17$ $\left(2 \mathrm{H}, \mathrm{t}, J=7.5 \mathrm{~Hz}, \mathrm{ImCH}_{2}\right.$ (dodec.)), $4.26\left(2 \mathrm{H}, \mathrm{t}, J=7.5 \mathrm{~Hz}, \mathrm{ImCH}_{2}\right), 7.22(1 \mathrm{H}, \mathrm{s}, \mathrm{ImH}), 7.38$ 7.55 (6H, m, ArH ), $7.71(1 \mathrm{H}, \mathrm{s}, \mathrm{ImH}), 7.77-7.91(4 \mathrm{H}, \mathrm{m}, \mathrm{ArH}), 8.72$ (1H, br s, NH), 10.00 
$(1 \mathrm{H}, \mathrm{s}, \mathrm{ImH}) .{ }^{13} \mathrm{C}$ NMR: $\delta 14.0,22.6,26.2,28.9,29.2,29.3,29.4,29.5,30.1,31.8,34.9,38.6$, 39.2, 46.3, 49.9, 122.0, 122.9, 128.6, 128.7, 131.0, 131.1, 131.6, 132.0, 132.6, 137.0, 165.7 . HRMS calculated for $\mathrm{C}_{32} \mathrm{H}_{48} \mathrm{~N}_{3} \mathrm{O}_{2} \mathrm{P} 536.3400[\mathrm{M}]^{+}$, found 536.3317 .

\section{1-(3-(2-(Diphenylphosphoryl)acetamido)propyl)-3-dodecyl-1H-imidazol-3-ium}

\section{hexafluorophosphate (9).}

9 was prepared according to the general procedure described above from CMPO-imidazolepropanamide-dodecyl bromide $(\mathbf{8})(20.0 \mathrm{~g}, 32.4 \mathrm{mmol})$ in anhydrous $\mathrm{CH}_{3} \mathrm{CN}(150 \mathrm{~mL})$ and $\mathrm{NaPF}_{6}(14.0 \mathrm{~g}, 83.1 \mathrm{mmol})$ in anhydrous $\mathrm{CH}_{3} \mathrm{CN}(35 \mathrm{~mL})$ as a gel $(21.2 \mathrm{~g})$ in $96 \%$ yield. ${ }^{1} \mathrm{H}$ NMR: $\delta 0.87\left(3 \mathrm{H}, \mathrm{t}, J=7.5 \mathrm{~Hz}, \mathrm{CH}_{3}\right), 1.13-1.36\left(18 \mathrm{H}, \mathrm{m},\left(\mathrm{CH}_{2}\right)_{9}\right), 1.71-1.87(2 \mathrm{H}, \mathrm{m}$, $\mathrm{ImCH}_{2} \mathrm{CH}_{2}$ (dodec.)), 1.87-2.04 (2H, m, $\left.\mathrm{ImCH}_{2} \mathrm{CH}_{2}\right), 3.04-3.16\left(2 \mathrm{H}, \mathrm{m}, \mathrm{NCH}_{2}\right), 3.44(2 \mathrm{H}, \mathrm{d}$, $\left.J=12.0 \mathrm{~Hz}, \mathrm{PCH}_{2} \mathrm{CO}\right), 3.97-4.09\left(4 \mathrm{H}, \mathrm{m}, \mathrm{ImCH}_{2}\right), 7.17(1 \mathrm{H}, \mathrm{s}, \mathrm{ImH}), 7.34(1 \mathrm{H}, \mathrm{br} \mathrm{s}, \mathrm{NH})$, $7.37(1 \mathrm{H}, \mathrm{s}, \mathrm{ImH}), 7.41-7.58(6 \mathrm{H}, \mathrm{m}, \mathrm{ArH}), 7.67-7.81(4 \mathrm{H}, \mathrm{m}, \mathrm{ArH}), 8.83(1 \mathrm{H}, \mathrm{s}, \mathrm{ImH}) .{ }^{13} \mathrm{C}$ NMR: $\delta$ 14.1, 22.7, 26.2, 28.9, 29.3, 29.4, 29.5, 29.6, 29.9, 31.9, 35.3, 38.4, 39.1, 46.4, 50.0, 122.0, 122.5, 128.9, 129.0, 130.8, 130.9, 131.2, 132.2, 132.4, 136.1, 165.7. HRMS calculated for $\mathrm{C}_{32} \mathrm{H}_{48} \mathrm{~F}_{6} \mathrm{~N}_{3} \mathrm{O}_{2} \mathrm{P}_{2} 682.3126\left[\mathrm{M}+\mathrm{PF}_{6}+\mathrm{H}\right]^{+}$, found 682.3185 .

\section{1-(3-(2-(Diphenylphosphoryl)acetamido)propyl)-3-dodecyl-1H-imidazol-3-ium} bis(trifluoromethane)sulfonimide (10).

10 was prepared according to the general procedure starting from 8 (20.0 g, $32.4 \mathrm{mmol})$ and bis(trifluoromethane)sulfonimide lithium salt $(22.1 \mathrm{~g}, 81.0 \mathrm{mmol})$ as a gel $(25.2 \mathrm{~g})$ in $97 \%$ yield. ${ }^{1} \mathrm{H}$ NMR: $\delta 0.87\left(3 \mathrm{H}, \mathrm{t}, J=7.5 \mathrm{~Hz}, \mathrm{CH}_{3}\right), 1.16-1.37\left(18 \mathrm{H}, \mathrm{m},\left(\mathrm{CH}_{2}\right)_{9}\right), 1.73-1.89(2 \mathrm{H}$, m, $\mathrm{ImCH}_{2} \mathrm{CH}_{2}$ (dodec.)), 1.90-2.03 (2H, m, $\left.\mathrm{ImCH}_{2} \mathrm{CH}_{2}\right), 3.10-3.20\left(2 \mathrm{H}, \mathrm{m}, \mathrm{NCH}_{2}\right), 3.47$ (2H, $\mathrm{d}, J=12.0 \mathrm{~Hz}, \mathrm{PCH}_{2} \mathrm{CO}$ ), $3.99\left(2 \mathrm{H}, \mathrm{t}, J=7.5 \mathrm{~Hz}, \mathrm{ImCH}_{2}\right.$ (dodec.)), 4.07 (2H, t, $J=7.5 \mathrm{~Hz}$, $\left.\mathrm{ImCH}_{2}\right), 7.17(1 \mathrm{H}, \mathrm{s}, \mathrm{ImH}), 7.37(1 \mathrm{H}, \mathrm{br} \mathrm{s}, \mathrm{NH}), 7.40(1 \mathrm{H}, \mathrm{s}, \mathrm{ImH}), 7.43-7.60(6 \mathrm{H}, \mathrm{m}, \mathrm{ArH})$, 7.72-7.84 (4H, m, ArH ), 8.97 (1H, s, ImH). ${ }^{13} \mathrm{C}$ NMR: $\delta$ 14.1, 22.6, 26.1, 28.8, 29.3, 29.5, 29.6, 29.9, 31.9, 35.3, 38.3, 38.9, 46.5, 50.0, 114.5, 118.2, 121.4, 122.2, 122.6, 124.6, 128.9, 129.0, 130.8, 130.9, 131.8, 132.6, 135.9, 166.0. HRMS calculated for $\mathrm{C}_{34} \mathrm{H}_{48} \mathrm{~F}_{6} \mathrm{~N}_{3} \mathrm{O}_{6} \mathrm{PS}_{2}$ 803.2621, $\left[\mathrm{M}+\mathrm{NTf}_{2}+\mathrm{H}\right]^{+}$found 803.2691. 


\subsubsection{Distribution studies}

The distribution studies were carried out by mixing $0.5 \mathrm{~mL}$ of pure of DGA-FILs, or 0.1 mol/L CMPO-FIL solution $(0.5 \mathrm{~mL})$ in RTILs with an equal volume of the aqueous phase containing the required radiotracers in a given concentration of $\mathrm{HNO}_{3}$. Studies with Am involved the use of ${ }^{241} \mathrm{Am}$ tracer and the concentration of the metal ion in a typical distribution experiment was $\sim 10^{-7} \mathrm{~mol} / \mathrm{L}$. The equilibration of the tubes was carried out in a thermostated water bath at $25+0.1{ }^{\circ} \mathrm{C}$ for a reasonable time which was optimized after the studies with varying equilibration time. After centrifugation, the phases were separated and assayed radiometrically. The distribution ratio, $D_{\mathrm{M}}$, was defined as the ratio of the activity per unit volume in the ionic liquid phase to that in the aqueous phase. The experiments were carried out in duplicate with a precision of $\pm 5 \%$.

\subsection{References}

1. Huddleston, J. G.; Willauer, H. D.; Swatloski, R. P.; Visser, A. N.; Rogers, R. D. Chem. Commun. 1998, 1765.

2. Blanchard, L. A.; Hancut, D.; Beckman, E. J.; Brennecke, J. F. Nature 1999, 399, 28.

3. Fadeev, A. G.; Meagher, M. M. Chem. Commun. 2001, 295.

4. Visser, A. E.; Rogers, R. D. J. Solid State Chem. 2003, 171, 109.

5. Nakashima, K.; Kubota, F.; Maruyama, T.; Goto, M. Ind. Eng. Chem. Res. 2005, 44, 4368.

6. Shen, Y. L.; Tan, X. W.; Wang, L.; Wu, W. S. Sep. Purif. Technol. 2011, 78, 298.

7. Turanova, A. N.; Karandashev, V. K.; Baulin, V. E. Solv. Extr. Ion Exch. 2008, 26, 77.

8. Ouadi, A.; Klimchuk, O.; Gaillard, C.; Billard, I. Green Chem. 2007, 9, 1160.

9. Odinets, I. L.; Sharova, E. V.; Artyshin, O. I.; Lyssenko, K. A.; Nelyubina, Y. V.; Myasoedova, G. V.; Molochnikova, N. P.; Zakharchenro, E. A. Dalton Trans. 2010, 39, 4170.

10. Rout, A.; Venkatesan, K. A.; Srinivasan, T. G.; Rao, P. R. V. Radiochim. Acta 2010, 98, 459.

11. Rout, A.; Karmakar, S.; Venkatesan, K. A.; Srinivasan, T. G.; Vasudeva Rao, P. R. Sep. Purif. Technol. 2011, 81, 109.

12. Djedovic, N.; Ferdani, R.; Harder, E.; Pajewska, J.; Pajewski, R.; Weber, M. E.;

Schlesinger, P. H.; Gokel, G. W. New J. Chem. 2005, 29, 291. 
13. Shimojo, K.; Kurahashi, K.; Naganawa, H. Dalton Trans. 2008, 5083.

14. Rout, A.; Venkatesan, K. A.; Srinivasan, T. G.; Vasudeva Rao, P. R. Sep. Purif. Technol. 2011, 76, 238.

15. Billard, I.; Gaillard, C.; Hennig, C. Dalton Trans. 2007, 4214.

16. Billard, I.; Ouadi, A.; Jobin, E.; Champion, J.; Gaillard, C.; Georg. S. Solv. Extr. Ion Exch. 2011, 29, 577.

17. Billard, I.; Ouadi, A.; Gaillard, C. Anal. Bioanal. Chem. 2011, 400, 1555.

18. Sengupta, A.; Mohapatra, P. K.; Iqbal, M.; Verboom, W.; Huskens. J. Dalton Trans. 2012, 41,6970 .

19. Djedovic, N.; Ferdani, R.; Harder, E.; Pajewska, J.; Pajewski, R.; Weber, M. E.;

Schlesinger, P. H.; Gokel, G. W. New. J. Chem. 2005, 29, 291. 



\section{Summary and Outlook}

The research presented in this thesis deals with the synthesis and evaluation of new potential ligands for the complexation of actinide and lanthanide ions either for their extraction from bulk radioactive waste or their stripping from an extracted organic phase for final processing of the waste. In particular, the aim of the work described here is the development of new ligands with improved separation and extraction efficiency. Separation of actinides (An) and lanthanides $(\mathrm{Ln})$ is a challenging issue in the field of nuclear waste management. The main objective in this work is to achieve separations by rather simple, completely incinerable, and easily accessible ligands, for different processes under development in the nuclear waste industry, and to develop environmentally friendly extraction systems, that might replace the conventional organic solvent system.

In the first Chapter, nuclear power is viewed for its present contribution to global energy and its possible growth as a future energy resource to combat environmental and climatological issues concerned with fossil fuels. A basic description and the current state of the management of nuclear waste is also given, since this is one of the most prominent problems for the expansion of nuclear energy.

Chapter 2 commences with a brief description of the properties of An and Ln, followed by an overview of various An/Ln ligands, that have been developed in the recent years and are being developed for various processes. Various $\mathrm{O}$ donor ligands are summarized for the coextraction of $\mathrm{An}(\mathrm{III})$ and $\mathrm{Ln}(\mathrm{III})$. For the separation of $\mathrm{An}(\mathrm{III})$ and $\mathrm{Ln}(\mathrm{III})$, ligands with soft $\mathrm{N}$ and $\mathrm{S}$ donor atoms, synergistic mixtures, and water-soluble ligands for the selective stripping of An(III) are described. Finally, recent developments are discussed towards the application of ionic liquids in nuclear waste treatment as green alternatives for conventional solvent systems.

Chapter 3 deals with structural modifications of the $N, N, N^{\prime}, N^{\prime}$-tetraoctyl diglycolamide (TODGA) skeleton, which include: a) the increase in chain length from one carbon to two carbons between the central ether oxygen atom and the amide moieties, b) the addition of substituents on the carbon between the central oxygen atom and the amide moieties on one or both sides of the central oxygen, c) the replacement of the central oxygen by a (substituted) nitrogen atom, and d) synthesis of a rigidified glycolamide. The effect of the structural modifications on their extraction behavior toward $\mathrm{Am}(\mathrm{III})$ and $\mathrm{Eu}(\mathrm{III})$ at various nitric acid 
concentrations was studied. In most cases, the distribution ratios $(D)$ of these ligands do not reach the values of TODGA in the entire acidity range of $0.001-4 \mathrm{~mol} / \mathrm{L} \mathrm{HNO}_{3}$, except for monomethyl-TODGA, which resembles that of TODGA at high nitric acid concentrations. However, at lower acidities its $D$ values are much lower, which may be beneficial for following back-extraction steps. The aza-tripodal ligands exhibited good $D_{\mathrm{Am}}$ values, but only at low nitric acid concentrations. Separation factors $\left(S F_{\mathrm{Am} / \mathrm{Eu}}\right)$ of 8 and 11 were obtained for aza-tripodal ligands bearing $n$-octyl and 2-ethylhexyl groups on the amidic nitrogen, respectively.

In Chapter 4 the synthesis of water-soluble ligands and their capability to complex trivalent actinides in solvent extraction studies is discussed. The hydrophilic diglycolamide (DGA) derivatives with a varying number of ethylene glycol units, or a sodium acetate moiety on the amidic nitrogen, showed a decrease in back-extraction efficiency with increasing number of ethylene glycol units at various $\mathrm{pH}$ of the aqueous phase. Among the $\mathrm{P}=\mathrm{S}$ donating ligands only the ligand with a malonamide backbone exhibited a high back-extraction efficiency, although, with no selectivity for Am(III). Amongst the water-soluble tripodal ligands, i.e. the amide derivatives of nitrilotriacid with $N, N$-dimethyl- and $N, N$-bis(hydroxyethyl) moieties, the former showed a pronounced selectivity for $\mathrm{Am}$ (III) over Eu(III), with a maximum separation factor of 11 , while the latter more efficiently complexed the radionuclides in the aqueous phase with a maximum separation factor of 5 . The extraction trends of various ligands were confirmed by determining their complexation strength using microcalorimetry.

Chapter 5 describes the effect of multivalency on the extraction of Am(III) and Eu(III) of diglycolamide-functionalized calix[4]arenes. On the narrow rim of the calix[4]arene platform, the DGA moiety was either 1,3-di- or tetrasubstituted with a varying spacer length between the oxygen and amide nitrogen atom. In addition, DGA groups were appended to the wide rim and to both rims of calix[4]arenes and their efficiencies were compared for Am(III) and $\mathrm{Eu}(\mathrm{III})$ extraction at different feed acidities. The extraction and separation efficiencies strongly depended on the $N$-alkyl substituent as well as the spacer length. 1,3-Disubstituted calix[4]arenes are inferior extractants to the corresponding tetrasubstituted ones. Narrow rim DGA-functionalized derivatives resulted in high extraction efficiencies, while the wide rim DGA-functionalized calix[4]arenes showed practically no extraction.

The synthesis of various organophosphorus ligands by combining different donor sites and their evaluation for the complexation of $\mathrm{An}(\mathrm{III}) / \mathrm{Ln}(\mathrm{III})$ is discussed in Chapter 6. Among the ligands with a glycolamide backbone, those with mixed amide and $\mathrm{P}=\mathrm{O}$ donor sites and a 190 
central oxygen or nitrogen atom, showed a reasonable extraction for $\mathrm{An}(\mathrm{III})$ and $\mathrm{Ln}(\mathrm{III})$. Ligands with a central oxygen atom exhibited selectivity towards Eu(III), and those with a central nitrogen atom towards $\mathrm{Am}(\mathrm{III})$. Ligands with $\mathrm{P}=\mathrm{S}$ donor sites and a glycolamide backbone did not show any reasonable extraction. Amongst the ligands with a malonamide backbone, a high extraction efficiency was observed for the ligand with electron-rich substituents on phosphorus, however, with almost no discrimination between Am(III) and $\mathrm{Eu}(\mathrm{III})$. The thermodynamics of the complexation of different ligands with $\mathrm{Eu}(\mathrm{III})$ was investigated by microcalorimetry.

Chapter 7 deals with the extraction of Am(III) from acidic feed solutions using a tripodal diglycolamide (T-DGA) and diglycolamide-substituted calix[4]arenes in room temperature ionic liquids (RTILs). The $D_{\mathrm{Am}}$ values for T-DGA decreased with increasing carbon chain length in the three studied RTILs, viz. $\left[\mathrm{C}_{\mathrm{n}} \mathrm{mim}^{+}\right]\left[\mathrm{NTf}_{2}{ }^{-}\right](\mathrm{n}=4,6,8)$, which was related to the solubility of the $\left[\mathrm{C}_{\mathrm{n}} \mathrm{mim}^{+}\right]$in the aqueous phase. The distribution behavior of $\mathrm{Am}$ (III) with calix [4] arene-4DGA (C4DGA) dissolved in $\mathrm{C}_{8} \mathrm{mim}^{+} \cdot \mathrm{PF}_{6}{ }^{-}$showed an improved extraction and separation efficiency. Apart from $\mathrm{Am}(\mathrm{III})$, the extraction of $\mathrm{Pu}(\mathrm{IV}), \mathrm{U}(\mathrm{VI}) \mathrm{O}_{2}, \mathrm{Eu}(\mathrm{III}), \mathrm{Sr}(\mathrm{II})$, and $\mathrm{Cs}(\mathrm{I})$ by T-DGA and C4DGA was investigated as well. The stoichiometry of the complexes was determined by slope analysis for both the T-DGA and C4DGA extractants. The use of RTILs led to selective separation of Am(III) from wastes containing a mixture of $\mathrm{U}(\mathrm{VI}) \mathrm{O}_{2}, \mathrm{Pu}(\mathrm{IV})$, and Am(III).

In Chapter 8 the synthesis of diglycolamide-functionalized ionic liquids (DGA-FILs) and CMPO-functionalized ionic liquids (CMPO-FILs) and their extraction behavior towards actinide and fission product ions is described. DGA-FILs exhibited exceptionally high distribution ratios for these metal ions in the order $\mathrm{Eu}(\mathrm{III})>\mathrm{Am}(\mathrm{III})>>\mathrm{Pu}(\mathrm{IV})>\mathrm{Np}$ (IV) with relatively insignificant extraction of $\mathrm{Cs}(\mathrm{I}), \mathrm{Sr}(\mathrm{II})$, and U(VI). CMPO-FILs dissolved in room temperature ionic liquids (RTILs) showed high distribution ratios for all tri-, tetra-, and hexavalent ions in the order $\mathrm{Pu}(\mathrm{IV})>\mathrm{U}(\mathrm{VI}) \mathrm{O}_{2}>\mathrm{Am}(\mathrm{III})>\mathrm{Eu}(\mathrm{III})$ with negligible extraction of $\mathrm{Cs}(\mathrm{I})$ and $\mathrm{Sr}(\mathrm{II})$. The excellent extraction properties of DGA-FILs and CMPO-FILs make them very suitable for long term applications in radioactive waste processing.

In conclusion, various ligands that were synthesized showed interesting extraction properties. For example, the diglycolamide derivatives (Chapter 3) showed an improvement over TODGA as they extract only at high nitric acid concentration and allow the stripping of An(III) at low nitric acid concentration. Diglycolamide-functionalized calix[4]arenes required only a very low ligand concentration as well as exhibited high $S F_{\text {Eu/Am }}$ values compared to 
TODGA. On the other hand, the hydrophilic tripodal ligands (Chapter 4) showed selectivity for Am(III). Combined properties of these ligands are of potential value in the "innovative SANEX" process aimed at $\mathrm{An}(\mathrm{III}) / \mathrm{Ln}$ (III) separation. However, the development of an extractant capable of selectively extracting An in a single extraction step still remains a challenge. The use of ionic liquids as medium and functionalized ionic liquids has demonstrated very encouraging results regarding the extraction efficiency as well as green alternatives of the conventional organic solvent systems. This opens up new avenues for the development of new, An-selective, N, S donor ligands as functionalized ionic liquids, which may yield optimized selectivities as well as favor the development of green separations in the nuclear fuel cycle. 


\section{Samenvatting en vooruitblik}

Het onderzoek beschreven in dit proefschrift betreft de synthese en evaluatie van nieuwe, potentiële liganden voor de complexering van actinide- en lanthanide-ionen uit kernafval. Deze liganden zijn bestudeerd zowel voor extractie van het radioactieve bulkafval als voor het verwijderen van actiniden (An) en lanthaniden ( Ln) uit de organische fase, als de laatste bewerkingsstap van kernafval. Het doel van het hier beschreven werk is voornamelijk de ontwikkeling van nieuwe liganden met een verbeterde scheidings- en extractie-efficiëntie. Scheiding van An en $\mathrm{Ln}$ is een grote uitdaging bij de opwerking van kernafval. De hoofddoelstelling van dit onderzoek is de scheiding van Ac en La met behulp van eenvoudige, volledig verbrandbare en makkelijk verkrijgbare liganden, voor het ontwikkelen van milieuvriendelijke extractiesystemen, die het huidige organisch oplosmiddelsysteem zouden kunnen vervangen.

In het eerste hoofdstuk wordt ingegaan op de huidige, mondiale bijdrage van kernenergie en haar rol als een mogelijke, toekomstige energiebron, die milieu- en klimatologische problemen gerelateerd aan fossiele brandstoffen vermijdt. Een beschrijving van de huidige verwerking van kernafval wordt gegeven, aangezien dit een van de hoofdproblemen is voor de uitbreiding van kernenergie als energiebron.

Hoofdstuk 2 begint met een korte beschrijving van de eigenschappen van An en Ln, gevolgd door een overzicht van verschillende An/Ln-liganden, die de afgelopen jaren voor verschillende processen ontwikkeld zijn. Vervolgens worden verschillende O-donerende liganden gepresenteerd voor de co-extractie van An(III) en Ln(III). Voor de scheiding van An(III) en Ln(III) worden liganden met zachte $\mathrm{N}$ - en S-donerende atomen beschreven en synergetische mengsels en wateroplosbare liganden voor de selectieve extractie van An(III). Tenslotte worden recente ontwikkelingen bediscussieerd in de toepassing van ionische vloeistoffen voor de behandeling van kernafval als een milieuvriendelijk alternatief voor oplosmiddel-gebaseerde systemen.

Hoofdstuk 3 behandelt de structurele modificatie van het $N, N, N^{\prime}, N^{\prime}$-tetraoctyldiglycolamide(TODGA-) skelet bestaande uit a) verlenging van de ketenlengte tussen de centrale etherzuurstof en de amidegroep van één naar twee koolstofatomen, b) additie van substituenten aan een of beide koolstofatomen tussen het centrale ether-zuurstofatoom en de amidegroep, c) vervanging van de centrale ether-zuurstof door een (gesubstitueerd) stikstofatoom, d) synthese 
van een rigide glycolamide. Het effect van de structurele modificaties van het ligand op het extractiegedrag van $\mathrm{Am}(\mathrm{III})$ en $\mathrm{Eu}(\mathrm{III})$ is bestudeerd bij verschillende salpeterzuurconcentraties. In de meeste gevallen bereikten de distributieverhoudingen $(D)$ van deze liganden niet de waarden van TODGA in het gehele zuurconcentratiegebied van 0.001-4 $\mathrm{mol} / \mathrm{L} \mathrm{HNO}_{3}$. Uitsluitend monomethyl-TODGA is vergelijkbaar met TODGA bij hoge $\mathrm{HNO}_{3^{-}}$ concentraties. Bij lage $\mathrm{HNO}_{3}$-concentraties daarentegen zijn de $D$-waarden van deze liganden veel lager, hetgeen voordelig kan zijn voor de daarop volgende terug-extractiestappen. Azatripodale liganden vertonen alleen bij lage $\mathrm{HNO}_{3}$-concentraties goede $D_{\mathrm{Am}}$-waarden. Scheidingsfactoren $\left(\mathrm{SF}_{\mathrm{Am} / \mathrm{Eu}}\right)$ van 8 en 11 zijn behaald voor aza-tripodale liganden gefunctionaliseerd met $n$-octyl- of 2-ethylhexylgroepen aan de amidestikstof.

In hoofdstuk 4 wordt de synthese van wateroplosbare liganden en hun complexeringsgedrag t.a.v. actiniden in oplosmiddelextracties bediscussieerd. De hydrofiele diglycolamide- (DGA-) derivaten, met een variërend aantal ethyleenglycoleenheden of een natriumacetaatgroep aan het amidestikstofatoom, laten bij verschillende $\mathrm{pH}$-waarden een afnemende terug-extractieefficiëntie zien bij een toenemend aantal ethyleenglycolgroepen. Onder de $\mathrm{P}=\mathrm{S}$-donerende liganden vertoont alleen het ligand met een malonamideskelet een hoge terug-extractieefficiëntie, echter zonder selectiviteit voor An(III). Onder de wateroplosbare liganden, te weten de amidederivaten van nitrilotriazijnzuur met $N, N$-dimethyl- en $N, N$ bis(hydroxyethyl)eenheden, laat de eerste een hoge selectiviteit voor An(III) over Eu(III) zien met een maximale scheidingsfactor van 11, terwijl het tweede ligand efficiënter het radionuclide in de waterige fase complexeert met een maximale scheidingsfactor van 5 . De trends in de extractie van verschillende liganden zijn bevestigd door de complexeringssterktes te bepalen m.b.v. microcalorimetrie.

Hoofdstuk 5 beschrijft het effect van multivalentie op de extractie van Am(III) en Eu(III) door diglycolamide-gefunctionaliseerde calix[4]arenen. Op de smalle rand van het calix[4]areen is de DGA-groep 1,3-di- of tetragesubstitueerd, met variërende afstanden tussen het zuurstof- en het amidestikstofatoom. Bovendien zijn de DGA-groepen ook gebonden aan de brede rand en aan beide randen van de calix[4]arenen, en tevens zijn de extractie-efficiënties t.a.v. Am(III) en Eu(III) vergeleken bij verschillende zuurgraden. De extractie- en scheidingsefficiënties zijn sterk afhankelijk van de $\mathrm{N}$-alkylsubstituent en de tussenliggende afstand. De extractie door 1,3-digesubstitueerde calix[4]arenen is slechter dan die door de overeenkomstige tetragesubstitueerde variant. Calix[4]arenen met DGA gefunctionaliseerd op de smalle rand 
hebben een hoge extractie-efficiëntie, in tegenstelling tot calix[4]arenen met DGA gefunctionaliseerd op de brede rand, waarmee vrijwel geen extractie plaatsvindt.

In hoofdstuk 6 is de synthese beschreven van verscheidene organofosforliganden, door het combineren van verschillende donorsites, en hun evaluatie t.a.v. de complexering van $\mathrm{An}(\mathrm{III}) / \mathrm{Ln}(\mathrm{III})$. Onder de liganden met een glycolamideskelet gaven de liganden met zowel amide- als $\mathrm{P}=\mathrm{O}$-donorsites en een centraal zuurstof- of stikstofatoom redelijke extracties van An(III) en Ln(III). Liganden met een centraal zuurstofatoom vertoonden enige selectiviteit voor $\mathrm{Eu}(\mathrm{III})$ en liganden met een centraal stikstofatoom voor $\mathrm{An}(\mathrm{III})$. Liganden met een $\mathrm{P}=\mathrm{S}$ donorgroep en een glycolamideskelet gaven geen noemenswaardige extractie. Voor liganden met een malonamideskelet en een elektronenrijke substituent op het fosforatoom werd een hoge extractie-efficiëntie gevonden, maar er was nauwelijks verschil tussen Am(III) en $\mathrm{Eu}(\mathrm{III})$. De thermodynamica van de complexering van verschillende liganden met Eu(III) is onderzocht met behulp van microcalorimetrie.

Hoofdstuk 7 beschrijft de extractie van Am(III) uit zure oplossingen, gebruikmakend van een tripodale diglycolamide (T-DGA) en diglycolamide-gesubstitueerde calix[4]arenen in ionische vloeistoffen bij kamertemperatuur (RTILs). De $D_{\mathrm{Am}}$-waarden voor T-DGA namen af met toenemende lengte van de koolstofketen in de bestudeerde RTILs, te weten $\left[\mathrm{C}_{\mathrm{n}} \mathrm{mim}^{+}\right]\left[\mathrm{NTf}_{2}{ }^{-}\right](\mathrm{n}=4,6,8)$. Dit is verklaard door middel van de afnemende oplosbaarheid van $\mathrm{C}_{\mathrm{n}} \mathrm{mim}^{+}$in de waterige fase. Het distributiegedrag van Am(III) met calix[4]areen-4DGA (C4DGA) opgelost in $\mathrm{C}_{8} \mathrm{mim}^{+} \cdot \mathrm{PF}_{6}^{-}$vertoonde een betere extractie- en scheidings-efficiëntie. Behalve voor Am(III) zijn ook de extracties van $\mathrm{Pu}(\mathrm{IV}), \mathrm{U}(\mathrm{VI}) \mathrm{O}_{2}, \mathrm{Eu}(\mathrm{III}), \mathrm{Sr}(\mathrm{II})$ en Cs(I) met T-DGA en C4DGA onderzocht. De stoechiometrie van de complexen met de T-DGA en C4DGA extractanten is bepaald m.b.v. een hellinganalyse. Het gebruik van RTILs kan leiden tot een selectieve scheiding van Am(III) uit kernafval dat een mengsel van $\mathrm{U}(\mathrm{VI}) \mathrm{O}_{2}, \mathrm{Pu}(\mathrm{IV})$ en Am(III) bevat.

In hoofdstuk 8 is de synthese van diglycolamide-gefunctionaliseerde ionische vloeistoffen (DGA-FILs) en CMPO-gefunctionaliseerde ionische vloeistoffen (CMPO-FILs) en hun extractiegedrag t.a.v. actiniden en ionen van splijtingsproducten beschreven. DGA-FILs vertoonden uitzonderlijk hoge distributieverhoudingen voor de verschillende metaalionen in de volgorde $\mathrm{Eu}(\mathrm{III})>\mathrm{Am}(\mathrm{III})>>\mathrm{Pu}(\mathrm{IV})>\mathrm{Np}(\mathrm{IV})$ met een te verwaarlozen extractie van $\mathrm{Cs}(\mathrm{I}), \mathrm{Sr}(\mathrm{II})$, en U(VI). CMPO-FILs opgelost in RTILs gaven hoge distributieverhoudingen voor alle tri-, tetra- en hexavalente ionen in de volgorde $\mathrm{Pu}(\mathrm{IV})>\mathrm{U}(\mathrm{VI}) \mathrm{O}_{2}>\mathrm{Am}(\mathrm{III})>$ $\mathrm{Eu}(\mathrm{III})$ met een verwaarloosbare extractie van $\mathrm{Cs}(\mathrm{I})$ en $\mathrm{Sr}(\mathrm{II})$. Vanwege de uitstekende 
extractie-eigenschappen van DGA-FILs en CMPO-FILs worden ze geschikt geacht voor de verwerking van kernafval op de lange termijn.

Concluderend kan er gesteld worden dat verschillende gesynthetiseerde liganden interessante extractieëigenschappen vertonen. Bijvoorbeeld de diglycolamidederivaten (hoofdstuk 3) laten een verbetering zien t.o.v. TODGA, omdat ze alleen extractie vertonen bij hoge salpeterzuurconcentraties en het strippen van An(III) mogelijk maken bij lage salpeterzuurconcentraties. Diglycolamide-gefunctionaliseerde calix[4]arenen hebben maar een lage ligandconcentratie nodig en geven hoge $\mathrm{SF}_{\mathrm{Eu} / \mathrm{Am}}$-waarden in vergelijking met TODGA. Anderzijds vertonen de hydrofiele tripodale liganden (hoofdstuk 4) selectiviteit voor Am(III), en als deze eigenschappen gecombineerd worden, kunnen ze van belang worden in het “innovative SANEX"-proces, dat zich richt op An(III)/Ln(III)-scheiding. De ontwikkeling van een extractant, die het mogelijk maakt om An in één enkele extractiestap te verwijderen, blijft echter nog een uitdaging. Het gebruik van gefunctionaliseerde ionische vloeistoffen en ionische vloeistoffen als oplosmiddel laat niet alleen veelbelovende resultaten zien voor de extractie-efficientië, maar biedt ook een groen alternatief voor de conventionele organisch oplosmiddelsystemen. Dit biedt mogelijkheden voor het ontwikkelen van nieuwe Anselectieve, N,S-donorliganden als gefunctionaliseerde ionische vloeistoffen, hetgeen kan resulteren in verhoogde selectiviteiten. Dit is ook een stap in de richting van milieuvriendelijke scheidingen in de kernafvalcyclus. 


\section{Acknowledgements}

Finally I finished my thesis, and I would like to thank many people for their help and support during these four years. I am very proud of the fact that now it is time to write the most important part of the thesis. It is not easy to summarize all my acknowledgements in a few words, but just I can say that I am really grateful to all people I met during my $\mathrm{PhD}$.

First of all, I express my sincere gratitude to my promotor and supervisor, Jurriaan. I thank you for giving me the opportunity to work as a PhD student and to be part of the MnF group. I have learnt so much from you in our work meetings and from your critical comments on the manuscripts and the chapter of the thesis. During meetings you always gave me lot of confidence, encouragement, and freedom. I am grateful for your supervision and guidance by raising crucial points, giving expert opinions and valuable comments during the whole thesis period. Thank you very much for everything you did for me.

I would like to acknowledge my daily supervisor, Wim, for giving me a chance to complete my master project, and then start my $\mathrm{PhD}$. It was a fantastic experience doing a $\mathrm{PhD}$ with you. You have spent maximum time for highly fruitful meetings as well as for correcting the chapters and manuscripts. Wim, thank you very much for being so kind, patient, and supportive as well as being always reachable even after office times via e-mails. The Monday morning 9 o'clock (earlier was also OK for you, but not for me) meetings worked as a catalyst to finish my $\mathrm{PhD}$ in time. In addition to that, your passion for chemical news on a daily basis also worked well. One of the most important things that I have learnt from you is to keep everything and everyone under control $:$.

During my PhD I had a chance to collaborate with other groups. I am really grateful to Dr. Modolo, Michal, Jana, and Andreas in Jülich, Germany for their nice collaboration. I would also like to thank Dr. Mohapatra and his team (Mumbai, India) for a very fruitful collaboration resulting in many joint publications. 
I would also like to thank all the staff members, Pascal, Jeroen, Melissa, and Natalie for discussions, nice questions and suggestions during presentations and meetings. My special "thanks" goes to Melissa also for proof reading my thesis and going so carefully through it. I would like to thank Regine, Marcel, Richard, Bianca, and Tieme for their technical support. The secretaries of MnF/BNT, Izabel and Nicole, are also acknowledged for taking care of all administrative work.

I will never forget our subgroup meetings where we had very nice discussions, and sometimes we enjoyed so much, the very special humor of Wim. I would like to thank all the brothzers and shisters of our cluub i.e. Roberto, Rajesh, Xumei, Nicolai, Laura, Vishwas, and Andrea. Thank you guys for the very nice times.

I would like to thank Deniz. We had nice times, nice discussions, and dinners. Deniz, you were always in the Lab on weekends and we could work together. I am also thankful for giving me a chance to be your paranimf so that I could practice the defense process. Carmen and Roberto, special thanks for being my paranimfs and for the organizational part of my defence. I cannot forget to acknowledge a very nice and gentle, but sometimes a bit aggressive lady, commonly known as Jealemy. Dear Jealemy, in your absence the lab looks like dead and if you are here we think a full crowd of people is there. I will remember our bullshit discussions on scientific, social, cultural, and political topics. Thanks to Albert for taking care of the invention of some new words in the lab and making a dictionary of it. Dear Albert, we are really missing the taste of people from the southz in the lab. My special thanks go Carmen (HC), she is extremely organized (like a real german) and still she is very outgoing and maybe too loud for a typical german lady. We had a lot of fun in our group outings. Your typical laughter style (of very high intensity) is something unforgettable, making the things most funny. Taking care, while walking, will be helpful for you and the rest of the people $:$. I also thank you, Rick, and Sven for translating my summary from English to Dutch. Another person to be acknowledged is Anna (banana). Anna I really appreciate you being friendly and helpful but sometimes.... Thanks for making the rest of the lab see that you are a relaxed, Pakistan friendly person, and of course for staying during the weekends so I could work, as 'required' by Wim. I would also like to acknowledge Richard and Neelish for doing their master and bachelor research projects, respectively with me. During my PhD, I have had the opportunity to meet a lot of fantastic people. I am thankful to Raquel (I will remember your very nice pronunciations of some words), Emanuella, Tibor, Kim, Jordi, 
Carlo, Pieter, Alijandro, Ina, Raluca, Jasper, Jenny, Rick, Liang, Bettina, Tomb, Zhen, Jiguand, Tushar, Alxis, Timon, Sirikrishnan, Sarah, Anne, Supitchaya, Rik, Martijn, Rindia, Xing Yi, Duan, Henk, Riccardo, Janet, Francesca, Ignacio, Andras, Huaping, Yiping, Arancha, Lanti, Peter, Alberto, Melanie, Vijay, Chien-Ching Wu, Martin, Christine, Dae June, Oya, Oktay, and many more... All the people I mentioned here have contributed to making the time I spent in Enschede an unforgettable time of my life.

I am grateful to the Pakistani friends Akram raza, Tauseef, Yawar, Salman, Rahim, Hammad Nazeer, Sohail Niazi, Amir, Khuram, Tamoor, Siafuallah, Adeel, Saqib, Nasir, Shujaa, Irfan Zafar, Irfan Ali, Abdulgani, Tariq Abbasi, Naveed Khalid, Kazmi, and all others for making my stay here memorable by cultural festivals and cricket matches. My special thanks to Abid for having very nice times, during your visits to Enschede.

I would like to thank and acknowledge the Higher Education Commission (HEC) of Pakistan for the financial support of my studies.

Finally, I would like to express my cordial gratitude to my family, especially my loving parents, for their unconditional love, care, and support throughout my life. I dedicate this thesis to my respectable parents whose prayers are a source of inspiration and confidence for me.

Mudassir Iqbal

August, 2012

Enschede 



\section{List of Publications}

1. M. Iqbal, J. Huskens, M. Sypula, G. Modolo, and W. Verboom, Synthesis and Am/Eu extraction of novel TODGA derivatives, Supramol. Chem. 2010, 22, 827.

2. P. K. Mohapatra, M. Iqbal, D. R. Raut, W. Verboom, J. Huskens, and V. K. Manchanda, Evaluation of a novel tripodal diglycolamide for actinide extraction: Solvent extraction and SLM transport studies, J. Membr. Sci. 2011, 375, 141.

3. M. Iqbal, J. Huskens, M. Sypula, G. Modolo, and W. Verboom, Synthesis and evaluation of novel water-soluble ligands for the complexation of metals during the partitioning of actinides, New J. Chem. 2011, 35, 2591.

4. P. K. Mohapatra, M. Iqbal, D. R. Raut, W. Verboom, J. Huskens, and S. V. Godbole, Complexation of novel diglycolamide functionalized calix[4]arenes: Unusual extraction behaviour, transport, and fluorescence studies, Dalton Trans. 2012, 41, 360.

5. A. Sengupta, P. K. Mohapatra, M. Iqbal, J. Huskens, and W. Verboom, A highly efficient solvent system containing functionalized diglycolamides and an ionic liquid for americium recovery from radioactive wastes, Dalton Trans. 2012, 41, 6970.

6. P. K. Mohapatra, M. Iqbal, D. R. Raut, J. Huskens, and W. Verboom, Unusual transport behaviour of actinide ions with a novel calix[4]arene-tetra-diglycolamide (C4DGA) extractant as the carrier, J. Membr. Sci. 2012, 411-412, 64.

7. M. Iqbal, P. K. Mohapatra, S. A. Ansari, J. Huskens, and W. Verboom, Preorganisation of TODGA on tripodal and calix[4]arene platforms for efficient separation of An(III) from nuclear waste streams, Tetrahedron 2012, 68, 7840.

8. A. Sengupta, P. K. Mohapatra, M. Iqbal, W. Verboom, J. Huskens and S. V. Godbole, Extraction of Am(III) using a tripodal diglycolamide in room temperature ionic liquids: A 'green' approach for radioactive waste processing, RSC Adv. 2012, 2, 7492. 
9. M. Iqbal, R. G. Struijk, J. Huskens, M. Sypula, A. Wilden, G. Modolo, and W. Verboom, Synthesis and evaluation of ligands with mixed amide and phosphonate, phosphinoxide, and phosphonothioate sites for $\mathrm{An}(\mathrm{III}) / \mathrm{Ln}(\mathrm{III})$ extraction, New J. Chem. 2012, 36, 2048.

10. S. D. Reilly, A. J. Gaunt, B. L. Scott, G. Modolo, M. Iqbal, W. Verboom, and M. J. Sarsfield, Plutonium(IV) complexation by diglycolamide ligands - coordination chemistry insight into TODGA-based actinide separations, Chem. Commun. 2012, 48, 9732.

11. S. A. Ansari, P. K. Mohapatra, M. Iqbal, W. Verboom, and J. Huskens, Novel diglycolamide functionalized calix[4]arenes for actinide extraction and supported liquid membrane studies: Part II. Role of substituents in the pendent arms and mass transfer modeling. (submitted)

12. A. Sengupta, M. Iqbal, W. Verboom, J. Huskens, and P. K. Mohapatra, Highly efficient novel diglycolamide-functionalized ionic liquids: Synthesis, extraction, radiation stability and fluorescence studies. (submitted)

13. A. Sengupta, P. K. Mohapatra, M. Iqbal, J. Huskens, S. V. Godbole, and W. Verboom, A highly efficient novel solvent system consisting of an octa-DGAfunctionalized calix[4]arene in a $\mathrm{C}_{8}$-ionic liquid: actinide extraction, radiolytic stability, and fluorescence studies. (submitted)

14. X. Wang, M. Iqbal, J. Huskens, and W. Verboom, Off-on fluorescent chemosensor for $\mathrm{Hg}^{2+}$ based on bis(rhodamine) in aqueous solution. (To be submitted)

Three more manuscripts are in preparation. 


\section{Curriculum Vitae}

Mudassir Iqbal was born on $1^{\text {st }}$ March 1980 in Sargodha, Pakistan. He obtained his Bachelor's degree from the University of Punjab, Pakistan. He studied Chemistry at the University of Sargodha, Pakistan, and he was awarded his Master's degree (M.Sc) with specialization in Organic Chemistry in 2005. Subsequently, he started his M.Phil degree at the University of Sargodha, where he completed the course work, while the required research work was carried out at the University of Twente, The Netherlands. He completed his M.Phil degree in January 2009 with a thesis entitled "Design and synthesis of novel ligands for the separation of actinides from nuclear waste."

In February 2009 he started as a Ph.D student at the University of Twente in the Molecular Nanofabrication group of Prof. Jurriaan Huskens with Dr. Willem Verboom as a supervisor. His research work was focused on the development of different types of ligands for the separation of actinides/lanthanides for nuclear waste processing. The results of his research work are described in this thesis. 